\title{
Impacts of developmental exposures to the harmful algal bloom toxin domoic acid on neural development and behavior
}

\author{
By \\ Jennifer Martinez Panlilio
}

B.A. in Anthropological Sciences, Stanford University, 2009

M.A. in Anthropology, Stanford University, 2010

B.S. in Biology and Marine Science, University of Miami, 2013

Submitted in partial fulfillment of the requirements for the degree of Doctor of Philosophy at the

MASSACHUSETTS INSTITUTE OF TECHNOLOGY and the WOODS HOLE OCEANOGRAPHIC INSTITUTION June 2019

(C) 2019 Jennifer Martinez Panlilio. All rights reserved.

The author hereby grants to MIT and WHOI permission to reproduce and to distribute publicly paper and electronic copies of this thesis document in whole or in part in any medium now known or hereafter created.

Signature of Author

Joint Program in Oceanography/Applied Ocean Science and Engineering Massachusetts Institute of Technology and Woods Hole Oceanographic Institution March 22, 2019

Certified by

Dr. Mark E. Hahn

Senior Scientist

Woods Hole Oceanographic Institution

Thesis Supervisor

Certified by

Dr. Neelakanteswar Aluru

Associate Scientist

Woods Hole Oceanographic Institution

Thesis Supervisor

Accepted by

Dr. Mick Follows

Chair, Joint Committee for Biological Oceanography Massachusetts Institute of Technology 


\title{
Impacts of developmental exposures to the harmful algal bloom toxin domoic acid on neural development and behavior
}

\author{
By \\ Jennifer Martinez Panlilio \\ Submitted to the MIT-WHOI Joint Program in Oceanography and Applied Ocean Science and \\ Engineering on March 22, 2019 in partial fulfillment of the requirements for the degree of \\ Doctor of Philosophy
}

\begin{abstract}
Harmful algal blooms (HABs) can produce potent neurotoxins that accumulate in seafood and affect human health. One HAB toxin of concern is domoic acid (DomA), a glutamate analog produced by the marine diatom Pseudo-nitzschia spp. Current regulatory limits are designed to prevent acute neurotoxicity in adult humans. However, research shows that low-level exposure during early life can lead to long-term changes in behavior, neural connectivity, and brain morphology. To determine the underlying mechanisms of developmental toxicity, this dissertation used zebrafish as a tool to: i) Establish the developmental window of susceptibility for DomA toxicity, ii) Characterize the behavioral consequences of exposures, and iii) Identify the cellular targets and processes perturbed by DomA. I found that DomA exposure particularly at 2 days post fertilization (dpf) led to altered startle response behavior, myelination defects, and the downregulation of axonal and myelin structural genes. Using vital dyes and immunolabeling, I assessed DomA-induced alterations in cells required for the startle response. I found no differences in the number of sensory neuromasts or in the sensory cranial ganglia structures that detect the acoustic stimuli. However, the majority of DomA-treated larvae lacked one or both Mauthner cells - hindbrain neurons critical for fast startle responses. DomA-treated larvae also had oligodendrocytes with fewer and shorter myelin sheaths, and appeared to aberrantly myelinate neuronal cell bodies. The loss of the Mauthner neurons and their axons may lead to a cellular environment where oligodendrocytes myelinate neuronal cell bodies in the absence of adequate axonal targets. Indeed, pharmacological treatment that reduced the oligodendrocyte number also led to the reduction in the number of these aberrant, myelinated cell bodies. These results indicate that exposure to DomA at a particular period in neural development targets specific cell types, disrupts myelination in the spinal cord, and leads to prolonged behavioral deficits. These mechanistic insights support hazard assessments of DomA exposures in humans during critical periods in early development.
\end{abstract}

Thesis Supervisors:

Dr. Mark E. Hahn

Senior Scientist in Biology

Woods Hole Oceanographic Institution

Dr. Neelakanteswar Aluru

Associate Scientist in Biology

Woods Hole Oceanographic Institution 


\section{FUNDING}

This work would simply not be possible without many generous funding sources. Funding for my research came from the Ocean Ventures Fund, Hill family foundation, Woods Hole Sea grant NA14OAR4170074, and the Woods Hole Center for Oceans and Human Health (COHH), which is jointly funded by the National Institutes of Health (P01ES02192, P01ES028938), and the National Science Foundation (OCE-1314642, OCE-1840381).

My funding came from the National Institutes of Health (NIH) P01ES021923-04S1, the Ocean Ridge Initiative Fellowship, the Von Damm Fellowship, and the MIT/WHOI Joint Program Academic Programs Office.

\section{ACKNOWLEDGMENTS}

This dissertation is the result of the support from a whole community of people.

To my advisors, Mark Hahn and Neel Aluru, who treated me like a colleague. I am immensely grateful for the latitude you gave me to explore ideas, build new devices, and forge my own path. I really grew into a scientist with this independence. Your thoughtfulness and deep kindness were especially critical when balancing life inside and out of the lab.

To my thesis committee, including Eduardo Rosa-Molinar and Hazel Sive. Ed, I began using startle behavior because of your advice to follow a more tractable behavior. Hazel, thank you for your critical feedback on ways to harness the zebrafish model, and for helping me identify crucial developmental processes in fish.

To Ann Tarrant who was not only my thesis defense chair, but also the chair for my general exam: thank you for your thoughtfulness, quick feedback, and reassurances when hiccups happened. These all made this process feel attainable.

I began science as a second career path, and I am grateful for the opportunities I was given by my undergraduate advisers at the University of Miami, Martin Grosell and Danielle McDonald. Thank you for introducing me to the toxicology world, treating me like a colleague, and pushing me to think critically about my experiments. Peter O'Day directed the summer undergraduate program that introduced me to zebrafish. It was in the program that I first truly knew that I wanted to explore science as a career. Aurélie Clément was my direct mentor in the program, who had the unique combination of being patient when things went awry, while still being relentless in her high expectations for me.

To the zebrafish community, particularly the Plavicki, Appel, Monk, Kucenas, Marsden, Lyons, Grunwald, Burgess, Kauffman, and Zon labs: my work would simply not be possible without your generosity in sharing transgenic lines, plasmids, and analysis software. It is due to your generosity that I have felt at home in the zebrafish community.

The WHOI Biology department is filled with brilliant people. To Andy Solow and Hanny Rivera, you were immensely helpful in selecting statistical tests and interpreting my results. Many thanks to Matt Salanga for teaching me how to clone, Ian Jones for calibrating my startle set-up, and to Nadja Brun for helping optimize my imaging. 
The MIT-WHOI Academic Programs Office has provided me with support through the years. A special thank you to Julia Westwater, Kris Kipp, Ronni Schwartz, Lea Fraser, Jim Yoder, Meg Tivey, and Christine Charette. You have made navigating graduate school so much easier.

To other members of the Tox group, including Jed Goldstone, John Stegeman, Sibel Karchner, Judy Luborski, and Rita Garrick. Thank you for your insights on my research, and for exposing me to different approaches in toxicology. To Diana Franks, thank you for keeping my fish safe.

To the members of the Woods Hole Center for Oceans and Human Health. My day-to-day scientific world was bound to the zebrafish and a lab bench. Being part of the COHH allowed me to place my work in the broader context of oceans and the organisms that produce the toxin I work on. Thank you for giving me this context, along with years of advice on my work.

To the MBL imaging facility, particularly Louis Kerr and Kasia Hammar. I am grateful for your patience in training me and working with me to address issues with the microscopes. There was no better person to share a giggle when looking at spinal cords than you, Kasia.

This work would not be possible without the support of Matt Salanga, Rebecca Helm, Nadja Brun, Keegan Krick, Rene Francolini, Francisco Mora, and Evie Fachon. Thank you all for being fantastic colleagues and friends. I could not think of better group of people to celebrate and moan every. single. experiment with. Your brilliant insights made me design better experiments, try new techniques in lab, and clarify confusing results. Your encouragement motivated me especially during times when optimizing experiments felt endless.

To my friends in Woods Hole - Megan, Laura, Rebecca, Hanny, Nadja, Rene, Evie, Casey, Cris, and Jenny. Thank you from the bottom of my heart. I will never forget the times you brought me "power snacks," started impromptu concerts and ran into flooded basements to save my artwork. I am so lucky to have you, and I cannot wait to see all the magic you create in the world.

To my friends from afar - Rebecca, Teresa, Munika, Elisa, and Maria. Thank you for all your incredible encouragement. For coming to visit when you could, for sending me supportive little notes when things were hard, and for opening your homes when it was time for a break from lab.

To my family, who gave me all the support in the world. To my dad, who introduced me to tidepools and provided me with access to the ocean, where I first fell in love with fish. To my mom, who gave me the courage to switch careers and pursue the sciences. To my sister, the first scientist in the family, who has been there since day one, from editing the first paper I ever wrote in undergrad to most recently being a home when I needed a safe place to regroup.

To my spouse, Ben. Thank you for patiently waiting outside when experiments invariably went over time, for fuddling about building experiments with me, for editing just about every piece of writing I have ever produced. Thank you for being my partner, and thank you for keeping our household together, especially towards the end. And, of course, to my dogs - Adobo and Potato, who always took advantage of the most opportune moments to either cheer me up or to compound an existing crisis. 


\section{TABLE OF CONTENTS}

Chapter 1: Introduction

1.1 Harmful algal blooms and their impacts of human health $16-17$

1.2 Human Exposures to Domoic acid $17-20$

1.3 Molecular mechanisms of Toxicity $20-22$

1.4 Toxicokinetics of Domoic acid $22-26$

1.5 Acute toxicosis from high-dose exposures to Domoic acid 26

1.6 Neurotoxicity from chronic exspoures to Domoic acid $27-28$

1.7 Domoic acid as a developmental neurotoxin 28-33

1.8 Dissertation rationale and overall approach $33-40$

1.9 Research impact

1.10 References 57-68

Chapter 2: Domoic acid as a developmental neurotoxin: critical exposure periods, structural phenotypes and behavioral effects

69-159

2.1 Graphical Abstract 69

2.2 Abstract 70

2.3 Introduction $71-73$

2.4 Methods 73-82

2.5 Results $82-91$

2.6 Discussion 91-101

2.7 Conclusion 101

2.8 References $154-160$

Chapter 3: Developmental exposure to domoic acid disrupts startle response behavior and circuitry 161-221

3.1 Graphical Abstract 161

3.2 Abstract 162

3.3 Introduction $163-165$

3.4 Methods $165-173$

3.5 Results $173-179$

3.6 Discussion 179-184

3.7 Conclusion 184

3.8 References 218-221 
Chapter 4: Developmental exposure to domoic targets specific neuronal populations and leads to aberrant myelination in the spinal cord

4.1 Graphical Abstract

4.2 Abstract $224-225$

4.3 Introduction 225- 227

4.4 Methods $227-234$

4.5 Results $234-241$

4.6 Discussion 241-244

4.7 Conclusion 244

4.8 References $268-272$

Chapter 5: Conclusions and Future Directions 273-288 Appendix: Public Thesis Synopsis 289-318 


\section{List of Figures}

\section{Chapter 1: Introduction}

Figure 1 Trophic transfer of DomA through the marine food web $\quad$ p. 42

Figure 2 Zebrafish startle response circuit is well-characterized and the p. 43 behavior is quantifiable

$\begin{array}{lll}\text { Figure } 3 \text { Dissertation approach } & \text { p. } 44\end{array}$

Chapter 2: Domoic acid as a developmental neurotoxin: critical exposure periods, structural phenotype and behavioral effects

Figure 1 Domoic acid-exposed larvae have morphological and acute p. 103 neurotoxic phenotypes that vary based on exposure time and dose

Figure 2 Domoic acid-exposed larvae at 2 dpf are less responsive to p. 104 auditory/vibrational stimuli

Figure 3 Exposure to domoic acid at 2 dpf (but not 1 or $4 \mathrm{dpf}$ ) alters p. 105 startle response bend angle at the lowest doses

Figure 4 Exposure to domoic acid doses at 2 dpf (but not 1 or 4 dpf) p. 106-107 consistently alters SLC startle response kinematics.

Figure 5 Exposure to domoic acid doses at 2 dpf (but not 1 or 4 dpf) p. 108-109 consistently alters LLC startle response kinematics.

Figure 6 Exposure to domoic acid at 2 dpf (but not 1 or $4 \mathrm{dpf}$ ) alters p. 110-111 myelin sheaths at $5 \mathrm{dpf}$

Figure 7 Exposures to domoic acid between 2- 2.5 dpf alters myelin p. 112-113 sheaths at $5 \mathrm{dpf}$

Figure $8 \quad$ Myelin sheath labeling defects persist until at least 7 dpf. p. 114-115

Figure 9 Domoic acid perturbs the initial formation of myelin sheaths p. 116

Figure 10 Domoic acid exposures at $2 \mathrm{dpf}$ lead to modest transcriptional p. 118-119 changes

Figure 11 Domoic acid exposures at 2 dpf leads to reduced expression of p. 120 key axonal and myelin structural proteins 


\section{Chapter 2: Supplemental figures}

Figure 1 Startle behavioral apparatus and behavioral classification p. 146

Figure 2 Startle kinematics and myelin sheath imaging of fish used for p. 147 RNASeq

$\begin{array}{lll}\text { Figure } 3 \quad \text { Qualitative myelin phenotypes } & \text { p. } 148\end{array}$

\section{Chapter 3: Developmental exposure to domoic acid disrupts startle response behavior and circuitry}

Figure 1 Domoic acid-exposed larvae are less responsive and preferentially perform LLC startles compared to controls when given auditory/vibrational stimuli

Figure 2 Domoic acid-exposed larvae have reduced bend angles and maximal angular velocities across all stimulus intensities tested when given auditory/vibrational stimuli

Figure 3 Kinematics deficits in LLC auditory/vibrational startle are correlated to myelin defects

Figure 4 Kinematics deficits in SLC auditory/vibrational startle are correlated to myelin defects

Figure 5 Domoic acid-exposed larvae have aberrant startle responses to direct electrical stimulation

Figure 6 Severity in myelin defects is correlated with startle kinematic deficits from direct electric field stimulation

Figure 7 The sensory inputs required for the startle response appeared intact in domoic acid-exposed fish

Figure 8 The majority of reticulospinal neurons required for startle responses are absent in domoic acid-exposed larvae

Figure 9 The majority of DomA-exposed larvae do not have Mauthner cells but have other hindbrain and midbrain structures

p. 203

Figure 10 Domoic acid exposure reduces axon collateral branching in primary motor neurons

Figure 11 DomA does not appear to reduce the overall population of
dorsal spinal neurons or neural precursors

Figure 11 DomA does not appear to reduce the overal
dorsal spinal neurons or neural precursors

p. 204-205

p. 206 


\section{Chapter 3: Supplemental figures}

$\begin{array}{lll}\text { Figure } 1 & \text { Startle behavioral apparatus and behavioral classification } & \text { p. } 213\end{array}$

Figure 2 SLC and LLC kinematics from replicate stimuli p. 214-215

$\begin{array}{lll}\text { Figure } 3 & \text { Range of classified myelin phenotypes } & \text { p. } 216\end{array}$

$\begin{array}{lll}\text { Figure } 4 & \text { Range of 3A10 staining observed in control larvae } 217\end{array}$

Chapter 4: Developmental exposures to domoic targets specific neuronal populations and leads to aberrant myelination in the spinal cord

Figure 1 Exposure to DomA at $2 \mathrm{dpf}$ did not reduce the number of $\quad$ p. 246-247 oligodendrocyte precursor cells prior to myelination

Figure 2 Exposure to DomA at $2 \mathrm{dpf}$ reduces the number of myelinating

p. $248-249$ oligodendrocytes in fish with severe myelin defects, and in a dose dependent manner

Figure 3 DomA reduces the length and number of myelin sheaths

p. $250-251$ produced by individual oligodendrocytes by $4 \mathrm{dpf}$

Figure 4 Exposure to DomA at $2 \mathrm{dpf}$ (but not $1 \mathrm{dpf}$ ) leads to the loss of the Mauthner neuron prior to myelination

Figure 5 Exposure to DomA at $2 \mathrm{dpf}$ does not alter selected sensory neuron structures or the main axons of two of the three primary motor neurons

Figure 6 DomA exposure at $2 \mathrm{dpf}$ leads to the formation of aberrant circular profiles that may be ectopically myelinated neuronal cell bodies

Figure 7 Treatment with GANT61, a small molecule that reduces the number of myelinating oligodendrocytes, also reduces the number of circular profiles in DomA treated fish

\section{Chapter 4: Supplemental figures}

Figure 1 Trial differences in oligodendrocyte precursor cell counts

p. 260

Figure 2 Trial differences in myelinating oligodendrocyte cell counts

p. $262-263$

Figure 3 Trial differences in Mauthner cell counts

p. 265

Figure 4 Trial differences in GANT61 treatment

p. $266-267$

\section{Chapter 5: Conclusions and Future Directions}

Figure 1 Research Summary

p. 281

Figure 2 Proposed model 1

p. 282

Figure 3 Proposed model 2

p. 283 


\section{List of Tables}

\section{Chapter 1: Introduction}

Table 1 Human health effects associated with exposures to different HAB p. 45 toxins

Table 2 Acute behavioral toxicity in different animal models following p. 46 intravenous (i.v.) exposures to DomA

Table 3 Acute behavioral toxicity in different animal models following p.47 intraperitoneal (i.p.) or intracoelemic (i.c.) exposures to DomA

Table 4 DomA toxicity from a single exposure to DomA intraperitoneally p. 48 in rats

Table 5 Behavioral effects from prenatal exposures to DomA

p. $49-50$

Table 6 Histological and neural activity endpoints from prenatal, p. 51 subcutaneous exposures to DomA in rats

Table 7 Behavioral effects from postnatal subcutaneous exposures to DomA in Sprague-Dawley rats

Table 8 Histological and neural activity endpoints from postnatal, subcutaneous exposures to DomA in rats

p. $54-55$

Table 9 Zebrafish transgenic lines used in the dissertation p. 56

Table 9 Zebrafish transgenic lines used in the dissertation p. 56

Chapter 2: Domoic acid as a developmental neurotoxin: critical exposure periods, structural phenotype and behavioral effects

Table 1 Percent mortality in $T g(m b p: E G F P-C A A X)$ larvae exposed to DomA by intravenous injection

Table 2 Mortality in $\operatorname{Tg}(\operatorname{sox} 10: m R F P)$ larvae exposed to DomA by intravenous injection

Table 3 Trials included for the swim bladder analysis

Table 4 Opaque brains in $T g(m b p: E G F P-C A A X)$ larvae exposed to DomA by intravenous injection

Table 5 Acute neurological phenotypes following developmental p. 125 exposures to DomA at $1 \mathrm{dpf}$

Table 6 Acute neurological phenotypes following developmental exposures to DomA at $2 \mathrm{dpf}$

Table 7 Acute neurological phenotypes following developmental exposures to DomA at $4 \mathrm{dpf}$

Table 8 Model coefficients for the prevalence of the lack of touch 
responses for fish exposed at $1 \mathrm{dpf}$ and $2 \mathrm{dpf}$

Table 9 Model coefficients for the prevalence of the convulsions and p. 129 pectoral fin flapping for fish exposed at $1 \mathrm{dpf}$ and $2 \mathrm{dpf}$

Table 10 Post-hoc pairwise Dunnett comparisons following binomial p. 130 modeling of percent responsiveness in startle behavior

Table 11 Nonparametric analysis of SLC kinematics following exposure p. 131 to different doses of DomA at $2 \mathrm{dpf}$

Table 12 Nonparametric analysis of LLC kinematics following exposure p. 132 to different doses of DomA at $2 \mathrm{dpf}$

Table 13 Median and Interquartile range for startle kinematic parameters p. 133 of fish exposed to different doses of DomA at $2 \mathrm{dpf}$

Table 14 Nonparametric analysis of SLC kinematics following exposure p. 134 to different doses of DomA at $1 \mathrm{dpf}$

Table 15 Nonparametric analysis of LLC kinematics following exposure p. 135 to different doses of DomA at $1 \mathrm{dpf}$

Table 16 Nonparametric analysis of SLC kinematics following exposure p. 136 to different doses of DomA at $4 \mathrm{dpf}$

Table 17 Nonparametric analysis of LLC kinematics following exposure p. 137 to different doses of DomA at $4 \mathrm{dpf}$

Table 18 Trials included to assess myelin labeling, imaged using confocal p. 138 microscopy at 3 and $5 \mathrm{dpf}$

Table 19 Trials included to assess myelin labeling, imaged using p. 139 widefield epifluorescence microscopy at $5 \mathrm{dpf}$

Table 20 Trials included to assess myelin labeling, imaged using p. 140 widefield microscopy at $6 \mathrm{dpf}$

Table 21 Trials included to assess myelin labeling, imaged using p. 141 widefield microscopy at $7 \mathrm{dpf}$

Table 22 Multinomial logistic regression model for the distribution of p. 142 myelin phenotypes in fish exposed to $0.14 \mathrm{ng}$ of DomA at different periods in development

Table 23 Multinomial logistic regression model for the distribution of myelin phenotypes in fish exposed to different doses of DomA at $2 \mathrm{dpf}$ and imaged at 5, 6, and $7 \mathrm{dpf}$

Table 24 Genes associated with the enriched GO term: biological p. 144 processes

Table 25 Human phenology phenotypes associated with differentially p. 145 expressed genes at $3 \mathrm{dpf}$ 


\section{Chapter 2: Supplemental Tables}

Table 1 Developmental time ranges in hours post fertilization (hpf) for fish p.149 included in each injection category

Table 2 Differentially expressed genes in DomA-exposed fish at 3 dpf (28 p.150-152 hours post exposure)

Table 3 Differentially expressed genes in DomA-exposed fish imaged at 7 p.153 $\mathrm{dpf}$ (exposed at $5 \mathrm{dpf}$ then imaged at $7 \mathrm{dpf}$ )

Chapter 3: Developmental exposure to domoic acid disrupts startle response behavior and circuitry

Table 1 Percentage of fish that performed LLCs, SLCs, or did not respond within a given treatment and to a selected stimulus intensity

Table 2 Multinomial logistic regression model for distribution of startle behaviors in DomA and control fish when given a $32 \mathrm{~dB}$ stimulus

Table 3 Multinomial logistic regression model for distribution of startle behaviors in DomA and control fish when given a $38 \mathrm{~dB}$ stimulus

Table 4 Multinomial logistic regression model for distribution of startle behaviors in DomA and control fish when given an $41 \mathrm{~dB}$ stimulus

$\begin{array}{lll}\text { Table } 5 \text { Multinomial logistic regression model for distribution of startle } & \text { p. } 208\end{array}$ behaviors in DomA and control fish when given a $43 \mathrm{~dB}$ stimulus

Table 6 Multinomial logistic regression model for distribution of startle behaviors in DomA and control fish that are subcategorized by myelin phenotype (from a $43 \mathrm{~dB} \mathrm{~A} / \mathrm{V}$ stimulus)

Table 7 Nonparametric multiple comparison tests for SLC startle kinematics by stimulus intensity

Table 8 Nonparametric multiple comparison tests for LLC startle kinematics by stimulus intensity

Table 9 Nonparametric multiple comparison tests for startle kinematics p. 212 following direct electric field stimulation 
CHAPTER 1

Introduction 


\subsection{HARMFUL ALGAL BLOOMS AND THEIR IMPACTS ON HUMANS}

Harmful algal blooms (HABs) are the mass accumulation of algae, defined by their adverse societal impacts rather than by any strict scientific definition. ${ }^{1,2}$ The term 'harmful' encompasses direct impacts on human health as well as threats to socioeconomic interests or to marine ecosystems that humans rely on.

HABs pose a real threat to food security and water quality. HABs can accumulate in seafood, making it unsafe for humans to eat. ${ }^{3-6}$ Freshwater cyanobacterial HABs can produce toxins and contaminate drinking water supplies. For example, in the 2014 event called the 'Toledo Water Crisis,' a massive Microcystis bloom in Lake Erie rendered the municipal water supply undrinkable for half a million people for 2 days. ${ }^{7,8}$ Even non-toxic coastal HABs can clog water intake systems, disrupting operations at water desalination plants in regions such as the United Arab Emirates where freshwater sources are limited. ${ }^{9,10}$

While some HABs are non-toxic, their sheer biomass (sometimes in the millions of cells per liter) can kill wildlife, damage marine ecosystems, and lead to economic losses. ${ }^{11}$ HABs can clog fish gills leading to mortality of commercially important fish. ${ }^{12,13}$ Algal mats can accumulate in recreational areas and lead to losses in tourism revenue. ${ }^{14,15}$ Finally, when blooms decay, they can generate areas with little to no oxygen in their bottom waters. This is turn can affect benthic ecosystems, and lead to the death of benthic marine fauna. ${ }^{16-18}$

Other HABs produce potent toxins that have direct impacts on human health. Globally, marine neurotoxic compounds are responsible for 50,000 to 500,000 acute intoxications annually with a $1.5 \%$ mortality rate. ${ }^{19,20}$ Human exposures to HAB toxins can occur through the ingestion of contaminated seafood or drinking water, skin contact with contaminated water, or inhalation of aerosolized toxins. ${ }^{1,21-23}$ There are seven clinical poisoning syndromes from human exposures to HAB toxins: Amnesic Shellfish Poisoning, Paralytic Shellfish Poisoning, Ciguatera Fish Poisoning, Diarrhetic Shellfish Poisoning, Neurotoxic Shellfish Poisoning, Azaspiracid Shellfish Poisoning, and Cyanobacterial poisoning (Table 1). ${ }^{1}$ These syndromes are caused by a diverse group of algae that produce chemically distinct toxins. 
The HAB toxin at the center of my research is Domoic Acid (DomA), a potent ionotrophic glutamate receptor agonist that can accumulate in shellfish. DomA is primarily produced by algae in the diatom genus Pseudo-nitzschia. DomA-producing Pseudo-nitzschia blooms are expected to increase in frequency and size as climate change drives more warm ocean temperature anomalies. ${ }^{24}$ Given this potential expansion, the possibility for incidental human exposures to DomA could increase dramatically, thus raising the need to understand the underlying mechanisms of toxicity.

\subsection{HUMAN EXPOSURES TO DOMA}

\section{Incidental human exposures}

The only confirmed outbreak of acute DomA poisoning in humans occurred in November 1987 in Eastern Canada. ${ }^{25}$ Within a four week period, 250 people sought medical attention for symptoms associated with consumption of blue mussels contaminated with DomA. ${ }^{26,27}$ Out of these, 107 people fit the strict case definition for the mussel-borne illness. Mild gastrointestinal symptoms that included nausea (77\% of the cases), vomiting (76\%), abdominal cramps, and diarrhea (42\%) occurred within 24 hours after exposure. More severe acute neurological symptoms such as confusion, motor deficits, seizures, and coma were founded in some patients by 48 hours post exposure. By 11-24 days post-exposure, three patients died, with two dying from septic shock and the other from pneumonia. Short-term memory losses occurred for $25 \%$ of the confirmed DomA toxicosis cases (24 out of 96 surveyed). It is due to this distinctive phenotype that illnesses associated with acute DomA exposures were named 'Amnesic Shellfish Poisoning Syndrome.' $28-30$

DomA could not be measured directly in humans who consumed the contaminated mussels. However, uneaten mussels from this outbreak were tested and had levels of DomA that ranged from 310 parts per million (ppm) to $1280 \mathrm{ppm}$. Based on consumption data, it was estimated that people who did not exhibit toxicity symptoms consumed 15-20 mg of DomA, those with mild gastrointestinal issues consumed 60-110 mg, and those with more severe symptoms received a

maximum dose that ranged between $200-300 \mathrm{mg}$ of DomA. ${ }^{31}$ Assuming the average weight of 50 $\mathrm{kg}$ (females) and $70 \mathrm{~kg}$ (males), the no-observed-adverse-effect-level (NOAEL) was identified to 
be $0.2-0.3 \mathrm{mg}$ of DomA $/ \mathrm{kg}$ body weight, while the lowest-observed-adverse-effect-level (LOAEL) was identified to be $0.9-2.0 \mathrm{mg} / \mathrm{kg} .{ }^{28}$

\section{Oral consumption as the sole exposure route}

The sole exposure route for DomA in adult humans is through the consumption of contaminated seafood. Common seafood that contains DomA includes shellfish (razor clams, scallops, mussels), planktivorous fish (sardines and anchovies), and benthic invertebrates (crabs). Shellfish and planktivorous fish can readily accumulate DomA through filter feeding on toxinproducing Pseudo-nitzschia, while benthic invertebrates obtain DomA through feeding on the seafloor where Pseudo-nitzschia cells have sunk and aggregated. ${ }^{32}$ Trophic transfer of DomA can also occur throughout the marine environment as predators such as large fish and marine mammals consume prey contaminated with the toxin (Fig. 1). ${ }^{33,34}$

Preparation of seafood affects the amount of DomA consumed by humans. In crabs, steaming and frying does very little to reduce the toxin in tissue while boiling and eviscerating crabs reduces the content to $5-10 \%$ of the original visceral DomA content. ${ }^{35}$ Sea scallops concentrate DomA in their digestive gland and do not accumulate it in their adductor muscle unless exposure durations are prolonged. ${ }^{36}$ Customarily in North America, it is common to only eat the adductor muscle, making it possible to avoid DomA contamination. Filter feeding fish like anchovies and sardines readily accumulate DomA in their guts, but the distribution to other tissue compartments appears to be extremely low (3-4 orders of magnitude difference in the gut versus other tissues). ${ }^{37}$ Even in bloom events when filter-feeding fish can accumulate DomA up to 1,800 $\mathrm{mg} / \mathrm{kg}$, proper gutting and cleaning techniques will likely protect human consumers as well. It is important to note that any processing that uses the whole fish, such as fish meal, could lead to food items that exceed the regulatory limits.

\section{Establishment of the current regulatory limits}

The U.S. Food and Drug Administration (FDA) has set the tolerable daily intake (TDI) of DomA to be $0.075 \mathrm{mg}$ of DomA $/ \mathrm{kg}$ body weight per day. This limit is based on oral toxicity studies in adult non-human primates where the NOAEL was $0.75 \mathrm{mg} / \mathrm{kg} .^{28}$ The TDI then incorporates a 10fold safety factor to account for sensitive individuals in the population. 
A set of consumption surveys were then done to assess the levels of DomA that were deemed tolerable within seafood. ${ }^{35}$ To calculate the tolerable level (TL) of DomA in razor clams, the following formula was used:

$$
T L=\frac{T D I * W_{O A}}{C L_{0.84}}
$$

TDI is the tolerable daily intake $(0.075 \mathrm{mg} / \mathrm{kg}) . W_{O A}$ is the average weight of an adult $(70.0 \mathrm{~kg})$. CL is the $84^{\text {th }}$ percentile of razor clam consumption level $(0.270 \mathrm{~kg})$. From this, the TL was calculated to be $19.4 \mathrm{mg}$ of DomA/kg razor clam, and regulatory health bodies nationally and internationally have widely accepted $20 \mathrm{mg}$ of DomA/kg seafood as the regulatory limit. ${ }^{38}$ The one exception to this is Dungeness crab, which has a TL set at $31.5 \mathrm{mg}$ of DomA $/ \mathrm{kg}$ tissue due to humans consuming relatively lower amounts of crab tissue compared to shellfish.

\section{Risks associated with the current regulatory limits}

It is important to note that the regulatory limit was set based on single acute exposures in adult non-human primates that resulted in overt phenotypes (vomiting, disorientation, seizures etc.). It does not take into account the effects from chronic exposures or effects from exposures during more sensitive life stages in humans. Furthermore, this limit does not include a safety factor that is applied for species-specific differences in sensitivity. From incidental exposure data in humans, the NOAEL $(0.2-0.3 \mathrm{mg} / \mathrm{kg})$ is lower than that defined in non-human primates $(0.75 \mathrm{mg} / \mathrm{kg}){ }^{28}$

Incidental human exposure data also suggests that there are sensitive populations. Those who had the most severe symptoms were men, elderly and people with renal dysfunction. Of the 107 patients who were diagnosed with ASP in Eastern Canada in 1987, 36\% were 60 years or older. All patients under 65 who exhibited severe neurological symptoms had other preexisting illnesses such as poor renal function. The odds of exhibiting memory loss symptoms were 4.4 times higher if the patient was male rather than female $(95 \% \mathrm{CI}-1.5,13.0)$, and 1.6 times higher for every 10-year increment in age starting at age $20(95 \% \mathrm{CI}-1.2,2.0)$. 
Indeed, a report jointly commissioned by the Food and Agriculture Organization of the United Nations (FAO), the World Health Organization (WHO), and the Intergovernmental Oceanographic Commission of UNESCO (IOC) raised concerns that the regulatory limit may not be protective for those who are chronically ill, elderly, or pregnant. ${ }^{39}$ The Washington State Department of Fish and Wildlife has also implemented an interim health advisory on eating razor clams. It encourages consumers to eat less than 15 clams per month, while pregnant women and the elderly are advised not to eat razor clams. ${ }^{40}$

Finally, the regulatory limit is calculated based on general consumption patterns and average body weights. Updated consumption surveys within Washington state show that participants in the surveyed population exceeded the TDI 7\% of the time through the combination of lower than expected body weights, high consumption rates, and consumption of clams with DomA levels near the regulatory limit. ${ }^{41}$ DomA exposures in Belgium were estimated by linking shellfish consumption data to measured concentrations of DomA in shellfish between 2004-2009. While less than $1 \%$ of the population is at risk of developing acute intoxication, up to $5-6 \%$ of the population is estimated to exceed the tolerable daily intake of $0.075 \mathrm{mg} / \mathrm{kg}$ body weight per day when taking into account consumption patterns over multiple days. ${ }^{42}$

\subsection{MOLECULAR MECHANISMS OF DOMA TOXICITY}

Receptor targets: ionotrophic glutamate receptors

DomA is water-soluble amino acid that is a structural analog of glutamate. It exerts its toxicity through binding to ionotrophic glutamate receptors, which leads to the opening of the ion pore and neural activation. ${ }^{43}$ DomA binds preferentially to alpha-amino-3-hydroxy-5-methyl-4isoxazole propionic acid (AMPA) and kainate (KA) receptor subtypes. DomA is considered a partial agonist as binding to these receptors leads to the incomplete opening of the ion channel

and to distinct conformational changes that make these receptors unable to desensitize. ${ }^{44-47}$ The inability of the receptors to desensitize leads to continued influx of ions and the protracted activation of these receptors. 


\section{Mechanisms of excitotoxicity following DomA exposure}

The aberrant activation of ionotrophic glutamate receptors leads to neural damage and cell death in a pathological condition called 'excitotoxicity'. Cell death occurs primarily through either apoptosis or necrosis depending on the duration and severity of the insult. ${ }^{48}$

Necrosis occurs when there is a sustained activation of glutamate receptors (through either prolonged DomA exposure or exposure to high DomA concentrations) that leads to the massive influx of ions, cellular swelling, disruptions to membrane integrity. ${ }^{48-50}$ During necrotic cell death, glutamatergic neurons can release aberrantly high amounts of glutamate into the extracellular space, leading to even further damage to the surrounding tissues.

Apoptosis occurs when there is a transient activation of the receptors that leads to the aberrant rise in intracellular calcium. High cytoplasmic levels of calcium lead to the persistent and pathological activation of calpains, protein kinases, phopholipases, proteases and endonucleases. ${ }^{51}$ The activation of these proteins in turn leads to formation of free radicals ${ }^{52}$ or to the degradation of key structural proteins. ${ }^{53,54}$ Conversely, the use of inhibitors of calpains, ${ }^{55,56}$ phospholipase c, ${ }^{57}$ and cysteine proteases ${ }^{58}$ along with mutations to the c-Jun amino-terminal kinase, ${ }^{59}$ all have been shown to reduce excitotoxicity.

The influx of calcium can also lead to the formation of free radicals, which play an important role in mediating neurotoxicity. ${ }^{60} \mathrm{Ca}^{2+}$ leads to the activation of nitric oxide synthase, which generates nitric oxide. As mentioned above, $\mathrm{Ca}^{2+}$ activates phospholipase A2 which then generates superoxide anions. ${ }^{49}$ The scavenging of these free radicals through the application of ascorbic acid, ${ }^{61}$ melatonin, ${ }^{62}$ or trans-resveratrol ${ }^{63}$ leads to neuroprotection from excitotoxicity following DomA or kainate exposures. Conversely, the depletion of the free radical scavenger glutathione exacerbates DomA toxicity in cerebellar granule neurons. ${ }^{62,64}$

DomA-induced excitotoxicity involves a rise in intracellular $\mathrm{Ca}^{2+}$ through various mechanisms. The activation of AMPA and KA receptors leads to the influx of $\mathrm{Na}^{+}$. This influx can result in the reversal of the mitochondrial $\mathrm{Na}^{+} / \mathrm{Ca}^{2+}$ exchanger, which releases mitochondrial calcium 
stores into the cytoplasm. ${ }^{65,66}$ Furthermore, the influx of $\mathrm{Na}^{+}$leads to the activation of voltagegated $\mathrm{Ca}^{2+}$ channels, which allows calcium to enter from the extracellular space.

Pharmacological inhibition of the L-type voltage-gated calcium channels has been shown to reduce intracellular $\mathrm{Ca}^{2+}$ concentrations following exposure to DomA in both hippocampal neuron cultures and mixed cortical cultures. ${ }^{65,67,68}$ In two out of the three of these studies, the reduced $\mathrm{Ca}^{2+}$ load resulted in neuroprotection ${ }^{67,68}$ while in the other it did not. ${ }^{6569,70}$ Finally, some AMPA and KA receptors are comprised of subunits that make them highly permeable to $\mathrm{Ca}^{2+}$; activation of these receptors leads directly to the influx of $\mathrm{Ca}^{2+} .69,70$

While DomA binds directly to AMPA and KA receptors, it also indirectly activates NMDA receptors - the other ionotrophic glutamate receptor subtype. It does this through binding to the AMPA and KA receptors, depolarizing the neuron, and ultimately leading to the relief of the NMDA $\mathrm{Mg}^{2+}$ block. This then poises the NMDA receptors to respond to endogenous glutamate. ${ }^{71}$ Several lines of evidence suggest that NMDA receptors may also play a role in DomA toxicity. Administration of the NMDA receptor antagonist, MK-901 led to the attenuation of the DomA-induced calcium influx, and ultimately to the neuroprotection in cerebellar granule neurons. ${ }^{72}$ Pretreatment with NMDA results in increased behavioral toxicity and reduced seizure latency following DomA administration, while combined treatments of the NMDA antagonist CPP and DomA ameliorated the behavioral deficits caused by DomA. ${ }^{73,74}$ However, there are instances of DomA neurotoxicity that are independent of the NMDA receptor involvement; for example ${ }^{75}$ retinal implants could be protected from DomA lesions with CNQX, a non-NMDA receptor antagonist, but not with the NMDA antagonist MK-801. ${ }^{47,75}$ These differences in responses may arise from differences in subunit composition between different neuronal populations at different developmental stages.

\section{Excitotoxicity: convergent mechanisms for various pathological conditions}

The overactivation of glutamate receptors occurs in a wide-range of brain pathologies including brain ischemia, trauma, stroke, epileptic seizures ${ }^{51,76,77}$ Excitotoxicity has also been implicated as a convergent pathway by which disease progression occurs in many neurodegenerative diseases, including Alzheimer's disease, Huntington's disease, and Amyotrophic Lateral 
Sclerosis (ALS) ${ }^{78,79}$ This suggests that further identifying the mechanisms by which DomA acts could provide fundamental insights into other diseases that share these convergent mechanisms.

\subsection{TOXICOKINETICS OF DOMA}

Toxicokinetics describes how the substance enters the body (absorption), gets to target tissues (distribution), is chemically transformed (metabolism), and is eventually removed from the system (excretion). These toxicological endpoints provide the mechanistic link between the dose of DomA consumed by humans, and the concentrations of DomA in target tissues that lead to the toxicity.

\section{DomA has low bioavailability following oral exposures}

Human consumption of food contaminated with DomA is the sole exposure scenario, and yet most DomA exposure studies are done using parenteral (outside the digestive tract) routes, resulting in limited data for oral absorption of DomA.

Evidence from non-human primate models indicates that the toxicokinetic parameters after oral exposures are distinct from intravenous exposures. Oral DomA exposures led to the limited but prolonged absorption of DomA.$^{80}$ Only $6 \% \pm 4$ of DomA reached the systemic circulation compared to when DomA was delivered intravenously (a measure of absolute bioavailability). This is in agreement with a 30-day oral exposure study in cynomolgus monkeys that showed that $4-7 \%$ of the orally administered DomA is absorbed (when given at the dose of $0.5 \mathrm{mg} / \mathrm{kg}$ per day for 15 days followed by $0.75 \mathrm{mg} / \mathrm{kg}$ per day for 15 days) ${ }^{81}$ Despite the low absorption rates, oral administration led to prolonged exposures. Following oral administration, the terminal half-life was $11.3 \pm 2.4$ hours - almost $10 \mathrm{x}$ the length of the half-life following intravenous exposure (1.2 \pm 1.1 hours). This indicates that humans may be exposed to DomA over longer periods of time than first expected.

\section{DomA is mostly excluded from the brain}

Radiolabelled DomA was intravenously injected into rats and then was measured in seven brain regions. DomA transfer constants were found to be similar to sucrose, indicating that DomA poorly penetrates the blood brain barrier and is likely not actively transported. ${ }^{82}$ Similar findings 
have been found following oral exposures in Coho salmon where DomA concentrations were highest in the kidney, followed by bile, and lowest in the brain. ${ }^{83}$

\section{DomA undergoes limited biotransformation}

One study has shown that following intravenous injections, DomA undergoes very little biotransformation, as $70-75 \%$ of the parent compound is excreted in the urine. ${ }^{84}$

\section{Excretion mechanisms differ based on exposure route}

Intravenous administration of DomA in rats $(0.5$ and $1 \mathrm{mg} / \mathrm{kg})$ and non-human primates $(0.05$ and $0.005 \mathrm{mg} / \mathrm{kg}$ ) leads to rapid serum elimination with terminal half-lives that range from 20 minutes (rats) to $72-110$ minutes (non-human primates). ${ }^{80,84,85}$ These clearance rates are not dose-dependent. ${ }^{84}$ The kidney is responsible for the majority of DomA clearance following intravenous exposures. In rodents, $100 \%$ of the intravenous injected dose of DomA was recovered in the urine by 160 minutes. ${ }^{84}$ Nephrectomized rats have lower clearance rates, further highlighting the importance of renal clearance mechanisms. ${ }^{82}$ In non-human primates, $42 \%$ of the intravenous DomA dose is recovered in the urine by 24 hours after exposure. ${ }^{80}$ The incomplete recovery of DomA in the urine in non-human primates suggests that DomA is either reabsorbed in the kidneys or that it is eliminated via other pathways.

Renal clearance mechanisms play a smaller role following oral exposures to DomA. In nonhuman primates, only $4 \pm 2 \%$ of the orally administered DomA dose was recovered in the urine by 24 hours. ${ }^{80,85}$ DomA delivered orally in rodents led to the excretion of the full DomA dose into feces $(102 \% \pm 17$ in mice, and $98 \% \pm 12$ in rats $) .{ }^{86}$ The recovery of DomA in feces could indicate that hepatic clearance mechanisms play a substantial role in removing DomA administered orally. However, the presence of DomA in the feces could also be due to the poor absorption of DomA through the GI tract.

\section{Toxicokinetics and species-specific sensitivities}

When DomA is delivered parenterally (outside of the digestive tract), the range of doses that lead to neurotoxicity is similar across different species. At the lowest doses tested $(0.025-0.5 \mathrm{mg} / \mathrm{kg})$, non-human primates were more sensitive than rodents to intravenous DomA exposures - 
exhibiting transient lip smacking and vomiting (Table 2). However, at higher doses (0.5-1 $\mathrm{mg} / \mathrm{kg}$, i.v.), the behavioral effects and neuronal degeneration pathologies are similar in nonhuman primates and rats. Doses required to elicit toxicity following intraperitoneal exposures were also fairly comparable across multiple species $(1-7.5 \mathrm{mg} / \mathrm{kg}$ ) (Table 3). One exception to this is mice, which show subtle changes in locomotion (hypoactivity) following intraperitoneal exposures to $0.24 \mathrm{mg} / \mathrm{kg}$ DomA.

In comparison, different species have wide range of sensitivities to DomA following oral exposures. In humans, acute neurotoxicity occurred after exposure to DomA that was estimated to range from $1.9-4.2 \mathrm{mg} / \mathrm{kg} .{ }^{87}$ Non-human primates have similar toxicity profiles, as they developed acute symptoms such as salivation, mastication, and lip smacking after exposure to DomA at doses of $5 \mathrm{mg} / \mathrm{kg}$ or higher. ${ }^{88}$ In contrast, rodents required much higher oral doses of DomA to exhibit neurotoxic symptoms (mice, $35 \mathrm{mg} / \mathrm{kg}$ and rats, $80 \mathrm{mg} / \mathrm{kg}$ ) ${ }^{86}$ Furthermore, fish did not exhibit any acute neurotoxic symptoms when orally gavaged with $10-13 \mathrm{mg} / \mathrm{kg}$ DomA. ${ }^{83}$ Wild-caught anchovy and sardines had up to $1000-1815 \mathrm{mg} / \mathrm{kg}$ of DomA within their viscera during mass bloom events. ${ }^{37,89}$ In fact, DomA concentrations in the fish viscera were correlated to Pseudo-nitzschia cell densities, suggesting that rather than avoiding DomA-producing cells, fish were tracking and feeding on these cells during algal blooms. ${ }^{37}$

All of this indicates that the species differences in sensitivity to DomA are more pronounced with oral administration compared to intravenous or intraperitoneal administration. It has been suggested that differences in GI tract length between species may account for the differences in toxicity, with species with longer GI tracts having greater overall absorption and greater sensitivity to DomA.$^{87}$ Fish that are the least sensitive to DomA toxicity also have comparatively shorter GI tracts. However, Coho salmon orally exposed to DomA had measurable DomA in all tissues tested 15 minutes post-gavage, with the highest concentrations found in the kidney and the bile. ${ }^{83}$ Authors suggest that rather than having a lower absorption capacity for DomA, fish may have more efficient first-pass excretion mechanisms in the liver, allowing them to rapidly sequester DomA in the bile prior to DomA entering into the circulation. This, in combination with highly efficient renal clearance mechanisms, may make fish less sensitive to DomA administered orally. While this may be true, the specific factors that lead to species differences in 
sensitivity have yet to be systematically tested.

\subsection{ACUTE TOXICOSIS FROM HIGH-DOSE EXPOSURES TO DOMA}

Exposure to DomA produces shared pathological symptoms across multiple animal models (fish, ${ }^{83,89,90}$ birds, mice, rats, ${ }^{91-98}$ sea lions, ${ }^{99,100}$ non-human primates ${ }^{81,101-103}$ ). The progression of symptoms of DomA toxicosis is consistent across animal species. Exposure to low doses of DomA initially leads to vomiting, nausea, and headaches in both humans and cynomolgus monkeys. ${ }^{93,101,104}$ Rodents, which are unable to vomit, first exhibit signs of hypoactivity and scratching. ${ }^{92,94,95}$ These symptoms subside and no other overt symptoms occur. At higher doses of DomA, humans exhibit neurotoxic symptoms including agitation, tremors, seizures, and coma. Anterograde amnesia - the inability to form new memories - is a characteristic lasting neurobehavioral symptom in humans after DomA exposure. Mice and rats exposed to DomA were also shown to have deficits in spatial and working memory, exhibiting delays in learning the location of the escape platform in the Morris water maze test. ${ }^{105,106}$ Stranded sea lions recovering from DomA toxicosis also had deficits in a spatial working memory (deficits in the delayed alternation task) and in spatial foraging behavior. ${ }^{107,108}$

DomA targets the same brain regions and leads to common neurodegenerative effects across multiple animal models. Examination of brain sections during autopsies of 4 patients who died 7 days to 3 months after DomA exposure revealed that DomA primarily targeted the limbic system, with most pronounced damage to the hippocampus and the amygdala. ${ }^{28}$ Histology of hippocampal sections from patients showed complete neuron loss in the CA1 and CA 3 regions of the hippocampus, pronounced losses in the CA4 region, and intermediate losses in the CA2 region. Lesions were also found in the claustrum, secondary olfactory areas, septal area, and the nucleus accumbens. DomA exposure led to similar pathologies in all mammalian species tested (rodents, sea lions, non-human primates). Histological analyses showed pronounced losses in hippocampal neurons in the CA1, CA3, and CA4 region following intravenous and intraperitoneal exposures to DomA in rodents and primates, and from incidental exposures in sea lions. ${ }^{86,95,98,103,109,110}$ 


\subsection{NEUROTOXICITY FROM CHRONIC EXPOSURES TO DOMA}

The current regulatory limit for DomA does not take into account toxicity that may occur with consumers who regularly consume shellfish and are exposed chronically to low-levels of DomA. Emerging evidence suggests that chronic exposure to DomA in humans is linked to cognitive deficits. Epidemiological studies show an association between heavy consumption $(\geq 15$ per month) of razor clams and subtle yet measurable decrements in memory. ${ }^{111,112}$ These deficits were first measured using standardized cognitive assessment tests, then through tests for 'everyday memory,' which are recalls necessary for daily living.

Research in animal models has sought to identify the underlying mechanisms from chronic exposures to DomA. Evidence from zebrafish, mice, and non-human primates indicates that chronic exposures to DomA can lead to distinct changes in gene expression, alterations to receptor densities, and aberrant neural connectivity, even in the absence of widespread structural damage. In particular, zebrafish chronically exposed to DomA (36 weeks) had dynamic changes in gene expression, impaired mitochondrial function, and upregulated immune responses. ${ }^{113,114}$ Mice chronically exposed to DomA for 22 weeks had higher expression of vesicular glutamate transporter proteins in the $\mathrm{CA} 1$ region of the hippocampus with no changes in overall neuron density. ${ }^{115}$ Mice exposed to DomA over a longer period ( 25 weeks), had measurable yet transient behavioral deficits that were abated 9 weeks after DomA exposure was stopped. ${ }^{116}$ All of this suggests that while chronic exposures to DomA may have important consequences for behavior and immune function, these effects may be reversible when DomA exposure is stopped.

While previous animal studies provide important mechanistic insights into chronic DomA exposures, all of these studies were done using doses and exposure routes that are not directly comparable to humans. Compelling new research in non-human primates show that chronic, oral exposures to DomA at or near the accepted human tolerable daily intake limits $(0.075 \mathrm{mg} / \mathrm{kg}$, $0.15 \mathrm{mg} / \mathrm{kg}$ body weight) led to intentional tremors during reaching and grasping tasks. ${ }^{117}$ Tremor scores were correlated with decreased fractional anisotropy (FA) - a measure of white matter integrity - during whole brain diffusion tensor imaging. This research suggests that chronic exposures to DomA at environmentally relevant exposure conditions (oral exposures, at 
or near the tolerable daily intake limit) can lead to structural damage and behavioral effects in adults.

\subsection{DOMOIC ACID AS A DEVELOPMENTAL NEUROTOXIN}

A myriad of well-coordinated, sequential, and co-dependent processes are required to build a functional nervous system. This makes the developing nervous system particularly sensitive to environmental insults. ${ }^{118}$ While glutamate is well-known for its role as an excitatory neurotransmitter at the synapse in mature nervous systems, during development, signaling through glutamatergic receptors has distinct functions for cell migration, proliferation, and survival. ${ }^{19,120}$ Domoic acid, which binds to and signals through ionotrophic glutamate receptors, may perturb these critical processes and have profound effects on the structure and function of the nervous system that are distinct from those following acute toxicity in adults.

\section{Developmental exposure routes}

Prenatal exposure to DomA can occur as DomA crosses the placental barrier, readily accumulates in amniotic fluid, and makes its way to the fetal brain. ${ }^{121-123}$ Following intravenous exposures in pregnant rodents, DomA is readily detected in both the fetal brain and the amniotic fluid. ${ }^{124}$ At early stages (gestational day (GD) 13), DomA is detected in the fetal brain at similar concentrations to those measured in the amniotic fluid. In contrast, by GD20, only half of DomA detected in the amniotic fluid was present in the fetal brain. ${ }^{121}$ This suggests that timing of exposure plays a role in determining the availability of DomA to its tissue targets in the fetus.

The amniotic fluid can also serve as a reservoir for DomA. DomA was also found to accumulate in the amniotic fluid by one hour post-exposure in rodents and be retained for up for 8 days in stranded sea lions. ${ }^{123}$ Retention in the amniotic fluid may provide a means by which fetuses can continue to be exposed to DomA after it is cleared from mother's plasma. It also provides a means by which chronic exposures in mothers may lead to the accumulation of DomA for fetuses.

Postnatal exposures to DomA can occur through consumption of contaminated breast milk. Following intraperitoneal injection into nursing rodent mothers, DomA concentrations were 16 
times lower in the milk relative to the maternal plasma. However, DomA had a longer retention in the milk relative to the mother's plasma, as DomA concentrations were 4 times higher in the milk versus the maternal plasma 8 hours later. ${ }^{125}$ Opportunistic sampling of stranded marine mammals has found detectable levels of DomA in the milk, further suggesting that neonatal DomA exposures can occur through lactational transfer. ${ }^{26}$

\section{Sensitivity to exposure during early development}

Rodents are more sensitive to DomA during early development compared to other life stages (Table 4). One tenth the dose of DomA is required to induce overt behavioral toxicity in postnatal rats compared to adults. ${ }^{96,127,128}$ Even within the postnatal period, rats are more sensitive at earlier postnatal stages. The dose of DomA that led to 50\% mortality (LD50) was almost 2.8 times lower at postnatal day 2 (PND2) versus PND10. ${ }^{128}$ Less than half the dose is required at PND 5 than at PND 14 to produce signs of toxicity (such as motor seizures, forelimb tremors, and mastication) in 50\% of the exposed animals (ED50), (PND 5 with an ED50 $=0.12$ $\mathrm{mg} / \mathrm{kg}$, PND 14 with an ED50 $=0.30 \mathrm{mg} / \mathrm{kg}$ ). It also takes less than one third of the dose of DomA at PND14 compared to PND 22 for animals to exhibit the same toxicity profiles in 50\% of the population (PND 22 with an ED50 $=1.06 \mathrm{mg} / \mathrm{kg}$ ). ${ }^{129}$

It is difficult to compare the sensitivity of prenatal versus postnatal stages to DomA, as the routes of exposures are distinct (exposing the pup directly versus the mother). One way to assess differences in sensitivity is by comparing phenotypes following exposure to the highest dose that does not cause overt behavioral phenotypes in exposed mothers (prenatal studies) or the pups (postnatal studies). Following subcutaneous exposures $(1.2 \mathrm{mg} / \mathrm{kg}$ of DomA) of mothers at gestational day 13 (GD13), pups had persistent behavioral deficits including delayed latencies to enter T-maze arms and changes in activity in the Figure- 8 maze. ${ }^{130}$ Rats exposed developmentally to DomA also had diminished capacity to cope with a second challenge (scopolamine exposure), which was reflected in significant reductions in choice accuracy in the Radial arm maze. In contrast, subcutaneous exposures to DomA $(0.05 \mathrm{mg} / \mathrm{kg})$ in postnatal pups (PND 1-2, twice daily) led only to a decrease in locomotor activity in the Figure-8 maze, with no other recorded behavioral deficits. ${ }^{131}$ This led the authors to conclude that the prenatal period is likely the more sensitive period for long-term behavioral deficits. Other prenatal and postnatal 
studies have been done, but using different species, varied exposure routes and disparate behavioral endpoints, making it impossible to draw any other further generalizations about differences in sensitivity prenatally versus postnatally.

\section{Sustained structural and behavioral deficits from prenatal exposures to domoic acid}

Prenatal exposure to DomA has been shown to lead to sustained behavioral deficits that include aberrant exploratory behaviors, ${ }^{130,132,133}$ subtle motor coordination deficits, ${ }^{133}$ and, in one out of two studies, deficits in contextual learning (Table 5). ${ }^{132,133}$ These behavioral changes were associated with extensive structural damage in the brain, altered neural connectivity, and sensitivity to chemically-induced stressors later in life (Table 6).

Extensive structural damage in specific brain regions has been reported in rodents exposed prenatally to doses of DomA. Prenatal exposures (GD11.5 and 14.5) led to decreased MAG labeling (myelin marker) and increased MAP2 staining (dendrite marker) in the cerebral cortex, suggesting that DomA can disrupt myelin formation and lead to abnormal dendrite sprouting. ${ }^{132}$ In another study, exposures at GD13 led to abnormal cell morphologies (swelling, distorted, pyknotic appearance) in the hippocampal CA3 region and dentate gyrus. ${ }^{134}$ These cellular disruptions were first observed at PND14, and increased in severity by PND 30.

Prenatal exposure to DomA has been shown to lead to aberrant neural activity and disrupted neuronal network connectivity. Mice exposed to DomA at GD13 $(0.6 \mathrm{mg} / \mathrm{kg}$ in mothers) showed abnormal spike activity measured via electroencephalogram at PND 10, with progressive worsening through PND 20 and PND $30 .{ }^{134}$ Another study showed that prenatally exposed rodents had altered connectivity patterns as assessed by fMRI, with some regions showing enhanced connectivity and others showing reduced connectivity. ${ }^{135}$

Exposure to DomA during early development has been shown to heighten sensitivity to chemically-induced disturbances later in life. ${ }^{130,134,136}$ Prenatal exposure to DomA sensitized mice to the second exposure to DomA, leading to reduced latencies to the first observed seizure, increased severity in seizure type, and increased excitability assessed by EEG. ${ }^{134}$ Similarly, developmental exposure to DomA in zebrafish prior to neurogenesis (1,000-cell stage) led to 
heightened sensitivity to another seizure-inducing chemical, pentylenetetrazol (PTZ) during larval stages. ${ }^{136}$ Finally, prenatal DomA exposures in rats led to increased susceptibility to scopolamine-induced memory impairments. ${ }^{130}$ The combination of an initial 'hit' of DomA prenatally $(1.2 \mathrm{mg} / \mathrm{kg})$ with the second 'hit' of scopolamine $(0.16 \mathrm{mg} / \mathrm{kg})$ led to the reduced choice accuracy in the radial-arm maze that was not observed in animals exposed to vehicle control.

\section{Latent and progressive dysfunction from postnatal exposures to domoic acid}

To assess the potential effects of DomA during the postnatal period, rodents were exposed to doses of DomA that do not lead to neurotoxic symptoms $(0.02$ or $0.05 \mathrm{mg} / \mathrm{kg})$ from postnatal day (PND) 8-14. PND 8-14 was selected, as it is a developmental period characterized by the exuberant outgrowth of excitatory axons in the hippocampus, which have the marked capacity to generate electrographic seizures. ${ }^{137}$ During this period, pups are susceptible to a variety of teratogenic agents, which can lead to permanent, irreversible dysfunction in the hippocampus.

Postnatal exposures to DomA led to long-term behavioral deficits that included novelty-induced seizures, ${ }^{127,138}$ alterations in drug seeking behaviors, ${ }^{139,140}$ and in some cases, the inability to filter out irrelevant stimuli (Table 7). ${ }^{141,142}$ In addition to behavioral changes, postnatal exposures also led to long-term changes in neuronal receptor densities, aberrant brain morphology, and increased susceptibility to seizures (reviewed in Table $8^{143,144}$ ).

Like prenatal exposures to DomA, postnatal exposures led to the enhanced susceptibility to seizures later in life. ${ }^{145}$ Exposure to DomA postnatally reduced the seizure thresholds of adults exposed to the seizure-inducing agent PTZ. Postnatal DomA exposure also led to lowered seizure thresholds after amygdala kindling - a model for inducing seizures through daily exposures to low amplitude electrical stimulations.

Postnatal exposures to DomA led to pronounced and lasting structural damage in regions of the hippocampus (Table 7). Mossy fiber sprouting refers to aberrant connections made by granule cells in the hippocampus that create seizure-inducing positive feedback loops. Like adults exposed to DomA, rodents exposed to DomA postnatally developed mossy fibers in the inner 
molecular layer of the hippocampus by PND 90, and this phenotype persisted until at least 15 months of age. ${ }^{127,146}$ Cellular damage was progressive; DomA-exposed rodents had no decreases in neuronal cell counts in the hippocampus at PND 90, but had significant decreases in the CA1 and portions of CA3 by 15 months of age. ${ }^{127,146}$

\section{Sex-specific effects from developmental domoic acid exposures}

Sex-specific behavioral deficits from developmental exposures to DomA have been identified. Females exposed postnatally to DomA have behavioral deficits that surface when the environment does not meet expectations. ${ }^{138,147,148}$ In the Morris water maze, there were no sex or treatment differences in the initial stages of learning (during the first trial where rats learned the initial location of the platform). It was only after the rats learned the initial location, and then were forced to learn a new location (a reversal task) that the female-specific deficits surfaced. ${ }^{127,148}$ Females exposed to DomA had significant delays in finding the platform in the reversal task. Females were also likely to have altered 'anxiety-like' behaviors relative to controls. In the water maze, they spent significantly more time in thigmotaxis (presented as circling the pool, and classified as anxiety-like behavior). However, when tested in an elevated plus maze, the same DomA-exposed females spent significantly more time in the open arm on the second test day relative to controls; increased time in the open arms is normally an indication of reduced anxiety.

Male-specific behavioral effects have been reported following both prenatal and postnatal exposures to DomA. These effects center primarily on social deficits. Male rats exposed postnatally to DomA spent less time in the social side of the arena, and more time in the isolated area compared to controls. ${ }^{149}$ Male mice exposed prenatally to DomA also had reduced social interactions, and reduced ultrasonic vocalizations. ${ }^{135,150}$ These social deficits depend on the developmental day mice were tested. In one study, male-specific reductions in sociality were observed at PND 25, but not at PND 35. ${ }^{135}$

None of the postnatal DomA exposure studies that reported sex-based differences in behavior assessed for histological endpoints or for changes in neural connectivity or activity. This makes it difficult to identify the underlying mechanisms for these sex-based differences. Only one 
postnatal study reported a sex-specific difference in histology. Only DomA-exposed females had increased glucocorticoid receptor density in the paraventricular region of the hypothalamus. ${ }^{138}$ However, both male and female rodents exposed to DomA showed 'novelty-induced seizures' when introduced to Morris water maze. Since this effect was independent of sex, it cannot be due to female-specific differences in GC receptor densities. Whether female-specific changes in the densities of GC receptors contribute to any female-specific behavioral responses observed in other studies is yet to be determined.

Prenatal DomA exposure studies also found no associations between the sex-based behavioral differences and histological or neural activity abnormalities. Both males and females exposed to DomA prenatally had significant increases in the parvalbumin positive neurons in the lateral amygdala, regardless of the male-specific social deficits observed in the study. ${ }^{150}$ Resting state connectivity (temporal patterns of neuronal activation patterns across brain regions) was altered in males exposed to DomA that had social deficits. Without any data on the resting state connectivity in females exposed to DomA, it is unclear whether any of these changes in connectivity were associated with observed male-specific behavioral deficits, or with other more generalized effects from DomA toxicity that occurs in both sexes. ${ }^{135}$

From the growing literature from developmental exposures to DomA, a few principle findings emerge:

- Some behavioral and histological phenotypes are latent and progressive.

- Subtle behavioral deficits emerge with increasing task complexity.

- Toxicity can be unmasked with a later life challenge.

- Sex-dependent behavioral deficits exist.

\subsection{DISSERTATION RATIONALE AND OVERALL APPROACH}

Current challenges in understanding the mechanisms of DomA-induced developmental toxicity Current regulations prevent the harvest and consumption of seafood contaminated by DomA at levels known to cause overt symptoms in adult humans. However, these regulations may not be sufficiently protective of sensitive populations such as developing fetuses or young children. 
Research in animal models suggests that developmental exposure to asymptomatic doses leads to long-term behavioral deficits, neural hyperexcitability, and pronounced changes in brain tissue architecture. However, the cellular and molecular mechanisms that drive these effects are unknown.

Developmental exposure studies in rodents have identified a broad range of behavioral deficits. However, the underlying cellular and molecular mechanisms that govern these deficits are generally unexplored. The rodent model has two major limitations for studying mechanisms of DomA toxicity. First, the neural networks that drive the assessed behaviors are complex and have not been completely characterized. The complexity in the neural circuits makes it extremely difficult to identify specific cellular or molecular changes induced by DomA that underlie these behavioral changes. Second, rodents develop in utero, making it very difficult to track the progression of structural and cellular damage in animals over the course of development.

\section{Zebrafish as a tool to identify mechanisms of toxicity}

My dissertation seeks to bridge this gap by using zebrafish as tools to identify the underlying cellular mechanisms of developmental toxicity. It takes advantage of unique attributes of zebrafish including external development, transparency, and the presence of simple, tractable neural circuits.

Zebrafish develop externally. While this means that I am unable to assess the impacts of DomA exposure using the exposure route most relevant for humans (in utero exposure following maternal consumption of DomA-containing food), external fertilization provides some distinct and important advantages. With external fertilization, I am able to use the same exposure route to deliver DomA over the course of early development (from early embryonic to late larval stages) and more directly compare sensitivity of fish at different stages. External fertilization and transparency also allow me to closely track cellular phenotypes and acute toxicity as they occur during early development.

The transparency of zebrafish embryos, their small size, and the availability of transgenic lines allow me to observe neurodevelopmental processes and visualize entire neural structures at 
defined developmental periods. ${ }^{151-154}$ This lets me characterize the progression of cellular and structural phenotypes that may underlie later behavioral deficits.

The zebrafish nervous system has enough complexity for findings to have potential translational impact, while remaining simple enough to allow tracking of specific cells and circuits that govern behavior.

Zebrafish have all the major sensory-motor regions and the same basic brain organization as mammals. ${ }^{155}$ While the zebrafish central nervous system (CNS) has considerable neuroanatomical differences from their mammalian counterparts, zebrafish do have regions that are functionally equivalent. For example, zebrafish do not have a hippocampus - the primary target for DomA toxicity in mammals - but instead have a medial pallium, which, like the hippocampus, is important for spatial learning. ${ }^{156,157}$ What this suggests is that while I cannot make direct comparisons between zebrafish and humans for the specific cell types and exact regions in the CNS affected by DomA exposure, I can still use zebrafish to identify the neural processes and general structures that are disrupted by DomA.

Larval zebrafish have a repertoire of behaviors that are simple enough to be characterized, yet complex enough to be used to assess the effects of toxicants on higher-order behaviors. Most of the larval locomotor behaviors were once considered innate, 'reflex' behaviors in response to different sensory cues, including touch, ${ }^{158,159}$ olfaction, ${ }^{160,161}$ auditory/vibrational inputs, ${ }^{162,163}$ heat, ${ }^{164}$ and noxious chemicals. ${ }^{165}$ However, emerging work has shown that many of these behaviors are not simply reflex behaviors, but can be tuned based on prior sensory information. Startle responses - responses to sudden and intense stimuli -were once considered reflex behavior. However, work has shown that the startle response can undergo habituation, a simple form of learning in which the response is suppressed by exposure to repeated inconsequential stimuli. ${ }^{166,167}$ It can also be modulated by weak pre-pulses (prepulse inhibition), demonstrating that the circuit has the capacity to filter out irrelevant stimuli. ${ }^{162,168}$ 
Using the forward genetic screens, many of these behaviors have been mapped to specific circuits, making it possible for toxicologists to use these behaviors as tools to link the functional endpoints to the underlying structural and cellular targets. ${ }^{169-171}$

Startle response: a tool for assessing perturbations to sensory and motor function For this dissertation, I used the larval startle responses to interrogate the functional effects from DomA toxicity. The startle response requires proper sensory processing and motor output, and serves as a tool to determine whether these processes are disrupted by exposures to DomA. ${ }^{172-175}$ Aberrant glutamate signaling alters startle response kinematics, further strengthening startle as an appropriate behavioral read-out for DomA-driven glutamate excitotoxicity. ${ }^{176,177}$

The kinematics of the startle response are stereotyped and possible to measure quantitavely. ${ }^{162}$ Responses are lateralized; a stimulus perceived by the fish on one side of the body leads to the contralateral movement away from this perceived threat. The first phase of the startle response occurs when the head and body bend with high angular velocities to create a "c" shape (Fig. 2B). In the second phase, the fish does a return flip, followed by swim patterns that allow the fish to move away from the stimulus. ${ }^{174,178}$

The neural circuits that drive startle behavior are fairly well-characterized, making it possible to link the startle behavioral data to the underlying structural and cellular targets (Fig. 2A). ${ }^{175,178}$ Following auditory/vibrational stimuli, the statoacoustic nerve transmits the primary sensory information from the hair cells in the inner ear. A secondary sensory input originates from the lateral line - an organ system that detects vibration and flow through a series of mechanoreceptors along the body. ${ }^{179,180}$ These sensory neurons project and synapse to reticulospinal neurons in the hindbrain. If a certain threshold is reached, a specific reticulospinal neuron called the Mauthner cell fires a single action potential. This action potential is propagated down the Mauthner axon that crosses and descends into the spinal cord where it synapses to motor neurons. The near simultaneous activation of the motor neurons all along the trunk on the contralateral side leads to the characteristic 'c-shape' bend angle (Fig. 2B). Knowing these specific components within the startle circuit allowed me to label cells in the circuit and assess for structural effects of DomA-exposure. 
Different stimulus intensities can bias towards one of two distinct startle responses. ${ }^{162}$ Short latency c-starts (SLCs) occur shortly after stimulus generation ( $<15$ milliseconds, with some dependency on temperature). SLCs have pronounced bend angles and large maximal angular velocities. Conversely, long latency c-starts (LLCs) occur later and have muted angular velocities and bend angles. Higher stimulus intensities increase the probability of eliciting SLCs rather than LLCs. SLCs require activation of the Mauthner cell while LLCs do not. ${ }^{181-183}$ Taking advantage of this, I used different stimulus intensities and measured kinematic attributes to see whether DomA preferentially alters SLCs or LLCs, which would provide me with information as to whether specific neuronal populations are targeted over others.

Different types of stimuli also activate distinct components of the startle circuit. Auditory and vibrational stimuli require sensory input. On the other hand, electrical stimulation bypasses the sensory system completely, directly activating the Mauthner neuron and the downstream pathways. ${ }^{184}$ By comparing startle response kinematics in DomA-exposed fish with electrical versus auditory/vibrational stimuli, I can identify potential components of the circuit disrupted by exposures. If DomA does not alter startle behavior following electrical stimulation, but does with auditory/vibrational stimulation, it suggests that DomA perturbs the sensory system. Conversely, if DomA alters startle behavior following both electrical and auditory/vibrational stimuli, it suggests that components downstream of the sensory circuit are affected by DomA and contribute to the startle response behavioral deficits.

\section{Novel tissue targets for DomA toxicity: hindbrain and spinal cord}

In addition to taking advantage of the zebrafish model to closely link neural circuits to behavior, this dissertation identifies novel cellular and regional targets for DomA toxicity, with the focus on the spinal cord and oligodendrocyte lineage.

The hippocampus is considered one of the primary brain regions targeted by DomA, due to the high densities of AMPA and KA receptors that are also highly permeable to $\mathrm{Ca}^{2+} \cdot{ }^{185,186}$ There are pronounced neuronal losses and structural deficits in the hippocampus in humans ${ }^{28}$ and sea 
lions ${ }^{110}$ incidentally exposed to DomA. This has been further corroborated by acute toxicological studies in non-human primates and rodents.

While the hippocampus is an important target for DomA-induced excitotoxicity, other evidence suggests that the spinal cord may be another target. Developmental exposures to DomA in rats showed that shortly after exposure, neuronal degeneration was observable in the spinal cord with no evidence of degeneration in any of the assessed brain regions. ${ }^{187}$ DomA administered orally to pregnant mothers led to offspring with gait deficits that are generally associated with spinal cord injuries. ${ }^{133}$ Following an incidental exposure in humans, the fourteen patients that suffered from severe DomA toxicosis showed unsteadiness and weakness. Following electromyography tests 46 months after exposure, 9 out of 10 patients tested had signs of denervated muscles, which were attributed to either neuronopathy in anterior horn cells or diffuse axonopathy in motor axons. ${ }^{28}$ While these reports suggest that the spinal cord is a target, no systematic studies have been done to track the progression of degeneration in the spinal cord following exposure to DomA. This dissertation characterizes the spinal cord as a target tissue for DomA toxicity and tracks the progression of degeneration over time. It also identifies lesions in the spinal cord that lead to disrupted motor output during startle behavior.

\section{Novel cellular targets for DomA toxicity: Oligodendrocyte lineage}

DomA is known to exert its toxicity by activating glutamate receptors in neurons, leading to ion influx and neuronal death. However, glial cells, critical support cells in the nervous system, also have glutamate receptors, making them vulnerable to DomA toxicity. Astrocytes - cells that remove glutamate from the extracellular space - are known targets from DomA toxicity. ${ }^{188,189}$ Toxicology studies with rodents and non-human primates have found damaged and necrotic astrocytes in the same hippocampal brain regions where neuronal loss occurs. ${ }^{97,102}$ DomA is also known to lead to reactive gliosis, the condition where cells including astrocytes and microglia develop characteristic morphologies and play distinct roles in response to injury and disease. ${ }^{97,190}$ However, no systematic characterization of glial targets has been done following developmental exposures to DomA. Furthermore, no work has looked at the effects of DomA on the oligodendrocyte lineage - cells in the central nervous system that are responsible for myelinating axons. 
Both myelinating oligodendrocytes and their precursors (oligodendrocyte precursor cells or OPCs) express functional ionotrophic glutamate receptors, potentially making them cellular targets for DomA. ${ }^{191,192}$ Previous studies have shown that KA, a structural analog of DomA, causes cell death in oligodendrocyte primary cell cultures at concentrations comparable to those affecting neurons by activating the receptors and disrupting calcium homeostasis. ${ }^{193-196}$ Furthermore, binding and activating AMPA receptors inhibits the proliferation and differentiation of OPCs into mature oligodendrocytes. ${ }^{197,198}$ Mature oligodendrocytes have also been shown to undergo demyelination after chronic direct infusion of KA on the optic nerves. ${ }^{199}$ All of this suggests that developmental exposure to DomA may target the oligodendrocyte lineage, and that exposure during early development may disrupt OPC proliferation, differentiation, and myelin sheath formation. This dissertation further studies the potential for DomA to perturb oligodendrocyte development to mediate observed behavioral deficits.

\section{General research questions}

This dissertation seeks to identify the impacts of developmental exposures to DomA on neural development and behavior. It utilizes the strengths of both zebrafish and the startle response circuit to characterize startle response deficits, and to identify the underlying cellular and molecular targets that lead to these deficits (Fig. 3). It takes advantage of the availability of transgenic lines in the zebrafish model (Table 9) to quantify cells in vivo, to track developmental processes, and to characterize circuit structure that may be perturbed by DomA exposures.

To identify the mechanisms of toxicity, the dissertation research had three main goals:

1. Identify specific developmental windows of susceptibility for DomA toxicity

2. Identify the functional consequences of DomA toxicity by assessing startle behavior - a behavior in response to an intense and sudden stimulus.

3. Identify the cellular targets and processes perturbed by DomA. 
Chapter 2: Domoic acid as a developmental neurotoxin: windows of susceptibility and structural and behavioral phenotypes

In this chapter, I established zebrafish as a tool for studying developmental DomA toxicity. I accomplished this by exposing zebrafish to a range of DomA doses during different development stages, then assessing the changes in behavior, neural structure, and gene expression. The specific objectives of this chapter were to: i) Identify periods in early development that are most sensitive to DomA toxicity, ii) Assess functional consequences of exposure by measuring startle response behavior, iii) Characterize structural changes in the startle circuit following exposures at different developmental periods, and iv) Identify transcriptional responses from DomA exposures.

\section{Chapter 3: Domoic acid exposure disrupts startle response circuitry and behavior}

The startle response behavior was used as a tool to assess the effects of DomA on sensory-motor function, and to identify specific neuronal populations targeted by DomA. Specifically, this chapter sought to: i) Characterize DomA-induced startle response deficits after exposure to either auditory/vibrational or direct electric field pulses, ii) Determine potential sensory deficits in the startle circuit, iii) Establish the presence and function of hindbrain neurons that integrate sensory information, and iv) Identify motor system deficits.

Chapter 4: Developmental exposures to domoic acid targets specific neuronal subpopulations and leads to aberrant myelination in the spinal cord This chapter examined the initial cellular targets of DomA by interrogating effects on specific cell types shortly after exposure to DomA (4-12 hours post injection). It focused particularly on cells in the oligodendrocyte lineage, hindbrain reticulospinal neurons, and primary motor neurons. All are required for startle response behavior (interrogated in chapter 3), and are shown to be disrupted by DomA at later larval stages. The objectives of this chapter were to: i) Quantify the oligodendrocyte precursor cells and mature oligodendrocytes following exposure, ii) Determine the presence or absence of neurons and structures necessary for startle responses, and iii) Identify DomA-induced perturbations in spinal cord cellular environment that contribute to myelination defects. 


\subsection{RESEARCH IMPACT}

Findings from this dissertation provide mechanistic insights into toxicity from low dose exposures to DomA. This research carries major health implications, especially for toxin exposure during pregnancy and early childhood development. Identifying the key neuronal cell types that are affected by DomA, and the potential downstream disruptions to neural circuit structure and behavior, will inform refined hazard assessments for exposures during susceptible periods in neurodevelopment. As a structural analog to glutamate, domoic acid also serves as a tool to identify broader mechanisms by which altered glutamate signaling during early development can lead to the development of neurological disorders. Last, the approaches applied in this dissertation that involve using both zebrafish and the startle response behavior as a tool could be readily adapted to best understand the mechanisms of toxicity for a wide variety of chemicals. 


\section{CHAPTER 1 FIGURES}

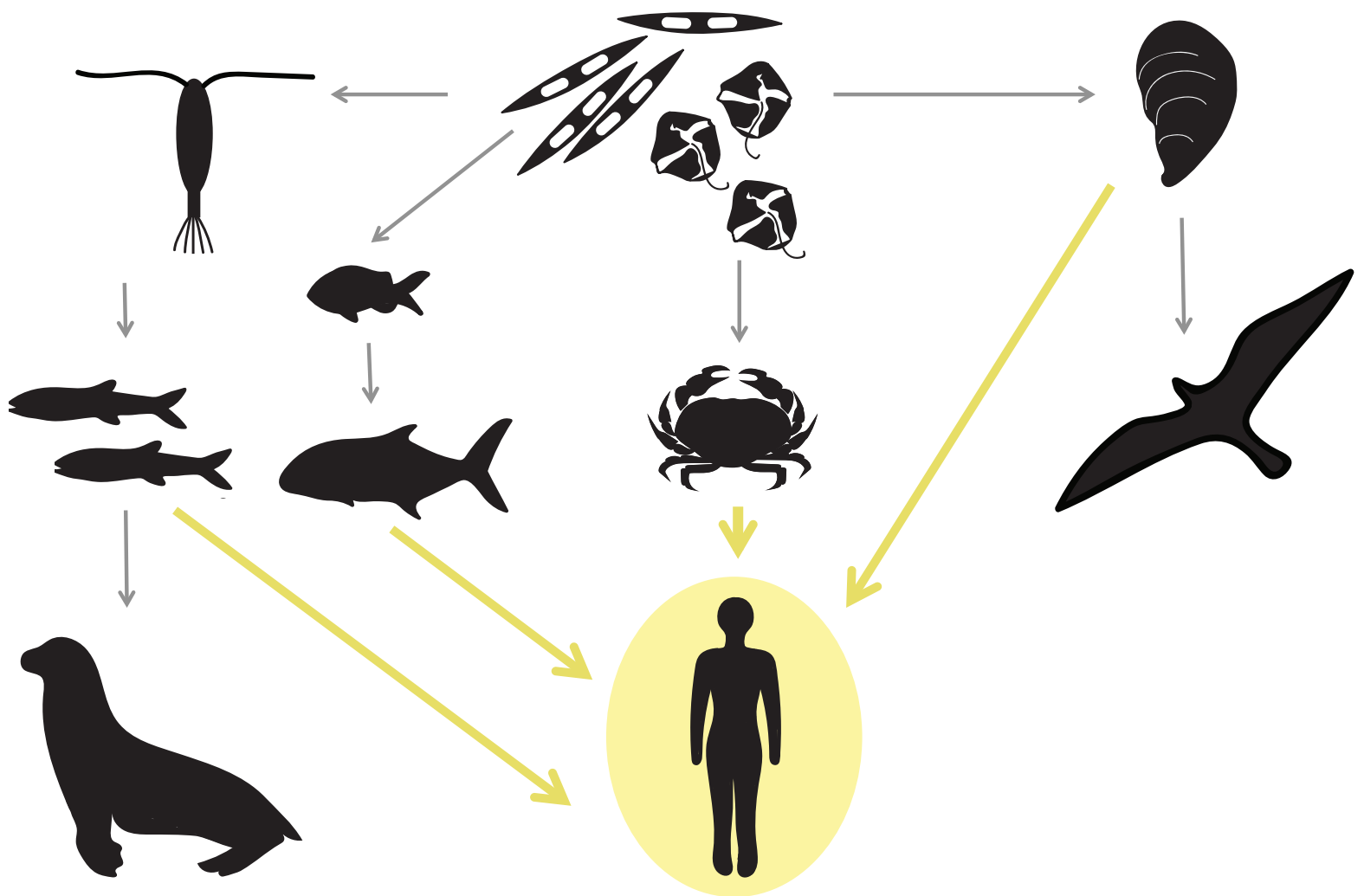

Figure 1: Trophic transfer of DomA through the marine food web.

DomA is produced primarily by the diatom genera Pseudo-nitzschia. The diatoms are consumed by zooplankton, plaktivorous fish, and shellfish. Diatoms and DomA can also sink to the bottom where they accumulate in benthic invertebrates such as crabs. DomA can also be passed up the marine food chain exposing sea birds and marine mammals who eat contaminated prey. Yellow arrows point to seafood consumed by humans which can accumulate DomA. 
A.

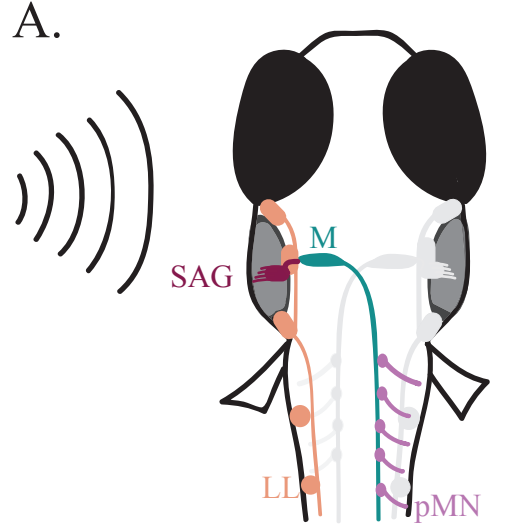

B.

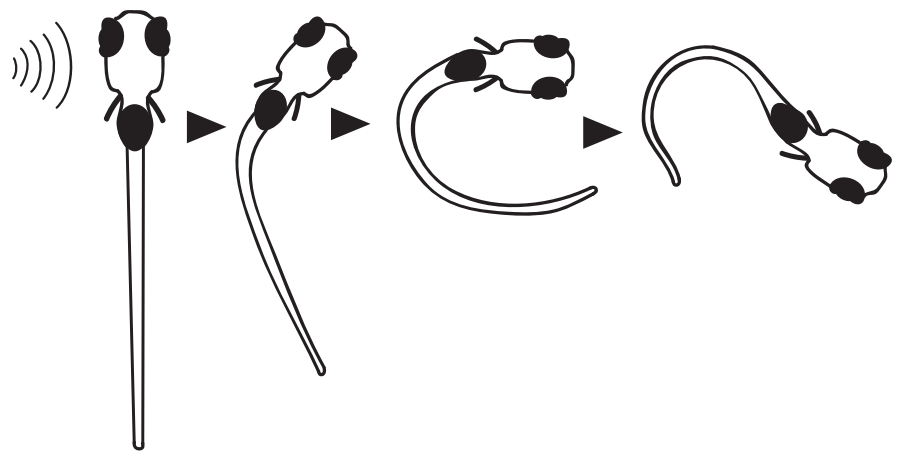

Figure 2: Zebrafish startle response circuit is well-characterized and the behavior is quantifiable.

(A) Auditory/vibrational (A/V) stimuli are perceived primarily through the statoacoustic nerve ganglion (SAG), and secondarily through inputs from the lateral line (LL). In Mauthner-dependent short latency c (SLC) type startles, the Mauthner (M) cell in the hindbrain receives the sensory inputs, and fires a single action potential that is propagated down its axons where it synapses to motor neurons. The near-simultaneous activation of the primary motor neurons ( $\mathrm{pMN}$ ) then leads to the contraction of the muscles, and the characteristic ' $c$ ' bend.

(B) An illustration of the stereotyped startle kinematics following exposure to $\mathrm{A} / \mathrm{V}$ stimuli. 


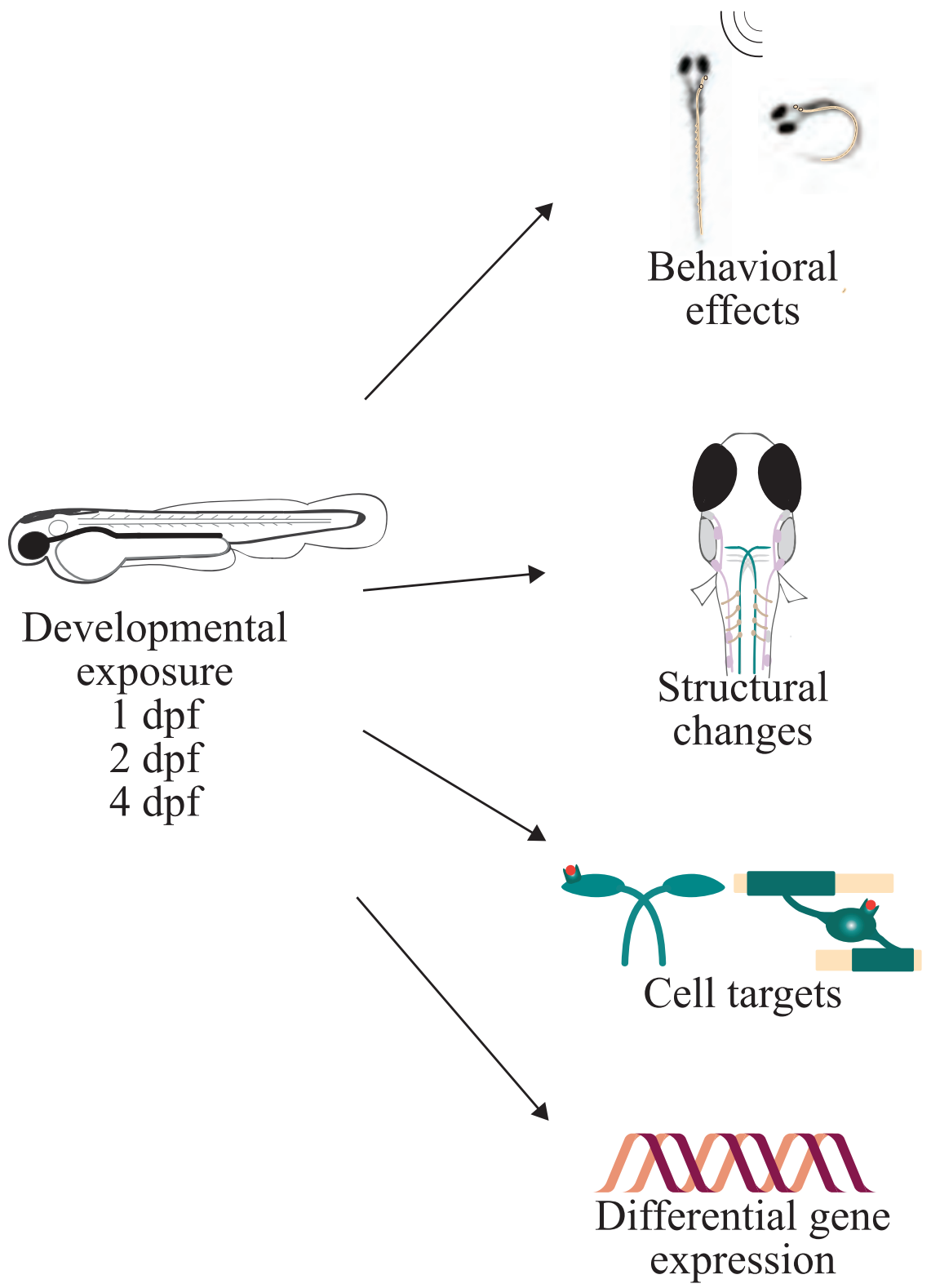

Figure 3: Dissertation approach

Zebrafish were exposed to DomA at different neurodevelopmental periods to identify the developmental window of susceptibility for toxicity. Startle behavior was characterized to assess the functional consequences of DomA exposure. To identify the underlying cellular changes that lead to DomA-induced behavioral deficits, neural structures and cell types that make up the startle circuit were imaged. Finally, whole embryo RNASeq was performed to identify the transcriptional responses resulting from DomA exposure. 


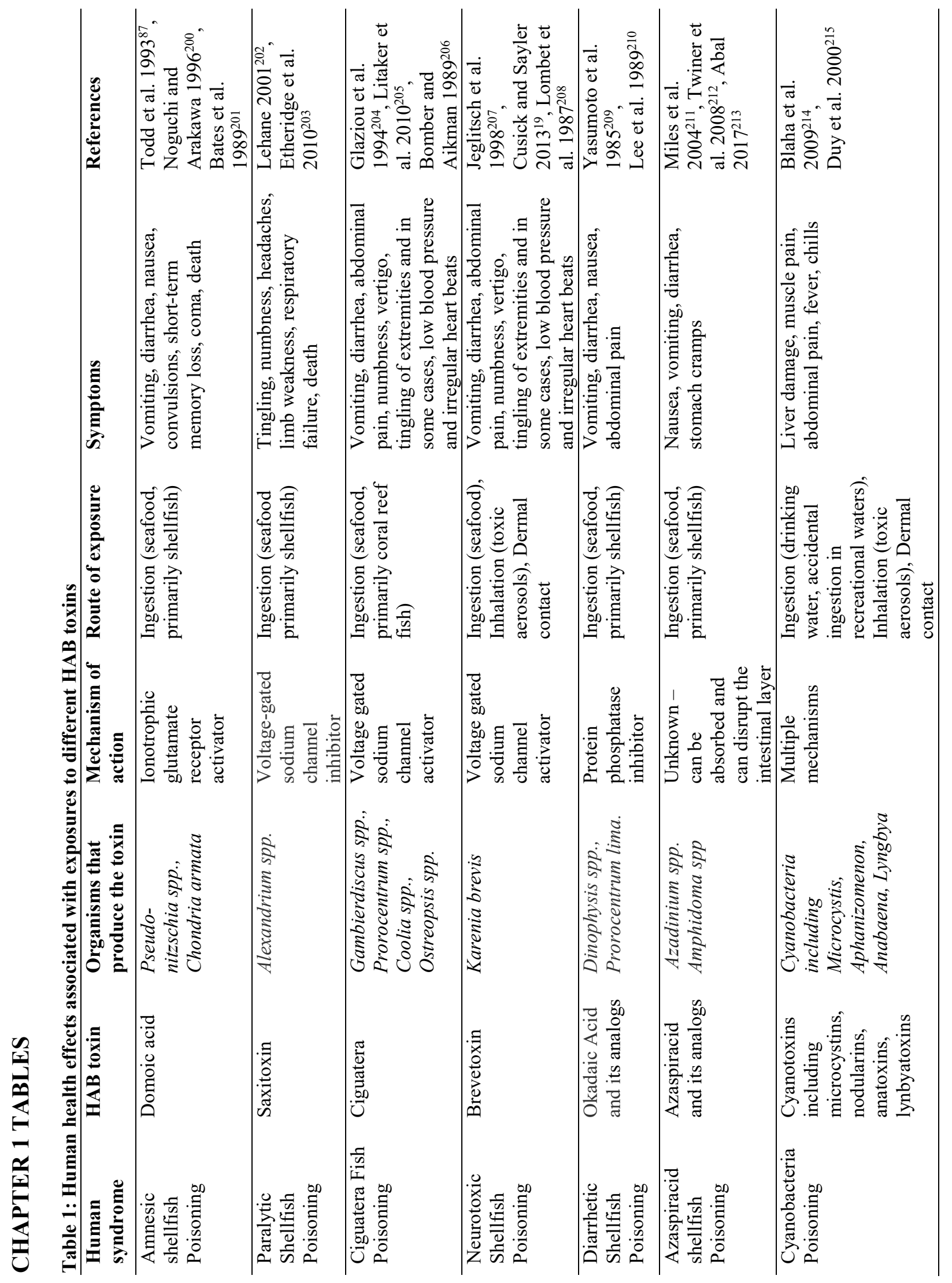




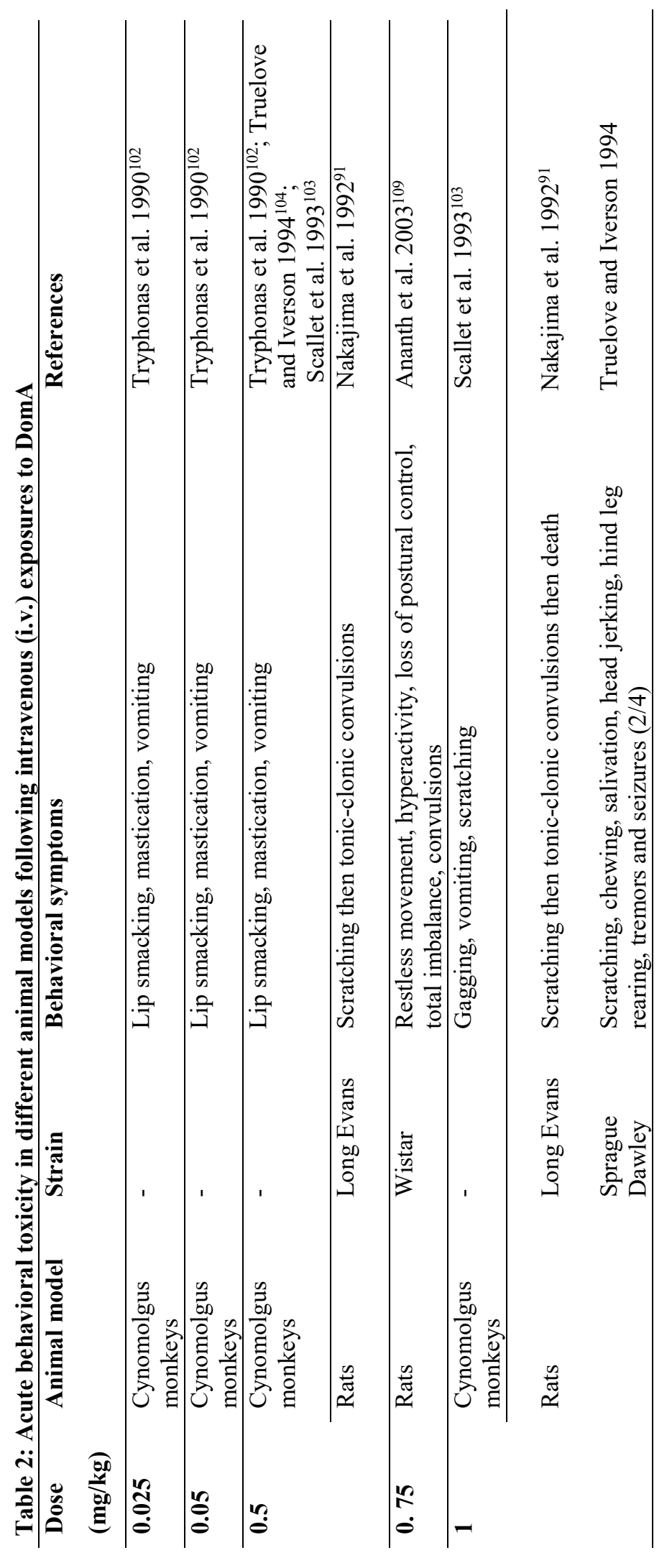




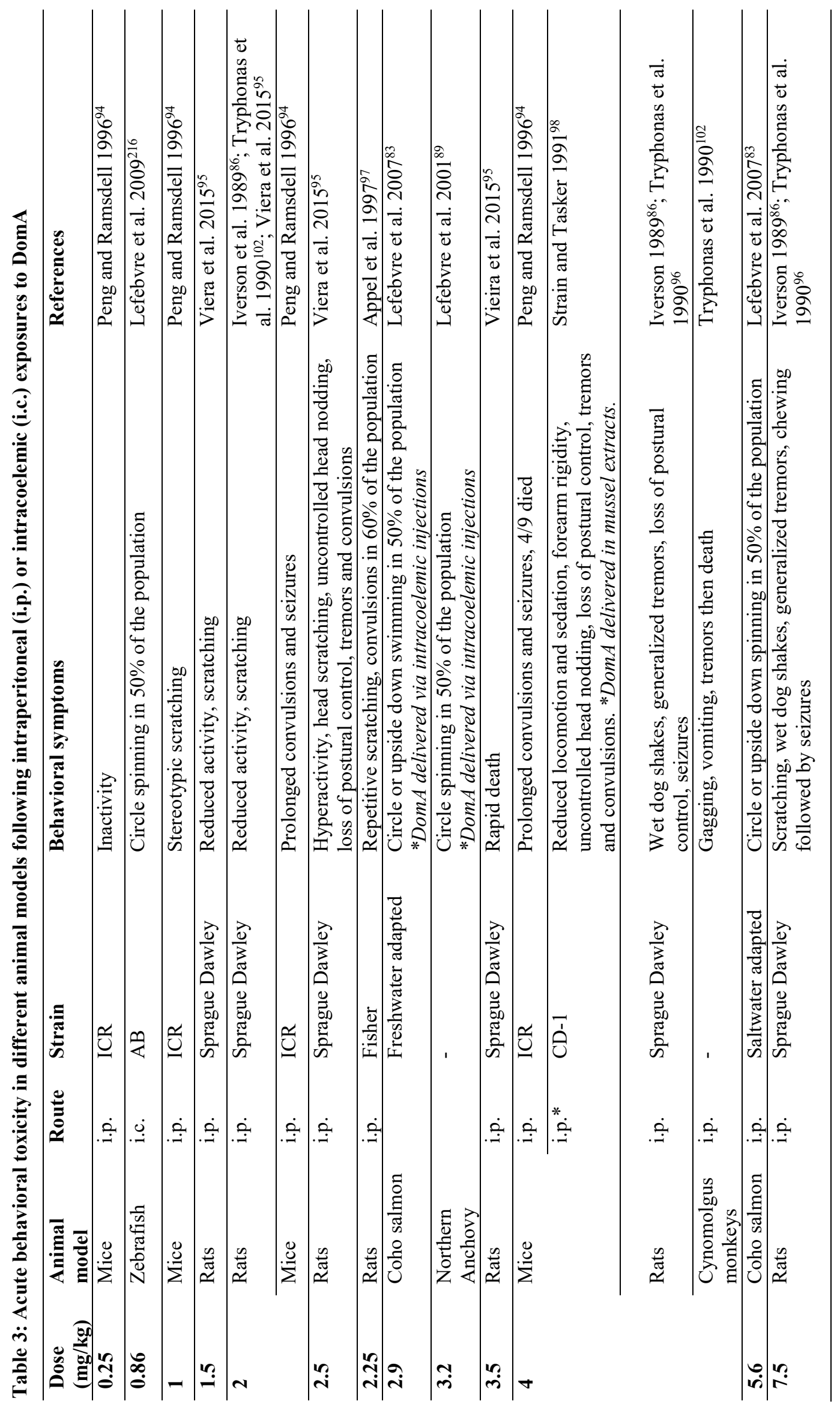


Table 4: DomA toxicity from a single exposure to DomA intraperitoneally in rats

\begin{tabular}{|c|c|c|c|c|c|}
\hline Strain & $\begin{array}{l}\text { Developmental } \\
\text { exposure day }\end{array}$ & ED50 & $\begin{array}{l}\mathbf{L T *} \\
(\mathrm{mg} / \mathrm{kg})\end{array}$ & $\begin{array}{c}\text { NT* } \\
(\mathrm{mg} / \mathrm{kg})\end{array}$ & Reference \\
\hline Long Evans & $\begin{array}{l}\text { PND 5, } \\
\text { PND } 10\end{array}$ & & $0.05 \mathrm{mg} / \mathrm{kg}$ & & Xi et al. $1997^{32}$ \\
\hline $\begin{array}{l}\text { Sprague- } \\
\text { Dawley }\end{array}$ & PND 0 & $\begin{array}{l}0.12 \pm \\
0.01\end{array}$ & & & $\begin{array}{l}\text { Doucette et } \\
\text { al. } 2000^{34}\end{array}$ \\
\hline $\begin{array}{l}\text { Sprague- } \\
\text { Dawley }\end{array}$ & PND 5 & $\begin{array}{l}0.15 \pm \\
0.01\end{array}$ & & & $\begin{array}{l}\text { Doucette et } \\
\text { al. } 2000^{34}\end{array}$ \\
\hline $\begin{array}{l}\text { Sprague- } \\
\text { Dawley }\end{array}$ & PND 14 & $\begin{array}{l}0.30 \pm \\
0.04\end{array}$ & & & $\begin{array}{l}\text { Doucette et } \\
\text { al. } 2000^{34}\end{array}$ \\
\hline $\begin{array}{l}\text { Sprague- } \\
\text { Dawley }\end{array}$ & PND 22 & $\begin{array}{l}1.06 \pm \\
0.11\end{array}$ & & & $\begin{array}{l}\text { Doucette et } \\
\text { al. } 2000^{34}\end{array}$ \\
\hline $\begin{array}{l}\text { Sprague- } \\
\text { Dawley }\end{array}$ & Adult & & $2 \mathrm{mg} / \mathrm{kg}$ & $1 \mathrm{mg} / \mathrm{kg}$ & $\begin{array}{l}\text { Tryphonas et } \\
\text { al. } 1990^{32}\end{array}$ \\
\hline
\end{tabular}

Notes: $\mathrm{LT}=$ lowest dose reported to cause overtly neurotoxic symptoms in a proportion of the population. $\mathrm{NT}=$ lowest dose reported that shows no signs of toxicity 


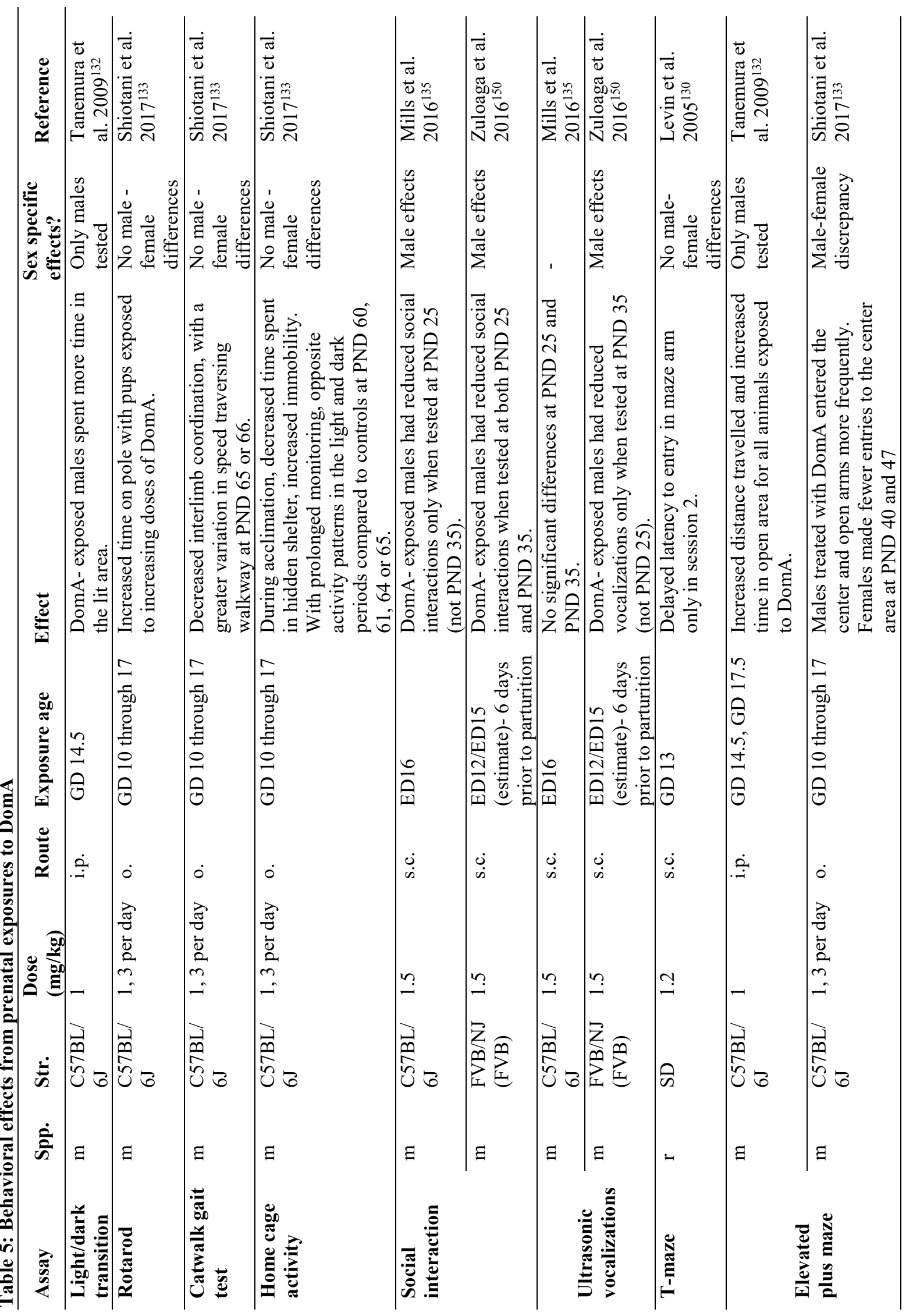




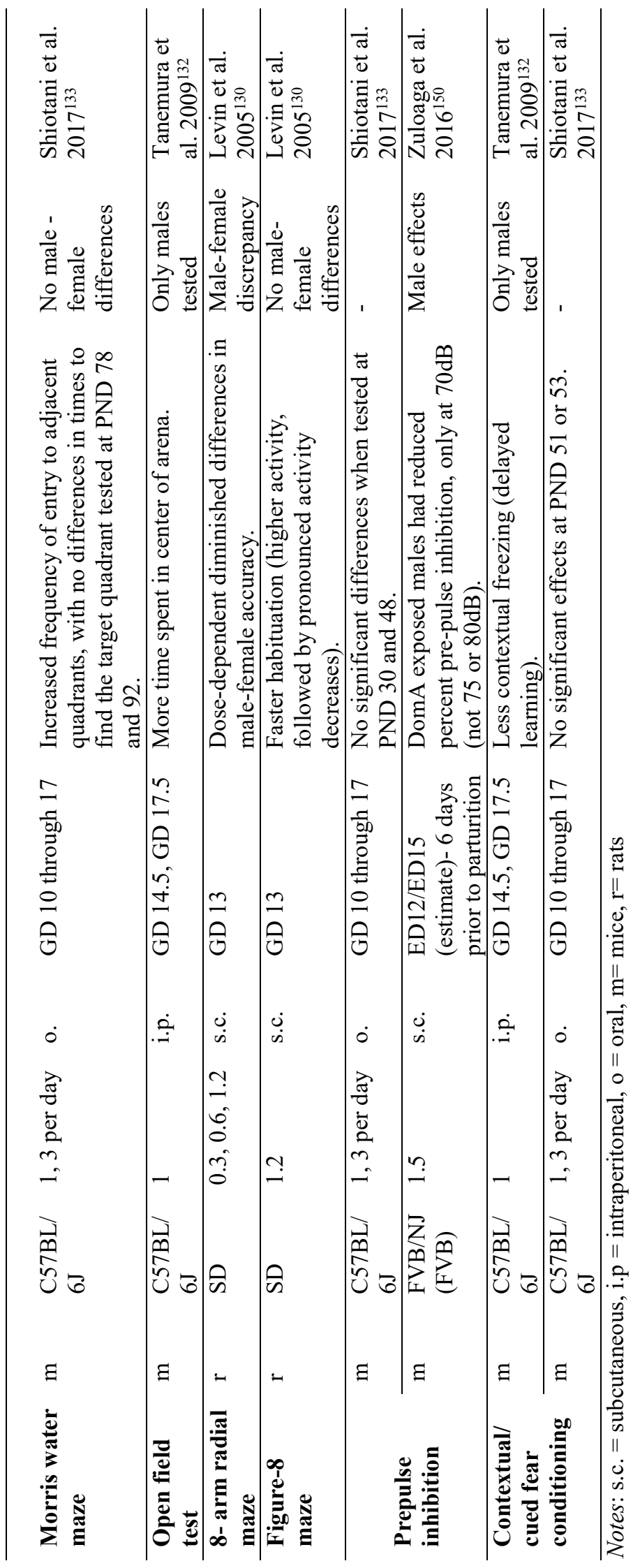




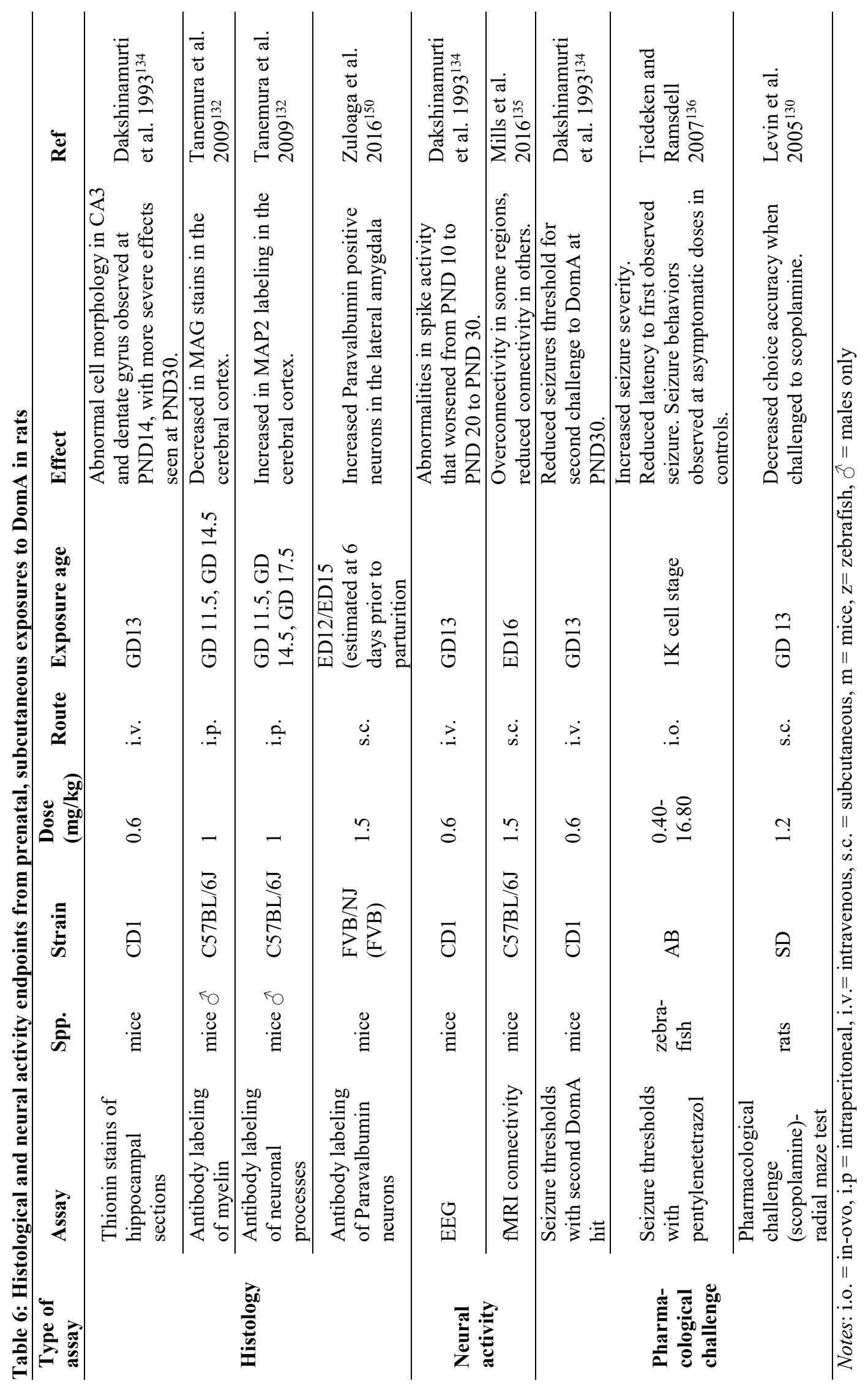




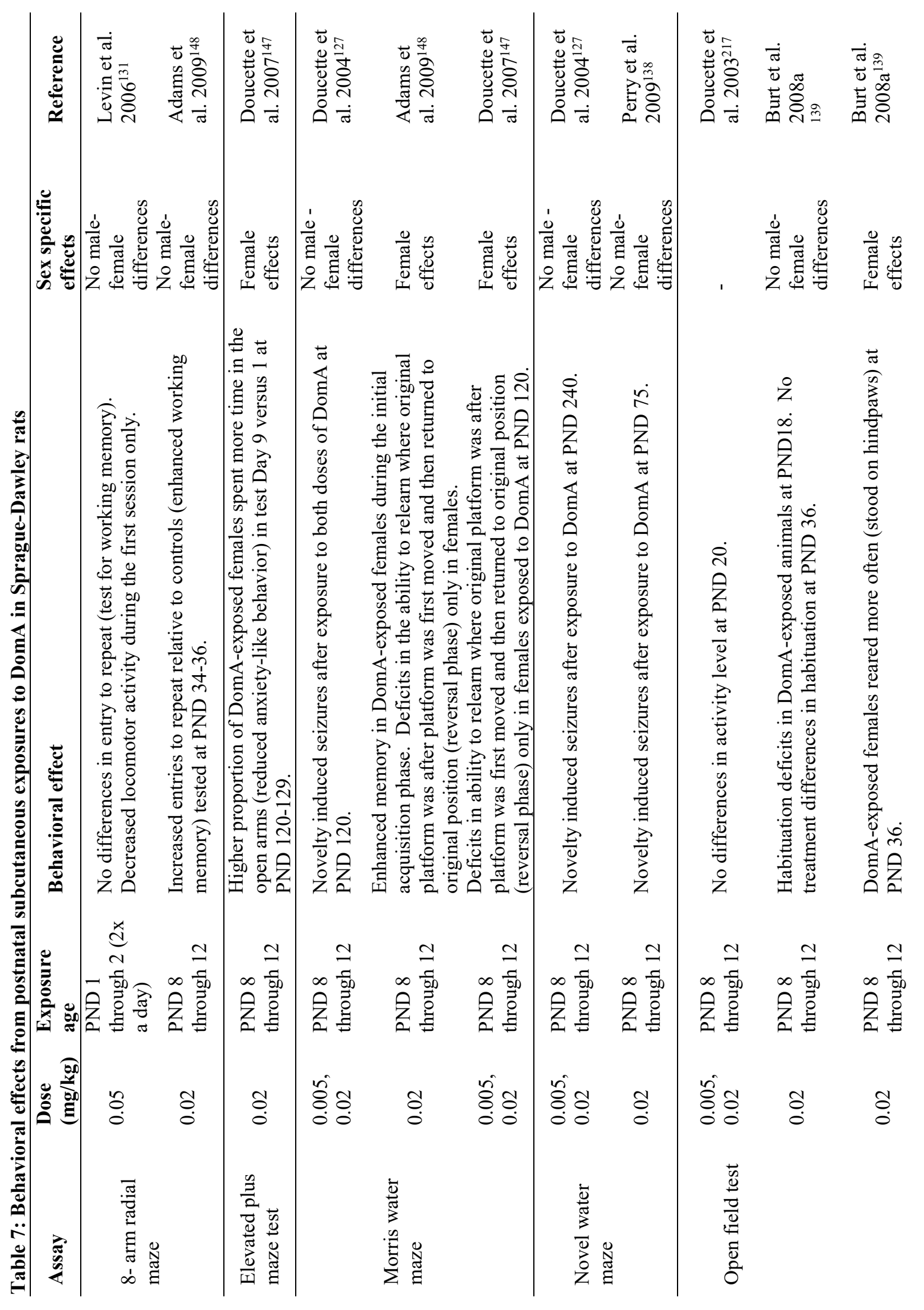




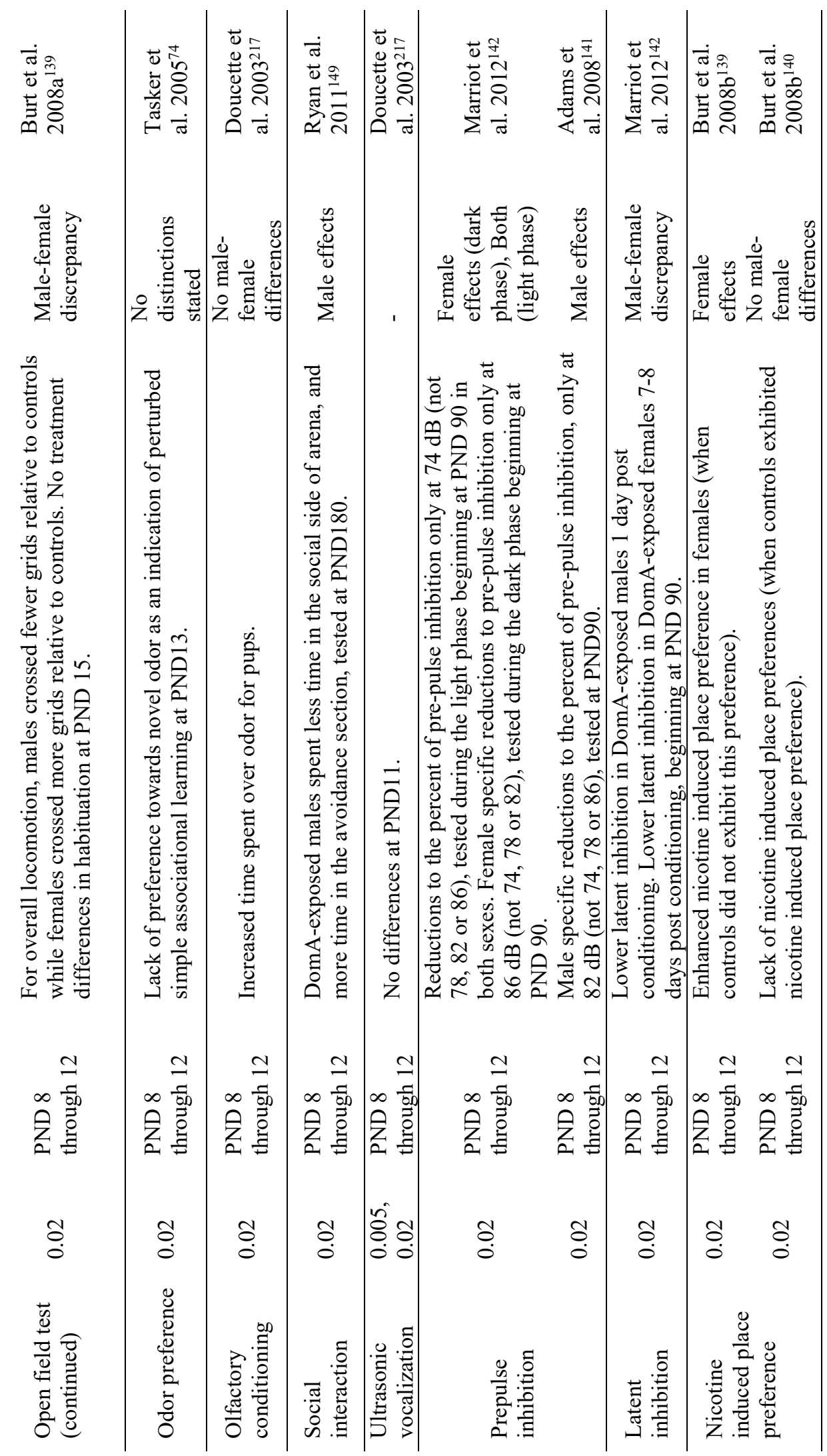




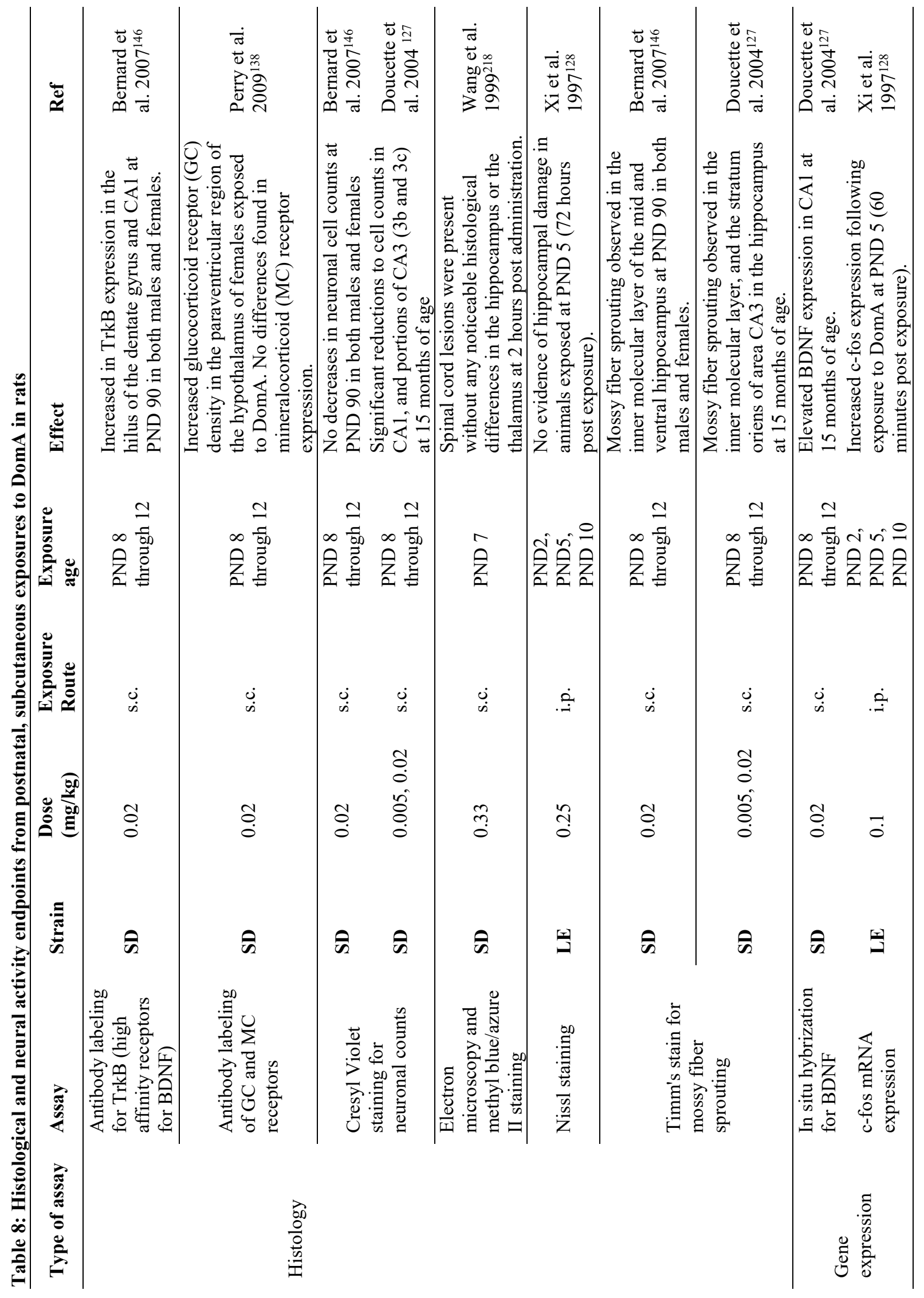




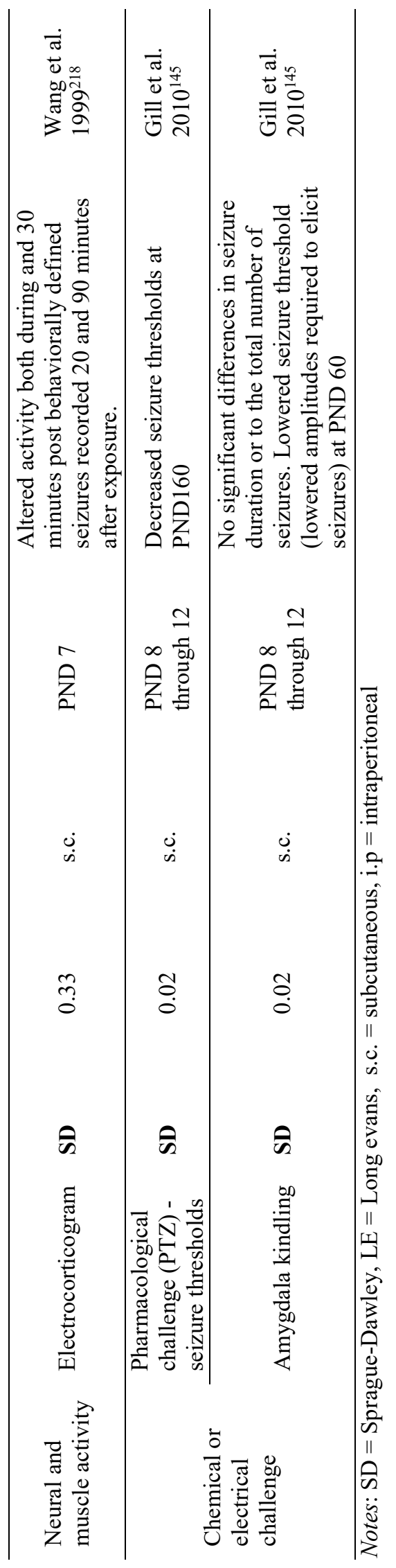




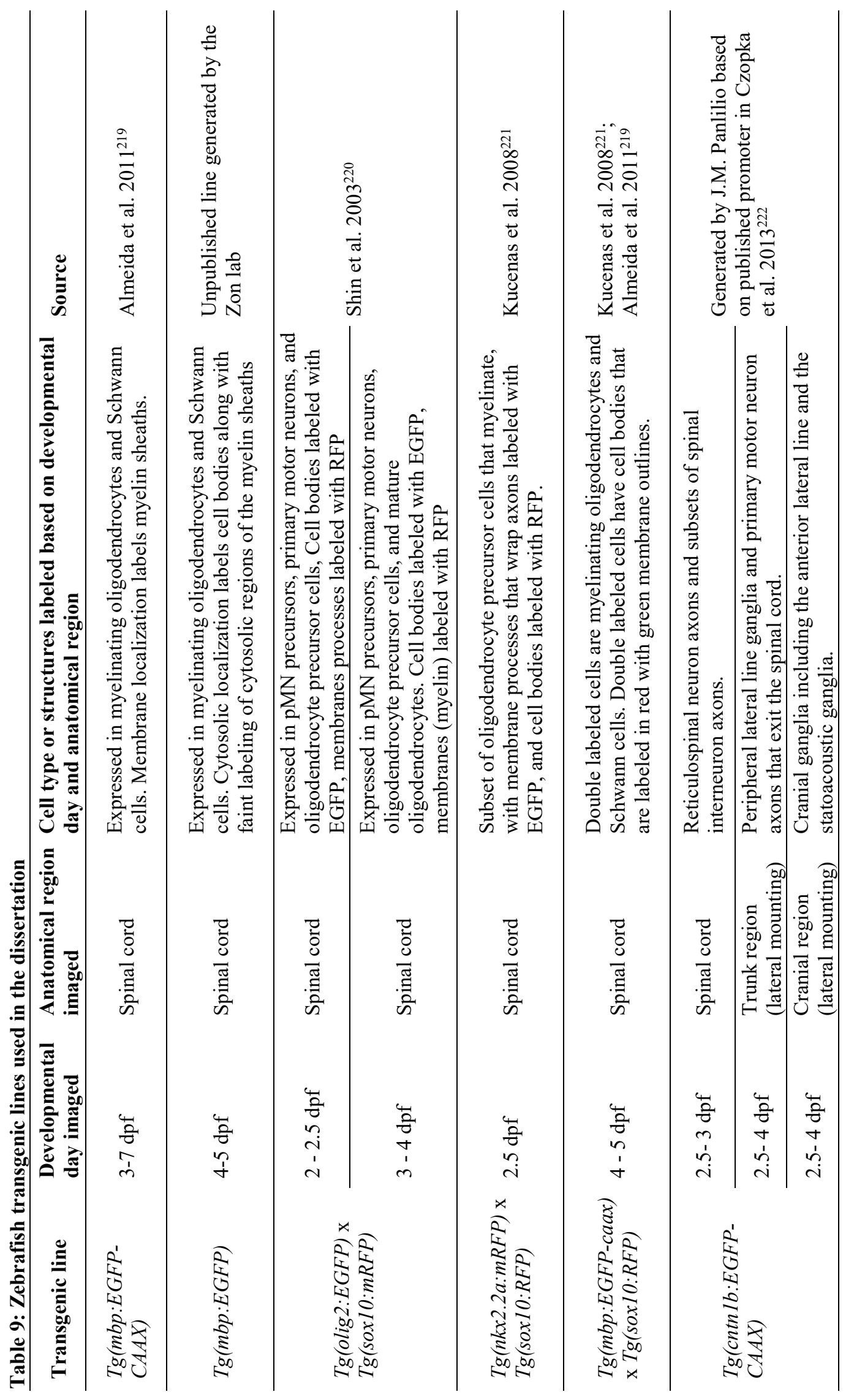




\subsection{REFERENCES}

1. Berdalet, E. et al. Marine harmful algal blooms, human health and wellbeing: challenges and opportunities in the 21st century. J. Mar. Biol. Assoc. United Kingdom 96, 61-91 (2016).

2. Anderson, D. M., Cembella, A. D. \& Hallegraeff, G. M. Progress in Understanding Harmful Algal Blooms: Paradigm Shifts and New Technologies for Research, Monitoring, and Management. Ann. Rev. Mar. Sci. 4, 143-176 (2012).

3. Naar, J. P. et al. Brevetoxins, like ciguatoxins, are potent ichthyotoxic neurotoxins that accumulate in fish. Toxicon 50, 707-723 (2007).

4. Geller, R. J., Olson, K. R. \& Senécal, P. E. Ciguatera fish poisoning in San Francisco, California, caused by imported barracuda. West. J. Med. 155, 639-42 (1991).

5. Wu, J.-Y., Zheng, L. \& Wang, J.-H. Contamination of shellfish from Shanghai seafood markets with paralytic shellfish poisoning and diarrhetic shellfish poisoning toxins determined by mouse bioassay and HPLC. Food Addit. Contam. 22, 647-651 (2005).

6. Vale, P. \& Sampayo, M. A. Domoic acid in Portuguese shellfish and fish. Toxicon 39, 893-904 (2001).

7. Jetoo, S. et al. The Toledo Drinking Water Advisory: Suggested Application of the Water Safety Planning Approach. Sustainability 7, 9787-9808 (2015).

8. Steffen, M. M. et al. Ecophysiological Examination of the Lake Erie Microcystis Bloom in 2014: Linkages between Biology and the Water Supply Shutdown of Toledo, OH. Environ. Sci. Technol. 51, 6745-6755 (2017).

9. Berktay, A. Environmental Approach and Influence of Red Tide to Desalination Process in the Middle East Region. International Journal of Chemical and Environmental Engineering 2, (2011).

10. Villacorte, L. O. et al. Seawater reverse osmosis desalination and (harmful) algal blooms. Desalination 360, 61-80 (2015).

11. Anderson, D. M. Turning back the harmful red tide. Nature 388, 513-514 (1997).

12. Jones, J. B. \& Rhodes, L. L. Suffocation of pilchards ( Sardinops sagax ) by a green microalgal bloom in Wellington Harbour, New Zealand. New Zeal. J. Mar. Freshw. Res. 28, 379-383 (1994).

13. Kent, M., Whyte, J. \& LaTrace, C. Gill lesions and mortality in seawater pen-reared Atlantic salmon Salmo salar associated with a dense bloom of Skeletonema costatum and Thalassiosira species. Dis. Aquat. Organ. 22, 77-81 (1995).

14. Hoagland, P., Anderson, D. M., Kaoru, Y. \& White, A. W. The economic effects of harmful algal blooms in the United States: Estimates, assessment issues, and information needs. Estuaries 25, 819-837 (2002).

15. Anderson, D. M., Hoagland, P., Kaoru, Y. \& White, A. W. Estimated annual economic impacts from harmful algal blooms (HABs) in the United States. (Woods Hole Oceanographic Institution, 2000). doi:10.1575/1912/96

16. Havens, K. E. in Cyanobacterial Harmful Algal Blooms: State of the Science and Research Needs (ed. Hudnell, H. K.) 733-747 (Springer, 2008). at $<$ https://link.springer.com/content/pdf/10.1007/978-0-387-75865-7_33.pdf $>$

17. Granéli, E. et al. in Novel Phytoplankton Blooms (eds. Cosper, E. M., Bricelj, V. M. \& Carpenter, E. J.) 407-427 (American Geophysical Union, 1989). doi:10.1007/978-3-64275280-3 24

18. Paerl, H. W., Fulton, R. S., Moisander, P. H. \& Dyble, J. Harmful freshwater algal 
blooms, with an emphasis on cyanobacteria. ScientificWorldJournal. 1, 76-113 (2001).

19. Cusick, K. D. \& Sayler, G. S. An overview on the marine neurotoxin, saxitoxin: genetics, molecular targets, methods of detection and ecological functions. Mar. Drugs 11, 9911018 (2013).

20. Van Dolah, F. M. Marine algal toxins: origins, health effects, and their increased occurrence. Environ. Health Perspect. 108 Suppl, 133-41 (2000).

21. Backer, L. et al. Cyanobacteria and Algae Blooms: Review of Health and Environmental Data from the Harmful Algal Bloom-Related Illness Surveillance System (HABISS) 2007-2011. Toxins (Basel). 7, 1048-1064 (2015).

22. Backer, L., LE, F., AD, R. \& DG, B. Epidemiology, public health and human diseases associated with harmful marine algae. (UNESCO Publishing, 2003).

23. Kite-Powell, H. L. et al. Linking the oceans to public health: current efforts and future directions. Environ. Health 7 Suppl 2, S6 (2008).

24. McKibben, S. M. et al. Climatic regulation of the neurotoxin domoic acid. Proc. Natl. Acad. Sci. U. S. A. 114, 239-244 (2017).

25. Perl, T. M. et al. An Outbreak of Toxic Encephalopathy Caused by Eating Mussels Contaminated with Domoic Acid. N. Engl. J. Med. 322, 1775-1780 (1990).

26. Bates, S. S., Garrison, D. L. \& Horner, R. A. in Physiological ecology of harmful algal blooms (eds. Anderson, D. M., Cembella, A. D. \& Hallegraeff, G. M.) 262-292 (SpringerVerlag, 1998).

27. Boyd, R. K. et al. Identification of domoic acid, a neuroexcitatory amino acid, in toxic mussels from eastern Prince Edward Island. Can. J. Chem. 67, 481 (1989).

28. Teitelbaum, J. S. et al. Neurologic sequelae of domoic acid intoxication due to the ingestion of contaminated mussels. N. Engl. J. Med. 322, 1781-7 (1990).

29. Zola-Morgan, S., Squire, L. R. \& Amaral, D. G. Human amnesia and the medial temporal region: enduring memory impairment following a bilateral lesion limited to field CA1 of the hippocampus. J. Neurosci. 6, 2950-67 (1986).

30. O'Keefe, J. \& Nadel, L. The hippocampus as a cognitive map. (Oxford University Press, 1978).

31. Wekell, J. C., Jurst, J. \& Lefebvre, K. a. The origin of the regulatory limits for PSP and ASP toxins in shellfish. J. Shellfish Res. 23, 927-930 (2010).

32. Kvitek, R., Goldberg, J., Smith, G., Doucette, G. \& Silver, M. Domoic acid contamination within eight representative species from the benthic food web of Monterey Bay, California, USA. Mar. Ecol. Prog. Ser. 367, 35-47 (2008).

33. Bargu, S. et al. Krill: a potential vector for domoic acid in marine food webs. Mar. Ecol. Prog. Ser. 237, 209-216 (2002).

34. F.Leandro, L., J.Teegarden, G., B.Roth, P., Wang, Z. \& J.Doucette, G. The copepod Calanus finmarchicus: A potential vector for trophic transfer of the marine algal biotoxin, domoic acid. Artic. J. Exp. Mar. Biol. Ecol. 382, 88-95 (2010).

35. Mariën, K. Establishing tolerable dungeness crab (Cancer magister) and razor clam (Siliqua patula) domoic acid contaminant levels. Environ. Health Perspect. 104, 1230-6 (1996).

36. Douglas, D. J. et al. Accumulation of domoic acid by the sea scallop ( Placopecten magellanicus ) fed cultured cells of toxic Pseudo-nitzschia multiseries. Can. J. Fish. Aquat. Sci. 54, 907-913 (1997).

37. Lefebvre, K., Silver, M., Coale, S. \& Tjeerdema, R. Domoic acid in planktivorous fish in 
relation to toxic Pseudo-nitzschia cell densities. Mar. Biol. 140, 625-631 (2002).

38. Food and Drug Adminstration. Bad Bug Book, Foodborne Pathogenic Microorganisms and Natural Toxins. (2012).

39. in Assessment and management of biotoxin risks in bivalve mollusks. (eds. Lawrence, J. et al.) 111-162 (Food and Agriculture Organization of the United Nations, 2011).

40. Washington State Department of Health. Domoic Acid in Razor Clams: Interim Health Advisory on Eating Razor Clams. (2016). at <www.doh.wa.gov>

41. Ferriss, B. E., Marcinek, D. J., Ayres, D., Borchert, J. \& Lefebvre, K. A. Acute and chronic dietary exposure to domoic acid in recreational harvesters: A survey of shellfish consumption behavior. Environ. Int. 101, 70-79 (2017).

42. Andjelkovic, M., Vandevijvere, S., Van Klaveren, J., Van Oyen, H. \& Loco, J. Van. Exposure to domoic acid through shellfish consumption in Belgium. Environ. Int. 49, 115-119 (2012).

43. Hampson, D. R., Huang, X., Wells, J. W., Walter, J. A. \& Wright, J. L. C. Interaction of domoic acid and several derivatives with kainic acid and AMPA binding sites in rat brain. Eur. J. Pharmacol. 218, 1-8 (1992).

44. Hampson, D. R. \& Manalo, J. L. The activation of glutamate receptors by kainic acid and domoic acid. Nat. Toxins 6, 153-8 (1998).

45. Hald, H. et al. Partial agonism and antagonism of the ionotropic glutamate receptor iGLuR5: structures of the ligand-binding core in complex with domoic acid and 2-amino3-[5-tert-butyl-3-(phosphonomethoxy)-4-isoxazolyl]propionic acid. J. Biol. Chem. 282, 25726-36 (2007).

46. Nanao, M. H., Green, T., Stern-Bach, Y., Heinemann, S. F. \& Choe, S. Structure of the kainate receptor subunit GluR6 agonist-binding domain complexed with domoic acid. Proc. Natl. Acad. Sci. U. S. A. 102, 1708-13 (2005).

47. Stewart, G. R., Zorumski, C. F., Price, M. T. \& Olney, J. W. Domoic acid: A dementiainducing excitotoxic food poison with kainic acid receptor specificity. Exp. Neurol. 110, 127-138 (1990).

48. Ankarcrona, M. et al. Glutamate-induced neuronal death: a succession of necrosis or apoptosis depending on mitochondrial function. Neuron 15, 961-73 (1995).

49. Bonfoco, E., Krainc, D., Ankarcrona, M., Nicotera, P. \& Lipton, S. A. Apoptosis and necrosis: two distinct events induced, respectively, by mild and intense insults with Nmethyl-D-aspartate or nitric oxide/superoxide in cortical cell cultures. Proc. Natl. Acad. Sci. 92, 7162-7166 (1995).

50. Nicholls, D. G. \& Ward, M. W. Mitochondrial membrane potential and neuronal glutamate excitotoxicity: mortality and millivolts. Trends Neurosci. 23, 166-174 (2000).

51. Arundine, M. \& Tymianski, M. Molecular mechanisms of calcium-dependent neurodegeneration in excitotoxicity. Cell Calcium 34, 325-337 (2003).

52. Brady, K. M., Texel, S. J., Kishimoto, K., Koehler, R. C. \& Sapirstein, A. Cytosolic phospholipase $\mathrm{A}_{2}$ alpha modulates NMDA neurotoxicity in mouse hippocampal cultures. Eur. J. Neurosci. 24, 3381-3386 (2006).

53. Chimura, T., Launey, T. \& Yoshida, N. Calpain-Mediated Degradation of Drebrin by Excitotoxicity In vitro and In vivo. PLoS One 10, e0125119 (2015).

54. Vosler, P. S., Brennan, C. S. \& Chen, J. Calpain-mediated signaling mechanisms in neuronal injury and neurodegeneration. Mol. Neurobiol. 38, 78-100 (2008).

55. Smith, A. W. et al. Calpain inhibition attenuates apoptosis of retinal ganglion cells in 
acute optic neuritis. Invest. Ophthalmol. Vis. Sci. 52, 4935-41 (2011).

56. Rami, A., Ferger, D. \& Krieglstein, J. Blockade of calpain proteolytic activity rescues neurons from glutamate excitotoxicity. Neurosci. Res. 27, 93-97 (1997).

57. Li, Y., Maher, P. \& Schubert, D. Phosphatidylcholine-specific phospholipase C regulates glutamate-induced nerve cell death. Proc. Natl. Acad. Sci. U. S. A. 95, 7748-53 (1998).

58. Hara, H. et al. Inhibition of interleukin 1beta converting enzyme family proteases reduces ischemic and excitotoxic neuronal damage. Proc. Natl. Acad. Sci. U. S. A. 94, 2007-12 (1997).

59. Yang, D. D. et al. Absence of excitotoxicity-induced apoptosis in the hippocampus of mice lacking the Jnk3 gene. Nature 389, 865-870 (1997).

60. Bondy, S. C. \& Lee, D. K. Oxidative stress induced by glutamate receptor agonists. Brain Res. 610, 229-233 (1993).

61. MacGregor, D. G. et al. Ascorbate attenuates the systemic kainate-induced neurotoxicity in the rat hippocampus. Brain Res. 727, 133-144 (1996).

62. Giordano, G. et al. Neurotoxicity of domoic Acid in cerebellar granule neurons in a genetic model of glutathione deficiency. Mol. Pharmacol. 70, 2116-26 (2006).

63. Gupta, Y. K., Briyal, S. \& Chaudhary, G. Protective effect of trans-resveratrol against kainic acid-induced seizures and oxidative stress in rats. Pharmacol. Biochem. Behav. 71, 245-249 (2002).

64. Giordano, G., White, C. C., Mohar, I., Kavanagh, T. J. \& Costa, L. G. Glutathione levels modulate domoic acid induced apoptosis in mouse cerebellar granule cells. Toxicol. Sci. 100, 433-44 (2007).

65. Berman, F. W., LePage, K. T. \& Murray, T. F. Domoic acid neurotoxicity in cultured cerebellar granule neurons is controlled preferentially by the NMDA receptor $\mathrm{Ca} 2+$ influx pathway. Brain Res. 924, 20-29 (2002).

66. Nijjar, M. S. \& Nijjar, S. S. Domoic acid-induced neurodegeneration resulting in memory loss is mediated by $\mathrm{Ca} 2+$ overload and inhibition of $\mathrm{Ca} 2++$ calmodulin-stimulated adenylate cyclase in rat brain (review). Int. J. Mol. Med. 6, 377-466 (2000).

67. Xi, D. \& Ramsdell, J. S. Glutamate receptors and calcium entry mechanisms for domoic acid in hippocampal neurons. Neuroreport 7, 1115-20 (1996).

68. Weiss, J. H., Hartley, D. M., Koh, J. \& Choi, D. W. The calcium channel blocker nifedipine attenuates slow excitatory amino acid neurotoxicity. Science 247, 1474-7 (1990).

69. Keller, B. U., Hollmann, M., Heinemann, S. \& Konnerth, A. Calcium influx through subunits GluR1/GluR3 of kainate/AMPA receptor channels is regulated by cAMP dependent protein kinase. EMBO J. 11, 891-6 (1992).

70. Hollmann, M., Hartley, M. \& Heinemann, S. Ca2+ permeability of KA-AMPA--gated glutamate receptor channels depends on subunit composition. Science 252, 851-3 (1991).

71. Novelli, A., Kispert, J., Ferna'ndez-Sa'nchez, M. T., Torreblanca, A. \& Zitko, V. Domoic acid-containing toxic mussels produce neurotoxicity in neuronal cultures through a synergism between excitatory amino acids. Brain Res. 577, 41-48 (1992).

72. Berman, F. W. \& Murray, T. F. Domoic acid neurotoxicity in cultured cerebellar granule neurons is mediated predominantly by NMDA receptors that are activated as a consequence of excitatory amino acid release. J. Neurochem. 69, 693-703 (1997).

73. Tasker, R. A. \& Strain, S. M. Synergism between NMDA and domoic acid in a murine model of behavioural neurotoxicity. Neurotoxicology 19, 593-7 
74. Tasker, R. A. R., Perry, M. A., Doucette, T. A. \& Ryan, C. L. NMDA receptor involvement in the effects of low dose domoic acid in neonatal rats. Amino Acids 28, 193196 (2005).

75. Bettler, B. \& Mulle, C. AMPA and kainate receptors. Neuropharmacology 34, 123-139 (1995).

76. Szydlowska, K. \& Tymianski, M. Calcium, ischemia and excitotoxicity. Cell Calcium 47, 122-129 (2010).

77. Lai, T. W., Zhang, S. \& Wang, Y. T. Excitotoxicity and stroke: Identifying novel targets for neuroprotection. Prog. Neurobiol. 115, 157-188 (2014).

78. Dong, X., Wang, Y. \& Qin, Z. Molecular mechanisms of excitotoxicity and their relevance to pathogenesis of neurodegenerative diseases. Acta Pharmacol. Sin. 30, 379387 (2009).

79. Salińska, E., Danysz, W. \& Łazarewicz, J. W. The role of excitotoxicity in neurodegeneration. Folia Neuropathol. 43, 322-39 (2005).

80. Jing, J. et al. Toxicokinetics and Physiologically Based Pharmacokinetic Modeling of the Shellfish Toxin Domoic Acid in Nonhuman Primates. Drug Metab Dispos 46, 155-165 (2018).

81. Truelove, J. et al. 30-day oral toxicity study of domoic acid in Cynomolgus monkeys: Lack of overt toxicity at doses approaching the acute toxic dose. Nat. Toxins 5, 111-114 (1998).

82. Preston, E. \& Hynie, I. Transfer constants for blood-brain barrier permeation of the neuroexcitatory shellfish toxin, domoic acid. Can. J. Neurol. Sci. 18, 39-44 (1991).

83. Lefebvre, K. A. et al. Uptake, tissue distribution and excretion of domoic acid after oral exposure in coho salmon (Oncorhynchus kisutch). Aquat. Toxicol. 81, 266-274 (2007).

84. Suzuki, C. A. M. \& Hierlihy, S. L. Renal clearance of domoic acid in the rat. Food Chem. Toxicol. 31, 701-706 (1993).

85. Truelove, J. \& Iverson, F. Serum domoic acid clearance and clinical observations in the cynomolgus monkey and Sprague-Dawley rat following a single IV dose. Bull. Environ. Contam. Toxicol. 52, (1994).

86. Iverson, F. et al. Domoic acid poisoning and mussel-associated intoxication: preliminary investigations into the response of mice and rats to toxic mussel extract. Food Chem. Toxicol. 27, 377-84 (1989).

87. Todd, E. C. D. Domoic Acid and Amnesic Shellfish Poisoning - A Review. J. Food Prot. 56, 69-83 (1993).

88. Tryphonas, L., Truelove, J., Todd, E., Nera, E. \& Iverson, F. Experimental oral toxicity of domoic acid in cynomolgus monkeys (Macaca fascicularis) and rats.: Preliminary investigations. Food Chem. Toxicol. 28, 707-715 (1990).

89. Lefebvre, K. A., Dovel, S. L. \& Silver, M. W. Tissue distribution and neurotoxic effects of domoic acid in a prominent vector species, the northern anchovy Engraulis mordax. Mar. Biol. 138, 693-700 (2001).

90. Lefebvre, K. A. et al. Gene expression profiles in zebrafish brain after acute exposure to domoic acid at symptomatic and asymptomatic doses. Toxicol. Sci. 107, 65-77 (2009).

91. Nakajima, S. \& Potvin, J. L. Neural and behavioural effects of domoic acid, an amnesic shellfish toxin, in the rat. Can. J. Psychol. 46, 569-81 (1992).

92. Ananth, C., Thameem Dheen, S., Gopalakrishnakone, P. \& Kaur, C. Domoic acid-induced neuronal damage in the rat hippocampus: Changes in apoptosis related genes (Bcl-2, Bax, 
caspase-3) and microglial response. J. Neurosci. Res. 66, 177-190 (2001).

93. Iverson, F., Truelove, J., Tryphonas, L. \& Nera, E. A. The toxicology of domoic acid administered systemically to rodents and primates. Can. Dis. Wkly. Rep. 16 Suppl 1E, 158-9 (1990).

94. Peng, Y. G. \& Ramsdell, J. S. Brain Fos induction is a sensitive biomarker for the lowest observed neuroexcitatory effects of domoic acid. Fundam. Appl. Toxicol. 31, 162-8 (1996).

95. Vieira, A. C. et al. Brain Pathology in Adult Rats Treated With Domoic Acid. Vet. Pathol. 52, 1077-1086 (2015).

96. Tryphonas, L., Truelove, J., Nera, E. \& Iverson, F. Acute Neurotoxicity of Domoic Acid in the Rat. Toxicol. Pathol. 18, 1-9 (1990).

97. Appel, N. M., Rapoport, S. I. \& O'callaghan, J. P. Sequelae of Parenteral Domoic Acid Administration in Rats: Comparison of Effects on Different Anatomical Markers in Brain. Synapse 25, (Wiley-Liss, Inc. $\dagger, 1997$ ).

98. Strain, S. M. \& Tasker, R. A. R. Hippocampal damage produced by systemic injections of domoic acid in mice. Neuroscience 44, 343-352 (1991).

99. Scholin, C. A. et al. Mortality of sea lions along the central California coast linked to a toxic diatom bloom. Nature 403, 80-84 (2000).

100. Gulland, F. M. D. et al. Domoic acid toxicity in Californian sea lions (Zalophus californianus): clinical signs, treatment and survival. Vet. Rec. 150, 475-80 (2002).

101. Schmued, L. C., Scallet, A. C. \& Slikker, W. Domoic acid-induced neuronal degeneration in the primate forebrain revealed by degeneration specific histochemistry. Brain Res. 695, 64-70 (1995).

102. Tryphonas, L. \& Truelove, J. Acute Parenteral Neurotoxicity of Domoic Acid in Cynomolgus Monkeys (M. fnscicuZaris)*. 18, (1990).

103. Scallet, A. C. et al. Domoic acid-treated cynomolgus monkeys (M. fascicularis): effects of dose on hippocampal neuronal and terminal degeneration. Brain Res. 627, 307-13 (1993).

104. Truelove, J. \& Iverson, F. Serum domoic acid clearance and clinical observations in the cynomolgus monkey and Sprague-Dawley rat following a single i.v. dose. Bull. Environ. Contam. Toxicol. 52, 479-86 (1994).

105. Petrie, B. F., Pinsky, C., Standish, N. M., Bose, R. \& Glavin, G. B. Parenteral domoic acid impairs spatial learning in mice. Pharmacol. Biochem. Behav. 41, 211-214 (1992).

106. Sutherland, R. J., Hoesing, J. M. \& Whishaw, I. Q. Domoic acid, an environmental toxin, produces hippocampal damage and severe memory impairment. Neurosci. Lett. 120, 221223 (1990).

107. Cook, P. F. et al. Algal toxin impairs sea lion memory and hippocampal connectivity, with implications for strandings. Science 350, 1545-7 (2015).

108. Cook, P., Reichmuth, C. \& Gulland, F. Rapid behavioural diagnosis of domoic acid toxicosis in California sea lions. Biol. Lett. 7, 536-8 (2011).

109. Ananth, C., Gopalakrishnakone, P. \& Kaur, C. Induction of inducible nitric oxide synthase expression in activated microglia following domoic acid (DA)-induced neurotoxicity in the rat hippocampus. Neurosci. Lett. 338, 49-52 (2003).

110. Silvagni, P. A., Lowenstine, L. J., Spraker, T., Lipscomb, T. P. \& Gulland, F. M. D. Pathology of Domoic Acid Toxicity in California Sea Lions ( Zalophus californianus ). Vet. Pathol. 42, 184-191 (2005).

111. Grattan, L. et al. Repeated Dietary Exposure to Low Levels of Domoic Acid and 
Problems with Everyday Memory: Research to Public Health Outreach. Toxins (Basel). 10, 103 (2018).

112. Grattan, L. M. et al. The association between razor clam consumption and memory in the CoASTAL cohort. Harmful Algae 57, 20-25 (2016).

113. Hiolski, E. M. et al. Chronic low-level domoic acid exposure alters gene transcription and impairs mitochondrial function in the CNS. Aquat. Toxicol. 155, 151-9 (2014).

114. Lefebvre, K. A. et al. A Novel Antibody-Based Biomarker for Chronic Algal Toxin Exposure and Sub-Acute Neurotoxicity. PLoS One 7, e36213 (2012).

115. Moyer, C. E. et al. Repeated low level domoic acid exposure increases CA1 VGluT1 levels, but not bouton density, VGluT2 or VGAT levels in the hippocampus of adult mice. Harmful Algae (2018). doi:10.1016/J.HAL.2018.08.008

116. Lefebvre, K. A. et al. Chronic low-level exposure to the common seafood toxin domoic acid causes cognitive deficits in mice. Harmful Algae 64, 20-29 (2017).

117. Petroff, R. et al. Chronic, Low-Level Oral Exposure to Marine Toxin, Domoic Acid, Alters Whole Brain Morphometry in Nonhuman Primates. Neurotoxicology (2019). doi:10.1101/439109

118. Rice, D. \& Barone, S. Critical periods of vulnerability for the developing nervous system: evidence from humans and animal models. Environ. Health Perspect. 108 Suppl, 511-33 (2000).

119. Mattson, M. P. Glutamate and neurotrophic factors in neuronal plasticity and disease. Ann. N. Y. Acad. Sci. 1144, 97-112 (2008).

120. Luján, R., Shigemoto, R. \& López-Bendito, G. Glutamate and GABA receptor signalling in the developing brain. Neuroscience 130, 567-80 (2005).

121. Maucher, J. M. \& Ramsdell, J. S. Maternal-fetal transfer of domoic acid in rats at two gestational time points. Environ. Health Perspect. 115, 1743-6 (2007).

122. Lefebvre, K. A. et al. Domoic acid in California sea lion fetal fluids indicates continuous exposure to a neuroteratogen poses risks to mammals. Harmful Algae (2018). doi:10.1016/J.HAL.2018.06.003

123. Brodie Frances M D Gulland Denise J Greig, E. C., Hunter, M., Jaakola, J., Leger, J. S. \& Leighfield Frances M Van Dolah, T. A. Domoic acid causes reproductive failure in california sea lions (Zalophus Californianus). Mar. Mammal Sci. 3, (22AD).

124. Maucher Fuquay, J., Muha, N., Wang, Z. \& Ramsdell, J. S. Toxicokinetics of domoic acid in the fetal rat. Toxicology 294, 36-41 (2012).

125. Maucher, J. M. \& Ramsdell, J. S. Domoic acid transfer to milk: evaluation of a potential route of neonatal exposure. Environ. Health Perspect. 113, 461-4 (2005).

126. Rust, L., Gulland, F., Frame, E. \& Lefebvre, K. Domoic acid in milk of free living California marine mammals indicates lactational exposure occurs. Mar. Mammal Sci. 30, 1272-1278 (2014).

127. Doucette, T. A. et al. Low doses of domoic acid during postnatal development produce permanent changes in rat behaviour and hippocampal morphology. Neurotox. Res. 6, 555563 (2004).

128. Xi, D., Peng, Y. G. \& Ramsdell, J. S. Domoic acid is a potent neurotoxin to neonatal rats. Nat. Toxins 5, 74-9 (1997).

129. Doucette, T. ., Strain, S. ., Allen, G. ., Ryan, C. . \& Tasker, R. A. . Comparative behavioural toxicity of domoic acid and kainic acid in neonatal rats. Neurotoxicol.

Teratol. 22, 863-869 (2000). 
130. Levin, E. D., Pizarro, K., Pang, W. G., Harrison, J. \& Ramsdell, J. S. Persisting behavioral consequences of prenatal domoic acid exposure in rats. Neurotoxicol. Teratol. 27, 719-25

131. Levin, E. D. et al. Persistent neurobehavioral effects of early postnatal domoic acid exposure in rats. Neurotoxicol. Teratol. 28, 673-680 (2006).

132. Tanemura, K. et al. Intrauterine environment-genome interaction and Children's development (2): Brain structure impairment and behavioral disturbance induced in male mice offspring by a single intraperitoneal administration of domoic acid (DA) to their dams. J. Toxicol. Sci. 34, SP279-SP286 (2009).

133. Shiotani, M. et al. Neurobehavioral assessment of mice following repeated oral exposures to domoic acid during prenatal development. Neurotoxicol. Teratol. 64, 8-19 (2017).

134. Dakshinamurti, K., Sharma, S., Sundaram, M. \& Watanabe, T. Hippocampal changes in developing postnatal mice following intrauterine exposure to domoic acid. J. Neurosci. 13, 4486-4495 (1993).

135. Mills, B. D. et al. Prenatal domoic acid exposure disrupts mouse pro-social behavior and functional connectivity MRI. Behav. Brain Res. 308, 14-23 (2016).

136. Tiedeken, J. A. \& Ramsdell, J. S. Embryonic exposure to domoic Acid increases the susceptibility of zebrafish larvae to the chemical convulsant pentylenetetrazole. Environ. Health Perspect. 115, 1547-52 (2007).

137. Galvan, C. D., Hrachovy, R. A., Smith, K. L. \& Swann, J. W. Blockade of Neuronal Activity During Hippocampal Development Produces a Chronic Focal Epilepsy in the Rat. 2904-2916 (2000). at <http://www.jneurosci.org/content/jneuro/20/8/2904.full.pdf >

138. Perry, M. A., Ryan, C. L. \& Tasker, R. A. Effects of low dose neonatal domoic acid administration on behavioural and physiological response to mild stress in adult rats. Physiol. Behav. 98, 53-59 (2009).

139. Burt, M. A., Ryan, C. L. \& Doucette, T. A. Altered responses to novelty and drug reinforcement in adult rats treated neonatally with domoic acid. Physiol. Behav. 93, 327336 (2008).

140. Burt, M. A., Ryan, C. L. \& Doucette, T. A. Low dose domoic acid in neonatal rats abolishes nicotine induced conditioned place preference during late adolescence. Amino Acids 35, 247-249 (2008).

141. Adams, A. L., Doucette, T. A. \& Ryan, C. L. Altered pre-pulse inhibition in adult rats treated neonatally with domoic acid. Amino Acids 35, 157-60 (2008).

142. Marriott, A. L., Ryan, C. L. \& Doucette, T. A. Neonatal domoic acid treatment produces alterations to prepulse inhibition and latent inhibition in adult rats. Pharmacol. Biochem. Behav. 103, 338-44 (2012).

143. Costa, L. G., Giordano, G. \& Faustman, E. M. Domoic acid as a developmental neurotoxin. Neurotoxicology 31, 409-23 (2010).

144. Doucette, T. A. \& Andrew Tasker, R. in Current topics in behavioral neurosciences 29, $87-110(2015)$.

145. Gill, D. A. et al. Neonatal exposure to low-dose domoic acid lowers seizure threshold in adult rats. Neuroscience 169, 1789-1799 (2010).

146. Bernard, P. B., MacDonald, D. S., Gill, D. A., Ryan, C. L. \& Tasker, R. A. Hippocampal mossy fiber sprouting and elevated trkB receptor expression following systemic administration of low dose domoic acid during neonatal development. Hippocampus 17, 1121-1133 (2007).

147. Doucette, T. A., Ryan, C. L. \& Tasker, R. A. Gender-based changes in cognition and 
emotionality in a new rat model of epilepsy. Amino Acids 32, 317-322 (2007).

148. Adams, A. L., Doucette, T. A., James, R. \& Ryan, C. L. Persistent changes in learning and memory in rats following neonatal treatment with domoic acid. Physiol. Behav. 96, 50512 (2009).

149. Ryan, C. L. et al. Altered social interaction in adult rats following neonatal treatment with domoic acid. Physiol. Behav. 102, 291-295 (2011).

150. Zuloaga, D. G. et al. Fetal domoic acid exposure affects lateral amygdala neurons, diminishes social investigation and alters sensory-motor gating. Neurotoxicology 53, 132 140 (2016).

151. Sumbre, G. \& de Polavieja, G. G. The world according to zebrafish: how neural circuits generate behavior. Front. Neural Circuits 8, 91 (2014).

152. Higashijima, S., Masino, M. A., Mandel, G. \& Fetcho, J. R. Imaging neuronal activity during zebrafish behavior with a genetically encoded calcium indicator. J. Neurophysiol. 90, 3986-97 (2003).

153. Fetcho, J. R. \& Higashijima, S.-I. Optical and genetic approaches toward understanding neuronal circuits in zebrafish. Integr. Comp. Biol. 44, 57-70 (2004).

154. Guo, S. Linking genes to brain, behavior and neurological diseases: what can we learn from zebrafish? Genes, Brain Behav. 3, 63-74 (2004).

155. Friedrich, R. W., Jacobson, G. A. \& Zhu, P. Circuit neuroscience in zebrafish. Curr. Biol. 20, R371-81 (2010).

156. Wullimann, M. F. \& Mueller, T. Teleostean and Mammalian Forebrains Contrasted: Evidence from Genes to Behavior. J. Comp. Neurol. 475, 143-162 (2004).

157. Mueller, T., Dong, Z., Berberoglu, M. A. \& Guo, S. The dorsal pallium in zebrafish, Danio rerio (Cyprinidae, Teleostei). Brain Res. 1381, 95-105 (2011).

158. Naganawa, Y. \& Hirata, H. Developmental transition of touch response from slow muscle-mediated coilings to fast muscle-mediated burst swimming in zebrafish. Dev. Biol. 355, 194-204 (2011).

159. Kohashi, T., Nakata, N. \& Oda, Y. Effective Sensory Modality Activating an Escape Triggering Neuron Switches during Early Development in Zebrafish. J. Neurosci. 32, 5810-5820 (2012).

160. Friedrich, R. W. \& Korsching, S. I. Combinatorial and Chemotopic Odorant Coding in the Zebrafish Olfactory Bulb Visualized by Optical Imaging. Neuron 18, 737-752 (1997).

161. Whitlock, K. E. The Sense of Scents: Olfactory Behaviors in the Zebrafish. Zebrafish 3, 203-213 (2006).

162. Burgess, H. A. \& Granato, M. Sensorimotor gating in larval zebrafish. J. Neurosci. 27, 4984-94 (2007).

163. Hale, M. E., Katz, H. R., Peek, M. Y. \& Fremont, R. T. Neural circuits that drive startle behavior, with a focus on the Mauthner cells and spiral fiber neurons of fishes. $J$. Neurogenet. 30, 89-100 (2016).

164. Haesemeyer, M., Robson, D. N., Li, J. M., Schier, A. F. \& Engert, F. The Structure and Timescales of Heat Perception in Larval Zebrafish. Cell Syst. 1, 338-348 (2015).

165. Prober, D. A. et al. Zebrafish TRPA1 Channels Are Required for Chemosensation But Not for Thermosensation or Mechanosensory Hair Cell Function. J. Neurosci. 28, 1010210110 (2008).

166. Roberts, A. C. et al. Habituation of the C-start response in larval zebrafish exhibits several distinct phases and sensitivity to NMDA receptor blockade. PLoS One 6, e29132 (2011). 
167. Wolman, M. A., Jain, R. A., Liss, L. \& Granato, M. Chemical modulation of memory formation in larval zebrafish. Proc. Natl. Acad. Sci. U. S. A. 108, 15468-73 (2011).

168. Bergeron, S. A., Carrier, N., Li, G. H., Ahn, S. \& Burgess, H. A. Gsx 1 expression defines neurons required for prepulse inhibition. Mol. Psychiatry 20, 974-985 (2015).

169. Arrenberg, A. B. \& Driever, W. Integrating anatomy and function for zebrafish circuit analysis. Front. Neural Circuits 7, 74 (2013).

170. Eddins, D., Cerutti, D., Williams, P., Linney, E. \& Levin, E. D. Zebrafish provide a sensitive model of persisting neurobehavioral effects of developmental chlorpyrifos exposure: comparison with nicotine and pilocarpine effects and relationship to dopamine deficits. Neurotoxicol. Teratol. 32, 99-108 (2010).

171. Wolman, M. \& Granato, M. Behavioral genetics in larval zebrafish: learning from the young. Dev. Neurobiol. 72, 366-72 (2012).

172. Levin, E. D. et al. Critical duration of exposure for developmental chlorpyrifos-induced neurobehavioral toxicity. Neurotoxicol. Teratol. 33, 742-751 (2011).

173. Rice, C., Ghorai, J. K., Zalewski, K. \& Weber, D. N. Developmental lead exposure causes startle response deficits in zebrafish. Aquat. Toxicol. 105, 600-8 (2011).

174. Kimmel, C. B., Patterson, J. \& Kimmel, R. O. The development and behavioral characteristics of the startle response in the zebra fish. Dev. Psychobiol. 7, 47-60 (1974).

175. Koch, M. The neurobiology of startle. Prog. Neurobiol. 59, 107-28 (1999).

176. Pogoda, H.-M. et al. A genetic screen identifies genes essential for development of myelinated axons in zebrafish. Dev. Biol. 298, 118-31 (2006).

177. McKeown, K. A., Moreno, R., Hall, V. L., Ribera, A. B. \& Downes, G. B. Disruption of Eaat2b, a glutamate transporter, results in abnormal motor behaviors in developing zebrafish. Dev. Biol. 362, 162-71 (2012).

178. Eaton, R. C., Bombardieri, R. A. \& Meyer, D. L. The Mauthner-initiated startle response in teleost fish. J. Exp. Biol. 66, 65-81 (1977).

179. Mirjany, M., Preuss, T. \& Faber, D. S. Role of the lateral line mechanosensory system in directionality of goldfish auditory evoked escape response. J. Exp. Biol. 214, 3358-3367 (2011).

180. Mirjany, M. \& Faber, D. S. Characteristics of the anterior lateral line nerve input to the Mauthner cell. J. Exp. Biol. 214, 3368-3377 (2011).

181. Hatta, K. \& Korn, H. Physiological Properties of the Mauthner System in the Adult Zebrafish. J. Comp. Neurol 395, 493-509 (1998).

182. Eaton, R. C., Lee, R. K. K. \& Foreman, M. B. The Mauthner cell and other identified neurons of the brainstem escape network of fish. Prog. Neurobiol. 63, 467-485 (2001).

183. Kohashi, T. \& Oda, Y. Initiation of Mauthner- or Non-Mauthner-Mediated Fast Escape Evoked by Different Modes of Sensory Input. J. Neurosci. 28, 10641-10653 (2008).

184. Tabor, K. M. et al. Direct activation of the Mauthner cell by electric field pulses drives ultra-rapid escape responses 23 4. J Neurophysiol (2014). doi:10.1152/jn.00228.2014

185. Werner, P., Voigt, M., Keinänen, K., Wisden, W. \& Seeburg, P. H. Cloning of a putative high-affinity kainate receptor expressed predominantly in hippocampal CA3 cells. Nature 351, 742-744 (1991).

186. Victor Nadler, J. \& Cuthbertson, G. J. Kainic acid neurotoxicity toward hippocampal formation: Dependence on specific excitatory pathways. Brain Res. 195, 47-56 (1980).

187. Wang, G. J. et al. Systemic administration of domoic acid-induced spinal cord lesions in neonatal rats. J. Spinal Cord Med. 23, 31-9 (2000). 
188. Ross, I. ., Johnson, W., Sapienza, P. . \& Kim, C. . Effects of the seafood toxin domoic acid on glutamate uptake by rat astrocytes. Food Chem. Toxicol. 38, 1005-1011 (2000).

189. Gill, S. et al. Regional Susceptibility to Domoic Acid in Primary Astrocyte Cells Cultured from the Brain Stem and Hippocampus. Mar. Drugs 6, 25-38 (2008).

190. Scallet, A. C., Schmued, L. C. \& Johannessen, J. N. Neurohistochemical biomarkers of the marine neurotoxicant, domoic acid. Neurotoxicol. Teratol. 27, 745-752 (2005).

191. Kolodziejczyk, K., Saab, A. S., Nave, K.-A. \& Attwell, D. Why do oligodendrocyte lineage cells express glutamate receptors? F1000 Biol. Rep. 2, 57 (2010).

192. Patneau, D. K., Wright, P. W., Winters, C., Mayer, M. L. \& Gallo, V. Glial cells of the oligodendrocyte lineage express both kainate- and AMPA-preferring subtypes of glutamate receptor. Neuron 12, 357-371 (1994).

193. Alberdi, E., Sánchez-Gómez, M. V., Marino, A. \& Matute, C. Ca(2+) influx through AMPA or kainate receptors alone is sufficient to initiate excitotoxicity in cultured oligodendrocytes. Neurobiol. Dis. 9, 234-43 (2002).

194. Matute, C., Domercq, M. \& Sánchez-Gómez, M.-V. Glutamate-mediated glial injury: mechanisms and clinical importance. Glia 53, 212-24 (2006).

195. Rosenberg, P. A. et al. Mature myelin basic protein-expressing oligodendrocytes are insensitive to kainate toxicity. J. Neurosci. Res. 71, 237-45 (2003).

196. Deng, W., Rosenberg, P. a, Volpe, J. J. \& Jensen, F. E. Calcium-permeable AMPA/kainate receptors mediate toxicity and preconditioning by oxygen-glucose deprivation in oligodendrocyte precursors. Proc. Natl. Acad. Sci. U. S. A. 100, 6801-6 (2003).

197. Gallo, V. et al. Oligodendrocyte progenitor cell proliferation and lineage progression are regulated by glutamate receptor-mediated K+ channel block. J. Neurosci. 16, 2659-2670 (1996).

198. Gudz, T. I., Komuro, H. \& Macklin, W. B. Glutamate stimulates oligodendrocyte progenitor migration mediated via an alphav integrin/myelin proteolipid protein complex. J. Neurosci. 26, 2458-66 (2006).

199. Matute, C. Characteristics of acute and chronic kainate excitotoxic damage to the optic nerve. Proc. Natl. Acad. Sci. U. S. A. 95, 10229-34 (1998).

200. Noguchi, T. \& Arakawa, O. Distribution of Domoic Acid In Seaweeds Occurring In Kagoshima, Southern Japan. Natural Toxins 2 Structure, Mechanism of Action, and Detection (Springer, Boston, MA, 1996). doi:10.1007/978-1-4613-0361-9_43

201. Bates, S. S. et al. Pennate Diatom Nitzschia pungens as the Primary Source of Domoic Acid, a Toxin in Shellfish from Eastern Prince Edward Island, Canada. Can. J. Fish. Aquat. Sci. 46, 1203-1215 (1989).

202. Lehane, L. Paralytic shellfish poisoning: a potential public health problem. Med. J. Aust. 175, 29-31 (2001).

203. Etheridge, S. M. Paralytic shellfish poisoning: Seafood safety and human health perspectives. Toxicon 56, 108-122 (2010).

204. Glaziou, P. \& Legrand, A.-M. The epidemiology of ciguatera fish poisoning. Toxicon 32, 863-873 (1994).

205. Litaker, R. W. et al. Global distribution of ciguatera causing dinoflagellates in the genus Gambierdiscus. Toxicon 56, 711-730 (2010).

206. Bomber, J. W. \& Aikman, K. E. The Ciguatera Dinoflagellates. Biol. Oceanogr. 6, 291311 (1989). 
207. Jeglitsch, G., Rein, K., Baden, D. G. \& Adams, D. J. Brevetoxin-3 (PbTx-3) and its derivatives modulate single tetrodotoxin-sensitive sodium channels in rat sensory neurons. J. Pharmacol. Exp. Ther. 284, 516-25 (1998).

208. Lombet, A., Bidard, J.-N. \& Lazdunski, M. Ciguatoxin and brevetoxins share a common receptor site on the neuronal voltage-dependent $\mathrm{Na}^{+}$channel. FEBS Lett. 219, 355-359 (1987).

209. Yasumoto, T. et al. Diarrhetic shellfish toxins. Tetrahedron 41, 1019-1025 (1985).

210. Lee, J.-S. et al. Determination of diarrhetic shellfish toxins in various dinoflagellate species. J. Appl. Phycol. 1, 147-152 (1989).

211. Miles, C. O. et al. A Novel Pectenotoxin, PTX-12, in Dinophysis Spp. and Shellfish from Norway. Chem. Res. Toxicol. 17, 1423-1433 (2004).

212. Twiner, M. J., Rehmann, N., Hess, P. \& Doucette, G. J. Azaspiracid shellfish poisoning: a review on the chemistry, ecology, and toxicology with an emphasis on human health impacts. Mar. Drugs 6, 39-72 (2008).

213. Abal, P. et al. Absorption and Effect of Azaspiracid-1 Over the Human Intestinal Barrier. Cell. Physiol. Biochem. 43, 136-146 (2017).

214. Bláha, L., Babica, P. \& Maršálek, B. Toxins produced in cyanobacterial water blooms toxicity and risks. Interdiscip. Toxicol. 2, 36-41 (2009).

215. Duy, T. N., Lam, P. K. S., Shaw, G. R. \& Connell, D. W. Toxicology and Risk Assessment of Freshwater Cyanobacterial (Blue-Green Algal) Toxins in Water. Rev Env. Con tam Toxicol 163, 113-186 (2000).

216. Lefebvre, K. a et al. Gene expression profiles in zebrafish brain after acute exposure to domoic acid at symptomatic and asymptomatic doses. Toxicol. Sci. 107, 65-77 (2009).

217. Doucette, T. A., Bernard, P. B., Yuill, P. C., Tasker, R. A. \& Ryan, C. L. Low doses of non-NMDA glutamate receptor agonists alter neurobehavioural development in the rat. Neurotoxicol. Teratol. 25, 473-479 (2003).

218. Jian Wang, G. H. et al. Systemic Administration of Domoic Acid-Induced Spinal Cord Lesions in Neonatal Rats. J. Spinal Cord Med. 23, 31-39 (2000).

219. Almeida, R. G., Czopka, T., Ffrench-Constant, C. \& Lyons, D. A. Individual axons regulate the myelinating potential of single oligodendrocytes in vivo. Development 138, 4443-50 (2011).

220. Shin, J., Park, H.-C., Topczewska, J. M., Mawdsley, D. J. \& Appel, B. Neural cell fate analysis in zebrafish using olig2 BAC transgenics. Methods Cell Sci. 25, 7-14 (2003).

221. Kucenas, S., Snell, H. \& Appel, B. nkx2.2a promotes specification and differentiation of a myelinating subset of oligodendrocyte lineage cells in zebrafish. Neuron Glia Biol. 4, 7181 (2008).

222. Czopka, T., Ffrench-Constant, C. \& Lyons, D. A. Individual oligodendrocytes have only a few hours in which to generate new myelin sheaths in vivo. Dev. Cell 25, 599-609 (2013). 


\section{CHAPTER 2}

Domoic acid as a developmental neurotoxin: critical exposure periods, structural phenotypes, and behavioral effects

\subsection{GRAPHICAL ABSTRACT}

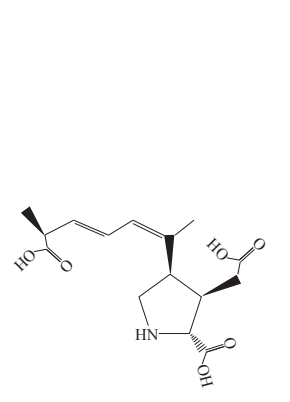

Domoic acid $(0.09-0.18 \mathrm{ng})$

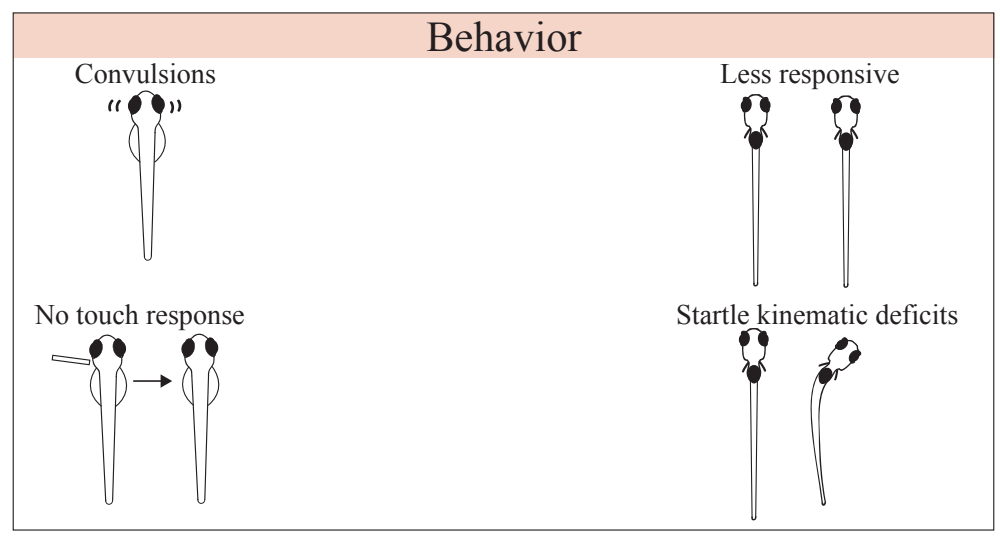

Structural targets

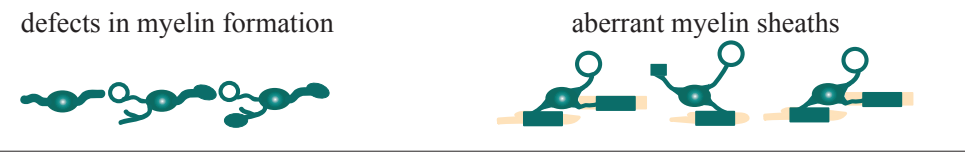

Gene expression

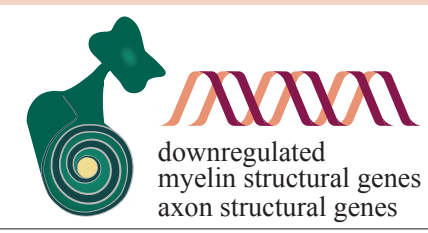

$3 \mathrm{dpf}$

$5 \mathrm{dpf}$

$7 \mathrm{dpf}$

*not 1 or 4 dpf 


\subsection{ABSTRACT}

Developmental exposure to Domoic acid (DomA) can lead to long-term changes in brain structure, increased seizure susceptibility, and aberrant behavior. However, the molecular and cellular mechanisms that underlie these effects are unknown. To determine how DomA affects the developing nervous system, the goals of this study were to 1) Identify windows of susceptibility to DomA exposure, 2) Characterize structural changes in the nervous system, and 3) Link these effects to behavioral changes. To identify potential windows of susceptibility, DomA $(0.09-0.18 \mathrm{ng})$ was delivered through caudal vein microinjection during three early neurodevelopmental periods: i) 1 day post fertilization (dpf), when the majority of the early sensory and motor neurons differentiate, ii) $2 \mathrm{dpf}$, when oligodendrocytes differentiate and begin axon wrapping, and iii) $4 \mathrm{dpf}$, a period after the early nervous system is specified. To assess the functional consequences of exposures, I quantified startle response behavior to acoustic/vibrational (A/V) stimuli. Larvae exposed to DomA at 2 dpf showed deficits in startle behavior; these effects were much less evident in larvae exposed at 1 or $4 \mathrm{dpf}$. Larvae exposed at $2 \mathrm{dpf}$ also showed structural changes in their myelin sheaths - the structures that enable the rapid signaling required for startle responses - as assessed in $\operatorname{Tg}(m b p: E G F P-C A A X)$ fish in which myelin is labeled by EGFP. Myelin deficits were visible as early as $3 \mathrm{dpf}$, when myelin sheaths first form, and persisted until at least $7 \mathrm{dpf}$, indicating that the initial formation of myelin is perturbed and does not recover within 4 days post-exposure. There was little evidence of myelination defects or behavioral phenotypes in larvae exposed to DomA at $4 \mathrm{dpf}$, a time point after the onset of myelination, suggesting that DomA does not affect established sheaths, but rather may perturb the formation of nascent myelin. Time-lapse imaging of $\operatorname{Tg}$ (nkx2.2a:mEGFP), $\operatorname{Tg}(\operatorname{sox} 10: m R F P)$ fish during the time of axon wrapping and nascent myelination (60-72 hpf) confirmed that DomA perturbs these initial stages of myelination. RNA sequencing of whole embryos was done to identify the molecular consequences of exposure to DomA at $2 \mathrm{dpf}$. DomA-treated larvae sampled at $3 \mathrm{dpf}$ showed down-regulation of genes that are required for maintaining myelin structure ( $m b p z, m b p a)$ and the axonal cytoskeleton (nefla, neflb, nefma, nefmb). Overall, I identified the developmental window of DomA susceptibility, defects in startle response behavior, and the underlying changes in myelin structure and gene expression. 
KEYWORDS: Domoic acid; HAB toxins; developmental toxicity; windows of susceptibility; startle response; myelination

\subsection{INTRODUCTION}

Domoic acid (DomA) is a potent neurotoxin that is produced by diatoms in the genus Pseudonitzschia. DomA is known to exert its toxicity through binding to and activating ionotrophic glutamate receptors, particularly the $\alpha$-amino-3-hydroxy-5-methyl-4-isoxazolepropionic acid (AMPA) and kainate (KA) subtypes. ${ }^{1}$ Human exposure to DomA occurs primarily through the consumption of contaminated seafood. High-dose exposure to DomA leads to Amnesic Shellfish Poisoning, with symptoms ranging from mild gastrointestinal issues to memory loss, seizures, coma, or even death. ${ }^{2-4}$ To protect adults from these acute symptoms, regulatory limits of $20 \mathrm{mg}$ of DomA per kilogram of shellfish tissue have been established. ${ }^{5,6}$ However, seafood with measurable levels of DomA below these regulatory limits is still widely harvested and consumed. This may have important public health consequences, especially for exposures that occur during embryonic and early postnatal development when animals are often more sensitive to neurotoxicants. ${ }^{7,8}$

Work in animal models has confirmed that early development is a sensitive period for exposure to DomA. In fact, only one-tenth the dose of DomA is required to induce overt behavioral toxicity in postnatal rats compared to adults. ${ }^{9-11}$ Even within the postnatal period, rats are generally more sensitive at earlier postnatal stages. The lethal dose of DomA that leads to $50 \%$ mortality (LD50) in rats is 2.8 times lower at postnatal day 2 (PND 2) compared to PND $10 .{ }^{11}$

DomA has been shown to readily cross the placental barrier, make its way into the fetal brains and accumulate in fetal fluids. DomA delivered intravenously into pregnant rodents is detectable in fetal brains. ${ }^{12}$ Furthermore, DomA has been detected in the fetal rat brain (GD13) at concentrations similar to those measured in both the maternal brain and the amniotic fluid. ${ }^{12,13}$ The amniotic fluid, in turn, can also serve as a reservoir for DomA. Studies in stranded sea lions show that DomA can remain in amniotic fluid for up to eight days - a period long after it has left the maternal circulation. ${ }^{14-16}$ The long residence times suggest that fetuses could be potentially exposed to DomA over more prolonged periods of time compared to their mothers. 
DomA can also be transferred to breast milk. DomA has been measured in the milk of stranded sea lions, indicating that consumption of DomA-contaminated seafood/prey can result in measurable levels of DomA in the milk. ${ }^{17}$ Furthermore, toxicological studies in rodents show that DomA that is injected intraperitoneally is detectable in both the maternal plasma and the milk of lactating rats. ${ }^{21}$ While the concentrations of DomA are initially 16 times lower in the milk than in the mother's plasma (two hours post exposure), DomA persists in the milk much longer, being four times higher in the milk versus the maternal plasma eight hours later. ${ }^{18}$ All of this suggests that lactation is a potential exposure route for neonates. However, it has yet to be determined whether the levels of DomA found in milk can result in any adverse effects to the neonatal nervous system.

A wide range of lasting behavioral deficits has been characterized following both prenatal and postnatal exposures to DomA. These effects occur even at doses that do not lead to overt signs of toxicity either in mothers (in the case of prenatal exposures) or in the pups themselves (for postnatal exposures). Rodents exposed prenatally to DomA had aberrant exploratory behaviors, ${ }^{19-21}$ subtle motor coordination deficits, ${ }^{20}$ and in some cases deficits in contextual learning. ${ }^{20,19}$ Rodents exposed postnatally had distinct seizures when exposed to novel environments, ${ }^{10,22}$ and also had aberrant drug-seeking behaviors as assessed by nicotine place preference tests. ${ }^{23,24}$

There is growing evidence that developmental exposures to DomA can also heighten sensitivity to chemically-induced stressors later in life. Prenatal exposures to DomA in mice led to reduced seizure thresholds when they were exposed to DomA again postnatally. Embryonic exposures in zebrafish led to reduced thresholds when exposed to the seizure-inducing agent, Pentylenetetrazol (PTZ). ${ }^{25}$ Similar to findings in zebrafish, postnatal DomA exposures in rats also reduced seizure thresholds with PTZ. ${ }^{26}$

All together, these studies indicate that developmental exposure to DomA leads to lasting behavioral deficits and increases sensitivity to other stressors later in life. ${ }^{19-21}$ However, the underlying cellular and molecular mechanisms that govern these deficits are poorly understood. 
First, the behaviors assessed in rodents are driven by complex, incompletely characterized neural networks. This makes it extremely difficult to identify the DomA-induced cellular or molecular disruptions that underlie behavioral deficits. Second, rodents are exposed to DomA in utero, making it impossible to track the progression of cellular phenotypes during early development.

To directly address these challenges, I used zebrafish as a tool to identify the mechanisms of toxicity from developmental exposures to DomA. Zebrafish have brain structures and sensorymotor pathways that are homologous to those of humans. ${ }^{27,28}$ This suggests that what we learn from the zebrafish model may provide insights into the mechanisms that may be occurring in humans. Furthermore, the transparency of zebrafish embryos and the availability of transgenic lines allow me to directly observe critical cellular processes during early development and visualize neural structures within their native tissue environment. ${ }^{29-32}$ Finally, larval zebrafish have simple behaviors that are driven by well-characterized neural circuits and comprised of known cell types. This allows me to link behavior to the underlying structural and cellular targets. ${ }^{33,34}$

The goal of this study was to identify the behavioral, structural, and transcriptional changes from low-dose exposures to DomA during critical periods in early development. Using intravenous microinjection, I was able to deliver single doses at specific developmental times that spanned late embryonic (1 day post fertilization, or dpf) to larval stages ( $4 \mathrm{dpf}$ ). I used the wellcharacterized startle response behavior to identify functional effects from domoic acid toxicity. To assess potential structural changes from exposures, transgenic lines that have fluorescentlylabeled myelin sheaths were used to assess changes in myelin structure over time. Finally, transcriptional changes resulting from exposures were identified using RNA Sequencing.

\subsection{METHODS}

\section{Fish husbandry and lines used}

These studies were approved by the Woods Hole Oceanographic Institution Animal Care and Use Committee (Assurance D16-00381 from the NIH Office of Laboratory Animal Welfare). Fish were maintained in recirculating tank systems that were specifically designed for zebrafish culture (Aquatic Habitats Inc., Apopka, FL). Temperature, lighting, and water quality were 
monitored daily and maintained according to recommendations from the Zebrafish International Resource Center. Fish were fed twice daily, once with live brine shrimp and once with the pellet feed Gemma Micro 300 (Skretting Inc., Tooele, UT). The afternoon before breeding, males and females were separated with a divider. The morning of the breeding, dividers were removed, and embryo collectors - containers with mesh on the top that let embryos filter to a catch basin were placed in tanks with multiple breeding pairs for batch breeding unless otherwise noted. Embryos were collected and placed in petri dishes or in individual wells in a multi-well plate with $0.3 x$ Danieau's medium. Embryos were maintained at $28.5^{\circ} \mathrm{C}$ with a $14: 10$ light dark cycle during the experimental period.

The transgenic line $\operatorname{Tg}(m b p: E G F P-C A A X)^{35}$ in the AB background was used for behavioral, RNAseq, and myelin labeling experiments, while the double transgenic, $\operatorname{Tg}(n k x 2.2 a: m E G F P),{ }^{36}$ $\operatorname{Tg}\left(\right.$ sox 10:mRFP), ${ }^{37}$ was used for time lapse microscopy experiments.

\section{Domoic acid exposure paradigm}

An initial pilot study was performed in which zebrafish embryos were exposed to DomA solutions (5- $40 \mu \mathrm{M}$ waterbourne exposures). The absence of expected acute neurotoxicity even at high concentrations (data not shown) raised questions about whether DomA was being taken up by the embryos. Because of this, I decided to use microinjection as the route of exposure.

Domoic acid (Sigma-Aldrich, MO) was dissolved in a diluted embryo medium (0.2x Danieau's) to generate stock concentrations of $0.68 \mu \mathrm{g} / \mu \mathrm{l}$ and $1.4 \mu \mathrm{g} / \mu \mathrm{l}$, and aliquots were stored at $-20^{\circ} \mathrm{C}$. Working solutions were prepared fresh prior to microinjection by diluting the stock to obtain the appropriate doses. Microinjection needles were prepared from glass capillary tubes $(0.58 \mathrm{~mm}$ inner diameter; World Precision Instruments, 1B100F-4) using a pipette puller (Sutter instrument model p-30, heat 750, pull=0). Microinjections were performed using a Narishige IM-300 microinjector. The microinjector was calibrated to consistently deliver $0.2 \mathrm{~nL}$ by adjusting the time (milliseconds) and pressure.

To determine the window of susceptibility for exposure at lower doses, DomA $(0.09,0.13,0.14$, $0.18 \mathrm{ng}$ nominal dose) was intravenously microinjected into the common posterior cardinal vein 
at different developmental stages. ${ }^{38}$ Chemical analyses of the actual concentrations of DomA are underway. Controls from the same breeding cohort were injected with the saline vehicle $(0.2 \mathrm{x}$ Danieau's). Supplemental Table 1 includes the developmental time ranges for each injection category. To perform intravenous microinjections, fish were dechorionated, anesthetized and placed laterally on dishes coated with $1.5 \%$ agarose. An injection was deemed successful if there was a visible displacement of blood cells. Following injections, zebrafish were placed back in clean embryo media and monitored daily.

\section{Assessment of gross morphological defects and acute neurological phenotypes}

Subsets of fish were imaged using brightfield microscopy to visualize potential gross morphological defects. The presence or absence of the swim bladder was scored blindly and then percentage was quantified for fish exposed to DomA at different doses and during different developmental stages. Images were white balance-corrected using Adobe Photoshop.

In a subset of the experiments, fish were kept individually in 48-well plates for phenotypic observation. Any mortalities, presence or absence of convulsions, pectoral flapping, and touch responses were recorded daily from the day after exposure to $5 \mathrm{dpf}$. Larvae were considered convulsing when whole body contractions were observed. Pectoral fin flapping was scored when larvae continued to rapidly move pectoral fins even when the fish were not actively swimming or attempting to right themselves. Touch responses were assessed using a tactile stimulus produced by an 'embryo poker' - a piece of fishing line $(0.41 \mathrm{~mm}$ diameter) glued to a glass pipette tip. Larvae were identified as having no touch response when they were unable to perform body bends and swim away following tactile stimulation.

Modeling the prevalence of neurotoxic phenotypes by dose, day of exposure, and day of observation.

Following daily observation, generalized estimating equations (GEE) were used to model the effects of both DomA dose (as a continuous factor) and the number of days post-exposure (categorical factor) on the presence of acute neurological phenotypes (convulsions, pectoral flapping, and the lack of touch responses) (gee(), geepack R package) ${ }^{49}$ Observations of the same fish over multiple days were treated as repeated measures and were clustered by the "id" 
term. Separate GEE models were created for exposure to DomA at two developmental periods (1 and $2 \mathrm{dpf})$.

There were only single observations for fish exposed at $4 \mathrm{dpf}$. To determine whether DomA dose alters the presence of neurotoxic phenotypes one day post-exposure, a generalized linear model was formulated containing the different doses as predictors, and the presence of phenotypes as the response. To account for variability amongst trials, dispersion was estimated using the quasibinomial link function rather than the binomial.

\section{Startle behavior set-up}

The custom-built startle behavior set-up is shown in Supplemental Fig. 1A. The system includes a speaker (Visaton BG20-8 8" Full-Range Speaker with Whizzer Cone, \#292-548) connected to an amplifier (100W TDA7498 Class-D Amplifier Board, \#320-303) as a source of auditory/vibrational stimuli. A cylinder with a flat base was 3D printed and glued to the base of the speaker to create a platform to rest the plate that contained the fish (radius $=50 \mathrm{~mm}$, height $=$ $50 \mathrm{~mm})$. A 16 -well acrylic plate $(4.83 \times 4.83 \mathrm{~cm})$ was then designed to contain 16 larvae individually. This plate was made based on a design from Wolman et al. (2011) that was comprised of laser cut acrylic pieces that were fused together using acrylic cement (Weld-On \#3; IPS) ${ }^{40}$

The intensity and frequency of the auditory/vibrational stimuli were controlled using a pulse generator (PulsePal, Sansworks). Stimuli were coded to deliver 3 millisecond pulses of $1000 \mathrm{~Hz}$ frequency.

Groups of 16 larvae were given 7 identical stimuli $(41 \mathrm{~dB})$ that were spaced 20 seconds apart to

prevent habituation. ${ }^{40} \mathrm{~A}$ high-speed video camera (Edgertronic) was set at a $10 \%$ pre-trigger rate to capture 13 frames prior to the stimulus being elicited, while recording larval movements at 1000 frames per second. 


\section{Measuring startle vibration}

Vibration was measured using a 3-axis accelerometer (PCB Piezotronics, model W356B11). The output signal was first conditioned (PCB Piezotronics, Model 480B31) then passed through a dual channel analog filter (Model 3382, Krohn-Hite Corporation) using a $10 \mathrm{kHz}$ low-pass cutoff frequency and $30 \mathrm{~dB}$ gain. Finally, the signal was collected by a data acquisition board (National Instruments Data Acquisition board, Model USB-6251). Raw voltage data were converted into acceleration units $\left(\mathrm{m} / \mathrm{s}^{2}\right)$ using manufacturer sensitivity values for each axis of the accelerometer. The Euclidian norm (vector sum) for the three acceleration signals was calculated to get the total acceleration. Individual peaks were identified, and metrics were calculated for the time window between 9 milliseconds prior to the peak to $50 \mathrm{~ms}$ after. The maximum value (peak) during each time window was taken as the zero to peak acceleration value for a given impulse, and this value was converted to $\mathrm{dB}$ using the following equation:

$$
L_{z-p k}=20 * \log _{10}(x)
$$

Where $L_{z-p k}$ is the zero-to-peak acceleration level in $\mathrm{dB}$ re $1 \mathrm{~m} / \mathrm{s}^{2}$, and $x$ is the maximum acceleration level (of the Euclidian norm) over the peak analysis window.

\section{Startle behavioral analysis}

High speed videos were converted into jpegs (.mov files with a minimal resolution of $720 \times 720$, 1/1008 shutter speed and a frame rate of 1000 frames/second). To reduce the noise and tracking errors, the background was filtered out using a custom script in MATLAB. FLOTE software was

then used to analyze the jpegs. ${ }^{41}$ Quantitative attributes of the startle response measured include startle responsiveness (whether larvae responded or not), latency (delay time prior to startle), maximal bend angle, and maximal angular velocity during startle. The identities of individual larvae across the multiple stimuli were distinguished based on their position on a grid.

\section{Statistical modeling of startle responsiveness}

Every fish was given 7 replicate auditory/vibrational stimuli, spaced 20 milliseconds apart. For all instances where a fish was successfully tracked, response rates were recorded, and the percent of times an individual fish responded to these replicate stimuli was calculated (\% responsiveness $=$ number of times the fish responded/ number of successfully tracked videos with a maximum of 7 tracks per individual fish). A mixed effects logistic regression model was used to identify 
treatment differences in percent responsiveness, with dose as a fixed effect and the replicate stimuli as a random effect using the 'glmer' function of lme4 package in $\mathrm{R}^{42} \mathrm{~A}$ Dunnett post-hoc test was used to identify potential treatment differences in responsiveness (glht(), multcomp R package). ${ }^{43}$

\section{Identifying SLC versus LLC responses using mixture models}

For all the fish that did respond, their startle responses were classified as either short latency cbends (SLCs) or long latency c-bends (LLCs) based on an empirically determined latency cutoff. Latency cut-offs have been known to vary based on environmental conditions such as temperature. ${ }^{41}$ To empirically determine the cut-offs, clustering was done using a Gaussian mixture model, which fits two Gaussian distributions, and assigns each latency data point a probability of belonging to either of the two distributions (R package, mixtools). ${ }^{44}$ The cut-off for assigning a response as a SLC was 13 milliseconds - the latency with a greater than 50\% probability of belonging to the first fitted Gaussian distribution (Supplemental Fig. 1B). Startle responses that had latencies greater than 13 milliseconds were classified as LLCs.

\section{Analysis of treatment differences in startle response kinematics}

There were several instances when individual fish performed a combination of LLC and SLC responses over the 7 replicate stimuli. For fish that did respond, their startle responses were classified as either SLCs ( $\leq 13$ milliseconds) or LLCs ( $>13$ milliseconds). Kinematic responses from the two types of startle responses (SLC v. LLC) were analyzed separately based on previous research that shows they are driven by distinct neural circuits and have distinct kinematic characteristics. ${ }^{41,45,46}$ Following this classification, the median response of individual fish for each startle type was then used to identity treatment-specific differences in kinematics.

I first checked for normality and variance homogeneity in the data being analyzed. I used the Bartlett test to test for homogeneity in variances (bartlett.test(), R), and the Shapiro-Wilk's method to test for normality (shapiro.test(), R). Kinematic data (bend angle, maximum angular velocity) showed departures from normality and had unequal variances. To account for this, I used nonparametric tests to determine whether fish exposed to various doses of DomA at different developmental periods had altered bend angles and maximal angular velocities. 
Kinematic data from fish exposed to DomA at different development days were analyzed separately. For trials that contained a single dose of DomA, nonparametric Behrens-Fisher t-tests were used to test the alternative hypothesis that kinematics of fish exposed to DomA were different from their control counterparts (npar.t.test(), nparcomp package, R). ${ }^{47}$ With trials that contained multiple doses, nonparametric analyses with Dunnett-type intervals were done to compare each of the doses to the control (nparcomp(), nonparam package, R). ${ }^{47}$

Functions in the nparcomp package estimates the relative effects, which range from 0 to 1 . Under the null hypothesis, the relative effect size is 0.5 - which represents a $50 \%$ probability (an equal probability) that the treated fish has a value greater than the control fish. The closer the estimated relative effect is to 1 , the higher the probability that the measured kinematics in the treated group has a larger value than the control. In contrast, the closer the estimated relative effect is to 0 , the higher the probability that the measured kinematic parameter in the treated group has a smaller value than the control.

\section{Startle kinematic analysis for interaction effects between dose and day of exposure}

I then directly tested whether exposures that occurred on distinct developmental days influenced startle kinematics differently - in other words, if there is an interaction between dose and day injected. To examine this, I analyzed the subset of trials that had fish that were collected from the same breeding cohort at day 0 and then exposed to DomA at different developmental days $(1,2$, or $4 \mathrm{dpf}$ ). Aligned Ranked Transformed ANOVA tests were done to determine whether there was an interaction of dose ( 0 versus $0.09 \mathrm{ng}$, or in a separate analysis, 0 versus $0.13 \mathrm{ng}$ DomA) and day of exposure $(1,2$, or $4 \mathrm{dpf})$ on startle kinematics ( $\operatorname{art}()$, ARTool R package). ${ }^{48}$ Difference-of-difference contrasts were then done to determine whether day of exposure affected treatment differences in kinematics (testContrasts(), Phia R package). ${ }^{49}$ In this way, I addressed questions such as, "is the difference in kinematics between DomA and control fish when exposed at $2 \mathrm{dpf}$ significant compared to the difference in kinematics between DomA and control fish when they are exposed at 1 or $4 \mathrm{dpf}$ ?" 


\section{Fluorescence microscopy}

Larvae were anesthetized in tricaine methanesulfonate (MS222) (0.16\%), mounted laterally, and then imaged using either widefield epifluorescence microscopy or confocal microscopy. For images collected on the confocal microscope, fish were anesthetized and mounted laterally in $1.5 \%$ low melt agarose within glass bottom microscopy dishes (Nunc Glass bottom dishes $27 \mathrm{~mm}$ ). 'Embryo pokers' were used to orient the embryos onto their sides. Once the embryos were oriented correctly, the agarose was allowed to harden, and the microscopy dish was flooded with MS222. Fish were then imaged using the confocal microscope (Zeiss LSM-710 and LSM780 ) with the $40 \mathrm{x}$ water objective (Zeiss $\mathrm{C}$ - Apochromat, $\mathrm{NA}=1.1$ ). Images were taken along the anterior spinal cord between $5^{\text {th }}$ and $10^{\text {th }}$ somites.

For images collected on the widefield epifluorescence microscope, a subset of fish were laterally mounted using $1.5 \%$ agarose. To allow for more rapid imaging of larvae, most larvae were oriented into custom-made acrylic molds that contained narrow channels where anesthetized larvae were positioned laterally using the embryo poker. Fish were imaged using the Zeiss inverted epifluorescence microscope with either a 20x (Fluar, NA $=0.75$ ) or 10x (Fluar, NA = $0.5)$ objective. Images were taken along the anterior to medial spinal cord between somites 5-15.

Analysis of the prevalence and severity of myelin phenotypes by dose and day of exposure $\operatorname{Tg}(\mathrm{mbp}: E G F P-C A A X)$ is a stable line in which EGFP is localized to myelin sheaths. I exposed $\operatorname{Tg}(m b p: E G F P-C A A X)$ fish to different doses of DomA at selected developmental times and then imaged their spinal cords. These images were classified qualitatively into categories 0 through 5 based on severity in the myelin defect observed (Supplemental Fig. 3). Multinomial regression was used to model the effect of both dose and day injected on the distribution of the myelin severity phenotypes (multinom(), nnet R package).$^{50}$

The overall significance of the dose and development day of exposures was obtained by performing an Analysis of Variance (ANOVA) on pairs of multinomial logistic regression models. The initial multinomial logistic regression model only included the dose of DomA as a predictor of the distribution of myelin phenotypes: $\beta_{0}+\beta_{\text {dose }}$. The alternative model incorporated

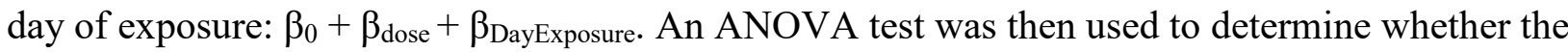


more complex alternative model was significantly better at capturing the data than the initial simpler one. Specifically, I compared the initial model to the alternative model to determine whether day of exposure influences the distribution of the myelin phenotypes (anova(initial model, first alternative model), car package, R). ${ }^{51}$

Multinomial models were constructed to identify the effects of increasing doses of DomA on the distribution of these myelin phenotypes. To accomplish this, I used imaging data from fish exposed to varying doses of DomA at $2 \mathrm{dpf}$.

\section{Time-lapse microscopy}

Embryos were exposed to DomA at $2 \mathrm{dpf}$, anesthetized, and mounted in 1.5\% low melt agarose at around $2.25 \mathrm{dpf}$. Images were acquired on the LSM710 using the 20x dry (Plan-Apochromat 20x/0.8) objective. Z-stacks were acquired every 13-17 minutes per embryo over the course of

12-13 hours. For each embryo observed, maximum intensity projections of the z-stacks were then generated and compiled over time to generate the movie file (ZEN blue, ZEN black imaging software, Zeiss Microscopy).

\section{Experimental animals used for RNASeq}

Three individual breeding tanks were set up with two males and one female per tank. Embryos collected from each tank were split so that some were injected with DomA (0.14 ng) and others with the saline vehicle control. Embryos were exposed to either the saline vehicle or to $0.14 \mathrm{ng}$ of DomA at 2 dpf (between 48.5- $51 \mathrm{hpf}$ ), then placed into 48-well plates for daily observation. Pools of 6 embryos from each of the three breeding sets were collected for RNAsequencing ( $n=3$ per treatment) at $3 \mathrm{dpf}(76 \mathrm{hpf})$. The remaining fish were used for imaging myelin at $5 \mathrm{dpf}$ and for assessing startle behavior at $7 \mathrm{dpf}$. At the end of the behavioral trial, a subset of the fish were snap frozen at $7 \mathrm{dpf}(124 \mathrm{hpf})$ for RNA sequencing.

\section{RNA Isolation and sequencing}

RNA was isolated using the Zymo Direct-Zol kit (Catlog \# R2062) and quantified using Nanodrop spectrophotometer. RNA quality was checked using the Bioanalyzer (Agilent technologies, CA) at the Harvard Biopolymers Facility, Cambridge, MA. RNA integrity number 
(RIN) of the samples was 8.2 or higher. Library preparation for single stranded RNAseq was done using the Illumina TruSeq total RNA library kit. Single-end 50 base pair sequencing was done on Illumina HiSeq2000 platform. Both library preparation and sequencing was performed at the Tufts University Core Facility (Boston, MA). Raw data files were assessed for quality using FastQC. ${ }^{52}$ Adapter trimming was done using Trimmomatic. ${ }^{53}$ Trimmed reads were aligned to the genome (GRCz10, version 84) using STAR aligner. ${ }^{53,54}$ HTSeq-count was used to count the number of reads mapped to the annotated regions of the genome. ${ }^{55}$ Differential gene expression (DGE) analysis was done using Bioconductor package, edgeR, following the DGE analysis pipeline outlined by Chen et al $2016 .{ }^{56,57}$

DGE analysis involved filtering genes with read counts less than $10 / \mathrm{n}$, where $\mathrm{n}$ is the minimal library size, and then normalizing read counts. Negative binomial models were used to account for gene-specific variability from biological and technical sources. Multi-dimensional scaling plots were used to visualize the leading fold-changes (largest $500 \log _{2}$ fold changes) between pairs of samples. False discovery rate of 5\% (Benjamini-Hochberg method) was used as a statistical cutoff for identifying differentially expressed genes. Gene annotation was done using BioMart with the latest genome (GRCz11), and only annotated genes were used in pathway analysis. gProfiler was then used to identify enriched Gene Ontology (GO) terms and human phenology phenotypes. ${ }^{58} \mathrm{GO}$ terms with evidence only from in silico curation methods were excluded from the enrichment analysis and a statistical significance level of less than or equal to 0.05 (adjusted p-value) was used.

\subsection{RESULTS}

\section{Mortality and gross morphological effects of DomA}

When DomA (0.09-0.14 ng) was injected intravenously into $\operatorname{Tg}(\mathrm{mbp}: E G F P-C A A X)$ embryos or larvae, there were no significant differences in mortality between DomA-exposed versus vehicletreated (control) fish for any of the developmental exposure time points tested (1-4 dpf) (Table $1)$.

I then assessed gross morphological defects from DomA exposure by screening for defects in swim bladder inflation and for massive cell die off in the brains (appearing as opaque brain 
tissue). A majority of the larvae exposed to DomA at $2 \mathrm{dpf}$ did not have inflated swim bladders at $5 \mathrm{dpf}$, in contrast to controls (Fig. 1A-B, Table 3). Furthermore, a subset of larvae injected at 4 dpf with the highest dose of DomA (0.18ng) had brains with a darkened appearance. These 'opaque' brains were signs of widespread apoptosis or necrosis, suggesting to me that this high dose could lead to widespread neurotoxicity (Table 4). Based on these results, DomA doses of $0.14 \mathrm{ng}$ or lower were primarily used to assess startle response behavior and gene expression analyses.

Notably, the sensitivity of zebrafish to these gross effects differed between strains. In $\operatorname{Tg}(\operatorname{sox} 10: m R F P)$, another transgenic line used in this thesis, there were significant increases in mortality when DomA (0.14 ng) was delivered at 2.5-3 dpf (Table 2).

\section{Acute neurotoxicity of DomA}

To characterize acute neurotoxic effects from DomA exposures, the lack of touch response and the presence of convulsions and rapid pectoral fin flapping were scored daily (up to $5 \mathrm{dpf}$ ) following exposure to DomA injected at 1, 2, or $4 \mathrm{dpf}$.

Fish exposed at $1 \mathrm{dpf}$ to all doses of DomA (0.09-0.18 ng) had significantly reduced touch responses as compared to controls (Table 5 and 8, Fig. 1C). Furthermore, the prevalence of reduced touch responsiveness increased as fish were exposed to higher doses of DomA ( $p=1.5 \mathrm{e}$ -8). This phenotype was transient as the prevalence of touch response deficits dropped after the first day post-exposure $(\mathrm{p}=0.0028)$.

Similar to what was observed with fish exposed at $1 \mathrm{dpf}$, fish exposed to all doses of DomA at 2 dpf also had a reduced touch response, with higher DomA doses also leading to a higher prevalence of no touch responses ( $p=1.5$ e -8 , Table 6 and 8, Fig. 1C). However, unlike fish exposed at $1 \mathrm{dpf}$, those exposed at $2 \mathrm{dpf}$ to a medium dose (0.13 ng of DomA) already had touch response deficits that were similar in prevalence to those exposed to the highest dose $(0.18 \mathrm{ng})$. Similar what was observed at $1 \mathrm{dpf}$, the reduced touch response phenotype was also transient, with significant reductions in touch response deficits after the first day post exposure $(p=6.6 \mathrm{e}-$ $6)$. 
Finally, when exposed at 4 dpf, only fish given the highest doses of DomA ( $0.18 \mathrm{ng})$ exhibited any touch response deficits (Table 7, Fig. 1C).

The presence of convulsions or pectoral fin flapping was also monitored (Fig. 1D and Table 9). Fish exposed to DomA at both 1 and $2 \mathrm{dpf}$ (but not at $4 \mathrm{dpf}$ ) had a higher prevalence of convulsions or pectoral fin flapping, with increasing doses of DomA leading to higher prevalence of this phenotype. ( $p=0.0001$ for $1 \mathrm{dpf}$ exposed fish, $\mathrm{p}=1.2 \mathrm{e}-7$ for $2 \mathrm{dpf}$ exposed fish). While exposure to DomA at both 1 and $2 \mathrm{dpf}$ led to these acute toxicity phenotypes, exposure to DomA at $2 \mathrm{dpf}$ led to a higher overall prevalence of the phenotype. Finally, like the no-touch response phenotype, convulsion and pectoral fin flapping phenotypes were transient, with the loss of convulsions and pectoral fin flapping by 2 days post exposure in $1 \mathrm{dpf}$ injected fish $(p=0.001)$, and by 1 day post exposure in 2 dpf injected fish $(p=2.2$ e -9$)$.

Overall, these results show that exposure to DomA (0.14 ng or lower) does not lead to appreciable mortality, but does result in transient acute neurotoxic phenotypes that last for a day after exposure. While fish exposed at both 1 and 2 dpf exhibited these neurotoxic phenotypes, fish exposed to DomA at $2 \mathrm{dpf}$ had these phenotypes at a higher prevalence than those exposed at $1 \mathrm{dpf}$.

\section{DomA effects on Responsiveness to Auditory/Vibrational stimuli}

I then assessed the functional impact of developmental DomA exposures by measuring startle response behavior during the larval stage (6-7 dpf).

I first assessed responsiveness, which is the ability of fish to react to auditory/vibrational (A/V) stimuli. Each fish was given 7 replicate stimuli, and a percent response was calculated for each individual. Fish exposed to DomA at $2 \mathrm{dpf}$ had reduced responsiveness to A/V stimuli at all doses tested $(0.09-0.18 \mathrm{ng})(\mathrm{p}<0.001)$. Fish exposed to DomA at $1 \mathrm{dpf}$ had reduced responsiveness when exposed to DomA doses above $0.09 \mathrm{ng}(\mathrm{p} \leq .001)$ (Table 10), while those exposed to DomA at $4 \mathrm{dpf}$ had reduced responsiveness only when exposed to the highest dose $(0.18 \mathrm{ng})$ tested $(\mathrm{p}<1 \mathrm{e}-4)$. 
All of this suggests that exposures to DomA can result in loss of responsiveness to A/V stimuli. Furthermore, fish exposed to DomA at $2 \mathrm{dpf}$ were more sensitive to DomA-driven disruptions in responsiveness than those exposed at 1 and $4 \mathrm{dpf}$; only fish exposed to DomA at $2 \mathrm{dpf}$ had significantly reduced responsiveness to A/V stimuli at the lowest dose tested (0.09 ng) (Fig. 2).

\section{DomA effects on startle response kinematics}

One of the defining movements of the larval startle response is the initial formation of a ' $c$ ' bend when the head and body bend together at a high angular velocity (Fig. 3A). Kinematics that underlie the 'c' bend include bend angle and maximal angular velocity, which I used to identify DomA-induced changes to startle behavior.

Exposure to DomA at $2 \mathrm{dpf}$ led to consistent kinematic deficits at all doses tested and in all trials (Table 11 and Table 12). Fish exposed to DomA at $2 \mathrm{dpf}$ had both reduced bend angles and slower maximal angular velocities (Mavs) relative to vehicle-injected controls (Fig. 3-5). These behavioral deficits occurred when fish performed both SLC-type and LLC-type startle responses (Fig. 4 and 5, Table 11-13).

In contrast to exposures at $2 \mathrm{dpf}$, those that occurred at 1 and 4 did not lead to any kinematic deficits with the lowest dose of DomA tested (0.09 ng). Fish exposed to $0.09 \mathrm{ng}$ DomA at 1 and $4 \mathrm{dpf}$ had bend angles and maximal angular velocities indistinguishable from those of their vehicle control counterparts for both types of startle responses (SLC and LLC-type) (Fig. 3-5).

At higher doses $(0.13-0.18 \mathrm{ng})$, exposures to DomA at $1 \mathrm{dpf}$ led to kinematic deficits that differed by startle response type (Table 14 and 15). Fish exposed to DomA at $1 \mathrm{dpf}$ had reduced angles and slower maximal angular velocities, particularly when they performed the LLC-type startle responses (Fig.5, Table 15). Fish exposed at $1 \mathrm{dpf}$ also had significant kinematic deficits when performing the SLC responses, but this was primarily due to reductions in bend angle, and not slower maximal angular velocities (Fig. 4, Table 14). 
Lastly, exposures to DomA at 4 dpf led to kinematic deficits that were inconsistent across repeated experimental trials. For example, in only 1 out of 3 trials did the fish exposed to 0.18 ng DomA at 4 dpf have significant reductions to bend angles during SLC-type startles (Table 16; Fig. 4). Furthermore, the type of kinematic deficits was inconsistent. In one of the three trials, fish exposed to $0.18 \mathrm{ng}$ DomA had reduced Mavs and bend angles with SLC-type startles but not LLC-type startles. In another trial, fish exposed to DomA at $0.13 \mathrm{ng}$ had deficits in LLC kinematics but not SLC kinematics (Table 16 and 17; Fig. 4 and 5). All of this suggests that while exposures to DomA at all developmental stages tested (1, 2, and $4 \mathrm{dpf}$ ) resulted in some kinematic deficits at higher doses, only those at $2 \mathrm{dpf}$ led to consistent kinematic deficits across the entire range of doses tested.

\section{Interaction effects between dose and day of exposure}

To directly compare the startle kinematics of fish exposed at different days, I performed a nonparametric multivariate factorial analysis to compare the effect of dose and day of exposure on bend angle or maximal angular velocities. For this analysis, I used the subset of trials that had fish from the same breeding cohort that were exposed to DomA on different days. Additionally, I focused on LLC-type startle responses because they were shown by the previous analysis to be more sensitive to treatment differences.

Startle kinematic parameters were significantly influenced by the interaction between lowest dose of DomA tested (0.09 ng) and day of exposure $(F(2,520)=21.7, p=9.6 \mathrm{e}-10$ for bend angle and $-F(2,520)=16.5, p=1.1$ e -7 for Mav) (Fig. 3B). Treatment effects from exposures to DomA at $2 \mathrm{dpf}$ were distinct from treatment effects from exposures at 1 or $4 \mathrm{dpf}\left(\mathrm{p}<1 \times 10^{-4}\right)$. On the other hand, there were no differences in effects of DomA from exposure at $1 \mathrm{dpf}$ versus 4 dpf, and the effects of DomA were not significantly different between DomA and their respective controls at these exposure times. This indicates that with the lowest doses of DomA $(0.09 \mathrm{ng})$, exposure at $2 \mathrm{dpf}$ led to distinct kinematic deficits that were not found at 1 or $4 \mathrm{dpf}$.

With exposure to the medium doses of DomA (0.13- $14 \mathrm{ng}$ ), the interaction between treatment and day of exposure persists for both startle bend angle $(F(2,422)=23.0,, p=3.4 \mathrm{e}-10)$ and for maximal angular velocity $(F(2,422)=18.8, p=1.4 \mathrm{x} \mathrm{e}-8)$ (Fig. 3C). Similar to exposures with 
the lowest dose, the exposures to DomA (0.126-0.135 ng) at $2 \mathrm{dpf}$ led significant decreases in kinematic parameters relative to exposures at 1 or $4 \mathrm{dpf}(\mathrm{p}<0.05)$. Additionally, at this dose, there were also differences in the effects of DomA when it was delivered at 1 versus $4 \mathrm{dpf}(1 \mathrm{dpf}$ $-4 \mathrm{dpf}$ comparison estimate $=102.73, \mathrm{p}=0.002$ ). This indicates that at higher doses, fish exposed at $1 \mathrm{dpf}$ also exhibit DomA-driven changes in kinematics. However, kinematic deficits that arise from exposures at $1 \mathrm{dpf}$ were still less pronounced compared to deficits following exposures at $2 \mathrm{dpf}(1 \mathrm{dpf}-2 \mathrm{dpf}$ comparison estimate $=81.23, \mathrm{p}=0.009)$.

\section{DomA effects on myelin sheaths in the spinal cord}

Startle response deficits can be due to a number of underlying reasons, including myelin defects. Startle responses are rapid, occurring between 5-50 milliseconds after the stimulus is given. ${ }^{41}$ Myelin is the structure that allows for these rapid responses, and mutations that disrupt myelin sheath structure have been shown to reduce angular velocities, lead to shallower bend angles, and increase latencies. $^{59}$

To screen for myelin defects that may underlie the DomA-induced startle response deficits, I exposed transgenic fish that have EGFP localized to the myelin sheaths to a range of DomA doses during different stages of development (Fig. 6A).

A subset of exposed fish was imaged at $5 \mathrm{dpf}$ using confocal microscopy, which provided the resolution needed for initial characterization of the myelin defects; representative images are shown in Fig. 6B. The severity of myelin defects was scored blindly on the scale of 0-4 (description of severity scores shown in Supplemental Fig. 2, Fig. 6D).

Imaging data suggested that prevalence and severity of myelin sheath phenotypes were influenced by day of exposure (Fig. 6, Table 18). Fish exposed to DomA at $1 \mathrm{dpf}$ had no visible myelin defects $(n=31)$. In contrast, a proportion of the population of fish exposed at $1.5 \mathrm{dpf}$ had visible myelin defects $(n=11$ out of 34$)$. Defects included the overall reduction in labeled myelin, along with the appearance of unusual circular membranes (Fig. 6B). The majority of fish exposed at $2 \mathrm{dpf}$ showed myelin defects $(\mathrm{n}=96$ out of 106). The prevalence of these defects remained high for fish exposed at $2.5 \mathrm{dpf}$, with 35 out of 40 exhibiting a myelin defect. 
However, these myelin phenotypes were less severe, with 2.5 dpf-exposed larvae having milder myelin sheath defects compared to those exposed to 2 dpf. Finally, most fish exposed to DomA at 4 dpf had "control-like" myelin sheaths ( $n=44$ out of 46$)$.

Confocal imaging data suggests that fish exposed at $2 \mathrm{dpf}$ had more severe and more prevalent myelin defects compared to those exposed to DomA at different developmental periods. To confirm this, additional experiments were done where fish were exposed to DomA (at various doses and times) and then imaged at $5 \mathrm{dpf}$ using widefield epifluorescence microcopy. This provided the increased throughput needed to statistically model the effects of DomA dose and the timing of exposure on myelin sheath defects (Fig. 7, Table 19).

To determine whether the day of exposure influenced the appearance and prevalence of myelin defects, I performed a pairwise ANOVA test to compare an initial model (with only DomA dose as the predictor) to an alternative model (with dose and day of exposure as predictors) (Fig. 7). Results indicate that incorporating day of exposure significantly improved its predictive power ( $p<1$ e -16), indicating that timing of DomA exposure influenced the myelin phenotypes.

I then determined whether DomA exposures that occurred during particular periods in development led to a higher prevalence of specific myelin defects. I found that the odds of fish exhibiting phenotypes from category 1-4 were higher when exposures occurred at 2, 2.5, and 3 dpf relative to exposures that occurred at $1 \operatorname{dpf}$ (Table 22, p $<1$ e -7 for $2 \mathrm{dpf}$ exposed). Of these time periods, exposures at $2 \mathrm{dpf}$ led to the highest odds of having fish with these myelin defects.

On the other hand, fish that were exposed to DomA at $2.5 \mathrm{dpf}$ or later were more likely than 2 dpf-exposed fish to have a distinct myelin defect labeled as category 5 . This myelin defect was also seen in conjunction with morphological defects such as brain edema with exposures to high doses of DomA (0.18 ng) at $4 \mathrm{dpf}$ (Table 4$)$. The defect was also generally not found in high proportions of the population (Fig. 7).

Finally, to determine whether these phenotypes persist, fish were also imaged at 6 and $7 \mathrm{dpf}$ (Fig. 8, Table 20, 21 and 23). Fish exposed to DomA at 2 dpf had significantly more myelin defects 
compared to control fish at both 6 and $7 \mathrm{dpf}$. Furthermore, the higher the dose of DomA delivered at $2 \mathrm{dpf}$, the more likely it was for the fish to exhibit all of the myelin phenotypes observed (Fig. 8A and 8B). All of this indicates that DomA exposure, particularly at 2 dpf, leads to myelin defects that persist for at least seven days after exposure.

\section{Time-lapse imaging of initial stages of myelin sheath formation}

I did not observe any myelination defects or behavioral phenotypes in larvae exposed to DomA at $4 \mathrm{dpf}$, a time point after the onset of myelination, suggesting that DomA does not affect established sheaths, but rather may perturb the formation of nascent myelin. DomA-exposed fish have perturbed myelin sheaths by $3 \mathrm{dpf}$, (the earliest development period myelin sheaths form) (Fig. 9A). To directly visualize the initial stages of myelin sheath formation, I performed timelapse imaging in double transgenic fish (Tg:sox 10:RFP; Tg:nkx2.2a:EGFP), in which cells of the oligodendrocyte lineage - the cells responsible for myelination in the central nervous system - are labeled, with EGFP in the cell membranes (myelin sheaths) and RFP in cell bodies. Imaging the axon wrapping and nascent myelin sheath formation from 2.5-3 dpf confirmed that oligodendrocytes in DomA-exposed larvae were unable to form elongated sheaths, but rather formed unusual circular membranes ( $n=5$ for controls, $n=6$ for DomA exposed) (Fig. 9B).

\section{Transcriptional changes following domoic acid exposure at 2 dpf}

To identify the gene expression changes that accompany the myelination and startle deficits, whole-embryo RNAseq was performed on embryos spawned from three different breeding tanks and then injected at $2 \mathrm{dpf}$ with $0.14 \mathrm{ng}$ of DomA and sampled at 3 and $7 \mathrm{dpf}$ for RNA (Fig. 10A).

To ensure effectiveness of the exposure, a subset of exposed fish were imaged to visualize myelin structure at $5 \mathrm{dpf}$ and then subjected to behavioral tests (startle response) at $7 \mathrm{dpf}$. Consistent with other experimental trials, there were also differences in behavior and myelin labeling between DomA-exposed fish and controls (Supplemental Fig. 2). Fish exposed to DomA at $2 \mathrm{dpf}$ had shorter bend angles (Estimate $=0.893,95 \% \mathrm{CI}[0.815,0.971], p<1 \mathrm{e}-6)$ and slower angular velocities (Estimate $=0.888,95 \%$ CI [ 0.779, 0.996], $p<1$ e -6 ,) relative to controls (Supplemental Fig. 2A and B). Also consistent with other experimental trials, only DomA-exposed larvae showed any visible myelin defects, with most of the fish having myelin 
defects that were in the second to highest severity (Category $3=21 / 49$, Supplemental Fig. 2C). Phenotypic analysis thus validated the use of RNAseq to identify potential transcriptional changes from exposures.

RNA sequencing yielded an average of 21 million raw reads per sample. Of these, $77.6 \%$ were uniquely mapped to the genome. A multidimensional scaling (MDS) plot revealed clustering by both developmental stage ( $3 \mathrm{dpf}$ vs. $7 \mathrm{dpf}$ ) and breeding tank ( 3 breeding trios) (Fig. 10B). This indicates that the differences in gene expression were driven primarily by developmental stage and breeding clutch, and less influenced by treatment (DomA versus control).

Statistical analysis revealed differential expression of 82 genes at $3 \mathrm{dpf}$ ( 28 hours post exposure), and 10 genes at $7 \mathrm{dpf}$ (Fig. 10C-D, Supplemental Tables 2 and 3). Among the 82 genes differentially expressed at $3 \mathrm{dpf}, 51$ genes were down-regulated and 31 were up-regulated in DomA-exposed larvae as compared to controls. Interestingly, glial fibrillary acidic protein ( $g f a p)$, a marker for astrocyte activation and neuronal injury response, was upregulated in DomA-exposed fish ( $\log 2$ fold change $=0.5)$.

Pathway analysis of the differentially expressed genes (DEGs) indicated an overrepresentation of the GO biological process terms, protein depolarization and microtubule depolarization. The genes represented under these GO terms include genes in the stathmin family of which 2 out of 3 stathmin genes were up-regulated, and 1 out of 3 were down-regulated in DomA-exposed fish (Table 24).

Significant human phenology phenotypes associated with the down-regulated genes included peripheral axonal degeneration, segmental peripheral demyelination/remyelination, and myelin outfoldings (Table 25). Several genes required for the maintenance of axonal and myelin structure (neflb, nefmb, nefma, nefla, mpba, mpz) were downregulated in DomA-exposed fish relative to controls, and were overrepresented in the human phenology phenotypes (Fig.11). There were no human phenology phenotypes associated with up-regulated genes. 
At $7 \mathrm{dpf}$, there were only ten DEGs, with nine down-regulated and one up-regulated in DomAexposed fish relative to the controls (Fig. 10D, Supplemental Table 3). Comparison of DEGs from 3 and $7 \mathrm{dpf}$ revealed four out of the ten genes to be common in both the time points. Among them, three were down-regulated and one up-regulated, with only two being annotated. Two of the four shared down-regulated genes were neurofilament genes required for maintaining axonal integrity (nefmb and neflb).

\subsection{DISCUSSION}

It is well known that early development is a sensitive period for DomA exposures, and that lowdoses of DomA can lead to persistent behavioral deficits. However, the mechanisms that drive these changes are largely unknown. This study identified a window of susceptibility to DomA toxicity in early development and characterized the resulting molecular, structural, and behavioral consequences of exposures during this period. The period around $2 \mathrm{dpf}$ was identified as the sensitive window of exposure. Exposures during this period led to changes in gene expression, disruption to myelin sheath formation, and aberrant startle behavior.

\section{A novel exposure method uncovers a window of susceptibility to lower doses of DomA}

This study established zebrafish as a tool for investigating the mechanisms of toxicity from lowdose exposures during development. Previous developmental DomA exposure studies in zebrafish were done by injecting DomA into the yolk during the early embryonic stages (5121000 cell stage). ${ }^{60,25}$ However, the DomA doses that led to behavioral phenotypes were also those that resulted in high mortality rates and lasting neurotoxic symptoms. To build on this work, I used a novel exposure method that delivered DomA intravenously at different periods in development - from the embryonic to the larval stages. Using this method, I was able to find a window of susceptibility for lower doses of DomA (3-260x lower than previously used doses) at which structural and behavioral effects occurred with no appreciable mortality and minimal gross morphological defects. In particular, the period around $2 \mathrm{dpf}$ was identified as the window of susceptibility for low doses of DomA (0.09-0.14 ng). 
Acute neurotoxic phenotypes are transient and dose- and exposure time-dependent Fish exposed to DomA at 1 and $2 \mathrm{dpf}$ exhibited all the acute neurotoxic symptoms previously described in developmental exposure studies in zebrafish. ${ }^{60}$ However, Tiedekken et al. (2005) found that high doses of DomA led to neurotoxic effects later in the larval stages. ${ }^{60}$ In contrast, my experiments showed that the loss of a touch response and presence of pectoral fin flapping and convulsions were transient, dropping off one day after exposure (Fig. 1C, 1D).

Both dose and timing of exposures significantly influenced the presence of acute neurotoxic symptoms. Fish exposed at $4 \mathrm{dpf}$ had no significant acute neurotoxic symptoms, while fish exposed at both 1 and $2 \mathrm{dpf}$ had touch response deficits. While exposures during both these developmental periods led to these neurotoxic symptoms, embryos exposed to DomA at $2 \mathrm{dpf}$ were more sensitive and showed more pronounced touch response deficits than those exposed at 1 dpf (Fig. 1D).

\section{Effects on startle responsiveness are dependent on timing of exposure}

Startle response behavior was used as a functional read out of toxicity. Fish exposed to DomA particularly at $2 \mathrm{dpf}$ (and not 1 and $4 \mathrm{dpf}$ ) had aberrant startle behavior across all doses tested. Fish exhibited reduced responsiveness, increased latency, slower maximal angular velocities, and reduced bend angles relative to controls.

Startle responsiveness - the ability to perceive and react to vibrational stimuli - was dependent on both dose and timing of exposure (Fig. 2A). Only fish exposed to DomA at $2 \mathrm{dpf}$ showed reduced responsiveness at all doses tested $(0.09-0.18 \mathrm{ng})$. Fish exposed to DomA at $1 \mathrm{dpf}$ showed reduced responsiveness in the higher doses $(0.13-0.18 \mathrm{ng})$. While fish exposed at $4 \mathrm{dpf}$ showed reduced responsiveness only at the highest dose $(0.18 \mathrm{ng})$, which was a dose that also led to morphological defects and mortality. All of this suggests that there is a window of susceptibility to low-dose $(<0.18 \mathrm{ng})$ DomA exposures at around $2 \mathrm{dpf}$.

\section{Exposure to DomA at 2 dpf leads to consistent deficits in startle response kinematics} In addition to affecting overall responsiveness to startle, exposure to DomA also led to reductions in bend angle and slower maximal angular velocities. Alterations in bend angles due 
to glutamate imbalances have been previously described in a genetic mutant for a glutamate reuptake transporter. ${ }^{61}$ In the genetic mutant, the increased availability of extracellular glutamate led to exaggerated body bending early in development (2-4 dpf) followed by the absence of a touch response later in development. While the phenotype is different from what was observed in my results, work with the genetic mutant shows that startle response kinematics are altered by perturbations to glutamate signaling.

The presence of kinematic deficits was influenced by the timing of DomA exposure, with patterns that are consistent with the startle responsiveness findings. Only larvae exposed at $2 \mathrm{dpf}$ had consistent kinematic deficits across all the doses tested (0.09-0.18 ng) (Fig. 4 and 5). Larvae exposed at $2 \mathrm{dpf}$ had reduced bend angles and slower maximal angular velocities when performing both SLC and LLC-type startles. Fish exposed to higher DomA doses (0.13 ng or higher) at $1 \mathrm{dpf}$ also had kinematic deficits, but these fish had less pronounced kinematic deficits compared to fish exposed at $2 \mathrm{dpf}$ (Fig. 3B, 3C). Finally, fish exposed to DomA at $4 \mathrm{dpf}$ also showed some measurable kinematic deficits at the highest dose tested $(0.18 \mathrm{ng})$. However, these deficits were found in either bend angle or maximal angular velocity, but not both. Deficits were also inconsistent across repeated experiments. Taken together, these kinematic results are also consistent with a window of greater susceptibility to DomA at around $2 \mathrm{dpf}$.

\section{DomA disrupts myelin formation}

Similar to the behavioral results, only fish exposed at $2 \mathrm{dpf}$ (but not 1 or $4 \mathrm{dpf}$ ) showed consistent defects in their myelin sheaths (Fig. 6B). DomA-exposed larvae had an overall reduction in labeled myelin, along with the appearance of unusual circular membranes (Fig. 6B). These deficits were visible as early as $3 \mathrm{dpf}$, when myelin sheaths first form (Fig. 9), and persisted until at least $7 \mathrm{dpf}$, indicating that initial formation of myelin is perturbed and does not recover within 4 days post-exposure (Fig. 8).

The window of susceptibility to DomA corresponds to the critical period for oligodendrocyte development

It is possible that $2 \mathrm{dpf}$ is the window of susceptibility because DomA perturbs specific developmental processes that occur within this time period. While most of the early neurons 
have already differentiated by $2 \mathrm{dpf}$, the oligodendrocyte lineage - the lineage that myelinates axons in the central nervous system - is just beginning to migrate and differentiate during this period. ${ }^{62,63}$ DomA exposures at $2 \mathrm{dpf}$ may perturb critical processes in oligodendrocyte development, leading to the observed disrupted myelination that was specific to this exposure time.

Both myelinating oligodendrocytes and their precursors express functional ionotrophic glutamate receptors, potentially making them cellular targets for DomA. ${ }^{64,65}$ Previous studies have shown that kainate, a structural analog of DomA, causes cell death in oligodendrocyte primary cell cultures, at concentrations comparable to those affecting neurons. ${ }^{66-69}$ Oligodendrocyte precursors (OPCs) have also been shown to be highly vulnerable to kainate-induced cell death due to the expression of ionotrophic glutamate receptors. ${ }^{68}$ Furthermore, binding and activating AMPA receptors inhibits the proliferation and differentiation of OPCs into mature oligodendrocytes in vitro. ${ }^{70,71}$ Mature oligodendrocytes have also been shown to undergo demyelination after chronic direct infusion of kainate on the optic nerves. ${ }^{72}$ All of this suggests that DomA may alter oligodendrocyte development, and that exposures to DomA at $2 \mathrm{dpf}$ may be disrupting critical processes important for OPC proliferation, differentiation, and myelin sheath formation.

This study characterized myelination defects that resulted from exposures to DomA that occur shortly before the stage in development when myelin sheaths first form (2.5-3 dpf). Previous studies have show that glutamate toxicity can perturb already established myelin sheaths. In particular, the overactivation of glutamate receptors by the glutamate receptor agonist, kainate, has been previously found to disrupt myelin sheaths prior to disrupting axons. Adult rats exposed to $5 \mathrm{nmol}$ of kainate had dismantled myelin sheaths that were loosely arranged around axons classified as 'healthy' as shown by ultrastructural analyses. ${ }^{73}$ In a separate study, exposures to kainate $(10 \mathrm{mg} / \mathrm{kg})$ in adult Sprague-Dawley rats also led to overall reductions in Black-Gold labeling (a myelin stain) in several brain regions including the neocortex, thalamus, and amygdala. ${ }^{74}$ These myelin defects preceded neurodegeneration, suggesting that myelin sheaths were primary targets of glutamate excitotoxicity. 
Of note, my findings suggest DomA (at the doses tested) does not seem to disrupt already established myelin sheaths but rather may perturb the initial formation of the myelin sheaths. I saw very few myelin defects at $4 \mathrm{dpf}$ - a time point after nascent myelin has been established (further discussion below).

Only one previous study has assessed myelin following developmental exposure to DomA. Eleven week-old juveniles exposed in utero during gestational days 11.5 and 14.5, but not 17.5, had a reduced staining for the myelin-associated glycoprotein (MAG) in their cerebral cortices. ${ }^{19}$ This suggests that there may be periods in early development that are more sensitive to exposure to DomA, leading to these myelination deficits. Indeed, it is possible that sensitivity at the early periods is due to disruptions to oligodendrocyte development, thereby altering their ability to form myelin sheaths during the postnatal period. ${ }^{75,76}$

\section{Extrinsic factors that may influence the critical window for DomA toxicity}

In zebrafish, $4 \mathrm{dpf}$ is a time period at which myelin sheaths are already established. The absence of a myelin phenotype following exposures at $4 \mathrm{dpf}$ suggests that DomA, at the doses used here, may not perturb already established sheaths but rather may perturb the initial formation of myelin sheaths. Time-lapse imaging of the initial stages of axon wrapping and nascent myelination (from 2.5-3 dpf), provides additional evidence that DomA affects the ability of oligodendrocytes to initially wrap axons and form elongated myelin sheaths (Fig. 9B). However, if oligodendrocyte differentiation and myelination are the critical processes perturbed by DomA, we would expect that exposures at $2.5 \mathrm{dpf}$, and not $2 \mathrm{dpf}$, would lead to the most pronounced phenotypes, as $2.5 \mathrm{dpf}$ is the period when axon wrapping and myelination occur. ${ }^{35}$ Thus, it is likely that the $2 \mathrm{dpf}$ window of susceptibility is also influenced by extrinsic factors that affect the distribution and availability of DomA to the cells and tissues of interest.

\section{Blood brain barrier development may limit DomA distribution at later developmental stages} One of the factors that influences DomA availability in the central nervous system is the bloodbrain barrier $(\mathrm{BBB})$ - a structure that separates the blood from the brain parenchyma. ${ }^{77}$ The BBB is composed of tight junctions between endothelial cells that seal the intercellular cleft and

prevent the diffusion of water-soluble molecules. ${ }^{78-80} \mathrm{BBB}$ formation has been studied by 
tracking the exclusion of differently sized fluorescently tagged proteins injected into the circulatory system over the course of development. It has been found that as the BBB forms, it progressively excludes smaller molecules over time. The fluorescent tracer, fluorescein, is similar in molecular weight to DomA $(\operatorname{DomA}=311.33 \mathrm{Da}$, Fluorescein $=332.311 \mathrm{Da})$ and has been shown to enter into the zebrafish brain over the course of 4-6 hours when injected at 8 dpf. ${ }^{78}$ However, injections of fluorescein at $3 \mathrm{dpf}$ have been shown to be excluded from the brain 50 minutes after injection. This suggests that even the immature blood brain barrier is able to exclude low-molecular weight dyes over these short timescales (50 minutes or less). ${ }^{80}$ If sizebased exclusion occurs similarly for DomA as for fluorescein, DomA may be excluded from the central nervous system to a greater degree during developmental periods past $2 \mathrm{dpf}$ due to maturation of the BBB.

DomA may also be less accessible to cell targets of interest later in development due to relatively higher excretion rates as the kidney matures. DomA is primarily cleared from the plasma via the kidneys, and nephrectomies in rodent models have been shown to increase the plasma half-life of DomA. ${ }^{81-83}$ In zebrafish, glomerular filtration begins at around $2 \mathrm{dpf}$ while full maturation of the kidney occurs by $4 \mathrm{dpf}^{84,85}$ Thus, DomA may be more readily cleared during periods in development after $2 \mathrm{dpf}$. To assess this, I would need to measure DomA concentrations in embryos over time. However, limited tissue from larvae has made this technically challenging.

\section{Transcriptional changes suggest defects in axon and myelin structures}

RNAseq data show an increase in glial fibrillary acidic protein ( $g f a p$ ) expression following exposure to DomA. gfap is an intermediate filament protein whose upregulation in mammals is a hallmark of reactive gliosis - the response of glial cells following mechanical injury, excitotoxicity, or ischemia. ${ }^{86,87}$ In zebrafish, $g f a p$ is expressed in ependymoglia glia, a population of neural progenitors in adult CNS ${ }^{88,89}$ Following mechanical injury in adult zebrafish, there is a delayed expression of $g f a p$ during the proliferation and recovery stages. ${ }^{90}$ The upregulation of $g f a p$ in $3 \mathrm{dpf}$ fish suggests that doses of DomA at these stages of development may lead to injury and trigger repair mechanisms associated with increased gfap expression. 
Protein depolarization and microtubule depolarization were the two significant gene ontology (GO) biological processes represented in the gene expression data. Stathmin genes were among the DEGs contributing to these GO terms. Stathmins destabilize microtubules by sequestering free tubulin. They are highly expressed in the developing nervous system and play critical roles in modulating neurite outgrowth and branching. ${ }^{91,92}$ It has been shown that the dysregulation of different stathmin genes (either through down- or up-regulation) can lead to alterations in microtubule density and axonal integrity. ${ }^{92-94}$

RNAseq analysis also identified genes and pathways that were consistent with the imaging and behavioral data. DomA exposure down-regulated genes required for maintaining myelin structure, including myelin protein zero ( $m p z)$ and $(m b p a)$, along with genes required for maintaining axonal structure (nefla, neflab, nefma, nefmb) (Fig. 11). Thus, it may be possible that DomA may be primarily targeting axons, and that the myelination defects may be a secondary effect. Alternatively, DomA may perturb oligodendrocyte development and myelin wrapping, leading to later axonal dysfunction. In subsequent chapters, I investigate potential axonal targets of DomA toxicity and assess the contribution of the axonal perturbations to the myelin sheath phenotypes that were characterized in this chapter.

\section{RNAsequencing shows few gene expression changes}

Overall, there were fewer gene expression changes associated with treatment (DomA vs. control) than with either the developmental period when sampling occurred or the breeding tanks the clutches came from. Gene expression differences due to developmental periods are well known, as distinctive clusters of expressed genes and pathways occur at different stages in development. ${ }^{95,96}$ Transcriptional changes based on clutches collected from different breeding tanks may be due to genetic differences in the trio parents that produced the clutches.

Alternatively, or in addition, there could be subtle differences among tanks in environmental parameters that influence gene expression.

The small number of genes differentially expressed in response to DomA exposure may be due to the nature of whole embryo RNAsequencing. Conceivably, if DomA is targeting a few specific cell types, more highly expressed genes in whole-embryo RNA samples may swamp out 
these modest but biologically relevant cell-specific changes in gene expression. Furthermore, if the same genes were differentially expressed across multiple tissues in different directions, so that in some tissues a gene is upregulated while in others it is downregulated, the overall expression would show no differences. These scenarios allow for false negatives in the context of whole embryo RNASeq. ${ }^{97}$ Future research could be done to identify transcriptional changes in specific cell types of interest, such as myelinating oligodendrocytes.

\section{Consequences for human health: timing and targets}

This study provides a careful examination of potential windows of susceptibility to DomA exposure. The identification of key processes disrupted during these windows of susceptibility has important implications for identifying hazards for early developmental exposures in humans. If myelination is a key process perturbed in DomA toxicity, the identification and protection of critical periods of myelination will be necessary to protect against DomA toxicity. Unlike zebrafish, myelination in humans occurs over a prolonged period, starting in utero and continuing into early childhood and adolescence. The progression of myelination is mostly conserved across species, with myelination commencing in the periphery, brainstem, and spinal cord, then progressing rostrally to the forebrain. ${ }^{98,99}$ The most widespread and rapid period of myelination in humans occurs within the first two years of infancy. ${ }^{100,101}$ While most of the major tracts are myelinated by 3-5 years of age, myelination is now known to continue into adulthood, especially in cortical regions where changes in myelination are associated with experience and learning new skills. ${ }^{102,103}$ Thus, for humans, there may not be a single window of susceptibility, but rather multiple windows; domoic acid may perturb myelin formation in specific regions of the nervous system in which myelination coincides with the timing of exposures.

It is also conceivable that the window of susceptibility might be influenced by changes in bioavailability and distribution of DomA to target regions in the nervous system. Earlier time periods may be more sensitive to DomA exposures, because clearance mechanisms and the blood brain barrier formation have yet to develop. If this is true, then DomA is more likely to perturb myelination processes that occur earlier in development, such as myelination in the periphery, brainstem, and spinal cord. 
In this study, myelination was shown to be perturbed in the spinal cord. Only two other studies in rodents have investigated the spinal cord as a target tissue for DomA exposures. Guang et al. (2000) found that postnatal exposures to high doses of DomA led to marked spinal cord lesions by 2 hours post exposure, even in the absence of any histological damage to selected brain regions, including the hippocampus. ${ }^{104}$ Gait deficits that were characterized following prenatal exposures (via oral administrations) were also posited to be due to spinal cord deficits. ${ }^{20}$ Future studies should look more carefully at the spinal cord as a target of DomA toxicity.

\section{Consequences for human health: behavioral deficit analogies}

This study also used startle response behavior as a functional read out of toxicity. Deficits in the kinematics of startle responses are reminiscent of motor deficits found in incidental human exposures, chronic exposures in primates, and developmental exposures in rodents. Adult humans acutely exposed to DomA developed sensorimotor neuropathy and axonopathy as assessed by electromyography. ${ }^{105}$ A subset of the primates exposed orally at or near the accepted daily tolerable dose of $0.075 \mathrm{mg} / \mathrm{kg}$ also developed visible hand tremors. ${ }^{106}$ Rodents prenatally exposed to DomA (PND 10-17) develop aberrant gait patterns including impaired interlimb coordination, aberrant step sequence patterns, and variability in maximal speeds. ${ }^{20}$ It is important to note that all the studies that have identified motor deficits used oral exposures to DomA, which is the sole exposure route in adult humans.

While there is evidence that DomA can perturb motor function, developmental exposures to DomA in rodents have not led to reductions in startle response amplitude during baseline conditions (prior to habituation or pre-pulse inhibition tests). ${ }^{20,107-109}$ This may be because exposures to DomA in these rodent models were done during a period that does not correspond to development of the startle circuit. Rodents and fish do share many of the basic neuronal components that underlie the startle response - an auditory nerve which senses the stimuli, the giant reticulospinal neurons that integrate the signal, and the spinal motor neurons that activate the muscles to elicit the response. ${ }^{110-112}$ However, there are some notable differences between rodent and fish startle. This includes distinct baseline startle kinematics (whole body displacement or not), differences in sensorimotor gating (PPI which leads to reduced amplitudes 
versus the extinction of the behavior itself), and variations in the specific neuronal subsets in the circuits.

\section{Consequences for human health: doses and toxicokinetics}

In all currently published developmental DomA exposure studies, 'low doses' have been defined based on the absence of acute neurotoxic symptoms, rather than by an absolute dose number. Low doses are those that do not lead to classical acute symptoms that include tremors, scratching, and convulsions either in mothers (prenatal exposures) or in the pups directly exposed to DomA (postnatal exposures). While this study used doses that were 3-260x lower than those used previously in zebrafish, these doses still led to transient neurotoxic effects in embryos. However, when directly comparing the weight-normalized amount of DomA, these doses are comparable to those used in the majority of the postnatal rodent studies. ${ }^{10,22,26,107,108,113-}$ ${ }^{115}$ Assuming a $1.4 \mathrm{mg}$ wet weight per embryo, ${ }^{60}$ the dosages at which embryos consistently exhibited myelin defects and behavioral deficits were 0.06-0.10 mg/kg DomA. Rodents who showed behavioral deficits following postnatal exposure were dosed subcutaneously with 7 injections of 0.005 and $0.020 \mathrm{mg} / \mathrm{kg}$ DomA between PND 8-14, leading to the exposure to a total of $0.035-0.14 \mathrm{mg} / \mathrm{kg}$.

The main challenge for translating findings in animal models to humans is the dearth of human exposure and toxicokinetic data. Human exposures to DomA are only estimated from consumption data, average weights of adults, and measured DomA concentrations in shellfish. Furthermore, the toxicokinetics are not well known. Oral exposure is the only route of exposure in adult humans. New evidence from non-human primate models indicates that the toxicokinetic behaviors of oral and intravenous doses are distinct. With oral exposures, while bioavailability is low $(6 \% \pm 4)$, the half-life is extended to $11.3 \pm 2.4$ hours - almost $10 x$ the length of the half-life following intravenous exposure $(1.2 \pm 1.1$ hours $) .{ }^{116}$ Due to the prolonged half-life, the chronic consumption of DomA may lead to plasma accumulation over time, which has important health implications for chronic shellfish consumers. In fact, new evidence shows that chronic exposures in non-human primates at or near the recognized tolerable daily intake level (0.075 and 0.150 $\mathrm{mg} / \mathrm{kg}$ ) leads to persistent hand tremors and disruptions to whole-brain connectivity. ${ }^{106}$ 
Even less information exists about the elimination and distribution in DomA in fetuses when mothers are exposed to DomA. One study in rodents showed that following intravenous injections, the same concentrations of DomA were found in fetal brains, amniotic fluid, and maternal brains when DomA was administered at GD13. ${ }^{12}$ This suggests that earlier in development, there are no barriers for DomA entry to the fetal brain as DomA in the brain reaches equilibrium concentrations with DomA in the amniotic fluid. Note that these measurements were taken 1 hour post exposure and do not take into account differences in distribution of DomA after longer periods post-exposure. Emerging evidence from marine mammals shows that DomA can remain in the fetal fluids (amniotic and allantoic fluids) over

prolonged periods of time. ${ }^{15,16}$ It is hypothesized that DomA is recirculated within the fetal fluid compartments, allowing for continuous exposures in fetuses, even when maternal plasma has reached undetectable levels of DomA.

\subsection{CONCLUSION}

DomA is a well-known developmental neurotoxin. However, few studies have been able to identify the cellular and molecular processes that underlie the observed behavioral deficits seen following developmental exposures. Using zebrafish, I was able to deliver DomA at specific developmental times and link behavioral deficits to structural changes in the neural circuit required for the behavior. The results from this study show that there is a critical window of susceptibility to DomA, and that exposures lead to altered expression of key axonal and myelin structural genes, disruptions to myelination, and later perturbations to startle behavior. 


\section{FUNDING}

This research was supported by the Oceans Venture Fund, the Von Damm Fellowship, the Ocean Ridge Initiative Fellowship, Woods Hole Sea grant (NA14OAR4170074), and the Woods Hole Center for Oceans and Human Health (NIH - P01ES021923 and NSF- OCE-1314642 to MEH and NA, P01ES021923-04S1 to JMP).

\section{ACKNOWLEDGEMENTS}

I would like to thank: Hanny E. Rivera and Andrew R. Solow for their advice on the statistical analysis, Harold Burgess for providing the FLOTE software for startle kinematic analysis, Ian T. Jones for measuring the vibrational output of the startle set-up, Benjamin G. Merrick for his help designing and building the startle apparatus, Louis Kerr for microscopy training and advice (MBL microscopy facility), and the labs who generously provided me with zebrafish transgenic lines to make this work possible - Bruce Appel (University of Colorado, Denver) and David Lyons (University of Edinburg). 


\section{CHAPTER 2 FIGURES}

A1.

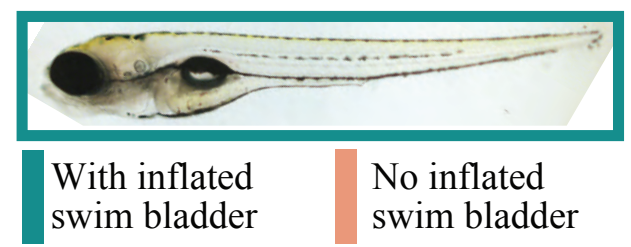

A2.

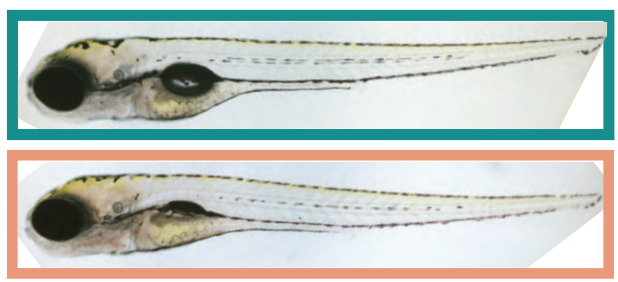

B.

B. $0 \mathrm{ng}$

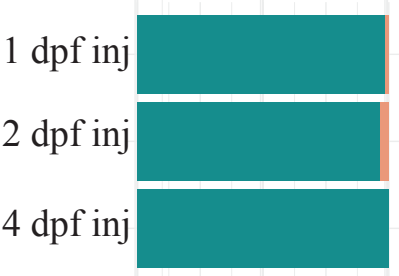

$0 \quad 50 \quad 1000$

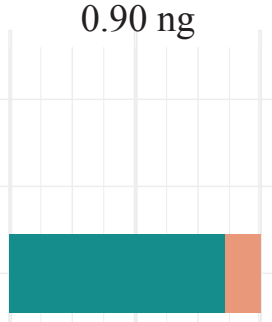

$0.13 \mathrm{ng}$

$0.14 \mathrm{ng}$

$0.18 \mathrm{ng}$
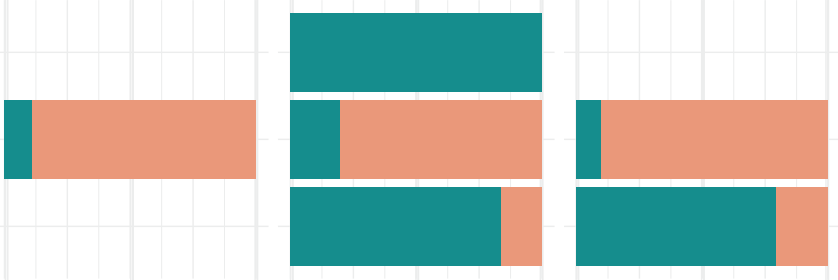

$50 \quad 100$

Percent

C.

$1 \mathrm{dpf} \quad 2 \mathrm{dpf} \quad 4 \mathrm{dpf}$
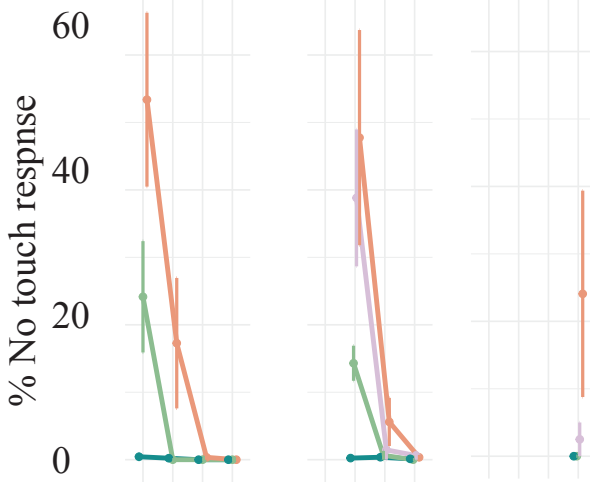

$$
\begin{array}{ccr}
2345 & 345 & 5 \\
\mathrm{dpf} & \mathrm{dpf} & \mathrm{dpf} \\
\hline \multicolumn{3}{c}{\text { day observed }}
\end{array}
$$

D.

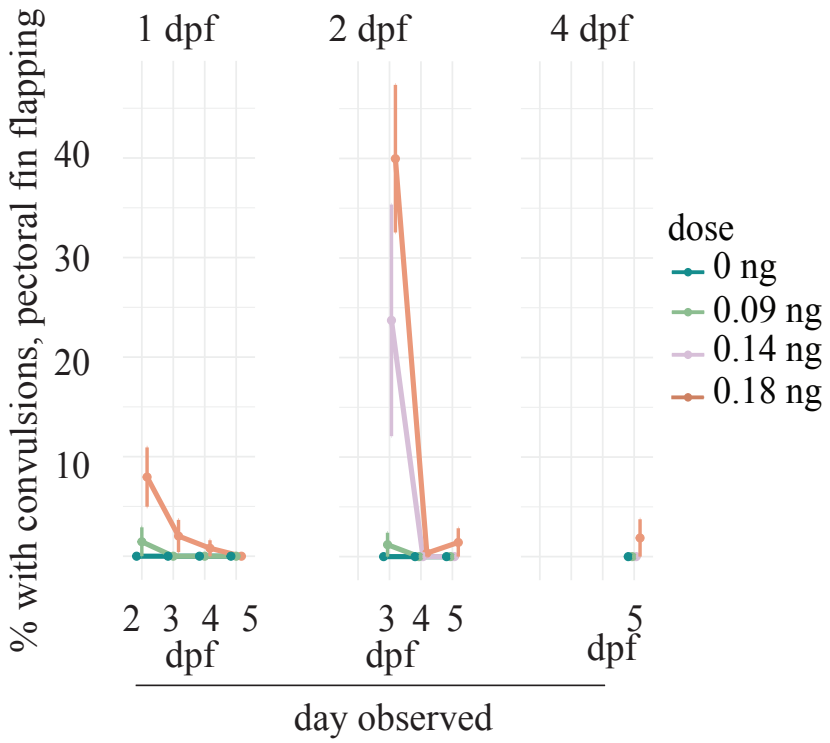

Figure 1: Domoic acid-exposed larvae have morphological and acute neurotoxic phenotypes that vary based on exposure time and dose.

(A1) Representative brightfield image of control larvae with an inflated swim bladder.

(A2) Representative brightfield image of DomA-exposed larvae with an inflated swim bladder (teal), and without an inflated swim bladder (peach).

(B) Presence or absence of the inflated swim bladder by treatment, dose and time of exposure.

(C) The percentage of embryos with touch response deficits were recorded one day post-exposure until $5 \mathrm{dpf}$, using experimental trials where individual fish were observed over several developmental days. Fish were exposed to different doses of DomA (0.09 ng- $0.18 \mathrm{ng})$ at 1, 2 and $4 \mathrm{dpf}$. Lines are dodged along the $\mathrm{x}$ axis. \pm standard errors of the mean between repeated experiments.

(D) The same fish population observed in Fig. 2C were also monitored for the presence of convulsions or pectoral fin flapping from one day post-exposure until $5 \mathrm{dpf}$. 


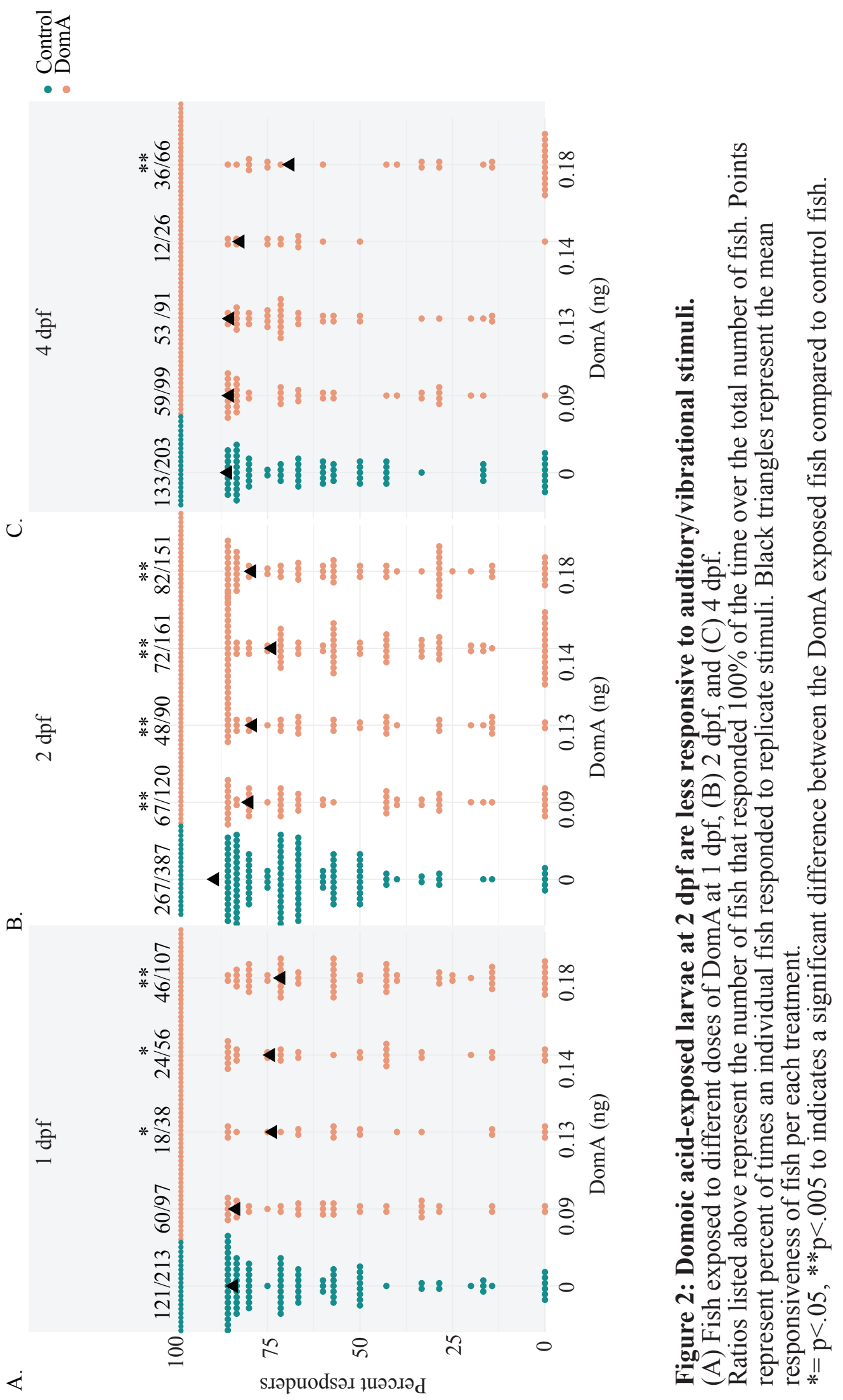



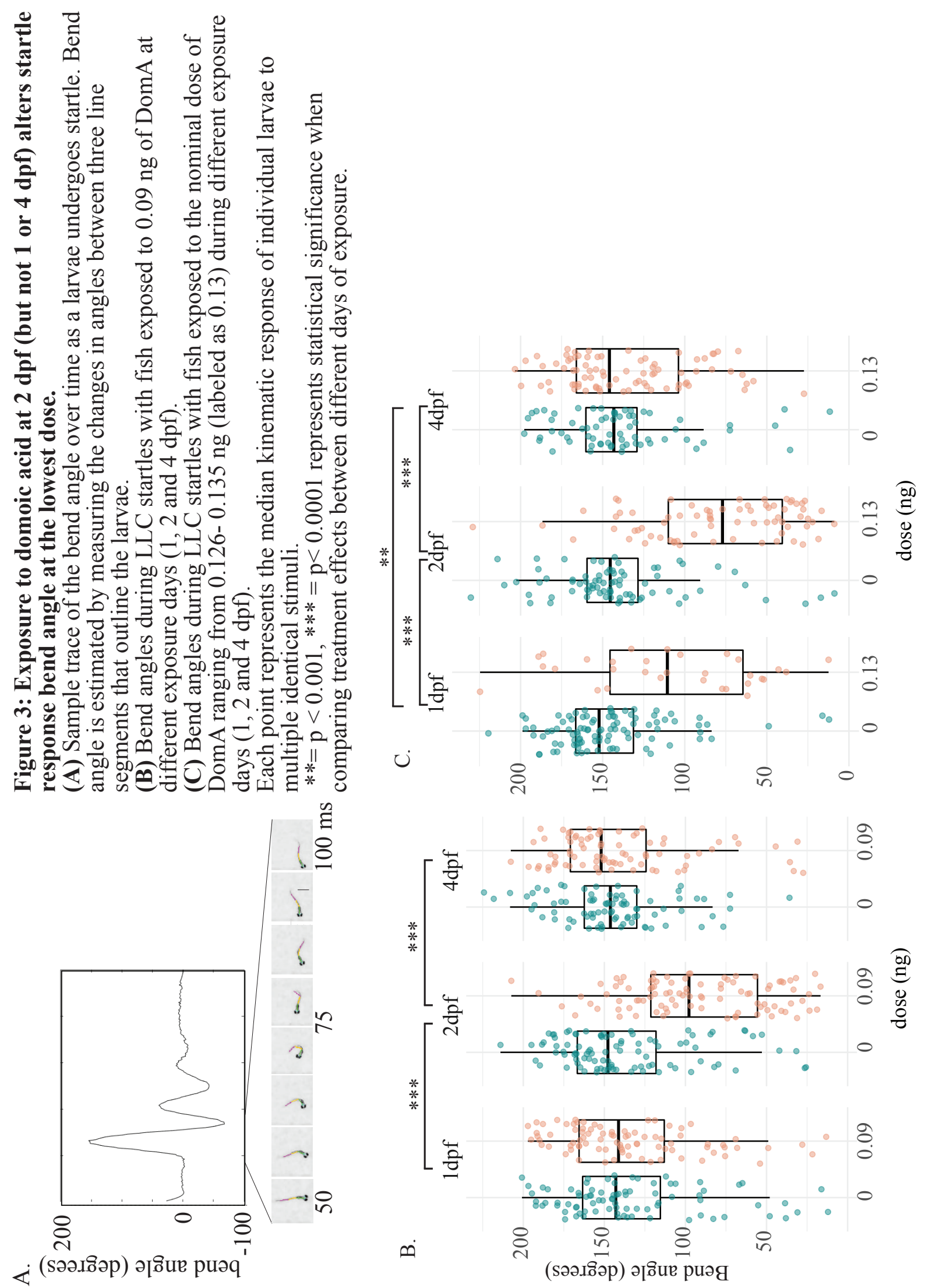
Figure 4: Exposure to domoic acid at 2 dpf (but not 1 or 4 dpf) consistently alters SLC startle response kinematics.

(A-C) Bend angle for fish undergoing SLC type startle response. Fish were exposed to different doses of DomA at $1 \mathrm{dpf}(\mathrm{A}), 2 \mathrm{dpf}(\mathrm{B})$, and, $4 \mathrm{dpf}(\mathrm{C})$.

(D- F) Maximal angular velocity obtained by DomA and control fish undergoing SLC type startle responses. Fish were exposed to different doses of DomA at $1 \mathrm{dpf}(\mathrm{D}), 2 \mathrm{dpf}(\mathrm{E})$, and $4 \mathrm{dpf}(\mathrm{F})$.

Each point represents the median of up to 7 responses for an individual fish. Boxplot show the group medians, upper $75 \%$ quantile, and the lower $25 \%$ quantile.

$* \mathrm{p}<0.05, * * \mathrm{p}<.001, * * * \mathrm{p}<.0001$ compared to control kinematic responses.

Ratios $=$ trials with statistically significant treatment effects/ total trials. 

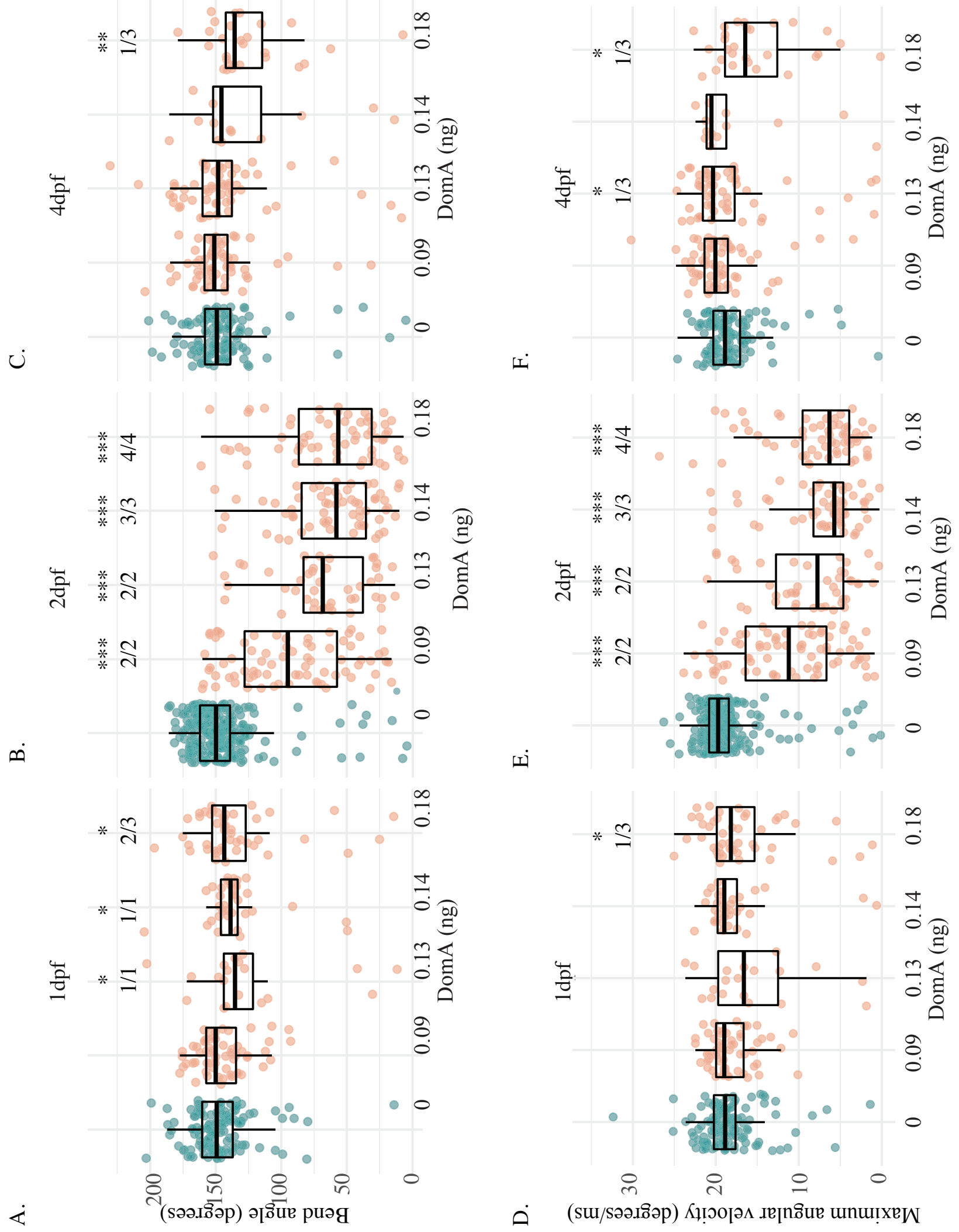
Figure 5: Exposure to domoic acid doses at 2 dpf (but not 1 or 4 dpf) consistently alters LLC startle response kinematics.

(A-C) Bend angle for fish undergoing LLC type startle response. Fish were exposed to different doses of DomA at $1 \mathrm{dpf}(\mathrm{A}), 2 \mathrm{dpf}(\mathrm{B})$, and $4 \mathrm{dpf}(\mathrm{C})$.

(D- F) Maximal angular velocity obtained by DomA and control fish undergoing LLC type LLC startle responses. Fish were exposed to different doses of DomA at $1 \mathrm{dpf}(\mathrm{D}), 2 \mathrm{dpf}(\mathrm{E})$, and $4 \mathrm{dpf}(\mathrm{F})$.

Each point represents the median of up to 7 responses for an individual fish. Boxplot show the group medians, upper $75 \%$ quantile, and the lower $25 \%$ quantile.

$* \mathrm{p}<0.05, * * \mathrm{p}<.001, * * * \mathrm{p}<.0001$ compared to control kinematic responses.

Ratios $=$ trials with statistically significant treatment effects/ total trials. 


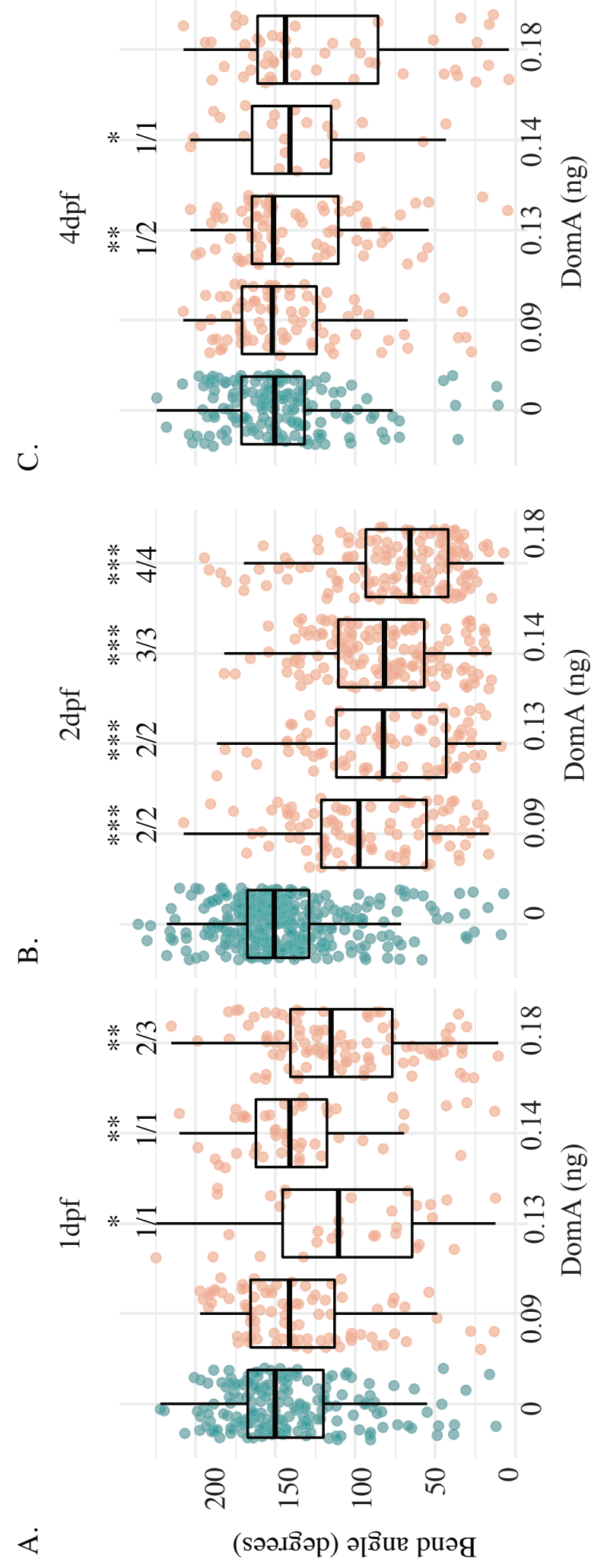

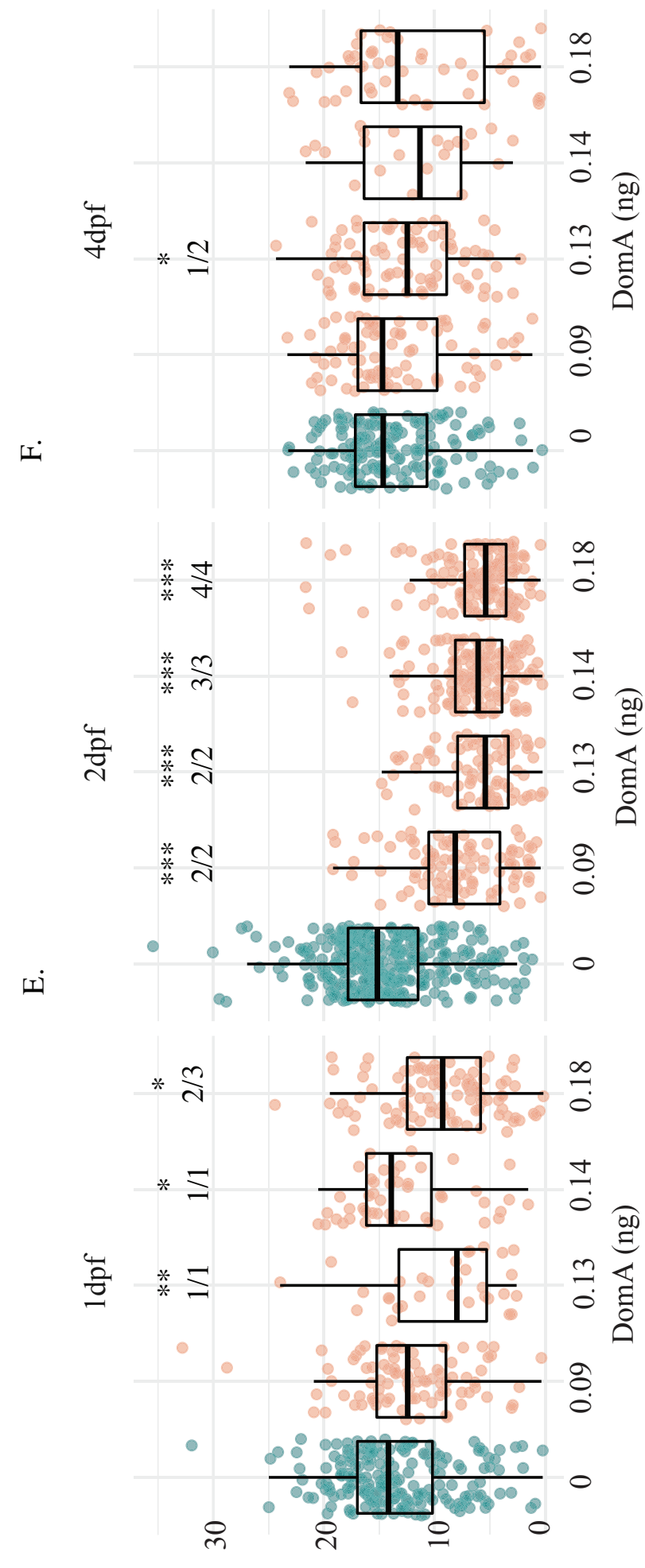

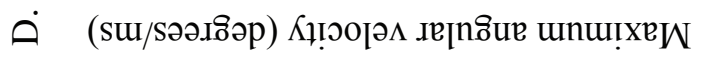


Figure 6: Exposure to domoic acid at 2 dpf (but not 1 or 4 dpf) alters myelin sheaths at 5 dpf

(A) $\operatorname{Tg}(\mathrm{mbp}: E G F P-C A A X)$ was used to visualize labeled myelin sheaths.

(B) Fish were exposed to DomA (0.14 ng) during discrete developmental periods (1- $4 \mathrm{dpf})$, then imaged at $5 \mathrm{dpf}$ using confocal microscopy. Arrows indicate the unusual circular membrane profiles.

(C) Stacked bar plots show the distribution of the different myelinphenotypes when fish were exposed to DomA at discrete developmental times. Multiple trials were combined to calculate the $\%$ distribution per phenotype observed. The trials represented along with the associated numbers of fish per trial are listed on Table 18.

(D) Representative confocal microscopy images of different myelin phenotypes that were observed. Each fish was blindly classified and assigned a category based on severity of the myelin deficit observed. Scale bar $=100 \mu \mathrm{m}$. 

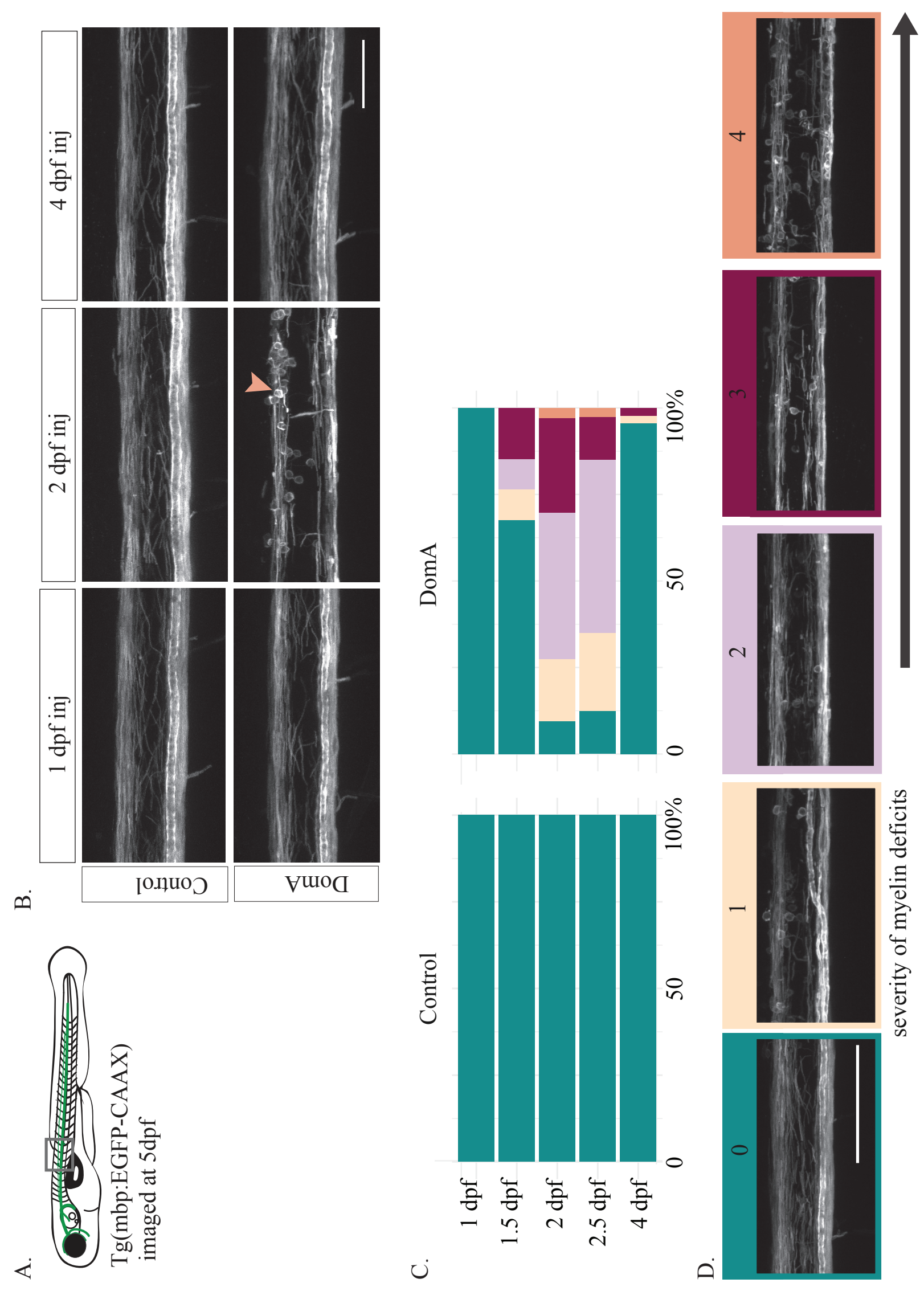
Figure 7: Exposures to domoic acid between 2- $2.5 \mathrm{dpf}$ alters myelin sheaths at 5 dpf

(A) $\operatorname{Tg}(\mathrm{mbp}: E G F P-C A A X)$ were exposed to DomA over a range of discrete developmental periods (1-4 dpf), then imaged at $5 \mathrm{dpf}$ using widefield epifluorescence microscopy. Images were blindly classified into 6 categories based on severity of the observed myelin phenotype. Arrows indicate the myelinated mauthner axon which is required for fast startle responses.

(B) Stacked bar plots show the distribution of the different phenotype per each dose of DomA delivered at discrete developmental times. Multiple trials were combined to calculate the \% distribution per phenotype observed. The trials represented along with the associated numbers of fish per trial can be found in Table 19.

Scale bar $=50$ microns 


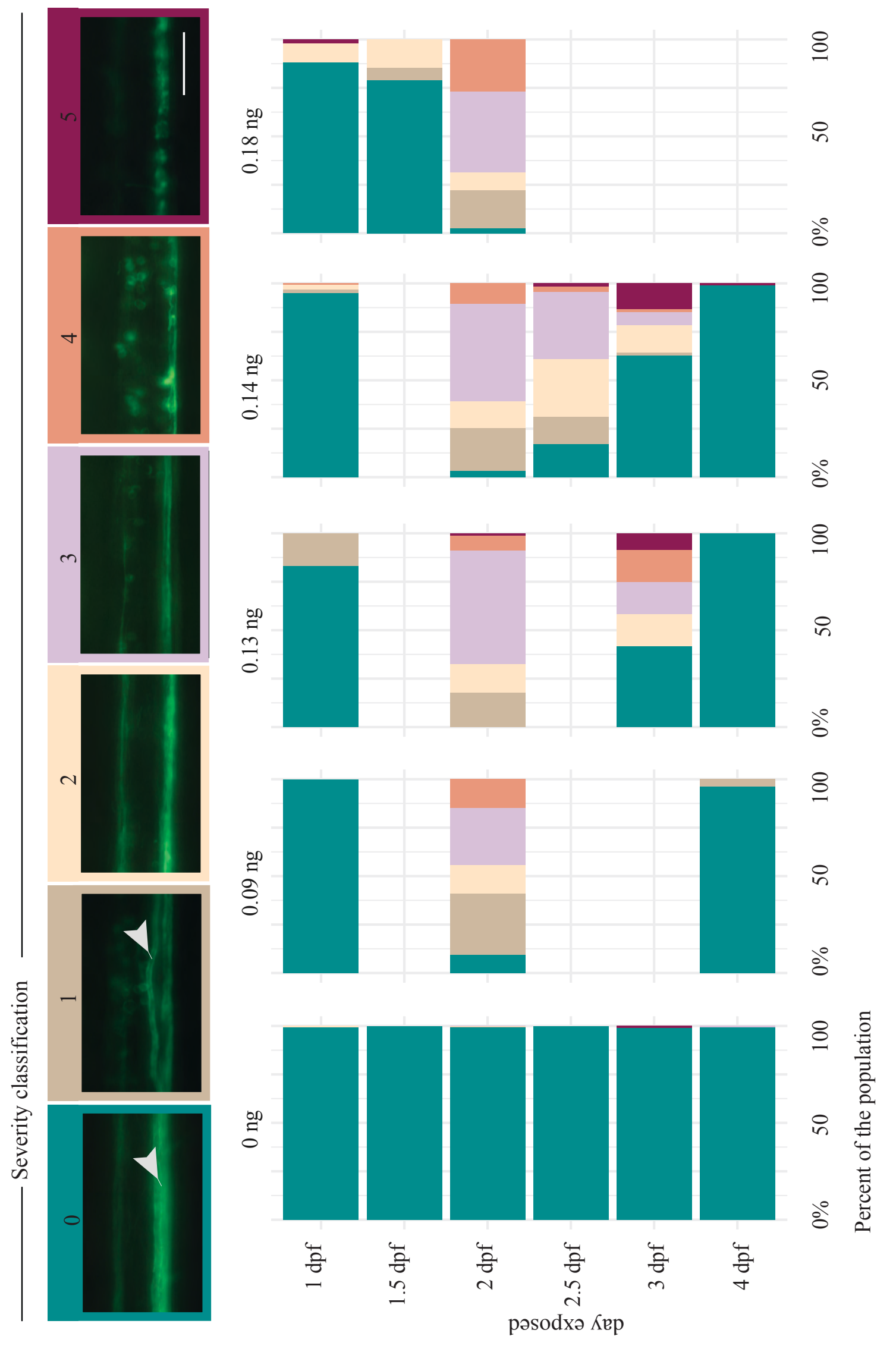


Figure 8: Myelin sheath labeling defects persist until at least $7 \mathbf{~ d p f}$.

(A) $\operatorname{Tg}(\mathrm{mbp}: E G F P-C A A X)$ were exposed to DomA during different developmental periods (1, 2 and $4 \mathrm{dpf}$ ), then imaged at $6 \mathrm{dpf}$ using widefield epifluorescence microscopy. Stacked bar plots show the distribution of the different phenotypes per each dose, which was delivered at discrete developmental times. Multiple trials were combined to calculate the \% distribution per phenotype observed. The trials represented along with the associated numbers of fish per trial can be found in Table 20.

(B) $\operatorname{Tg}(\mathrm{mbp}: E G F P-C A A X)$ imaged at $7 \mathrm{dpf}$ using widefield microscopy. The trials represented along with the associated numbers of fish per trial can be found in Table 21. 

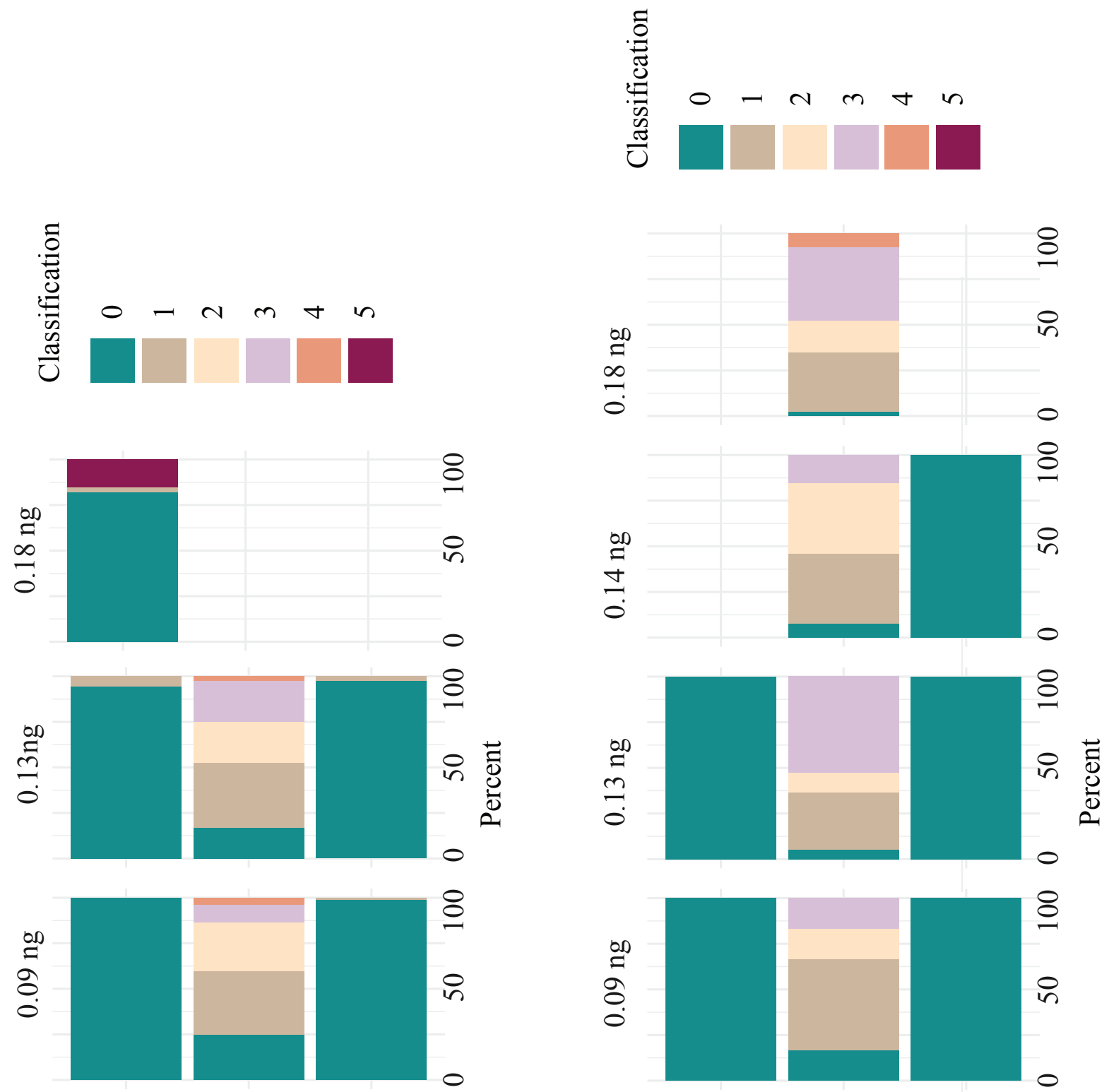
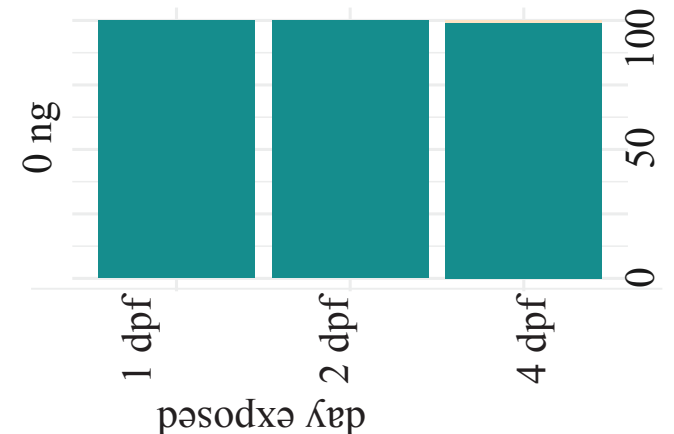

4
$\frac{n}{7}$

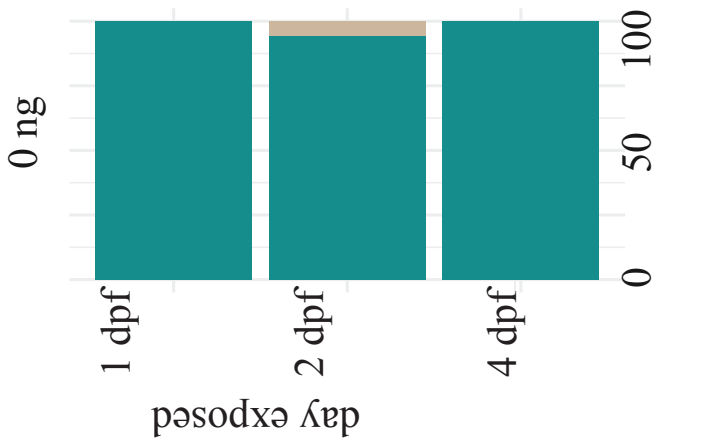



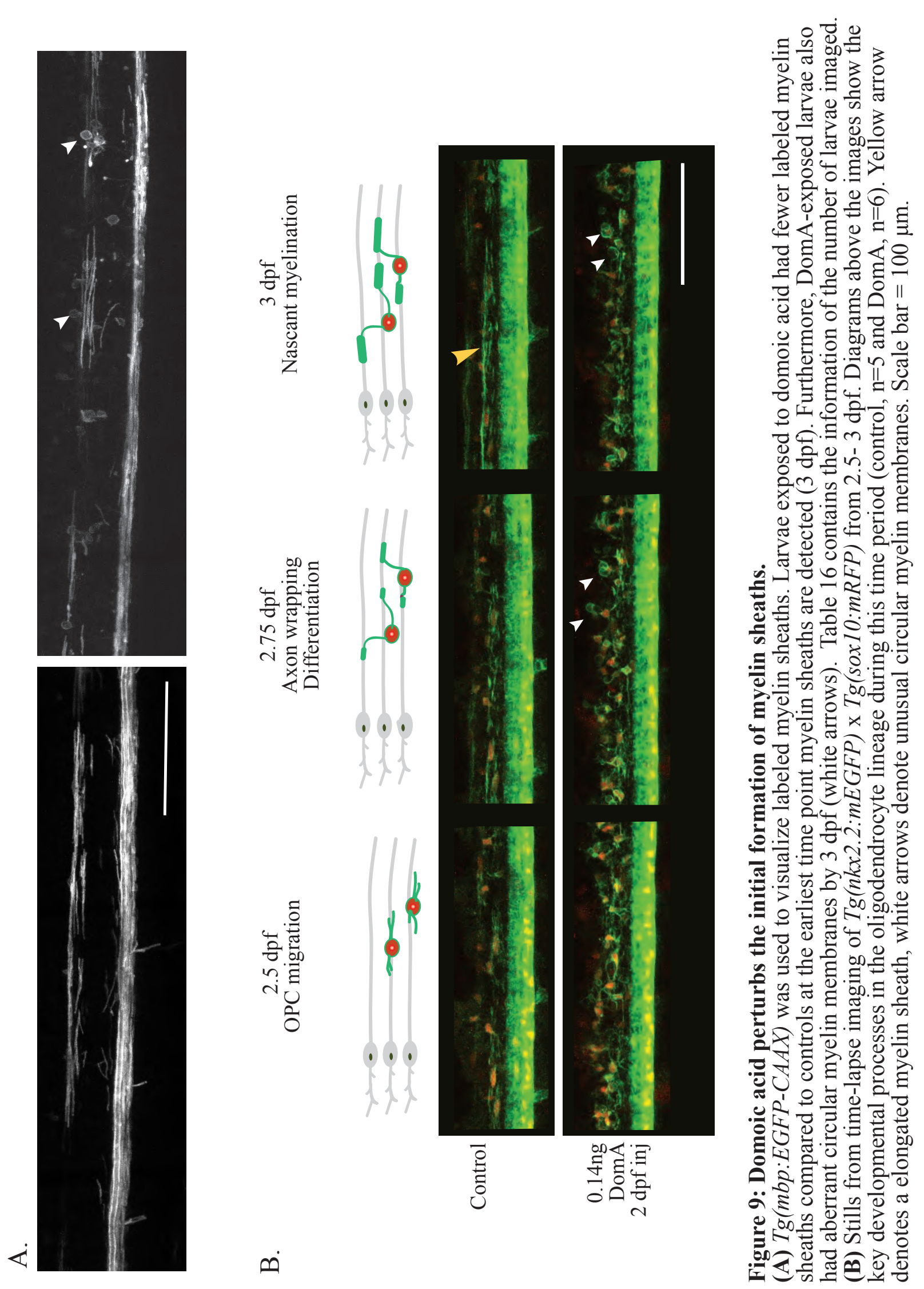
Figure 10: Domoic acid exposures at 2 dpf lead to modest transcriptional changes

(A) Experimental design. Tanks of 3 (2 females, 1 male) $\operatorname{Tg}(\mathrm{mbp}: E G F P-C A A X)$ were bred and exposed to DomA or vehicle (saline) at $2 \mathrm{dpf}$. Pools of 6 embryos within a given treatment from each tank were then sampled at $3 \mathrm{dpf}$ and $7 \mathrm{dpf}$ for RNAsequencing. For functional analyses, myelin sheath labeling was assessed at $5 \mathrm{dpf}$ (for the fish in the trial not sampled at $3 \mathrm{dpf}$ ), and startle response was assessed at $7 \mathrm{dpf}$ prior to RNAsequencing.

(B) MDS plot shows clustering of samples based on overall differences in expression profiles.

(C) Mean-difference (MD) plots to compare the log fold changes of genes in DomA exposed versus control fish at the $3 \mathrm{dpf}$ sampling point.

(D) Mean-difference (MD) plots to compare the log fold changes of genes in DomA exposed versus control fish at the $7 \mathrm{dpf}$ sampling point. 


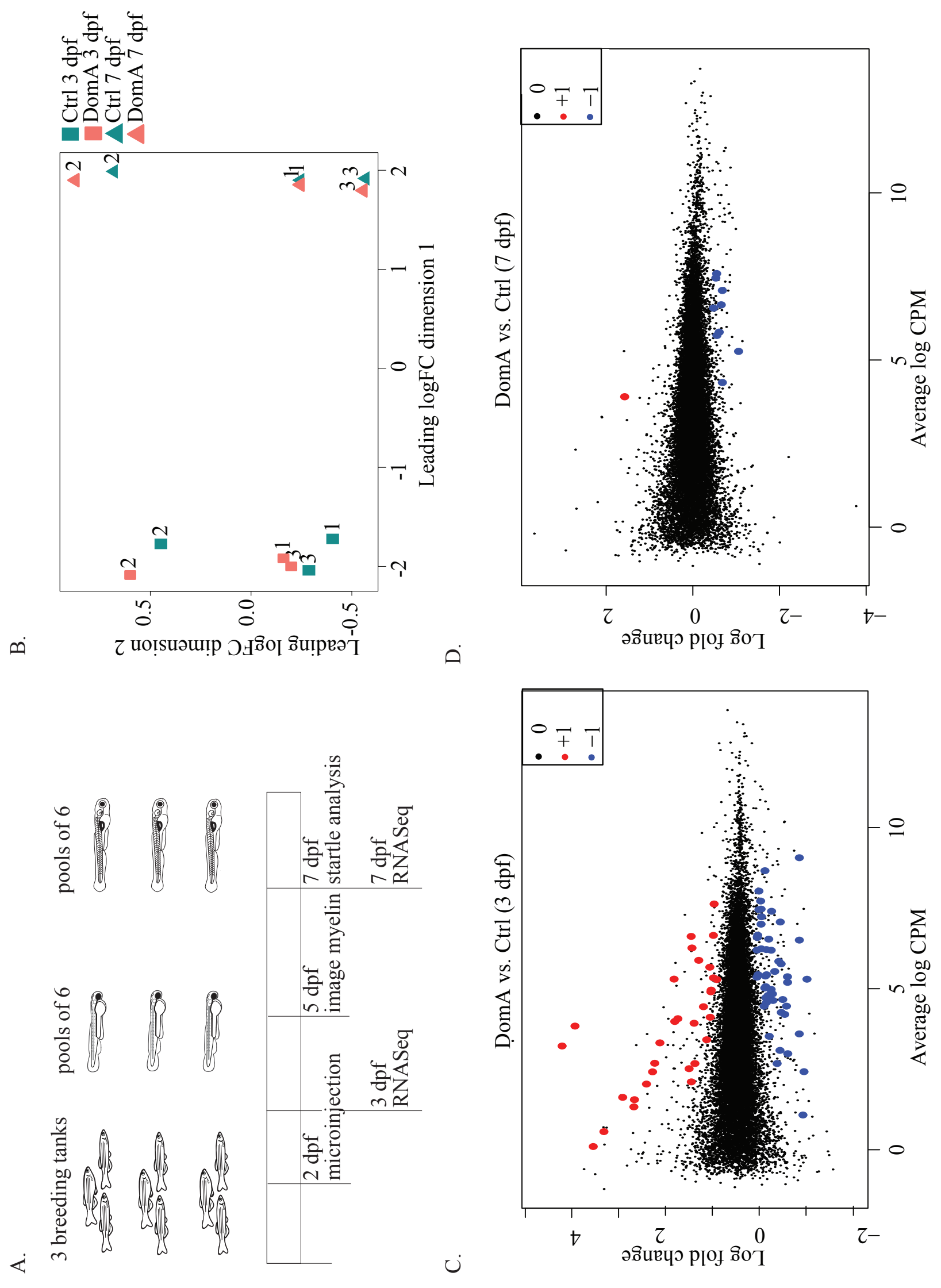


A.

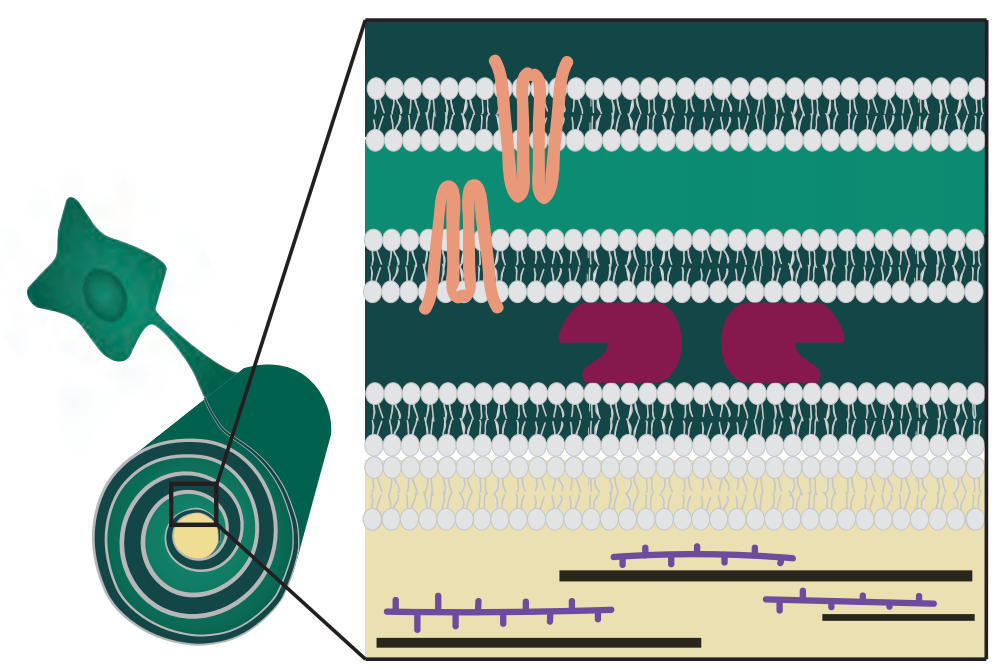

B.

Myelin structural proteins
\begin{tabular}{|c|c|}
\hline gene name & $\operatorname{logFC}$ \\
\hline mpz & -0.75 \\
\hline mbpa & $-\mathbf{0 . 5 1}$ \\
\hline
\end{tabular}

Axonal structural proteins

\begin{tabular}{|c|c|}
\hline gene name & $\operatorname{logFC}$ \\
\hline nefla & -1.33 \\
\hline neflb & -0.94 \\
\hline nefma & -0.80 \\
\hline nefmb & -1.08 \\
\hline
\end{tabular}

Figure 11: Domoic acid exposure at 2 dpf leads to reduced expression of key axonal and myelin structural proteins.

(A) Schematic of Axon-Myelin interface, with a focus on selected myelin and axon structural proteins that are differentially expressed in DomA exposed fish.

(B) A table with the myelin and structural proteins that are differentially expressed with the $\log$ fold change $(\log \mathrm{FC})$. (-) indicates that the gene was downregulated in DomA-exposed fish relative to controls. 


\section{CHAPTER 2 TABLES}

Table 1: Mortality in $T g(m b p: E G F P-C A A X)$ larvae exposed to DomA by intravenous injection

\begin{tabular}{|c|c|c|c|c|c|}
\hline \multirow{2}{*}{$\begin{array}{l}\text { Day injected } \\
1 \mathrm{dpf}\end{array}$} & \multirow{2}{*}{$\begin{array}{l}\text { Control } \\
\mathbf{0} \mathbf{~ n g} \\
0.4 \% \pm 0.4 \\
(\mathrm{n}=6)\end{array}$} & \multicolumn{4}{|c|}{ DomA (nominal dose) } \\
\hline & & $\begin{array}{l}\mathbf{0 . 0 9} \text { ng } \\
1.9 \% \pm 1.3 \\
(\mathrm{n}=3)\end{array}$ & $\begin{array}{l}\text { 0.13 ng } \\
3.1 \%\end{array}$ & $0.14 \mathrm{ng}$ & $\begin{array}{l}\text { 0.18 ng } \\
0.8 \% \pm 0.5 \\
(n=5)\end{array}$ \\
\hline $1.5 \mathrm{dpf}$ & $\begin{array}{l}0 \% \\
(n=1)\end{array}$ & & & & $\begin{array}{l}0 \% \\
(n=1)\end{array}$ \\
\hline $2 \mathrm{dpf}$ & $\begin{array}{l}1.2 \% \pm 0.7 \\
(n=13)\end{array}$ & $\begin{array}{l}0.4 \% \pm 0.4 \\
(n=3)\end{array}$ & $2.9 \%, 0 \%$ & $\begin{array}{l}0 \% \pm 0 \\
(n=6)\end{array}$ & $\begin{array}{l}6.2 \% \pm 3.9 \\
(n=6)\end{array}$ \\
\hline $2.5 \mathrm{dpf}$ & $\begin{array}{l}0 \% \\
(n=1)\end{array}$ & & & $\begin{array}{l}0 \% \\
(n=1)\end{array}$ & \\
\hline 3 dpf & $\begin{array}{l}0 \% \\
(n=1)\end{array}$ & & & $\begin{array}{l}0 \% \\
(n=1)\end{array}$ & \\
\hline $4 \mathrm{dpf}$ & $\begin{array}{l}0.2 \% \pm 0.2 \\
(n=8)\end{array}$ & $\begin{array}{l}0 \% \\
(n=3)\end{array}$ & $0 \%, 0 \%$ & $0 \%, 0 \%$ & $\begin{array}{l}0.9 \% \pm 0.6 \\
(n=4)\end{array}$ \\
\hline
\end{tabular}

Note: Mortality was recorded at 5 days post fertilization (dpf). $\mathrm{n}=$ number of repeated trials \pm standard error (SE) of the mean when there are more than two repeated experiments. Otherwise, raw percentage is listed per trial 
Table 2: Mortality in $\operatorname{Tg}(\operatorname{sox} 10: m R F P)$ larvae exposed to DomA by intravenous injection

\begin{tabular}{|c|c|c|}
\hline \multirow[b]{2}{*}{$\begin{array}{l}\text { Day } \\
\text { injected }\end{array}$} & \multirow{2}{*}{$\begin{array}{l}\text { Control } \\
0 \mathrm{ng} \\
\end{array}$} & DomA (nominal dose) \\
\hline & & $0.14 \mathrm{ng}$ \\
\hline $2 \mathrm{dpf}$ & $0 \%(\mathrm{n}=1)$ & $\begin{array}{l}0 \% \\
(n=1)\end{array}$ \\
\hline $2.25 \mathrm{dpf}$ & $0 \%(\mathrm{n}=1)$ & $\begin{array}{l}0 \% \\
(\mathrm{n}=1)\end{array}$ \\
\hline $2.50 \mathrm{dpf}$ & $0 \%(\mathrm{n}=1)$ & $\begin{array}{l}6.3 \% \\
(n=1)\end{array}$ \\
\hline $2.5 \mathrm{dpf}$ & $0 \%(\mathrm{n}=1)$ & $\begin{array}{l}53.2 \% \\
(\mathrm{n}=1)\end{array}$ \\
\hline $2.75 \mathrm{dpf}$ & $0 \%(\mathrm{n}=1)$ & $\begin{array}{l}42.1 \% \\
(n=1)\end{array}$ \\
\hline $3 \mathrm{dpf}$ & $(0 \%, 0 \%)$ & $\begin{array}{l}16.0 \% \pm 2.9 \\
(\mathrm{n}=3)\end{array}$ \\
\hline $4 \mathrm{dpf}$ & $0 \%(\mathrm{n}=1)$ & $\begin{array}{l}0 \% \\
(n=1)\end{array}$ \\
\hline
\end{tabular}

Note: Mortality was recorded at 5 days post fertilization (dpf). $\mathrm{n}=$ number of repeated trials \pm standard error (SE) of the mean when there are more than two repeated experiments. Otherwise, raw percentage is listed per trial 
Table 3: Trials included for the swim bladder analysis

\begin{tabular}{|c|c|c|c|c|c|}
\hline \multirow{5}{*}{ 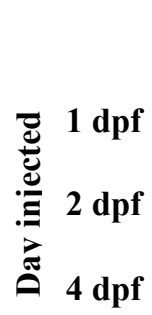 } & \multirow{2}{*}{$\begin{array}{l}\text { Control } \\
\mathbf{0} \text { ng }\end{array}$} & \multicolumn{4}{|c|}{ DomA (nominal dose) } \\
\hline & & $0.09 \mathrm{ng}$ & $0.13 \mathrm{ng}$ & $0.14 \mathrm{ng}$ & $0.18 \mathrm{ng}$ \\
\hline & $\mathrm{n}=20$ & - & - & $\mathrm{n}=24$ & - \\
\hline & $\begin{array}{l}\mathrm{n}=4,25,8,26, \\
24,47\end{array}$ & - & $\mathrm{n}=9$ & $\begin{array}{l}\mathrm{n}=38,12,7,26,27 \\
40\end{array}$ & $\mathrm{n}=10$ \\
\hline & $\mathrm{n}=25,10$ & $\mathrm{n}=19,9$ & - & $\mathrm{n}=25$ & $\mathrm{n}=12,10$ \\
\hline
\end{tabular}

Note: $\mathrm{n}$ corresponds to the number of fish within a trial that were exposed to a given dose of DomA administered at the specified developmental time period. Fish were imaged from 5- $7 \mathrm{dpf}$. Fish injected at $4 \mathrm{dpf}$ with $0.18 \mathrm{ng}$ DomA that also had 'opaque brains' were excluded from this analysis (See Table 4 for opaque brain phenotype breakdown). 
Table 3: Opaque brains in $T g(m b p: E G F P-C A A X)$ larvae exposed to DomA by intravenous injection

\begin{tabular}{|c|c|c|c|c|c|}
\hline \multirow[t]{2}{*}{ Day injected } & \multirow{2}{*}{$\begin{array}{l}\text { Control } \\
\mathbf{0} \mathrm{ng} \\
\end{array}$} & \multicolumn{4}{|c|}{ DomA (nominal dose injected) } \\
\hline & & $0.09 \mathrm{ng}$ & 0.13 & 0.14 & $0.18 \mathrm{ng}$ \\
\hline $1 \mathrm{dpf}$ & $0 \% \pm 0(\mathrm{n}=6)$ & $0 \% \pm 0(n=3)$ & $0 \%(n=1)$ & & $0 \%(n=5)$ \\
\hline $1.5 \mathrm{dpf}$ & $(0 \%, 0 \%)$ & & & & $0 \%$ \\
\hline $2 \mathrm{dpf}$ & $0 \% \pm 0(\mathrm{n}=13)$ & $0 \% \pm 0(n=3)$ & $0 \%, 0 \%$ & $0 \% \pm 0(\mathrm{n}=6)$ & $0 \% \pm 0(n=6)$ \\
\hline $2.5 \mathrm{dpf}$ & $(0 \%, 0 \%)$ & & & $0 \%(n=1)$ & \\
\hline $3 \mathrm{dpf}$ & $0 \%(n=1)$ & & & $0 \%(n=1)$ & \\
\hline $4 \mathrm{dpf}$ & $0 \% \pm 0(n=8)$ & $0.5 \% \pm 0.5(n=3)$ & $0 \%, 2.2 \%$ & $(0 \%, 5 \%)$ & $40.6 \% \pm 9.5(n=4)$ \\
\hline
\end{tabular}

Note: Percent of the population with opaque brains were recorded at 5 days post fertilization (dpf) $\pm=$ the standard error of the mean. $\mathrm{n}=$ number of trials included. 
Table 5: Acute neurological phenotypes following developmental exposures to DomA at 1 dpf

\begin{tabular}{|c|c|c|c|c|}
\hline $\begin{array}{l}\text { Dose } \\
\text { (ng) }\end{array}$ & Trials & Day observed & $\begin{array}{l}\text { Convulsions mean } \\
\% \pm \mathrm{SE} \text { or } \\
(\mathrm{mean} \% \text {, mean } \%)\end{array}$ & $\begin{array}{l}\text { No touch response } \\
\text { mean } \% \pm \text { SE or } \\
(\text { mean } \% \text {, mean } \%)\end{array}$ \\
\hline \multirow{4}{*}{0} & \multirow{4}{*}{$\mathrm{n}=5$} & $2 \mathrm{dpf}$ & 0 & $0.4 \pm 0.4$ \\
\hline & & $3 \mathrm{dpf}$ & 0 & $0.2 \pm 0.2$ \\
\hline & & $4 \mathrm{dpf}$ & 0 & 0 \\
\hline & & $5 \mathrm{dpf}$ & 0 & 0 \\
\hline \multirow{4}{*}{0.09} & \multirow{4}{*}{$\mathrm{n}=2$} & $2 \mathrm{dpf}$ & $(2.9,0)$ & $(32.4,15.9)$ \\
\hline & & $3 \mathrm{dpf}$ & $(0,0)$ & $(0,0)$ \\
\hline & & $4 \mathrm{dpf}$ & $(0,0)$ & $(0,0)$ \\
\hline & & $5 \mathrm{dpf}$ & $(0,0)$ & $(0,0)$ \\
\hline \multirow{4}{*}{0.18} & \multirow{4}{*}{$\mathrm{n}=4$} & $2 \mathrm{dpf}$ & $8.0 \pm 3.0$ & $53.4 \pm 12.9$ \\
\hline & & $3 \mathrm{dpf}$ & $2.0 \pm 1.6$ & $17.3 \pm 9.7$ \\
\hline & & $4 \mathrm{dpf}$ & $0.8 \pm 0.8$ & $0.4 \pm 0.4$ \\
\hline & & $5 \mathrm{dpf}$ & 0 & 0 \\
\hline
\end{tabular}

Note: Percent of fish exhibiting convulsions or no touch responses tracked daily post exposure until $5 \mathrm{dpf}$ (2-5 dpf). $\mathrm{n}=$ number of repeated trials $\pm \mathrm{SE}$ of the mean when there are greater than two repeated experiments. Otherwise, raw percentage is listed per trial. Within an individual treatment group, the minimum number of fish was 31 , and the maximum number was 92 . 
Table 6: Acute neurological phenotypes following developmental exposures to DomA at $2 \mathrm{dpf}$

\begin{tabular}{|c|c|c|c|c|}
\hline Dose (ng) & Trials & $\begin{array}{l}\text { Day } \\
\text { observed }\end{array}$ & $\begin{array}{l}\text { Convulsion } \\
\text { mean } \pm \text { SE or } \\
\text { (mean, mean) }\end{array}$ & $\begin{array}{l}\text { No touch response } \\
\text { mean } \pm \mathrm{SE} \text { or } \\
\text { (mean, mean) }\end{array}$ \\
\hline \multirow{3}{*}{0} & \multirow{3}{*}{$\mathrm{n}=11$} & $3 \mathrm{dpf}$ & 0 & $0.2 \pm 0.2$ \\
\hline & & $4 \mathrm{dpf}$ & 0 & $0.4 \pm 0.3$ \\
\hline & & $5 \mathrm{dpf}$ & 0 & $0.1 \pm 0.1$ \\
\hline \multirow{3}{*}{0.09} & \multirow{3}{*}{$\mathrm{n}=2$} & $3 \mathrm{dpf}$ & $(2.4,0)$ & $(16.9,11.7)$ \\
\hline & & $4 \mathrm{dpf}$ & $(0,0)$ & $(0,1.3)$ \\
\hline & & $5 \mathrm{dpf}$ & $(0,0)$ & $(0,0)$ \\
\hline \multirow{3}{*}{0.14} & \multirow{3}{*}{$\mathrm{n}=6$} & $3 \mathrm{dpf}$ & $23.7 \pm 11.6$ & $38.8 \pm 10.3$ \\
\hline & & $4 \mathrm{dpf}$ & 0 & $1.5 \pm 1.5$ \\
\hline & & $5 \mathrm{dpf}$ & 0 & $0.6 \pm 0.6$ \\
\hline \multirow{3}{*}{0.18} & \multirow{3}{*}{$\mathrm{n}=4$} & $3 \mathrm{dpf}$ & $40.0 \pm 7.4$ & $47.8 \pm 16.0$ \\
\hline & & $4 \mathrm{dpf}$ & $0.4 \pm 0.4$ & $5.6 \pm 3.6$ \\
\hline & & $5 \mathrm{dpf}$ & $1.4 \pm 1.4$ & $0.4 \pm 0.4$ \\
\hline
\end{tabular}

Note: Percent of fish exhibiting convulsions or no touch responses tracked over the course of development (3-5 dpf). $\mathrm{n}=$ number of repeated trials. $\pm \mathrm{SE}$ of the mean when there are more than 2 repeated experiments. Otherwise, raw percentage data is listed per trial. Within an individual treatment group, the minimum number of fish was 20 , and the maximum number was 96. 
Table 7: Acute neurological phenotypes following developmental exposures to DomA at 4 dpf

\begin{tabular}{llll}
\hline Dose (ng) & Trials & $\begin{array}{l}\text { Convulsions mean \% } \pm \\
\text { SE or (mean \%, mean\%) }\end{array}$ & $\begin{array}{l}\text { No touch response mean \% } \pm \\
\text { SE or (mean \%, mean\%) }\end{array}$ \\
\hline $0 \mathrm{ng}$ & $\mathrm{n}=8$ & $0 \pm 0$ & $0 \pm 0$ \\
\hline $0.09 \mathrm{ng}$ & $\mathrm{n}=3$ & $0 \pm 0$ & $0 \pm 0$ \\
\hline $0.14 \mathrm{ng}$ & $\mathrm{n}=2$ & $(0,0)$ & $(0,5.0)$ \\
\hline $0.18 \mathrm{ng}$ & $\mathrm{n}=4$ & $1.9 \pm 1.9$ & $24.1 \pm 15.3$ \\
\hline
\end{tabular}

Note: Percent of fish exhibiting convulsions or no touch responses assessed at $5 \mathrm{dpf}$. $=$ number of repeated trials \pm $\mathrm{SE}$ of the mean when there are greater than two repeated experiments. Otherwise, raw percentage is listed per trial. Within an individual treatment group, the minimum number of fish was 32, and the maximum number was 96. 
Table 8: Model coefficients for the prevalence of the lack of touch responses for fish exposed at $1 \mathrm{dpf}$ and 2 dpf

$\frac{\text { Exposure at } 1 \text { dpf }}{\text { Est Coef (Std. error) }}$

\begin{tabular}{c} 
Exposure at 2 dpf \\
\hline Est Coef (Std. error) \\
\hline$-4.2(0.4) * * *$ \\
\hline$+23.7(4.2) * * *$ \\
\hline$-2.9(0.6)^{* * *}$ \\
\hline$-4.9(0.6)^{* * *}$ \\
$N A$ \\
\hline ror $* *=\mathrm{p}<0.01 * * *=\mathrm{p}<0.0001$
\end{tabular}

\section{Intercept}

Dose

$-3.8(0.5)^{* * *}$

2 days post exposure

$+22.1(3.9)^{* * *}$

$-2.2(0.7)^{* * *}$

3 days post exposure

$-5.8(1.3)^{* *}$

$-46.3(0.5) * * *$

4 days post exposure

Note: Est Coef $=$ estimated coefficients, Std. error $=$ standard error $* *=p<0.01 * * *=p<0.0001$ 
Table 9: Model coefficients for the prevalence of the convulsions and pectoral fin flapping for fish exposed at $1 \mathrm{dpf}$ and $2 \mathrm{dpf}$

\begin{tabular}{|c|c|c|}
\hline \multirow[b]{3}{*}{ Intercept } & Exposure at $1 \mathrm{dpf}$ & Exposure at $2 \mathrm{dpf}$ \\
\hline & Est Coef (Std. error) & Est Coef (Std. error) \\
\hline & $-7.3(1.2) * * *$ & $-6.6(1.1) * * *$ \\
\hline Dose & $+26.3(6.8)^{* * *}$ & $+35.1(6.6)^{* * *}$ \\
\hline 2 days post exposure & $-1.2(0.8)$ & $-5.4(0.9)^{* * *}$ \\
\hline 3 days post exposure & $-3.0(0.9)^{* *}$ & $-4.3(0.7)^{* * *}$ \\
\hline 4 days post exposure & $-44.8(0.8)^{* * *}$ & $N A$ \\
\hline
\end{tabular}


Table 10: Post-hoc pairwise Dunnett comparisons following binomial modeling of percent responsiveness in startle behavior

\begin{tabular}{|c|c|c|c|c|c|c|}
\hline \multirow[b]{2}{*}{$\begin{array}{l}\text { Dose comparisons } \\
\text { (DomA - Control) }\end{array}$} & \multicolumn{2}{|c|}{ Exposures at $1 \mathrm{dpf}$} & \multicolumn{2}{|c|}{ Exposures at 2 dpf } & \multicolumn{2}{|c|}{ Exposures at $4 \mathrm{dpf}$} \\
\hline & $\begin{array}{c}\text { Coef (Std. } \\
\text { Er) }\end{array}$ & $\operatorname{Pr}(>|z|)$ & $\begin{array}{c}\text { Coef (Std. } \\
\text { Er) }\end{array}$ & $\operatorname{Pr}(>|z|)$ & $\begin{array}{c}\text { Coef (Std. } \\
\text { Er) }\end{array}$ & $\operatorname{Pr}(>|z|)$ \\
\hline $0.18 \mathrm{ng}-0 \mathrm{ng}==\mathbf{0}$ & $-0.8(0.1)$ & $<1$ e -4 & $-0.7(0.2)$ & $<1$ e -8 & $-0.9(0.1)$ & $<1$ e -4 \\
\hline $0.14 n g-0 \mathrm{ng}==0$ & $-0.5(0.1)$ & 0.001 & $-0.8(0.2)$ & $<1$ e -8 & $-0.2(0.1)$ & 0.75 \\
\hline $0.13 \mathrm{ng}-0 \mathrm{ng}==\mathbf{0}$ & $-0.8(0.2)$ & $<1$ e -4 & $-1.0(0.2)$ & $<1$ e -8 & $0.1(0.2)$ & 0.98 \\
\hline $0.09 n g-0 \mathrm{ng}==\mathbf{0}$ & $-0.1(0.1)$ & 0.988 & $-0.8(0.2)$ & $<1$ e -8 & $0.0(0.1)$ & 0.997 \\
\hline
\end{tabular}

Notes: Coef $=$ estimated coefficient, $\operatorname{Std}$. $\mathrm{Er}=$ standard error, $\operatorname{Pr}(>|z|)=\mathrm{p}$-value of the $\mathrm{z}$-statistic. Significant values are in red font. 


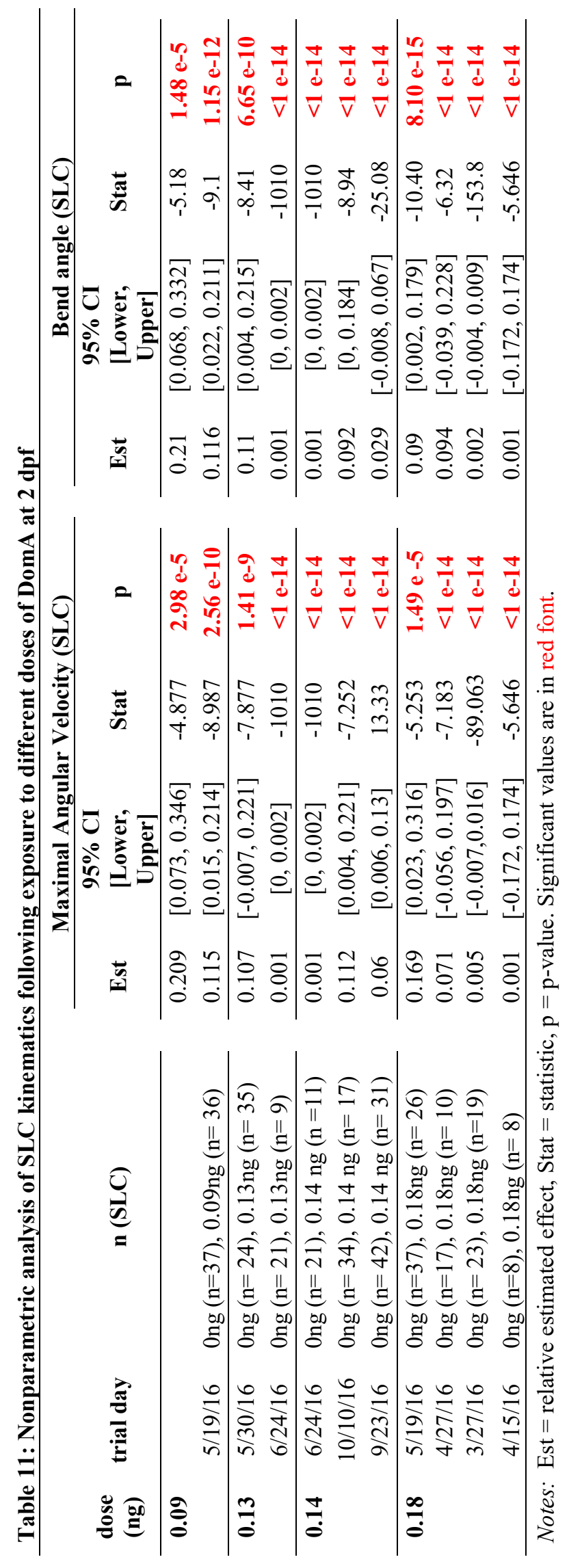




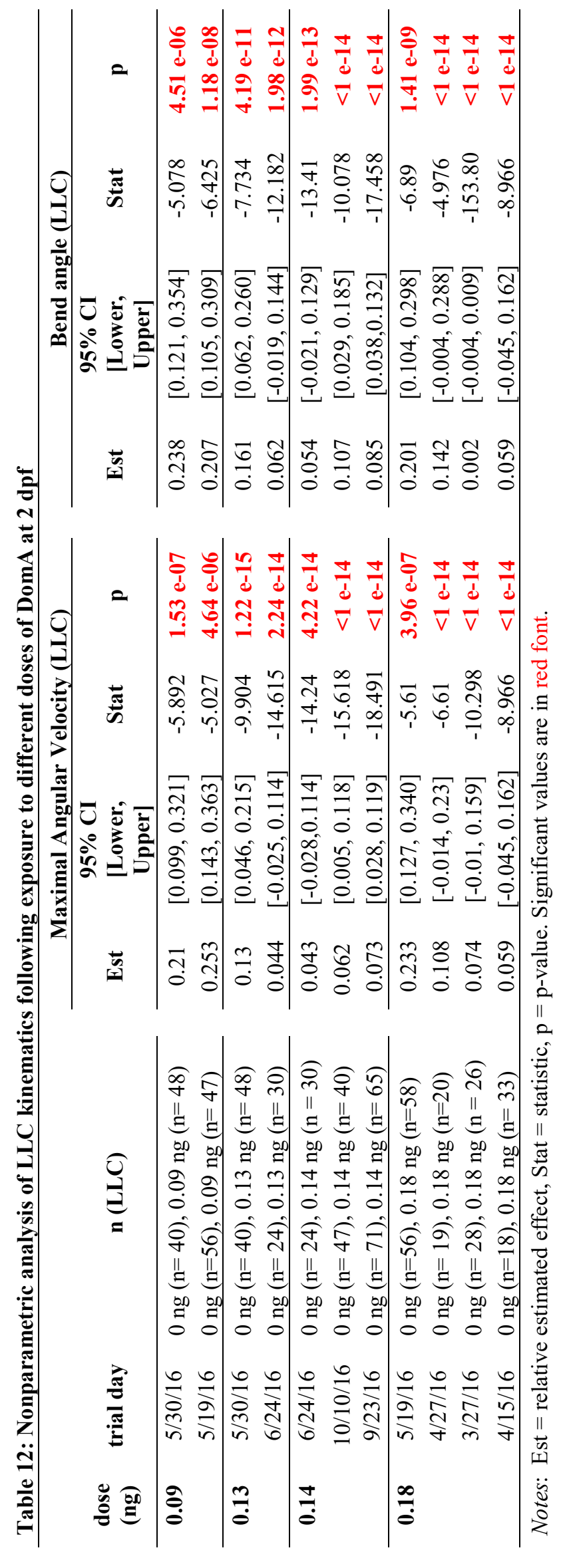




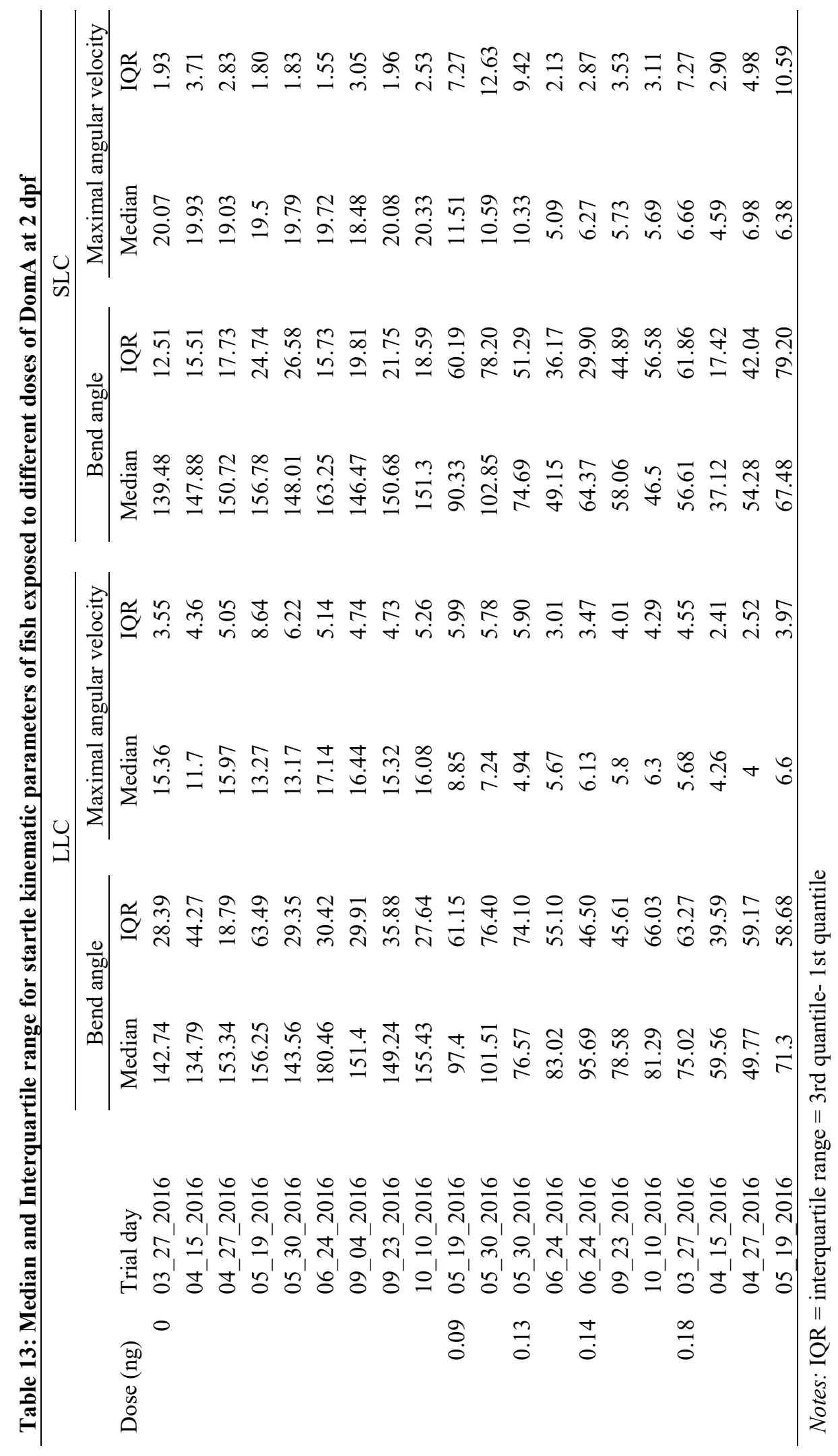




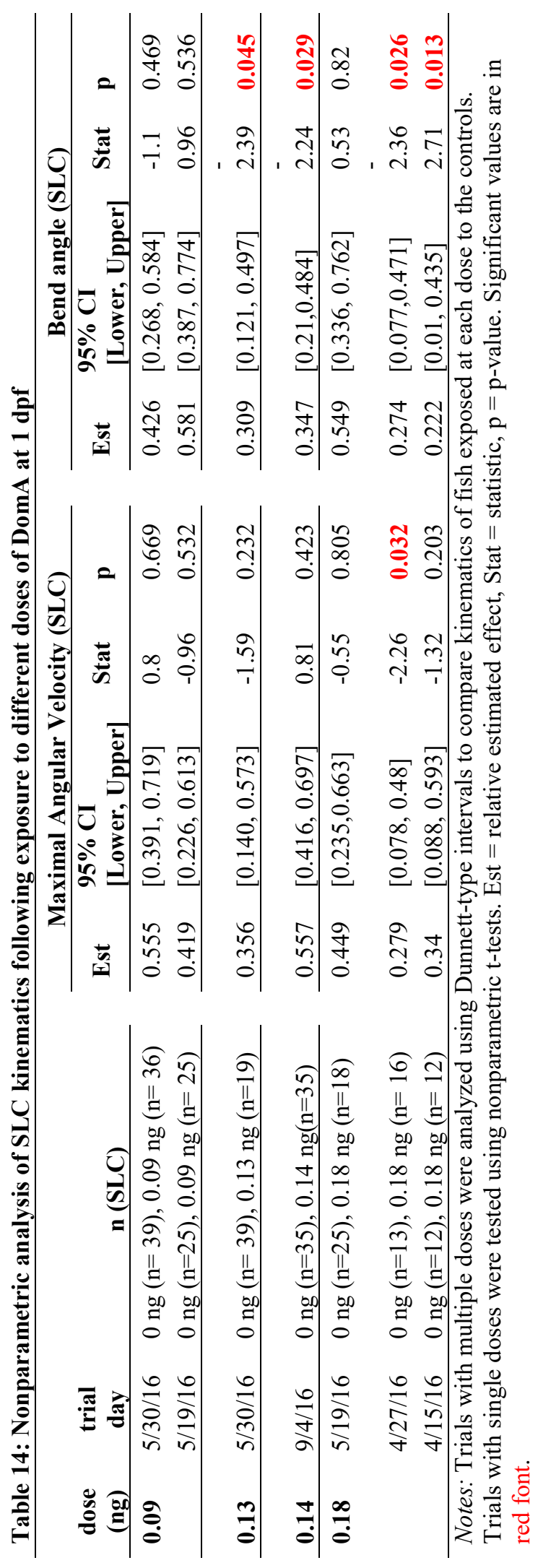




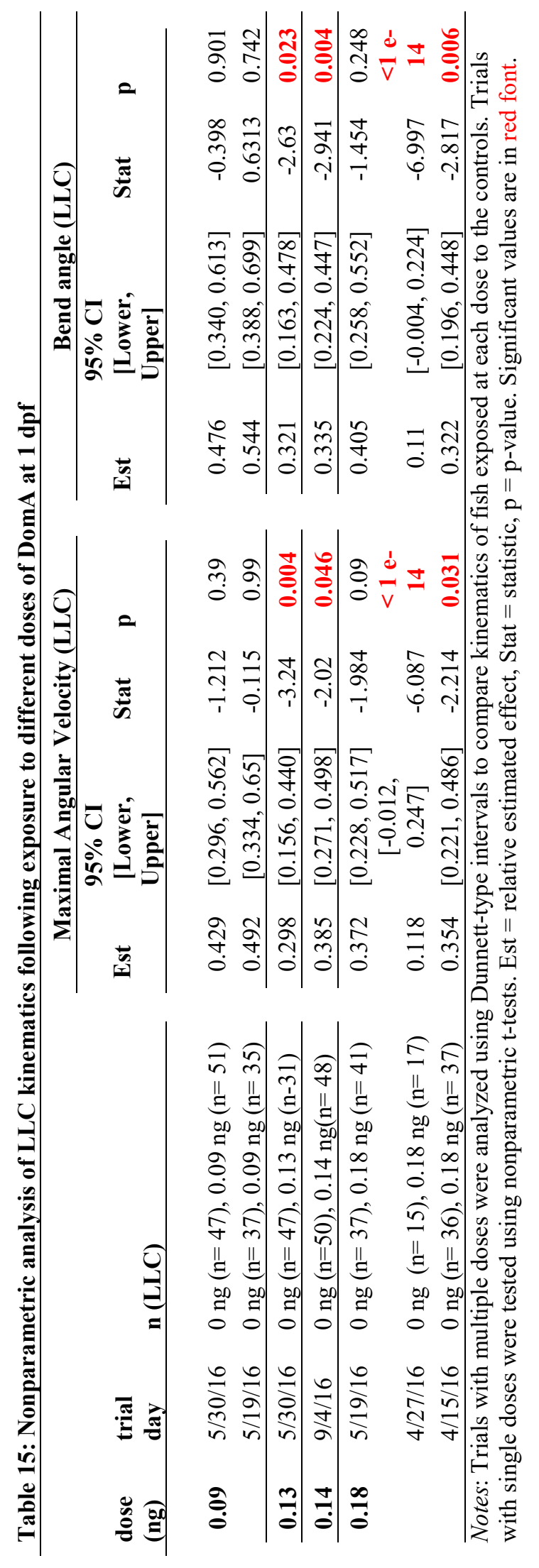




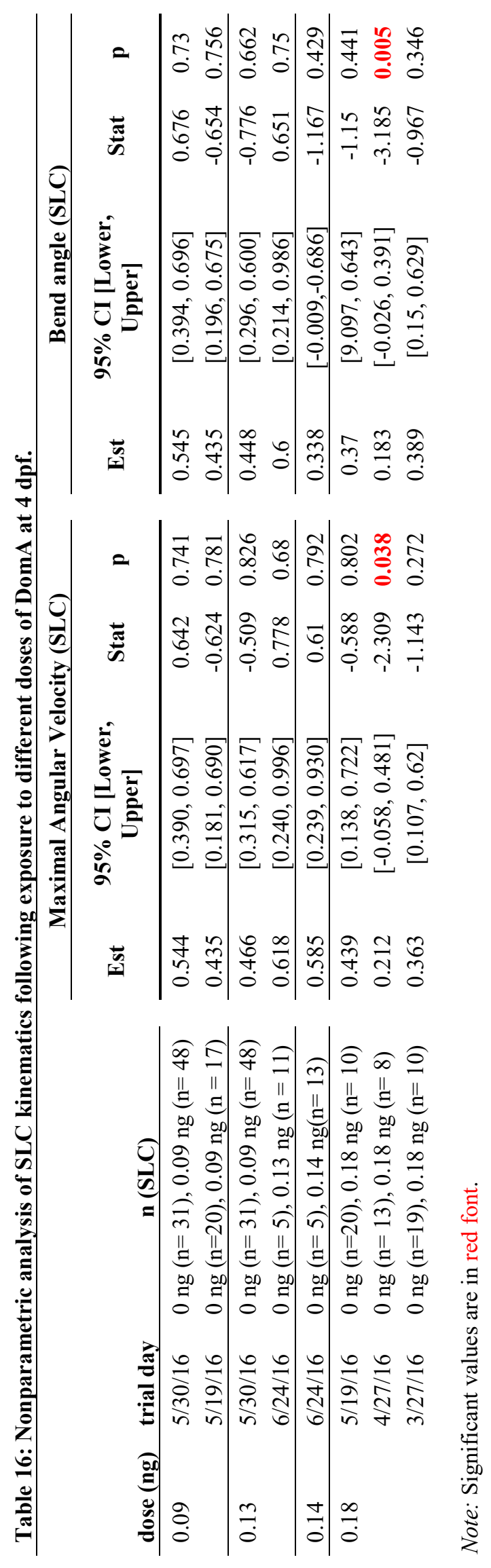




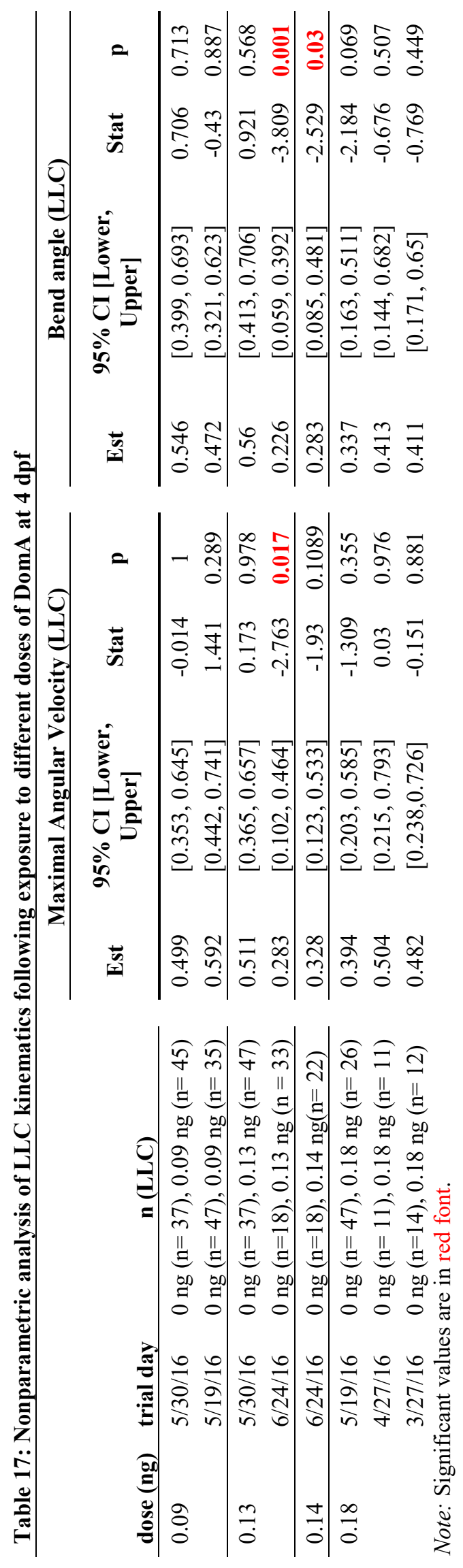


Table 18: Trials included to assess myelin labeling, imaged using confocal microscopy at 5 dpf

\begin{tabular}{|c|c|c|}
\hline & Control & DomA \\
\hline $1 \mathrm{dpf}$ & $\mathrm{n}=15,7$ & $n=25,6$ \\
\hline $1.5 \mathrm{dpf}$ & $\mathrm{n}=18,16$ & $n=23,11$ \\
\hline 2 dpf & $\mathrm{n}=7,8,24,22$ & $\mathrm{n}=31,32,36,7$ \\
\hline $2.5 \mathrm{dpf}$ & $\mathrm{n}=21,8$ & $\mathrm{n}=33,7$ \\
\hline $4 \mathrm{dpf}$ & $\mathrm{n}=6,10,24$ & $\mathrm{n}=14,24,8$ \\
\hline
\end{tabular}

Note: $\mathrm{n}$ corresponds to the number of fish within a trial that were exposed to DomA administered at the specified developmental time period (leftmost column). DomA- exposed larvae were given a nominal dose that ranged from 0.13- $0.14 \mathrm{ng}$. 


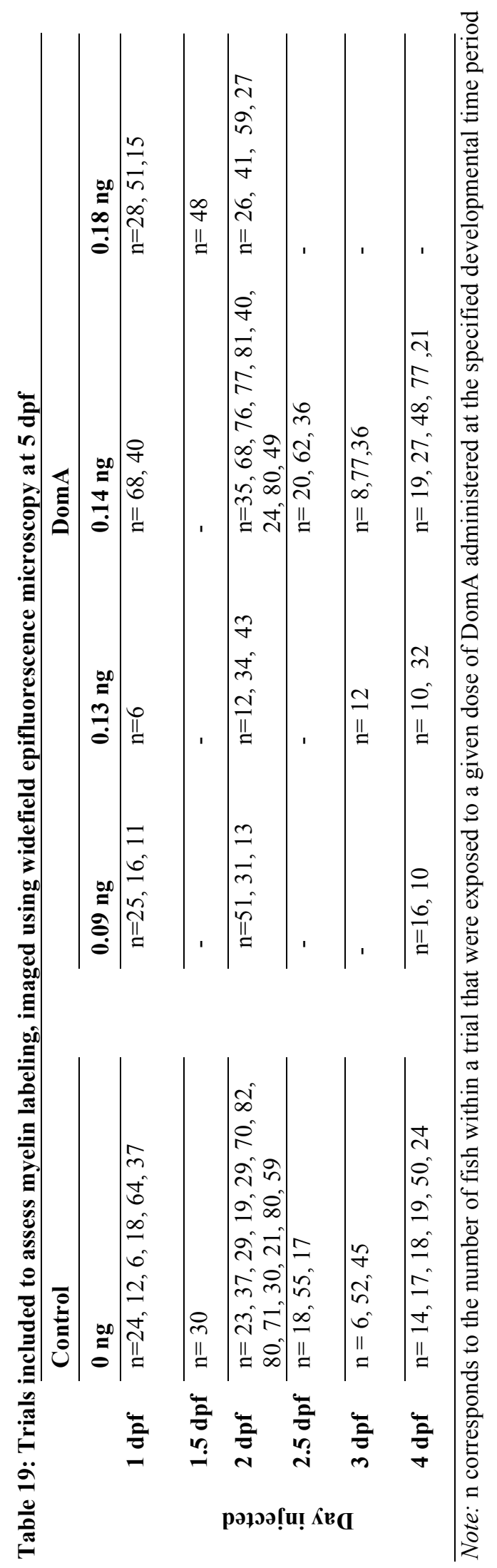


Table 20: Trials included to assess myelin labeling, imaged using widefield microscopy at 6 dpf

\begin{tabular}{|c|c|c|c|c|}
\hline \multirow{2}{*}{$\begin{array}{c}\text { Day } \\
\text { injected }\end{array}$} & \multirow{2}{*}{$\begin{array}{l}\text { Control } \\
\text { o ng }\end{array}$} & \multicolumn{3}{|c|}{ DomA } \\
\hline & & $0.09 \mathrm{ng}$ & $0.13 \mathrm{ng}$ & $0.18 \mathrm{ng}$ \\
\hline $1 \mathrm{dpf}$ & $\mathrm{n}=27,10,32$ & $\mathrm{n}=22,36$ & $\mathrm{n}=36$ & $\mathrm{n}=25,14$ \\
\hline $2 \mathrm{dpf}$ & $\mathrm{n}=22,15$ & $\mathrm{n}=52$ & $\mathrm{n}=41$ & - \\
\hline 4 dpf & $\mathrm{n}=31,24,34$ & $\mathrm{n}=35,13,41$ & $n=37$ & - \\
\hline
\end{tabular}

Note: $\mathrm{n}$ corresponds to the number of fish within a trial that were exposed to a given dose of DomA administered at the specified developmental time period. 
Table 21: Trials included to assess myelin labeling, imaged using widefield microscopy at $7 \mathrm{dpf}$

\begin{tabular}{|c|c|c|c|c|c|}
\hline \multirow{3}{*}{$\begin{array}{c}\text { Day } \\
\text { injected } \\
1 \text { dpf }\end{array}$} & \multirow{3}{*}{$\begin{array}{l}\text { Control } \\
\mathbf{0 ~ n g} \\
\mathrm{n}=23\end{array}$} & \multicolumn{4}{|c|}{ DomA } \\
\hline & & 0.09 ng & $0.13 \mathrm{ng}$ & $0.14 \mathrm{ng}$ & $0.18 \mathrm{ng}$ \\
\hline & & $\mathrm{n}=18$ & $\mathrm{n}=17$ & - & - \\
\hline $2 \mathrm{dpf}$ & $\mathrm{n}=14,10,2,28$ & $\mathrm{n}=18$ & $\mathrm{n}=19$ & $n=39$ & $\mathrm{n}=19,21$ \\
\hline $4 \mathrm{dpf}$ & $\mathrm{n}=21,16$ & $\mathrm{n}=19$ & $\mathrm{n}=20$ & $\mathrm{n}=25$ & - \\
\hline
\end{tabular}




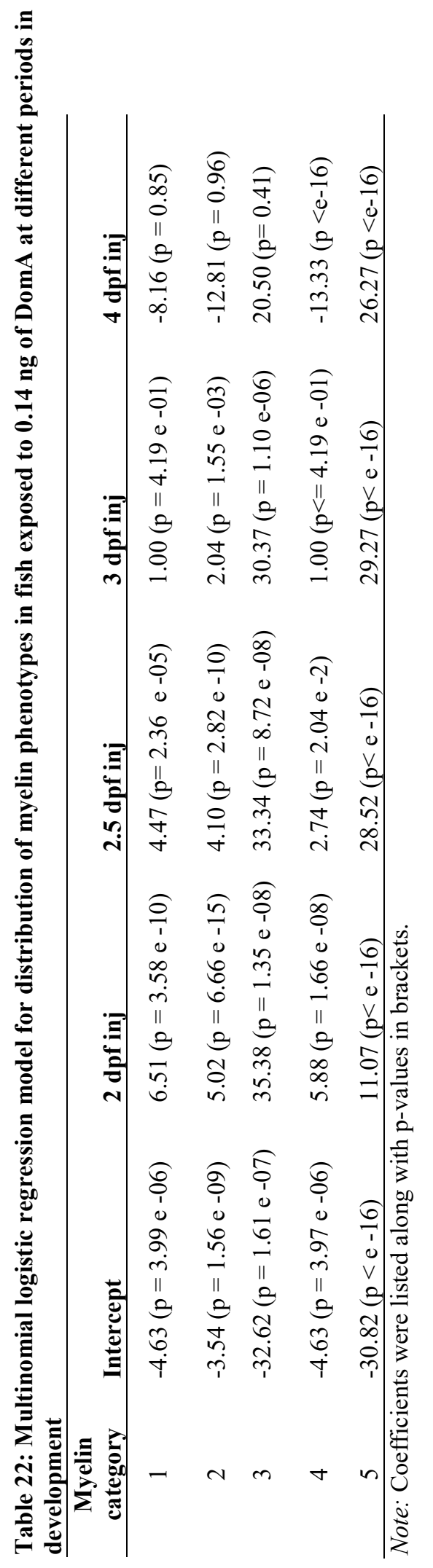




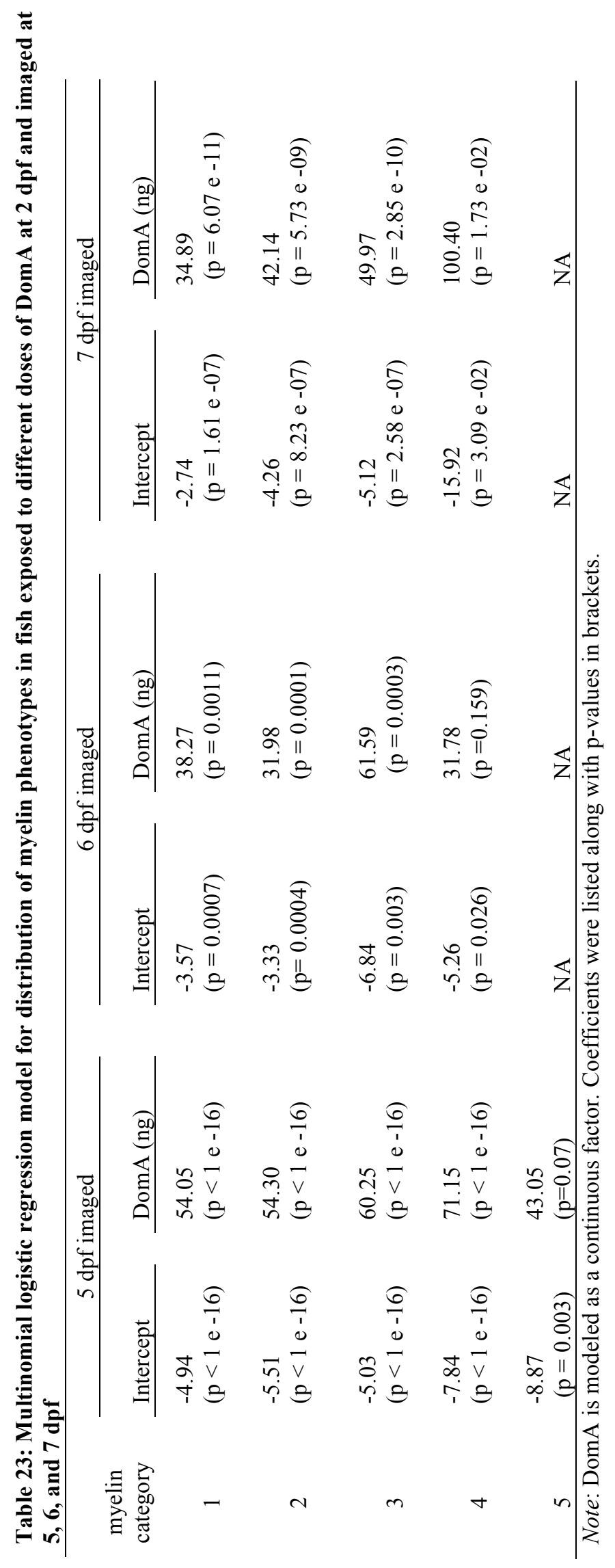


Table 24: Genes associated with the enriched GO term: biological processes

\begin{tabular}{lllllr}
\hline GO term & term ID & pvalue & ENSEMBL_gene & gene name & logFC \\
\hline $\begin{array}{l}\text { Protein } \\
\text { depolymerization }\end{array}$ & GO:0051261 & $3.49 \mathrm{E}-02$ & ENSDARG00000030106 & stmn4 & 0.5066 \\
\hline $\begin{array}{l}\text { Microtubule } \\
\text { depolymerization }\end{array}$ & GO:0007019 & $8.58 \mathrm{E}-03$ & ENSDARG00000038465 & stmn3 & -0.6122 \\
\hline
\end{tabular}




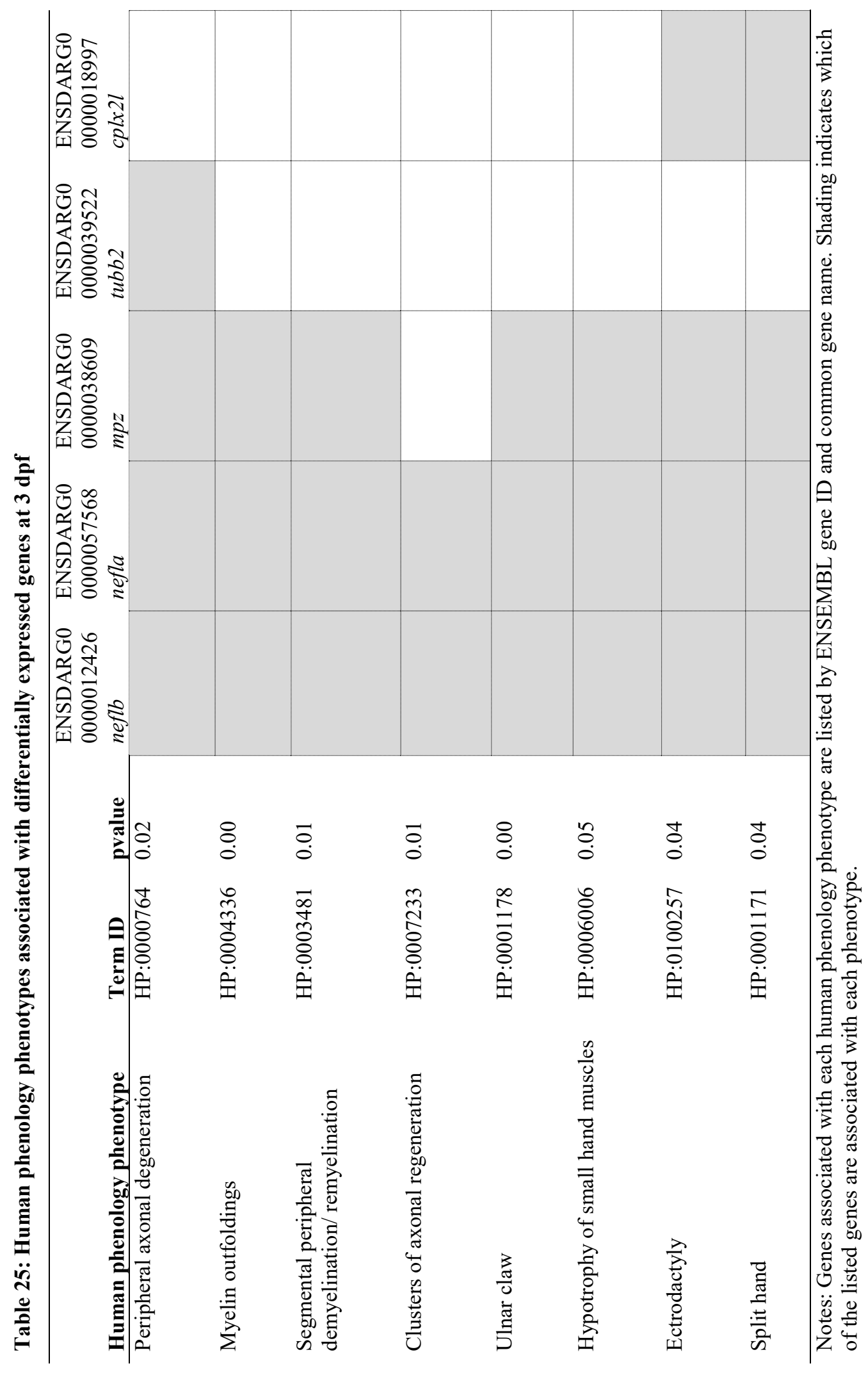




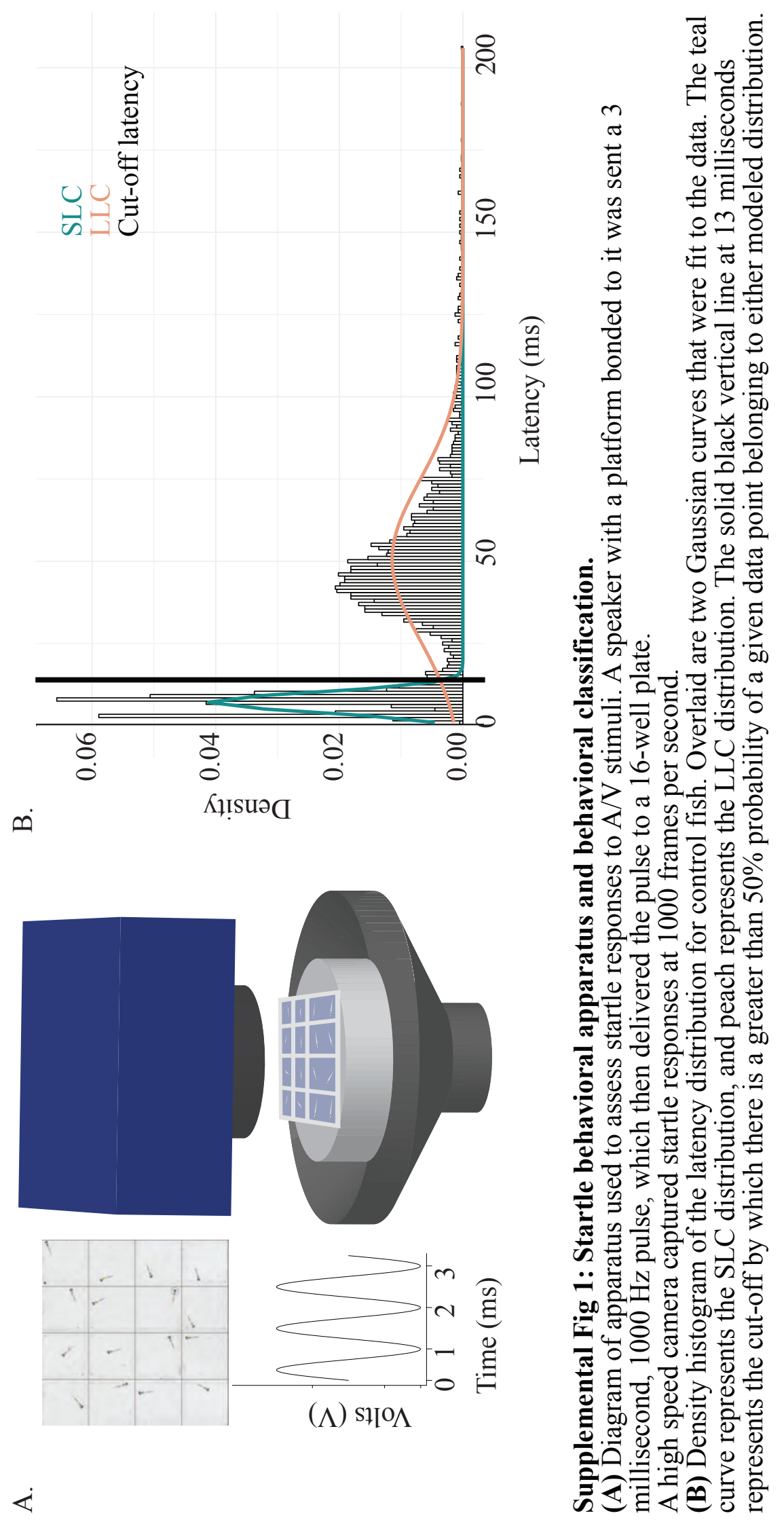



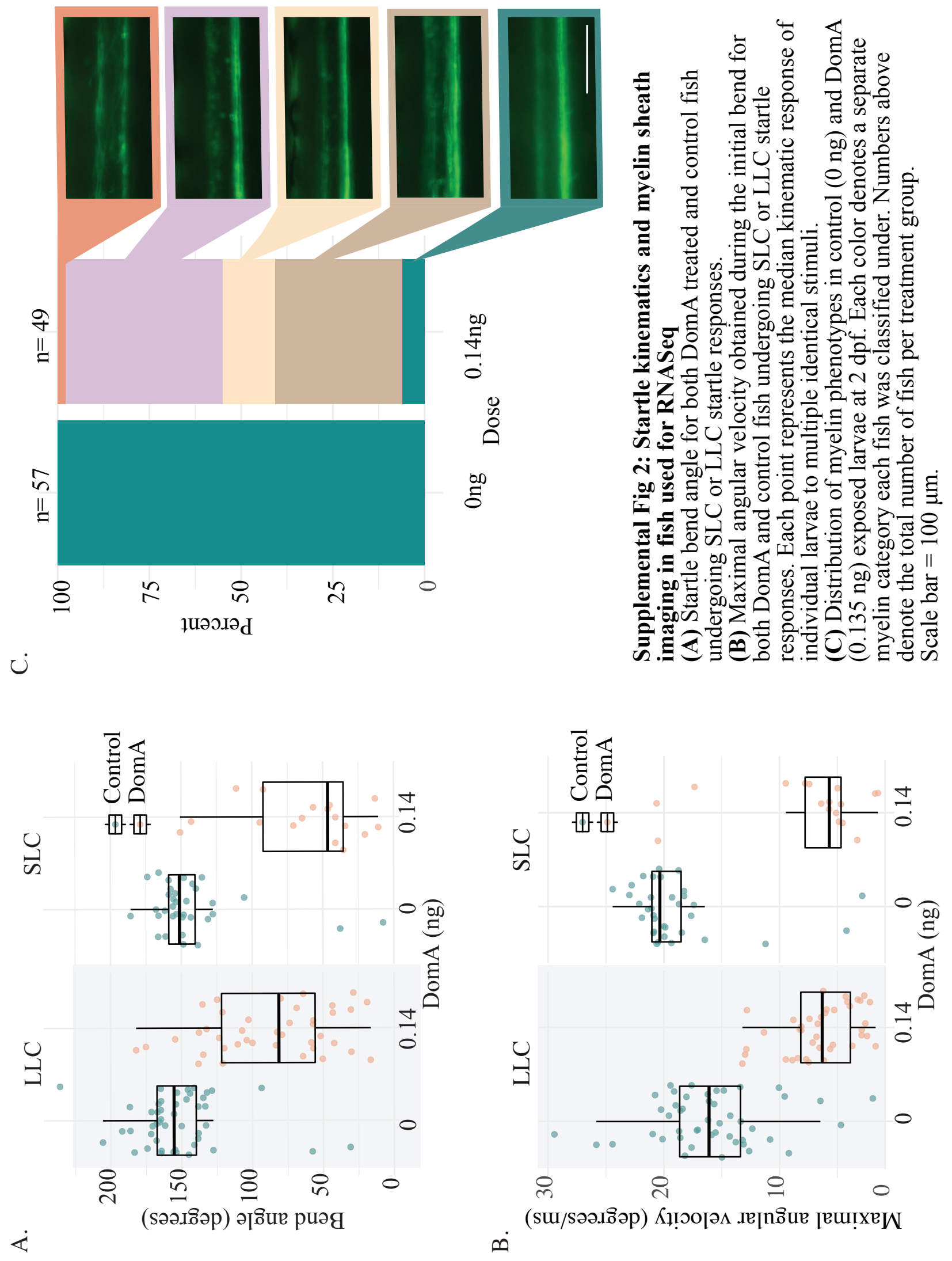


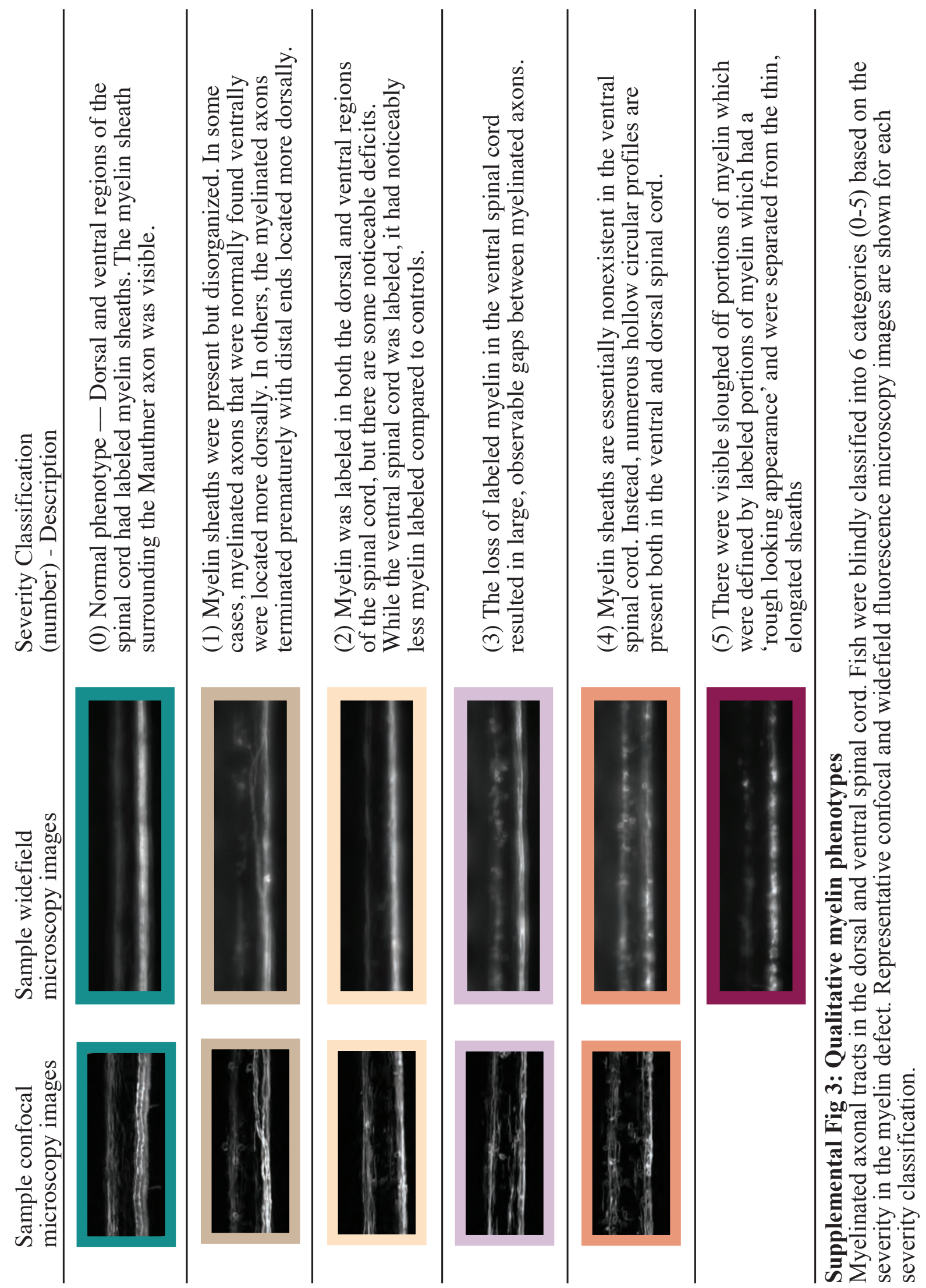




\section{CHAPTER 2 SUPPLEMENTAL TABLES}

Supplemental Table 1: Developmental time ranges in hours post fertilization (hpf) for fish included in each injection category

\begin{tabular}{|l|l|}
\hline Injection label & Time range (hpf) \\
\hline $1 \mathrm{dpf}$ & $28-32.5$ \\
\hline $2 \mathrm{dpf}$ & $47-52$ \\
\hline $4 \mathrm{dpf}$ & $100-105$ \\
\hline $1.5 \mathrm{dpf}$ & $37-39$ \\
\hline $2.25 \mathrm{dpf}$ & $54-55$ \\
\hline $2.50 \mathrm{dpf}$ & $59.5-60$ \\
\hline $2.5 \mathrm{dpf}$ & $62-64$ \\
\hline $2.75 \mathrm{dpf}$ & $66-67$ \\
\hline $3 \mathrm{dpf}$ & $71-74.5$ \\
\hline
\end{tabular}


Supplemental Table 2: Differentially expressed genes in DomA-exposed fish at $3 \mathrm{dpf}$ (28 hours post exposure)

\begin{tabular}{|c|c|c|c|c|c|c|}
\hline ENSEMBL_gene & Gene name & $\operatorname{logFC}$ & $\log \mathrm{CPM}$ & $\mathrm{F}$ & PValue & FDR \\
\hline ENSDARG00000031588 & si:dkey-239b22.1 & 3.460 & 3.901 & 255.669 & $1.47 \mathrm{E}-10$ & $3.08 \mathrm{E}-06$ \\
\hline ENSDARG00000077799 & egr4 & -1.491 & 5.356 & 157.207 & $1.53 \mathrm{E}-09$ & $1.59 \mathrm{E}-05$ \\
\hline ENSDARG00000028804 & ankrd9 & -1.074 & 5.434 & 129.297 & $6.06 \mathrm{E}-09$ & 4.22E-05 \\
\hline ENSDARG00000074311 & mast2 & 0.964 & 6.326 & 122.873 & 8.64E-09 & $4.51 \mathrm{E}-05$ \\
\hline ENSDARG00000097110 & si:dkey-56f14.4 & -0.973 & 4.725 & 115.655 & $1.32 \mathrm{E}-08$ & $5.49 \mathrm{E}-05$ \\
\hline ENSDARG00000027744 & gadd $45 b a$ & -0.921 & 7.133 & 97.579 & $4.21 \mathrm{E}-08$ & 0.000146422 \\
\hline ENSDARG00000012426 & neflb & -0.943 & 4.326 & 89.623 & $7.46 \mathrm{E}-08$ & 0.000222491 \\
\hline ENSDARG00000041140 & $d d b 2$ & -0.624 & 6.277 & 84.308 & $1.12 \mathrm{E}-07$ & 0.000292604 \\
\hline ENSDARG00000097528 & si:dkey-7j14.5 & -0.727 & 6.256 & 82.711 & $1.27 \mathrm{E}-07$ & 0.000295223 \\
\hline ENSDARG00000043697 & $n e f m b$ & -1.076 & 5.258 & 83.299 & $1.74 \mathrm{E}-07$ & 0.000362796 \\
\hline ENSDARG00000021351 & nefma & -0.803 & 5.597 & 77.039 & 2.03E-07 & 0.000385537 \\
\hline ENSDARG00000012281 & $z g c: 65851$ & -1.016 & 4.264 & 70.095 & $3.75 \mathrm{E}-07$ & 0.000629667 \\
\hline ENSDARG00000004187 & zgc: 122979 & -0.939 & 5.832 & 70.292 & $3.92 \mathrm{E}-07$ & 0.000629667 \\
\hline ENSDARG00000029795 & $\operatorname{fam} 213 b$ & -0.602 & 5.455 & 58.698 & $1.16 \mathrm{E}-06$ & 0.001724981 \\
\hline ENSDARG00000075891 & sall1b & 1.805 & 2.480 & 57.120 & $1.40 \mathrm{E}-06$ & 0.00194405 \\
\hline ENSDARG00000052917 & si:ch211-202f3.3 & 0.719 & 4.502 & 52.467 & $2.31 \mathrm{E}-06$ & 0.002836386 \\
\hline ENSDARG00000078567 & lonrf1l & -0.675 & 6.601 & 52.458 & $2.31 \mathrm{E}-06$ & 0.002836386 \\
\hline ENSDARG00000074390 & tmem176l.4 & 0.819 & 5.943 & 53.861 & $2.72 \mathrm{E}-06$ & 0.002836386 \\
\hline ENSDARG00000057568 & nefla & -1.326 & 3.659 & 52.984 & $2.76 \mathrm{E}-06$ & 0.002836386 \\
\hline ENSDARG00000102899 & cremb & 2.204 & 1.392 & 50.913 & 2.77E-06 & 0.002836386 \\
\hline ENSDARG00000094860 & gpr186 & -1.053 & 4.520 & 51.072 & $2.86 \mathrm{E}-06$ & 0.002836386 \\
\hline ENSDARG00000019498 & cry 5 & -0.718 & 5.029 & 50.294 & 2.99E-06 & 0.002836386 \\
\hline ENSDARG00000087303 & cebpd & -0.594 & 8.725 & 49.658 & $3.23 \mathrm{E}-06$ & 0.002929668 \\
\hline ENSDARG00000053475 & $\begin{array}{l}n g b \\
\text { si:ch211- }\end{array}$ & -0.590 & 5.084 & 48.579 & $3.68 \mathrm{E}-06$ & 0.003203961 \\
\hline ENSDARG00000104919 & $\begin{array}{l}153 b 23.3 \\
\text { No longer in }\end{array}$ & 2.442 & 1.688 & 48.068 & 4.90E-06 & 0.004090186 \\
\hline ENSDARG00000037910 & database & 0.583 & 5.730 & 45.645 & 5.34E-06 & 0.004285939 \\
\hline ENSDARG00000040135 & fosaa & -1.080 & 3.044 & 43.812 & $6.79 \mathrm{E}-06$ & 0.005252425 \\
\hline ENSDARG00000075048 & lonrf1 & -0.510 & 7.065 & 44.833 & $9.23 \mathrm{E}-06$ & 0.006667837 \\
\hline ENSDARG00000103199 & si:dkey-247k7.2 & 1.346 & 5.359 & 57.858 & $9.26 \mathrm{E}-06$ & 0.006667837 \\
\hline ENSDARG00000031702 & prkglb & -0.890 & 5.910 & 45.425 & $9.66 \mathrm{E}-06$ & 0.006726869 \\
\hline ENSDARG00000030106 & stmn 4 & 0.507 & 6.716 & 40.426 & $1.08 \mathrm{E}-05$ & 0.007291357 \\
\hline ENSDARG00000089429 & si:dkey-205h13.2 & -0.511 & 7.533 & 41.178 & $1.35 \mathrm{E}-05$ & 0.00840476 \\
\hline ENSDARG00000038639 & $\begin{array}{l}\text { elovl6l } \\
\text { No longer in }\end{array}$ & -0.725 & 5.040 & 38.875 & $1.35 \mathrm{E}-05$ & 0.00840476 \\
\hline ENSDARG00000074642 & database & 1.935 & 2.101 & 39.526 & $1.37 \mathrm{E}-05$ & 0.00840476 \\
\hline ENSDARG00000082789 & $\begin{array}{l}\text { NC_002333.18 } \\
\text { No longer in }\end{array}$ & -0.655 & 4.798 & 37.108 & $1.76 \mathrm{E}-05$ & 0.010498015 \\
\hline ENSDARG00000091715 & database & 1.265 & 4.131 & 38.860 & $2.15 \mathrm{E}-05$ & 0.012487411 \\
\hline ENSDARG00000005085 & ggctb & 0.505 & 5.399 & 35.011 & $2.43 \mathrm{E}-05$ & 0.013723445 \\
\hline ENSDARG00000039754 & $x p c$ & -0.624 & 4.628 & 34.711 & $2.55 \mathrm{E}-05$ & 0.014010615 \\
\hline
\end{tabular}




\begin{tabular}{|c|c|c|c|c|c|c|}
\hline ENSDARG00000069951 & eeflall2 & -0.609 & 5.125 & 33.215 & $3.24 \mathrm{E}-05$ & 0.017088134 \\
\hline ENSDARG00000069377 & si:dkey-242g16.2 & -0.766 & 4.696 & 33.161 & $3.27 \mathrm{E}-05$ & 0.017088134 \\
\hline ENSDARG00000036045 & penkb & 1.025 & 2.579 & 32.876 & $3.43 \mathrm{E}-05$ & 0.017282185 \\
\hline ENSDARG00000095294 & si:dkey-200l5.4 & -1.427 & 2.485 & 32.795 & $3.48 \mathrm{E}-05$ & 0.017282185 \\
\hline ENSDARG00000038465 & $\begin{array}{l}\text { stmn3 } \\
\text { si:ch211- }\end{array}$ & -0.612 & 4.781 & 32.440 & $3.69 \mathrm{E}-05$ & 0.017904601 \\
\hline ENSDARG00000068951 & $219 a 15.4$ & 2.843 & 0.624 & 33.394 & $3.81 \mathrm{E}-05$ & 0.018078068 \\
\hline ENSDARG00000053130 & $p c p 4 a$ & -0.661 & 4.852 & 31.938 & 4.01E-05 & 0.018606096 \\
\hline ENSDARG00000094990 & si:dkey-91f15.1 & 1.648 & 3.381 & 35.342 & $4.25 \mathrm{E}-05$ & 0.019295365 \\
\hline ENSDARG00000038359 & enosf1 & 0.560 & 4.965 & 30.600 & 5.04E-05 & 0.022385334 \\
\hline ENSDARG00000036186 & $m b p a$ & -0.513 & 6.301 & 30.383 & $5.23 \mathrm{E}-05$ & 0.022761232 \\
\hline ENSDARG00000007769 & sult5al & 2.191 & 1.616 & 33.786 & $5.35 \mathrm{E}-05$ & 0.022820274 \\
\hline ENSDARG00000005372 & camk4 & -0.575 & 4.518 & 34.802 & $5.63 \mathrm{E}-05$ & 0.023516933 \\
\hline ENSDARG00000017490 & cel.1 & -1.327 & 9.128 & 42.716 & $6.18 \mathrm{E}-05$ & 0.025296181 \\
\hline ENSDARG00000088711 & lgals1l1 & 1.331 & 4.044 & 36.251 & $6.49 \mathrm{E}-05$ & 0.026064889 \\
\hline ENSDARG00000023082 & $k r t 1-19 d$ & -0.520 & 7.295 & 28.834 & $6.88 \mathrm{E}-05$ & 0.027120305 \\
\hline ENSDARG00000034503 & per2 & -0.502 & 7.783 & 28.599 & $7.18 \mathrm{E}-05$ & 0.027774121 \\
\hline ENSDARG00000062788 & irgll & 1.759 & 2.747 & 33.320 & $7.73 \mathrm{E}-05$ & 0.029316922 \\
\hline ENSDARG00000100515 & duspl & -0.735 & 7.465 & 31.726 & $7.86 \mathrm{E}-05$ & 0.029316922 \\
\hline ENSDARG00000077982 & elf 3 & 0.918 & 3.989 & 29.039 & 8.00E-05 & 0.029316922 \\
\hline ENSDARG00000052779 & $\begin{array}{l}\text { zgc: } 153932 \\
\text { si:ch211- }\end{array}$ & 3.735 & 3.282 & 45.407 & 8.54E-05 & 0.029942295 \\
\hline ENSDARG00000039393 & $240 l 19.5$ & -0.628 & 5.495 & 27.647 & $8.55 \mathrm{E}-05$ & 0.029942295 \\
\hline ENSDARG00000023228 & vsnlla & -0.439 & 6.721 & 39.331 & $8.60 \mathrm{E}-05$ & 0.029942295 \\
\hline ENSDARG00000018542 & hapln4 & -1.405 & 1.140 & 27.273 & $9.17 \mathrm{E}-05$ & 0.031397972 \\
\hline ENSDARG00000018997 & cplx $2 l$ & -0.433 & 6.652 & 34.489 & $9.69 \mathrm{E}-05$ & 0.032634538 \\
\hline ENSDARG00000025301 & gfap & 0.491 & 7.693 & 26.790 & $1.00 \mathrm{E}-04$ & 0.033296277 \\
\hline ENSDARG00000090297 & ldlrad2 & 0.977 & 2.169 & 26.435 & $1.07 \mathrm{E}-04$ & 0.035067226 \\
\hline ENSDARG00000058992 & cers $2 b$ & 0.574 & 4.176 & 29.458 & $1.10 \mathrm{E}-04$ & 0.035237692 \\
\hline ENSDARG00000038609 & $\begin{array}{l}m p z \\
\text { si:ch211- }\end{array}$ & -0.754 & 4.901 & 27.377 & $1.14 \mathrm{E}-04$ & 0.036081227 \\
\hline ENSDARG00000058206 & $153 b 23.5$ & 0.554 & 5.018 & 25.854 & $1.20 \mathrm{E}-04$ & 0.037009979 \\
\hline ENSDARG00000002758 & dedd1 & -0.465 & 7.515 & 25.840 & $1.21 \mathrm{E}-04$ & 0.037009979 \\
\hline ENSDARG00000026726 & anxala & -0.465 & 8.092 & 25.727 & $1.23 \mathrm{E}-04$ & 0.037097551 \\
\hline ENSDARG00000003146 & $\begin{array}{l}\text { si:dkey-27m7.4 } \\
\text { KRT18 (1 of }\end{array}$ & -0.914 & 3.146 & 25.582 & $1.27 \mathrm{E}-04$ & 0.037097551 \\
\hline ENSDARG00000058332 & many) & -0.853 & 2.739 & 25.547 & $1.28 \mathrm{E}-04$ & 0.037097551 \\
\hline ENSDARG00000039522 & $t u b b 2$ & -0.424 & 5.420 & 30.844 & $1.28 \mathrm{E}-04$ & 0.037097551 \\
\hline ENSDARG00000098256 & si:ch73-329n5.6 & 3.071 & 0.161 & 25.221 & $1.36 \mathrm{E}-04$ & 0.038906766 \\
\hline ENSDARG00000045269 & $p t s$ & 0.649 & 3.477 & 25.212 & $1.43 \mathrm{E}-04$ & 0.040450174 \\
\hline ENSDARG00000004748 & zgc: 100868 & 0.981 & 6.686 & 32.601 & $1.48 \mathrm{E}-04$ & 0.041174301 \\
\hline ENSDARG00000016238 & arl6ip $5 b$ & -0.445 & 5.497 & 24.511 & $1.57 \mathrm{E}-04$ & 0.043044942 \\
\hline ENSDARG00000086705 & probl & 0.901 & 2.738 & 24.384 & $1.61 \mathrm{E}-04$ & 0.043586532 \\
\hline ENSDARG00000055752 & npas4a & -1.327 & 6.570 & 34.241 & $1.65 \mathrm{E}-04$ & 0.043786913 \\
\hline ENSDARG00000030980 & csrplb & -0.684 & 3.584 & 27.065 & $1.66 \mathrm{E}-04$ & 0.043786913 \\
\hline
\end{tabular}




\begin{tabular}{|c|c|c|c|c|c|c|}
\hline ENSDARG00000043932 & $\begin{array}{l}\text { stmn4l } \\
\text { si:ch211- }\end{array}$ & 0.433 & 5.347 & 28.293 & $1.78 \mathrm{E}-04$ & 0.045886342 \\
\hline ENSDARG00000070442 & $113 \mathrm{~g} 11.6$ & -0.424 & 6.260 & 33.027 & $1.78 \mathrm{E}-04$ & 0.045886342 \\
\hline ENSDARG00000039502 & eeflala & -0.430 & 6.668 & 37.536 & $1.84 \mathrm{E}-04$ & 0.046899431 \\
\hline
\end{tabular}

Note: $\log 2$ fold change reported $(\log F C)$ in which + indicates a gene that is up-regulated in DomA-exposed fish relative to controls, and - indicates a gene that is down-regulated in DomA-exposed fish relative to controls.

False discovery rate (FDR) applied for multiple testing correction using the Benjamini-Hochberg method. 


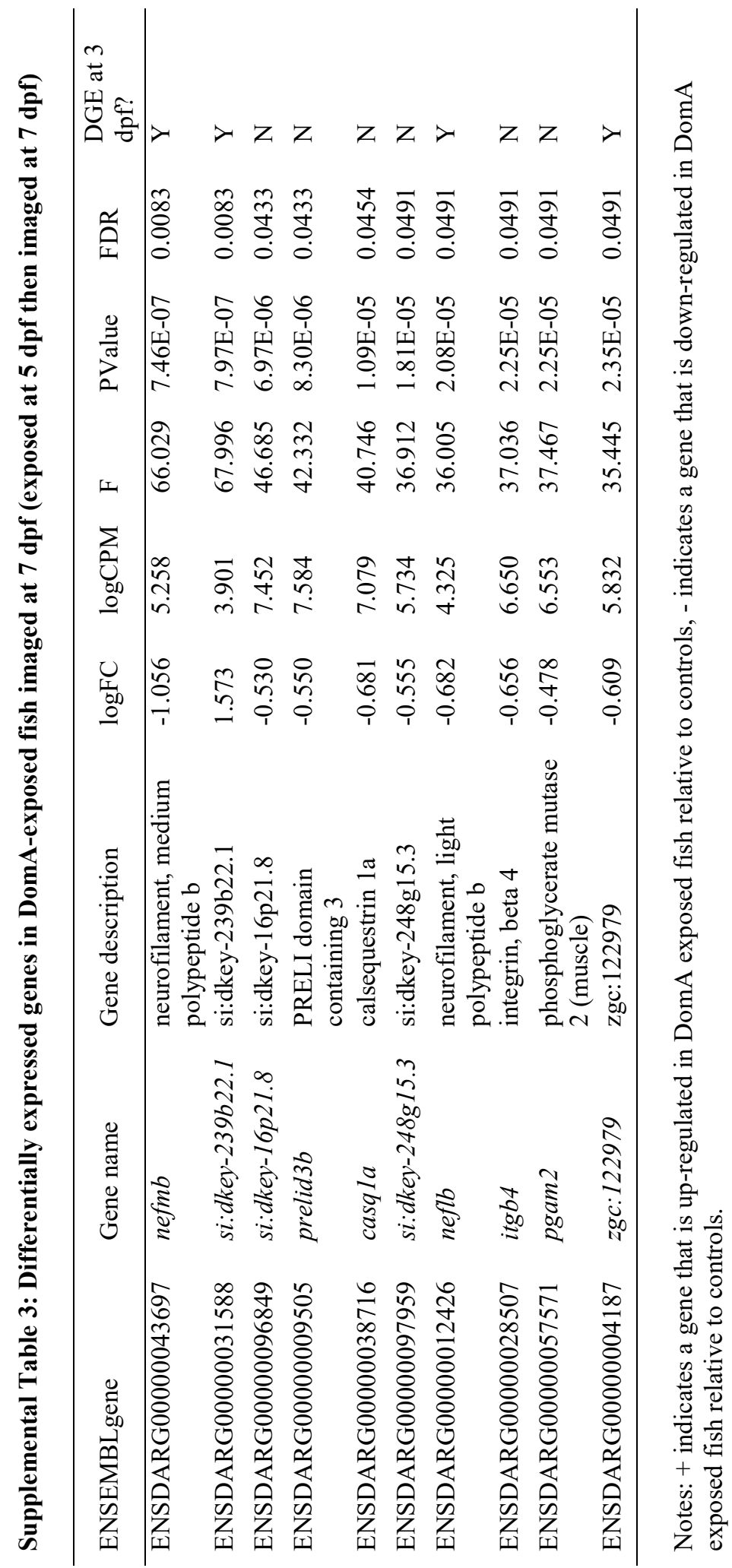




\subsection{REFERENCES}

1. Hampson, D. R., Huang, X., Wells, J. W., Walter, J. A. \& Wright, J. L. C. Interaction of domoic acid and several derivatives with kainic acid and AMPA binding sites in rat brain. Eur. J. Pharmacol. 218, 1-8 (1992).

2. Lefebvre, K. A. \& Robertson, A. Domoic acid and human exposure risks: a review. Toxicon 56, 218-30 (2010).

3. Perl, T. M. et al. An outbreak of toxic encephalopathy caused by eating mussels contaminated with domoic acid. N. Engl. J. Med. 322, 1775-80 (1990).

4. Jeffery, B., Barlow, T., Moizer, K., Paul, S. \& Boyle, C. Amnesic shellfish poison. Food Chem. Toxicol. 42, 545-57 (2004).

5. Wekell, J. C., Jurst, J. \& Lefebvre, K. a. The origin of the regulatory limits for PSP and ASP toxins in shellfish. J. Shellfish Res. 23, 927-930 (2010).

6. Mariën, K. Establishing tolerable dungeness crab (Cancer magister) and razor clam (Siliqua patula) domoic acid contaminant levels. Environ. Health Perspect. 104, 1230-6 (1996).

7. Grandjean, P. \& Landrigan, P. J. Neurobehavioural effects of developmental toxicity. Lancet Neurol. 13, 330-338 (2014).

8. Andersen, H. R., Nielsen, J. B. \& Grandjean, P. Toxicologic evidence of developmental neurotoxicity of environmental chemicals. Toxicology 144, 121-127 (2000).

9. Tryphonas, L., Truelove, J., Nera, E. \& Iverson, F. Acute Neurotoxicity of Domoic Acid in the Rat. Toxicol. Pathol. 18, 1-9 (1990).

10. Doucette, T. A. et al. Low doses of domoic acid during postnatal development produce permanent changes in rat behaviour and hippocampal morphology. Neurotox. Res. 6, 555563 (2004).

11. Xi, D., Peng, Y. G. \& Ramsdell, J. S. Domoic acid is a potent neurotoxin to neonatal rats. Nat. Toxins 5, 74-9 (1997).

12. Maucher, J. M. \& Ramsdell, J. S. Maternal-fetal transfer of domoic acid in rats at two gestational time points. Environ. Health Perspect. 115, 1743-6 (2007).

13. Ahrens, M. B. et al. Brain-wide neuronal dynamics during motor adaptation in zebrafish. Nature 485, 471-7 (2012).

14. Scholin, C. A. et al. Mortality of sea lions along the central California coast linked to a toxic diatom bloom. Nature 403, 80-84 (2000).

15. Brodie Frances M D Gulland Denise J Greig, E. C., Hunter, M., Jaakola, J., Leger, J. S. \& Leighfield Frances M Van Dolah, T. A. Domoic acid causes reproductive failure in california sea lions (Zalophus Californianus). Mar. Mammal Sci. 3, (22AD).

16. Lefebvre, K. A. et al. Domoic acid in California sea lion fetal fluids indicates continuous exposure to a neuroteratogen poses risks to mammals. Harmful Algae (2018). doi:10.1016/J.HAL.2018.06.003

17. Rust, L., Gulland, F., Frame, E. \& Lefebvre, K. Domoic acid in milk of free living California marine mammals indicates lactational exposure occurs. Mar. Mammal Sci. 30, 1272-1278 (2014).

18. Maucher, J. M. \& Ramsdell, J. S. Domoic acid transfer to milk: evaluation of a potential route of neonatal exposure. Environ. Health Perspect. 113, 461-4 (2005). 
19. Tanemura, K. et al. Intrauterine environment-genome interaction and Children's development (2): Brain structure impairment and behavioral disturbance induced in male mice offspring by a single intraperitoneal administration of domoic acid (DA) to their dams. J. Toxicol. Sci. 34, SP279-SP286 (2009).

20. Shiotani, M. et al. Neurobehavioral assessment of mice following repeated oral exposures to domoic acid during prenatal development. Neurotoxicol. Teratol. 64, 8-19 (2017).

21. Levin, E. D., Pizarro, K., Pang, W. G., Harrison, J. \& Ramsdell, J. S. Persisting behavioral consequences of prenatal domoic acid exposure in rats. Neurotoxicol. Teratol. 27, 719-25

22. Perry, M. A., Ryan, C. L. \& Tasker, R. A. Effects of low dose neonatal domoic acid administration on behavioural and physiological response to mild stress in adult rats. Physiol. Behav. 98, 53-59 (2009).

23. Burt, M. A., Ryan, C. L. \& Doucette, T. A. Altered responses to novelty and drug reinforcement in adult rats treated neonatally with domoic acid. Physiol. Behav. 93, 327336 (2008).

24. Burt, M. A., Ryan, C. L. \& Doucette, T. A. Low dose domoic acid in neonatal rats abolishes nicotine induced conditioned place preference during late adolescence. Amino Acids 35, 247-249 (2008).

25. Tiedeken, J. A. \& Ramsdell, J. S. Embryonic exposure to domoic Acid increases the susceptibility of zebrafish larvae to the chemical convulsant pentylenetetrazole. Environ. Health Perspect. 115, 1547-52 (2007).

26. Gill, D. A. et al. Neonatal exposure to low-dose domoic acid lowers seizure threshold in adult rats. Neuroscience 169, 1789-1799 (2010).

27. Howe, K. et al. The zebrafish reference genome sequence and its relationship to the human genome. Nature 496, 498-503 (2013).

28. Tropepe, V. \& Sive, H. L. Can zebrafish be used as a model to study the neurodevelopmental causes of autism? Genes. Brain. Behav. 2, 268-81 (2003).

29. Sumbre, G. \& de Polavieja, G. G. The world according to zebrafish: how neural circuits generate behavior. Front. Neural Circuits 8, 91 (2014).

30. Higashijima, S., Masino, M. A., Mandel, G. \& Fetcho, J. R. Imaging neuronal activity during zebrafish behavior with a genetically encoded calcium indicator. J. Neurophysiol. 90, 3986-97 (2003).

31. Fetcho, J. R. \& Higashijima, S.-I. Optical and genetic approaches toward understanding neuronal circuits in zebrafish. Integr. Comp. Biol. 44, 57-70 (2004).

32. Guo, S. Linking genes to brain, behavior and neurological diseases: what can we learn from zebrafish? Genes, Brain Behav. 3, 63-74 (2004).

33. Arrenberg, A. B. \& Driever, W. Integrating anatomy and function for zebrafish circuit analysis. Front. Neural Circuits 7, 74 (2013).

34. Eddins, D., Cerutti, D., Williams, P., Linney, E. \& Levin, E. D. Zebrafish provide a sensitive model of persisting neurobehavioral effects of developmental chlorpyrifos exposure: comparison with nicotine and pilocarpine effects and relationship to dopamine deficits. Neurotoxicol. Teratol. 32, 99-108 (2010).

35. Almeida, R. G., Czopka, T., Ffrench-Constant, C. \& Lyons, D. A. Individual axons regulate the myelinating potential of single oligodendrocytes in vivo. Development 138, 4443-50 (2011).

36. Kucenas, S., Snell, H. \& Appel, B. nkx2.2a promotes specification and differentiation of a myelinating subset of oligodendrocyte lineage cells in zebrafish. Neuron Glia Biol. 4, 71- 
81 (2008).

37. Takada, N., Kucenas, S. \& Appel, B. Sox 10 is necessary for oligodendrocyte survival following axon wrapping. Glia 58, 996-1006 (2010).

38. Cianciolo Cosentino, C., Roman, B. L., Drummond, I. A. \& Hukriede, N. A. Intravenous microinjections of zebrafish larvae to study acute kidney injury. J. Vis. Exp. (2010). doi: $10.3791 / 2079$

39. Halekoh, U., Højsgaard, S. \& Yan, J. The $R$ Package geepack for Generalized Estimating Equations. J. Stat. Softw. 15, 1-11 (2006).

40. Wolman, M. A., Jain, R. A., Liss, L. \& Granato, M. Chemical modulation of memory formation in larval zebrafish. Proc. Natl. Acad. Sci. U. S. A. 108, 15468-73 (2011).

41. Burgess, H. a \& Granato, M. Modulation of locomotor activity in larval zebrafish during light adaptation. J. Exp. Biol. 210, 2526-39 (2007).

42. Bates, D., Mächler, M., Bolker, B. \& Walker, S. Fitting Linear Mixed-Effects Models using lme4. (2014). at $<$ http://arxiv.org/abs/1406.5823>

43. Hothorn, T., Bretz, F. \& Westfall, P. The multcomp Package Title Simultaneous Inference for General Linear Hypotheses. (2007). at

$<$ http://132.180.15.2/math/statlib/R/CRAN/doc/packages/multcomp.pdf $>$

44. Benaglia, T., Chauveau, D., Hunter, D. \& Young, D. mixtools: An R Package for Analyzing Finite Mixture Models. J. Stat. Softw. 32, 1-29 (2009).

45. O’Malley, D. M., Kao, Y. H. \& Fetcho, J. R. Imaging the functional organization of zebrafish hindbrain segments during escape behaviors. Neuron 17, 1145-55 (1996).

46. Marsden, K. C. \& Granato, M. In Vivo Ca(2+) Imaging Reveals that Decreased Dendritic Excitability Drives Startle Habituation. Cell Rep. 13, 1733-40 (2015).

47. Konietschke, F., Placzek, M., Schaarschmidt, F. \& Hothorn, L. A. nparcomp : An $R$ Software Package for Nonparametric Multiple Comparisons and Simultaneous Confidence Intervals. J. Stat. Softw. 64, (2015).

48. Wobbrock, J. O., Findlater, L., Gergle, D. \& Higgins, J. J. The aligned rank transform for nonparametric factorial analyses using only anova procedures. in Proceedings of the 2011 annual conference on Human factors in computing systems - CHI '11 143 (ACM Press, 2011). doi:10.1145/1978942.1978963

49. Rosario-Martinez, H. De, Fox, J. \& Team, R. C. Post-Hoc Interaction Analysis [R package phia version 0.2-1]. at <https://cran.r-project.org/web/packages/phia/index.html >

50. Ripley, B. \& Venables, W. Package 'nnet'. (2016). at $<$ http://www.stats.ox.ac.uk/pub/MASS4/>

51. Fox, J. et al. in An R Companion to Applied Regression (SAGE Publications, 2018). at $<\mathrm{ftp}$ //ftp.math.ethz.ch/sfs/pub/Software/CRAN/web/packages/car/car.pdf $>$

52. Andrews, S. FastQC: a quality control tool for high throughput sequence data. (2010).

53. Bolger, A. M., Lohse, M. \& Usadel, B. Trimmomatic: a flexible trimmer for Illumina sequence data. Bioinformatics 30, 2114-20 (2014).

54. Dobin, A. et al. STAR: ultrafast universal RNA-seq aligner. Bioinformatics 29, 15-21 (2013).

55. Anders, S., Pyl, P. T. \& Huber, W. HTSeq--a Python framework to work with highthroughput sequencing data. Bioinformatics 31, 166-9 (2015).

56. Robinson, M. D., McCarthy, D. J. \& Smyth, G. K. edgeR: a Bioconductor package for differential expression analysis of digital gene expression data. Bioinformatics 26, 139-40 (2010). 
57. Chen, Y., Lun, A. T. L. \& Smyth, G. K. From reads to genes to pathways: differential expression analysis of RNA-Seq experiments using Rsubread and the edgeR quasilikelihood pipeline. F1000Research 5, 1438 (2016).

58. Reimand, J. et al. g:Profiler - a web server for functional interpretation of gene lists (2016 update). Nucleic Acids Res. 44, W83-W89 (2016).

59. Pogoda, H.-M. et al. A genetic screen identifies genes essential for development of myelinated axons in zebrafish. Dev. Biol. 298, 118-31 (2006).

60. Tiedeken, J. A., Ramsdell, J. S. \& Ramsdell, A. F. Developmental toxicity of domoic acid in zebrafish (Danio rerio). Neurotoxicol. Teratol. 27, 711-717 (2005).

61. McKeown, K. A., Moreno, R., Hall, V. L., Ribera, A. B. \& Downes, G. B. Disruption of Eaat $2 \mathrm{~b}$, a glutamate transporter, results in abnormal motor behaviors in developing zebrafish. Dev. Biol. 362, 162-71 (2012).

62. Kirby, B. B. et al. In vivo time-lapse imaging shows dynamic oligodendrocyte progenitor behavior during zebrafish development. Nat. Neurosci. 9, 1506-11 (2006).

63. Brösamle, C. \& Halpern, M. E. Characterization of myelination in the developing zebrafish. Glia 39, 47-57 (2002).

64. Kolodziejczyk, K., Saab, A. S., Nave, K.-A. \& Attwell, D. Why do oligodendrocyte lineage cells express glutamate receptors? F1000 Biol. Rep. 2, 57 (2010).

65. Patneau, D. K., Wright, P. W., Winters, C., Mayer, M. L. \& Gallo, V. Glial cells of the oligodendrocyte lineage express both kainate- and AMPA-preferring subtypes of glutamate receptor. Neuron 12, 357-371 (1994).

66. Alberdi, E., Sánchez-Gómez, M. V., Marino, A. \& Matute, C. Ca(2+) influx through AMPA or kainate receptors alone is sufficient to initiate excitotoxicity in cultured oligodendrocytes. Neurobiol. Dis. 9, 234-43 (2002).

67. Matute, C., Domercq, M. \& Sánchez-Gómez, M.-V. Glutamate-mediated glial injury: mechanisms and clinical importance. Glia 53, 212-24 (2006).

68. Rosenberg, P. A. et al. Mature myelin basic protein-expressing oligodendrocytes are insensitive to kainate toxicity. J. Neurosci. Res. 71, 237-45 (2003).

69. Deng, W., Rosenberg, P. a, Volpe, J. J. \& Jensen, F. E. Calcium-permeable AMPA/kainate receptors mediate toxicity and preconditioning by oxygen-glucose deprivation in oligodendrocyte precursors. Proc. Natl. Acad. Sci. U. S. A. 100, 6801-6 (2003).

70. Gallo, V. et al. Oligodendrocyte progenitor cell proliferation and lineage progression are regulated by glutamate receptor-mediated K+ channel block. J. Neurosci. 16, 2659-2670 (1996).

71. Gudz, T. I., Komuro, H. \& Macklin, W. B. Glutamate stimulates oligodendrocyte progenitor migration mediated via an alphav integrin/myelin proteolipid protein complex. J. Neurosci. 26, 2458-66 (2006).

72. Matute, C. Characteristics of acute and chronic kainate excitotoxic damage to the optic nerve. Proc. Natl. Acad. Sci. U. S. A. 95, 10229-34 (1998).

73. Dusart, I., Marty, S. \& Peschanski, M. Demyelination, and remyelination by Schwann cells and oligodendrocytes after kainate-induced neuronal depletion in the central nervous system. Neuroscience 51, 137-148 (1992).

74. Hopkins, K. J., Wang, G.-J. \& Schmued, L. C. Temporal progression of kainic acid induced neuronal and myelin degeneration in the rat forebrain. Brain Res. 864, 69-80 (2000). 
75. Verity, A. N. \& Campagnoni, A. T. Myelination and its Underlying Mechanisms Regional Expression of Myelin Protein Genes in the Developing Mouse Brain: In Situ Hybridization Studies. Journal of Neuroscience Research 21, (1988).

76. Foran, D. R. \& Peterson, A. C. Myelin acquisition in the central nervous system of the mouse revealed by an MBP-Lac Z transgene. J. Neurosci. 12, 4890-7 (1992).

77. Eliceiri, B. P., Gonzalez, A. M. \& Baird, A. in Methods in molecular biology (Clifton, N.J.) 686, 371-378 (2011).

78. Fleming, A., Diekmann, H. \& Goldsmith, P. Functional Characterisation of the Maturation of the Blood-Brain Barrier in Larval Zebrafish. PLoS One 8, e 77548 (2013).

79. Jeong, J.-Y. et al. Functional and developmental analysis of the blood-brain barrier in zebrafish. Brain Res. Bull. 75, 619-628 (2008).

80. Xie, J., Farage, E., Sugimoto, M. \& Anand-Apte, B. A novel transgenic zebrafish model for blood-brain and blood-retinal barrier development. BMC Dev. Biol. 10, 76 (2010).

81. Preston, E. \& Hynie, I. Transfer constants for blood-brain barrier permeation of the neuroexcitatory shellfish toxin, domoic acid. Can. J. Neurol. Sci. 18, 39-44 (1991).

82. Suzuki, C. A. M. \& Hierlihy, S. L. Renal clearance of domoic acid in the rat. Food Chem. Toxicol. 31, 701-706 (1993).

83. Lefebvre, K. A. et al. Uptake, tissue distribution and excretion of domoic acid after oral exposure in coho salmon (Oncorhynchus kisutch). Aquat. Toxicol. 81, 266-274 (2007).

84. Drummond, I. A. \& Davidson, A. J. Zebrafish Kidney Development. Methods Cell Biol. 100, 233-260 (2010).

85. Drummond, I. A. Kidney Development and Disease in the Zebrafish. J Am Soc Nephrol 16, 299-304 (2005).

86. Burtrum, D. \& Silverstein, F. S. Excitotoxic Injury Stimulates Glial Fibrillary Acidic Protein mRNA Expression in Perinatal Rat Brain. Exp. Neurol. 121, 127-132 (1993).

87. Nielsen, A. L. \& Jørgensen, A. L. Structural and functional characterization of the zebrafish gene for glial fibrillary acidic protein, GFAP. Gene 310, 123-132 (2003).

88. Lam, C. S., März, M. \& Strähle, U. gfap and nestin reporter lines reveal characteristics of neural progenitors in the adult zebrafish brain. Dev. Dyn. 238, 475-486 (2009).

89. Hui, S. P., Nag, T. C. \& Ghosh, S. Characterization of Proliferating Neural Progenitors after Spinal Cord Injury in Adult Zebrafish. PLoS One 10, e0143595 (2015).

90. Grupp, L., Wolburg, H. \& Mack, A. F. Astroglial structures in the zebrafish brain. $J$. Comp. Neurol. 518, 4277-4287 (2010).

91. Grenningloh, G., Soehrman, S., Bondallaz, P., Ruchti, E. \& Cadas, H. Role of the microtubule destabilizing proteins SCG10 and stathmin in neuronal growth. J. Neurobiol. 58, 60-69 (2004).

92. Wen, H.-L. et al. Stathmin, a microtubule-destabilizing protein, is dysregulated in spinal muscular atrophy†. Hum. Mol. Genet. 19, 1766-1778 (2010).

93. Cheng, H. W., Jiang, T., Mori, N. \& McNeill, T. H. Upregulation of stathmin (p19) gene expression in adult rat brain during injury-induced synapse formation. Neuroreport $\mathbf{8}$, 3691-5 (1997).

94. Wen, H.-L., Ting, C.-H., Liu, H.-C., Li, H. \& Lin-Chao, S. Decreased stathmin expression ameliorates neuromuscular defects but fails to prolong survival in a mouse model of spinal muscular atrophy. Neurobiol. Dis. 52, 94-103 (2013).

95. Mathavan, S. et al. Transcriptome Analysis of Zebrafish Embryogenesis Using Microarrays. PLoS Genet. 1, e29 (2005). 
96. White, R. J. et al. A high-resolution mRNA expression time course of embryonic development in zebrafish. Elife 6, (2017).

97. Johnson, B. R., Atallah, J. \& Plachetzki, D. C. The importance of tissue specificity for RNA-seq: highlighting the errors of composite structure extractions. BMC Genomics 14, 586 (2013).

98. Rice, D. \& Barone, S. Critical periods of vulnerability for the developing nervous system: evidence from humans and animal models. Environ. Health Perspect. 108 Suppl, 511-33 (2000).

99. Tanaka, S., Mito, T. \& Takashima, S. Progress of myelination in the human fetal spinal nerve roots, spinal cord and brainstem with myelin basic protein immunohistochemistry. Early Hum. Dev. 41, 49-59 (1995).

100. Kinney, H. C. Myelination Events. Volpe's Neurol. Newborn 176-188 (2018). doi:10.1016/B978-0-323-42876-7.00008-9

101. Kinney, H. C., Ann brody, B., Kloman, A. S. \& Gilles, F. H. Sequence of Central Nervous System Myelination in Human Infancy. II. Patterns of Myelination in Autopsied Infants. $J$. Neuropathol. Exp. Neurol. 47, 217-234 (1988).

102. Fields, R. D. Myelination: an overlooked mechanism of synaptic plasticity? Neuroscientist 11, 528-31 (2005).

103. Pajevic, S., Basser, P. J. \& Fields, R. D. Role of myelin plasticity in oscillations and synchrony of neuronal activity. Neuroscience 276, 135-147 (2014).

104. Wang, G. J. et al. Systemic administration of domoic acid-induced spinal cord lesions in neonatal rats. J. Spinal Cord Med. 23, 31-9 (2000).

105. Teitelbaum, J. S. et al. Neurologic Sequelae of Domoic Acid Intoxication Due to the Ingestion of Contaminated Mussels. N. Engl. J. Med. 322, 1781-1787 (1990).

106. Petroff, R. et al. Chronic, Low-Level Oral Exposure to Marine Toxin, Domoic Acid, Alters Whole Brain Morphometry in Nonhuman Primates. Neurotoxicology (2019). doi:10.1101/439109

107. Adams, A. L., Doucette, T. A. \& Ryan, C. L. Altered pre-pulse inhibition in adult rats treated neonatally with domoic acid. Amino Acids 35, 157-60 (2008).

108. Marriott, A. L., Ryan, C. L. \& Doucette, T. A. Neonatal domoic acid treatment produces alterations to prepulse inhibition and latent inhibition in adult rats. Pharmacol. Biochem. Behav. 103, 338-44 (2012).

109. Zuloaga, D. G. et al. Fetal domoic acid exposure affects lateral amygdala neurons, diminishes social investigation and alters sensory-motor gating. Neurotoxicology 53, 132 140 (2016).

110. Koch, M. The neurobiology of startle. Prog. Neurobiol. 59, 107-28 (1999).

111. Eaton, R. C., Lee, R. K. K. \& Foreman, M. B. The Mauthner cell and other identified neurons of the brainstem escape network of fish. Prog. Neurobiol. 63, 467-485 (2001).

112. Yeomans, J. S. \& Frankland, P. W. The acoustic startle reflex: neurons and connections. Brain Res. Rev. 21, 301-314 (1995).

113. Bernard, P. B., MacDonald, D. S., Gill, D. A., Ryan, C. L. \& Tasker, R. A. Hippocampal mossy fiber sprouting and elevated trkB receptor expression following systemic administration of low dose domoic acid during neonatal development. Hippocampus 17, 1121-1133 (2007).

114. Ryan, C. L. Hippocampal mossy fiber sprouting and elevated trkB receptor expression following systemic administration of low dose domoic acid during neonatal development. 
(2007). doi:10.1002/hipo.20342

115. Tasker, R. A. R., Perry, M. A., Doucette, T. A. \& Ryan, C. L. NMDA receptor involvement in the effects of low dose domoic acid in neonatal rats. Amino Acids 28, 193196 (2005).

116. Jing, J. et al. Toxicokinetics and Physiologically Based Pharmacokinetic Modeling of the Shellfish Toxin Domoic Acid in Nonhuman Primates. Drug Metab Dispos 46, 155-165 (2018). 


\section{CHAPTER 3}

Developmental exposure to domoic acid disrupts startle response behavior and circuitry

\subsection{GRAPHICAL ABSTRACT}

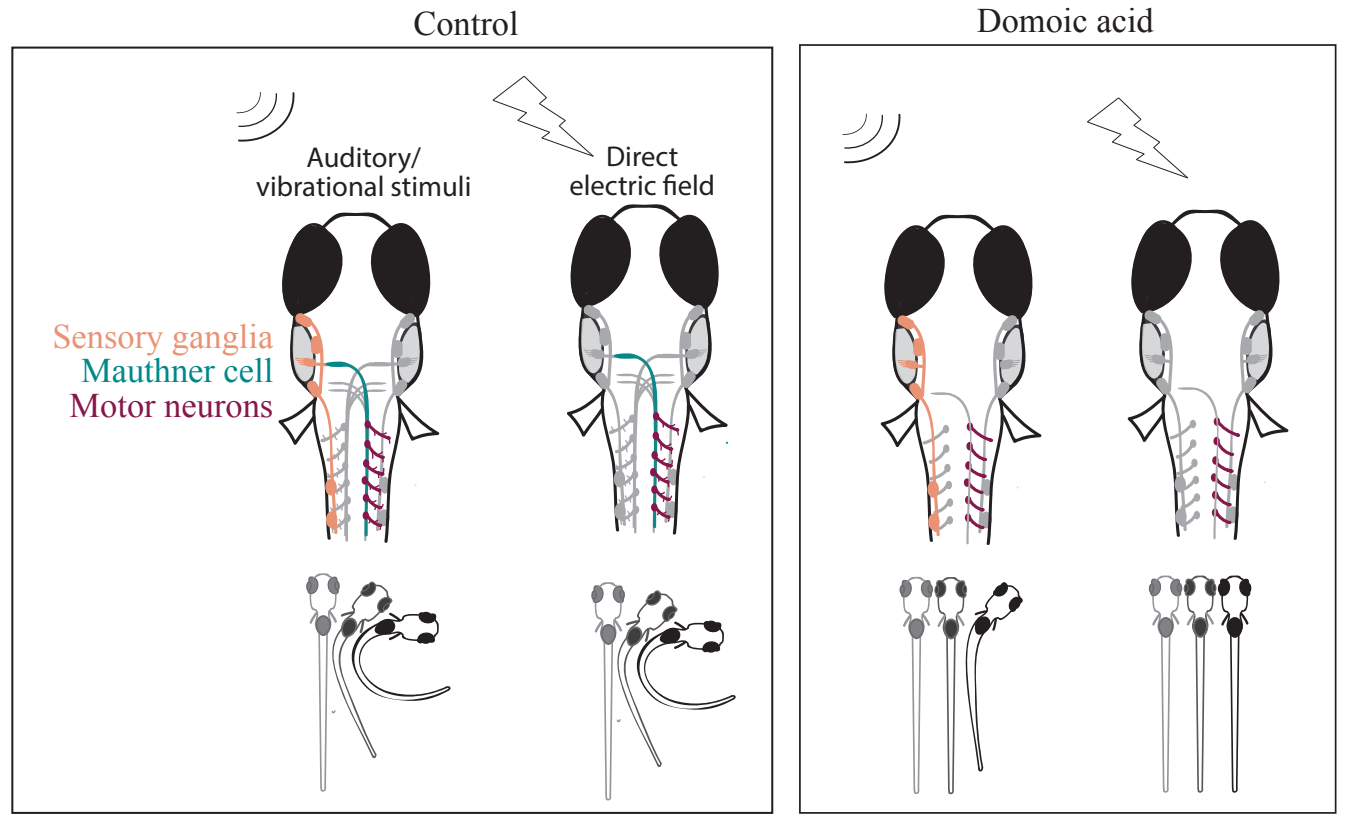




\subsection{ABSTRACT}

In this chapter I investigated the cellular and molecular mechanisms that underlie DomA toxicity at 2 days post-fertilization - the window of susceptibility identified in Chapter 2. In particular, I examined the effects of low-level DomA exposure on the well-characterized startle response. Using a variety of stimulus intensities and modalities, I identified different components of the startle circuit that were disrupted following exposure to DomA. Exposure to DomA reduced the probability of eliciting a startle in response to auditory/vibration (A/V) stimuli at all intensities. Even at the highest stimulus intensities, a majority of DomA-exposed larvae did not exhibit short latency c-start (SLC) responses, which require the hindbrain Mauthner neuron. Furthermore, DomA-exposed larvae had altered startle kinematics, exhibiting shallower bend angles and slower maximal angular velocities. Electrical stimulation bypasses the sensory system to directly activate the Mauthner neuron in the hindbrain. Strikingly, the majority of DomA-exposed larvae were nonresponsive to electrical stimulation, and those that did respond had significantly reduced bend angles. These startle deficits suggest that components downstream of the sensory circuit are affected by DomA. Using vital dye staining, immunolabelling, and live imaging of transgenic lines, I assessed DomA-induced alterations to both the sensory inputs and motor outputs necessary for the startle response. I found no differences in the number of sensory neuromasts that detect the acoustic stimuli or in the presence of sensory cranial ganglia. This is consistent with the behavioral data that suggested that deficits were downstream of the sensory system. In contrast, a majority of DomA-treated larvae lacked one or both Mauthner neurons that are required for the SLC and electrical-induced startles. DomA-treated larvae also showed significantly reduced primary motor neuron axon collaterals. Overall, these results show that developmental exposure to DomA leads to startle deficits by targeting specific subsets of neurons. These findings provide mechanistic insights into the neurodevelopmental effects of excess glutamate signaling caused by exposure to DomA, supporting hazard assessments for DomA exposures in humans during critical periods in early development.

KEYWORDS: Domoic acid; harmful algal bloom toxins; startle response; escape response; Mauthner cells 


\subsection{INTRODUCTION}

Harmful algal blooms (HABs) - the proliferation of algae that produce toxins - have increased in frequency, duration, and geographic extent in recent decades. ${ }^{1,2}$ Some HABs produce potent toxins that contaminate drinking water, seafood, and air quality. Domoic acid (DomA) is a HAB toxin that is produced primarily by algae in the genus Pseudo-nitzschia. It is a structural analog of glutamate, and is known to exert its toxicity by binding and activating ionotrophic glutamate receptors. ${ }^{3,4}$ Exposure to DomA occurs primarily through consumption of contaminated seafood. To prevent acute toxicity in adults, a regulatory limit has been set at $20 \mathrm{mg}$ of DomA per $\mathrm{kg}$ shellfish tissue. ${ }^{5}$

DomA is an established developmental neurotoxin. It has been shown to cross the placental barrier, readily accumulate in amniotic fluids, and distribute to fetal brains. ${ }^{6-8}$ This provides a means by which fetuses can continue to be exposed to DomA after it is cleared from the mother's plasma. ${ }^{8}$ Further exposures may occur in postnatal development through consumption of contaminated breast milk. ${ }^{9,10}$

While regulatory limits have been established, evidence shows that developmental exposures to doses of DomA that do not lead to acute neurotoxic phenotypes ("asymptomatic doses") can have long-term consequences. Both prenatal and postnatal exposures to these asymptomatic doses have been shown to lead to changes in receptor densities, ${ }^{11}$ altered neural connectivity, ${ }^{12,13}$ aberrant brain morphology, ${ }^{14-16}$ and ultimately behavioral deficits through adulthood. Behavioral deficits include impaired interlimb coordination, ${ }^{16,17}$ aberrant exploratory behavior, ${ }^{17,18}$ reduced socialization, ${ }^{13,19}$ and the inability to cope with novel environments. ${ }^{11,15}$ Some behavioral and histological phenotypes are latent and progressive, either appearing for the first time or increasing in severity as the animal ages. Developmental DomA toxicity also can be silent until unmasked by further challenge. Exposures to either a second dose of domoic acid, ${ }^{12}$ PTZ (GABA antagonist and convulsant), ${ }^{20,21}$ or scopolamine ${ }^{22}$ (muscarinic receptor antagonist) led to decreased seizure latencies and memory deficits in animals developmentally exposed to DomA. 
While behavioral deficits have been extensively characterized, the cellular and molecular mechanisms that underlie these behaviors are mostly unknown. Findings from chapter 2 showed that developmental exposures to DomA in zebrafish during a specific period in neurodevelopment led to transcriptional, structural, and behavioral deficits. Exposures occurring at 2 days post fertilization (dpf) led to myelin sheath defects and aberrant startle behavior. For this chapter, I extend these findings by using startle response behavior and the underlying neural circuitry as tools to i) determine whether sensory and motor processes are disrupted by DomA exposure, and ii) identify specific neuronal populations within the circuit that are disrupted by DomA toxicity.

The startle response is an appropriate tool to investigate the behavioral effects of DomA exposure. Aberrant startle response patterns have been associated with myelination defects, ${ }^{23}$ which as I showed in Chapter 2 are a feature of DomA toxicity. In addition, aberrant glutamate signaling also alters startle response kinematics, further strengthening the selection of startle response as a read-out for DomA-driven glutamate excitotoxicity. ${ }^{23,24}$

Startle responses are elicited by a variety of stimuli with different intensity levels. Auditory vibrational stimuli can lead to either of the two types of startle responses - a short latency c(SLC) type startle response or a long latency c-(LLC) type startle response, which are distinguished by their onset time and kinematics. SLC-type responses occur shortly after exposure to a stimulus (about $15 \mathrm{~ms}$ or less, depending on temperature) and lead to pronounced bend angles, while LLCs occur later and produce shallower bend angles. Increasing the intensity of auditory/vibrational stimuli biases fish to perform SLC-type responses, which requires the activation of a hindbrain neuron called the Mauthner cell. Conversely, lower intensity stimuli are more likely to lead to LLC-type startles, which are Mauthner cell independent.

These underlying startle response circuits are also well known, making it possible to link startle behavioral data to underlying structural and cellular targets. Auditory/vibrational stimuli are perceived by the statoacoustic ganglia, which transmits sensory information from the hair cells in the inner ear. ${ }^{25,26}$ Sensory information is also perceived by the lateral line, an organ system that detects vibration and changes in flow through a series of mechanoreceptors along the body. ${ }^{27}$ For 
SLC-type startles, the statoacoustic ganglia cranial nerve synapses to the Mauthner cell in the hindbrain, which fires a single action potential that is propagated down its axons in the spinal

cord and synapses to the primary motor neurons. ${ }^{28,29}$ The near-simultaneous activation of the primary motor neurons results in a 'c' shaped bend. In contrast, direct-electric field stimulation bypasses the sensory system altogether, directly activating the Mauthner neurons and the downstream circuits. ${ }^{30}$

The goal of this study was to use the startle response circuit as a tool to identify cell targets and nervous system processes that were perturbed by DomA. By varying stimulus intensities, I was able to determine whether DomA-exposed fish had the capacity to perceive changes in sensory stimuli and to switch from LLC-type to SLC-type startles. By using direct electric fields which bypasses the sensory system, I was then able to determine whether DomA-induced startle deficits occurred downstream of the sensory system. Finally, I used vital dyes, transgenics, and immunolabeling to identify specific neuronal populations comprising that circuit that may be affected by DomA.

\subsection{METHODS}

Fish husbandry and lines used

These studies were approved by the Woods Hole Oceanographic Institution Animal Care and Use Committee (Assurance D16-00381 from the NIH Office of Laboratory Animal Welfare). Embryos were maintained at $28.5^{\circ} \mathrm{C}$ with a $14: 10$ light dark cycle during the experimental period in 0.3x Danieau's medium. The following transgenic lines were used: $\operatorname{Tg}(m b p: E G F P-C A A X)^{31}$, $\operatorname{Tg}(\operatorname{sox} 10: R F P)^{32}, \operatorname{Tg}(-8.4 \mathrm{~kb}$ neurog 1:mGFP) (gift from E. Linney, Duke University, Durham, NC), and $\operatorname{Tg}(m b p: E G F P)$ (gift from Charles Kaufman in the laboratory of Len Zon, Harvard Medical School, Boston, MA).

\section{Generation of the Tg(cntn1b:EGFP-CAAX) line}

The $\operatorname{Tg}$ (cntn 1b:EGFP-CAAX) line was generated by Gibson assembly. ${ }^{33}$ Using previously published primers, the $c n t n 1 b$ promoter region was amplified from AB wildtype fish. ${ }^{33}$ The $c n t n 1 b$ promoter was then assembled with EGFP-CAAX (cloned out of $\operatorname{Tg}(m b p: E G F P-C A A X)$ fish) into the vector backbone pkHR7, which contains ISCe-1 restriction sites. ${ }^{34}$ To generate the 
stable line, the plasmid containing cntn1b:EGFP-CAAX was co-injected along with ISCe-1 in the 1-2 cell stage. ${ }^{35,36}$ Injected fish were grown up, and their progeny that carried the transgene were used to generate embryos for experimental use.

\section{Domoic acid exposure paradigm}

Domoic acid (Sigma-Aldrich, MO) was dissolved in a diluted embryo medium (0.2x Danieau's) to generate the nominal stock concentrations of $0.675 \mu \mathrm{g} / \mu \mathrm{l}$ and $1.4 \mu \mathrm{g} / \mu \mathrm{l}$, and aliquots were stored at $-20^{\circ} \mathrm{C}$. Working solutions were prepared fresh prior to microinjection by diluting the stock to obtain the appropriate doses. Microinjection needles were prepared from glass capillary tubes ( $0.58 \mathrm{~mm}$ inner diameter; World Precision Instruments, FL; 1B100F-4) using a pipette puller (Sutter instrument model p-30, heat 750, pull $=0$ ). Microinjections were then performed using Narishige IM-300 microinjector, which was calibrated to consistently deliver 0.2-nL by adjusting the time (milliseconds) and the pressure.

DomA was intravenously microinjected into the common posterior cardinal vein at 48-52 hpf. The nominal concentration of $0.14 \mathrm{ng}$ was injected. Chemical analyses of the concentrations are currently underway. Controls from the same breeding cohort were injected with the saline vehicle ( $0.2 \mathrm{x}$ Danieau's). To perform intravenous microinjections, fish were anesthetized with $0.10 \%$ Tricaine mesylate (MS222), and placed laterally on dishes coated with $1.5 \%$ agarose. ${ }^{37}$ An injection was deemed successful if there was a visible displacement of blood cells. Following injections, zebrafish were placed back in clean embryo media and monitored daily.

\section{Imaging myelin sheaths in the spinal cord following domoic acid exposures}

Findings in Chapter 2 showed pronounced myelin defects following exposures to DomA at 2 dpf. To determine whether there is a relationship between the behavioral findings and the myelin phenotype, $\operatorname{Tg}(m b p$ :EGFP-CAAX) embryos were exposed to DomA at 2 dpf and screened for myelin defects at $5 \mathrm{dpf}$ prior to behavioral analyses at $7 \mathrm{dpf}$. The severity of the myelin defect was qualitatively scored (Supplemental Fig. 3). To simplify the analyses, four categories were used: (0) Normal phenotype - dorsal and ventral regions had labeled myelin sheaths. The myelin sheath surrounding the Mauthner axon was visible. (1) Myelin sheaths were present but disorganized. In some cases, myelinated axons that were normally found ventrally were located 
more dorsally. In others, the myelinated axons terminated prematurely with distal ends located more dorsally. (2) Myelin was labeled in both the dorsal and ventral regions of the spinal cord, but there are some noticeable deficits. While the ventral spinal cord was labeled, it had noticeably less myelin labeled compared to controls. (3) The loss of labeled myelin in the ventral spinal cord resulted in large, observable gaps between myelinated axons. Numerous circular myelin membranes were present both in the ventral and dorsal spinal cord.

Two different types of stimuli were used to elicit startle behavior: auditory/vibrational (A/V) and direct electric field stimuli.

\section{Auditory/Vibrational $(A / V)$ stimulation}

A/V stimuli were generated by a minishaker (Brüel \& Kjaer, Vibration Exciter 4810) connected to an amplifier (Brüel \& Kjaer, Power Amplifier Type 2718). This set-up was designed based on the one developed in the Granato lab. ${ }^{38,39}$ Larvae were tested in a 16 -well acrylic plate $(40 \mathrm{x} 40$ $\mathrm{mm})$ that rested in a petri dish $(100 \mathrm{~mm} \times 100 \mathrm{~mm})$. The 16 -well plate itself was made based on designs from Wolman et al. 2011 that comprised of laser cut acrylic pieces that were fused together using acrylic cement (Weld-On \#3; IPS). ${ }^{39}$ An LED backlight was placed below the dish to illuminate the well plate (Adafruit Cat \#1622). The petri dish was epoxied to a thumbscrew (\#10-32 UNF threads) that was fitted to the base of the minishaker (Supplemental Fig. 1A).

Groups of larvae were subjected to four stimulus intensities $(32,38,41$, and $43 \mathrm{~dB})$ that were given in increasing order $(1000 \mathrm{~Hz}, 3$ millisecond pulses). Each stimulus intensity was given four times, spaced 20 milliseconds apart to prevent habituation (Supplemental Fig. 1B and 1C). ${ }^{39}$ For a given stimulus intensity, startle kinematics were shown to be statistically indistinguishable between the $1^{\text {st }}$ and the $4^{\text {th }}$ stimulus given, showing that the 20 millisecond wait period was sufficient (Supplemental Fig. 2). A high-speed video camera (Edgertronic) was set at a 10\% pretrigger rate to capture 13 frames prior to the stimulus being elicited, while recording larval movements at 1000 frames per second. 


\section{Startle behavioral analysis}

High speed videos were converted into jpegs (.mov files with a minimal resolution of 720x720, 1/1008 shutter speed and a frame rate of 1000 frames/second). To reduce the noise and tracking errors, the background was filtered out using a custom script in MATLAB. FLOTE software was then used to analyze the jpegs (Burgess and Granato 2007). Quantitative attributes of the startle response measured include startle responsiveness (whether larvae responded or not), latency (delay time prior to startle), and the maximal bend angle during startle. The identities of individual larvae across the multiple stimuli were distinguished based on their position on a grid. The median response of each of the startle kinematics attributes was obtained for each fish per given stimulus intensity (with 4 being the maximum number of average responses if the fish was tracked perfectly across all 4 replicate stimuli within a stimulus intensity).

\section{Measuring startle vibration}

Vibration was measured using a 3-axis accelerometer (PCB Piezotronics, model W356B11). The output signal was first conditioned (PCB Piezotronics, Model 480B31) then passed through a dual channel analog filter (Model 3382, Krohn-Hite Corporation) using a $10 \mathrm{kHz}$ low-pass cutoff frequency and $30 \mathrm{~dB}$ gain. Finally, the signal was collected by a data acquisition board (National Instruments Data Acquisition board, Model USB-6251). Raw voltage data were converted into acceleration units $\left(\mathrm{m} / \mathrm{s}^{2}\right)$ using manufacturer sensitivity values for each axis of the accelerometer. The Euclidian norm (vector sum) for the three acceleration signals was calculated to get the total acceleration. Individual peaks were identified, and metrics were calculated for the time window between 9 milliseconds prior to the peak to $50 \mathrm{~ms}$ after. The maximum value (peak) during each time window was taken as the zero to peak acceleration value for a given impulse, and this value was converted to $\mathrm{dB}$ using the following equation:

$$
L_{z-p k}=20 * \log _{10}(x)
$$

Where $L_{z-p k}$ is the zero-to-peak acceleration level in $\mathrm{dB}$ re $1 \mathrm{~m} / \mathrm{s}^{2}$, and $x$ is the maximum acceleration level (of the Euclidian norm) over the peak analysis window.

\section{Using mixture models to identify latency cut-offs for SLC versus LLC responses}

Startle responses were classified as either short latency c-bends (SLCs) or long latency c-bends (LLCs) based on an empirically determined latency cut-off. Latency cut-offs have been known to 
vary based on environmental conditions such as temperature. ${ }^{38}$ To empirically determine the cutoffs, clustering was done using a Gaussian mixture model, which fitted two Gaussian distributions, and assigned each latency data point a probability of belonging to either of the two distributions (R package, mixtools). ${ }^{40}$ The cut-off for assigning a response as an SLC was 14 milliseconds - the latency with a greater than $50 \%$ probability of belonging to the first fitted Gaussian distribution (Supplemental Fig. 1D). Startle responses that had latencies greater than 14 milliseconds were classified as LLCs.

\section{Differences in the distribution of startle response type due to treatment}

Animals exposed to stimuli did one of three things: did not perform a startle response (a nonresponse), performed an SLC-type startle, or performed an LLC-type startle. To simplify the model, only responses to the first replicate stimulus within a given intensity were analyzed. Multinomial logistic regression analyses were done to model the effect of treatment on distribution of larvae that were non-responsive, performed SLC-type startles, or performed LLCtype startles (multinom(), nnet R package). The references for this analysis (the intercept) were vehicle-injected controls $(0 \mathrm{ng})$ that performed LLC responses. Odds ratios were calculated to determine how likely it would be that the DomA-exposed larvae would perform a given behavior versus an LLC, in comparison to the control. For example, I calculated the odds of a DomAexposed larvae not responding versus performing an LLC response, compared to controls. The higher the odds, the more likely it was that DomA-exposed larvae would not respond rather than perform an LLC, as compared to a control fish.

\section{Analysis of treatment differences in startle response kinematics}

Kinematic responses from the two types of startle responses (SLC v. LLC) were analyzed separately based on previous research showing that they are driven by distinct neural circuits and have distinct kinematic characteristics. ${ }^{38,41,42}$ The median response of individual fish for each startle type was then calculated for each intensity level.

To determine whether DomA alters kinematic responses to startle, I performed a nonparametric multivariate response analysis, with treatment group as fixed factors (DomA vs. control). Nine separate repeated experiments (trials) were combined for the analysis ( $\operatorname{art}()$, ARTool R 
package) ${ }^{43}$ To account for potential differences in response due to the variations between trials, the experimental trial was incorporated into the model as a random factor.

To determine whether there was an association between the myelin phenotypes and the startle kinematics, I used a nonparametric multiple comparisons test (nonpartest(), npmv R package). ${ }^{44}$

\section{Direct electric field stimulation}

Larvae were head mounted in $35 \mathrm{~mm}$ glass bottom dishes that were modified by gluing jumper wires to the edges of the dish to create a defined surface area for the electric field. Larvae were positioned rostral-caudally to the electrodes to provide the highest probability of eliciting a startle. ${ }^{30}$ To head-mount, fish were anesthetized in MS222 (0.1\%) and mounted upright (ventral side on the dish) using 1.5\% low-melt agarose. Once the agarose solidified, an insect pin (size $\# 000$ ) was used to carefully carve out the agarose so that the region below the head was free to move. The dish was then flooded with embryo media (0.3x Danieau's) and the fish were allowed to recover in a water bath heated to approximately $26^{\circ} \mathrm{C}$. Using an "embryo poker" (a piece of $0.41 \mathrm{~mm}$ fishing line glued to a glass pipette tip), the fish's tail was brushed lightly to confirm that it recovered from anesthesia prior to beginning the trial. A $4.4 \mathrm{~V} / \mathrm{cm}, 2$ millisecond square wave was generated by the stimulus generator, PulsePal (Sansworks). This was delivered simultaneously as a TTL pulse that triggered the high-speed camera (Edgertronic), which recorded the tail movements at 1000 frames per second. Tail bend angle and latency were then tracked using the FLOTE software. ${ }^{45}$

\section{Statistical modeling of startle responsiveness}

Every fish was given 4 replicate auditory/vibrational stimuli, spaced 20 milliseconds apart. For all instances where a fish was successfully tracked, response rates were recorded, and the percent of times an individual fish responded to these replicate stimuli was calculated (\% responsiveness $=$ number of times the fish responded/ number of successfully tracked videos with a maximum of 4 tracks per individual fish). A mixed effects logistic regression model was used to identify treatment differences in percent responsiveness, with treatment (DomA vs. control) as a fixed factor and the replicate stimuli as a random factor using the 'glmer' function of lme4 package in R. A Dunnett post-hoc test was used to identify potential treatment differences in responsiveness 
(glht(), multcomp R package) ${ }^{46}$ To correlate the myelin phenotypes to responsiveness, larvae were classified based on their assigned myelin category. Responsiveness was then modeled based on myelin phenotype and treatment as predictors, with the presence or absence of startle as the response. Repeated measures from multiple stimuli were accounted for using a logistic regression with a quasibinomial distribution, which estimates the overdispersion parameter $\left(\mathrm{g} \operatorname{lm}(\right.$ family$=$ quasibinomial(link= "logit"), multcomp package $) .{ }^{46}$

\section{Immunohistochemistry}

Fish were anesthetized and fixed in $4 \%$ paraformaldehyde overnight at $4^{\circ} \mathrm{C}$. In some cases, whole brains were dissected (Fig. 8), and in other cases whole embryos were used (Fig. 10). Antigen retrieval was done by placing tissue in $150 \mathrm{mM}$ Tris $\mathrm{HCl}(\mathrm{pH} 9.0)$ in a $70^{\circ} \mathrm{C}$ water bath for 15 minutes. Brain tissue was permeabilized using proteinase $\mathrm{K}$ ( $10 \mu \mathrm{g} / \mathrm{ml}$ for 3 minutes) then post-fixed for 20 minutes using 4\% paraformaldehyde. Whole mount embryos were permeabilized using ice-cold acetone ( 7 minutes). Samples were then blocked in 10\% normal goat serum and $1 \%$ DMSO, followed by 1-3 day incubations in primary antibodies ( $\alpha-3 \mathrm{~A} 10$ 1:100 dilution, Developmental Studies Hybridoma Bank; $\alpha$-acetylated tubulin - 1:500 dilution, Santa Cruz Biotechnology). After several washes in phosphate buffered saline with $0.01 \%$ triton$\mathrm{X}$, samples were then incubated in secondary antibodies (1:400 Alexa Fluor 488 Goat $\alpha$-mouse or Alexa Fluor 596 Goat $\alpha$-mouse; Abcam). Samples were then placed in antifade mountant (Prolong or SlowFade Diamond mountant, Invitrogen), and placed between bridged \#1.5 coverslips for imaging.

Acetylated tubulin was used to assess primary neuron axons, early sensory neurons (Rohon beard cells), and the peripheral lateral line, while 3A10 was used to characterize Mauthner cell bodies and hindbrain and midbrain axonal tracks. Brain dissections required for 3A10 staining sometimes led to reticulospinal axons (those that extend down to the spinal cord) being removed from the preparation. (Supplemental Fig. 4 shows the range of phenotypes found in controls.) Thus, for the antibody labeling experiment, the presence or absence of the Mauthner cell was determined solely based on the presence of the Mauthner cell body and lateral dendrite. 


\section{DASPEI labeling}

Sensory neuromasts were labeled using the vital dye, DASPEI ((2-(4-(dimethylamino)styryl) -Nethylpyridinium iodide, Biotium Inc., Freemont, CA). Zebrafish larvae (5 dpf) were incubated in $0.005 \%$ DASPEI for 15-20 minutes, then washed and placed in anesthetic (0.16\% MS222). Larvae were then mounted in low-melt agarose (1-1.5\%) either laterally or ventrally and imaged with a widefield microscope. Neuromasts were manually counted on blinded files. To identify treatment differences in counts, a random coefficient Poisson regression was done, with repeated experiments (trials) modeled as random factors (glmer(), lme4 R package). ${ }^{47}$

\section{Reticulospinal backfills}

Larvae (7 dpf) were anesthetized using MS222 (0.16\%), then mounted ventrally in $1.5 \%$ low melt agarose. Dissection spring scissors were dipped into Texas red dextran (3000 MW) and the spinal cord was transected at the level of the anus. ${ }^{48}$ Larvae were placed back into anesthetic, and screened for dye uptake using epifluorescence microscopy (Zeiss Axiovert inverted microscope). Roughly an hour after spinal cord transections, larvae were placed in $4 \%$ paraformaldehyde and fixed overnight in $4^{\circ} \mathrm{C}$. Following several washes in PBS, the whole brain was dissected. ${ }^{49}$ Whole brains were mounted using the same technique used with brains that were labeled with antibodies. The presence of the Mauthner cell, along with any labeled neurons in rhombomere 5 (r5) and rhombomere 6 (r6) - the location of MiDcm2, MiDcm3 - were identified. Following this classification, ordered logistic regression was done to determine whether treatment alters the number of neurons found in each location (polr(), Mass R package, R). ${ }^{50}$

\section{Primary motor neuron live imaging and tracing}

$\operatorname{Tg}$ (cntn 1b:EGFP-CAAX) embryos were exposed to DomA at 48-52 hpf. Larvae (2.5 dpf) were then anesthetized ( $0.16 \%$ MS222), and embedded laterally in 1-1.5\% low-melt agarose in glass bottom microscopy dishes. Larvae were imaged using a confocal microscope (LSM 710 or LSM 780 ) with the 40x water immersion objective (C-Apochromat, 40x, NA 1.1). Primary motor neurons located approximately at myotome 20-23 were imaged. Images were blinded prior to image analysis. A single primary motor neuron from the image stack was chosen. Its main axon along with the axon collaterals were traced from the image stacks using the ImageJ plugin, Simple Neurite Tracer. ${ }^{51}$ The total length of the traced axon collaterals was calculated from the 
tracing files. One-way analysis of variance was used to determine whether there was an effect of treatment on the total length of the axon collaterals (oneway.test(), R).

\subsection{RESULTS}

DomA-exposed fish were less responsive, and less likely to perform SLC-type startles

All successfully tracked fish were classified into one of three categories: non-responders (did not move within the $250 \mathrm{~ms}$ tracked time), responders that performed SLC-type startles (latency less than $14 \mathrm{~ms}$ ), or responders that performed LLC-type startles (latency $\geq 14 \mathrm{~ms}$ ). Multinomial models were then used to determine whether the DomA-treated fish population had a different distribution for these three types of responses for each stimulus intensity provided.

At the lowest stimulus intensity tested $(32 \mathrm{~dB})$, most of control and DomA-treated fish did not respond (Fig. 1A, Table 1). The likelihoods of fish not responding rather than performing LLCs were similar for DomA-treated and control fish $(\mathrm{p}=0.083$, Table 1 and 2). For the fish that did respond, they were more likely to perform LLC-type rather than SLC-type startles. While this was true for both groups, DomA-treated fish were even less likely to perform SLC-type startles compared to controls (odds ratio of $0.35, \mathrm{p}=8.5$ e -05 ) (Table 2).

With higher intensities ( $38 \mathrm{~dB}$ and up), a larger proportion of both the control and DomA-treated fish performed LLC-type startles rather than not responding (Fig. 1A, Table 3-5). Furthermore, a higher proportion of control fish preferentially performed SLC-type startles rather than LLC-type startles. The opposite was true for DomA-exposed fish. Significantly higher proportion of the DomA-exposed population performed LLC-type startles rather than SLC-type startles compared to the controls at all intensities tested (odd ratio of 0.3 or lower, Table 3-5).

This is further illustrated by calculating population-level biases for the types of startle responses that are elicited by different stimulus intensities. Since individual fish were provided 4 replicate stimuli for each stimulus intensity, the proportion of startle types (startle bias) was calculated for each fish (Fig. 1B). A fish with the value of +1 always responded with an SLC startle while a fish with a value of -1 always responded with an LLC startle. At the lowest stimulus intensity (32 $\mathrm{dB}$ ), both control and DomA-treated fish were LLC-biased. By $38 \mathrm{~dB}$ and higher, control fish 
preferentially performed SLC startle responses. In contrast, DomA-exposed fish preferentially performed LLC (rather than SLC) responses at all stimulus intensities.

Fish with myelin defects were less likely to perform SLC-type startles than those without defects Larvae were classified based on myelin defects observed prior to the behavioral assessment. This made it possible to assess the relationship between startle bias and the observed myelin sheath defects. Similar to the control group, DomA-exposed fish with normal myelin sheaths performed more SLC startles with higher stimulus intensities (38 dB and higher) (Fig. 1C). In contrast, fish that exhibited any defects in myelin sheaths performed more LLC than SLC startles, regardless of stimulus intensity. This is reflected in a multinomial analysis that was done with DomA further subcategorized by myelin phenotypes (Table 6). Even at the highest stimulus intensity, fish with any noticeable myelin defects (Category 1-3) had a reduced likelihood of switching from LLC to SLC compared to controls ( $p<0.01$, with an odds ratio of $0.07-0.3$ ).

DomA-exposed fish that had myelin defects also had startle response kinematic deficits For the fish that responded to a given stimulus, the startle kinematics (bend angle, maximal angular velocity) were assessed. In agreement with findings in Chapter 2, DomA-exposed animals had reduced bend angles and lower maximal angular velocities (Mav) compared to control animals $(\mathrm{p}<2.22 \mathrm{e}-16)$. This was true for all intensities tested (Fig. 2).

I then assessed the relationship between the classified myelin phenotypes and startle response kinematics. A majority of DomA-exposed larvae were classified as category 3 (with most severe myelin defects). These larvae also had significantly reduced bend angles and Mavs for all stimuli tested. This was true for both SLC and LLC-type startle responses (Fig. 3 and 4, Table 7 and 8).

DomA-treated fish with control-like myelin sheaths (category 0) also had kinematic deficits when performing SLC-type startle responses (Fig. 4, Table 7). In contrast, DomA-treated fish with category 0 phenotypes did not have any measurable kinematic deficits when performing LLC-type startle responses across all stimulus intensities tested (Fig. 3, Table 8). 
Fish with intermediate myelin defects (category 1-2) also consistently had kinematic deficits when performing SLC-type startles across all stimulus intensities tested (Fig. 4). However, it was only at higher stimulus intensities ( $38 \mathrm{~dB}$ or higher) that larvae in these categories had kinematic deficits during LLC-type startles (Fig. 3, Table 8).

These results suggest that exposures to DomA that led to distinguishable myelin defects also led to startle deficits. The severity of the myelin defect was correlated with the severity of the behavioral deficit; fish with the most severe myelin defects were also less likely to perform SLCs, and had shallower bend angles and slower maximal angular velocities.

DomA-exposed fish were less responsive, and had aberrant kinematics following direct-electric field stimulation

To assess the contribution of the sensory system to observed startle deficits, I used direct electric field stimulation, which bypasses the sensory system to directly activate the Mauthner cell. Using electrical stimulation allowed me to identify whether neural components downstream of the sensory system were affected by DomA.

Previous findings from chapter 2 identified swim bladder defects in larvae exposed to DomA at 2 dpf. An uninflated swim bladder could impede kinematics of the startle behavior independent of the neural circuit that drives it. Assessing the startle response to electrical stimuli requires the use of head-mounted fish, and fish mounted in this way do not require an inflated swim bladder to maintain the upright posture necessary to perform the bend angles during startle. Thus, the electrical stimulation assay also served as an alternative way to determine whether startle deficits observed were solely due to an uninflated swim bladder (Fig. 5A).

DomA-treated larvae were significantly less responsive to electrical stimulation than control fish ( $p<1$ e -16 ). A majority of control fish (69/74) responded to all of the 7 replicate stimuli (Fig. 5B), while a majority of the DomA-treated fish (47/74) did not respond to any of the replicate stimuli. Furthermore, control fish responded rapidly to electrical stimulation, with a median latency of 2 milliseconds (Fig. 5C). Strikingly, when DomA-treated fish did respond, they had 
significantly longer latencies (Control median $=2 \mathrm{~ms}$, DomA median $=49 \mathrm{~ms}, \mathrm{IQR}=2 \mathrm{~s}$, estimated relative effect (est) $=0.78,95 \%$ CI [0.633, 0.927], $\mathrm{p}=0.001]$ ).

Fish that were treated with DomA also had shallower bend angles when performing startles compared to controls (median $=83.5^{\circ}$ in controls, median $=41.9^{\circ}$ in DomA treated larvae, Coefficient $=0.173,95 \%$ CI $[0.059,0.286], \mathrm{p}<\mathrm{e}-16)$. Furthermore, DomA-exposed fish also had slower maximal angular velocities (median $=3.78 \% \mathrm{~ms}$ in controls, median $=2.28 \% \mathrm{~ms}$ in DomA treated larvae, Coefficient $=0.21,95 \%$ CI $[0.083,0.338], p<e-16)$ (Fig. 5D and 5E). Even when head mounted, DomA-exposed fish still had reduced bend angles, suggesting that kinematic differences are not solely due to balance defects from swim bladder deflations.

Since fish were screened for myelin defects, I could test for a correlation between the observed myelin defects and the startle deficits (Fig. 6). DomA-treated fish that had no visible myelin defects had no differences in responsiveness (Fig. 6E). However, DomA-treated fish that had any visible myelin defect, even in its least severe form (category 1), had significantly reduced responsiveness compared to controls (Dunnet post-hoc test, $\mathrm{p}<\mathrm{e}-9$ ) (Fig. 6E).

DomA-exposed animals with no visible myelin defects did not have significantly different bend angles, Mavs, or differences in latencies compared to controls (Fig. 6C and 6D). While DomAexposed fish that had milder defects (category 2 ) had significantly longer latencies (est $=0.989$, $95 \% \mathrm{CI}=[0.946,1.032], \mathrm{p}<3.28 \mathrm{e}-13)$, the same fish showed no deficits in bend angle or Mav (Fig 6C and D, Table 9). In contrast, DomA-exposed fish that had more severe myelin defects (category 2) also had significantly lower bend angles and longer latencies compared to controls (Table 9).

To further identify the potential cellular and structural defects underlying these observed behavioral deficits, I imaged different components of the startle circuit, starting with the sensory system. 


\section{Sensory inputs for acoustic/vibrational startle}

Hair cells are mechanoreceptor cells in both the inner ear and lateral line that sense auditory/vibrational stimuli. When activated, these hair cells lead to the firing of the statoacoustic ganglia (in the inner ear) or the lateral line ganglia, which in turn send the sensory information to the hindbrain where the information is integrated. ${ }^{52,53}$ To assess DomA-induced effects on the sensory system, I determined whether DomA reduced the number of neuromasts or disrupted statoacoustic ganglia or lateral line structures.

I first assessed whether DomA reduced the number of neuromasts - sensory bundles of hair cells and support cells - that comprise the lateral line. To accomplish this, I used the vital dye DASPEI, to label sensory neuromasts in $5 \mathrm{dpf}$ larvae that were exposed to DomA at $2 \mathrm{dpf}$ in 4 experimental trials (Fig 7A). There were no differences in the number of neuromasts in both the cranial and the trunk region following DomA exposures (Fig. 7B).

In the transgenic line $\operatorname{Tg}$ (cntn1b:EGFP-CAAX), subsets of axons, including those that comprise the peripheral lateral line and the statoacoustic ganglia, express EGFP (Fig. 7C). Fish exposed to DomA and then imaged at 4-5 dpf showed no differences in the presence of these structures compared to controls (Fig. 7D,E).

\section{Reticulospinal neurons integrate sensory information}

Spinal backfills were done at $7 \mathrm{dpf}$ to determine whether DomA disrupts the formation of hindbrain reticulospinal neurons that are known to be important for eliciting startle response. The Mauthner is required for eliciting SLC responses, while MiDcm2 and MiDcm3 are known to be active in LLC responses. ${ }^{41,42}$ Most of the control larvae contained both Mauthner cells and all neurons within rhombomere 5 (r5) and rhombomere 6 (r6) (Fig. 8). In contrast, a majority of DomA-exposed larvae had 0 (out of the 2) Mauthner cells, and 0 or 1 (out of the 4) neurons in positions r5 and r6. In fact, fish exposed to DomA were 99.3\% less likely than controls to have 2 Mauthner cells (odds ratio $(\mathrm{OR})=0.007$, confidence interval $(\mathrm{CI})$ for odds ratio $=[-6.17,0.024]$, $\mathrm{p}=1.7 \mathrm{e}-14)$, and had an even lower probability of having 4 neurons at $\mathrm{r} 5$ and $\mathrm{r} 6(\mathrm{OR}=0.001$, $\mathrm{CI}=[4.96$ e $-05,6.45$ e -03$], \mathrm{p}=1.6$ e -09$)$. 
Spinal backfills have some disadvantages. The absence of labeled neurons may be an indication that the neurons are not present. Alternatively, neurons may still be there but they may just not have axons that extend to the cut site where the axons can take up dye. Furthermore, if axonal transport mechanisms are defective, axons may be present but unable to take up the dye efficiently enough to stain the neuronal cell bodies. To address these potential pitfalls, I also performed whole brain 3A10 antibody staining, which labels the Mauthner cell bodies along with other midbrain and hindbrain axonal tracks (1-7) (Fig. 9A). In agreement with the spinal backfill data, a majority of DomA-exposed larvae did not have Mauthner cell bodies, while a majority of controls did (Fig. 9B and 9C). Importantly, other axonal tracts in the midbrain and hindbrain appeared to be intact in both control and exposed animals. Both control and DomA-exposed animals had medial longitudinal fasciculi, and had no significant differences in the number of hindbrain axonal tracts (Fig. 9B and 9D).

\section{Primary motor neurons that innervate muscles that generate the response}

Axons from each Mauthner cell cross the midline and extend into the spinal cord where they form synapses with primary motor neurons that innervate the muscles required for the startle responses. To determine whether DomA alters primary motor neuron axons shortly after exposure, I stained the trunk region using anti-acetylated tubulin (Fig. 10A). There were no differences in the presence of the primary motor neurons in DomA-exposed larvae versus controls (Fig. 10B). Furthermore, there were also no differences in the appearance of sensory neuron cell bodies or the peripheral lateral line (Fig. 10B).

By using the transgenic line $\operatorname{Tg}$ (cntn $1 b: E G F P-C A A X)$, I was able examine the axon collaterals that branch from the main caudal primary motor neuron axon. While staining with $\alpha$-acetylated tubulin showed no differences in the presence of the main branches in primary motor neurons (Fig. 10B), tracings of the motor neuron axon collaterals in DomA-treated $\operatorname{Tg}$ (cntn1b:EGFPCAAX) larvae showed a significant reduction in the length of the branches (Fig. 10C and 10D) $($ Control $($ mean $\pm \mathrm{SE})=1460 \mu \mathrm{m} \pm 42, \operatorname{DomA}(\operatorname{mean} \pm \mathrm{SE})=527 \mu \mathrm{m} \pm 29, \mathrm{~F}(1,77)=326.92$, $\mathrm{p}<2.2$ e-16). 


\section{Neural precursors}

To determine whether DomA alters ventral spinal cord interneurons or neural precursors, I performed live imaging with the neuronal and neuronal precursor line $\operatorname{Tg}$ (neurog:EGFP) at 4 and $5 \mathrm{dpf}$. There were no substantial differences in labeling between DomA-treated and control animals (Fig. 11).

\subsection{DISCUSSION}

Overview and significance

This study used the startle response as a tool to identify neural systems and cell targets that are preferentially altered by DomA exposures. Through behavioral assays, I found that DomA exposures altered motor function, by reducing responsiveness to stimuli and altering startle kinematics. Imaging of neuronal components of the circuit, I found that DomA exposures led to the loss of reticulospinal neurons, and to the reduction in axonal collaterals in caudal primary motor neurons. Taken together, these results suggest that DomA exposure targets specific cell types, and has functional consequences on motor function.

\section{Startle behavioral findings provide insights into cell types targeted by DomA}

DomA-exposed larvae were less responsive and less likely to perform SLC-type Mauthner mediated startle responses compared to controls. DomA-exposed larvae subjected to direct electrical stimulation were also markedly less responsive than controls. Since direct-electric field stimulation bypasses the sensory system, these observed startle deficits cannot be due to DomAinduced deficits in the sensory system. Furthermore, both A/V and direct-electric field stimulation require Mauthner cell activation, suggesting that DomA is targeting the Mauthner cell to mediate these phenotypes. ${ }^{28,30,42}$

DomA-exposed larvae also had reduced bend angles and slower maximal angular velocities in all the types of startle responses observed (A/V mediated SLC, LLC-type startle response, and electric field-mediated startle responses). I hypothesized that this shared phenotype (altered startle kinematics) may be a result of disruptions to a cell target that is common in the neural circuits of all three types of startle responses. Since electrical stimulation bypasses the sensory system, and the LLC does not require that Mauthner cell, I further assessed the effects of DomA 
on the caudal primary motor neurons (findings below). Building off of these behavioral results, I then identified specific neural populations within the startle circuit that were preferentially altered by DomA exposures.

\section{Sensory system as an unlikely primary target for DomA}

It is well known that sensory information is integrated prior to Mauthner cell activation, and that different sensory cues influence the probability of startle responses occurring. For example, preexposure to a non-startling tone leads to a reduced responsiveness to an otherwise startling tone in a phenomenon called "prepulse inhibition." ${ }^{.5,54}$ It is thus conceivable that the overall reduced responsiveness observed in DomA-exposed animals could be due to disruptions of the sensory system.

However, when assessing different components of the sensory circuit, I found that DomA did not reduce the number of neuromasts nor disrupt the presence of important sensory ganglia necessary for perceiving A/V stimuli, supporting the idea that DomA may not target the sensory system. It is important to note that I assessed the presence versus absence of the specific sensory structures rather than their function. Previous studies have shown that the application of the ionotrophic glutamate receptor agonist, AMPA, reduced the firing of afferent sensory neurons and neurons' responsiveness to hair cell activation. ${ }^{55}$ Furthermore, I assessed gross morphological features. I quantified the number of neuromasts present rather than more detailed structural features such as number of hair cells within a neuromast or the number of branches in afferent neuron terminals that synapse to the hair cells. Indeed, zebrafish hair cells express AMPA and KA receptors to which DomA binds, making them potential targets for toxicity. Exposure to high doses of ionotrophic glutamate agonists have been shown to lead to both the loss of hair cells within a single neuromast (with $300 \mu \mathrm{M} \mathrm{KA}$ or AMPA), ${ }^{56}$ and to the swelling of hair cell afferent synapses (with $100 \mu \mathrm{M}$ AMPA).

It is thus conceivable that DomA may bind to ionotrophic glutamate receptors in hair cells and at the mature hair cell ribbon synapse. DomA-induced overactivation of these receptors could then lead to hair cell death with individual neurons, and to the reduced firing capacity of afferent sensory neurons. Both of these events could contribute to the overall reduced responsiveness 
observed in DomA-exposed larvae. However, a more detailed analysis of individual hair cells and hair cell afferent synapse following DomA exposure would be necessary to determine whether this occurs.

It is also important to note that even if DomA-exposed larvae responded less often than control larvae, they still had the capacity to respond to A/V stimuli. Furthermore, their responsiveness increased with increasing stimulus intensities, suggesting that larvae have the ability to encode graded sensory information that translates to different degrees of responsiveness.

Taken together, these results indicate that DomA does not alter the overall structures in the sensory system, nor does it affect the ability of larvae to increase their responsiveness with higher stimulus intensities. However, DomA-exposed fish are generally less responsive relative to control fish, and this behavioral phenotype may be due to subtle perturbations to sensory systems that have yet to be investigated.

DomA exposure leads to the loss of the Mauthner cell and alters other reticulospinal neurons Behavioral results show that DomA-exposed larvae are less likely than controls to perform SLCtype startles even with high-intensity stimuli. Strikingly, DomA-exposed larvae rarely performed startles when given direct electric field pulses. Since both these behaviors require the Mauthner cell, I hypothesized that the Mauthner cell may be disrupted by DomA exposures. Imaging results support this hypothesis. A majority of DomA-treated larvae did not have Mauthner cells, as assessed by immunohistochemistry with $\alpha 3 \mathrm{~A} 10$ at $5 \mathrm{dpf}$, and by reticulospinal backfills at 7 dpf.

DomA disrupts reticulospinal neurons and reduces caudal primary motor neuron branching DomA-exposed larvae showed kinematic deficits such as reduced bend angles and slower Mavs when performing A/V-induced startle responses (of the SLC and LLC-type) and electric-field induced startles. I hypothesized that this shared phenotype (altered startle kinematics) may be a result of disruptions to a cell target that is common in the neural circuits of all three types of startle responses. 
One of the shared neuronal components is the downstream primary motor neuron that activates the trunk and tail muscles required for the startle responses. While the main caudal primary motor neuron axons are present in DomA-exposed fish, the collaterals of these motor neuron axons are severely reduced. The reduction in the collaterals could translate to fewer synapses to the muscles, and the observed shallower bend angles. ${ }^{57}$ Furthermore, new evidence shows that motor neurons may not just be passive recipients of upstream circuit activity, but rather control the firing threshold and recruitment of spinal interneurons during locomotion via gap junctions. Any defects in motor neurons could also lead to locomotor defects. ${ }^{58}$

\section{Behavioral deficits are correlated with the severity of myelin phenotypes}

By imaging $T g(m b p: E G F P-C A A X)$ larvae prior to behavioral tests, I was able to correlate structural and behavioral phenotypes - fish with more severe axonal and myelin defects also had more severe behavioral defects.

$\operatorname{Tg}(m b p: E G F P-C A A X)$ fish have EGFP localized to the myelin sheaths. Aberrant EGFP expression patterns could be due to a combination of myelin sheath deficits (the loss of myelin while the axons they wrap are present) or due to axonal loss (which would also result in no myelin labeling). Imaging results (Fig. 8 and 9) indicate that reticulospinal neurons are lost in DomA-exposed fish, suggesting that at least some of the loss of EGFP labeling is due to the loss of axons. Thus, fish with aberrant EGFP expression were considered to have "myelin and axonal defects."

It is important to note that since myelin defects were characterized in the larval stages (5- $7 \mathrm{dpf}$ ), this chapter did not determine whether the loss of myelin earlier in development led to later axonal losses, or whether the axonal losses preceded the loss of myelin. Chapter 4 delves further into this question by characterizing the initial cellular targets of DomA.

When I compared the imaging to behavioral data, I found that only DomA-exposed larvae with the normal axon and myelin phenotypes (category 0) were consistently responsive to the electric field-induced stimuli, while the fish with any axon and myelin defects (category 1-3) were not. 
Furthermore, only fish with "control-like phenotypes" (category 0) also had "control-like" LLC kinematic responses.

DomA-exposed larvae with "control-like" axons and myelin sheaths had fewer kinematic deficits relative to DomA-exposed fish with axon or myelin defects. However, these fish with "controllike" axons and myelin sheaths did not always have "control-like" kinematics, especially during SLC-type startles. There are two potential explanations: 1) Fish that had "control-like" myelin sheaths and axons actually had subtle myelin sheath and axonal defects that I could not distinguish from controls at the resolution I was imaging. These subtle defects then contributed to some of the observed SLC behavioral deficits, or 2) Fish actually had myelin sheaths and axons that were indistinguishable from controls, but had other neuronal defects that I did not correlate to behavior. To test the latter scenario, I could take the subset of the fish that had "control-like" myelin sheaths and assess for effects on other cell targets, including motor axon collateral branching and ionotrophic glutamate receptor activity in hair cells.

\section{DomA is not just a general neurotoxin}

Findings from this chapter suggest that DomA isn't simply a general neurotoxin that targets all neural precursors and neurons, but instead targets specific neuronal subtypes. The most apparent targets of DomA were the reticulospinal neurons in the hindbrain (including the Mauthner cell) and the caudal primary motor neurons. DomA had no apparent effects on other neurons and neuronal precursors examined including the medial longitudinal fasciculus, the hindbrain axonal tracts, the Rohon-beard sensory cell bodies, or the neural precursors in the spinal cord. While both Mauthner cells and caudal primary motor neurons have the ionotrophic glutamate receptors to which DomA binds, ${ }^{59-61}$ so too do other neurons and glial cells. ${ }^{62}$

The sensitivity of these two neuronal classes to DomA may be due their intrinsic properties. Both hindbrain reticulospinal neurons and motor neurons have large axons which can have extensive axonal arbors, located in either the spinal cord or periphery. Due to their axon size, both neuron classes have high energetic requirements that are associated with transporting cargo long distances and maintaining axonal arbors. Any perturbation that disrupts homeostatic mechanisms 
such as slowing of axonal transport mechanisms could have important effects on these neurons, making these cells more susceptible to disease and environmental insults.

In fact, motor neurons are known for being selectively vulnerable to insult because they are large, continuously active and heavily rely on mitochondrial respiration processes. ${ }^{63}$

\section{Implications for human health}

These findings have implications for human health. Behavioral data indicates that DomA exposure in fish disrupts motor control. Motor deficits have been previously characterized from both incidental human exposures and animal exposure models. ${ }^{17,64,65}$ Adult humans acutely exposed to DomA developed sensorimotor neuropathy and axonopathy as assessed by electromyography. ${ }^{64}$ A subset of primates exposed orally at or near the accepted daily tolerable dose of $0.075 \mathrm{mg} / \mathrm{kg}$ developed visible hand tremors. ${ }^{66}$ Finally, rodents prenatally exposed to DomA (PND 10-17) developed aberrant gait patterns. ${ }^{17}$ All of this suggests that motor deficits may be an important functional endpoint for DomA toxicity.

Findings also show that DomA selectively targets specific motor neurons and reticulospinal neuron subclasses. While humans do not have Mauthner cells, the intrinsic characteristics that make this cell type more vulnerable to toxins may be shared with other neurons that humans do have. Identifying these general characteristics (large axons, extensive arbors, location in the spinal cord) may also provide some useful hints as to which neurons are targeted in humans. While the identification of other candidates would be necessary in other animal models, this work provides a stepping point for further investigation.

\subsection{CONCLUSION}

This chapter further characterized the effects of developmental DomA exposures on the startle response. The startle response circuit is well characterized, and the neural circuits that drive it are well known. Utilizing this knowledge, I was able to identify specific neural populations that may be more sensitive to exposure to DomA. Furthermore, this study illustrates the potential of using the startle response circuit as a tool to identify neuronal populations targeted by toxin or toxicant exposures. 


\section{FUNDING}

This research was supported by the MIT/WHOI Academic Programs Office, Oceans Venture Fund, the Woods Hole Sea grant (NA14OAR4170074), and the Woods Hole Center for Oceans and Human Health (NIH - P01ES021923 and NSF - OCE-1314642 to MEH and NA, P01ES021923-04S1 to JMP, NIH - P01ES028938 and NSF - OCE-1840381 to MEH and NA)

\section{ACKNOWLEDGEMENTS}

I would like to thank: Hanny E. Rivera for her advice on the statistical analysis, Harold Burgess (NIH) for providing the FLOTE software for startle kinematic analysis, Ian T. Jones for measuring the vibrational output of the startle apparatus, Benjamin G. Merrick for his help designing and building the startle apparatus, Louis Kerr and Nadja Brun for microscopy training and advice (MBL microscopy facility), and the labs who generously provided me with zebrafish transgenic lines to make this work possible - Elwood Linney (Duke University), Sarah Kucenas (University of Virginia), Bruce Appel (University of Colorado, Denver), and David Lyons (University of Edinburg). 


\section{CHAPTER 3 FIGURES}

Figure 1: Domoic acid-exposed larvae are less responsive and preferentially perform LLC startles compared to controls when given auditory/vibrational stimuli.

(A) Distribution of fish that did one of three behaviors: 1) no response, 2) LLC-type startle, or 3) SLC-type startle per each stimulus intensity. Large dark dots represent the proportion of the population that performed one of the three behaviors within a given stimulus intensity. Individual points represent single fish that performed each behavior. Control fish are colored in teal and DomA fish colored in peach. Arrows denote pairwise comparisons between two behaviors. Asterisks denote whether the proportion of two behaviors (the given behavior versus LLC) was significantly different from controls.

(B) Relative Startle Bias Index was calculated for all fish that were responsive. Individual fish were provided with 4 replicate stimuli within a given stimulus intensity. Bias per individual was calculated as the (frequency of SLC - frequency of LLC)/ total responses. +1 represents the value in which all responses were SLC-type startles, and -1 represents the value in which all were LLC-type startles. Mean behavioral biases for a treatment group per stimulus intensity were graphed.

(C) Domoic acid-treated larvae plotted in B were further subdivided by myelin category (0-3), which was graphically depicted above the data. Startle bias per myelin phenotype was plotted. 
A.

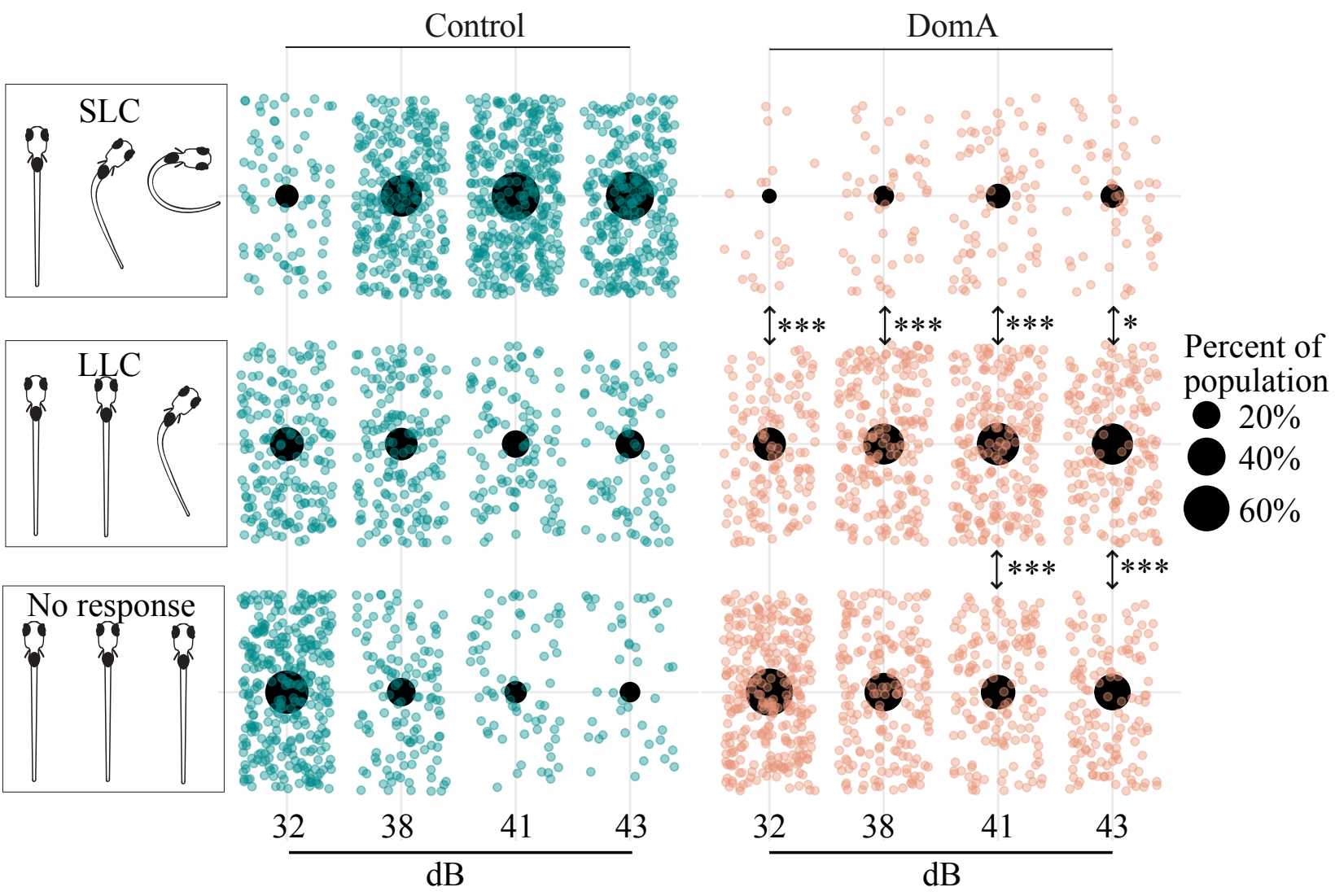

B.

C.

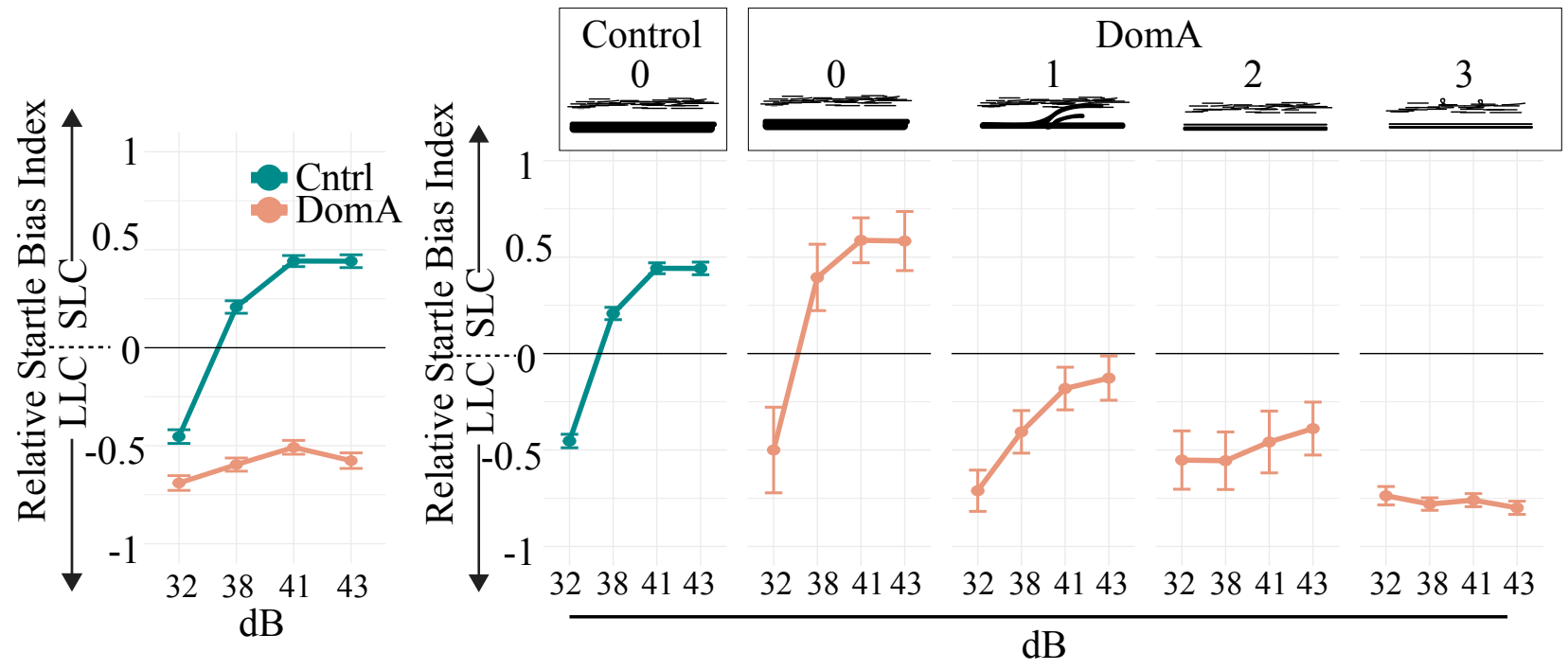


Figure 2: Domoic acid-exposed larvae have reduced bend angles and maximal angular velocities across all stimulus intensities tested when given auditory/vibrational stimuli.

(A) Bend angles during LLC-type startles with increasing stimulus intensities

(B) Maximal angular velocities during LLC-type startles with increasing stimulus intensities

(C) Bend angles during SLC-type startles with increasing stimulus intensities

(D) Maximal angular velocities during SLC-type startles with increasing stimulus intensities Individual points represent the median kinematic response of a single fish for up to 4 replicate stimuli.

$* * * \mathrm{p}<1$ e -6 compared to control responses. 
A.
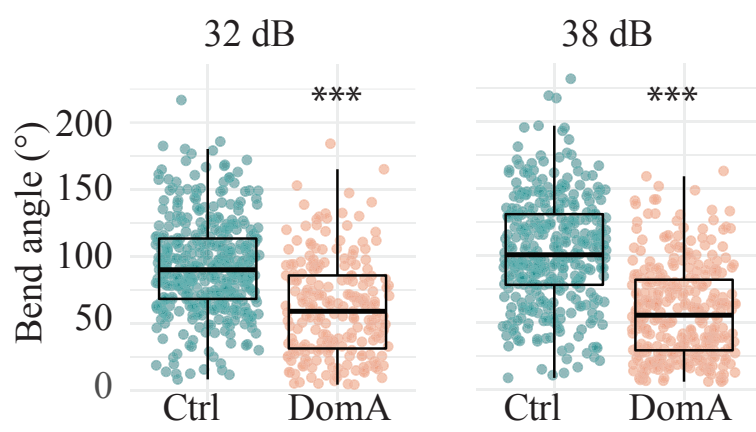

$41 \mathrm{~dB}$

$43 \mathrm{~dB}$

B.
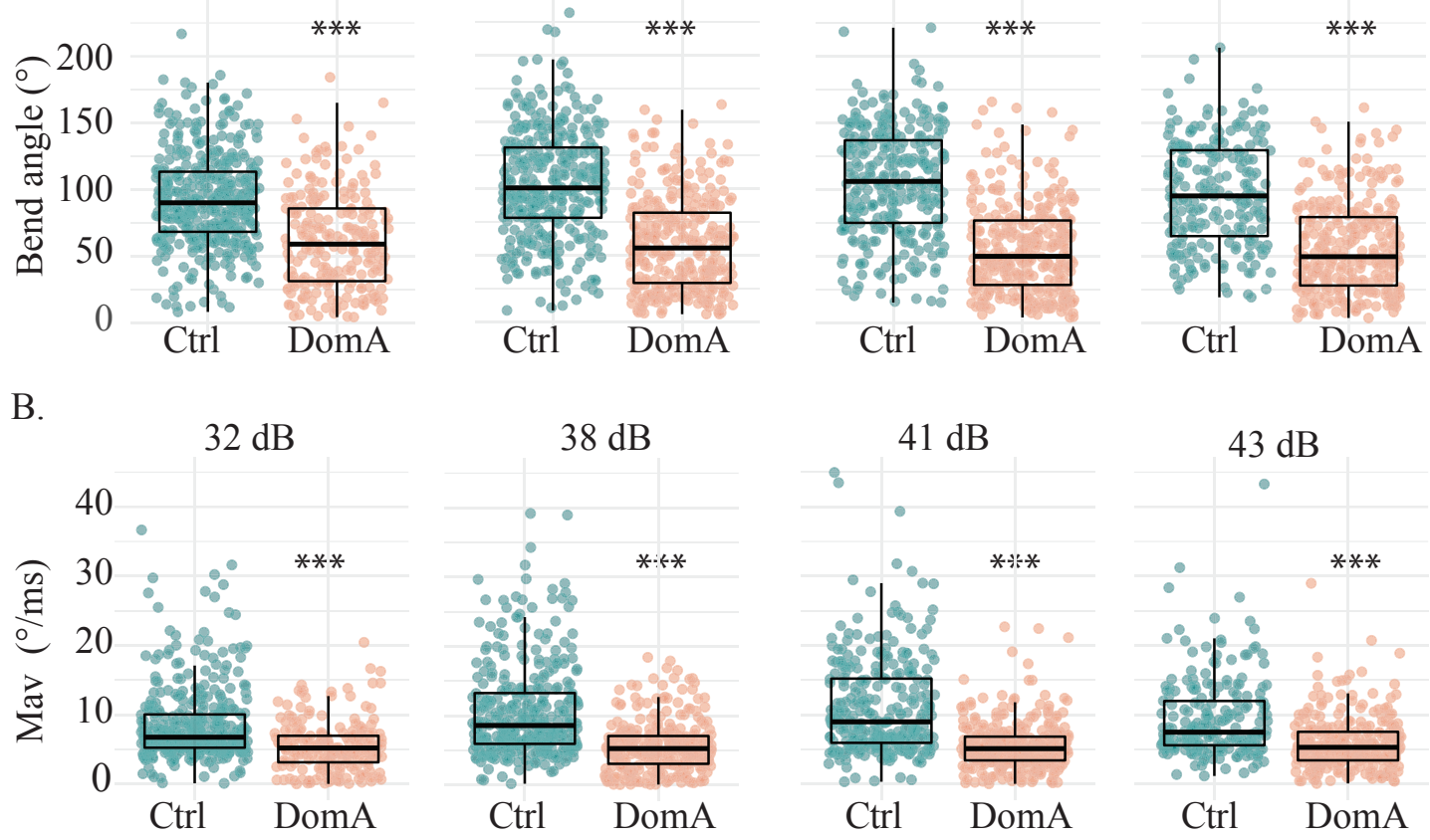

C.
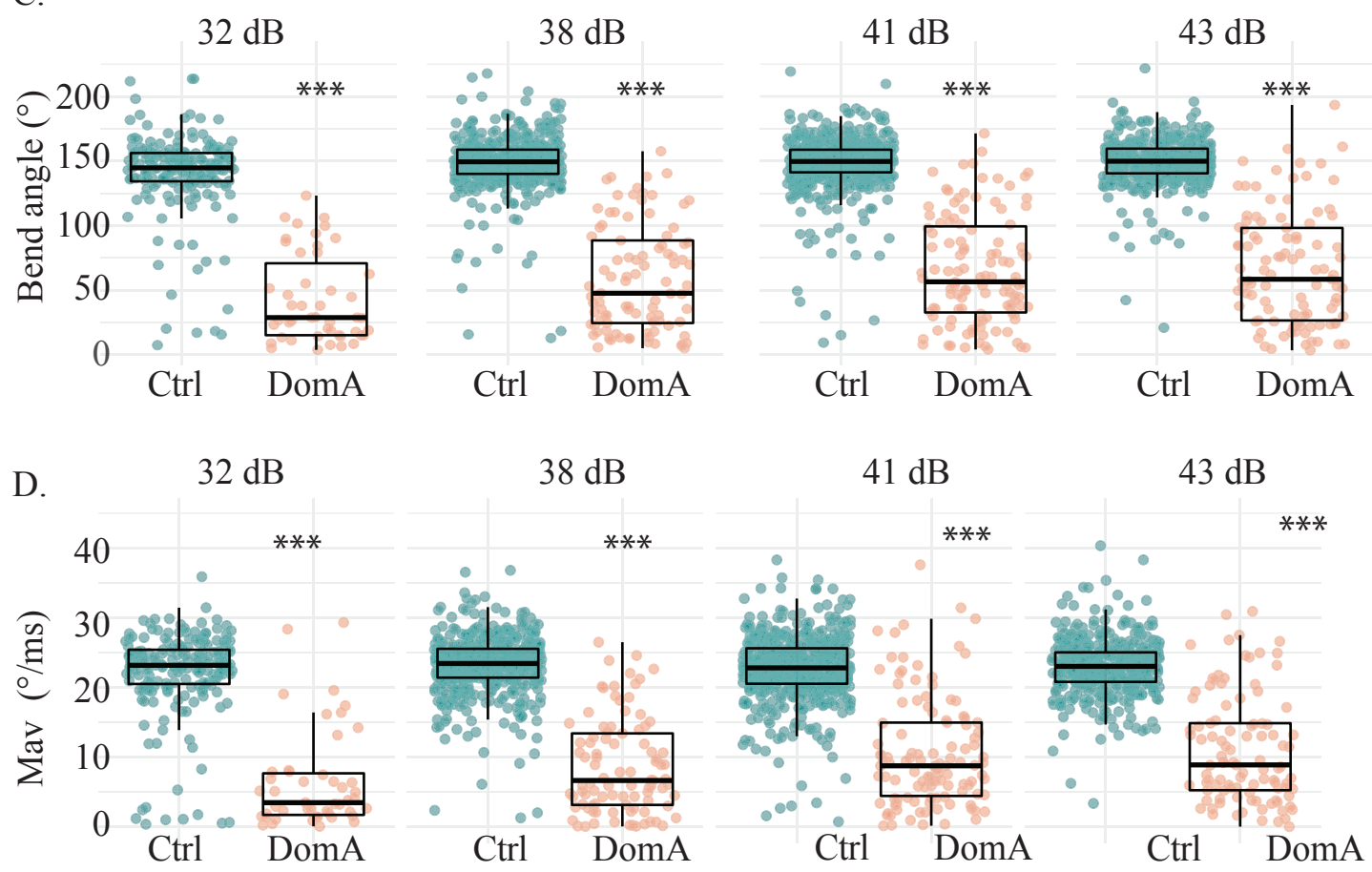
Figure 3: Kinematic deficits in LLC auditory/vibrational startle are correlated to myelin defects.

(A) DomA-treated fish were further subcategorized by myelin sheath defects. $0=$ control-like myelin sheaths to $3=$ the most severe myelin defect observed. Bend angles during LLC-type startles were plotted with increasing stimulus intensities.

(B) Maximal angular velocities during LLC-type startles with increasing stimulus intensities. $*=\mathrm{p}<0.05, * *=\mathrm{p}<0.001, * * *=\mathrm{p}<0.0001$ compared to control. 

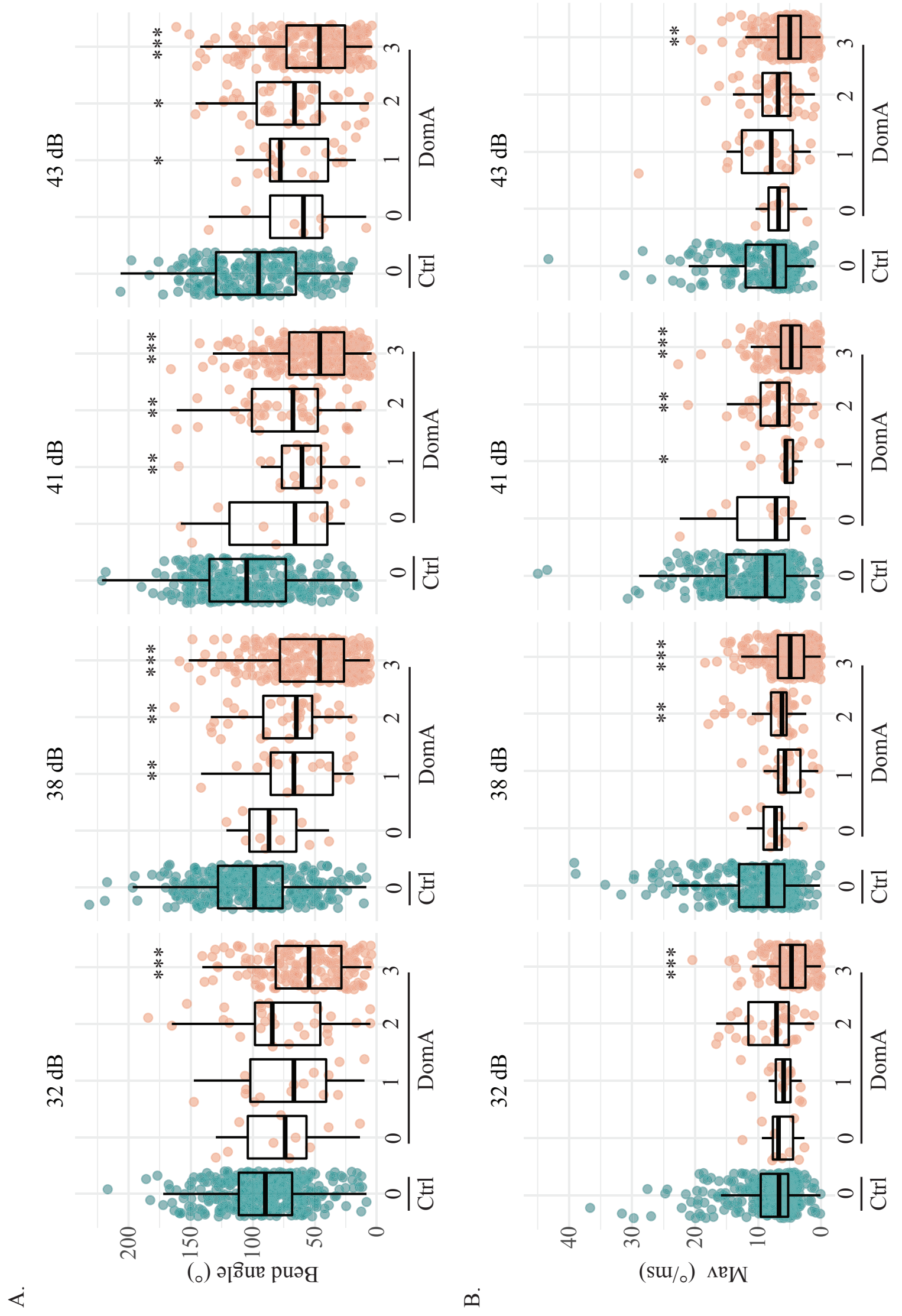
Figure 4: Kinematics deficits in SLC auditory/vibrational startle are correlated to myelin defects.

(A) DomA-treated fish were further subcategorized by myelin sheath defects. $0=$ control-like myelin sheaths to $3=$ the most severe myelin defect observed. Bend angles during SLC-type startles were plotted with increasing stimulus intensities.

(B) Maximal angular velocities during SLC-type startles with increasing stimulus intensities. $*=\mathrm{p}<0.05, * *=\mathrm{p}<0.001, * * *=\mathrm{p}<0.0001$ compared to control. 

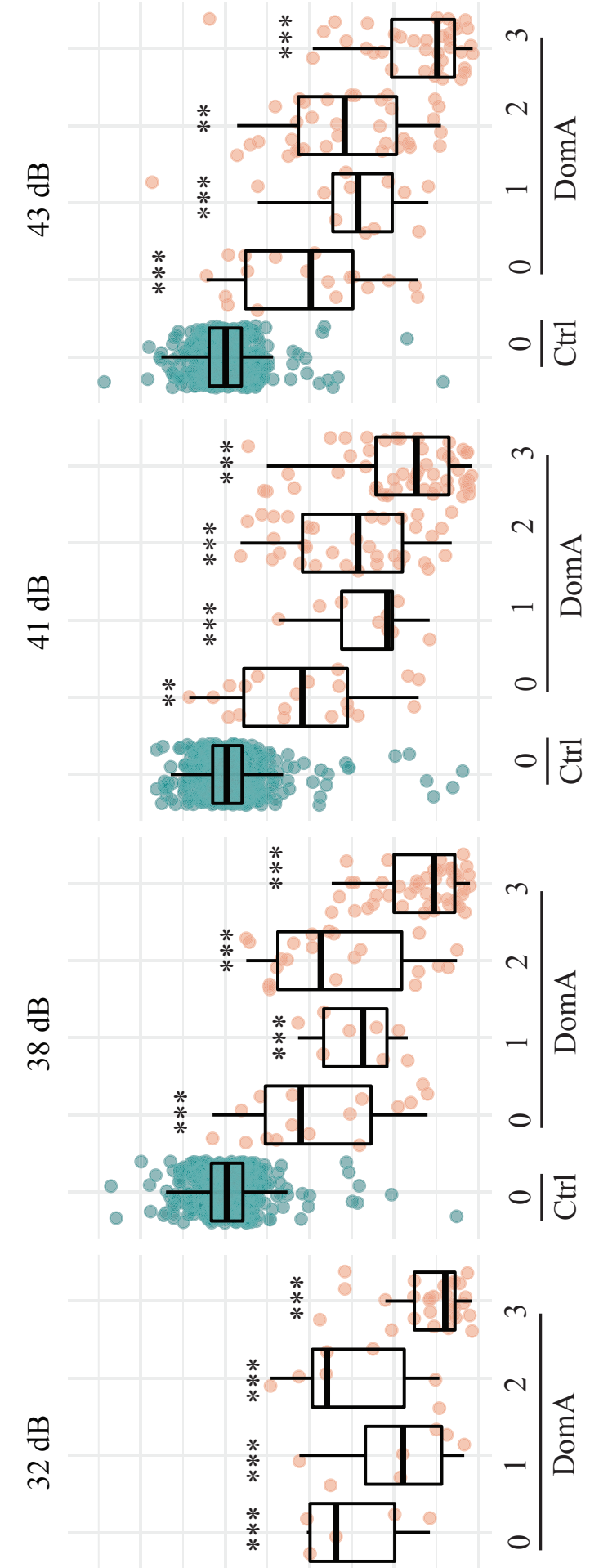

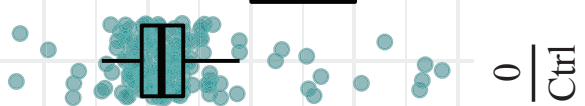

\& $\&$ in

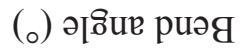
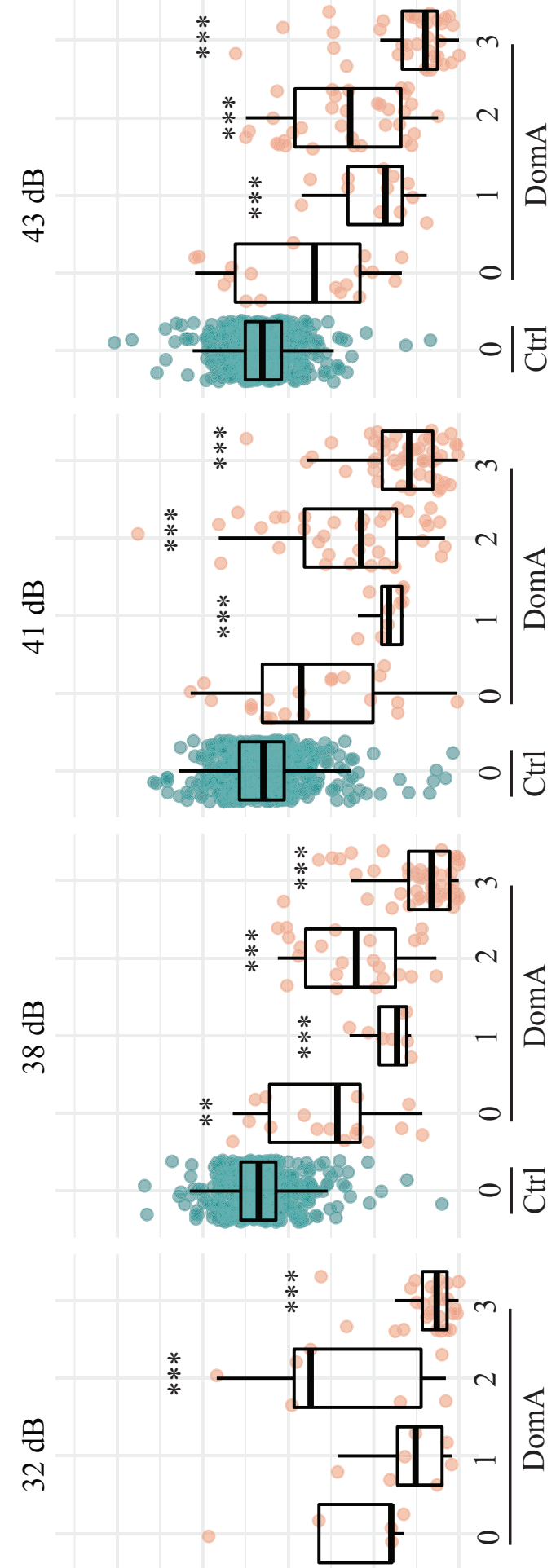

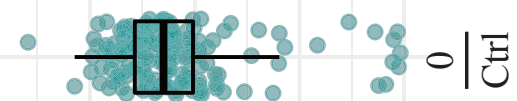

유 유 으 0 $(\mathrm{su} / \%) \Lambda \mathcal{E} \mathrm{W}$ 
Figure 5: Domoic acid-exposed larvae have aberrant startle responses to direct electrical stimulation.

(A) Larvae were head-mounted in agar and positioned rostral-caudally to the electrodes. Control larvae were mounted on the left, DomA-exposed larvae on the right. Larvae were then provided with a $4.4 \mathrm{~V} / \mathrm{cm}, 2$ millisecond $(\mathrm{ms})$ square pulse. Their tail movements were captured using a high-speed video camera.

(B) Percent responsiveness of individual larvae to 7 identical electric field pulses. Points represent the percent of times an individual fish responded to replicate stimuli. 69/74 of the control population responded $100 \%$ of the time while $47 / 74$ of DomA-treated population responded $0 \%$ of the time.

(C) Latency distributions for control and DomA-treated fish. Count represents the number of fish that have latencies within each of the $2 \mathrm{~ms}$ time bins. The majority of the control fish responded within $2 \mathrm{~ms}$ after stimulus was produced (51/74). Control fish had a median latency of 1-2 ms. Not shown=1 DomA-treated larvae which responded at $179 \mathrm{~ms}$.

(D) Maximum bend angle for control $(n=74)$ versus DomA-treated fish $(n=27)$. Each point represents the median response of an individual fish.

(E) Maximal angular velocity for control versus DomA-treated fish. Each point represents the median response of an individual fish.

$* * * \mathrm{p} \leq 0.0001$ indicates significant difference between DomA exposed larvae compared to control. 


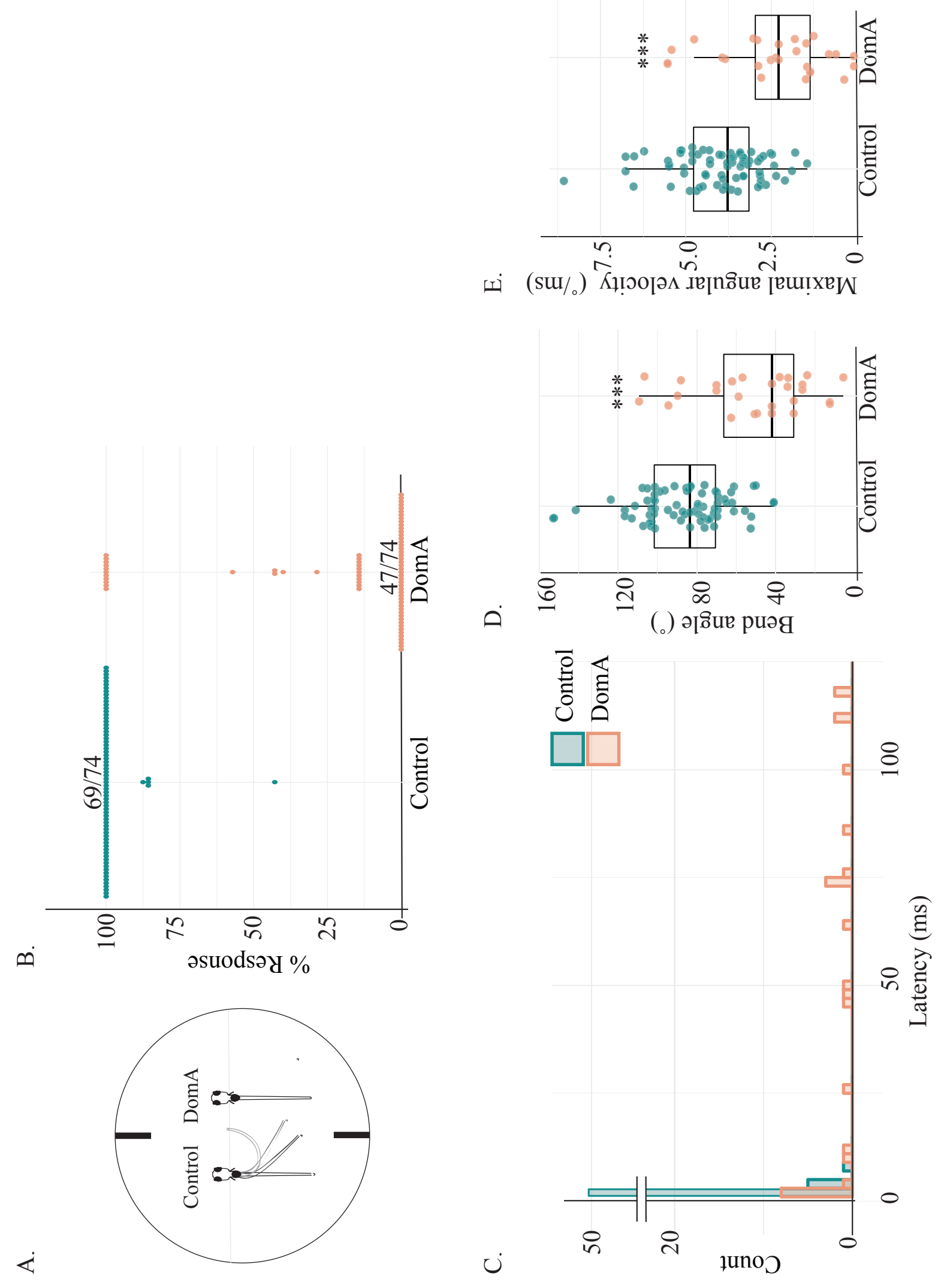


Figure 6: Severity in myelin defects is correlated with startle kinematic deficits from direct electric field stimulation.

(A) Representative widefield epifluoresence images of the myelin sheath phenotypes categorized, with (0) having no myelin defect to (3) having the most myelin severe defect observed. $\mathrm{C}=$ Control, $\mathrm{D}=$ DomA treated.

(B) Latency distributions for control and DomA-treated fish. The same data used in Figure 2C, but further categorized by myelin sheath imaging categories.

(C) Maximum bend angle for control $(n=74)$ versus DomA-treated fish $(n=25)$. Fish with myelin defects in category 1 were excluded from this analysis due to low sample size $(n=2)$.

(D) Maximal angular velocity for control versus DomA-treated fish.

(E) Percent responsiveness of individual larvae to 7 identical electric field pulses. Points represent the percent of times an individual fish responded to replicate stimuli. Ratios listed above a group of points represent the ratio of fish that responded to that stimulus over the total within that percentage bracket. Ratios were listed when all the individual points could not fit on the graph.

$* * * \mathrm{p} \leq 0.0001$ indicates significant difference between DomA exposed larvae compared to control. 


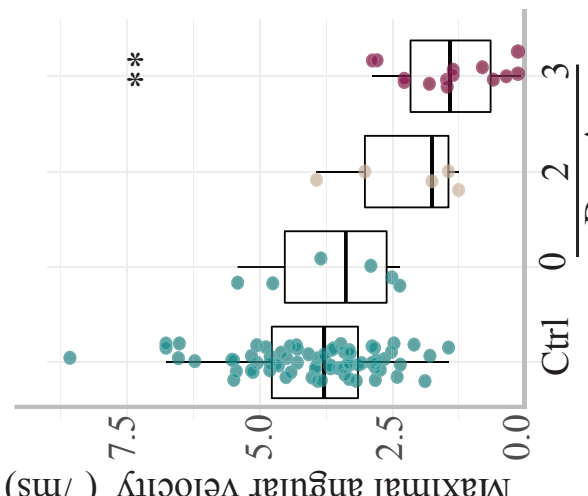

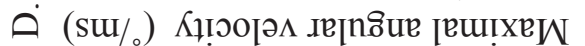
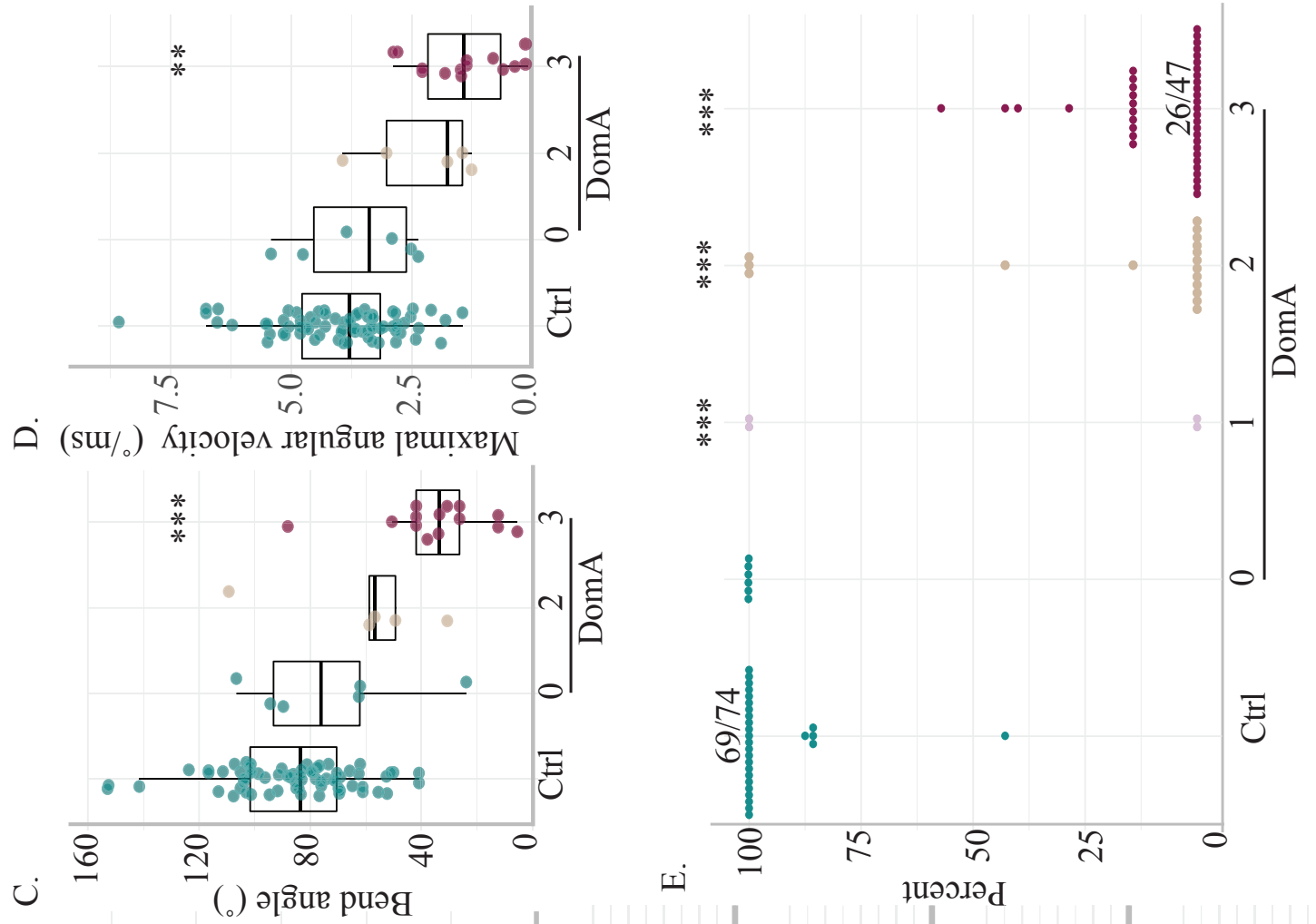

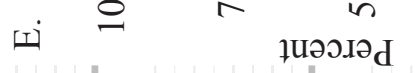

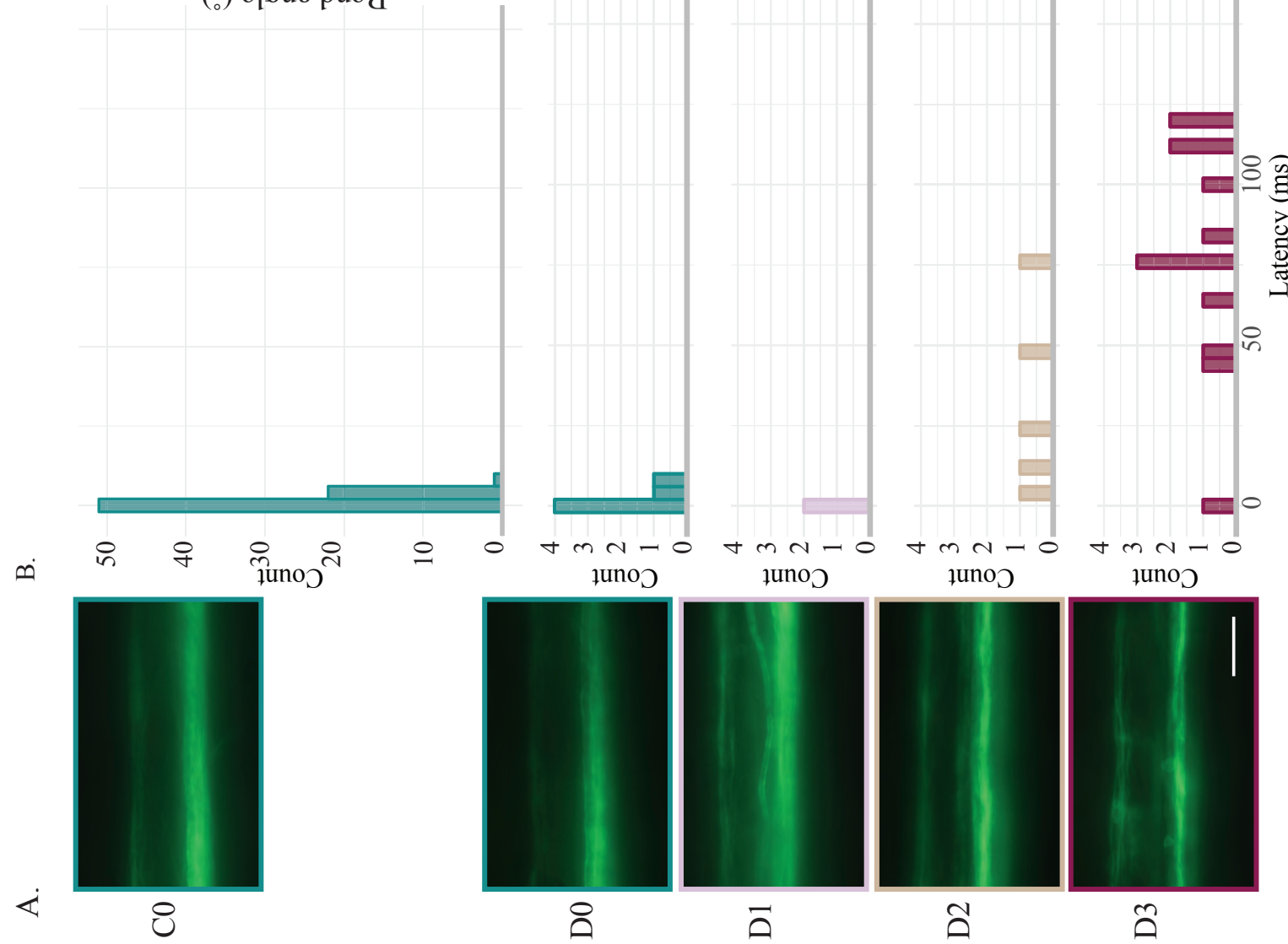


Figure 7: The sensory inputs required for the startle response appeared intact in domoic acid-exposed fish.

(A-B) DASPEI labeling of sensory neuromasts in 5 dpf larvae.

(A) Representative widefield fluorescence images of DASPEI-stained control and DomAexposed larvae.

(B) Diagram of a $5 \mathrm{dpf}$ larvae with head neuromasts colored in teal and trunk neuromasts colored in peach. Head and trunk neuromast counts for control and DomA-exposed. Single points represent individual larvae. Black bar represents standard error of the mean (SE).

For cranial region, control - mean $=19, \pm 2(\mathrm{SD})$, DomA - mean $=19 \pm 3(\mathrm{SD})$

For trunk region, control - mean $=17, \pm 2(\mathrm{SD}), \mathrm{DomA}-$ mean $=17 \pm 3(\mathrm{SD})$

(C-E) Imaging of sensory ganglia in $5 \mathrm{dpf} \operatorname{Tg}$ (cntn $1 b$ :EGFP-CAAX) larvae.

(C) Diagram of a laterally mounted $\operatorname{Tg}$ (cntn $1 b: E G F P-C A A X)$ larvae, with green boxes that indicate approximate areas imaged.

(D) Representative confocal images from the cranial region of laterally mounted $T g$ (cntn 1b:EGFP-CAAX) control $(\mathrm{n}=26)$ and DomA-exposed $(\mathrm{n}=35)$ larvae. Inner ear is outlined in teal.

(E) Representative confocal images from anterior spinal cord in laterally mounted $T g$ (cntn 1b:EGFP-CAAX) control $(\mathrm{n}=27)$ and DomA-exposed $(\mathrm{n}=35)$ larvae. $\mathrm{SAG}=$ statoacoustic ganglion, $\mathrm{PLL}=$ peripheral lateral line. Scale bar: $100 \mu \mathrm{m}$. 


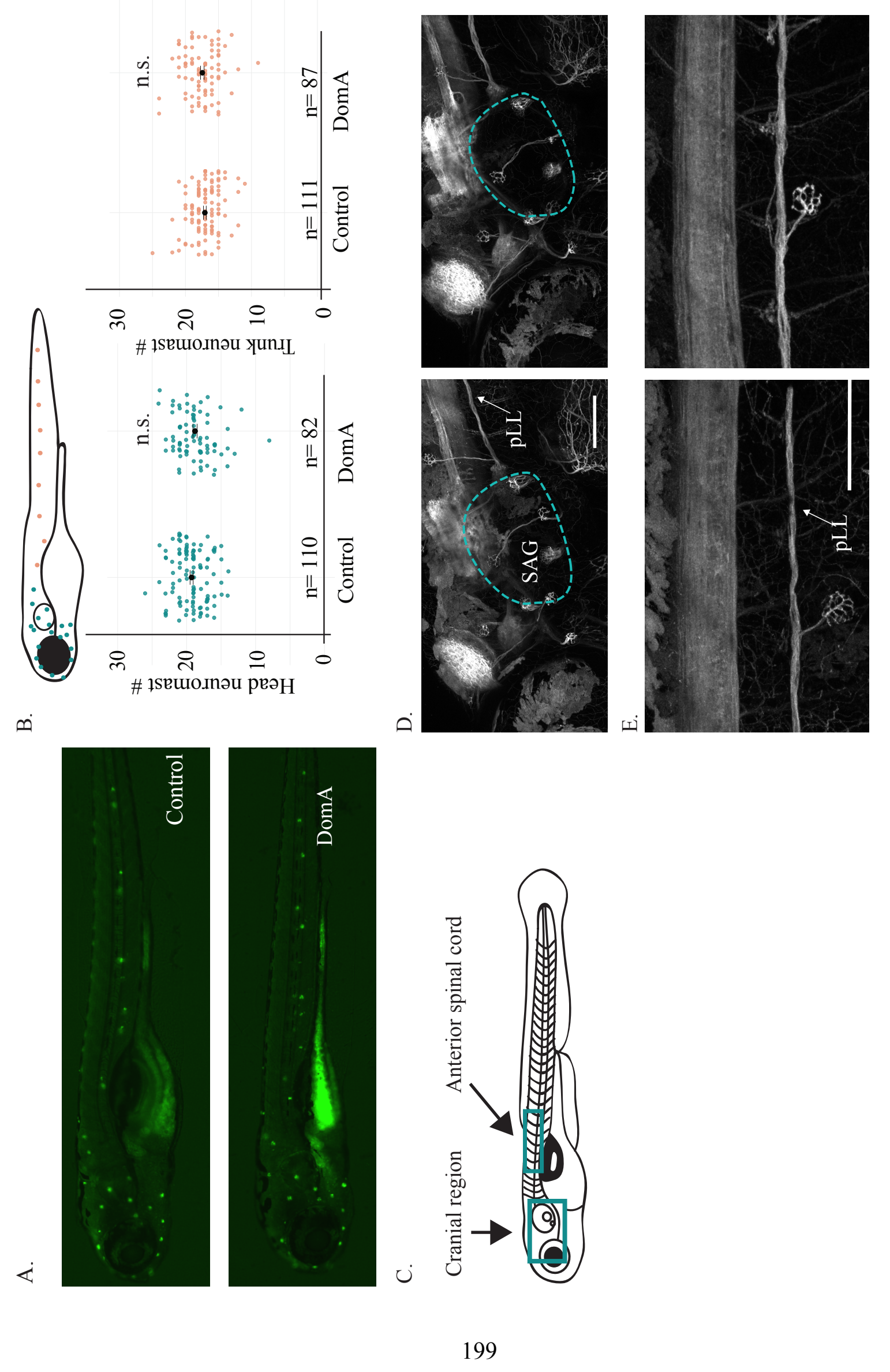


Figure 8: The majority of reticulospinal neurons required for startle responses are absent in domoic acid-exposed larvae.

(A) 7 dpf larvae were backfilled with Texas Red dextran through spinal cord transections. The images represent the range of phenotypes observed in control and DomA-injected fish. Teal arrows mark Mauthner cells and magenta arrows mark backfilled neurons in rhombomere 5 (r5) and rhombomere (r6).

(B) Mauthner cells on the two lateral sides were scored per fish. A majority of DomA-exposed fish (51 out of 60) did not have any Mauthner cells.

(C) Other reticulospinal neurons involved in startle responses (MiD2cm, MiD3) are located in $\mathrm{r} 5$ and r6. The presence of any neuron backfilled in r5 and r6 on the two lateral sides was scored. A majority of DomA-exposed fish had one or no neurons that were backfilled in r5 and r6 (44 out of 60). Scale bar $=100 \mu \mathrm{m}$. 

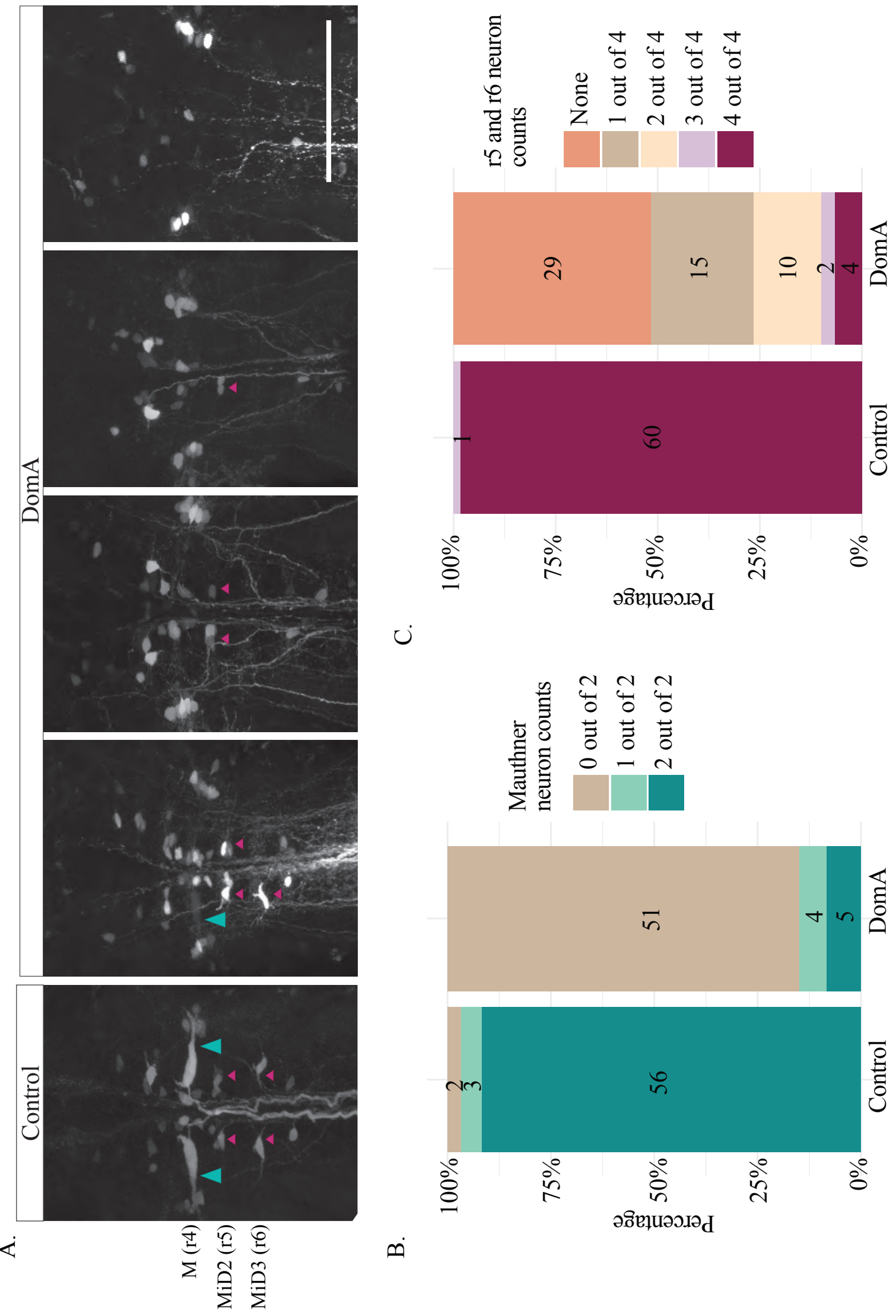


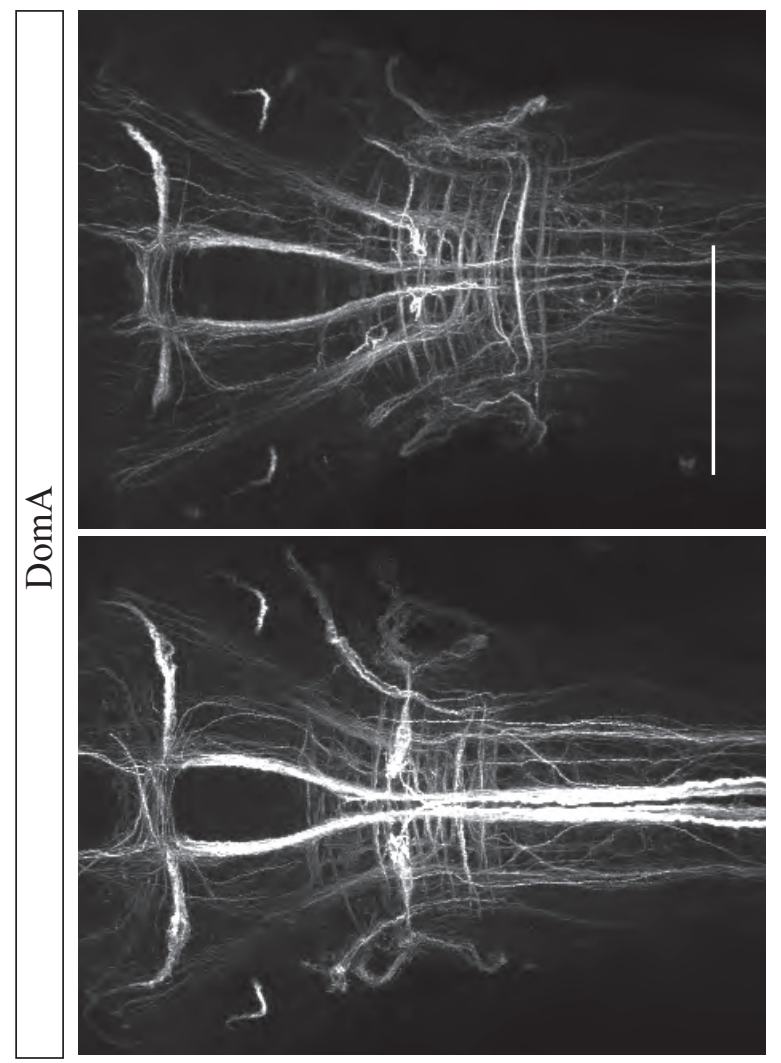

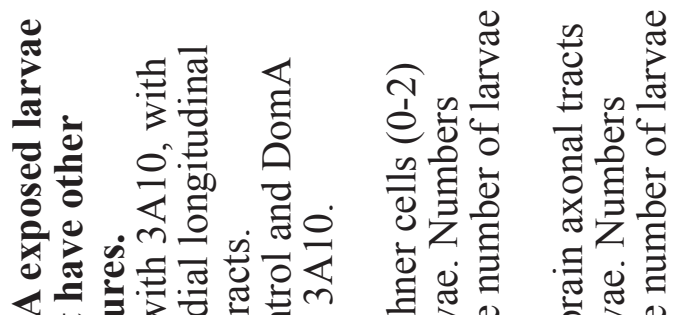

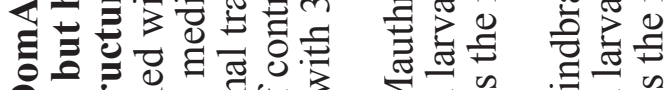

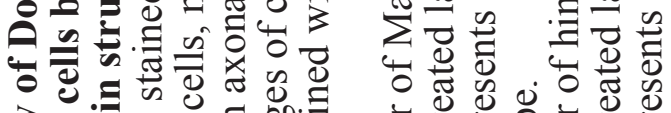
已.

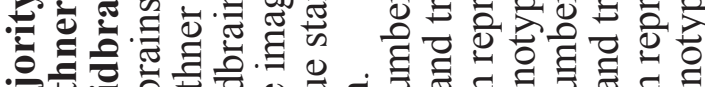

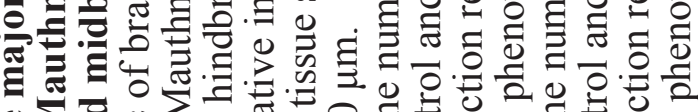

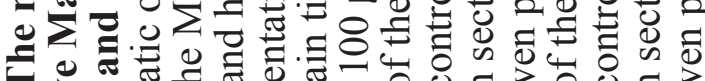
F ดั

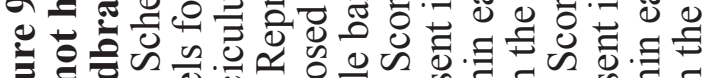

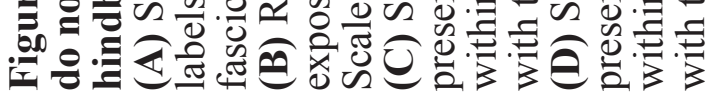

$\dot{n}$
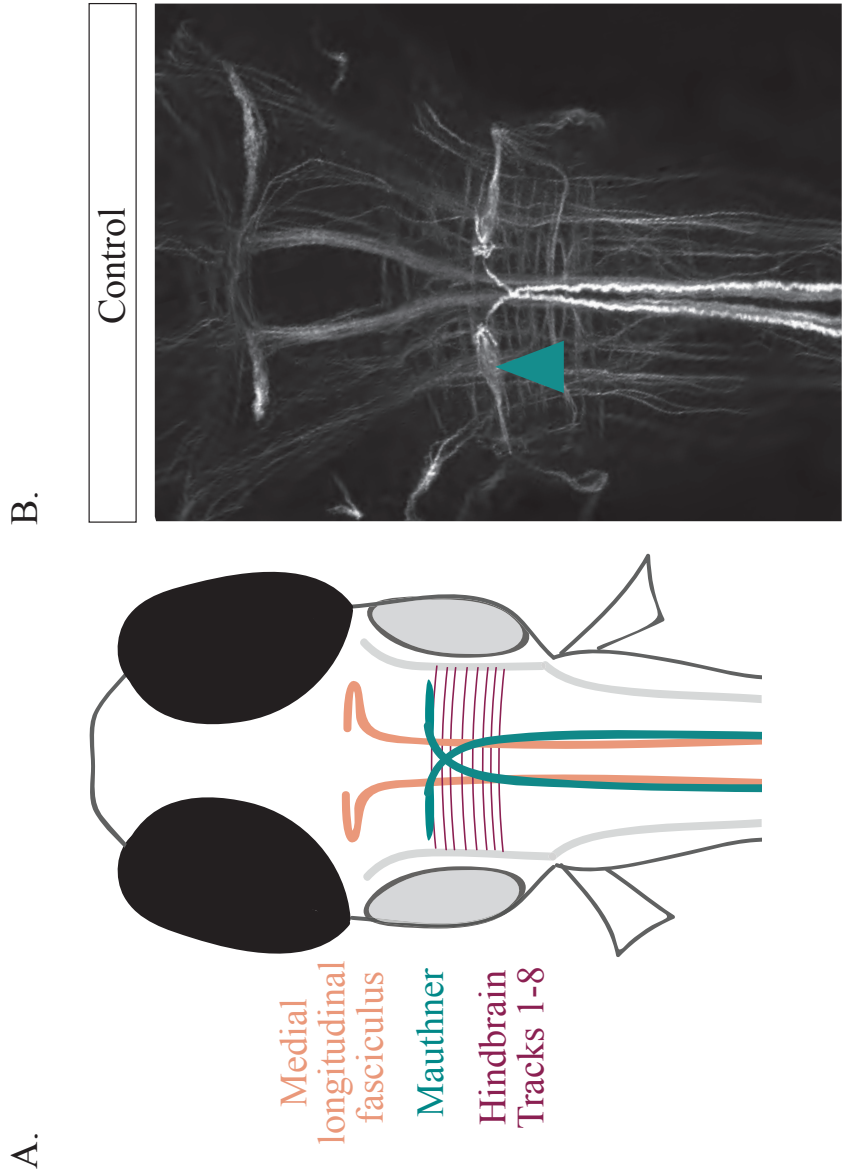
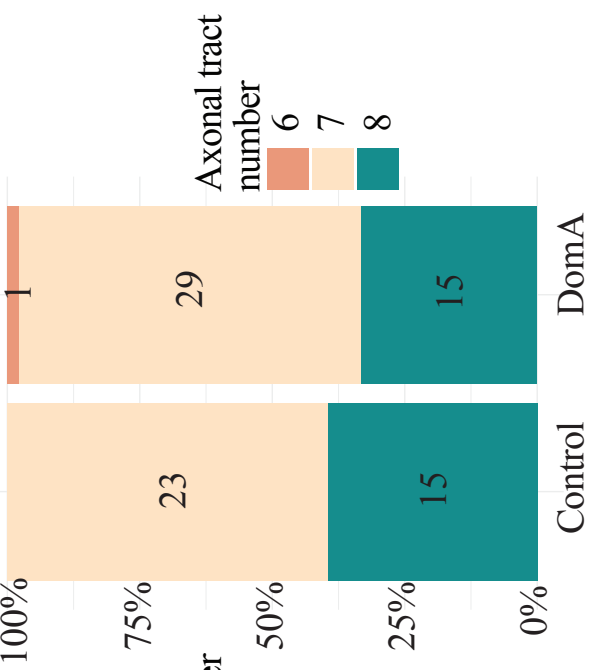

离离
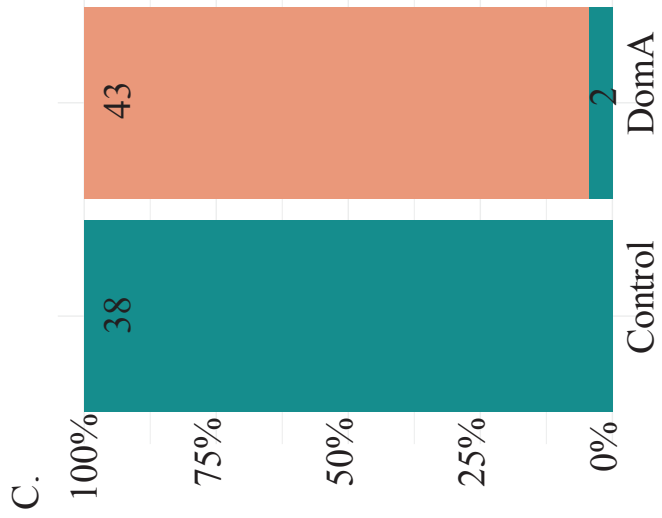


\section{Figure 10: Domoic acid exposure reduces axon collateral branching in primary motor}

neurons.

(A-B) Immunostaining for acetylated tubulin in $2.5 \mathrm{dpf}$ embryos

(A) Diagram of a 2.5 dpf larvae laterally mounted and immunostained with anti-acetylated tubulin. Anatomical features of interest are highlighted.

(B) Immunostaining with anti-acetylated tubulin at $2.5 \mathrm{dpf}$. DomA-exposed embryos $(\mathrm{n}=34)$ had visible caudal primary $(\mathrm{CaP})$ motor neuron axons, sensory neuron cell bodies, and peripheral lateral lines that were indistinguishable from controls $(n=31)$.

(C-D) Imaging of primary motor neuron branching in $2.5 \mathrm{dpf} \operatorname{Tg}$ (cntn 1 b:EGFP-CAAX) embryos

(C) Representative images from $5 \mathrm{dpf} T g(c n t n 1 b: E G F P-C A A X)$ controls and DomA-exposed larvae. Green arrow points to motor neuron axons that were traced to estimate the total axonal length in a single caudal primary motor neuron within a fish. Tracings are false colored. The main primary motor neuron axon was traced in green, and the axon collaterals were traced with magenta.

(D) Quantification of total lengths from axonal tracings (inclusive of the main motor neuron in green and axon collaterals in magenta). Each point represents a single axon in one fish (control $n=44$, DomA $n=45$ ). Error bars represent standard error of the mean. $* * *=\mathrm{p}<2.2 \mathrm{e}-16$. Scale bars $=100 \mu \mathrm{m}$. 


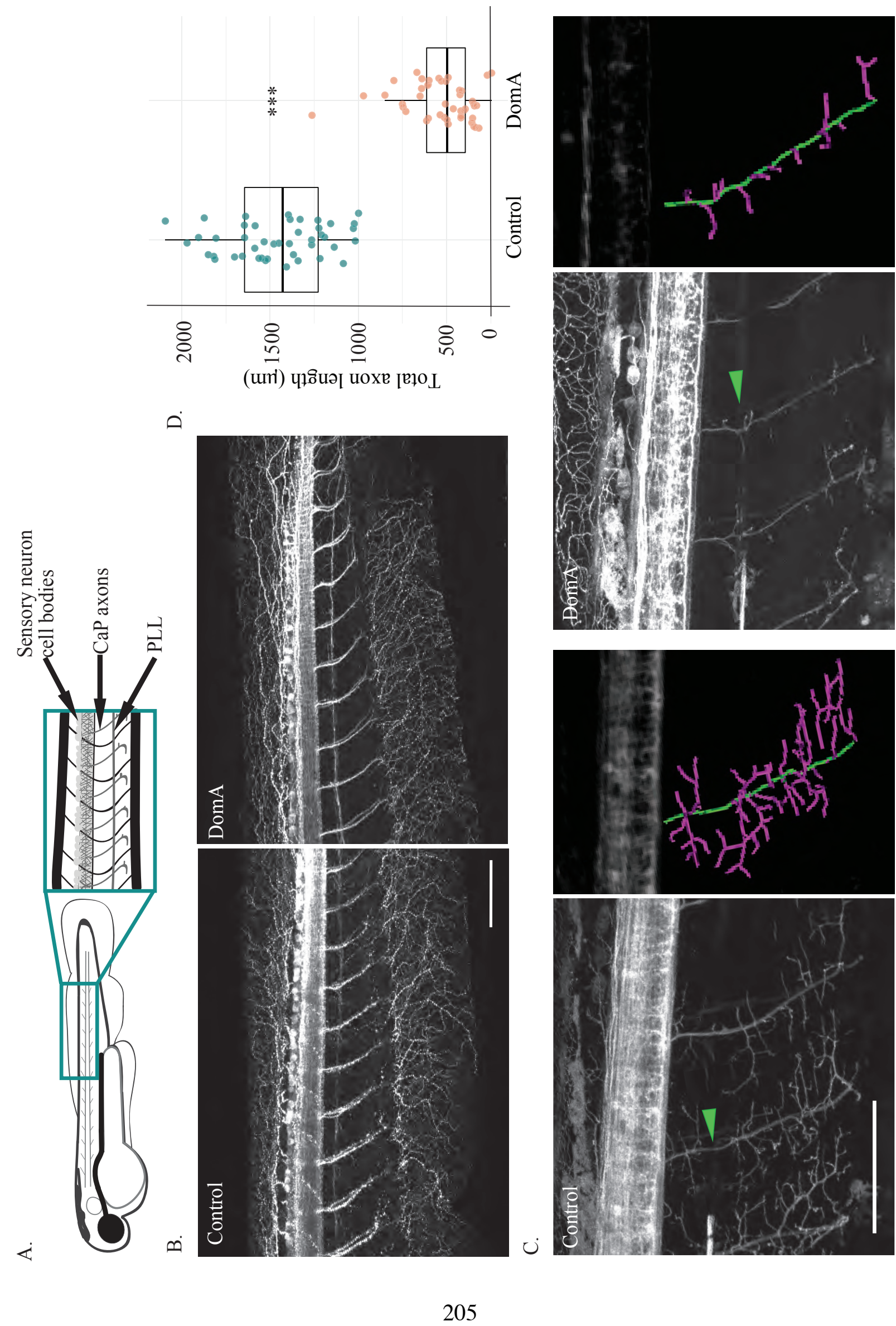


A.

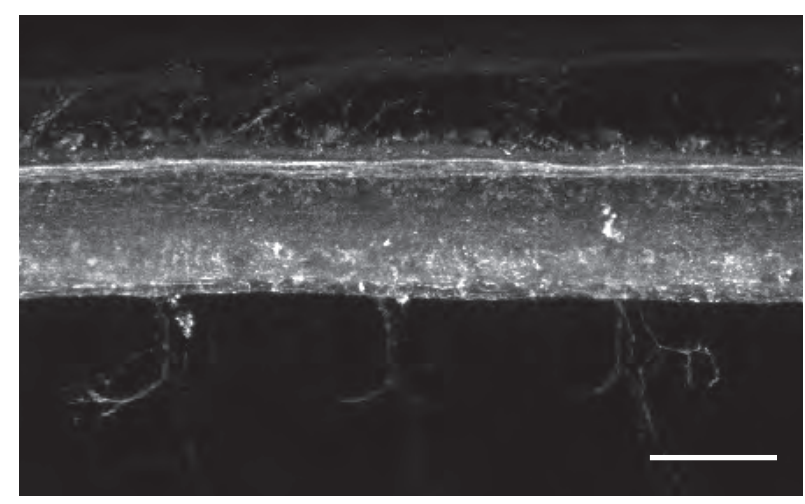

B.

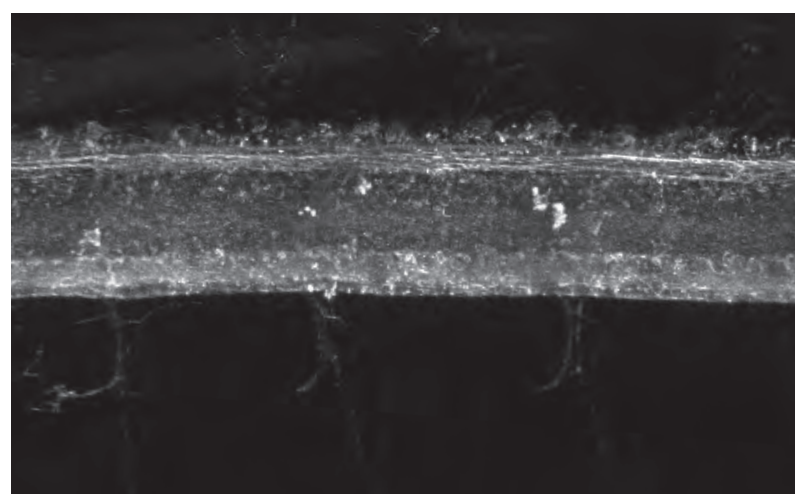

Figure 11: DomA does not appear to reduce the overall population of dorsal spinal neurons or neural precursors.

(A) $\mathrm{Tg}(-8.4 \mathrm{kbneurog} 1: \mathrm{mEGFP})$ live imaging in $5 \mathrm{dpf}$ control larvae $(\mathrm{n}=16)$ and in (B) DomA-exposed larvae (2 dpf injected) $(\mathrm{n}=14)$. Scale bar $=50 \mu \mathrm{m}$. 


\section{CHAPTER 3 TABLES}

Table 1: Percentage of fish that performed LLCs, SLCs, or did not respond within a given treatment and to a selected stimulus intensity

\begin{tabular}{|c|c|c|c|c|c|c|c|c|}
\hline Stimulus & \multicolumn{4}{|c|}{ Control } & \multicolumn{4}{|c|}{ DomA } \\
\hline Intensity & $\%$ LLC & $\%$ NR & $\%$ SLC & $\mathrm{N}$ & $\%$ LLC & $\% \mathrm{NR}$ & $\% \mathrm{SLC}$ & $\mathrm{N}$ \\
\hline $32 \mathrm{~dB}$ & $31.9 \%$ & $54.2 \%$ & $13.9 \%$ & 583 & $30.2 \%$ & $65.2 \%$ & $4.6 \%$ & 460 \\
\hline $38 \mathrm{~dB}$ & $29.1 \%$ & $21.9 \%$ & $49.0 \%$ & 567 & $47.8 \%$ & $41.9 \%$ & $10.4 \%$ & 454 \\
\hline $41 \mathrm{~dB}$ & $19.9 \%$ & $12.7 \%$ & $67.4 \%$ & 528 & $51.6 \%$ & $33.0 \%$ & $15.5 \%$ & 446 \\
\hline $43 \mathrm{~dB}$ & $22.3 \%$ & $10.3 \%$ & $67.4 \%$ & 417 & $48.5 \%$ & $37.1 \%$ & $14.4 \%$ & 361 \\
\hline
\end{tabular}


Table 2: Multinomial logistic regression model for distribution of startle behaviors in DomA and Control fish when given a $32 \mathrm{~dB}$ stimulus

\begin{tabular}{cccc}
\hline & \multicolumn{2}{c}{ Coefficients } & \\
\cline { 2 - 3 } $\begin{array}{c}\text { Behavior } \\
\text { type }\end{array}$ & $\begin{array}{c}\text { (Intercept) -- Control, } \\
\text { LLC }\end{array}$ & DomA & $\begin{array}{c}\text { Odds } \\
\text { Ratios }\end{array}$ \\
\hline NR & $0.530(\mathrm{p}=9.8 \mathrm{e}-09)$ & $0.239(\mathrm{p}=0.083)$ & \\
SLC & $-0.832(\mathrm{p}=4.2 \mathrm{e}-10)$ & $-1.058(\mathrm{p}=8.5 \mathrm{e}-05)$ & \\
\hline
\end{tabular}

Notes: $\mathrm{NR}=$ no response, $\mathrm{SLC}=$ Short latency c-bend startles, $\mathrm{LLC}=$ Long latency c-bend startles

Table 3: Multinomial logistic regression model for distribution of startle behaviors in DomA and Control fish when given a $38 \mathrm{~dB}$ stimulus

\begin{tabular}{cccc}
\hline & \multicolumn{2}{c}{ Coefficients } & \\
\cline { 2 - 3 } $\begin{array}{c}\text { Behavior } \\
\text { type }\end{array}$ & $\begin{array}{c}\text { (Intercept) -- Control, } \\
\text { LLC }\end{array}$ & DomA & $\begin{array}{c}\text { Odds } \\
\text { Ratios }\end{array}$ \\
\hline NR & $-0.286(\mathrm{p}=0.016)$ & $0.152(\mathrm{p}=0.32)$ & \\
SLC & $0.522(\mathrm{p}=1.1 \mathrm{e}-7)$ & $-2.05(\mathrm{p}<\mathrm{e}-13)$ & \\
\hline
\end{tabular}

Notes: $\mathrm{NR}=$ no response, $\mathrm{SLC}=$ Short latency c-bend startles, $\mathrm{LLC}=$ Long latency c-bend startles

Table 4: Multinomial logistic regression model for distribution of startle behaviors in DomA and Control fish when given an $41 \mathrm{~dB}$ stimulus

\begin{tabular}{|c|c|c|c|}
\hline \multirow[b]{2}{*}{$\begin{array}{c}\text { Behavior } \\
\text { type }\end{array}$} & \multicolumn{2}{|c|}{ Coefficients } & \multirow[b]{2}{*}{$\begin{array}{l}\text { Odds } \\
\text { Ratios }\end{array}$} \\
\hline & $\begin{array}{c}\text { (Intercept) -- Control, } \\
\text { LLC }\end{array}$ & DomA & \\
\hline NR & $-0.449(p=0.004)$ & $-0.448(p=2.2 e-5)$ & 0.64 \\
\hline SLC & $1.221(\mathrm{p}<\mathrm{e}-13)$ & $-1.204(\mathrm{p}<\mathrm{e}-13)$ & 0.30 \\
\hline
\end{tabular}

Notes: $\mathrm{NR}=$ no response, $\mathrm{SLC}=$ Short latency c-bend startles, $\mathrm{LLC}=$ Long latency c-bend startles

Table 5: Multinomial logistic regression model for distribution of startle behaviors in DomA and Control fish when given a $43 \mathrm{~dB}$ stimulus

\begin{tabular}{cccc}
\hline & \multicolumn{2}{c}{ Coefficients } & \\
\cline { 2 - 3 } $\begin{array}{c}\text { Behavior } \\
\text { type }\end{array}$ & $\begin{array}{c}\text { (Intercept) -- Control, } \\
\text { LLC }\end{array}$ & DomA & $\begin{array}{c}\text { Odds } \\
\text { Ratios }\end{array}$ \\
\hline NR & $-0.771(\mathrm{p}=2.9 \mathrm{e}-5)$ & $0.504(\mathrm{p}=0.02)$ & \\
SLC & $1.106(\mathrm{p}<\mathrm{e}-13)$ & $-2.319(\mathrm{p}<\mathrm{e}-13)$ & \\
\hline
\end{tabular}

Notes: $\mathrm{NR}=$ no response, $\mathrm{SLC}=$ Short latency c-bend startles, $\mathrm{LLC}=$ Long latency c-bend startles 
Table 6: Multinomial logistic regression model for distribution of startle behaviors in Control and DomA fish that are subcategorized by myelin phenotype (from a $43 \mathrm{~dB} \mathrm{~A} / \mathrm{V}$ stimulus)

\begin{tabular}{|c|c|c|c|c|c|c|c|c|c|}
\hline \multirow{3}{*}{$\begin{array}{l}\text { Beha- } \\
\text { vior }\end{array}$} & \multicolumn{5}{|c|}{ Coefficients } & \multicolumn{4}{|c|}{ Odds Ratios } \\
\hline & (Intercept) -- & \multicolumn{4}{|c|}{$\operatorname{DomA}$} & \multicolumn{4}{|c|}{ DomA } \\
\hline & 0 & 0 & 1 & 2 & 3 & 0 & 1 & 2 & 3 \\
\hline NR & $\begin{array}{c}-0.771 \\
(p=2.88 \text { e- }-05)\end{array}$ & $\begin{array}{c}0.995 \\
(p=0.15)\end{array}$ & $\begin{array}{c}0.717 \\
(p=0.057)\end{array}$ & $\begin{array}{c}-0.209 \\
(p=-.765)\end{array}$ & $\begin{array}{c}0.484 \\
(p=0.030)\end{array}$ & 2.7 & 2.0 & 0.8 & 1.6 \\
\hline SLC & $\begin{array}{c}1.106 \\
(\mathrm{p}<\mathrm{e} 10-13)\end{array}$ & $\begin{array}{c}0.073 \\
(p=0.90)\end{array}$ & $\begin{array}{c}-1.217 \\
(p=5.9 \text { e }-4)\end{array}$ & $\begin{array}{c}-1.393 \\
(p=0.012)\end{array}$ & $\begin{array}{l}-3.303 \\
(p<e-13)\end{array}$ & 1.1 & 0.3 & 0.3 & 0.04 \\
\hline
\end{tabular}

Notes: $\mathrm{Ctrl}=$ Control (0 ng vehicle Control). Numbers 0-3 denote myelin categories into which fish were classified prior to behavioral analyses. $0=$ phenotype indistinguishable from control to $3=$ most severe phenotype observed. See Methods and Supplemental Fig. 3 for details 


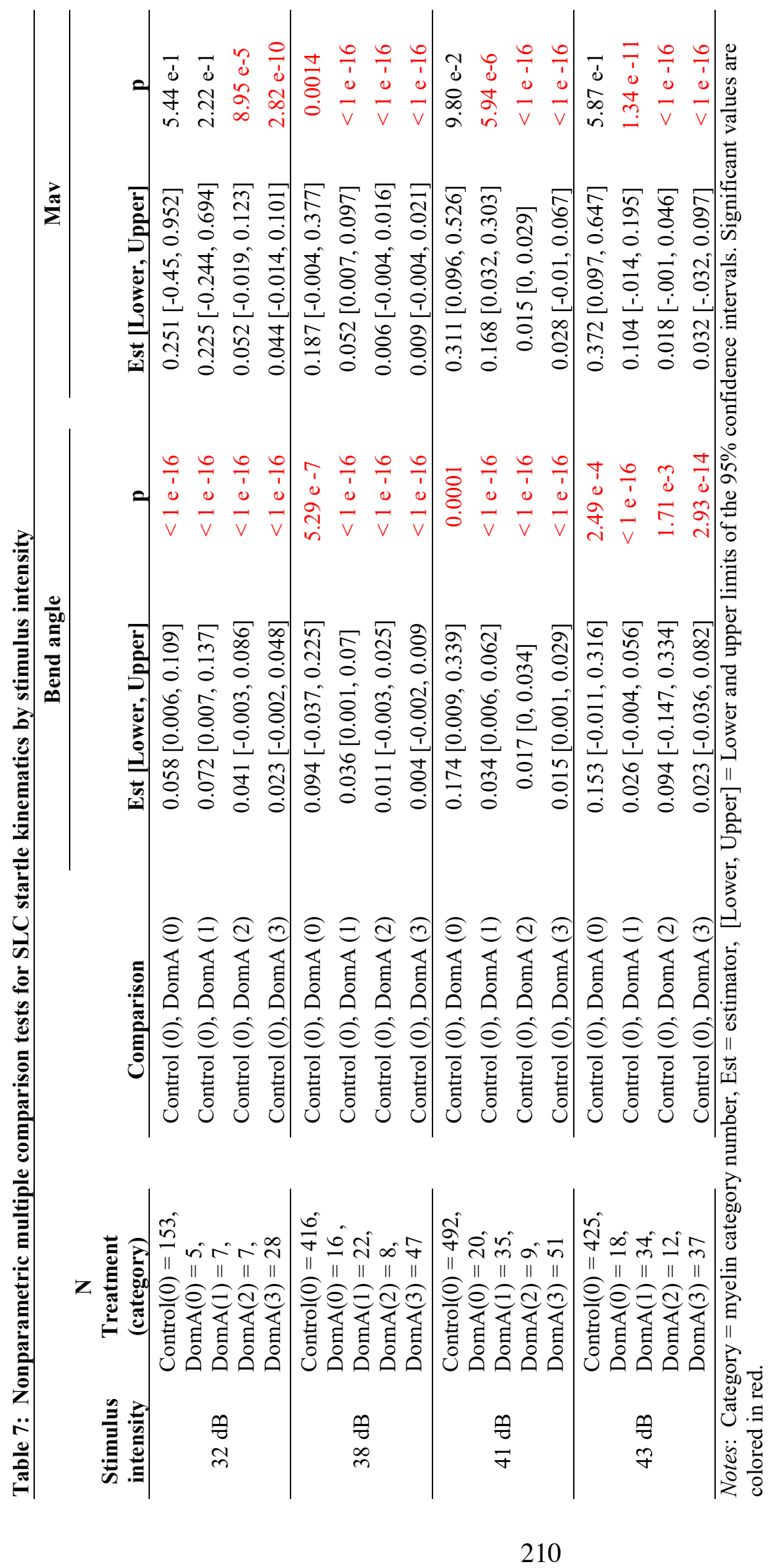




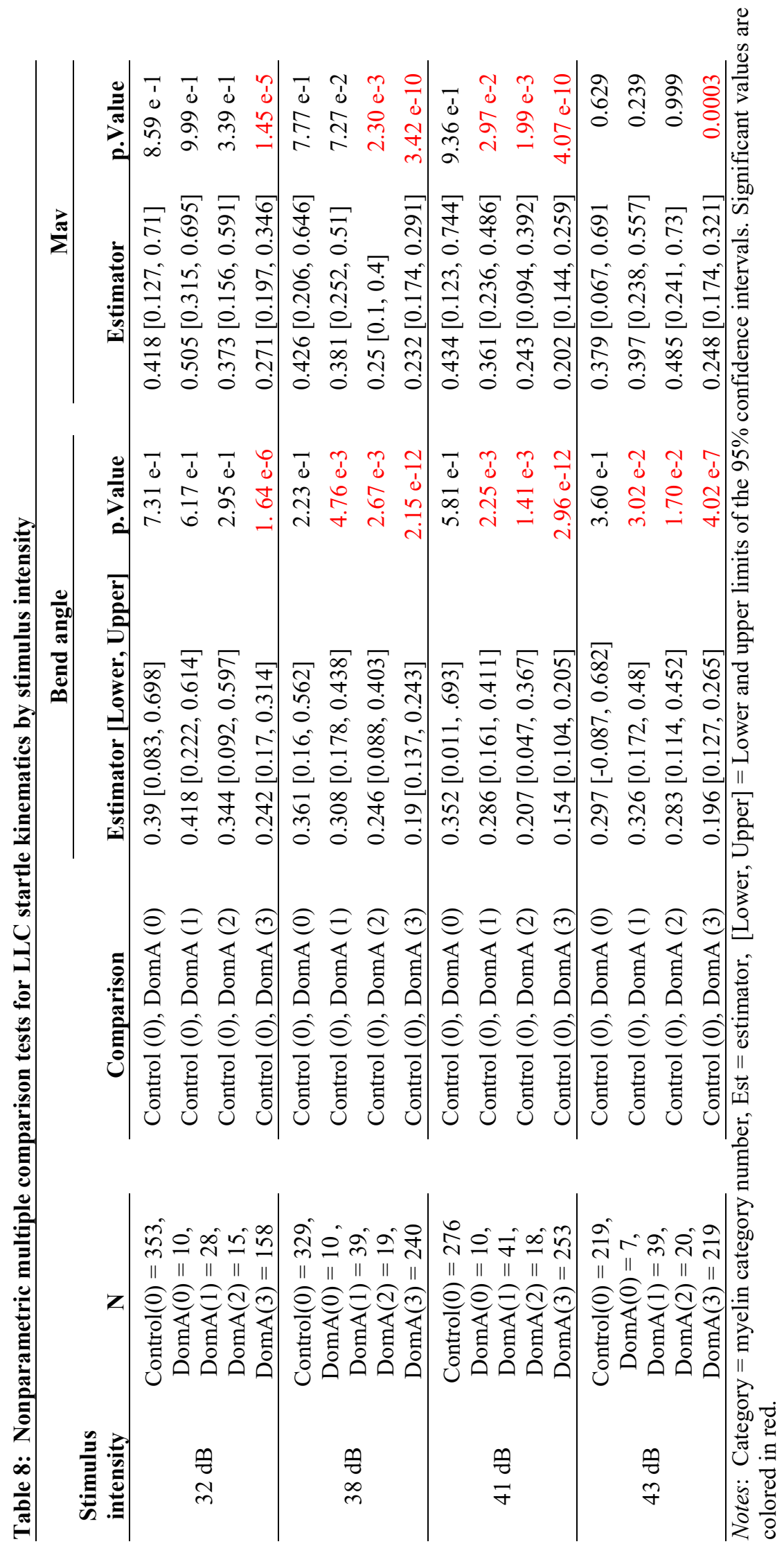




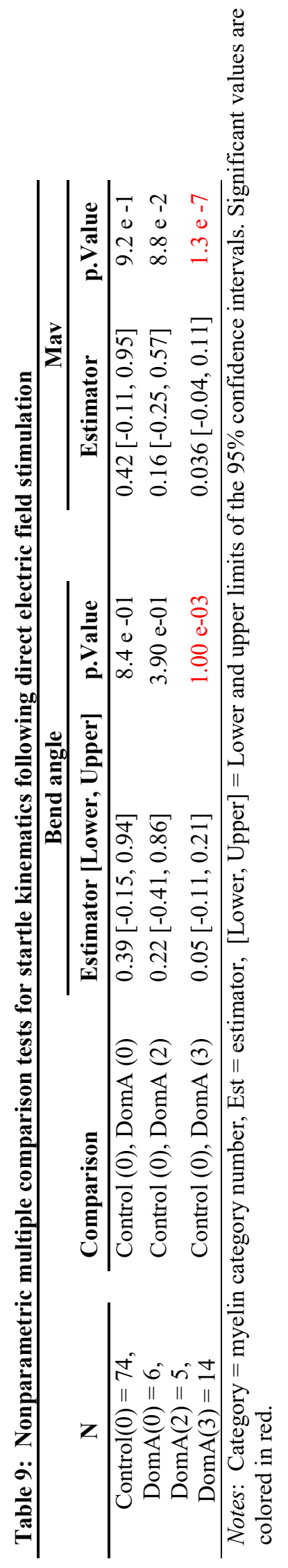




\section{CHAPTER 3 SUPPLEMENTAL INFORMATION}

A.

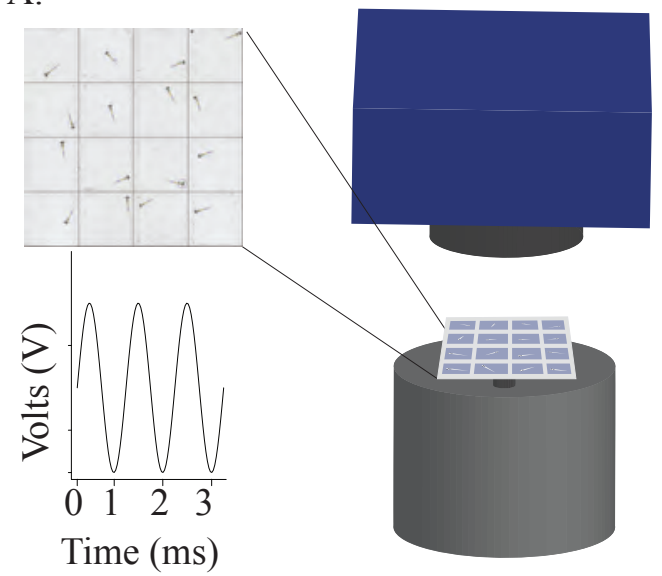

D.

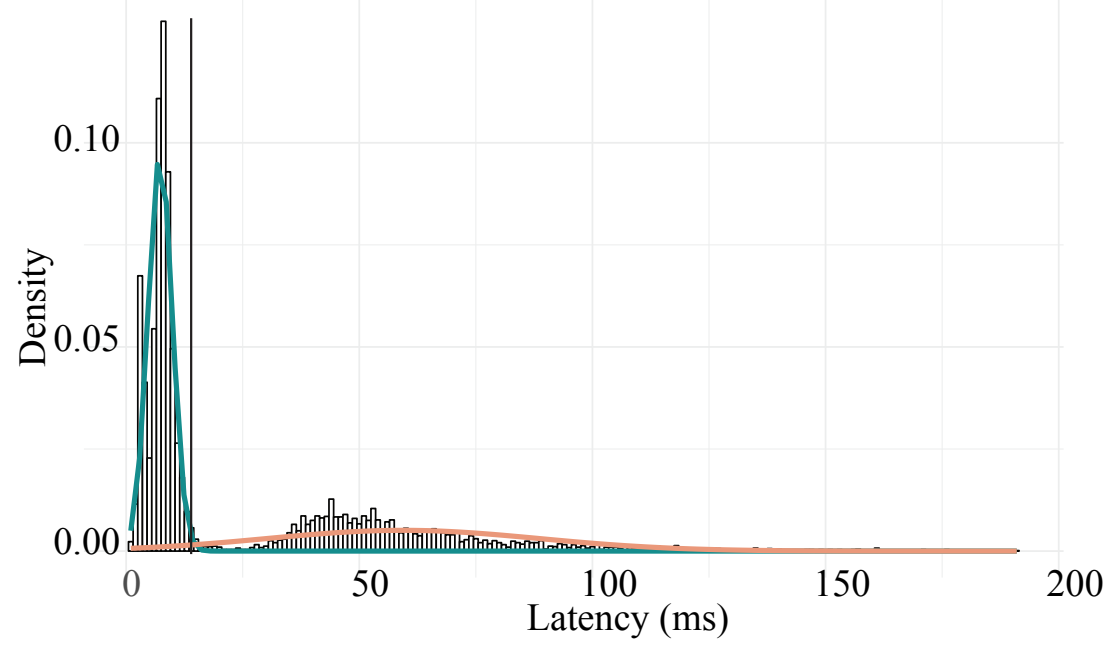

Supplemental Figure 1: Startle behavioral apparatus and behavioral classification (A) Diagram of apparatus used to assess startle responses to A/V stimuli. A vibrational exciter delivers a 3 millisecond, $1000 \mathrm{~Hz}$ pulse to 16-well plate that contains single larvae within individual wells. The amplitude of the pulse was varied to deliver a range of stimulus intensities. A high-speed camera captured startle responses at 1000 frames per second. (B) Graphical representation of the pulse sent to the vibrational apparatus. Each plate was given a total of 16 stimuli, with 4 intensities delivered 4 times each. The stimuli were spaced 20 seconds apart (interstimulus interval (ISI) of 20s).

(C) Temporal projections of the Mauthner-dependent short latency c-startle response (SLC) which initiates within $14 \mathrm{~ms}$ or less after the delivery of the stimuli. Temporal projections of the long latency c-startle response (LLC) which initiates more than 14 milliseconds after the stimulus.

(D) Density histogram of the latency distribution for control fish. Overlaid are two Gaussian curves that were fit for the data. The teal curve represents SLC responses, and peach curve represents LLC responses. The solid black vertical line at 14 milliseconds represents the cut-off by which there is a greater than $50 \%$ probability of a given data point belonging to either the modeled SLC or LLC distribution. 


\section{Supplemental Fig 2: SLC and LLC kinematics from replicate stimuli}

(A) Bend angle during LLC-type startles for control fish across the replicate stimuli (1-4) within each intensity $(32 \mathrm{~dB}, 38 \mathrm{~dB}, 41 \mathrm{~dB}, 43 \mathrm{~dB})$.

(B) Bend angle during LLC-type startles for DomA-exposed fish across the replicate stimuli (1-4) within each intensity.

(C) Bend angle during SLC-type startles for control fish across the replicate stimuli (1-4) within each intensity.

(D) Bend angle during SLC-type startles for DomA-exposed fish across the replicate stimuli (1-4) within each intensity. 


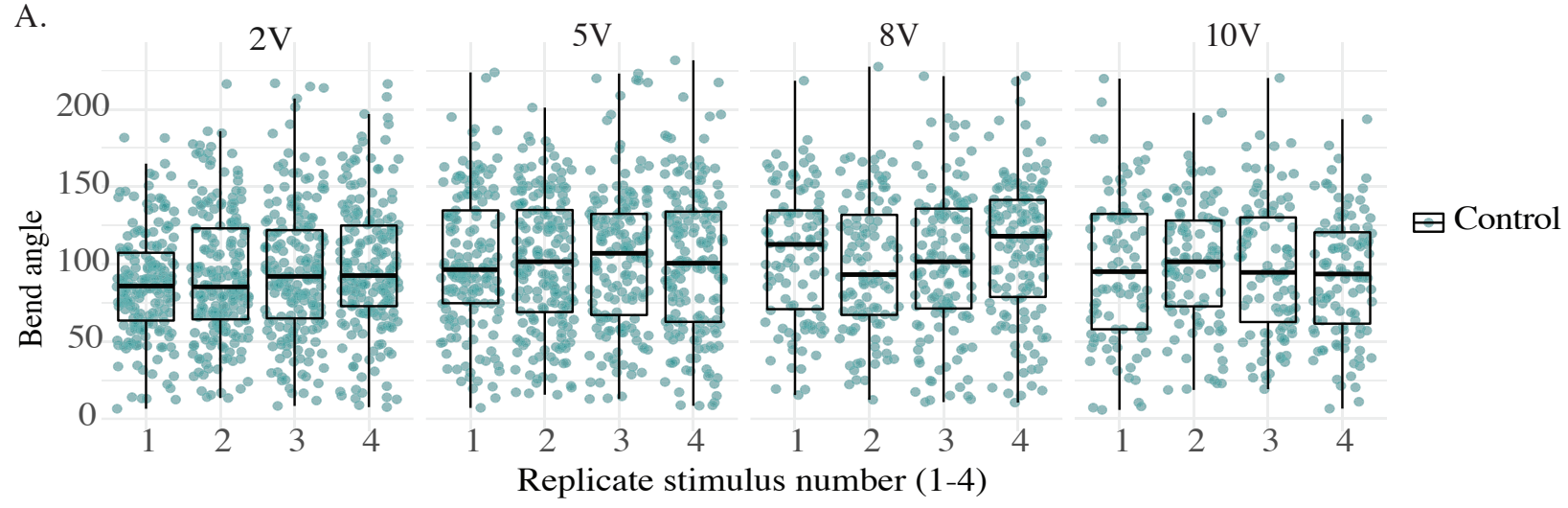

B.

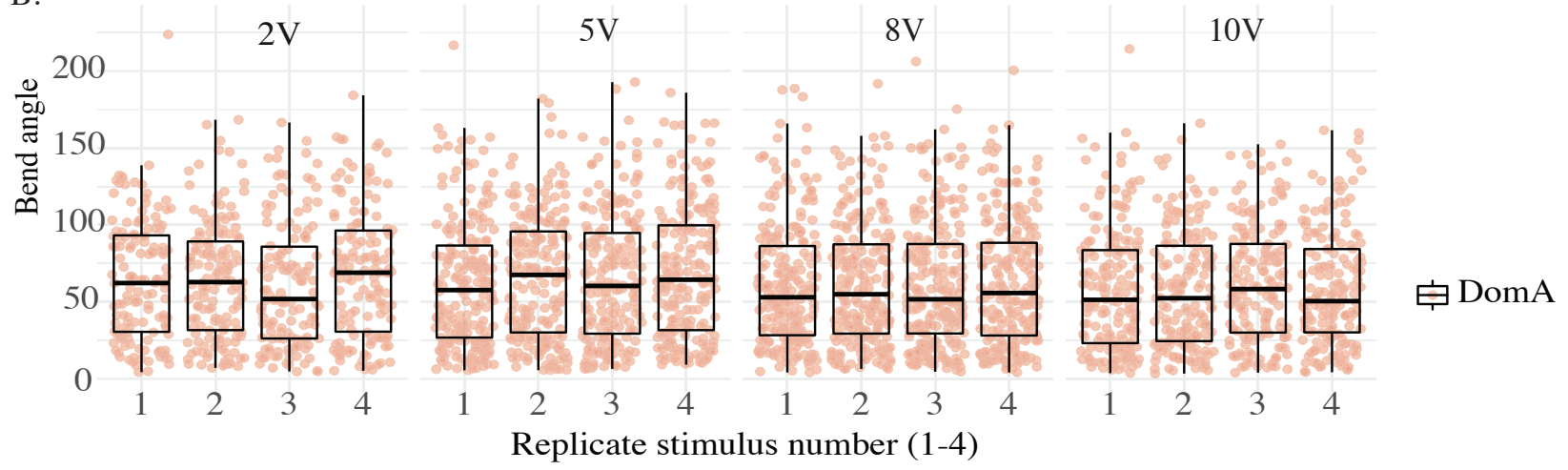

C.

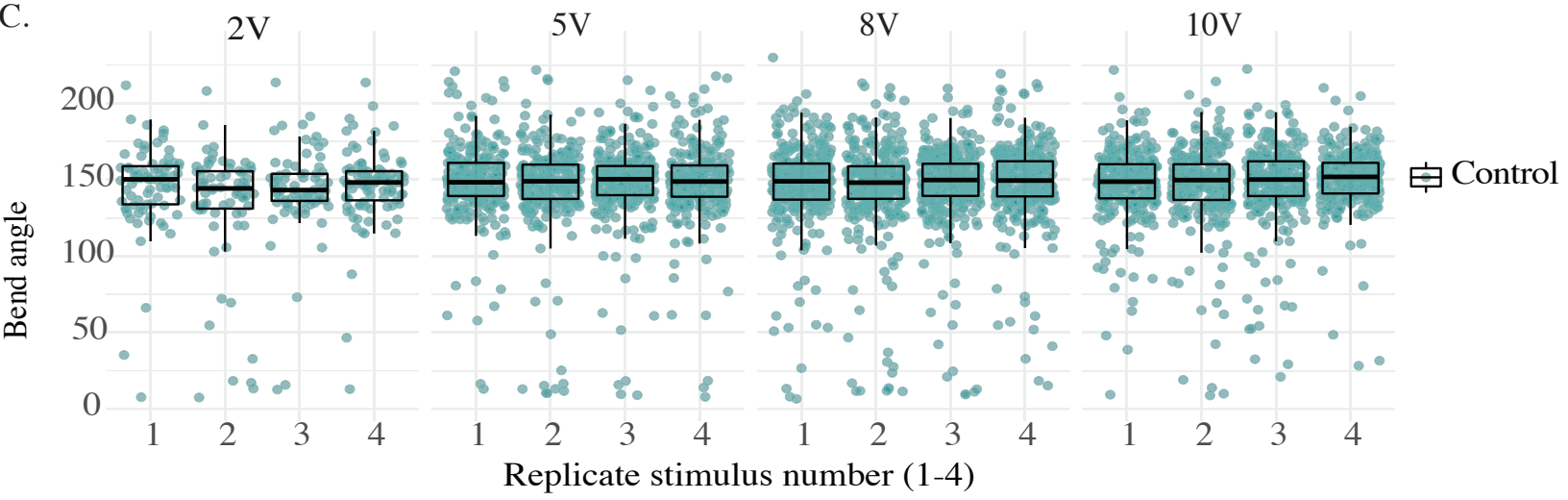

D.

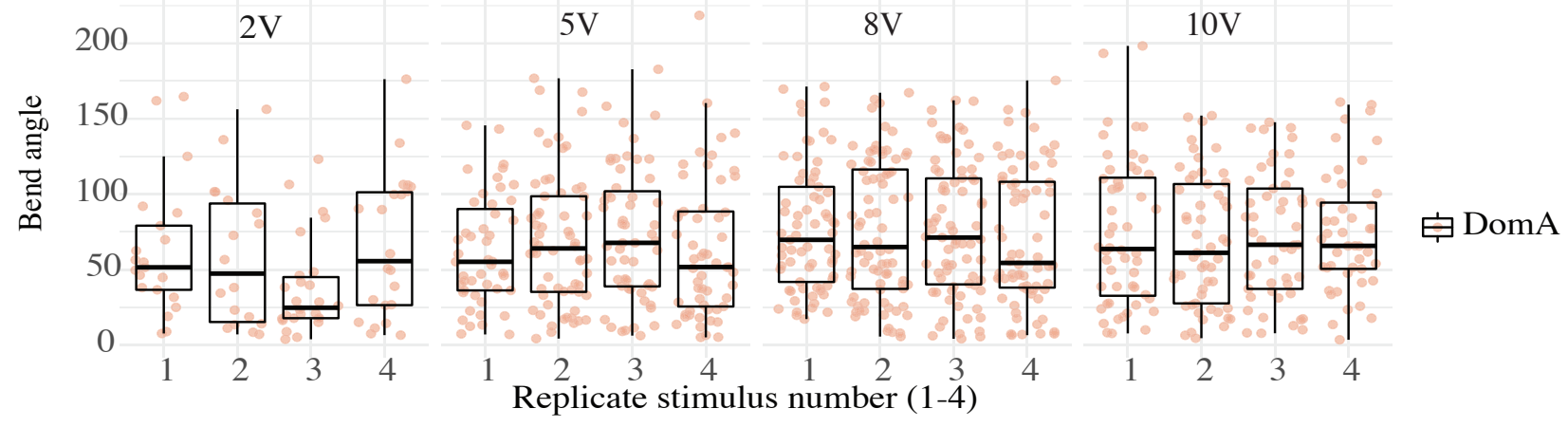




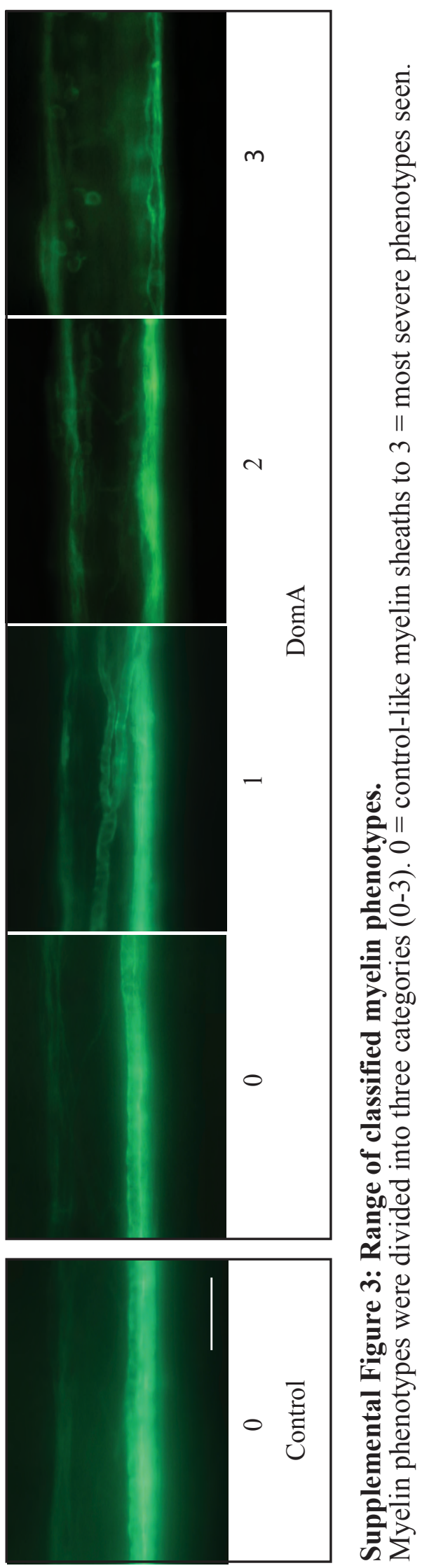


A. 1

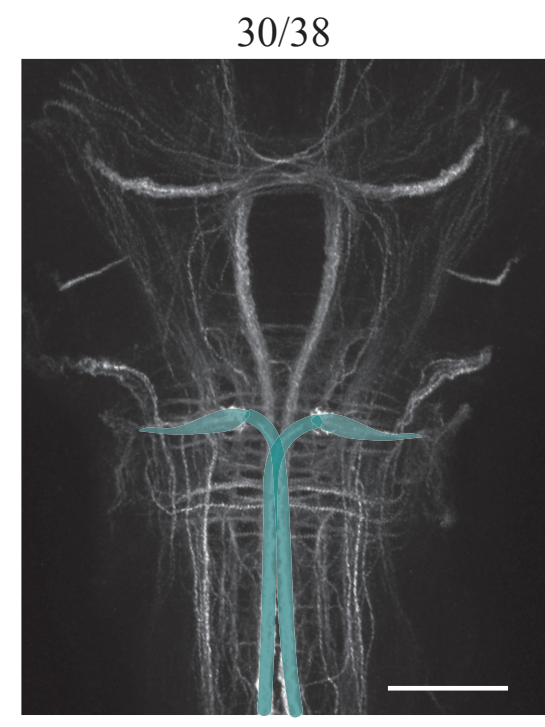

B.

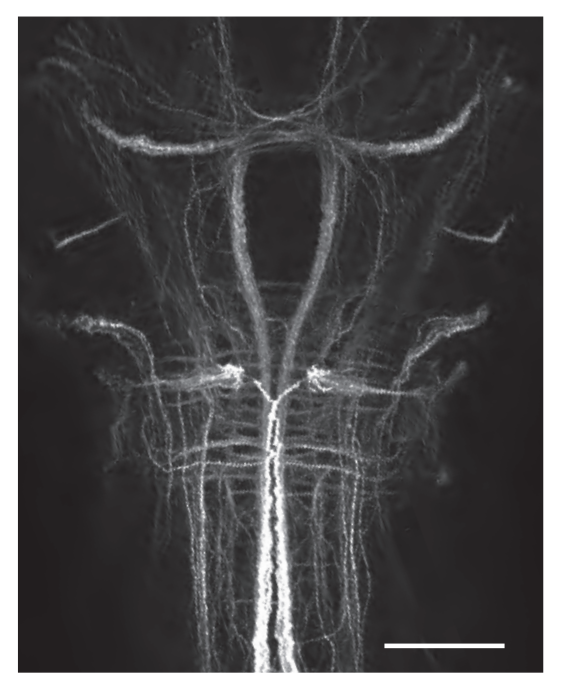

A.2
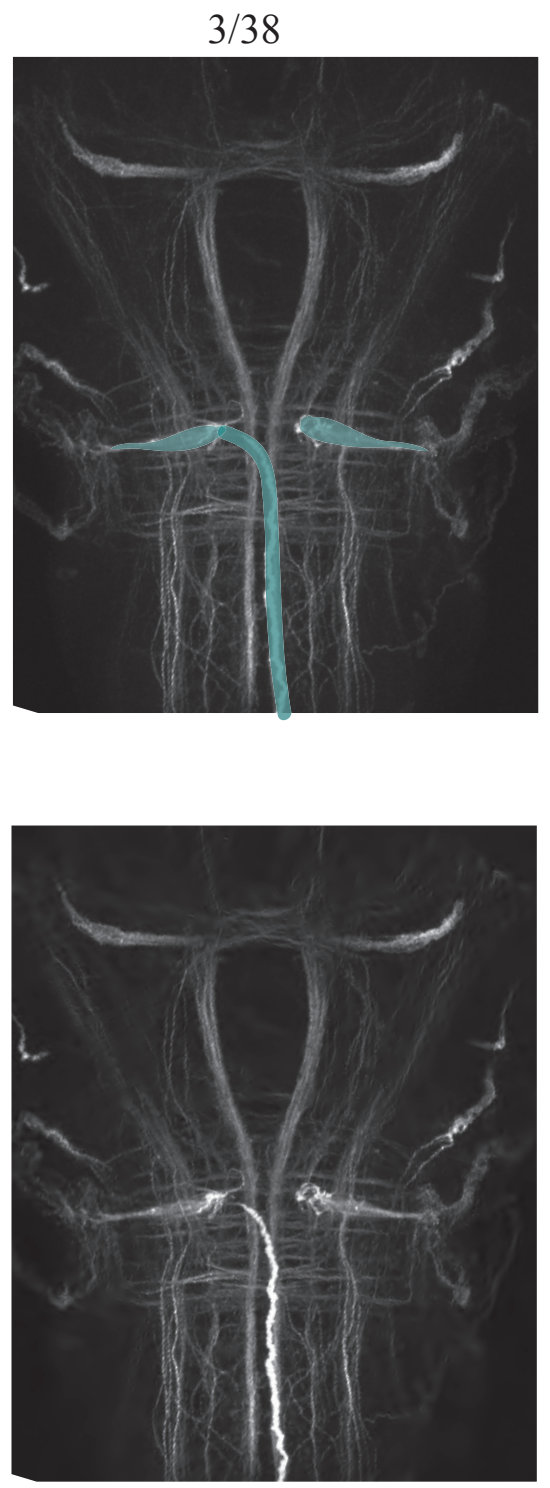

A.3
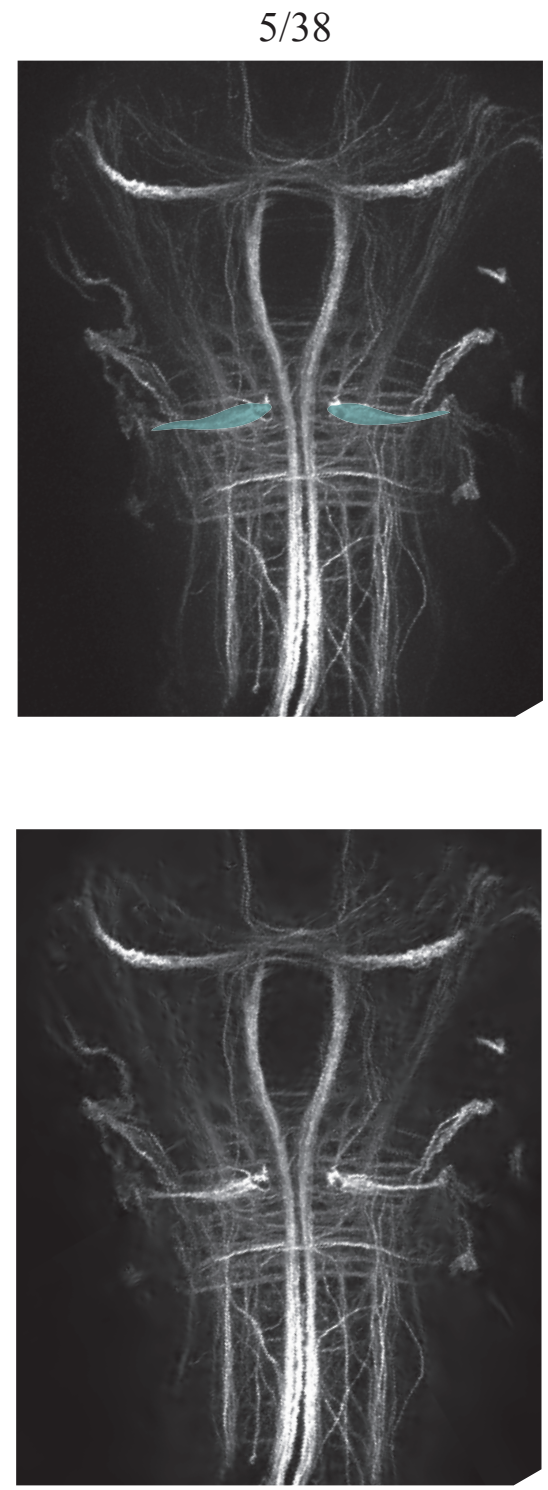

Supplemental Figure 4: Range of 3A10 staining observed in control larvae.

(A) Larval brains were dissected then stained using the 3A10 antibody. In a subset of the control animals, the Mauthner cell bodies were present, but one (A2) or both (A3 and A4) of the Mauthner axons were absent, likely as an artifact of the dissections. Mauthner cell bodies and axons are highlighed in teal. Ratios above each image show the number of control fish with each of these phenotypes. Scale bar $=100 \mu \mathrm{m}$.

(B) The same images without the highlighted regions. 


\subsection{REFERENCES}

1. Anderson, D. M., Glibert, P. M. \& Burkholder, J. M. Harmful algal blooms and eutrophication: Nutrient sources, composition, and consequences. Estuaries 25, 704-726 (2002).

2. Anderson, D. M. Harmful algal blooms and eutrophication: nutrient sources, composition, and consequences. Estuaries and coasts 25, (2002).

3. Hampson, D. R. \& Manalo, J. L. The activation of glutamate receptors by kainic acid and domoic acid. Nat. Toxins 6, 153-8 (1998).

4. Cendes, F., Andermann, F., Carpenter, S., Zatorre, R. J. \& Cashman, N. R. Temporal lobe epilepsy caused by domoic acid intoxication: Evidence for glutamate receptor-mediated excitotoxicity in humans. Ann. Neurol. 37, 123-126 (1995).

5. Wekell, J. C., Jurst, J. \& Lefebvre, K. a. The origin of the regulatory limits for PSP and ASP toxins in shellfish. J. Shellfish Res. 23, 927-930 (2010).

6. Maucher, J. M. \& Ramsdell, J. S. Maternal-fetal transfer of domoic acid in rats at two gestational time points. Environ. Health Perspect. 115, 1743-6 (2007).

7. Lefebvre, K. A. et al. Domoic acid in California sea lion fetal fluids indicates continuous exposure to a neuroteratogen poses risks to mammals. Harmful Algae (2018).

doi:10.1016/J.HAL.2018.06.003

8. Brodie Frances M D Gulland Denise J Greig, E. C., Hunter, M., Jaakola, J., Leger, J. S. \& Leighfield Frances M Van Dolah, T. A. Domoic acid causes reproductive failure in california sea lions (Zalophus Californianus). Mar. Mammal Sci. 3, (22AD).

9. Maucher, J. M. \& Ramsdell, J. S. Domoic acid transfer to milk: evaluation of a potential route of neonatal exposure. Environ. Health Perspect. 113, 461-4 (2005).

10. Rust, L., Gulland, F., Frame, E. \& Lefebvre, K. Domoic acid in milk of free living California marine mammals indicates lactational exposure occurs. Mar. Mammal Sci. 30, 1272-1278 (2014).

11. Perry, M. A., Ryan, C. L. \& Tasker, R. A. Effects of low dose neonatal domoic acid administration on behavioural and physiological response to mild stress in adult rats. Physiol. Behav. 98, 53-59 (2009).

12. Dakshinamurti, K., Sharma, S., Sundaram, M. \& Watanabe, T. Hippocampal changes in developing postnatal mice following intrauterine exposure to domoic acid. J. Neurosci. 13, 4486-4495 (1993).

13. Mills, B. D. et al. Prenatal domoic acid exposure disrupts mouse pro-social behavior and functional connectivity MRI. Behav. Brain Res. 308, 14-23 (2016).

14. Bernard, P. B., MacDonald, D. S., Gill, D. A., Ryan, C. L. \& Tasker, R. A. Hippocampal mossy fiber sprouting and elevated trkB receptor expression following systemic administration of low dose domoic acid during neonatal development. Hippocampus 17, 1121-1133 (2007).

15. Doucette, T. A. et al. Low doses of domoic acid during postnatal development produce permanent changes in rat behaviour and hippocampal morphology. Neurotox. Res. 6, 555563 (2004).

16. Jian Wang, G. H. et al. Systemic Administration of Domoic Acid-Induced Spinal Cord Lesions in Neonatal Rats. J. Spinal Cord Med. 23, 31-39 (2000).

17. Shiotani, M. et al. Neurobehavioral assessment of mice following repeated oral exposures 
to domoic acid during prenatal development. Neurotoxicol. Teratol. 64, 8-19 (2017).

18. Tanemura, K. et al. Intrauterine environment-genome interaction and Children's development (2): Brain structure impairment and behavioral disturbance induced in male mice offspring by a single intraperitoneal administration of domoic acid (DA) to their dams. J. Toxicol. Sci. 34, SP279-SP286 (2009).

19. Zuloaga, D. G. et al. Fetal domoic acid exposure affects lateral amygdala neurons, diminishes social investigation and alters sensory-motor gating. Neurotoxicology 53, 132 140 (2016).

20. Gill, D. A. et al. Neonatal exposure to low-dose domoic acid lowers seizure threshold in adult rats. Neuroscience 169, 1789-1799 (2010).

21. Tiedeken, J. A. \& Ramsdell, J. S. Embryonic exposure to domoic Acid increases the susceptibility of zebrafish larvae to the chemical convulsant pentylenetetrazole. Environ. Health Perspect. 115, 1547-52 (2007).

22. Levin, E. D., Pizarro, K., Pang, W. G., Harrison, J. \& Ramsdell, J. S. Persisting behavioral consequences of prenatal domoic acid exposure in rats. Neurotoxicol. Teratol. 27, 719-25

23. Pogoda, H.-M. et al. A genetic screen identifies genes essential for development of myelinated axons in zebrafish. Dev. Biol. 298, 118-31 (2006).

24. McKeown, K. A., Moreno, R., Hall, V. L., Ribera, A. B. \& Downes, G. B. Disruption of Eaat2b, a glutamate transporter, results in abnormal motor behaviors in developing zebrafish. Dev. Biol. 362, 162-71 (2012).

25. Faber, D. S., Fetcho, J. R. \& Korn, H. Neuronal Networks Underlying the Escape Response in Goldfish. Ann. N. Y. Acad. Sci. 563, 11-33 (1989).

26. Kohashi, T., Nakata, N. \& Oda, Y. Effective Sensory Modality Activating an Escape Triggering Neuron Switches during Early Development in Zebrafish. J. Neurosci. 32, 5810-5820 (2012).

27. Mirjany, M., Preuss, T. \& Faber, D. S. Role of the lateral line mechanosensory system in directionality of goldfish auditory evoked escape response. J. Exp. Biol. 214, 3358-3367 (2011).

28. Kohashi, T. \& Oda, Y. Initiation of Mauthner- or Non-Mauthner-Mediated Fast Escape Evoked by Different Modes of Sensory Input. J. Neurosci. 28, 10641-10653 (2008).

29. Korn, H. \& Faber, D. S. The Mauthner cell half a century later: a neurobiological model for decision-making? Neuron 47, 13-28 (2005).

30. Tabor, K. M. et al. Direct activation of the Mauthner cell by electric field pulses drives ultra-rapid escape responses 23 4. J Neurophysiol (2014). doi:10.1152/jn.00228.2014

31. Almeida, R. G., Czopka, T., Ffrench-Constant, C. \& Lyons, D. A. Individual axons regulate the myelinating potential of single oligodendrocytes in vivo. Development 138, 4443-50 (2011).

32. Kucenas, S. et al. CNS-derived glia ensheath peripheral nerves and mediate motor root development. Nat. Neurosci. 11, 143-151 (2008).

33. Czopka, T., Ffrench-Constant, C. \& Lyons, D. A. Individual oligodendrocytes have only a few hours in which to generate new myelin sheaths in vivo. Dev. Cell 25, 599-609 (2013).

34. Hoshijima, K., Jurynec, M. J. \& Grunwald, D. J. Precise Editing of the Zebrafish Genome Made Simple and Efficient. Dev. Cell 36, 654-67 (2016).

35. Thermes, V. et al. I-SceI meganuclease mediates highly efficient transgenesis in fish. Mech. Dev. 118, 91-8 (2002).

36. Soroldoni, D., Hogan, B. M. \& Oates, A. C. in 117-130 (Humana Press, 2009). 
doi:10.1007/978-1-60327-977-2_8

37. Cianciolo Cosentino, C., Roman, B. L., Drummond, I. A. \& Hukriede, N. A. Intravenous microinjections of zebrafish larvae to study acute kidney injury. J. Vis. Exp. (2010). doi:10.3791/2079

38. Burgess, H. a \& Granato, M. Modulation of locomotor activity in larval zebrafish during light adaptation. J. Exp. Biol. 210, 2526-39 (2007).

39. Wolman, M. A., Jain, R. A., Liss, L. \& Granato, M. Chemical modulation of memory formation in larval zebrafish. Proc. Natl. Acad. Sci. U. S. A. 108, 15468-73 (2011).

40. Benaglia, T., Chauveau, D., Hunter, D. \& Young, D. mixtools: An R Package for Analyzing Finite Mixture Models. J. Stat. Softw. 32, 1-29 (2009).

41. O'Malley, D. M., Kao, Y. H. \& Fetcho, J. R. Imaging the functional organization of zebrafish hindbrain segments during escape behaviors. Neuron 17, 1145-55 (1996).

42. Marsden, K. C. \& Granato, M. In Vivo Ca(2+) Imaging Reveals that Decreased Dendritic Excitability Drives Startle Habituation. Cell Rep. 13, 1733-40 (2015).

43. Wobbrock, J. O., Findlater, L., Gergle, D. \& Higgins, J. J. The aligned rank transform for nonparametric factorial analyses using only anova procedures. in Proceedings of the 2011 annual conference on Human factors in computing systems - CHI '11 143 (ACM Press, 2011). doi:10.1145/1978942.1978963

44. Harrar, S. W., Bathke, A. C., Ellis, A. R. \& Burchett, W. W. Nonparametric Inference for Multivariate Data: The R Package npmv. J. Stat. Softw. 76, (2017).

45. Burgess, H. A. \& Granato, M. Sensorimotor gating in larval zebrafish. J. Neurosci. 27, 4984-94 (2007).

46. Hothorn, T., Bretz, F. \& Westfall, P. The multcomp Package Title Simultaneous Inference for General Linear Hypotheses. (2007). at

$<$ http://132.180.15.2/math/statlib/R/CRAN/doc/packages/multcomp.pdf>

47. Bates, D., Mächler, M., Bolker, B. \& Walker, S. Fitting Linear Mixed-Effects Models using lme4. (2014). at $<$ http://arxiv.org/abs/1406.5823>

48. Moens, C. B., Yan, Y. L., Appel, B., Force, A. G. \& Kimmel, C. B. valentino: a zebrafish gene required for normal hindbrain segmentation. Development 122, 3981-90 (1996).

49. Turner, K. J., Bracewell, T. G. \& Hawkins, T. A. in 197-214 (Humana Press, Totowa, NJ, 2014). doi:10.1007/978-1-62703-655-9_14

50. Ripley, B. et al. Package 'MASS'. (2018). at $<$ http://www.stats.ox.ac.uk/pub/MASS4/>

51. Longair, M. H., Baker, D. A. \& Armstrong, J. D. Simple Neurite Tracer: open source software for reconstruction, visualization and analysis of neuronal processes.

Bioinformatics 27, 2453-2454 (2011).

52. Nicolson, T. et al. Genetic Analysis of Vertebrate Sensory Hair Cell Mechanosensation: the Zebrafish Circler Mutants. Neuron 20, 271-283 (1998).

53. Nicolson, T. The genetics of hair-cell function in zebrafish. J. Neurogenet. 31, 102-112 (2017).

54. Bergeron, S. A., Carrier, N., Li, G. H., Ahn, S. \& Burgess, H. A. Gsx1 expression defines neurons required for prepulse inhibition. Mol. Psychiatry 20, 974-985 (2015).

55. Sebe, J. Y. et al. Ca 2-Permeable AMPARs Mediate Glutamatergic Transmission and Excitotoxic Damage at the Hair Cell Ribbon Synapse. (2017). doi:10.1523/JNEUROSCI.3644-16.2017

56. Sheets, L. Excessive activation of ionotropic glutamate receptors induces apoptotic haircell death independent of afferent and efferent innervation. Sci. Rep. 7, 41102 (2017). 
57. McLean, D. L. \& Dougherty, K. J. Peeling back the layers of locomotor control in the spinal cord. Curr. Opin. Neurobiol. 33, 63-70 (2015).

58. Song, J., Ampatzis, K., Björnfors, E. R. \& El Manira, A. Motor neurons control locomotor circuit function retrogradely via gap junctions. Nature 529, 399-402 (2016).

59. Patten, S. A. \& Ali, D. W. AMPA receptors associated with zebrafish Mauthner cells switch subunits during development. J. Physiol. 581, 1043-56 (2007).

60. Warp, E. et al. Emergence of patterned activity in the developing zebrafish spinal cord. Curr. Biol. 22, 93-102 (2012).

61. Todd, K. J., Slatter, C. A. B. \& Ali, D. W. Activation of Ionotropic Glutamate Receptors on Peripheral Axons of Primary Motoneurons Mediates Transmitter Release at the Zebrafish NMJ. J. Neurophysiol. 91, 828-840 (2004).

62. Hoppmann, V., Wu, J. J., Søviknes, A. M., Helvik, J. V. \& Becker, T. S. Expression of the eight AMPA receptor subunit genes in the developing central nervous system and sensory organs of zebrafish. Dev. Dyn. 237, 788-99 (2008).

63. Lewinski, F. von \& Keller, B. U. Ca2+, mitochondria and selective motoneuron vulnerability: implications for ALS. Trends Neurosci. 28, 494-500 (2005).

64. Teitelbaum, J. S. et al. Neurologic Sequelae of Domoic Acid Intoxication Due to the Ingestion of Contaminated Mussels. N. Engl. J. Med. 322, 1781-1787 (1990).

65. Wang, G. J. et al. Systemic administration of domoic acid-induced spinal cord lesions in neonatal rats. J. Spinal Cord Med. 23, 31-9 (2000).

66. Petroff, R. et al. Chronic, Low-Level Oral Exposure to Marine Toxin, Domoic Acid, Alters Whole Brain Morphometry in Nonhuman Primates. Neurotoxicology (2019). doi:10.1101/439109 


\section{CHAPTER 4}

Developmental exposure to domoic acid targets specific neuronal populations and leads to aberrant myelination in the spinal cord

\subsection{GRAPHICAL ABSTRACT}
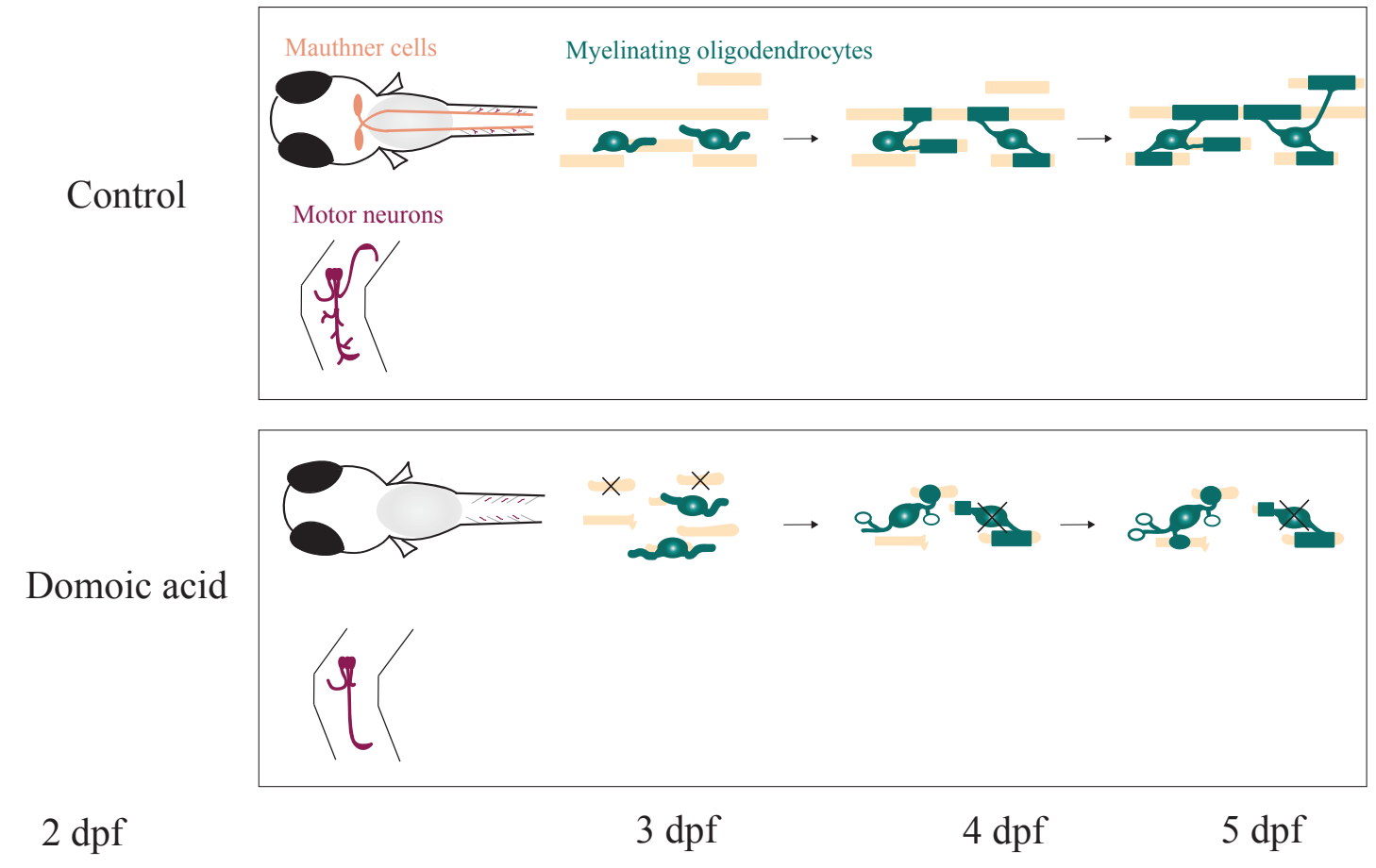


\subsection{ABSTRACT}

Exposure to the HAB toxin domoic acid (DomA) during key periods in neurodevelopment can have long-lasting adverse effects on nervous system function. However, the initiating cellular and molecular mechanisms that underlie these changes are largely unknown. Findings from chapters 2 and 3 show that exposure to DomA at a critical period in development leads to myelin defects and to the loss of reticulospinal neurons. However, it is unclear whether DomA primarily targets myelin sheaths, leading to the loss of reticulospinal neurons or, conversely, whether DomA primarily targets reticulospinal neurons leading to myelin defects. To identify the initial targets for DomA, zebrafish were exposed at $2 \mathrm{dpf}$ to $0.14 \mathrm{ng}$ of DomA via intravenous microinjections, and the effects on selected cell types were characterized shortly after exposure (4-12 hours post injection). To determine whether myelination deficits were driven by a decrease in supply of available oligodendrocyte precursor cells or myelinating oligodendrocytes, I quantified these cell types in exposed larvae. Exposure to DomA at $2 \mathrm{dpf}$ did not reduce the number of oligodendrocyte precursor cells prior to myelination. However, DomA exposures that led to severe myelin defects also led to significantly fewer myelinating oligodendrocytes. These effects were dose-dependent; higher doses of DomA led to fewer myelinating oligodendrocytes and more severe myelin phenotypes. Furthermore, DomA exposure resulted in fish with individual oligodendrocytes that had fewer and shorter myelin sheaths, while having more aberrant circular myelin membranes. I then assessed the effects of DomA on hindbrain reticulospinal neurons and found that a majority of the larvae treated with DomA at 2 dpf lacked one or both Mauthner cells - the hindbrain neurons critical for startle responses. These effects were observed prior to myelination, suggesting that the loss of the axons is not secondary to myelin defects. Using a double transgenic line that labels neurons and myelin, I showed that in DomA-treated larvae, circular oligodendrocyte membranes surround neuronal cell bodies, suggesting that these circular features were ectopically myelinated neuronal cell bodies. The loss of the Mauthner neurons and their axons potentially led to a cellular environment where oligodendrocytes inappropriately myelinated neuronal cell bodies in the absence of adequate axonal targets. Pharmacological treatment (25-40 $\mu \mathrm{M}$ GANT61) that reduced the number of oligodendrocytes led to a reduction in the number of ectopically myelinated cell bodies, further 
supporting the hypothesis that these circular myelin membranes arise due to a mismatch of available axonal surface area to the number of oligodendrocytes. These results suggest that DomA alters reticulospinal neurons first and the loss of the axons contributes to observed aberrant myelination. The identification of cell types targeted and the cellular processes that are perturbed provides a mechanistic understanding of how DomA alters neurodevelopment to lead to observed structural and behavioral phenotypes.

KEYWORDS: Harmful algal blooms, Domoic acid, Reticulospinal neurons, Mauthner neuron, Myelin, Oligodendrocytes, Oligodendrocyte precursor cells

\subsection{INTRODUCTION}

Harmful algal blooms (HABs) are the mass accumulation of algae that cause harm. HABs are a growing global threat that can lead to socioeconomic losses, ${ }^{1,2}$ illnesses in humans and wildlife, and disruptions in marine ecosystems. ${ }^{3-5}$ Some HABs can produce potent toxins that directly affect human health. ${ }^{6-8}$ Domoic acid (DomA) is a neurotoxin and ionotrophic glutamate receptor agonist $^{9,10}$ that is produced by some species in the diatom genus, Pseudo-nitzschia. ${ }^{11,12}$ With the formation of toxicogenic Pseudo-nitzschia blooms, DomA can accumulate in seafood, making it unsafe for consumption. The consumption of DomA-contaminated seafood at high doses leads to a syndrome called 'Amnesic Shellfish Poisoning' with symptoms that include memory loss, seizures, coma, and even death in the most severe cases. ${ }^{13-16}$ To protect people against this syndrome, a regulatory limit has been set at $20 \mathrm{mg}$ of DomA per $\mathrm{kg}$ shellfish tissue. ${ }^{17,18}$ However, this limit was designed to protect adults from acute symptoms of toxicity. Mounting evidence suggests that developmental exposures to doses of DomA that do not cause overt symptoms can lead to lasting changes in tissue morphology, ${ }^{19-22}$ neural activity patterns, ${ }^{23,24}$ and behavior. ${ }^{25-33}$ However, the cellular targets and initiating molecular events that underlie these changes are largely unknown. The objective of this chapter was to identity the initial cellular targets and disrupted developmental processes shortly after developmental exposure to DomA.

Previous chapters have implicated DomA as a neurotoxin that targets both myelin in the spinal cord and reticulospinal neurons in the hindbrain. Exposure to DomA at $2 \mathrm{dpf}$ leads to the disruption of the initial stages of myelination, to the overall reduction in myelin sheaths, and to 
the appearance of aberrant circular myelin membranes. RNAseq results show that by 28 hours post exposure ( $3 \mathrm{dpf}$ ), DomA downregulates genes required for maintaining myelin sheath and axonal structure. Furthermore, DomA also has been shown to lead to the loss of reticulospinal neurons and their axons by 5-7 dpf. While these results highlight the effects of DomA on reticulospinal neurons and myelin, it is unclear whether DomA primarily targets axons, leading to deficits in myelination, or whether DomA primarily targets myelinating oligodendrocytes, leading to later axonal degeneration. I have taken a temporal approach to identify the initial targets of DomA. By assessing the effects of DomA on these cellular targets close to the time of exposure, I was able to determine which cell types are the first to be perturbed by DomA exposure.

DomA-induced disruptions to myelin sheath formation in the central nervous system led me to look into the effects of DomA on the oligodendrocyte lineage - the cell lineage that is responsible for myelinating the central nervous system. ${ }^{34,35}$ Successful myelination requires oligodendrocyte precursor cells (OPCs) to form, migrate, differentiate into oligodendrocytes, and then initiate the process of myelination in different regions within the nervous system. ${ }^{36,37}$ Both OPCs and mature oligodendrocytes express functional ionotrophic glutamate receptors, making them potential cellular targets for DomA. ${ }^{38,39}$ Previous studies have shown that kainate, a structural analog of DomA, causes cell death in OPC cultures at concentrations comparable to those affecting neurons. ${ }^{40-43}$ OPCs have also been shown to be more vulnerable to kainateinduced cell death than mature oligodendrocytes, potentially due to the higher expression of ionotrophic receptors in OPCs. ${ }^{42}$ Furthermore, binding and activation of AMPA receptors inhibits the proliferation and differentiation of OPCs into mature oligodendrocytes. ${ }^{4,45}$ While generally less sensitive to kainate-induced cell death, ${ }^{42}$ mature oligodendrocytes have also been shown to undergo demyelination after chronic direct infusion of kainate on the optic nerves. ${ }^{46}$ All of this suggests that developmental exposure to DomA may target the oligodendrocyte lineage, and that exposure during early development may inhibit OPC differentiation, disrupt myelin sheath formation, and lead to cell death in the oligodendrocyte lineage.

Findings from Chapter 2 and 3 also show that exposure to DomA at 2 dpf downregulates critical axonal structural genes and leads to the loss of both hindbrain reticulospinal neurons and primary 
motor neuron axon collaterals. Both hindbrain reticulospinal neurons and primary motor neurons contain AMPA receptors, making them potential targets for DomA toxicity. ${ }^{47,48}$ Furthermore, both the hindbrain reticulospinal neurons and primary motor neurons rely on glutamatergic signaling to elicit early motor behavior, including embryonic tactile tail coiling responses and larval escape responses. ${ }^{49-51}$

The objective of this study was to identify the initial cellular targets of DomA. To accomplish this, I exposed fish to DomA at $2 \mathrm{dpf}$ and then assessed the effects on candidate cell types prior to myelination between 4 to 8 hours post-exposure. Candidate cell types included OPCs, reticulospinal neurons, sensory neurons, and motor neurons. I also further characterized DomA's effects on the oligodendrocyte lineage that is responsible for myelination by quantifying cells from the oligodendrocyte lineage and characterizing the myelination capacity of individual oligodendrocytes. Results from this chapter identify initial cellular targets for DomA, and further characterize the developmental processes perturbed by DomA exposures.

\subsection{METHODS}

Fish husbandry and lines used

These studies were approved by the Woods Hole Oceanographic Institution Animal Care and Use Committee (Assurance D16-00381 from the NIH Office of Laboratory Animal Welfare). Embryos were maintained at $28.5^{\circ} \mathrm{C}$ with a $14: 10$ light dark cycle during the experimental period in 0.3x Danieau's medium. The following published transgenic lines were used: $\mathrm{Tg}(\mathrm{mbp}: E G F P$ $C A A X)^{52}, \operatorname{Tg}(m b p: E G F P)$ (gift from Charles Kaufman in the Zon lab), $\operatorname{Tg}(\operatorname{sox} 10: R F P)^{53}$, $\operatorname{Tg}(\text { olig2:EGFP })^{5}, \operatorname{Tg}(n k x 2.2 a: m E G F P)^{54}$, and $\operatorname{Tg}(\operatorname{sox} 10: m R F P) .{ }^{55}$

\section{Generation of the Tg(cntn1b:EGFP-CAAX) line}

The $\operatorname{Tg}$ (cntn1b:EGFP-CAAX) line was generated by Gibson assembly. ${ }^{56}$ Using previously published primers, the $c n t n 1 b$ promoter region was amplified from AB wildtype fish. ${ }^{56}$ The $c n t n 1 b$ promoter was then assembled with EGFP-CAAX (cloned out of $\operatorname{Tg}(m b p: E G F P-C A A X)$ fish) into the vector backbone pkHR7, which contains ISCe-1 restriction sites. ${ }^{57}$ To generate the stable line, the plasmid containing cntn1b:EGFP-CAAX was co-injected along with ISCe-1 in 
the 1-2 cell stage. ${ }^{58,59}$ Injected fish were grown up, and their progeny that carried the transgene were grown up and used to generate embryos for experimental use.

\section{Domoic acid exposure paradigm}

Domoic acid (Sigma-Aldrich, MO) was dissolved in a diluted embryo medium (0.2x Danieau's) to generate stock concentrations of $0.676 \mu \mathrm{g} / \mu \mathrm{l}$ and $1.4 \mu \mathrm{g} / \mu \mathrm{l}$ and aliquots $(10 \mu \mathrm{L}$ each) were stored at $-20^{\circ} \mathrm{C}$. Working solutions were prepared fresh prior to microinjection by diluting the stock to obtain the appropriate doses. Microinjection needles were prepared from glass capillary tubes (0.58 mm inner diameter; World Precision Instruments, FL; 1B100F-4) using a pipette puller (Sutter instrument model p-30, heat 750, pull=0). Microinjections were performed using Narishige IM-300 microinjector that was calibrated to consistently deliver $0.2 \mathrm{~nL}$ by adjusting the time (milliseconds) and the pressure.

DomA was intravenously microinjected into the common posterior cardinal vein at 48-52 hpf. $^{60}$ A nominal dose of $0.14 \mathrm{ng}$ was injected, as exact concentrations need to be measured using analytical chemistry techniques. Controls from the same breeding cohort were injected with the saline vehicle (0.2x Danieau's). To perform intravenous microinjections, fish were anesthetized with $0.10 \%$ Tricaine methanesulfonate (MS222), and placed laterally on dishes coated with $1.5 \%$ agarose. An injection was deemed successful if there was a visible displacement of blood cells. Following injections, zebrafish were placed back in clean embryo media and monitored daily.

\section{Live imaging with confocal microscopy}

All transgenic fish used in the study were live imaged using confocal microscopy. To accomplish this, fish were anesthetized in Tricaine methanesulfonate (MS222) (0.16\%), and then embedded laterally in 1-1.5\% low melt agarose in glass bottom microscopy dishes. Fish were then imaged on the confocal microscope (LSM 710 or LSM 780) with the 40x water immersion objective (CApochromat, 40x, NA 1.1).

\section{OPC cell counts}

Two different double transgenic lines were used to assess potential effects of DomA on OPC counts - $(T g(\operatorname{sox} 10: m R F P)$ x $T g($ olig2:EGFP) and $T g(n k x 2.2 a: m E G F P) \times \operatorname{Tg}(\operatorname{sox} 10: R F P)$. 
Following DomA exposure at $2 \mathrm{dpf}$, fish were laterally mounted at $2.5 \mathrm{dpf}$, and the anterior spinal cord imaged (within a region from somites 8-12).

Imaging files were first blinded (treatment information was removed from the file). For a given imaging file, the OPCs within the dorsal spinal cord were quantified within the imaged area. To accomplish this, image stacks were made into 3D projections in ImageJ. The stacks were then rotated along the $\mathrm{x}$-axis, and cell bodies were counted. I used the double transgenic line, $\operatorname{Tg}(\operatorname{sox} 10: m R F P) \times \operatorname{Tg}($ olig2:EGFP) to quantify all OPCs and pre-myelinating oligodendrocytes in the dorsal spinal cord. In particular, I counted cells that had membranes labeled with RFP and cell bodies labeled with EGFP. I used the double transgenic line, $\operatorname{Tg}(n k x 2.2 a: m E G F P) \mathrm{x}$ $\operatorname{Tg}(\operatorname{sox} 10: R F P)$ to quantify the subset of OPCs that were fated to differentiate into myelinating oligodendrocytes. To accomplish this, I counted cells that had membranes labeled with EGFP, and cell bodies labeled with RFP.

Experiments were performed twice (Supplemental Fig. 1A and 1B). The two experimental trials done using $\operatorname{Tg}(n k x 2.2 a: m E G F P)$ x $\operatorname{Tg}(\operatorname{sox} 10: R F P)$ fish yielded significantly different OPC counts, with the second trial having fish with 1.6 times more OPCs than fish in the first trial, regardless of treatment group $(\mathrm{CI}=[1.461 .74], \mathrm{p}=<2 \mathrm{e}-16$, Supplemental Fig 1B). To address this, I compared DomA to controls within the same experiment.

\section{Myelinating oligodendrocyte cell counts}

Similar procedures were used for counting myelinating oligodendrocytes. Transgenic fish that expressed GFP in myelinating cells, $\operatorname{Tg}(\mathrm{mbp}: E G F P)$, were laterally mounted at $4 \mathrm{dpf}$, and their anterior spinal cords were imaged (somites 6-10). Imaging files were then blinded. For a given imaging file, myelinating oligodendrocytes were counted by rotating image stacks along the $\mathrm{x}$ axis. To determine whether DomA alters the number of myelinating oligodendrocytes, I constructed a negative binomial model, with dose $(0,0.14$ or $0.18 \mathrm{ng}$ of DomA) as a predictor using the 'glm.nb' function of the MASS package in R. ${ }^{61}$

Cytoplasmic localized GFP also faintly labels the innermost and outermost of the myelin sheath layers. This allowed me to qualitatively classify myelin defects in addition to quantifying the 
number of oligodendrocytes for every individual fish (Fig. 2C). To see whether there were significant differences in oligodendrocyte number based on myelin phenotype, I constructed negative binomial models with the myelin classification as predictors. When assessing data from multiple experimental trials, a negative binomial regression model with random effects was used, with repeat trials taken into account as random factors (glmer.nb(), lme 4 package, R).

\section{Mosaic labeling of myelin sheaths from individual oligodendrocytes}

To obtain the mbp:EGFP-CAAX construct, I used the $T g(m b p: E G F P-C A A X)$ genomic DNA as a template, and then amplified the region that contained the $m b p$ promoter along with the membrane bound EGFP. To accomplish this, I used the previously published forward primer for the $m b p$ promoter ${ }^{62}$ and the reverse primer for the CAAX box sequence. ${ }^{63}$ The sequence was then placed into the vector backbone pkHR7 which contains ISCe-1 restriction sites. ${ }^{57}$ To mosaically express EGFP-CAAX in a few oligodendrocytes, the construct was injected along with ISCe-1 into 2-4 cell stage transgenic embryos that expressed RFP in oligodendrocyte cell bodies (Tg:sox10:RFP).

Embryos were then exposed to either DomA or the saline vehicle. Embryos with sparsely labeled oligodendrocytes were then imaged at $4 \mathrm{dpf}$. Some embryos had multiple labeled oligodendrocytes. Oligodendrocytes from the same fish were assigned the same 'fish ID' number, allowing me to distinguish multiple oligodendrocytes that came from the same fish from oligodendrocytes that came from different fish.

Images of individual oligodendrocytes were then blinded prior to image analysis. To perform the analysis, myelin sheaths from individual oligodendrocytes were traced using the ImageJ plugin Simple Neurite Tracer. ${ }^{64}$ An individual oligodendrocyte was identified by a single cell body that was labeled with RFP, with surrounding myelin sheaths labeled with EGFP. For each individual oligodendrocyte, the total number of myelin sheaths and the number of circular profiles were counted. The length of the individual myelin sheaths was approximated.

To determine whether there was an effect of treatment on the average length of the myelin sheaths per oligodendrocyte, a nonparametric multivariate response analysis was performed 
(art(), ARTool R package). ${ }^{65}$ To account for potential variations in responses from different individual fish, fish ID numbers were included in the model as random effects.

To determine whether DomA altered the number of myelin sheaths or the number of circular myelin membranes, I used generalized linear mixed effects models with a negative binomial distribution (glmer.nb(), lme4 R package), assigning treatment (Control or DomA) as the fixed effect and fish ID as the random effect.

\section{DomA effect on primary motor neuron axons}

$\operatorname{Tg}($ olig2:EGFP) x $\operatorname{Tg}(\operatorname{sox} 10: m R F P)$ double transgenic fish were also used to assess the effects of DomA on primary motor neurons. The presence or absence of the main axons for three primary motor neuron classes were noted for each fish. Following this classification, mixed effects logistic regression analyses were performed to determine whether exposure to DomA led to significant differences in the presence of main axons in these three types of primary motor neurons.

\section{Immunohistochemistry}

Fish used for imunohistochemical analyses were placed in $75 \mu \mathrm{M}$ 1-phenyl-2-thiourea treatment (PTU) at $24 \mathrm{hpf}$ and onwards to inhibit pigment formation. This allowed for imaging of axonal structures within different brain regions. The dose was chosen based on prior studies showing that it was effective to prevent pigmentation while having minimal detrimental effects on the development in the embryos. ${ }^{66}$

Embryos were anesthetized and then fixed in $4 \%$ paraformaldehyde overnight at $4{ }^{\circ} \mathrm{C}$. Antigen retrieval was done by placing tissue in $150 \mathrm{mM}$ Tris $\mathrm{Hcl}(\mathrm{pH} 9.0)$ in a $70^{\circ} \mathrm{C}$ water bath for 15 minutes. ${ }^{67}$ Tissues were processed as whole mounts, and permeabilized using ice-cold acetone (7 minutes). Samples were then blocked in 10\% normal goat serum, 1\% BSA and 1\% DMSO, followed by 1-3 day incubations in primary antibodies (neurofilament associated antigen ( $\alpha$ 3A10) -1:100 dilution, Developmental Studies Hybridoma Bank; $\alpha$-acetylated tubulin - 1:500 dilution, Santa Cruz Biotechnology). After several washes with phosphate buffered saline (1X PBS, pH 7.2- 7.4), samples were then incubated in secondary antibodies (1:400 Alexa Fluor 488 
Goat $\alpha$-mouse or Alexa Fluor 596 Goat $\alpha$-mouse; Abcam). Samples were then placed in antifade mountant (Prolong or SlowFade Diamond mountant, Invitrogen) and placed between bridged \#1.5 coverslips for imaging.

Embryos stained with 3A10 were mounted dorsally in order to image the Mauthner cell bodies and hindbrain and midbrain axonal tracks. Embryos stained with $\alpha$-acetylated tubulin were mounted laterally to assess the sensory neuron ganglia and lateral line structures. Following antibody staining and mounting, fixed larvae were imaged on the confocal microscope using either 20x air objective (Plan-Apochromat 20x NA 0.8) or the 40x water immersion objective (C-Apochromat, 40x, NA 1.1).

\section{DomA effect on Mauthner cells}

Following 3A10 staining and confocal imaging, the number of Mauthner cells $(0,1$, or 2$)$ was noted for each fish. To determine whether DomA alters Mauthner cell number, ordinal logistic regression analyses were done (polr(), MASS R package), with treatment (DomA vs. control) as a categorical predictor.

\section{Characterization of circular myelin features}

$T g$ (cntn 1b:EGFP-CAAX) x $T g(\operatorname{sox} 10: m R F P)$ are double transgenic fish that express RFP in myelin sheaths and EGFP in the membranes of specific neurons. After exposure to DomA or vehicle, a $131 \mu \mathrm{m}$ long region between somites 6-10 was imaged at $5 \mathrm{dpf}$. Image stacks were then examined to see whether the circular oligodendrocyte membranes labeled with RFP surrounded neuronal cell bodies labeled in EGFP.

\section{Scanning Electron microscopy}

$T g$ (mbp:EGFP-CAAX) fish were exposed to $0.14 \mathrm{ng}$ of DomA (nominal dose) at $2 \mathrm{dpf}$. At 5 dpf, fish were screened using the widefield epifluorescence microscope for myelin defects. DomA-exposed fish with a high prevalence of circular membranes were preselected for electron microscopy. Control fish with normal myelin were also selected. 
The scanning electron microscopy (SEM) work, including fixation, embedding, and cutting, were done by the Marine Biological Laboratory Central Microscopy facility. Using a modified ROTO protocol, larvae were fixed in a 5\% glutaraldehyde and $2 \%$ paraformaldehyde in $0.2 \mathrm{M}$ cacodylic buffer with $3 \%$ sucrose for 2.5 hours. Following washes in 0.2 cacodylic buffer, samples were postfixed in $1 \%$ osmium and $0.75 \%$ potassium ferricyanide for 30 minutes. Samples were then washed in water and incubated in $0.25 \%$ thiocarbohydrazide for $10 \mathrm{~min}$. Following another wash, they were embedded in agarose and cut. Samples were then post-treated with osmium tetraoxide for 30 minutes, and washed once more with water prior to being incubated overnight in $1 \%$ uranyl acetate at $4^{\circ} \mathrm{C}$. A series of dehydration steps $(50,70,80,90$, then $95 \%$ ethanol) were done prior to embedding in Epon/Alardite plastic. Infiltration took place over the course of 2 days. The embedded samples were then polymerized in the $60^{\circ} \mathrm{C}$ over for 4 days.

Sections were cut $\sim 70 \mathrm{~nm}$ thick and collected on a continuous feed of Kapton tape using an RMC Boeckleler ATUMtome tape collecting ultramicrotome. Sections $(\sim 200 \mathrm{~nm})$ were then cut into strips and affixed to a $10 \mathrm{~cm}$ in diameter silicon wafer.

Samples were then imaged using the Zeiss SMT Supra 40VP SEM at 7KV and 8mm WD using Zeiss 4QBSD pneumatically driven detector and ATLAS 5 software version 5.1. Whole spinal cords were imaged at $10 \mathrm{~nm}$ resolution to screen for these circular myelin membranes over roughly 50 slices in the medial spinal cord. When areas of interest were identified, smaller regions were imaged at $5 \mathrm{~nm}$ resolution.

\section{GANT61 pharmacology}

GANT61 (2,2'-[[dihydro-2-(4-pyridinyl)-1,3(2H,4H)-pyrimidinediyl]bis(methylene)]bis[N,Ndimethyl-benzenamine) is a GLI antagonist that was used to reduce the number of oligodendrocytes. ${ }^{68,69}$ GANT61 (Sigma-Aldrich, MO) was dissolved in 100\% DMSO to create 10,25 , and $40 \mathrm{mM}(1000 \mathrm{x})$ stock solutions. Working solutions were prepared fresh prior to the experimental trials. Double transgenic fish (Tg(mbp:EGFP) x $\operatorname{Tg}(m b p: E G F P-C A A X))$ were used for these experiments. The use of the double transgenic allowed me to distinguish aberrant circular membrane profiles (which only had labeled membranes) from oligodendrocyte cell 
bodies (which had both labeled cytoplasm and the membranes). These double transgenic fish were bath-treated with 0, 10, 25, or $40 \mu \mathrm{M}$ GANT61 from 1.5- 4 dpf. A subset of GANT61treated fish were also exposed to either $0.14 \mathrm{ng}$ of DomA or vehicle saline $(0.2 \mathrm{x}$ Danieau's $)$ at 2 dpf. Larvae were then live imaged at $4 \mathrm{dpf}$ using the confocal microscope (LSM 710) using the techniques outlined above.

The number of oligodendrocyte cell bodies in the dorsal spinal cord was determined. GANT61 treatment in both control and DomA-treated fish led to the labeling of several cells in the most dorsal regions of the spinal cord that could not be identified (peach arrows in Fig. 7E and Fig. 7F). Additionally, the large myelin sheaths present in the ventral region of the spinal cord occluded some oligodendrocyte cell bodies, making it difficult to accurately quantify the oligodendrocytes in this region. Given these two considerations, I simplified the analysis by only quantifying oligodendrocytes and circular myelin features in the dorsal region of the spinal cord.

\section{Statistical analyses of data from GANT61-treated fish}

To identify the effects of GANT61 treatment on the number of oligodendrocytes and circular bodies, Poisson or negative binomial regression models with random effects were used. Treatments $(0,10,25$, and $40 \mu \mathrm{M}$ GANT61) were considered fixed effects in the model, while repeated experimental trials were considered random effects. Negative binomial distributions were used instead of Poisson distributions when the conditional variance exceeded the conditional mean (glmer.nb(), lme4 R package). To assess the potential correlation between oligodendrocyte counts and circular cell body counts, a Spearman correlation was performed (cor.test(), stats R package).

\subsection{RESULTS}

\section{Oligodendrocyte precursor cell number is unaltered by DomA exposure}

Exposure to DomA at $2 \mathrm{dpf}$ led to myelin deficits along with the appearance of unusual circular profiles (chapter 2). Myelin deficits could be due to a decrease in the number of oligodendrocytes available to myelinate the axons, or the inability of existing oligodendrocytes to properly myelinate axons in the spinal cord. To determine whether myelination deficits are driven by a decrease in supply of available oligodendrocyte precursor cells (OPCs) or 
myelinating oligodendrocytes, I quantified these cells in exposed larvae using transgenic lines (Fig. 1A).

Using the $\operatorname{Tg}(\operatorname{sox} 10: m R F P)$ x $\operatorname{Tg}($ olig2:EGFP) line, I was able to quantify the number of oligodendrocyte precursor cells (OPCs). DomA did not reduce the number of OPCs in the ventral spinal cord prior to myelination (Fig. 1B, 1C). To determine whether DomA selectively targets OPCs that are fated to become myelinating oligodendrocytes, I used another double transgenic line $(\operatorname{Tg}(\operatorname{sox} 10: R F P) \times \operatorname{Tg}(n k x 2.2 a: m E G P))$, and also found no differences in OPC count between control and DomA-treated larvae (Fig. 1D, 1E).

\section{DomA exposure leads to a reduction of myelinating oligodendrocytes in a dose-dependent} manner

OPCs differentiate into myelinating oligodendrocytes starting at $2.5 \mathrm{dpf}$. I used the $\operatorname{Tg}$ (mbp:EGFP) line to determine the effect of DomA exposure at $2 \mathrm{dpf}$ on the number of myelinating oligodendrocytes at $4 \mathrm{dpf}$ (Fig. 2A). DomA reduced the number of myelinating oligodendrocytes in a dose-dependent manner. Exposure to $0.14 \mathrm{ng}$ led to a $12.9 \%$ decrease relative to controls, while $0.18 \mathrm{ng}$ DomA led to a $31.8 \%$ decrease in the number of oligodendrocytes relative to controls (0.14 ng DomA -- Coefficient $=-0.138$, Error $=0.030, \mathrm{p}=$ 3.09 e- 06; $0.18 \mathrm{ng}$ DomA -- Coefficient $=-0.383$, Error $=0.048, \mathrm{p}=1.61 \mathrm{e}-15)($ Fig. $2 \mathrm{~B})$.

In addition to oligodendrocyte count, individual fish from these experiments were classified by the severity in myelin defects observed (Fig. 2C). This allowed me to determine the relationship between the severity of myelin defects and the number of oligodendrocytes present (Fig. 2D). Fish that had the least severe myelin defects (category 1) did not have significantly different oligodendrocyte numbers compared to fish with no myelin defects (category 0$)($ Coefficient $=$ -0.053 , Error $=0.028, p=0.057)$. This indicates that reductions to oligodendrocyte numbers are not necessary for the appearance of the least severe myelin phenotypes. In contrast, fish with higher myelin severity phenotypes (category 2 and 3 ) had significantly reduced oligodendrocyte numbers. Furthermore, fish that had the most severe defects (category 3 ) also had the lowest oligodendrocyte numbers $(42 \%$ reduction relative to controls $($ Coefficient $=-0.544$, Error $=$ 
$0.035, \mathrm{p}=<2 \mathrm{e}-16))$. Fish with category 2 phenotypes had an $18 \%$ reduction in oligodendrocytes compared to controls $($ Coefficient $=-0.195$, Error $=0.032, p=1.35$ e -09$)$.

There were among-trial differences in the effects of DomA (0.14 ng) on oligodendrocyte number. In 2 out of the 3 trials, DomA treatment significantly reduced the number of oligodendrocytes (Supplemental Fig. 2A). DomA-exposed larvae (0.14 ng) had a 23.5\% reduction in the number of oligodendrocytes relative to controls in Trial 1 and a $12.7 \%$ reduction in the number relative to controls in Trial 3 (Coefficient $=-0.268$, Error $=0.043, p=$ 6.13 e -10 and Coefficient $=-0.136$, Error $=0.059, p=0.02$ respectively $)($ Supplemental Fig. 2A) . Comparatively, DomA-exposed larvae in Trial 2 exhibited no differences to the controls. Fish from each trial were also classified by their myelin phenotype, and the relationship between myelin defects and oligodendrocyte number was examined. A majority of the fish from Trial 2 had the least severe myelin phenotypes (25/34), and none of the fish from this trial displayed the most severe phenotype (Supplemental Fig. 2B).

These results suggests that exposure to DomA reduced the number of myelinating oligodendrocytes at the high doses that also led to the most severe myelin phenotypes observed. However, there are instances when exposure to DomA results in less severe myelin phenotypes and that do not result in the reduction in oligodendrocyte number.

\section{Individual oligodendrocytes have aberrant myelin sheaths following exposure to DomA} Myelin deficits could also result from the inability of individual oligodendrocytes to myelinate axons appropriately. To address this, I sparsely labeled oligodendrocytes and counted myelin sheath length and number for individual oligodendrocytes (Fig. 3A). DomA-exposed larvae had oligodendrocytes with significantly fewer myelin sheaths $($ Coefficient $=-1.00$, Std. Error $=$ $0.110, p<2$ e-16) that were also shorter relative to controls $(F(1,142.32)=66.13, p=1.88$ e -13$)$ (Fig. 3B, 3C). This suggests that DomA exposure perturbs the ability of the remaining oligodendrocytes to myelinate axons in the spinal cord. In addition to the myelin deficits, DomAexposed fish also had a higher number of unusual circular profiles instead of the characteristic long and thin myelin sheaths (Coefficient $=2.293$, Std. Error $=0.296, p=9.58$ e -15) $($ Fig. 3D). 


\section{DomA exposure at 2 dpf leads to the loss of the Mauthner neuron prior to myelination}

Aberrant myelination may result from both the loss of myelinating oligodendrocytes and the inability of the remaining oligodendrocytes to myelinate axons. However, the effects of DomA on the oligodendrocytes do not rule out the possibility that DomA leads to the loss of neurons and their axons prior to these observed myelin defects. The loss of the neurons and their axons, in turn, could have secondary effects on the oligodendrocytes. To determine whether DomA first targets the axons prior to myelination, I performed immunohistochemistry and live imaging of specific neuronal populations prior to myelination. A loss in axons that occurs prior to myelination would suggest that DomA affects neurons and their axons, and that neuronal loss is not simply due to the myelin defects observed.

The first goal was to determine whether reticulospinal neurons lost at $7 \mathrm{dpf}$ (chapter 3) were present at the time of exposure, making these neurons potential targets for DomA. All the major reticulospinal neurons, including the Mauthner cells, were present by $52 \mathrm{hpf}$, which was within the $2 \mathrm{dpf}$ injection exposure period (Fig. 4A,B). However, by 8 hours post exposure (60 hpf) Mauthner neurons were lost in a majority of fish exposed to DomA (40/72) (Fig. 4C, Fig. 4D, p $<\mathrm{e}-13)$. In contrast, both Mauthner neurons were found in all control fish tested (76/76). Since Mauthner neurons were present by the time of exposure, DomA did not inhibit the formation of the Mauthner neurons but rather led to the loss of these neurons.

The degree of DomA-induced losses in Mauthner cells varied among repeated experiments. In 3 out of 4 trials, a majority of DomA-exposed fish had 0 out of the 2 Mauthner cells (Supplemental Fig. 3). Nonetheless, in all trials, DomA-exposed fish show Mauthner cell numbers that were significantly different from controls $\left(\mathrm{p}<10^{-13}\right)$.

\section{DomA exposure at 1 dpf does not lead to losses in the Mauthner neuron}

Findings from Chapter 2 show that exposure at $2 \mathrm{dpf}$ (injected at 48-52 hpf) led to startle response deficits and myelin defects. In contrast, exposures at $1 \mathrm{dpf}$ (injected at 28-32 hpf) did not lead to any of these effects. I then sought to determine whether, similar to the behavioral and myelin phenotypes described, exposure at $1 \mathrm{dpf}$ does not lead to the loss of Mauthner cells even if exposure at $2 \mathrm{dpf}$ does. 
Mauthner neurons were present by $32 \mathrm{hpf}$, which is within the $1 \mathrm{dpf}$ time period that DomA is delivered (Fig. 4E). Even though the Mauthner cells were present at the time of exposure, fish exposed to DomA at $1 \mathrm{dpf}$ did not lose Mauthner cells by either 8 hours post exposure (40 hpf) or by $60 \mathrm{hpf}$. These two time points - 8 hours post-exposure and $60 \mathrm{hpf}$ - were significant because they were the times when 2-dpf-injected fish were observed to have lost one or both Mauthner cells (Fig. 4 F, G).

Consistent with the behavioral and imaging data, exposures to DomA at $2 \mathrm{dpf}$ (but not $1 \mathrm{dpf}$ ) led to the loss of Mauthner cells prior to myelination $(60 \mathrm{hpf})$ and these losses persist until the larval stages (7 dpf, chapter 3).

\section{DomA exposure does not lead to any apparent effects in selected sensory system structures}

Chapter 3 established that exposure to DomA at $2 \mathrm{dpf}$ led to no apparent changes in sensory system structures or to the main axon of the caudal primary motor neurons by $5 \mathrm{dpf}$. I thus assessed whether exposure to DomA altered specific sensory and motor neuron structures at 2.5 dpf, a time period shortly after exposure to DomA; doing so allowed me to rule out the possibility that DomA perturbs these neuronal subtypes early in development even if they eventually recover in the larval stages.

DomA exposure did not lead to any apparent changes in selected sensory structures in the brain as assessed by staining for acetylated tubulin (Fig. 5A). In particular, DomA did not alter the sensory ganglia that comprise the anterior lateral line; this included the nADso, nVDI, nADb and nAVm ganglia (Fig. 5B). Consistent with results from the anti-acetylated tubulin staining, neurofilament staining with anti-3A10 antibody also showed that domoic acid exposure did not lead to any apparent deficits in the anterior lateral line or the medial longitudinal fasciculus prior to myelination (Fig. 4D).

\section{DomA exposure alters the main axons in one of the three primary motor neuron classes}

The $\operatorname{Tg}($ olig2:EGFP) line labels motor neurons in addition to the oligodendrocyte lineage. Therefore, in addition to being used to quantify OPC cells (Fig. 1), fish from this transgenic line 
were also used to determine whether DomA alters primary motor neurons at $2.5 \mathrm{dpf}$, prior to myelination (Fig. 5C-E). In particular, the transgenic line was used to determine whether DomA alters the main axons of the caudal $(\mathrm{CaP})$, middle $(\mathrm{MiP})$, and the rostral (RoP) primary motor neurons (Fig. 5E). In agreement with findings from Chapter 3, DomA did not alter the main caudal and rostral primary motor neurons (Fig. 5D,E). However, it significantly reduced the number of observed MiP primary motor neurons (Estimate $=-3.03$, Std. Error $=0.78, \mathrm{p}=9.93 \mathrm{e}$ -05) (Fig. 5D,E). Together, these results show that DomA does not alter selected sensory structures or $\mathrm{CaP}$ and $\mathrm{RoP}$ main primary motor neuron axons in embryos soon after DomA exposure - a finding that is consistent with effects described in the larval stages ( $5 \mathrm{dpf}$ ).

\section{Domoic acid-induced axonal losses may contribute to observed myelin defects}

DomA led to the loss of reticulospinal neurons and their axons prior to myelination (Fig. 4). The loss of subsets of hindbrain reticulospinal neurons and spinal interneurons would likely result in an overall decrease in axonal surface area. It is conceivable that changes to the axonal surface area could alter the spinal cord cellular environment and in turn lead to the myelin defects observed.

One of the characteristics of DomA-induced myelin defects is appearance of the unusual circular profiles rather than thin elongated myelin sheaths (chapter 2). These circular profiles have also been found recently in a genetic model, a mutant for kifl binding protein (kiflbp), which has 50$80 \%$ reductions in axon surface areas within the spinal cord. ${ }^{69}$ The circular profiles were identified as myelin that ectopically wrapped neuronal cell bodies. I thus sought to determine whether DomA-induced circular profiles were also ectopically myelinated neuronal cell bodies.

One possibility that needed to be excluded is that the circular features I observed in DomAexposed fish were oligodendrocyte cell bodies. To accomplish this, I used the double transgenic, $\operatorname{Tg}(m b p: E G F P-C A A X) \times \operatorname{Tg}(m b p: E G F P)$, which allowed me to distinguish oligodendrocyte cell bodies, which are round but contain EGFP in the cytoplasm, from circular membranes that are also round but contain EGFP only in their membranes. Control fish had labeled oligodendrocyte cell bodies but rarely had the unusual circular features. In contrast, DomA-exposed fish had labeled oligodendrocyte cell bodies along with many of these circular features (Fig. 6A). These 
findings were also corroborated by experiments in which $\operatorname{Tg}(\operatorname{sox} 10: R F P)$ fish, which express RFP in all oligodendrocyte cell bodies, were injected with the mbp:EGFP-CAAX plasmid to produce mosaic expression of EGFP in myelin sheaths. Fish exposed to DomA showed numerous circular profiles that contained EGFP in the membrane, but with no RFP in their cytoplasm (Fig. 6B). These findings using two separate fish lines indicate that these circular features were not oligodendrocyte cell bodies.

To determine whether these circular features were ectopically myelinated neuronal cell bodies, I used a double transgenic line $(\operatorname{Tg}(\operatorname{cntn} 1 b: E G F P-C A A X) \times \operatorname{Tg}(\operatorname{sox} 10: m R F P))$ that labels both neuronal membranes in the spinal cord and oligodendrocyte membranes. Using this line, I showed that the circular membrane features were in similar locations to the neuron cell bodies, suggesting that these circular membranes were indeed myelin that ectopically wrapped neuronal cell bodies (Fig. 6C).

Scanning electron microscopy was then used to image selected regions in the spinal cord in one control and one DomA- exposed fish (Fig. 6D). The DomA-exposed fish had several neuronal cell bodies that were surrounded by additional membranes (Fig 6D2- D3). Due to resolution limitations $(5 \mathrm{~nm})$, I am unable to definitively identify these features as myelin that ectopically wrap the neuronal cell bodies, but together with the light microscopy data, it strengthens the possibility that they are.

Pharmacologically reducing the number of oligodendrocytes results in lower numbers of circular myelin profiles

Findings from the genetic model ( $k i f 1 b p-/-)$ suggest that oligodendrocytes begin to wrap neuronal cell bodies when there is relatively less axonal surface area to myelinate ${ }^{69}$ Thus, these circular profiles are said to result from the mismatch between the available myelinating oligodendrocytes and the axonal surface area.

If the appearance of ectopically myelinated neuronal cell bodies was due to a higher number of oligodendrocytes relative to the axonal surface area, it stands to reason that reducing the number of oligodendrocytes would also lead to the reduction in these ectopically myelinated neuronal 
cell bodies. To test this hypothesis, I used the small molecule GLI antagonist GANT61 in (Tg(mbp:EGFP) x $\operatorname{Tg}(m b p: E G F P-C A A X))$ fish to pharmacologically reduce the number of oligodendrocytes.

I first assessed whether GANT61 treatment successfully reduced the number of dorsal oligodendrocytes in the spinal cord. In control fish, exposure to all doses of GANT61 (10, 25, and $40 \mu \mathrm{M}$ ) led to significant reductions in oligodendrocyte number (Fig. 7A). For DomAexposed fish, application of 25 and $40 \mu \mathrm{M}$ GANT61 led to significant reductions in the oligodendrocyte numbers, while $10 \mu \mathrm{M}$ did not (Fig. 7A).

I then characterized the effects of GANT61 treatment on the number of ectopically myelinated neuronal cell bodies (Fig.7B). As expected, there were very few ectopically myelinated cell bodies in control fish, regardless of GANT61 treatment. In contrast, a majority of DomAexposed fish had ectopically myelinated cell bodies. The number of these ectopically-myelinated cell bodies was influenced by GANT61 treatment. DomA-exposed fish that were treated with 25 and $40 \mu \mathrm{M}$ GANT61 had fewer ectopically-myelinated neuronal cell bodies compared to those that were not treated with GANT61.

Lastly, there was a slight positive correlation between the numbers of dorsal oligodendrocytes and the number of ectopically-myelinated neuronal cell bodies (Fig. 7D). As the number of oligodendrocytes increased in DomA treated fish, so did the number of ectopically-myelinated neuronal cell bodies ( $\mathrm{rho}=0.61$, Pearson correlation, $\mathrm{p}=3.96 \mathrm{e}-13$ ). The positive correlation was significant for both experimental trials. However, the first trial showed a stronger correlation between the number of oligodendrocytes and the ectopically-myelinated neuronal cell bodies than the second one (Trial $1-\mathrm{rho}=0.65, \mathrm{p}=4.06 \mathrm{e}-08$, Trial $2-\mathrm{rho}=0.36, \mathrm{p}=0.007$, Supplemental Fig. 4C, 4D).

\subsection{DISCUSSION}

Findings from this chapter show that DomA exposure at 2 dpf leads to the loss of specific neural populations, including the Mauthner cells, prior to myelination (at $60 \mathrm{hpf}$ ). This indicates that the loss of these neurons does not occur secondarily as a result of the myelin defects observed later 
in development (from $3.5 \mathrm{dpf}$ onwards). Instead, the loss of reticulospinal neurons may contribute to the observed aberrant myelination pattern, which is characterized by both the overall reduction in myelin and the appearance of unusual circular myelin membranes. The overall reduction in myelin was partially due to the loss of the Mauthner neuron which, when present, is the largest neuron that has the most heavily myelinated axon in zebrafish. ${ }^{70,71}$

The loss of the Mauthner axon may also have contribute to the appearance of the unusual circular myelin membranes, which were tentatively identified as myelin that ectopically wrapped neuronal cell bodies. The appearance of these circular membranes may be due to the loss of axonal surface area for oligodendrocytes to myelinate, which would then lead oligodendrocytes to myelinate neuronal cell bodies instead. I hypothesized this because of findings from a recent study that used a genetic model that lacks reticulospinal axons ( $k i f 1 b p-/-)$, and also had an excess of ectopic myelin wrapping neuronal cell bodies. ${ }^{69}$ The authors posited that the oligodendrocytes myelinate neuronal cell bodies in cellular environments where there were fewer axons to myelinate relative to the number of oligodendrocytes present. To test this, they manipulated oligodendrocyte numbers using a pharmacological approach; decreasing the number of oligodendrocytes numbers reduced the number of ectopically myelinated neuronal cell bodies while increasing the number of oligodendrocytes increased the number of these ectopic features. Because I hypothesized that DomA-induced axonal loss led to these circular myelin membranes, I used the same approach. I treated DomA-exposed fish with GANT61 - a small molecule that decreases oligodendrocyte number - and found a reduction in the number of oligodendrocytes as well as the circular myelin membranes. This supports the hypothesis that these circular myelin membranes arise in DomA-treated fish due to the loss of reticulospinal axons and the resulting mismatch of axonal surface area to oligodendrocyte number causes oligodendrocytes to ectopically myelinate neuronal cell bodies.

It is notable that DomA exposure at $2 \mathrm{dpf}$ (and not $1 \mathrm{dpf}$ ) leads to the loss of Mauthner neurons. While Mauthner neurons are present at both 1 and $2 \mathrm{dpf}$, they have different intrinsic properties at different developmental stages. These properties, in turn, alter the neurons' excitability and susceptibility to excitotoxicity. Previous work has shown that Mauthner cells are more excitable at $48 \mathrm{hpf}$ versus $30 \mathrm{hpf}$ as measured by higher frequency and amplitude in their AMPA miniature 
excitatory postsynaptic potentials (mEPSPs). ${ }^{48}$ Furthermore, the authors attributed these changes to a switch in AMPA receptor subunit composition between $30 \mathrm{hpf}$ and $48 \mathrm{hpf}$. It is conceivable that the developmental switch in AMPA receptor subunits of Mauthner neurons make these neurons more sensitive to DomA exposures later in development. It is well-known that DomA has different binding affinities for different AMPA and KA receptors that are composed of homomeric subunits. ${ }^{10}$ The switches in AMPA receptor subunits in development could change the sensitivity of neurons to DomA, making it more excitotoxic for the Mauthner neuron at the 2 dpf timepoint compared to the $1 \mathrm{dpf}$ timepoint.

While DomA exposure at $2 \mathrm{dpf}$ led to the loss of the Mauthner cell at $2.5 \mathrm{dpf}$, it did not lead to any apparent losses in selected sensory neuron structures or the main axons of both the CaP and the RoP primary motor neurons. However, exposure to DomA did lead to significant losses in the MiP primary motor neurons. Primary motor neuron subtypes - $\mathrm{CaP}$, RoP and MiP - have different axonal trajectories, innervate different regions of the muscle mass and have different electrical membrane properties. ${ }^{72,73}$ This may explain how DomA may preferentially alter the main primary motor neuron axon of one subtype and not the other.

DomA exposure also affected the oligodendrocyte lineage. After the initial stages of myelination (2.5- $3 \mathrm{dpf}$ ), DomA exposure led to significantly fewer myelinating oligodendrocytes in fish that also had severe myelin defects. DomA exposure also resulted in individual oligodendrocytes that had fewer and shorter elongated myelin sheaths, while having more aberrant circular myelin membranes, which may be ectopically myelinating neuronal cell bodies. In contrast, DomA did not alter the number of OPCs. DomA might have caused a reduction in OPC count in a number of ways, including disrupting OPC specification, inhibiting OPC proliferation, or causing OPC cell death. The lack of DomA-induced reductions in OPC counts suggests that these processes are not affected by DomA exposure. However, it is possible that DomA alters these processes in opposite ways that result in no changes to total number of OPCs - for example, if DomA exposure increased OPC specification while also causing higher cell death rates. Further experiments that directly investigate critical cellular processes including specification, migration, and differentiation would be necessary to confirm that DomA does not perturb OPC development. 
It is possible that the effects of DomA on the oligodendrocyte lineage are secondary to the loss of reticulospinal neurons. Results from experiments using the kiflbp mutant ${ }^{74}$ were similar to those from my study. The loss of kifl $b p$, and the resulting reduction in axonal surface area, did not lead to fewer OPCs, nor did the loss alter OPC specification, migration, or timing of differentiation. In contrast, the loss of kiflbp led to the impaired survival and the overall reduction in myelination in the posterior region on the spinal cord where the axonal loss was more pronounced. This illustrates how target axons regulate oligodendrocyte development, and conversely how the loss of axons leads to perturbations in oligodendrocyte survival, as well as oligodendrocyte loss.

Although my results strongly suggest that reticulospinal neurons are initial targets of DomA and that their loss contributes to the myelination defects observed, I cannot rule out the possibility that DomA also targets the oligodendrocyte lineage directly. It is possible that DomA directly binds to ionotrophic glutamate receptors in oligodendrocytes, potentially inhibiting their ability to myelinate the remaining axons and leading to cell death. This in turn may lead to further axonal loss.

\subsection{CONCLUSION}

This chapter identified neuronal subpopulations (Mauthner cell, MiP primary motor neurons) as the initial targets for DomA exposure that occurs during a specific developmental period. The loss of these neurons, and the resulting changes to the axonal environment in the spinal cord, contributed to the pronounced myelin defects observed. DomA also led to loss of oligodendrocytes and the inability of remaining oligodendrocytes to appropriately myelinate targets in the spinal cord. This may be a secondary effect due to the initial loss of neurons or to direct effects of DomA on oligodendrocytes. These results suggest that DomA exposure can target specific neuronal populations and alter cellular environments that lead to pronounced and lasting structural and behavioral phenotypes. 


\section{FUNDING}

This research was supported by the WHOI/MIT Academic Programs Office, the Woods Hole Sea grant (NA14OAR4170074), and the Woods Hole Center for Oceans and Human Health (NIH - P01ES021923 and NSF- OCE-1314642 to MEH and NA, P01ES021923-04S1 to JMP, NIH - P01ES028938 and NSF- OCE-1840381 to MEH and NA).

\section{ACKNOWLEDGEMENTS}

I would like to thank Matthew Salanga (Northern Arizona University) for training and advice regarding plasmid design and transgenic line generation, Louis Kerr and Nadja Brun for light microscopy training and advice (MBL microscopy facility), Eduardo Rosa-Molinar, Noraida Marinez-Rivera, and Irma Torres-Vazquez (University of Kansas) for electron microscopy training and advice, Kasia Hamar for electron microscopy sample preparation and imaging (MBL microscopy facility), and the labs for generously providing me with zebrafish transgenic lines to make this work possible - Sarah Kucenas (University of Virginia), Bruce Appel (University of Colorado, Denver), Kelly Monk (Vollum Institute, Oregon Health and Sciences University), Charles Kaufman (University of Washington St. Louis), David Lyons (University of Edinburg) and David Grunwald (University of Utah). 


\section{CHAPTER 4 FIGURES}

\section{Figure 1: Exposure to DomA at 2 dpf did not reduce the number of oligodendrocyte precursor cells prior to myelination}

(A) Diagram of laterally mounted double transgenic larvae. The rectangle delineates the approximate location within the spinal cord where the image was acquired.

(A1) $\operatorname{Tg}($ olig2:EGFP) x $T g(\operatorname{sox} 10: m R F P)$ double transgenic fish used for (B,C) (A2) $\operatorname{Tg}(n k x 2.2 a: m E G F P) \times \operatorname{Tg}(\operatorname{sox} 10: R F P)$ double transgenic fish used for (D,E)

(B) Representative images of the double transgenic fish, $\operatorname{Tg}(\operatorname{sox} 10: m R F P) \mathrm{x}$

Tg(olig2:EGFP), imaged at $2.5 \mathrm{dpf}$. Control (B1) and DomA-exposed fish (B2). White arrow points to a dorsal OPC.

(C) Oligodendrocyte precursor cell (OPC) count in dorsal spinal cords of the control and DomA-exposed double transgenic fish - $T g(\operatorname{sox} 10: m R F P)$ x $T g(o l i g 2: E G F P)$. Each point represents the number of OPCs counted within the $403.1 \mu \mathrm{M}$ imaging area in a single fish $($ Control - median $=26, \mathrm{IQR}=6$; DomA - median $=25, \mathrm{IQR}=9)$.

(D) Representative images of the double transgenic fish, $\operatorname{Tg}(\operatorname{sox} 10: R F P) \mathrm{x}$ $\operatorname{Tg}(n k x 2.2 a: m E G F P)$, imaged at $2.5 \mathrm{dpf}$ in Control (D1) and DomA exposed fish (D2). White arrow points to a dorsal OPC in the myelinating lineage.

(E) Counts of OPCs that are fated to be myelinating oligodendrocytes in the dorsal spinal cord in double transgenic fish - Tg(nkx2.2a:mEGFP) x $\operatorname{Tg}(\operatorname{sox} 10: R F P)$. Each point represents the number of OPCs counted within the $354.3 \mu \mathrm{M}$ imaging area in a single fish. $($ Control - median $=33, \mathrm{IQR}=15$; DomA - median $=36, \mathrm{IQR}=13)$.

Data shown are from two trials. See Supplemental Fig. 1 for data from individual trials. 
A1.

$\operatorname{Tg}($ olig2:EGFP) х $\operatorname{Tg}(\operatorname{sox} 10: m R F P)$

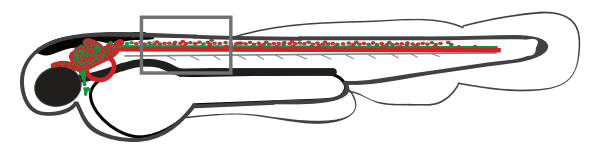

B1.

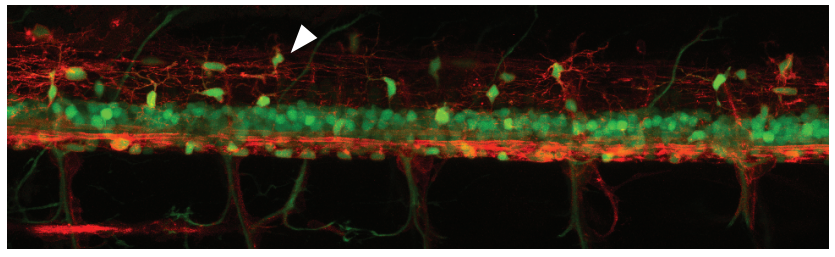

B2.

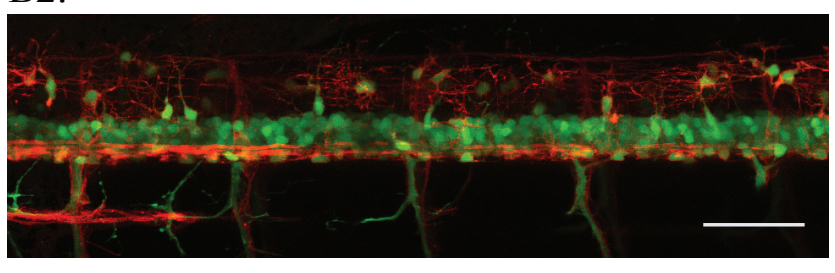

D1.

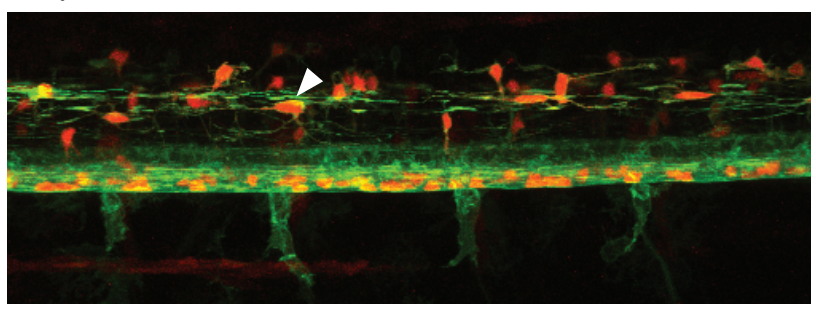

D2.

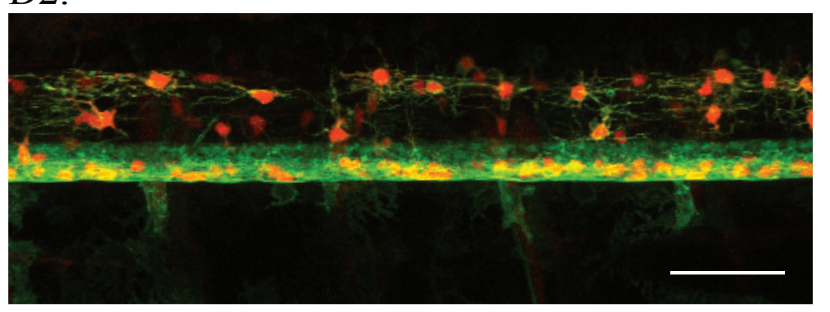

A2.

$\operatorname{Tg}(n k x 2.2 a: m E G F P) \times \operatorname{Tg}(\operatorname{sox} 10: R F P)$

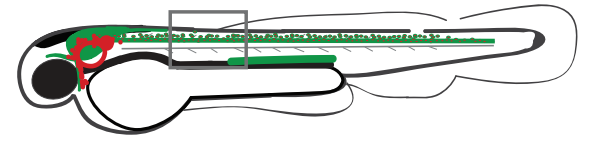

C.

$$
\mathrm{n}=54 \quad \mathrm{n}=59
$$

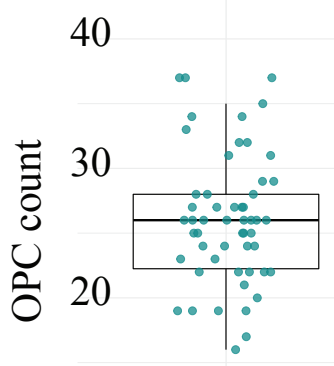

10

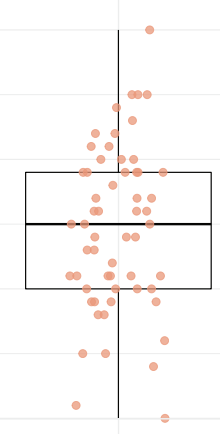

0

Control

DomA

E.

$$
n=64 \quad n=63
$$

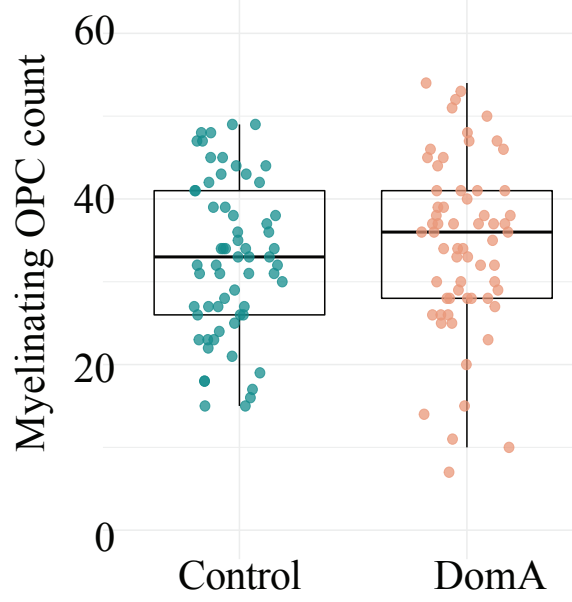


Figure 2: Exposure to DomA at 2 dpf reduces the number of myelinating oligodendrocytes in fish with severe myelin defects, and in a dose dependent manner

(A) $T g(m b p: E G F P)$ transgenic line was used to quantify myelinating oligodendrocytes at 4 dpf. The rectangle delineates the approximate location within the spinal cord where the image was acquired (somites 6-10).

(B) Number of myelinating oligodendrocytes quantified at $4 \mathrm{dpf}$ in the spinal cords of fish exposed to different doses of DomA at $2 \mathrm{dpf}$. Each point represents the number of myelinating oligodendrocytes within the $403.9 \mu \mathrm{M}$ imaging area in a single fish. (Control $(0 \mathrm{ng})-$ median $=70, \mathrm{IQR}=13$; DomA 0.14ng - median $=62, \mathrm{IQR}=20 ;$ DomA $0.18 \mathrm{ng}-$ median $=47, \mathrm{IQR}=20$ ).

(C) Representative images of laterally mounted $\operatorname{Tg}(\mathrm{mbp}$ :EGFP) fish classified by myelin severity, ranging from 0 , representing control-like myelin sheaths, to 3 , representing the most severe myelin phenotype observed.

(D) Fish used in Fig 2B were further subdivided by the severity of the myelin defect observed. The myelinating oligodendrocyte counts were then plotted against the myelin severity score.

Scale bar $=100 \mu \mathrm{m}, \mathrm{n} . \mathrm{s} .=$ not significant, $* * * \mathrm{p}<1$ e -3 using a generalized mixed effects models with a negative binomial distribution 

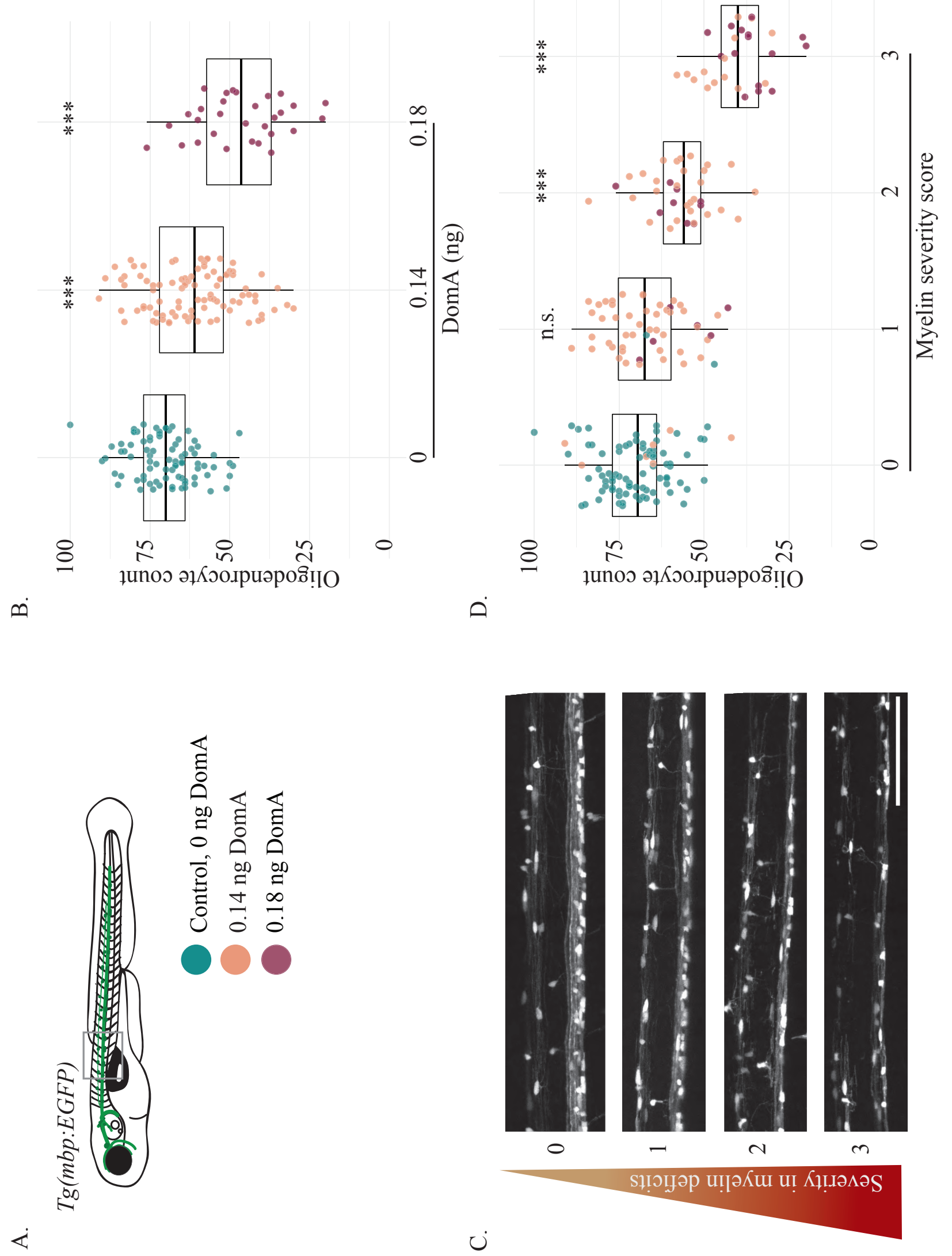
Figure 3: DomA reduces the length and number of myelin sheaths produced by individual oligodendrocytes by 4 dpf

(A) Representative images of mosaically labeled oligodendrocytes in DomA-exposed (2 dpf) and control fish following 1 cell injections of the reporter construct, mbp:EGFP-CAAX into $\operatorname{Tg}(\operatorname{sox} 10: R F P)$ background. Only the EGFP channel is presented to highlight the sparsely labeled myelin sheaths. Asterisks mark oligodendrocyte cell bodies. Peach arrow points to aberrant circular profiles. Scale bar $=50 \mu \mathrm{m}$

(B) Number of myelin sheaths produced by single oligodendrocytes (OL) in both control and DomA exposed fish.

(C) Median length of the myelin sheaths produced by individual oligodendrocytes (OL) in $\mu \mathrm{m}$. Oligodendrocytes without myelin sheaths were excluded from this graph.

(D) Number of circular myelin membranes produced by individual oligodendrocytes (OL). 

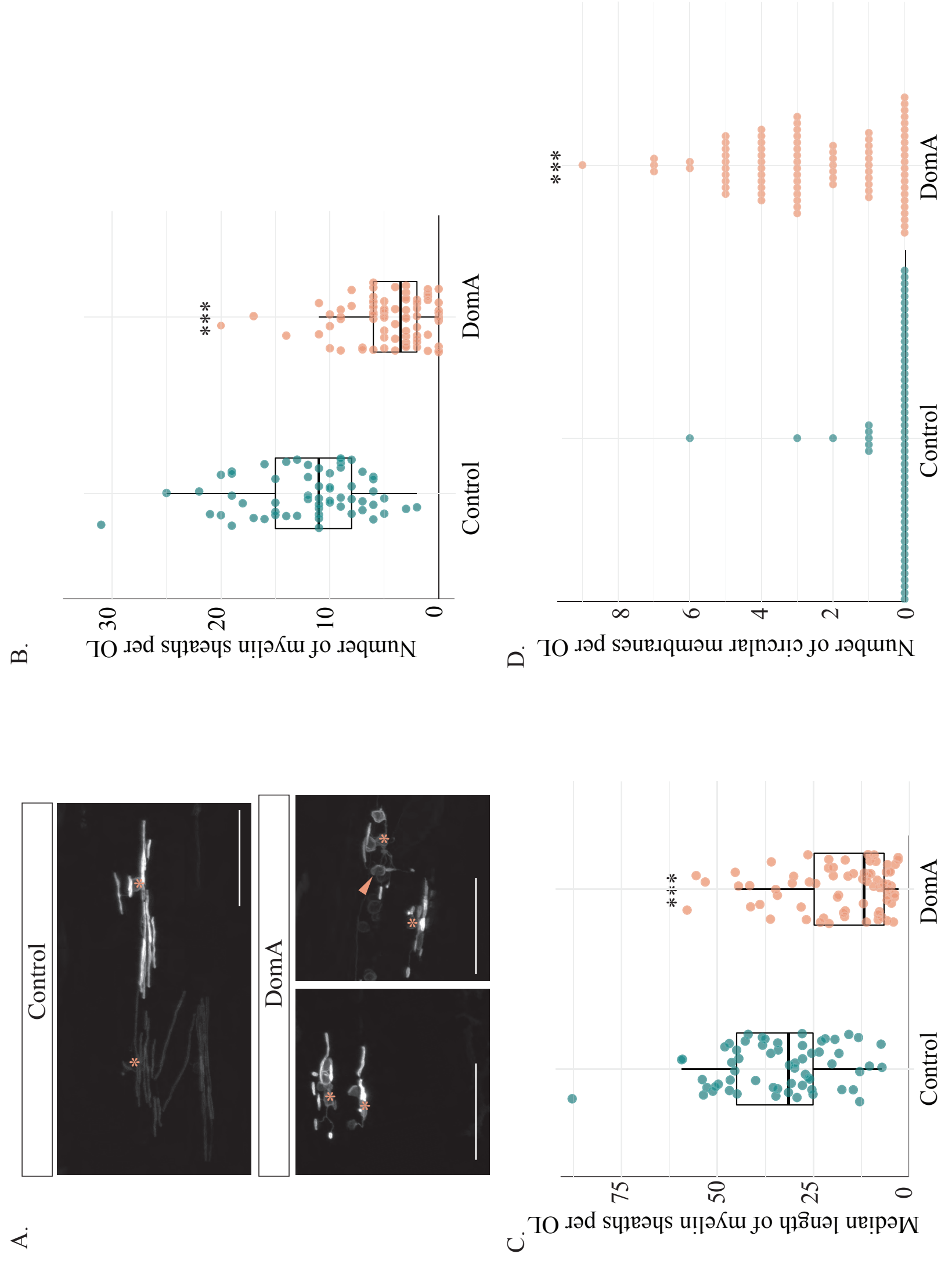
Figure 4: Exposure to DomA at 2 dpf (but not 1 dpf) leads to the loss of the Mauthner neuron prior to myelination

(A) Diagram of $2.5 \mathrm{dpf}$ embryos that were dorsally mounted and immunostained with anti3A10. The rectangle delineates the approximate area that was imaged. Mauthner cells are labeled in teal.

(B) 3A10 immunostaining of $52 \mathrm{hpf}$ non-injected (NI) embryos confirmed that all the major reticulospinal neurons were present by the $2 \mathrm{dpf}$ injection period $(\mathrm{n}=26)$. Mauthner neuron labeled with a teal arrow.

(C) Percentage of embryos exposed to DomA ( $2 \mathrm{dpf}$ ) or vehicle that had 0 , 1, or 2 Mauthner cells after being stained for anti-3A10 at 60 hpf. Numbers of larvae with each phenotype are listed within each bar. Data was aggregated from four trials. See Supplemental Fig. 3 for individual trial data.

(D) 3A10 immunostaining of embryos that were exposed to DomA at $2 \mathrm{dpf}(52 \mathrm{hpf})$, then processed 8 hours post exposure $(60 \mathrm{hpf})$. Teal arrows label Mauthner cells, peach arrows label the anterior lateral line, and magenta arrows label the medial longitudinal fasciculus. Scale bar $=100 \mu \mathrm{m}$.

(E) 3A10 immunostaining of 32 hpf non-injected embryos confirmed that all the major reticulospinal neurons were present by 1 dpf injection period $(n=10)$.

(F) 3A10 immunostaining of embryos that were exposed to DomA at $1 \mathrm{dpf}$ (32 hpf), then processed 8 hours post exposure $(40 \mathrm{hpf})$. Control embryos $(\mathrm{n}=38)$; DomA exposed embryos $(n=38)$, with $n=37$ fish with 2 Mauthner cells, and 1 fish with 1 Mauthner cell.

(G) 3A10 immunostaining of embryos that were exposed to DomA at $1 \mathrm{dpf}$ (32 hpf), then processed at 60 hpf. Control embryos $(n=35)$, DomA exposed embryos $(n=41)$. 

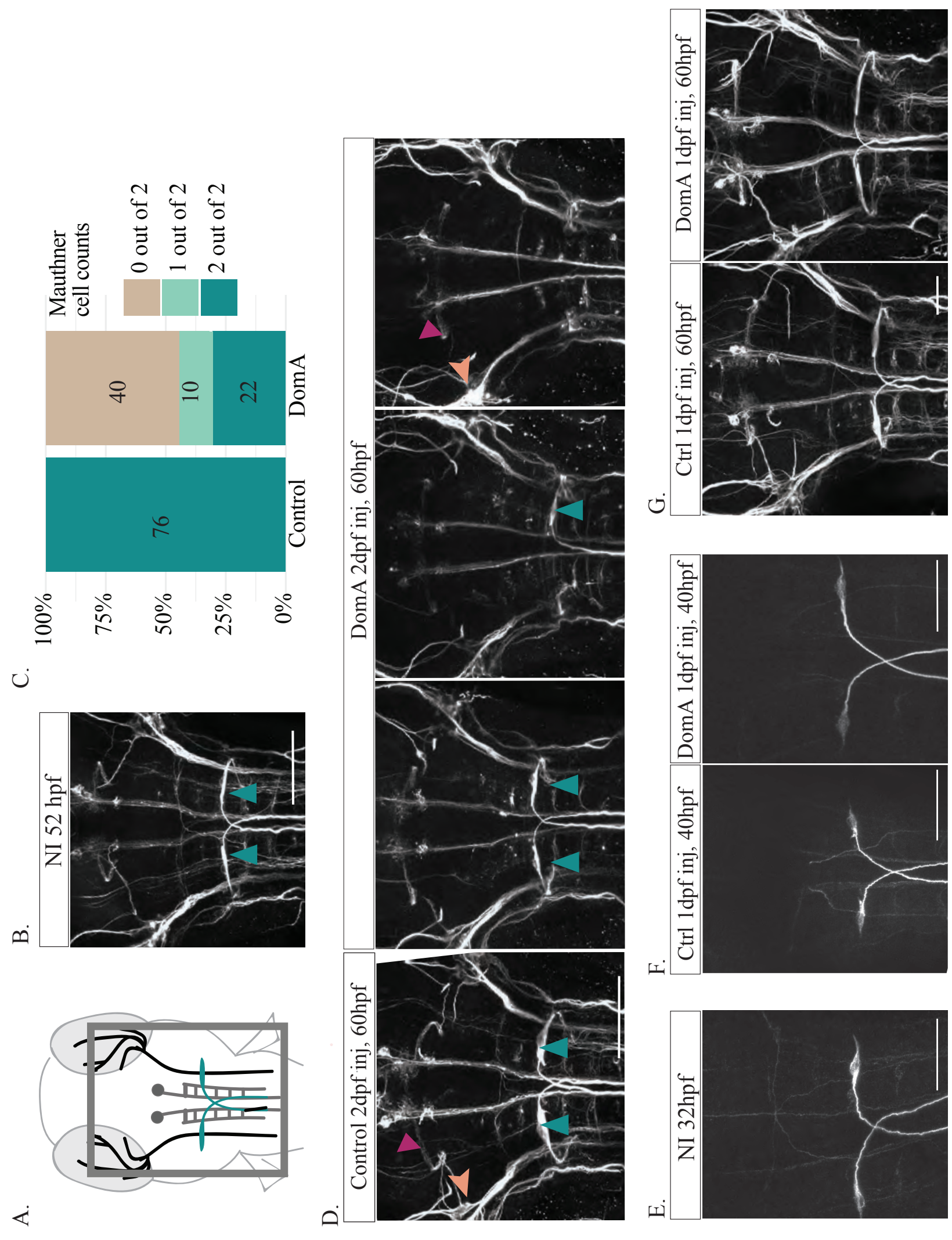
Figure 5: Exposure to DomA at 2 dpf does not alter selected sensory neuron structures or the main axons of two of the three primary motor neurons

(A) Diagram of $2.5 \mathrm{dpf}$ embryos laterally mounted and immunostained with anti-acetylated tubulin. The rectangle delineates the approximate area that was imaged.

(B) Representative images of control $(n=35)$ and DomA-exposed larvae $(n=29)$ Immunostained with anti-acetylated tubulin. Labels point to both the sensory ganglia that comprise the anterior lateral line (nADso, nVDI, nADb, nAVm) and to the peripheral lateral line ( $p L L)$. These fish were a subset of those stained for acetylated tubulin in the trunk region (Chapter 3, Fig. 10B).

(C) Diagram of the axonal trajectories in the three types of primary motor neurons assessed in $\operatorname{Tg}($ olig 2:EGFP) fish.

(D) Representative images of $\operatorname{Tg}($ olig2:EGFP) exposed to vehicle or to DomA at $2 \mathrm{dpf}$ then imaged at $2.5 \mathrm{dpf}$. Peach arrows point to the MiP main axons, magenta arrows point to the RoP main axons, and teal arrows point to the $\mathrm{CaP}$ main axons. Scale bar $=50 \mu \mathrm{m}$. These fish were the same fish used in Figure 1.

(E) Presence or absence of the main axons of the three types of primary motor neurons in control and DomA-exposed fish. $* * * p<0.001$

Abbreviations: superior opthalmic ramus of the anterodorsal lateral line nerve (nADso), dorsolateral nerve of the trigeminal ganglion (nVDI), buccal ramus of the anterodorsal lateral line nerve (nADb) or the mandibular ramus of the anterior lateral line nerve (nAVm). Middle primary motor neuron $(\mathrm{MiP})$, rostral primary motor neuron $(\mathrm{RoP})$, caudal primary motor neurons $(\mathrm{CaP})$. 

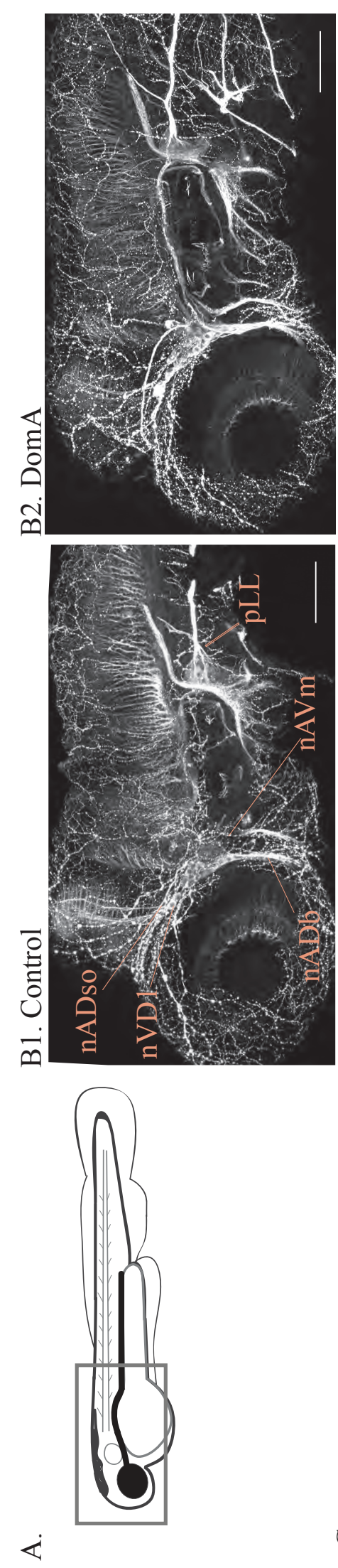
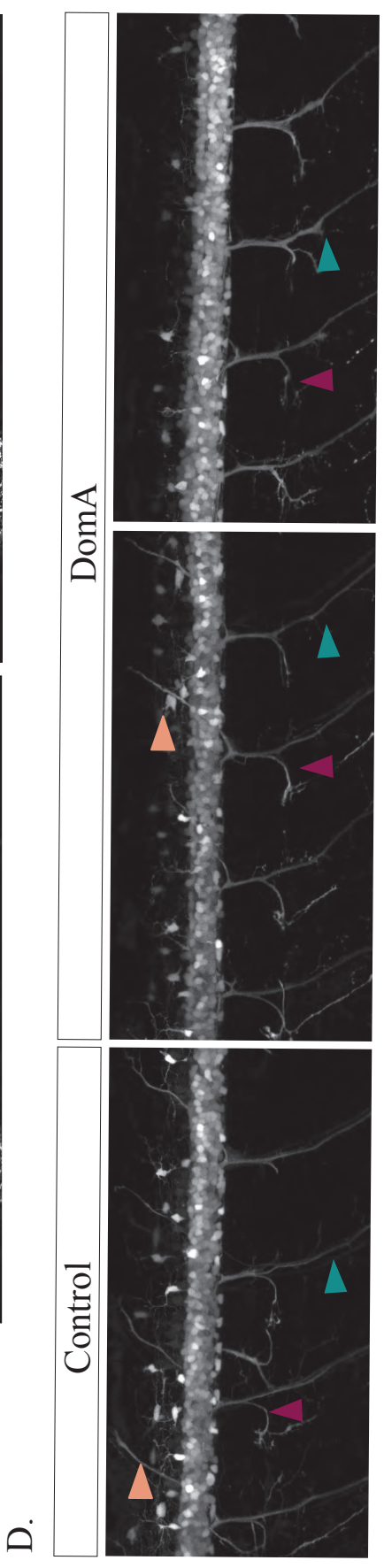

$\sum$
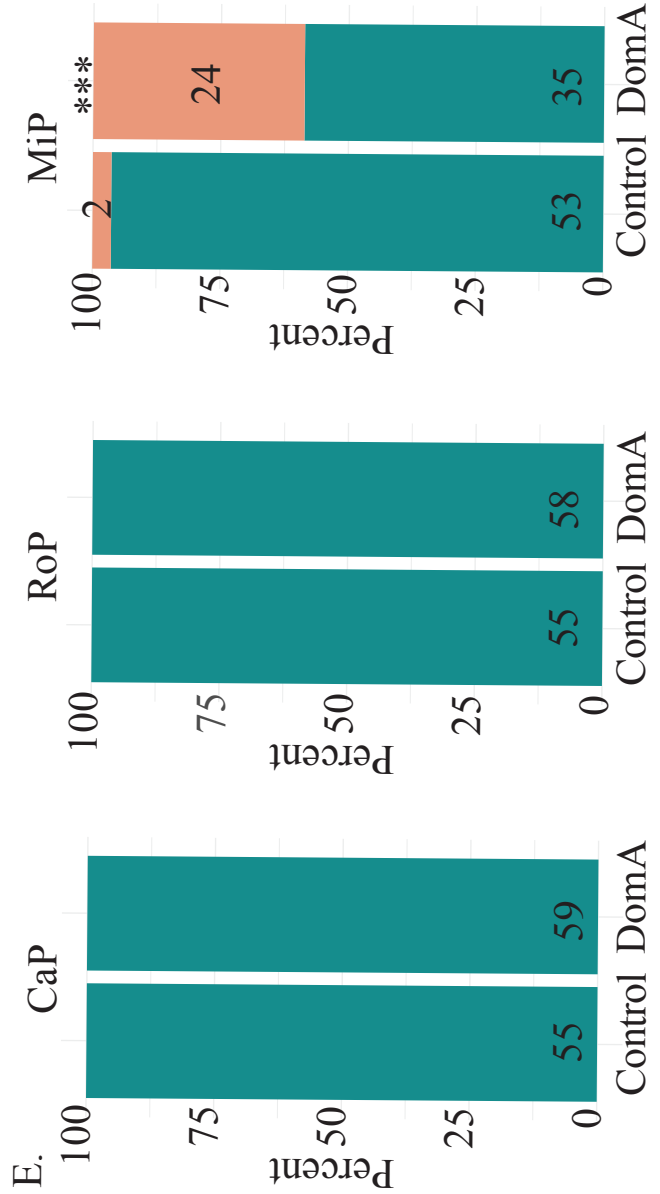
Figure 6: DomA exposure at 2 dpf leads to the formation of aberrant circular profiles that may be ectopically myelinated neuronal cell bodies

(A) $\operatorname{Tg}(m b p: E G F P) \times T g(m b p: E G F P-C A A X)$ double transgenic line labels both oligodendrocyte cell bodies and myelin sheaths. Asterisks mark oligodendrocyte cell bodies. Arrows label circular myelin membranes, which are commonly found in DomAexposed larvae and rarely observed in controls. Scale bar $=100 \mu \mathrm{m}$.

(B) $\operatorname{Tg}(\operatorname{sox} 10: R F P)$ fish with mosaic expression of mbp:EGFP-CAAX fish imaged at $4 \mathrm{dpf}$. Oligodendrocyte cell bodies are labeled in red. Arrows mark circular myelin membranes which are outlined in green. These were the same fish used in Fig. 3, but with the red channel present to show the oligodendrocyte cell body. Scale bar $=50 \mu \mathrm{m}$.

(C) $\operatorname{Tg}(\operatorname{sox} 10: m R F P)$ x $T g(c n t n 1 b: E G F P-C A A X)$ double transgenic line exposed to DomA at $2 \mathrm{dpf}$ then imaged at $5 \mathrm{dpf}$. mRFP labels myelin sheaths and oligodendrocyte membranes. EGFP-CAAX labels the membrane of subpopulations of spinal cord neurons. Two examples of DomA exposed double transgenic fish. Circular myelin membranes labeled in RFP (C1), neuronal cell bodies outlined in EGFP (C2). Images were then merged (C3). Asterisks mark neuronal cell bodies that are potentially associated with circular myelin membranes in the imaging plane. Fish were a subset of those used in chapter 3 to assess the effect of DomA on cranial sensory structures (Chapter 3, Fig. 7D). Scale bar $=20 \mu \mathrm{m}$.

(D) Scanning electron micrograph of neuronal cell bodies in the spinal cords of $T g(m b p: E G F P-C A A X)$ fish. DomA exposed larvae were prescreened for myelin defects prior to processing. (D1) Example neuronal cell body in a control fish shows no evidence of myelin surrounding it. (D2-D3) Example neuronal cell bodies in DomA-exposed fish that may be wrapped by myelin. Arrows point to the putative myelin that surrounds the cell body ( $n=1$ control, $n=1$ DomA-exposed). Scale bar $=2 \mu \mathrm{m}$. 

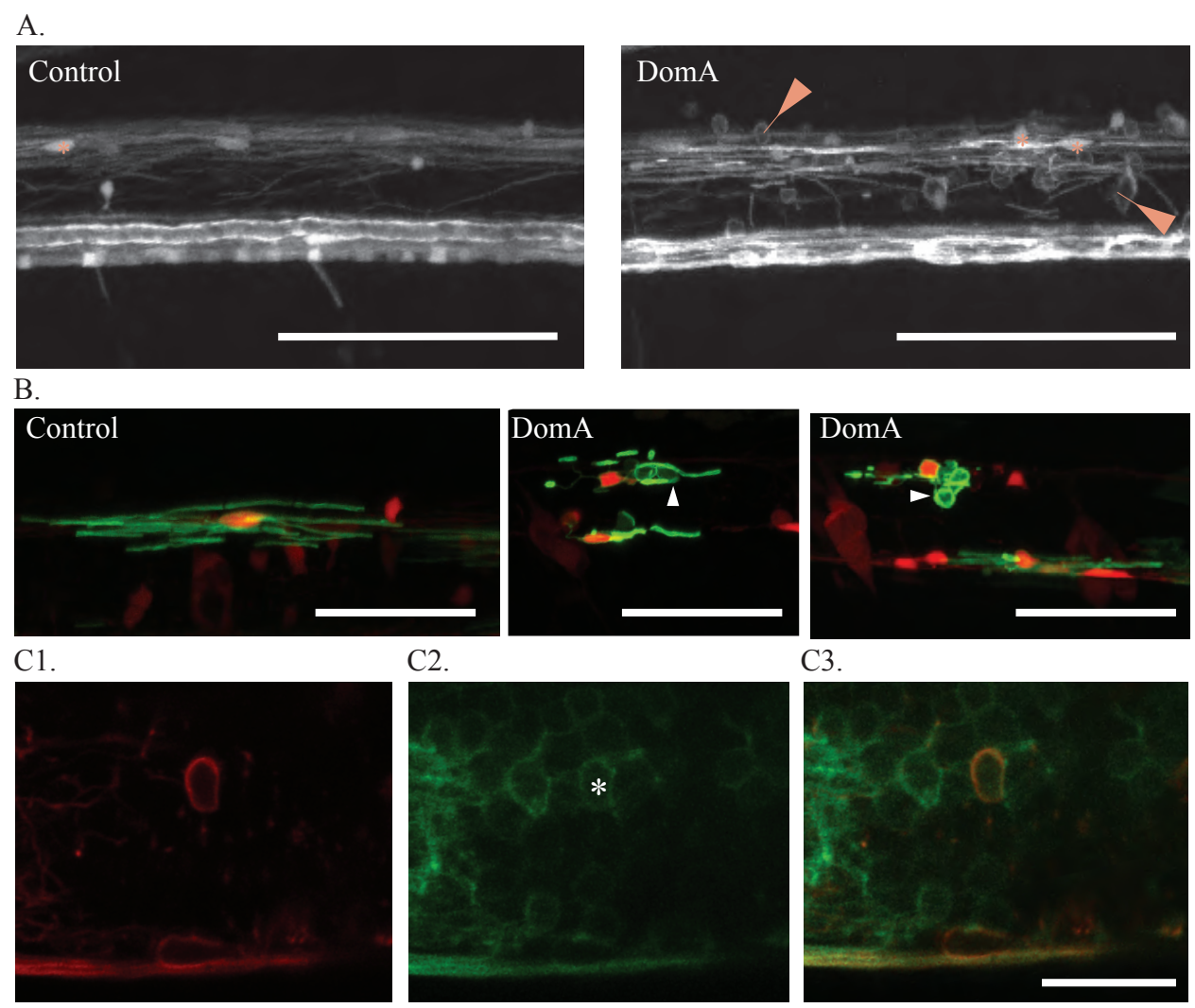

C2.
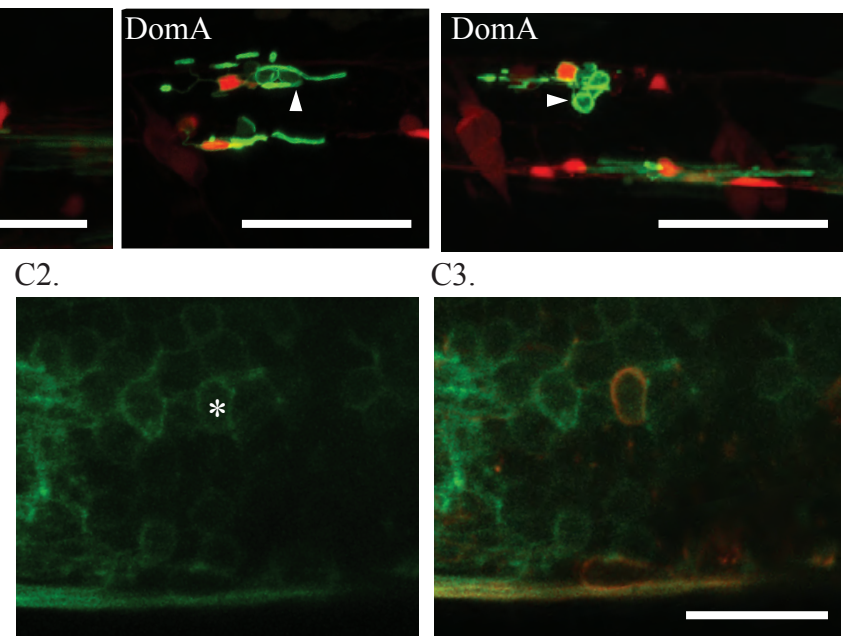

C3.
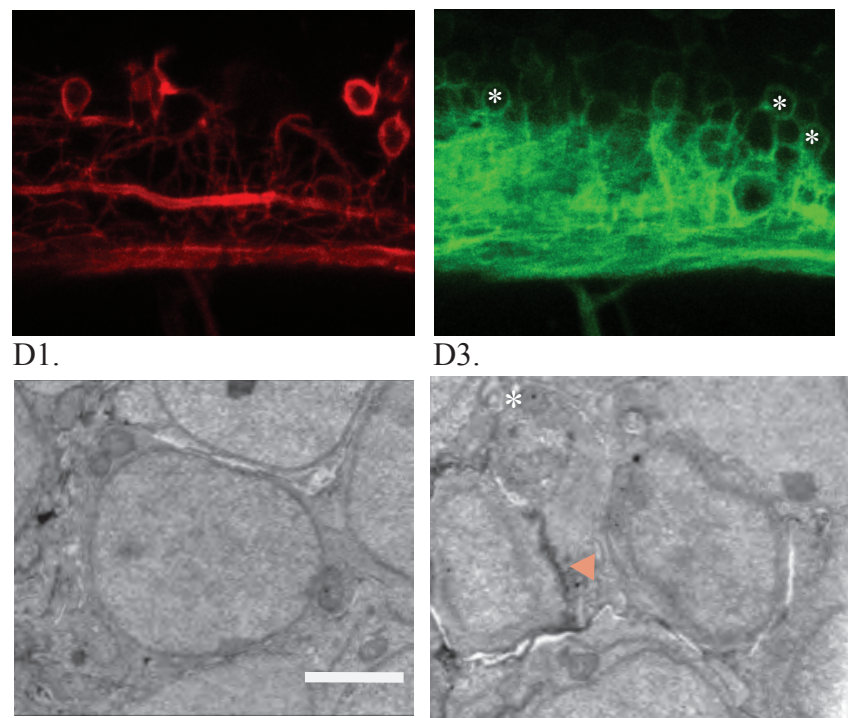

D3.
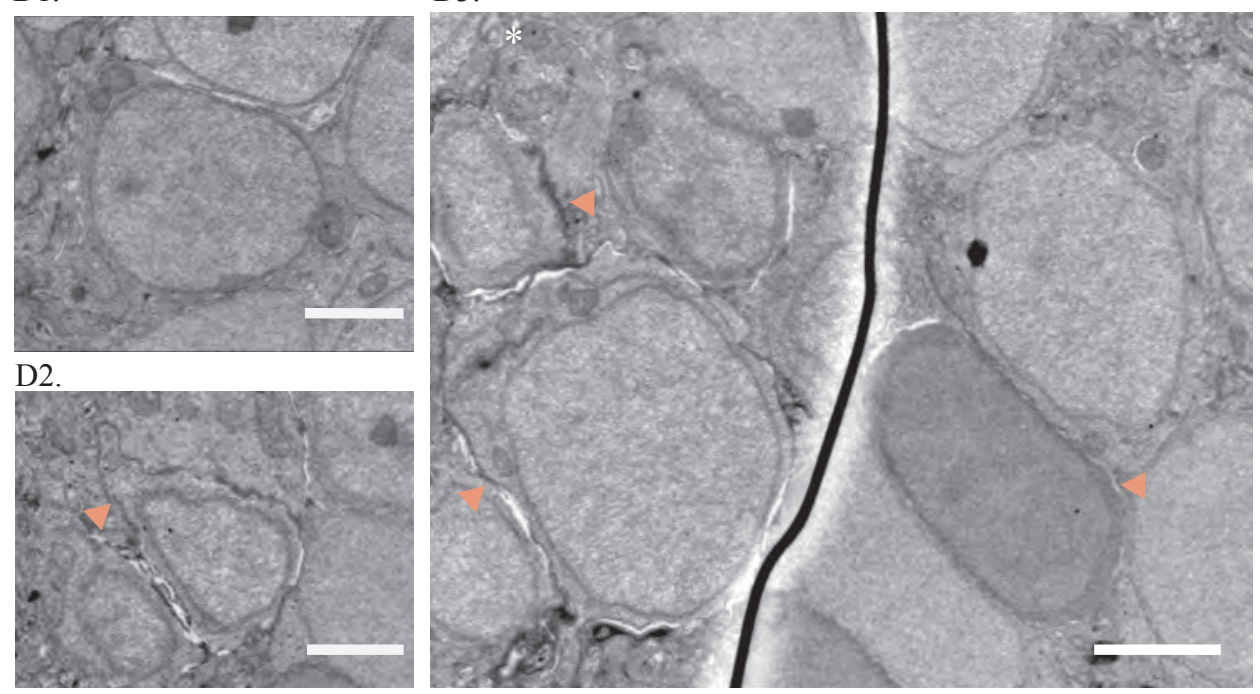
Figure 7: Treatment with GANT61, a small molecule that reduces the number of myelinating oligodendrocytes, also reduces the number of circular profiles in DomA treated fish

(A) $T g(m b p: E G F P)$ x $T g(m b p: E G F P-C A A X)$ double transgenic line were exposed to DomA or vehicle at $2 \mathrm{dpf}$. Myelinating oligodendrocytes in dorsal spinal cord were counted for control and DomA-exposed fish that were exposed to different concentrations of GANT61 (1.5- 4 dpf). Each point represents the number of oligodendrocytes counted within a $269 \mu \mathrm{M}$ imaging area in a single fish.

(B) Number of circular myelin features in dorsal spinal cord of the same control and DomA exposed fish plotted in Fig7A.

(C) The number of circular features per oligodendrocytes in dorsal spinal cord of the control and DomA-exposed fish that were exposed to different concentrations of GANT61.

(D) The number of circular features plotted against the number of dorsal oligodendrocytes.

(E) Images of control fish exposed to $0 \mu \mathrm{M}$ (E1), $10 \mu \mathrm{M}$ (E2), $25 \mu \mathrm{M}$ (E3) and $40 \mu \mathrm{M}$ GANT61 (E4).

(F) Images of DomA fish exposed to $0 \mu \mathrm{M}$ (F1), $10 \mu \mathrm{M}$ (F2), $25 \mu \mathrm{M}$ (F3) and $40 \mu \mathrm{M}$ GANT61 (F4). Scale bar $=50 \mu \mathrm{m}$.

$*=\mathrm{p}<0.05, * *=\mathrm{p}<0.01, * * * \mathrm{p}<0.001$ by generalized mixed models with a Poisson or a negative binomial distribution.

Data were aggregated from two trials. See Supplemental Fig. 4 for data from individual trials. 


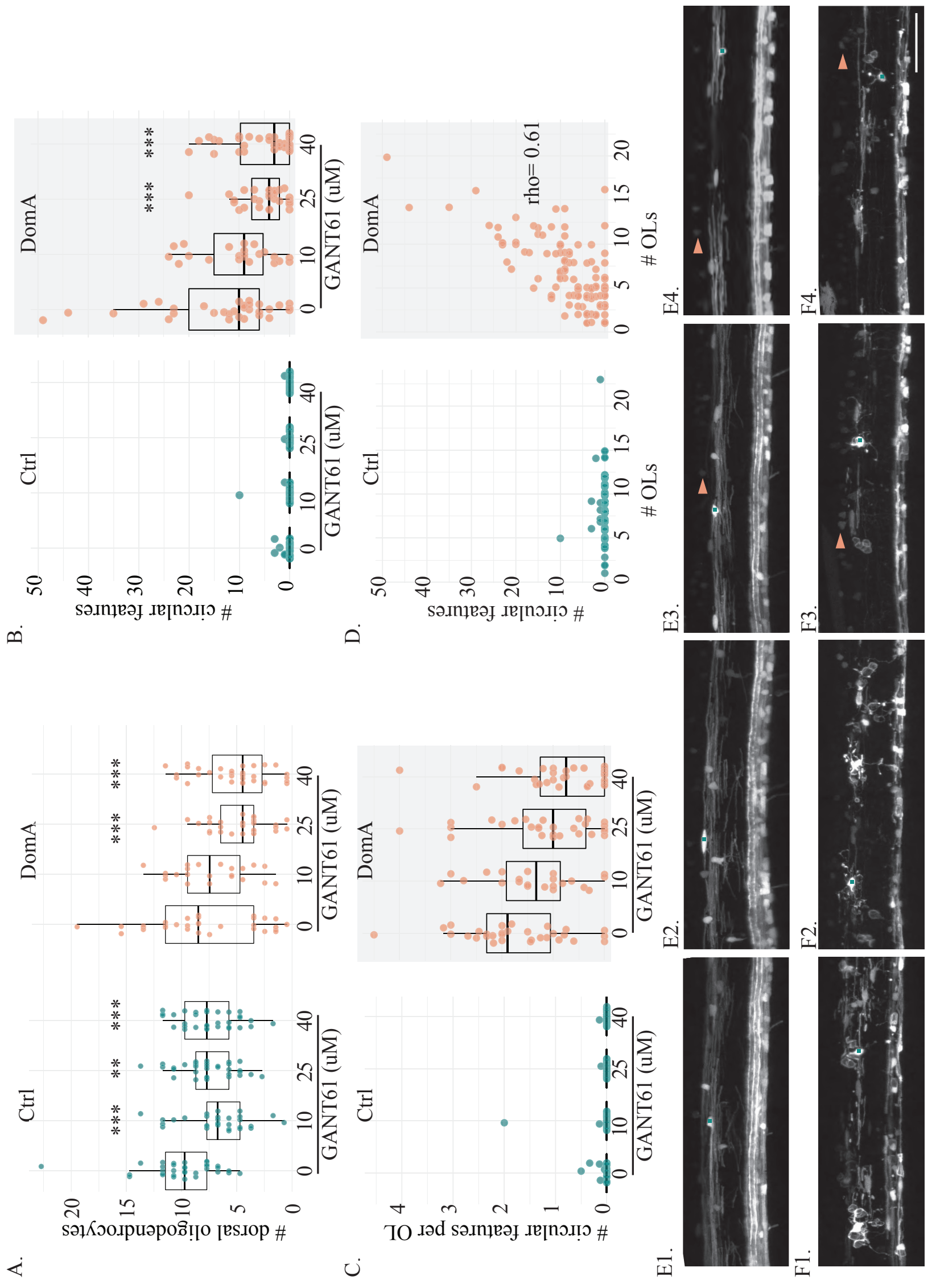




\section{CHAPTER 4: SUPPLEMENTAL INFORMATION}

A.

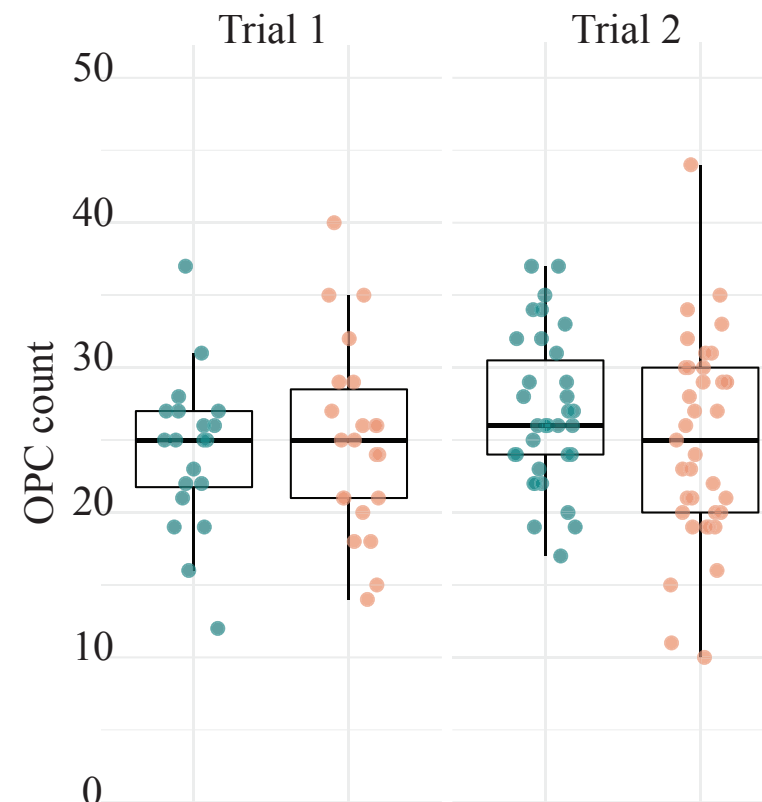

Control DomA Control DomA
B.

Trial 1

Trial 2

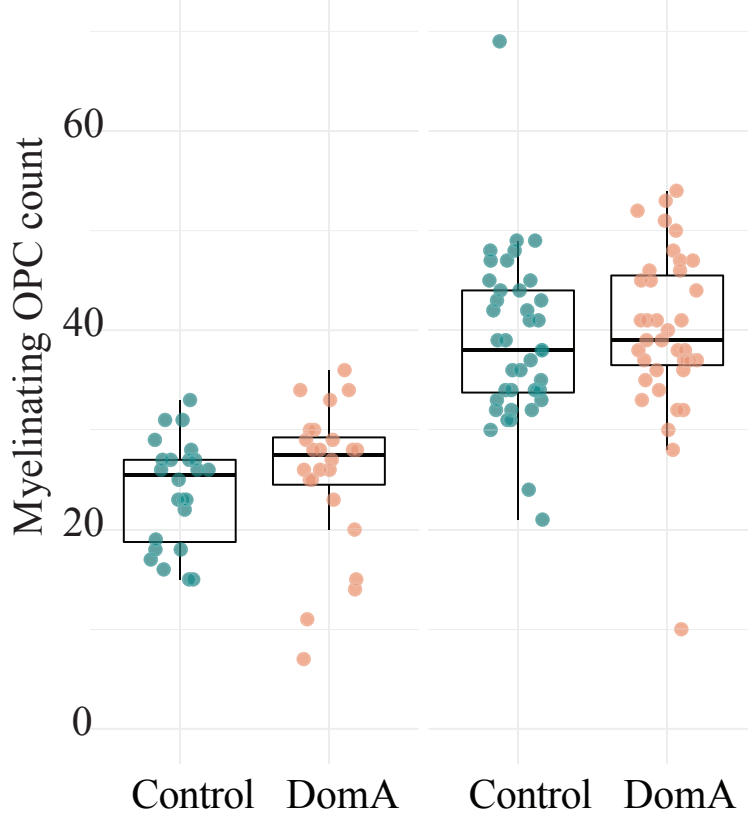

Supplemental Figure 1: Trial differences in oligodendrocyte precursor cell counts.

(A) Oligodendrocyte precursor cell (OPC) count in the dorsal spinal cord in the double transgenic fish, $\operatorname{Tg}(\operatorname{sox} 10: m R F P)$ x $T g(\operatorname{lig} 2: E G F P)$, imaged at $2.5 \mathrm{dpf}$. Each point represents the number of OPCs counted within the $403.1 \mu \mathrm{M}$ imaging area in a single fish. There were no differences in OPC count based on treatment or on repeat trials.

(B) Oligodendrocyte precursor cell (OPC) count in dorsal spinal cord of the $354.3 \mu \mathrm{M}$ long region in the dorsal spinal cords in the double transgenic fish, $\operatorname{Tg}(\operatorname{sox} 10: R F P) \times \operatorname{Tg}(n k x 2.2 a: m E G F P)$, imaged at $2.5 \mathrm{dpf}$. Each point represents the number of OPCs counted within the $354.3 \mu \mathrm{M}$ imaging area in a single fish. While there were no differences in OPC count based on treatment, there were differences in OPC count between the two repeat trials. 


\section{Supplemental Figure 2: Trial differences in myelinating oligodendrocyte cell counts}

(A) Myelinating oligodendrocyte count in the spinal cords. $\operatorname{Tg}(m b p: E G F P)$ imaged at $2.5 \mathrm{dpf}$. Individual points represents myelinating oligodendrocyte counts within the $403.9 \mu \mathrm{M}$ imaging area in a single fish. Trial 2 shows no significant differences between control $(0$ ng DomA) and domoic acid exposed fish (0.14 ng DomA)

(B) Fish were further subdivided by the severity of the myelin defect observed. No treated fish in Trial 2 had myelin with the most severe score (category 3 ). 
A.

Trial 1

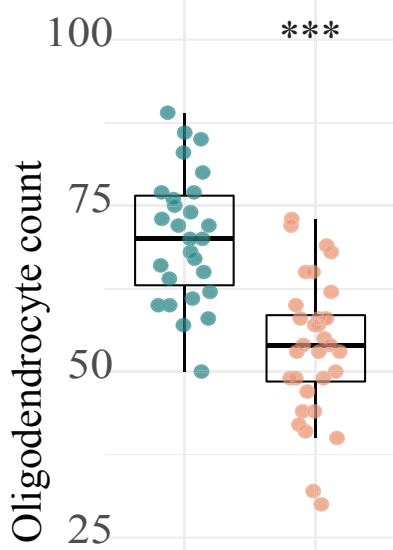

0

0.14

$\bullet$

Trial 2

Trial 3

n.s.
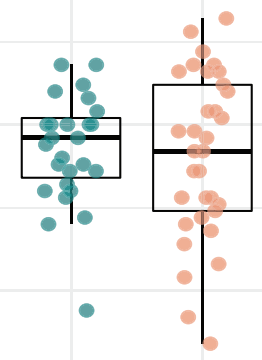

*

***

•
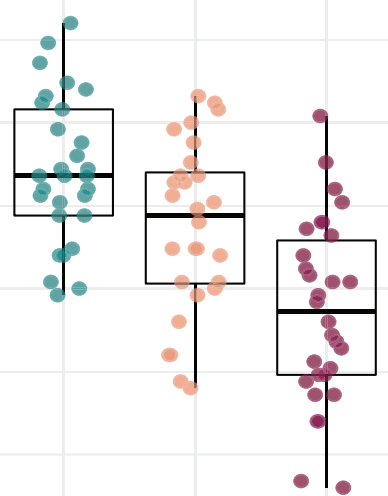

B.

Trial 1

Trial 2

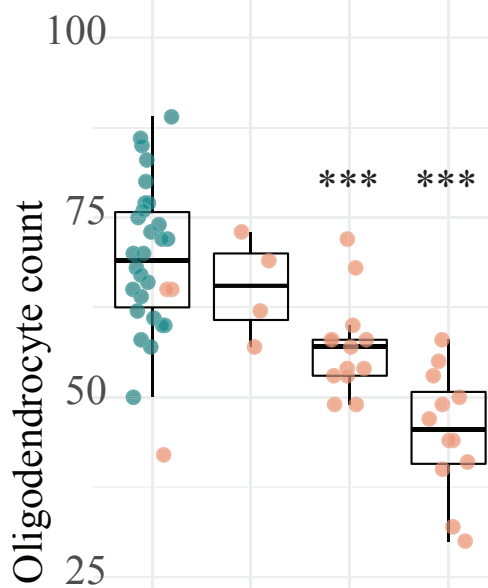

0
Trial 3

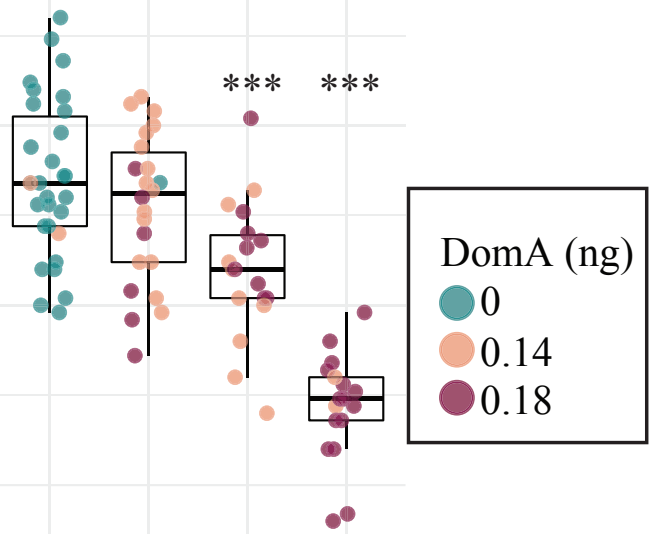

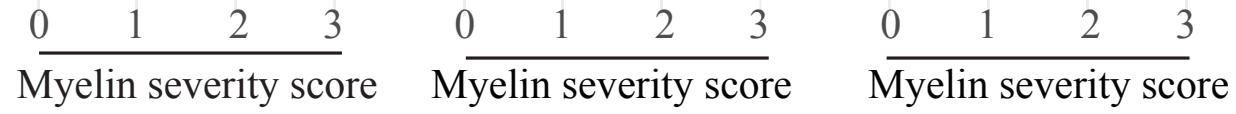




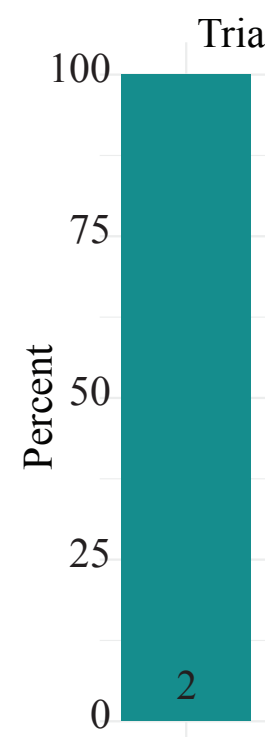

Ctrl DomA
Trial 2

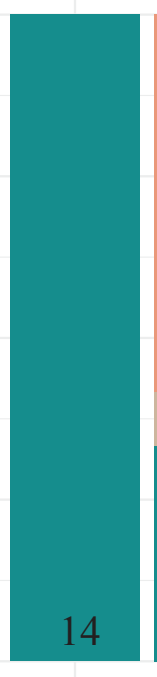

Ctrl

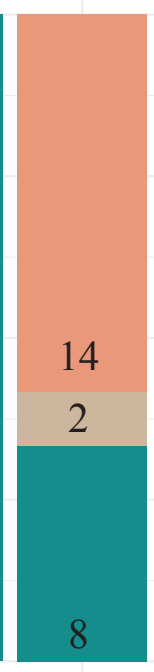

DomA
Trial 3

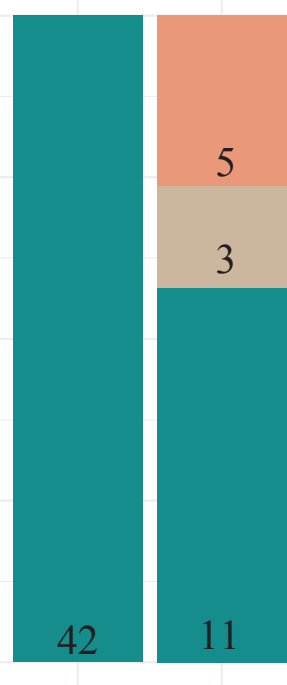

Trial 4

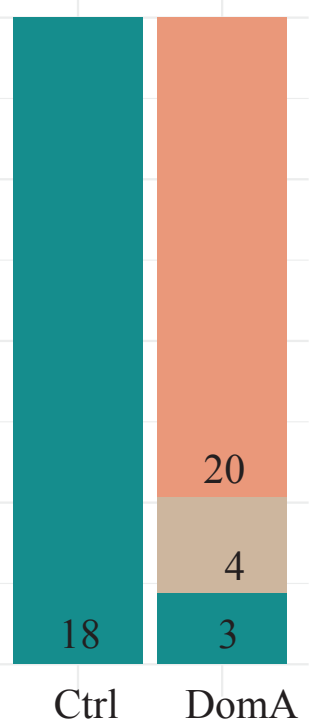

\# Mauthner cells

0
1
2

\section{Supplemental Figure 3: Trial differences in Mauthner cell counts}

Percentage of embryos exposed to DomA ( $2 \mathrm{dpf}$ ) or vehicle that had 0 , 1, or 2 Mauthner cells after being stained for anti-3A10 at $60 \mathrm{hpf}$. Numbers of larvae with each phenotype are listed within each bar. Data are shown by each individual experimental trial. 


\section{Supplemental Figure 4: Trial differences in GANT61 treatment}

(A) Number of myelinating oligodendrocytes in dorsal spinal cord of control and DomAexposed fish that were also exposed to different concentrations of GANT61 (1.5- $4 \mathrm{dpf}$ ). Each point represents the number of oligodendrocytes counted within a $269 \mu \mathrm{M}$ imaging area in a single fish.

(B) Total number of circular features in dorsal spinal cord of the control and DomA-exposed fish that were exposed to different concentrations of GANT61.

(C) The number of circular features plotted against the number of dorsal oligodendrocytes in Trial 1

(D) The number of circular features plotted against the number of dorsal oligodendrocytes in Trial 2

$*=\mathrm{p}<0.05, * *=\mathrm{p}<0.01, * * * \mathrm{p}<0.001$ by generalized mixed models with a Poisson or a negative binomial distribution. 


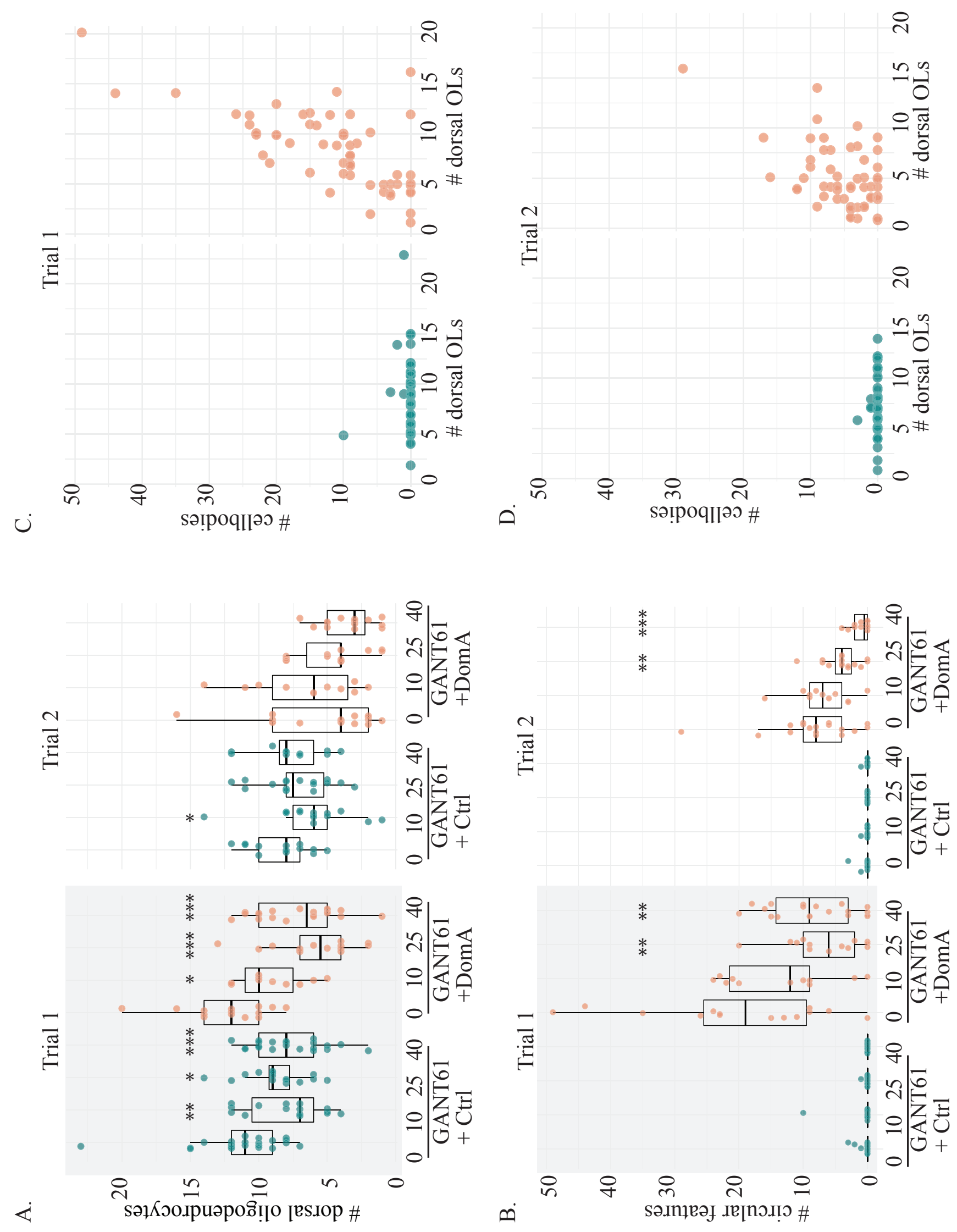




\subsection{REFERENCES}

1. Hoagland, P., Anderson, D. M., Kaoru, Y. \& White, A. W. The economic effects of harmful algal blooms in the United States: Estimates, assessment issues, and information needs. Estuaries 25, 819-837 (2002).

2. Anderson, D. M., Hoagland, P., Kaoru, Y. \& White, A. W. Estimated annual economic impacts from harmful algal blooms (HABs) in the United States. (Woods Hole Oceanographic Institution, 2000). doi:10.1575/1912/96

3. Sunda, W. G., Graneli, E. \& Gobler, C. J. Positive feedback and the development and persistence of ecosystem disruptive algal blooms. J. Phycol. 42, 963-974 (2006).

4. Dolah, F. M. Van, Roelke, D. \& Greene, R. M. Health and Ecological Impacts of Harmful Algal Blooms: Risk Assessment Needs. Hum. Ecol. Risk Assess. An Int. J. 7, 1329-1345 (2001).

5. Moore, S. K. et al. Impacts of climate variability and future climate change on harmful algal blooms and human health. Environ. Health 7 Suppl 2, S4 (2008).

6. Berdalet, E. et al. Marine harmful algal blooms, human health and wellbeing: challenges and opportunities in the 21st century. J. Mar. Biol. Assoc. United Kingdom 96, 61-91 (2016).

7. Grattan, L. M., Holobaugh, S. \& Morris, J. G. Harmful algal blooms and public health. Harmful Algae 57, 2-8 (2016).

8. Backer, L., LE, F., AD, R. \& DG, B. Epidemiology, public health and human diseases associated with harmful marine algae. (UNESCO Publishing, 2003).

9. Stewart, G. R., Zorumski, C. F., Price, M. T. \& Olney, J. W. Domoic acid: A dementiainducing excitotoxic food poison with kainic acid receptor specificity. Exp. Neurol. 110, 127-138 (1990).

10. Hampson, D. R. \& Manalo, J. L. The activation of glutamate receptors by kainic acid and domoic acid. Nat. Toxins 6, 153-8 (1998).

11. Bates, S. S. et al. Pennate Diatom Nitzschia pungens as the Primary Source of Domoic Acid, a Toxin in Shellfish from Eastern Prince Edward Island, Canada. Can. J. Fish. Aquat. Sci. 46, 1203-1215 (1989).

12. Fritz, L., Quilliam, M. A., Wright, J. L. C., Beale, A. M. \& Work, T. M. An outbreak of domoic acid poisoning attributed to the pennate diatom Pseudonitzchia australis . J. Phycol. 28, 439-442 (1992).

13. Nakajima, S. \& Potvin, J. L. Neural and behavioural effects of domoic acid, an amnesic shellfish toxin, in the rat. Can. J. Psychol. 46, 569-81 (1992).

14. Todd, E. C. D. Domoic Acid and Amnesic Shellfish Poisoning - A Review. J. Food Prot. 56, 69-83 (1993).

15. Pulido, O., Pulido \& M., O. Domoic Acid Toxicologic Pathology: A Review. Mar. Drugs 6, 180-219 (2008).

16. Perl, T. M. et al. An outbreak of toxic encephalopathy caused by eating mussels contaminated with domoic acid. N. Engl. J. Med. 322, 1775-80 (1990).

17. Wekell, J. C., Jurst, J. \& Lefebvre, K. a. The origin of the regulatory limits for PSP and ASP toxins in shellfish. J. Shellfish Res. 23, 927-930 (2010).

18. Mariën, K. Establishing tolerable dungeness crab (Cancer magister) and razor clam (Siliqua patula) domoic acid contaminant levels. Environ. Health Perspect. 104, 1230-6 (1996). 
19. Tanemura, K. et al. Intrauterine environment-genome interaction and Children's development (2): Brain structure impairment and behavioral disturbance induced in male mice offspring by a single intraperitoneal administration of domoic acid (DA) to their dams. J. Toxicol. Sci. 34, SP279-SP286 (2009).

20. Zuloaga, D. G. et al. Fetal domoic acid exposure affects lateral amygdala neurons, diminishes social investigation and alters sensory-motor gating. Neurotoxicology 53, 132 140 (2016).

21. Doucette, T. A. et al. Low doses of domoic acid during postnatal development produce permanent changes in rat behaviour and hippocampal morphology. Neurotox. Res. 6, 555563 (2004).

22. Bernard, P. B., MacDonald, D. S., Gill, D. A., Ryan, C. L. \& Tasker, R. A. Hippocampal mossy fiber sprouting and elevated trkB receptor expression following systemic administration of low dose domoic acid during neonatal development. Hippocampus 17, 1121-1133 (2007).

23. Mills, B. D. et al. Prenatal domoic acid exposure disrupts mouse pro-social behavior and functional connectivity MRI. Behav. Brain Res. 308, 14-23 (2016).

24. Dakshinamurti, K., Sharma, S., Sundaram, M. \& Watanabe, T. Hippocampal changes in developing postnatal mice following intrauterine exposure to domoic acid. J. Neurosci. 13, 4486-4495 (1993).

25. Levin, E. D. et al. Persistent neurobehavioral effects of early postnatal domoic acid exposure in rats. Neurotoxicol. Teratol. 28, 673-680 (2006).

26. Aldridge, J. E., Levin, E. D., Seidler, F. J. \& Slotkin, T. A. Developmental exposure of rats to chlorpyrifos leads to behavioral alterations in adulthood, involving serotonergic mechanisms and resembling animal models of depression. Environ. Health Perspect. 113, 527-531 (2005).

27. Adams, A. L., Doucette, T. A., James, R. \& Ryan, C. L. Persistent changes in learning and memory in rats following neonatal treatment with domoic acid. Physiol. Behav. 96, 50512 (2009).

28. Burt, M. A., Ryan, C. L. \& Doucette, T. A. Altered responses to novelty and drug reinforcement in adult rats treated neonatally with domoic acid. Physiol. Behav. 93, 327336 (2008).

29. Marriott, A. L., Ryan, C. L. \& Doucette, T. A. Neonatal domoic acid treatment produces alterations to prepulse inhibition and latent inhibition in adult rats. Pharmacol. Biochem. Behav. 103, 338-44 (2012).

30. Tasker, R. A. R., Perry, M. A., Doucette, T. A. \& Ryan, C. L. NMDA receptor involvement in the effects of low dose domoic acid in neonatal rats. Amino Acids 28, 193 196 (2005).

31. Doucette, T. A., Bernard, P. B., Yuill, P. C., Tasker, R. A. \& Ryan, C. L. Low doses of non-NMDA glutamate receptor agonists alter neurobehavioural development in the rat. Neurotoxicol. Teratol. 25, 473-479 (2003).

32. Matsugami, T. R. et al. From the Cover: Indispensability of the glutamate transporters GLAST and GLT1 to brain development. Proc. Natl. Acad. Sci. U. S. A. 103, 12161-6 (2006).

33. Shiotani, M. et al. Neurobehavioral assessment of mice following repeated oral exposures to domoic acid during prenatal development. Neurotoxicol. Teratol. 64, 8-19 (2017).

34. Nave, K.-A. \& Trapp, B. D. Axon-Glial Signaling and the Glial Support of Axon 
Function. Annu. Rev. Neurosci. 31, 535-561 (2008).

35. Nave, K.-A. Myelination and support of axonal integrity by glia. Nature 468, 244-52 (2010).

36. Le Bras, B. et al. Oligodendrocyte development in the embryonic brain: the contribution of the plp lineage. Int. J. Dev. Biol. 49, 209-20 (2005).

37. Park, H.-C., Shin, J., Roberts, R. K. \& Appel, B. An olig2 reporter gene marks oligodendrocyte precursors in the postembryonic spinal cord of zebrafish. Dev. Dyn. 236, 3402-7 (2007).

38. Kolodziejczyk, K., Saab, A. S., Nave, K.-A. \& Attwell, D. Why do oligodendrocyte lineage cells express glutamate receptors? F1000 Biol. Rep. 2, 57 (2010).

39. Patneau, D. K., Wright, P. W., Winters, C., Mayer, M. L. \& Gallo, V. Glial cells of the oligodendrocyte lineage express both kainate- and AMPA-preferring subtypes of glutamate receptor. Neuron 12, 357-371 (1994).

40. Alberdi, E., Sánchez-Gómez, M. V., Marino, A. \& Matute, C. Ca(2+) influx through AMPA or kainate receptors alone is sufficient to initiate excitotoxicity in cultured oligodendrocytes. Neurobiol. Dis. 9, 234-43 (2002).

41. Matute, C., Domercq, M. \& Sánchez-Gómez, M.-V. Glutamate-mediated glial injury: mechanisms and clinical importance. Glia 53, 212-24 (2006).

42. Rosenberg, P. A. et al. Mature myelin basic protein-expressing oligodendrocytes are insensitive to kainate toxicity. J. Neurosci. Res. 71, 237-45 (2003).

43. Deng, W., Rosenberg, P. a, Volpe, J. J. \& Jensen, F. E. Calcium-permeable AMPA/kainate receptors mediate toxicity and preconditioning by oxygen-glucose deprivation in oligodendrocyte precursors. Proc. Natl. Acad. Sci. U. S. A. 100, 6801-6 (2003).

44. Gallo, V. et al. Oligodendrocyte progenitor cell proliferation and lineage progression are regulated by glutamate receptor-mediated K+ channel block. J. Neurosci. 16, 2659-2670 (1996).

45. Gudz, T. I., Komuro, H. \& Macklin, W. B. Glutamate stimulates oligodendrocyte progenitor migration mediated via an alphav integrin/myelin proteolipid protein complex. J. Neurosci. 26, 2458-66 (2006).

46. Matute, C. Characteristics of acute and chronic kainate excitotoxic damage to the optic nerve. Proc. Natl. Acad. Sci. U. S. A. 95, 10229-34 (1998).

47. Hoppmann, V., Wu, J. J., Søviknes, A. M., Helvik, J. V. \& Becker, T. S. Expression of the eight AMPA receptor subunit genes in the developing central nervous system and sensory organs of zebrafish. Dev. Dyn. 237, 788-99 (2008).

48. Patten, S. A. \& Ali, D. W. AMPA receptors associated with zebrafish Mauthner cells switch subunits during development. J. Physiol. 581, 1043-56 (2007).

49. Hatta, K. \& Korn, H. Physiological Properties of the Mauthner System in the Adult Zebrafish. J. Comp. Neurol 395, 493-509 (1998).

50. Hackett, J. T. \& Faber, D. S. Mauthner axon networks mediating supraspinal components of the startle response in the goldfish. Neuroscience 8, 317-331 (1983).

51. Pietri, T., Manalo, E., Ryan, J., Saint-Amant, L. \& Washbourne, P. Glutamate drives the touch response through a rostral loop in the spinal cord of zebrafish embryos. Dev. Neurobiol. 69, 780-95 (2009).

52. Almeida, R. G., Czopka, T., Ffrench-Constant, C. \& Lyons, D. A. Individual axons regulate the myelinating potential of single oligodendrocytes in vivo. Development 138, 
4443-50 (2011).

53. Kucenas, S. et al. CNS-derived glia ensheath peripheral nerves and mediate motor root development. Nat. Neurosci. 11, 143-151 (2008).

54. Kucenas, S., Snell, H. \& Appel, B. nkx2.2a promotes specification and differentiation of a myelinating subset of oligodendrocyte lineage cells in zebrafish. Neuron Glia Biol. 4, 7181 (2008).

55. Takada, N., Kucenas, S. \& Appel, B. Sox10 is necessary for oligodendrocyte survival following axon wrapping. Glia 58, 996-1006 (2010).

56. Czopka, T., Ffrench-Constant, C. \& Lyons, D. A. Individual oligodendrocytes have only a few hours in which to generate new myelin sheaths in vivo. Dev. Cell 25, 599-609 (2013).

57. Hoshijima, K., Jurynec, M. J. \& Grunwald, D. J. Precise Editing of the Zebrafish Genome Made Simple and Efficient. Dev. Cell 36, 654-67 (2016).

58. Thermes, V. et al. I-SceI meganuclease mediates highly efficient transgenesis in fish. Mech. Dev. 118, 91-8 (2002).

59. Soroldoni, D., Hogan, B. M. \& Oates, A. C. in 117-130 (Humana Press, 2009). doi:10.1007/978-1-60327-977-2_8

60. Cianciolo Cosentino, C., Roman, B. L., Drummond, I. A. \& Hukriede, N. A. Intravenous microinjections of zebrafish larvae to study acute kidney injury. J. Vis. Exp. (2010). doi:10.3791/2079

61. Ripley, B. et al. Package 'MASS'. (2018). at <http://www.stats.ox.ac.uk/pub/MASS4/>

62. Jung, S.-H. et al. Visualization of myelination in GFP-transgenic zebrafish. Dev. Dyn. 239, 592-597 (2010).

63. Kwan, K. M. et al. The Tol2kit: A multisite gateway-based construction kit forTol2 transposon transgenesis constructs. Dev. Dyn. 236, 3088-3099 (2007).

64. Longair, M. H., Baker, D. A. \& Armstrong, J. D. Simple Neurite Tracer: open source software for reconstruction, visualization and analysis of neuronal processes.

Bioinformatics 27, 2453-2454 (2011).

65. Wobbrock, J. O., Findlater, L., Gergle, D. \& Higgins, J. J. The aligned rank transform for nonparametric factorial analyses using only anova procedures. in Proceedings of the 2011 annual conference on Human factors in computing systems - CHI'11 143 (ACM Press, 2011). doi:10.1145/1978942.1978963

66. Karlsson, J., von Hofsten, J. \& Olsson, P.-E. Generating Transparent Zebrafish: A Refined Method to Improve Detection of Gene Expression During Embryonic Development. Mar. Biotechnol. 3, 0522-0527 (2001).

67. Inoue, D. \& Wittbrodt, J. One for all--a highly efficient and versatile method for fluorescent immunostaining in fish embryos. PLoS One 6, e19713 (2011).

68. Agyeman, A., Jha, B. K., Mazumdar, T. \& Houghton, J. A. Mode and specificity of binding of the small molecule GANT61 to GLI determines inhibition of GLI-DNA binding. Oncotarget 5, 4492-503 (2014).

69. Almeida, R. G. et al. Myelination of Neuronal Cell Bodies when Myelin Supply Exceeds Axonal Demand. Curr. Biol. 28, 1296-1305.e5 (2018).

70. Brösamle, C. \& Halpern, M. E. Characterization of myelination in the developing zebrafish. Glia 39, 47-57 (2002).

71. Kimmel, C. B., Sessions, S. K. \& Kimmel, R. J. Morphogenesis and synaptogenesis of the zebrafish mauthner neuron. J. Comp. Neurol. 198, 101-120 (1981).

72. Babin, P. J., Goizet, C. \& Raldúa, D. Zebrafish models of human motor neuron diseases: 
Advantages and limitations. Prog. Neurobiol. 118, 36-58 (2014).

73. Moreno, R. L. \& Ribera, A. B. Zebrafish motor neuron subtypes differ electrically prior to axonal outgrowth. J. Neurophysiol. 102, 2477-84 (2009).

74. Almeida, R. \& Lyons, D. Oligodendrocyte Development in the Absence of Their Target Axons In Vivo. PLoS One 11, e0164432 (2016). 


\section{CHAPTER 5 \\ Conclusions and Future Directions}


Harmful algal blooms (HABs) can produce potent neurotoxins that accumulate in seafood and affect human health. One HAB toxin of concern is domoic acid (DomA), a glutamate analog produced by the marine diatom Pseudo-nitzschia spp. Research in animal models shows that developmental exposure to DomA can lead to long-term changes in brain structure, neural connectivity, and behavior. However, the molecular and cellular mechanisms that lead to these later life effects are largely unknown.

This dissertation sought to address these knowledge gaps. It utilized the strengths of both zebrafish and the startle response circuit to provide insights into the cell types targeted by DomA and to characterize the downstream effects on neural circuit formation and behavior (Fig. 1). In chapter 2, I established zebrafish as a tool to study the developmental effects of DomA exposure. I identified a window of susceptibility during development that led to behavioral, structural, and transcriptional effects. In chapter 3, I used the startle response behavior as a way to determine whether DomA alters sensory processing and motor control. The neural circuit that underlies startle behavior is well characterized, which also made it possible for me to identify specific neuronal components in the circuit that were affected by DomA. Lastly, in Chapter 4, I identified cell types that are targeted by DomA shortly after exposure, and proposed a series of cellular events that lead to the later aberrant startle behavior.

\section{Chapter 2: Developmental windows of susceptibility to low doses of DomA}

In Chapter 2, I established zebrafish as a tool for investigating the mechanisms of toxicity following low-dose exposure to DomA during development. Previous developmental DomA exposures in zebrafish were done through yolk injections at early embryonic stages (512-1000 cell stage). ${ }^{1}$ However, only high doses of DomA produced measurable behavioral effects, and these effects occurred in conjunction with high mortality rates and acute neurotoxic effects in the larval stages. To build on this work, I applied a novel exposure method that delivered DomA intravenously at different developmental time points - from the embryonic to the larval stages (1-4 dpf). I also took an integrative approach to identifying the window of susceptibility to DomA by assessing behavior, structural targets, and gene expression changes - something that has not been done in any other developmental DomA exposure study. 
I found that exposure to DomA, particularly at $2 \mathrm{dpf}$, led to changes in behavior, tissue structure, and gene expression. These changes occurred at doses (0.09-0.135 ng) that did not lead to mortality or to prolonged acute neurological phenotypes. More specifically, DomA exposure resulted in startle response behavioral deficits, myelin defects, and down-regulation of axonal and myelin structural genes. Furthermore, time-lapse microscopy showed that DomA exposure disrupted the initial stages of axon wrapping and nascent myelination - cellular processes that have not been previously identified to be disrupted by DomA.

Findings from this chapter identify $2 \mathrm{dpf}$ as the window of susceptibility to DomA exposure. It may be the developmental period when there are cells that are intrinsically more sensitive to DomA exposure. Some of these cell types could conceivably be present earlier in development (prior to $2 \mathrm{dpf}$ ), but are more excitable, and thus more sensitive to DomA, at $2 \mathrm{dpf}$. One example of this is the Mauthner neuron, which is more excitable at $2 \mathrm{dpf}$ even if it is present earlier in development. ${ }^{2}$ Additionally, there may be critical cellular processes that occur during the $2 \mathrm{dpf}$ time period that are perturbed by DomA. Indeed, oligodendrocyte precursor cells (OPCs) proliferate at $2 \mathrm{dpf}$, and begin to wrap axons and myelinate by $2.5 \mathrm{dpf}$.

Furthermore, there could also be extrinsic factors that govern this 2 dpf window of susceptibility. The pronephros develops at $2 \mathrm{dpf}, 3,4$ potentially allowing for faster clearance of DomA injected after $2 \mathrm{dpf}$. The rapid loss of DomA in the system could in turn translate to lower toxicity. Furthermore, the blood brain barrier begins to form by $2 \mathrm{dpf}$ and progressively excludes smaller molecules as development proceeds.$^{5-7}$ Conceivably, with the blood brain barrier present after 2 dpf, DomA has limited access to target tissues.

While the 2 dpf time period is specific to zebrafish, some general principles arise that may also be relevant for human exposures. Findings from this chapter reinforce our knowledge that DomA toxicity is dependent on the timing of exposure. Exposure timing matters - consumption of contaminated seafood may have different effects for developing fetuses or young children depending on when the exposure happens. Furthermore, while 2 dpf may be specific to zebrafish, the developmental processes that occur at this time may be targets of DomA in human exposures. 
The $2 \mathrm{dpf}$ time point corresponds to the period shortly before cells in the oligodendrocyte lineage wrap and myelinate axons. Myelination may be a cellular process that is disrupted by DomA in humans as well. Since myelination in humans occurs over a prolonged period, starting around the third trimester and continuing into young adulthood, the potential exposure windows of susceptibility to DomA in humans may also be extended as well. ${ }^{8,9}$

\section{Chapter 3: Startle response as a tool to identify cell targets of toxicants}

Once the developmental model was established, I then sought to identify the specific cell types targeted by DomA exposure. In Chapter 3, I used the startle response circuit as a tool to identify cell targets and nervous system processes that were perturbed by DomA.

Startle responses are elicited by a variety of stimuli at different intensity levels. The intensity and type of stimulus results in the activation of distinct neural circuits that underlie startle responses. Increasing auditory/vibrational stimuli biases fish to perform the Mauthner-mediated short latency c-start (SLC) response rather than the slower non-Mauthner-mediated long latency c-start response (LLC). Direct electric field stimulation bypasses the sensory system altogether and directly activates the Mauthner neuron to produce very rapid startle responses. I took advantage of this previous knowledge. By providing different types of stimuli and at varied intensities, I was able to pinpoint which portions of the neural circuits were most likely affected by DomA exposure. I then imaged specific components of the startle circuit to identify potential DomAinduced perturbations.

I found that a majority of DomA-treated fish did not perform SLC-type startles in response to auditory/vibrational stimuli, nor did they respond to electric field pulses. Since both of these behaviors require the Mauthner neuron, the loss of these behaviors suggests that there were DomA-induced effects on the Mauthner cells. Indeed, imaging results show that at $7 \mathrm{dpf}$ the majority of DomA-treated fish had lost their Mauthner cells and other major reticulospinal neurons.

Furthermore, DomA altered startle kinematics, leading to changes in bend angle and maximal angular velocities. To further assess whether these kinematic deficits were driven in part by 
defects in the motor system, I imaged caudal primary motor neuron axons that synapse to the ventral muscle fibers. I found that DomA-exposed fish had significantly reduced axon collaterals. Such losses could conceivably contribute to the reduced bend angle as fewer muscle fibers are recruited for the startle response. ${ }^{10}$

Findings from this chapter indicate that DomA exposure in fish disrupts motor control during startle. Motor deficits have been characterized previously during both incidental exposures in humans and in experimental work with animal models. Humans acutely exposed to DomA developed sensorimotor neuropathy and axonopathy. ${ }^{10}$ A proportion of non-human primates that were chronically exposed to DomA at or near the tolerable doses developed visible hand tremors. ${ }^{11}$ Mice exposed to DomA prenatally (prenatal day (PND) 10-17) developed aberrant gait patterns later in life. ${ }^{12}$ Taken together, these findings suggest that motor deficits may be an important functional consequence of DomA toxicity.

This chapter also identified two neuronal populations that were altered by DomA exposures - the Mauthner neurons and the caudal primary motor neurons. Both of these neuronal populations have long axons, while caudal primary motor neurons have extensive axonal arbors. The maintenance of long, arborized axons, in turn, requires tightly controlled processes that are sensitive to toxicants and genetic disorders. Processes such as axonal transport mechanisms, mitochondrial respiration and lipid homeostasis are all vulnerable to disturbances, and disruption of these processes can lead to axonopathy and neuronal degeneration. ${ }^{13-15}$

Identifying the potential neuronal targets of DomA in zebrafish can provide clues as to which cell types are targeted in humans. The specific cell type need not be the same in fish and humans to glean insights as to the potential cell targets of DomA in developing humans. Humans do not have Mauthner cells, but instead have giant neurons in the caudal pontine reticular formation in the brainstem, which are thought to have functionally replaced the Mauthner cells and their homologs. ${ }^{16}$ Thus, the Mauthner cell may be viewed as a model for studying the effects of DomA on neurons. Rather than identifying specific types of neurons, it may be possible to identify general characteristics that make some cell types more sensitive to DomA toxicity as compared to others. Perhaps neurons that are larger, have extensive arbors, and are located in the 
spinal cord are more sensitive to DomA exposure. This hypothesis would require testing in a variety of models, but it provides a direction for further investigation.

More generally, work from this chapter illustrates the advantages of using well-characterized behavior with known underlying neural circuits. The startle response served as a valuable tool for identifying disrupted sensory and motor processes, and for determining which specific neuronal populations were targeted by DomA. While this work was done with DomA, the startle response behavior and circuit can serve as tools for identifying mechanisms of action for a variety of neurodevelopmental toxicants. The work presented here provides a framework for how to do this.

\section{Chapter 4: Initial cell targets and perturbed processes after toxicant exposures}

Chapter 4 identified the cell types that were targeted first, and suggested a series of initiating events that may underlie the changes observed. In this chapter, I attempted to disentangle the effects of DomA on neuronal targets from the myelin defects observed. Findings from this chapter show that the Mauthner cell was lost prior to myelination. This indicates the loss of the Mauthner cell is not simply a downstream event from DomA-induced perturbations to myelin. In fact, it is possible that the loss of the Mauthner cell itself led to downstream processes such as myelination deficits, myelination of inappropriate targets, and disruptions to the oligodendrocyte lineage. I propose a model for developmental exposure that expounds more on this.

This chapter also highlighted how a specific cell type may be more sensitive to a toxicant at one developmental time point as compared to another. Mauthner cells were lost following exposure to DomA at $2 \mathrm{dpf}$ but not $1 \mathrm{dpf}$. Developmental variations in sensitivities could be due to intrinsic features that change during development. Mauthner cells are postulated to switch AMPA subunits and are generally more excitable at $2 \mathrm{dpf}$ versus $1 \mathrm{dpf}^{2}$ Axons that are preparing for myelination are also known to be more sensitive than both smaller axons that are myelinated later and previously-myelinated axons when subjected to hypoxia-induced ischemia - a process that involves the release of glutamate and results in excitotoxicity. ${ }^{17}$ 
Integrating Chapter findings: Proposed mechanisms for developmental toxicity of DomA Integrating the findings from all three chapters, I propose a model for how developmental exposures may lead to the observed structural and behavioral effects (Fig. 2). The simplest proposed model involves progression of phenotypes that stem from a single initiating event. Exposure to DomA at $2 \mathrm{dpf}$ leads to the loss of Mauthner cells prior to myelination, presumably through binding to the Mauthner cells' ionotrophic glutamate receptors. The loss of the biggest axon in the spinal cord reduces the axonal surface area available for myelination. When myelination commences at $2.5 \mathrm{dpf}$ onwards, oligodendrocytes have less axonal surface area to wrap, and inappropriately myelinate neuronal cell bodies instead. This results in myelin defects that include both the overall reduction in myelin along with the appearance of circular myelin features that occur when myelin ectopically wraps neuronal cell bodies. In addition to aberrant myelination, the severe loss of axonal surface area may also have feedback mechanisms that lead to a reduction of the number of oligodendrocytes.

The loss of the Mauthner cells and the aberrant myelination can in turn lead to larval behavioral deficits. The loss of the Mauthner cells translates to the loss of startle behaviors that require the Mauthner cell. Furthermore, the reduction in axon collaterals in caudal primary motor neurons conceivably results in fewer synapses to muscle fibers. This in turn could lead to reduced bend angles, as fewer muscle fibers are recruited during the startle response.

Alternatively, it is possible that DomA does not have a single cellular target and DomA toxicity does not start from one initiating event (Fig. 3). While the loss of the Mauthner cells occurs first, DomA could also be targeting the oligodendrocyte lineage in concert. DomA did not reduce the number of oligodendrocyte precursor cells (OPCs), suggesting that processes such as OPC cell death and/or proliferation may not be altered by DomA. However, DomA could still directly bind to ionotrophic glutamate receptors on OPCs or immature oligodendroyctes and lead to disruptions in other fundamental developmental processes such as differentiation and the initial stages of axon-wrapping. Evidence from ischemia models shows that immature oligodendrocytes express glutamate receptors in their processes that are interacting with pre-myelinated axons, and suggests that glutamate-induced injury of these oligodendrocyte processes could have downstream consequences for the pre-myelinated axons that are about to be wrapped. ${ }^{17-19}$ 
Furthermore, DomA also may bind to glutamate receptors in newly differentiated myelinating oligodendrocytes starting at $2.5 \mathrm{dpf}$, leading to the myelinating oligodendrocyte cell loss that persists until $4 \mathrm{dpf}$. All of these effects would contribute to the observed aberrant myelination and the behavioral deficits.

\section{Future directions}

These findings lead to more questions that can be addressed to further our understanding of the mechanisms of DomA toxicity.

\section{Toxicokinetics after DomA exposure}

One of the important findings of this research is that there is a critical period for exposure to DomA. To further understand the implications of this finding for human exposures, it will be necessary to understand the toxicokinetics of DomA after exposures in the developing zebrafish and how they may contribute to defining the windows of sensitivity. Since DomA was delivered intravenously, questions would primarily focus on how much DomA enters the nervous system and how long it stays in the fish.

New assays are being developed to measure DomA at low concentrations in animal tissues. ${ }^{20}$ Using an assay sensitive enough to measure DomA in embryonic and larval fish, one could sample DomA-exposed fish at different times after injection to determine the half-life of DomA. One could then compare the half-lives of DomA injected at different developmental times. This information would provide insights into the mechanisms that underlie the $2 \mathrm{dpf}$ critical period. For example, the shorter half-life DomA at later developmental stages ( $2.5 \mathrm{dpf}$ or onwards) could be due to more efficient clearance mechanisms, which may in turn contribute to the lower sensitivity to DomA toxicity at these stages.

Tissue-specific measurements of DomA would allow one to characterize the distribution of DomA in different tissue compartments following intravenous exposures. ${ }^{21}$ One could determine whether DomA is able to reach target tissues in the central nervous system at all days injected, or whether it is excluded as the blood brain barrier develops after $2 \mathrm{dpf}$. The exclusion of DomA at later stages would suggest that the critical period is driven at least in part by the exclusion of 
DomA from the central nervous system. Tissue-specific measurements could also provide insights into whether DomA is sequestered in other organs like the liver when it is present in later larval stages.

\section{Axon and myelin loss in the spinal cord}

Mauthner cells are lost prior to myelination, and by $5 \mathrm{dpf}$, many other reticulospinal neurons are also absent. However, it is still unclear how much of the axonal surface area is lost due to DomA. Using electron microscopy, one could quantitatively assess DomA-induced reductions in the number of axons in the spinal cord. Findings from this work would allow one correlate the extent of the axonal loss with the other cellular and structural phenotypes observed after DomA exposure, including the appearance of ectopically-myelinated cell bodies and loss of oligodendrocytes.

In addition to axon loss, one would also quantify the extent of demyelination, and determine whether myelin loss is proportional to axon loss. Imaging at higher resolution, one then could determine whether there are ultrastructural defects in myelin wrapping around axons still present in the spinal cord.

\section{Mechanisms of neuronal loss}

Findings show that DomA leads to the loss of the Mauthner neuron. However, the mechanisms that underlie the neuronal loss are not known; it is unclear whether retrograde axonal degeneration precedes the loss of the neuronal cell body or whether the loss of the axons is a consequence of neuronal cell death. Determining which processes occur first will provide greater insights into the cellular mechanisms that underlie DomA-induced neuronal degeneration. The most straightforward way to accomplish this would be through time-lapse imaging of the transgenic line with fluorescently-labeled Mauthner cells (hspGFP130DMCA:UAS-gap43citrine). ${ }^{22}$ Fish with this transgenic background would be exposed to DomA and then immediately imaged until $60 \mathrm{hpf}$ - the time point by which Mauthner cells are lost. 
Functional consequences for ectopically-myelinated neuronal cell bodies

One intriguing finding is that DomA exposure led to the appearance of ectopically-myelinated neuronal cell bodies. I posit that these ectopically-myelinated cell bodies arise due to DomAmediated axonal losses; the loss of the axons results in a cellular environment where oligodendrocytes have fewer axons to myelinate, and thus myelinate neuronal cell bodies instead. The presence of these myelinated neuronal cell bodies is a novel phenotype following toxicant exposure, and further work to understand the potential functional consequences of this phenotype would be intriguing. Does myelin surrounding these cell bodies lead to aberrant neuronal activity patterns? Does this ectopic myelin lead to eventual neuronal cell death? Are the mistargeted neuronal cell bodies eventually demyelinated over time? These questions would enable us to understand not only the functional consequences for DomA exposure, but also provide insights into mechanisms that may occur with any toxicant that reduces axons prior to myelination.

\section{Cell-specific gene expression}

Whole embryo RNA-Seq analysis identified genes that are required for axonal and myelin sheath structure, and are downregulated following DomA exposure. It also identified changes in expression for genes within the stathmin family that are important for regulating microtubule structure, and axonal integrity. ${ }^{23,24}$ These changes were consistent with the imaging results showing the loss of axons in select neuronal populations along with myelin defects in the spinal cord.

While whole embryo RNA-Seq identified important gene targets, it may have missed other target genes that are differentially expressed in a cell-specific manner. Fluorescence-Activated Cell Sorting (FACS) could be used to isolate cells from the oligodendrocyte lineage during the initial stages of myelination. It would be a powerful tool to determine what transcriptional changes underlie disruptions in myelin wrapping in the spinal cord during this critical period. As an unbiased transcriptomic approach, it will also be used to identify other pathways that may be altered in the oligodendrocyte lineage. 


\section{Long-term effects in adults}

Work here showed that embryonic exposures to DomA ( $2 \mathrm{dpf}$ ) can lead to behavioral deficits that occur into the larval stages (5-7 dpf). However, it is unknown if these behavioral deficits persist over time or whether other new deficits arise in adulthood. Evidence from rodent models show that developmental exposures lead to increased seizure susceptibility ${ }^{25,26}$ and long-term changes in neural activity ${ }^{26,27}$ and behavior ${ }^{28-32}$ well into adulthood. It would be critical to determine whether there are long-term effects from developmental exposures to DomA in fish. Do the startle deficits and myelin defects persist? Are there other behavioral or histological phenotypes that arise with age? Are fish more sensitive to other chemical compounds as a result of their previous exposure to DomA? Work to expose fish at $2 \mathrm{dpf}$ then raise them to adulthood for behavioral tests and histological analyses will be necessary to better understand longer-term effects from developmental exposures to DomA.

\section{Broader implications for human health}

Ultimately, this body of work provides mechanistic insights into the cell targets and developmental processes perturbed by DomA. These mechanistic insights can be extrapolated to support hazard assessments for low-dose DomA exposures in humans during the periods of pregnancy and early childhood development. As a structural analog of glutamate, domoic acid also serves as a tool to identify broader mechanisms by which altered glutamate signaling during early development can lead to the development of neurological disorders. 


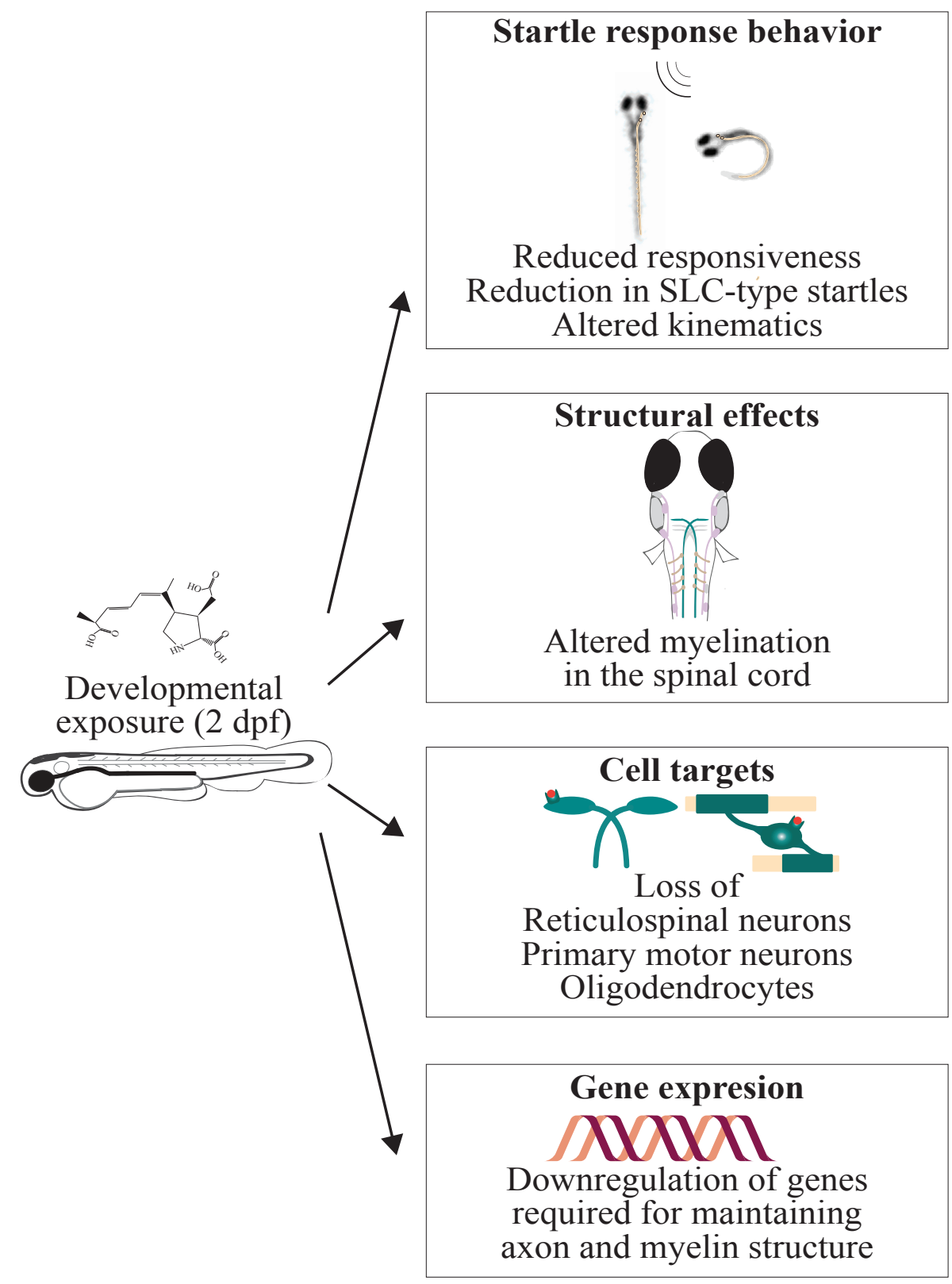

Figure 1: Research Summary

Developmental exposure to DomA at $2 \mathrm{dpf}$ alters behavior, disrupts nervous system structure, perturbs cellular development, and alters gene expression. 

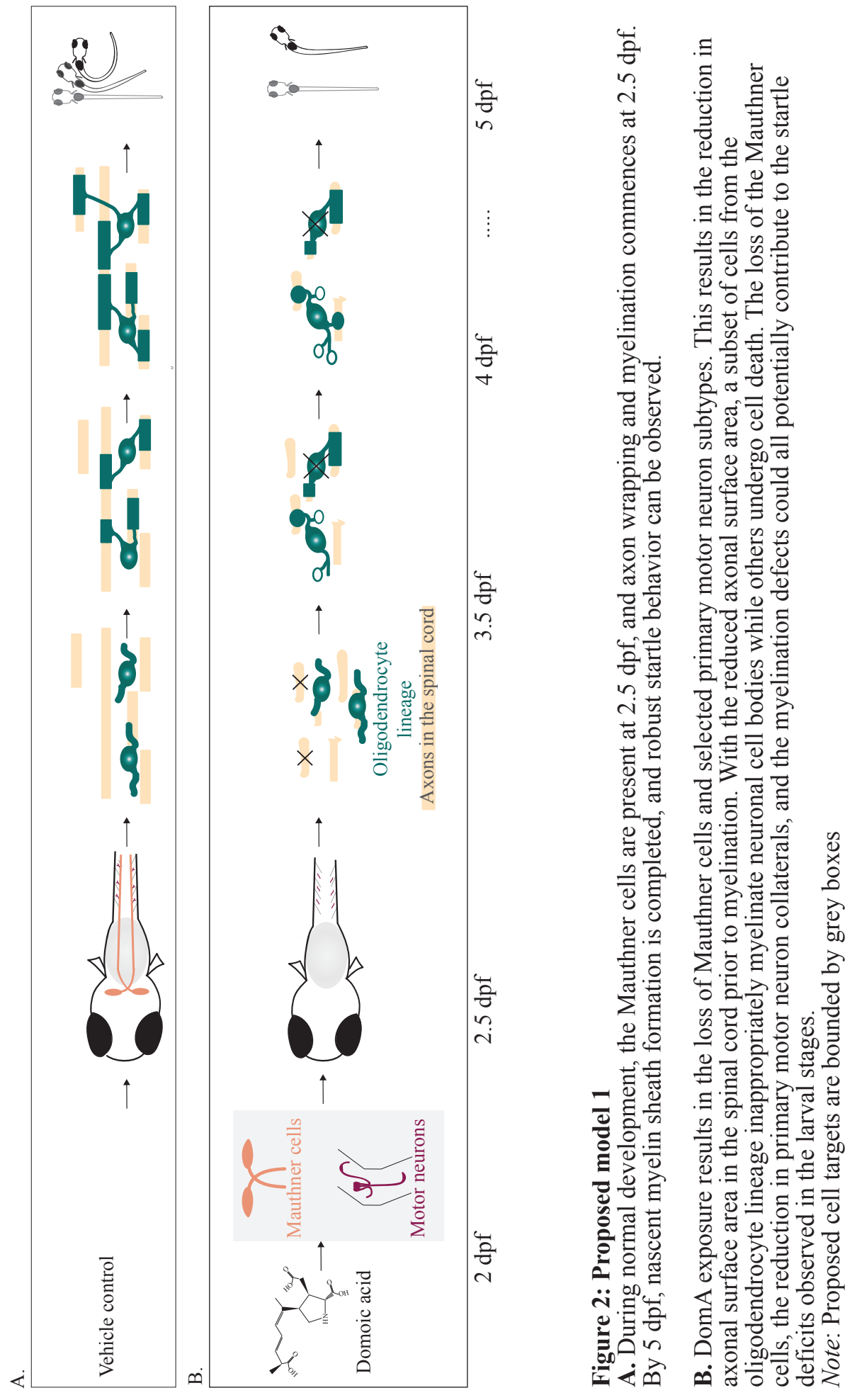


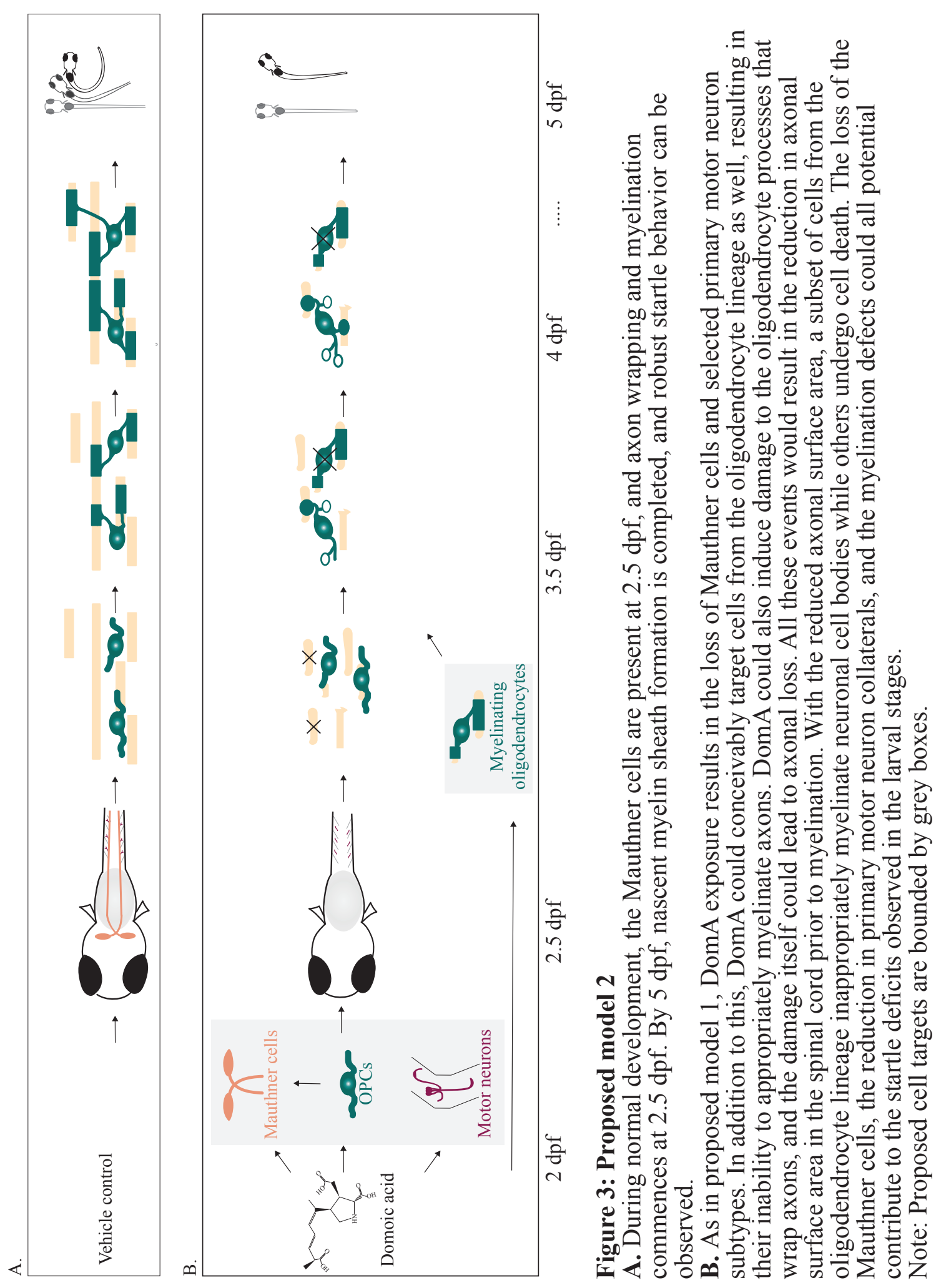




\section{REFERENCES}

1. Tiedeken, J. A., Ramsdell, J. S. \& Ramsdell, A. F. Developmental toxicity of domoic acid in zebrafish (Danio rerio). Neurotoxicol. Teratol. 27, 711-717 (2005).

2. Patten, S. A. \& Ali, D. W. AMPA receptors associated with zebrafish Mauthner cells switch subunits during development. J. Physiol. 581, 1043-56 (2007).

3. Drummond, I. A. \& Davidson, A. J. Zebrafish Kidney Development. Methods Cell Biol. 100, 233-260 (2010).

4. Drummond, I. A. Kidney Development and Disease in the Zebrafish. J Am Soc Nephrol 16, 299-304 (2005).

5. Xie, J., Farage, E., Sugimoto, M. \& Anand-Apte, B. A novel transgenic zebrafish model for blood-brain and blood-retinal barrier development. BMC Dev. Biol. 10, 76 (2010).

6. Fleming, A., Diekmann, H. \& Goldsmith, P. Functional Characterisation of the Maturation of the Blood-Brain Barrier in Larval Zebrafish. PLoS One 8, e77548 (2013).

7. Jeong, J.-Y. et al. Functional and developmental analysis of the blood-brain barrier in zebrafish. Brain Res. Bull. 75, 619-628 (2008).

8. Hasegawa, M. et al. Development of myelination in the human fetal and infant cerebrum: A myelin basic protein immunohistochemical study. Brain Dev. 14, 1-6 (1992).

9. Jakovcevski, I., Filipovic, R., Mo, Z., Rakic, S. \& Zecevic, N. Oligodendrocyte development and the onset of myelination in the human fetal brain. Front. Neuroanat. 3, 5 (2009).

10. Teitelbaum, J. S. et al. Neurologic Sequelae of Domoic Acid Intoxication Due to the Ingestion of Contaminated Mussels. N. Engl. J. Med. 322, 1781-1787 (1990).

11. Petroff, R. et al. Chronic, Low-Level Oral Exposure to Marine Toxin, Domoic Acid, Alters Whole Brain Morphometry in Nonhuman Primates. Neurotoxicology (2019). doi:10.1101/439109

12. Shiotani, M. et al. Neurobehavioral assessment of mice following repeated oral exposures to domoic acid during prenatal development. Neurotoxicol. Teratol. 64, 8-19 (2017).

13. Babin, P. J., Goizet, C. \& Raldúa, D. Zebrafish models of human motor neuron diseases: Advantages and limitations. Prog. Neurobiol. 118, 36-58 (2014).

14. Lewinski, F. von \& Keller, B. U. Ca2+, mitochondria and selective motoneuron vulnerability: implications for ALS. Trends Neurosci. 28, 494-500 (2005).

15. Spencer, P. S. \& Schaumburg, H. H. Distal axonopathy: one common type of neurotoxic lesion. Environ. Health Perspect. 26, 97-105 (1978).

16. Korn, H. \& Faber, D. S. Escape behavior - brainstem and spinal cord circuitry and function. Curr. Opin. Neurobiol. 6, 826-832 (1996).

17. Alix, J. J. P. et al. Central axons preparing to myelinate are highly sensitivity to ischemic injury. Ann. Neurol. 72, 936-951 (2012).

18. McCarran, W. J. \& Goldberg, M. P. White Matter Axon Vulnerability to AMPA/Kainate Receptor-Mediated Ischemic Injury Is Developmentally Regulated. J. Neurosci. 27, 4220 4229 (2007).

19. Alix, J. J. P. \& Fern, R. Glutamate receptor-mediated ischemic injury of premyelinated central axons. Ann. Neurol. 66, 682-693 (2009).

20. Huang, C. et al. Effective Extraction of Domoic Acid from Seafood Based on Postsynthetic-Modified Magnetic Zeolite Imidazolate Framework- 8 Particles. Anal. Chem. (2018). 
21. Lefebvre, K. A. et al. Uptake, tissue distribution and excretion of domoic acid after oral exposure in coho salmon (Oncorhynchus kisutch). Aquat. Toxicol. 81, 266-274 (2007).

22. Kawakami, K. et al. Gal4 Driver Transgenic Zebrafish: Powerful Tools to Study Developmental Biology, Organogenesis, and Neuroscience. (2016). doi:10.1016/bs.adgen.2016.04.002

23. Wen, H.-L. et al. Stathmin, a microtubule-destabilizing protein, is dysregulated in spinal muscular atrophy†. Hum. Mol. Genet. 19, 1766-1778 (2010).

24. Liedtke, W., Leman, E. E., Fyffe, R. E. W., Raine, C. S. \& Schubart, U. K. StathminDeficient Mice Develop an Age-Dependent Axonopathy of the Central and Peripheral Nervous Systems. Am. J. Pathol. 160, 469-480 (2002).

25. Gill, D. A. et al. Neonatal exposure to low-dose domoic acid lowers seizure threshold in adult rats. Neuroscience 169, 1789-1799 (2010).

26. Dakshinamurti, K., Sharma, S., Sundaram, M. \& Watanabe, T. Hippocampal changes in developing postnatal mice following intrauterine exposure to domoic acid. J. Neurosci. 13, 4486-4495 (1993).

27. Mills, B. D. et al. Prenatal domoic acid exposure disrupts mouse pro-social behavior and functional connectivity MRI. Behav. Brain Res. 308, 14-23 (2016).

28. Doucette, T. A. et al. Low doses of domoic acid during postnatal development produce permanent changes in rat behaviour and hippocampal morphology. Neurotox. Res. 6, 555563 (2004).

29. Tasker, R. A. R., Perry, M. A., Doucette, T. A. \& Ryan, C. L. NMDA receptor involvement in the effects of low dose domoic acid in neonatal rats. Amino Acids 28, 193 196 (2005).

30. Zuloaga, D. G. et al. Fetal domoic acid exposure affects lateral amygdala neurons, diminishes social investigation and alters sensory-motor gating. Neurotoxicology 53, 132 140 (2016).

31. Tanemura, K. et al. Intrauterine environment-genome interaction and Children's development (2): Brain structure impairment and behavioral disturbance induced in male mice offspring by a single intraperitoneal administration of domoic acid (DA) to their dams. J. Toxicol. Sci. 34, SP279-SP286 (2009).

32. Aldridge, J. E., Levin, E. D., Seidler, F. J. \& Slotkin, T. A. Developmental exposure of rats to chlorpyrifos leads to behavioral alterations in adulthood, involving serotonergic mechanisms and resembling animal models of depression. Environ. Health Perspect. 113, 527-531 (2005). 


\section{APPENDIX \\ Public Dissertation Synopsis}


We live in a world full of chemicals - some of which are harmful when we are exposed to them in high doses. A subset of these chemicals are be made by humans, such as industrial waste, emissions from vehicles, and pesticides. Others are produced naturally by living organisms like bacteria, fungi, and algae (Fig. 1).
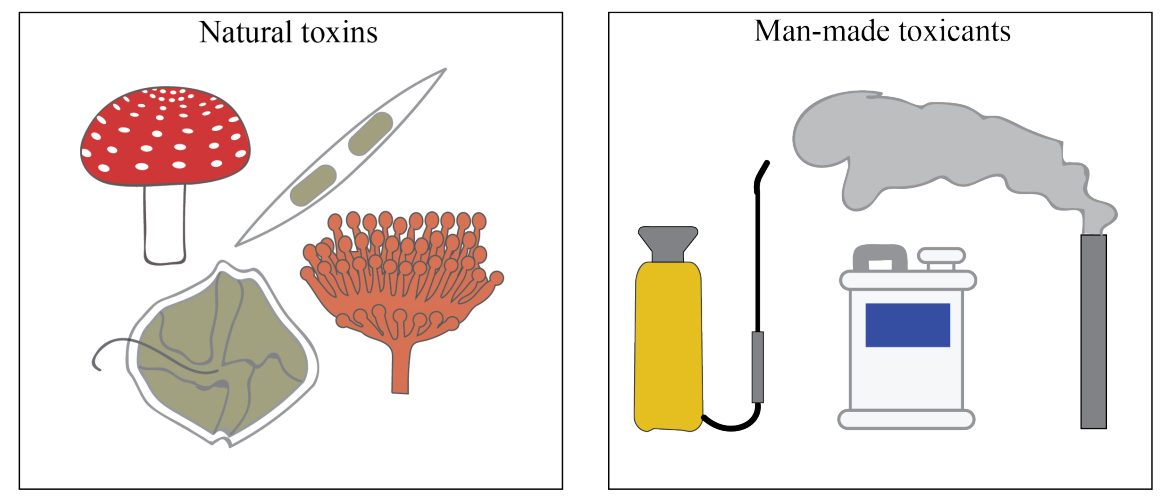

Figure 1: Examples of natural toxins and human-made toxicants

\section{Chemicals during early development}

A growing body of evidence shows that exposures that occur during early development can lead to later life consequences for human health. A hypothesis known as the "developmental origins of human disease" was first conceived when researchers observed that infants with lower birth weights had an increased risk of developing diseases including diabetes, heart disease, and high blood pressure. ${ }^{1,2}$ This led to the hypothesis that lack of nutrition in the fetal environment permanently changes a person's tissues and metabolism to lead to these diseases later in life. Further studies are showing that exposures to specific chemicals during fetal development can also lead to worse health outcomes later in life. ${ }^{3,4}$

\section{Disproportionate exposures to chemicals}

No scientific and environmental issue can be isolated from the social realm. When we think about toxicology and exposures in humans, we know that not everyone is exposed to contaminants equally.

Low-income communities of color are disproportionately exposed to a number of contaminants. These communities are more likely to be surrounded by industrial facilities, landfills, and hazardous material dumping sites. ${ }^{5-7}$ Consequently, people of color, especially those with lower 
incomes, also have a higher risk for diseases associated with the increase in exposures to these contaminants.

Even when we consider natural toxins, specific people are affected more than others. Subsistence fisherfolk and farmers are the hardest hit when natural toxins contaminate the seafood they fish or the crops they produce. Mycotoxins - toxins produced by fungi - can contaminate crops, pose a big threat to food security, and bring substantial health risks for subsistence farmers. ${ }^{8-10}$

For this dissertation, I focused on a specific natural toxin that is produced by algae and can accumulate in seafood during harmful algal blooms.

\section{Harmful algal blooms and human health}

Harmful algal blooms (HABs) are mass accumulations of algae that have damaging effects on human health and the social and economic systems on which we rely. ${ }^{11,12}$ Colloquially, harmful algal blooms are called "red tides" because some of blooms cause the water to turn red. However, in reality, blooms can appear brownish, greenish, or reddish, with the color of bloom depending on the species of algae comprising it.

HABs can cause harm by directly affecting human health. ${ }^{13-15}$ During a bloom, there are large collections of cells that produce toxins, which are then present in the water. In some cases, humans can get exposed to the toxins through breathing in sea spray with aerosolized toxins. If you were swimming in a body of water with these blooms, you could be exposed through your skin, or by accidentally swallowing water. You might also ingest drinking water contaminated with toxins. Finally, you can be exposed to it by eating seafood contaminated with the toxin (Fig. 2). 

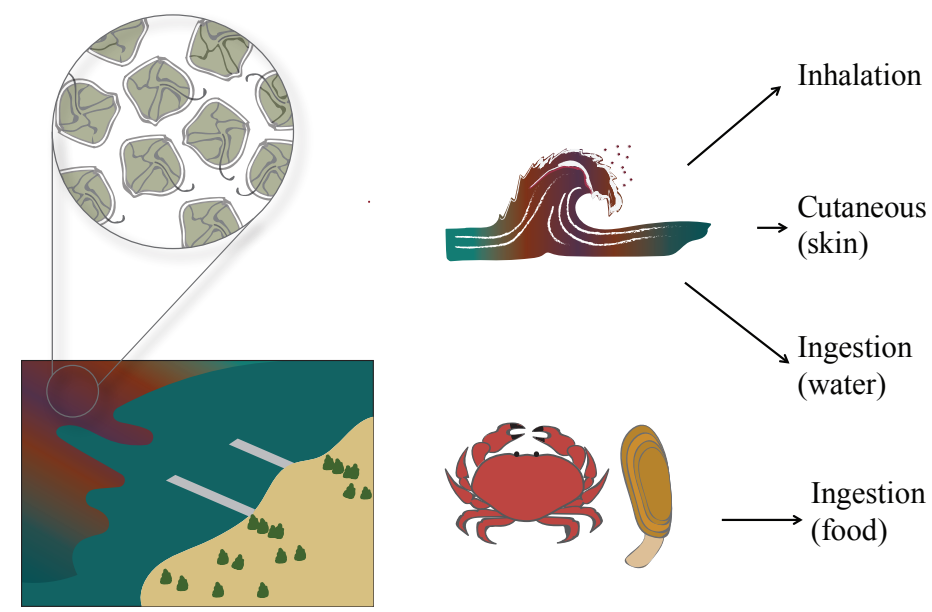

Figure 2: Human exposure routes to harmful algal bloom toxins

So how do these toxins end up in our seafood? First, algae produce the toxin (top of Fig. 3). Then filter feeders like blue mussels, quahogs, and razor clams eat the algae and the toxins. These filter feeders are in turn eaten by animals like seabirds (red arrows in Fig. 3a). When algae occur in massive amounts, they can adhere to each other and sink to the bottom, where they can accumulate in bottom feeders like Dungeness crabs (green arrow). Algae are also eaten by small animals like zooplankton, which are then eaten by other fish and marine mammals. The algae can also be directly eaten by filter-feeding fish and passed up the food chain (blue arrows). Finally, we are linked to this food web by the seafood we eat-the shellfish, crabs, and fish- and so have the potential to accumulate these harmful algal bloom toxins (orange arrows).

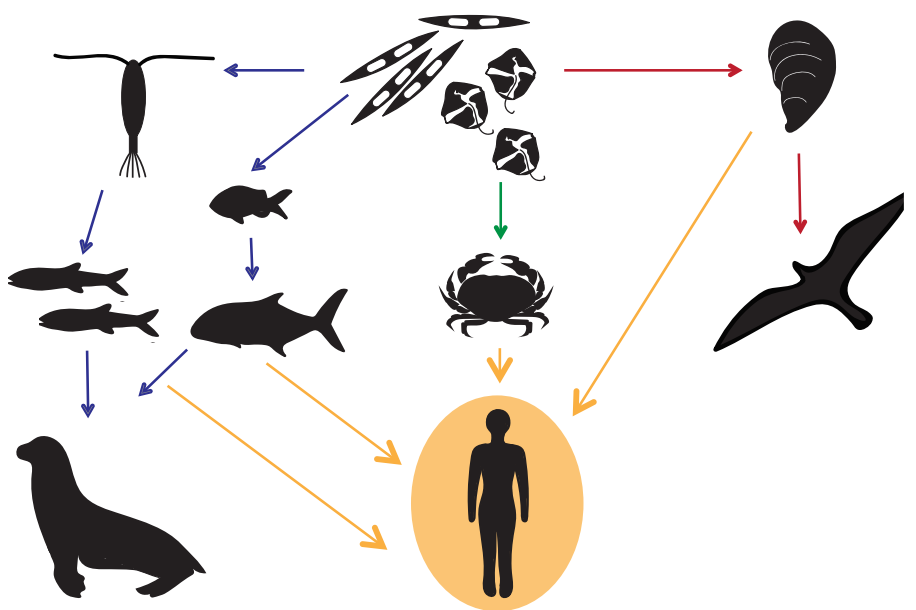

Figure 3: Harmful algal bloom toxins and their transfer in marine food webs 


\section{Domoic acid as a harmful algal bloom neurotoxin}

There are many types of algae that make up harmful algal blooms, and there are also many types of harmful algal bloom toxins. This dissertation specifically focuses on effects of one type of harmful algal bloom toxin called domoic acid (DomA). This toxin is produced by the phytoplankton genus known as Pseudo-nitzschia. During Pseudo-nitzschia blooms, shellfish and other seafood accumulate DomA, which we in turn consume.

When DomA is consumed in high enough doses, it causes a syndrome called 'Amnesic Shellfish Poisoning' (Fig. 4). Symptoms of this syndrome include gastrointestinal issues, memory loss, coma, and even death in the most severe cases. These symptoms occur through DomA binding to and activating glutamate receptors on specific cells in the nervous system.

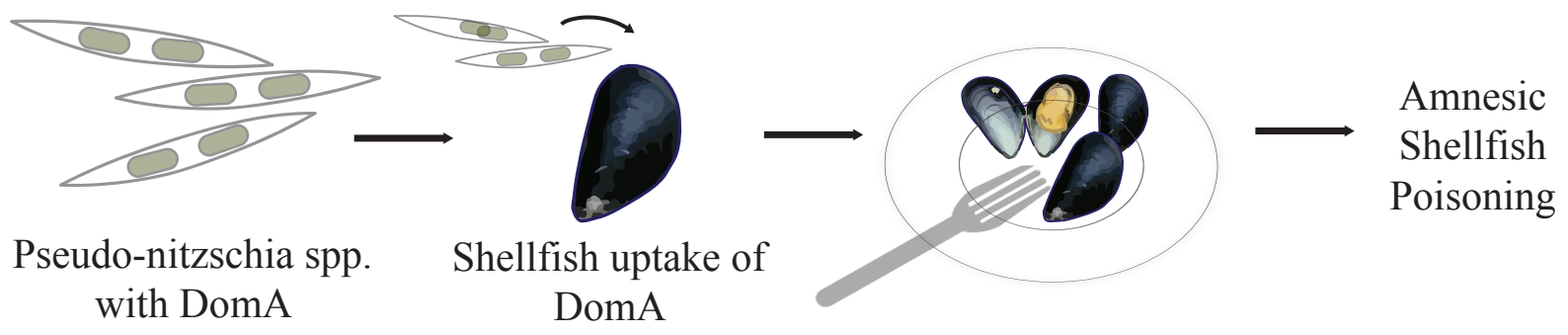

Figure 4: Human exposure route to DomA

To prevent this syndrome, a regulatory limit of $20 \mathrm{mg}$ of DomA per $\mathrm{kg}$ of shellfish tissue has been set; when seafood accumulate DomA that exceeds these limits, fisheries are closed and people do not get exposed (Fig. 5). This regulatory limit was decided based on incidental exposure in humans, along with controlled toxicology studies in non-human primates. It is at this limit that a single exposure to DomA does not lead to overt symptoms in adults.

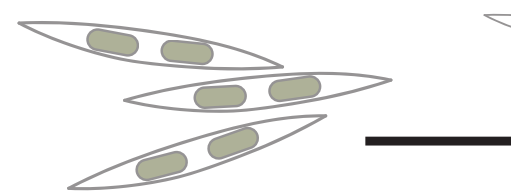

Pseudo-nitzschia spp. with DomA

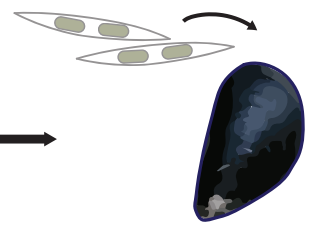

Shellfish uptake of DomA

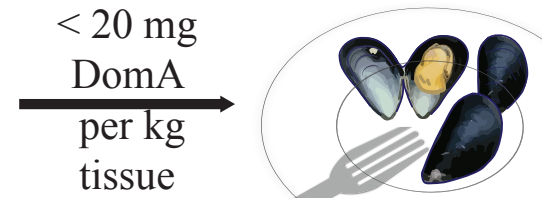

Figure 5: Regulatory limits for DomA exposure 


\section{DomA exposures as an expanding problem}

Human exposures to DomA are a real and growing concern. Pseudo-nitzschia blooms that produce DomA are regularly recorded in the West Coast of the United States. However, in 2015, an anomalous warm 'blob' allowed for a bloom to form from Vancouver all the way to Southern California. ${ }^{16}$ Such massive blooms are expected to become even more common if sea surface temperatures rise. ${ }^{17}$ Furthermore, DomA contamination has become an emerging problem on the East Coast of the United States as well. 2016 was the first year that shellfisheries were closed in parts of New England due to DomA accumulation. Since then, DomA-induced fisheries closures have occurred in 2017 and then again in 2018. To emphasize, these shellfish closures occurred in a region that had never previously had blooms that led to DomA accumulation in shellfish to these levels. These events suggest that DomA exposure is an expanding geographic problem.

While these larger events trigger fishery closures, we also know that humans are regularly exposed to measurable levels of DomA that are below this regulatory limit. Washington State regularly monitors DomA concentrations in shellfish, and makes this data available publicly

online. ${ }^{18}$ During blooms, DomA levels accumulate in shellfish beyond the regulatory limit, leading to the closure of the fisheries and the protection of the public. However, data also show that shellfish regularly have DomA concentrations below this limit. During these periods, shellfish continue to be harvested, and consumers are exposed to these lower levels of DomA (Fig.6).

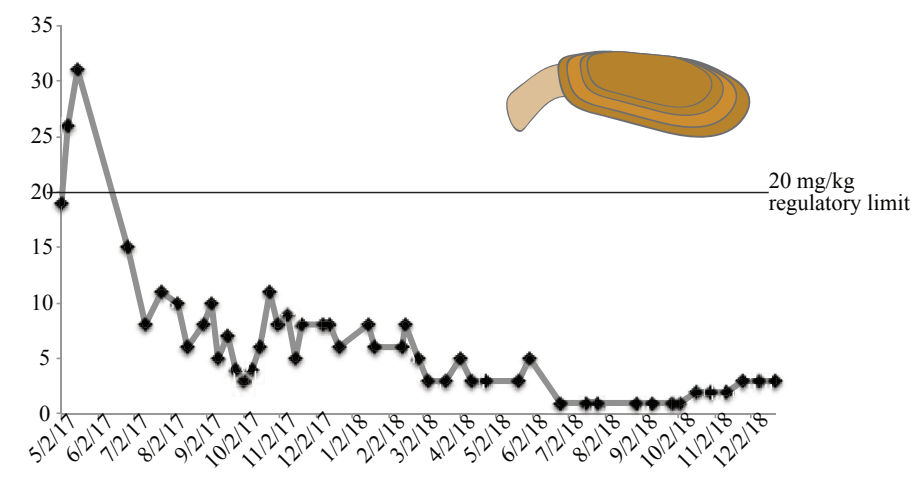

Figure 6: DomA concentrations in razor clams in Mocrocks beach in Washington state 
The question then becomes - are these regulatory limits safe? Particularly, are they safe for people who eat a lot of shellfish (chronic consumers)? Are they safe for people who get exposed to these levels of DomA during sensitive life stages?

\section{Health effects for chronic consumers}

Emerging research suggests that exposure to DomA below the regulatory limit can have measurable effects on learning and memory. An epidemiological study involving a cohort of three Native American tribes in the Pacific Northwest was done, in which adults were categorized into three groups: high consumers, medium, and non-consumers. After adjusting for sex, age, education level, and employment status, high consumers who ate 15 razor clams or more per month had measurable decrements in specific memory tests. In a follow-up study, researchers found that high consumers had measurable decrements in 'everyday memory,' which is the memory necessary for everyday life. An example of this would be forgetting to grab milk in the grocery store. Furthermore, work in non-human primates shows that daily ingestion of domoic acid at or near the currently regulatory limits led to noticeable hand tremors. ${ }^{19}$ All of this suggests that the currently regulatory limit may not be safe for chronic consumers.

\section{Early nervous system development is sensitive to toxicant exposures.}

We also know that exposures during sensitive life stages could also lead to persistent and longterm health effects. Development is a sensitive time because building a brain and a spinal cord requires highly coordinated and tightly regulated processes..$^{20} \mathrm{~A}$ human brain has an incredible number of folds and layers. To get to this form, first, there is a step called proliferation where a vast number of neurons are created. To give you a sense for just how many neurons are made, in a human fetus at 12-14 weeks, 15 million neurons are generated per hour in the developing brain. ${ }^{21}$ Once there is a pool of neurons, these neurons need to migrate to specific locations all over the brain. Neurons also begin the process of differentiation and polarization where these cells change shape and develop specialized roles. Neurons also start forming connections with one another to communicate and execute specific tasks. Finally, it is time for glial cells to develop. Glial cells are other cell types in the nervous system that have important specialized functions. My research focuses on a specific glial cell called the oligodendrocyte (Fig. 7). 


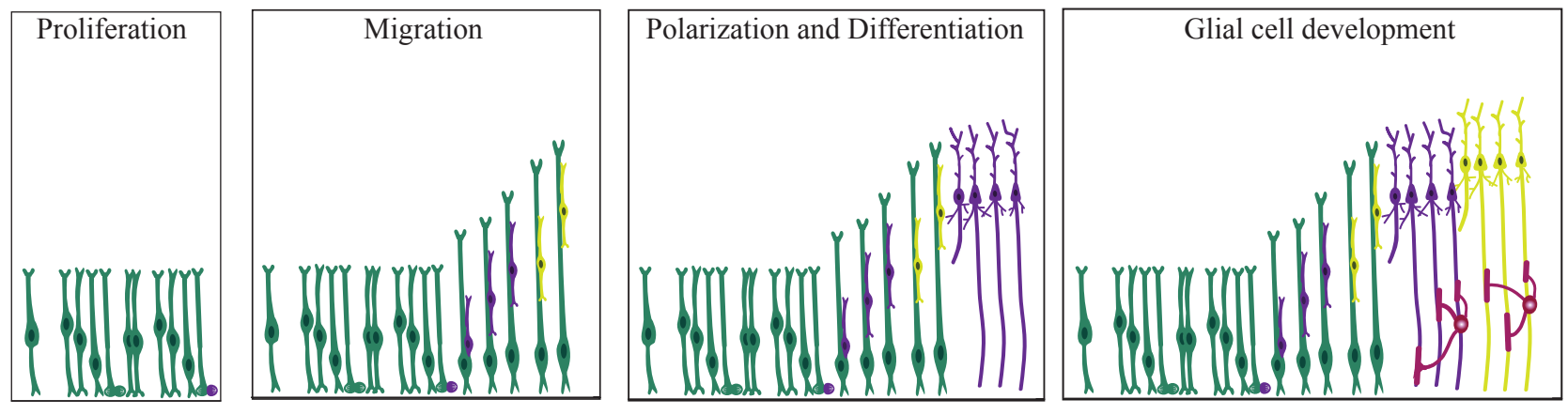

Figure 7: Important neurodevelopmental processes

All of this is to say that nervous system development is complex, and that chemicals that disrupt any of these processes could lead to lasting neurological deficits. In fact, because chemicals may target one specific process, the specific timeframe in development in which you are exposed matters a great deal. For example, if a chemical primarily targets proliferation, then early periods in neurodevelopment when proliferation happens may be the most sensitive to the toxicant.

To understand how chemicals affect development in people, we use animal systems as models. By exposing animals and studying the impacts of a given chemical with the model, we may get a deeper understanding of what happens in humans. This is also true for studies with DomA. Exposures to DomA have been done during early development in both rodents (mice and rats) and zebrafish.

\section{DomA as a developmental neurotoxin}

There is ample evidence that DomA is a developmental neurotoxin and that developmental exposures to DomA can have long-lasting consequences.

Both prenatal and postnatal exposures to DomA in rodents has been shown to lead to long-term behavioral defects, aberrant neural activity, and pronounced changes in brain tissue architecture. Furthermore, developmental exposure to DomA has also been shown to heighten sensitivity to other toxicants later in life. 
Earlier studies in zebrafish have also shown that developmental exposures to high doses of DomA causes behavioral phenotypes consistent with those we see in adult humans acutely exposed to DomA (Fig. 8). The similarity in behavioral phenotypes suggests that we can use zebrafish to understand what may be happening in humans. However, no zebrafish studies have been done on lower-level doses that may be more relevant in humans. Furthermore, there are no studies that determine potential windows of susceptibility for exposures.

Domoic acid is a developmental neurotoxin

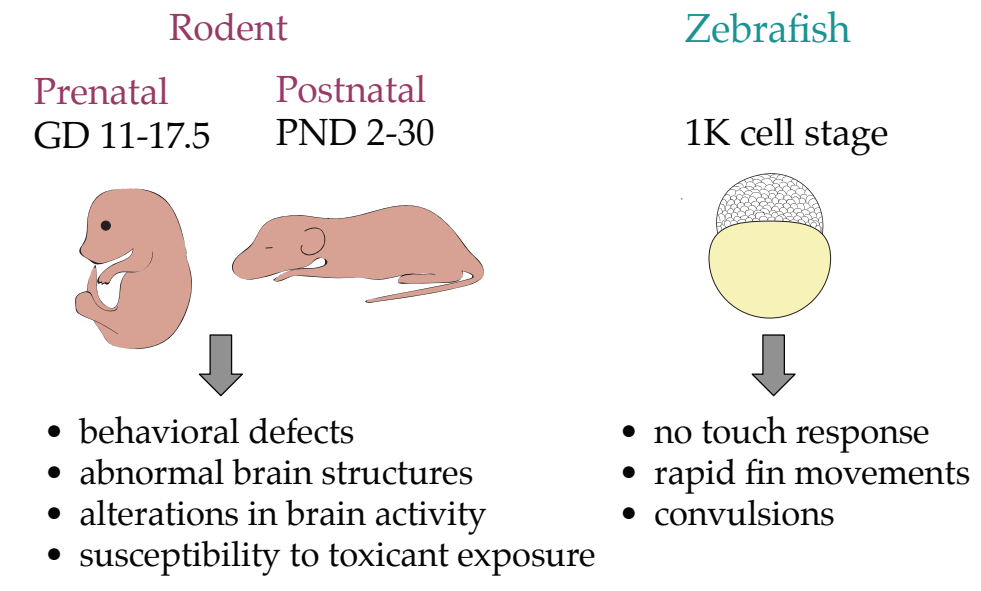

Figure 8: Developmental exposure to DomA in animal models

To fill these knowledge gaps, I use zebrafish as a model system. Zebrafish have all the major organ systems found in humans. $70 \%$ of protein-encoded genes in humans have an obvious counterpart in zebrafish. Furthermore, zebrafish have analogous brain regions and all major chemical signaling molecules found in humans.

Aside from these commonalities, zebrafish also have some unique attributes; my dissertation takes advantage of several of them (Fig. 9). Zebrafish develop externally and rapidly. This allows me to closely track cellular phenotypes and acute toxicity throughout different stages in early development. To emphasize how rapid development is, zebrafish develop from a single cell to a swimming and feeding organism in just 5 days. The rapidity allows me to track phenotypes through development on the order of days rather than months or years. The availability of transgenic lines that label different types of cells in the nervous system allows me to observe neural circuit structures and neurodevelopmental processes as they occur in living animals. 
Finally, zebrafish have behaviors that are quantifiable and driven by well-known neural circuits, which enables me to connect what I learn from my behavioral tests to the underlying neural circuits and cell types that drive these behaviors.

\section{Zebrafish as a model to identify mechanisms of toxicity}

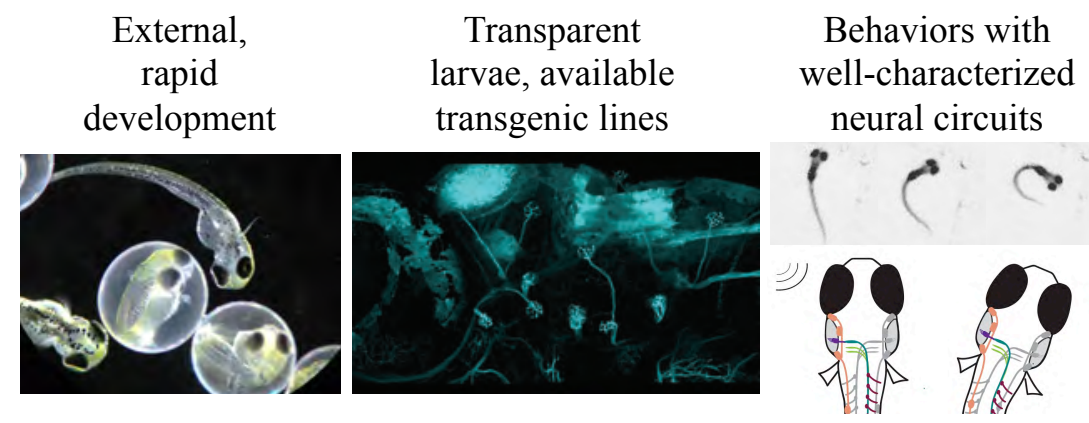

Figure 9: Unique attributes of the zebrafish model

\section{Goals of this study}

Using the zebrafish model, I address two main questions. First, when during development is exposure to DomA important? I sought to identify the specific developmental windows of susceptibility to DomA exposures. Second, what are the impacts from exposures during this period? I sought to identify the underlying mechanism of toxicity for DomA during this window by taking a multi-level approach (Fig. 10). At the highest level, I measured animal behavior. Doing this is important for understanding whether exposure to DomA has a functional effect on the level of the whole organism. Underlying this behavior are neural circuits, or populations of neurons, that communicate with each other to fulfill specific tasks. I looked at whether DomA causes any potential structural changes in the neural circuits that drive this behavior. Finally, these neural circuits are made up of individual players - different neurons and glial cells that make this possible. Thus, I also interrogate specific cell types that are altered during exposure to DomA.

During my first set of studies, I found that exposures at a particular period in early development ( 2 dpf or days post fertilization) led to changes in behavior, gene expression, and neural structures - all at doses that were much lower than previously used in zebrafish. Knowing the 
timing ( $2 \mathrm{dpf}$ ) and the dose of domoic acid was critical for taking a deeper dive into understanding the mechanisms of exposure.

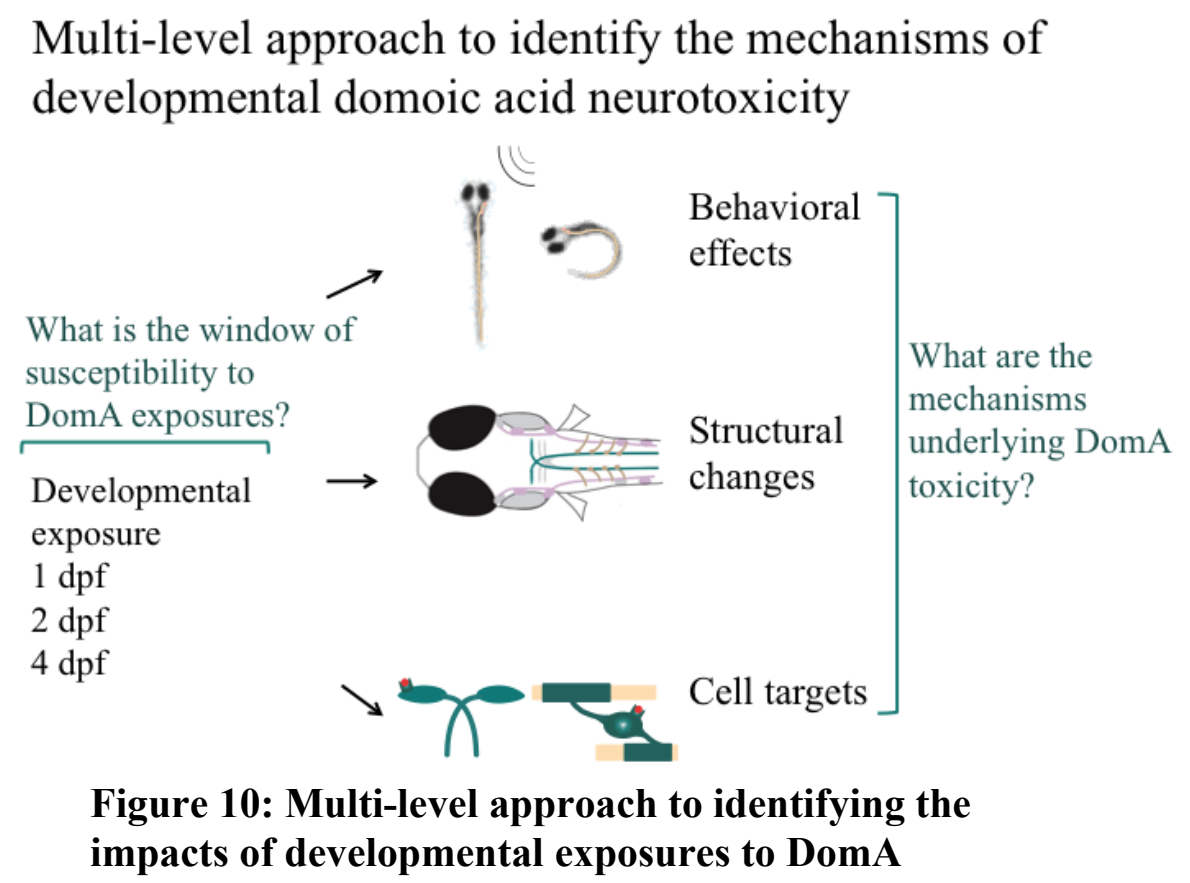

Startle behavior as a functional read out of toxicity

To assess the functional effects of DomA exposure, I looked at the startle response behavior.

The larval startle behavior occurs in response to sudden and intense stimuli. We actually undergo startle ourselves. If you ever shivered when a car backfires near you, or a balloon pops, you have experienced the human equivalent of the startle response.

Startle behavior is quantifiable, and is driven by underlying neural circuits that are well known. This makes it a really powerful behavior to study because I can link my behavioral results to the anatomy and the cell types involved in the circuit.

To assess startle behavior, larvae (recently hatched fish) were placed in 16 well plates above a speaker, and their startle responses were recorded using high-speed videography. To track their movements, larvae are divided into three segments, as coded by three different colors (Fig. 11). Changes in curvature are estimated by the changes in angle between the segments. 


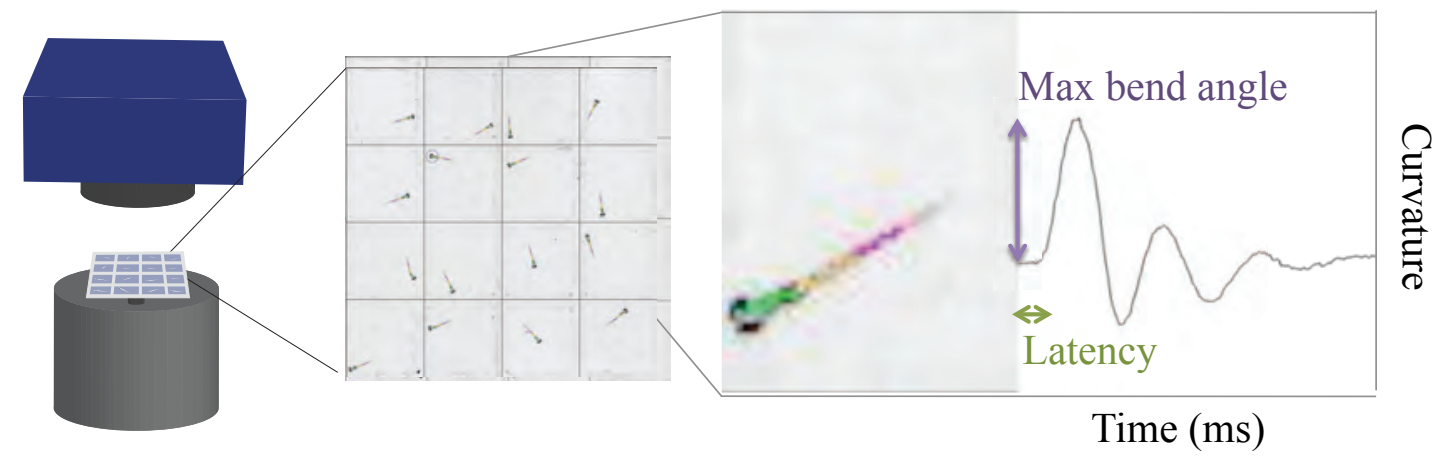

Figure 11: Startle response set up

Neural components that drive startle behaviors

Before delving into the neural circuit that drives this behavior, I want to introduce the most basic unit of the neural circuit - the neuron. Below is a neuron, which contains a projection called an axon that transmits information in the nervous system (Fig.12). Myelin is a structure that wraps axons and allows for the rapid transmission of this information. Myelin itself is made by another cell type known as the oligodendrocyte.

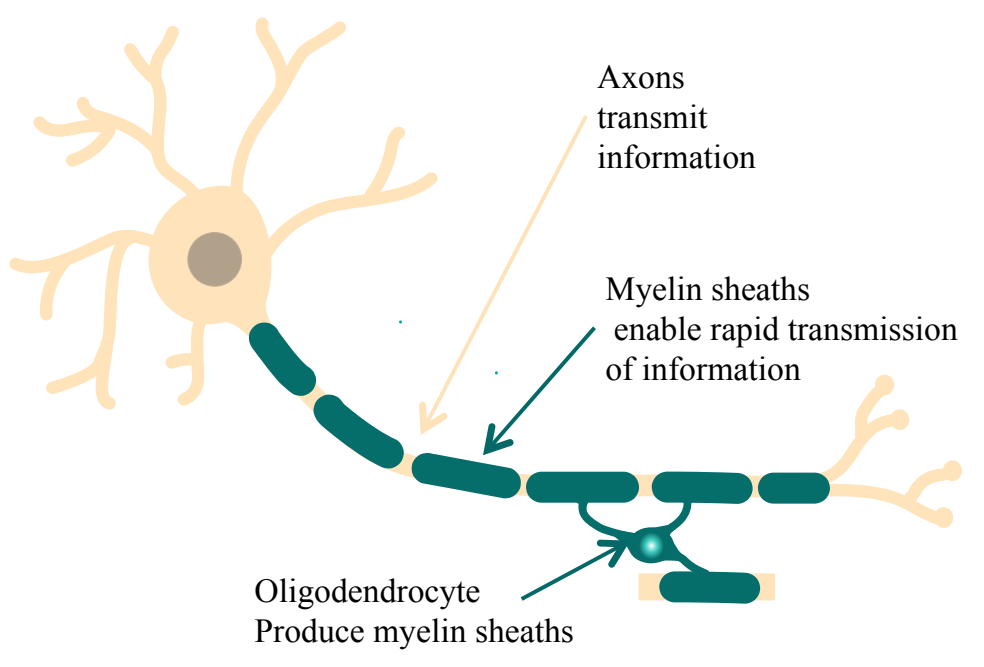

Figure 12: Parts of a neuron and an oligodendrocyte 
The beauty of using the startle response behavior is that we know a lot about the specific cell types and structures that are necessary for driving the startle response. Auditory/vibrational stimuli are first detected by hair cells within the neuromasts and within the inner ear. The mechanical deflection of these hair cells then leads to the activation of sensory neurons. These sensory neurons in turn send information to the hindbrain. In the hindbrain, a special cell called the Mauthner cell, along with other neurons, then integrates all of this sensory information. If the Mauthner reaches a certain threshold, it produces a signal that gets propagated down the axons within the spinal cord. As the signal rapidly travels down the spinal cord, it activates primary motor neurons along the way. Because the signal propagates so quickly, there are resulting fast unilateral muscle contractions and deep bend angles (Fig. 13).

\section{Startle response as a model for assessing sensory processing and motor control}

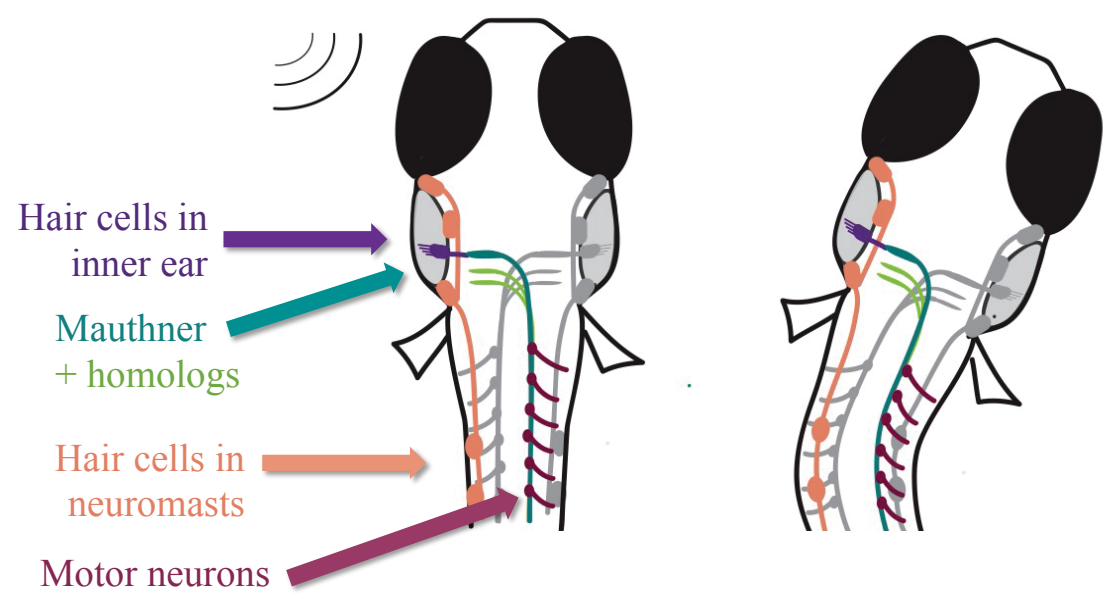

Figure 13: Components of the startle response circuit

We heard about the signal propagating down the axons, and how rapidly this has to occur. To give you a sense for just how fast this is, startle responses occur between 5-50 milliseconds after a stimulus is generated. Recall that myelin sheaths are structures that surround axons and enable this rapid signaling. Indeed, that startle response circuit contains heavily myelinated neurons (Fig 14). 


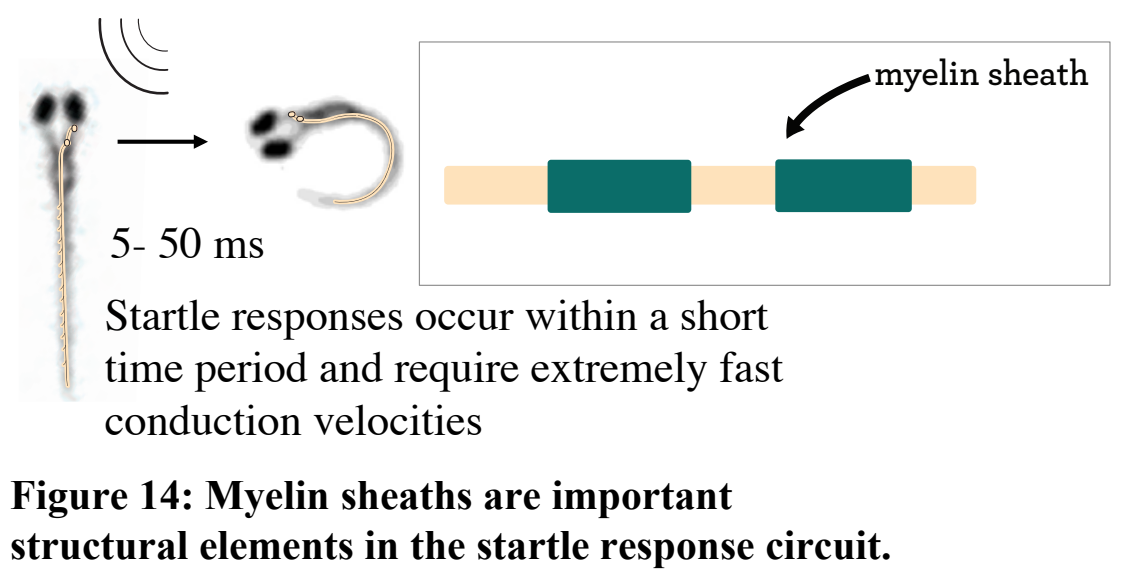

Now that we know a little about the players that make up the circuit, let's talk a bit more about the types of startle behaviors that occur. Previous work has shown that following auditory/vibrational stimuli, two types of startle responses can occur, and different stimulus intensities can bias towards one form of startle over another. Higher intensity stimuli preferentially activate the Mauthner cell. This in turn leads to specific startle response type called the short latency c-startle (SLC) response. This response occurs rapidly (within 14 milliseconds after a stimulus is produced). It is also known for producing very pronounced bend angles.

Lower intensity stimuli biases toward the activation of Mauthner-independent pathways and tend to lead to long latency c-start responses, or LLCs. LLCs occur more than 14 milliseconds after the stimulus in given, and produces much shallower bend angles (Fig. 15).

Stimulus intensity biases which neurons get activated, and which startle response is elicited

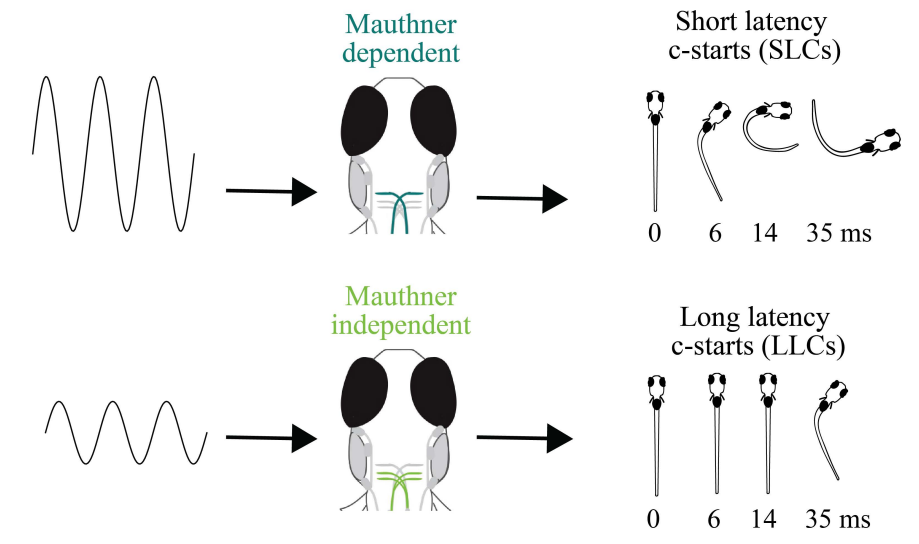

Figure 15: Two types of startle responses to auditory/vibrational stimuli 


\section{Types of auditory/vibrational stimuli}

When an auditory/vibrational stimulus is given to a larval fish, there are three possible outcomes. They could either not respond, respond with an LLC startle that is Mauthner independent, or respond with an SLC startle that is Mauthner mediated (Fig. 16).

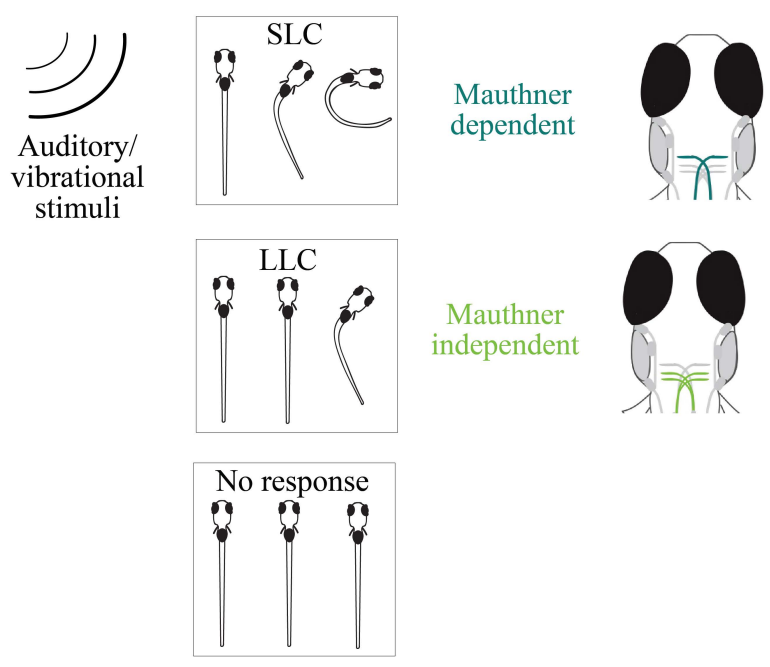

Figure 16: Three "behavioral choices"

Startle behavioral deficits with DomA exposure

I investigated how larvae "chose" different behaviors depending on the intensity of the vibrational stimuli I used. In Figure 17, each point represents an individual fish that either does not respond, performs an LLC, or performs an SLC. In control fish, as the stimulus intensity increases, fewer fish are non-responsive - or put another way, more fish begin to respond with higher stimulus intensities. Furthermore, fish that did respond preferentially performed SLC-type startles at higher intensity levels compared to at lower intensity levels.

For DomA-treated larvae, there were fewer non-responsive fish as the stimulus intensity increased. However, the percent of nonresponsive fish at the higher intensity levels is still greater in DomA-treated animals relative to control animals. Furthermore, DomA-exposed fish that do respond preferentially perform these non-Mauthner-mediated LLC-type startle responses at all stimulus intensities tested (Fig. 17). 


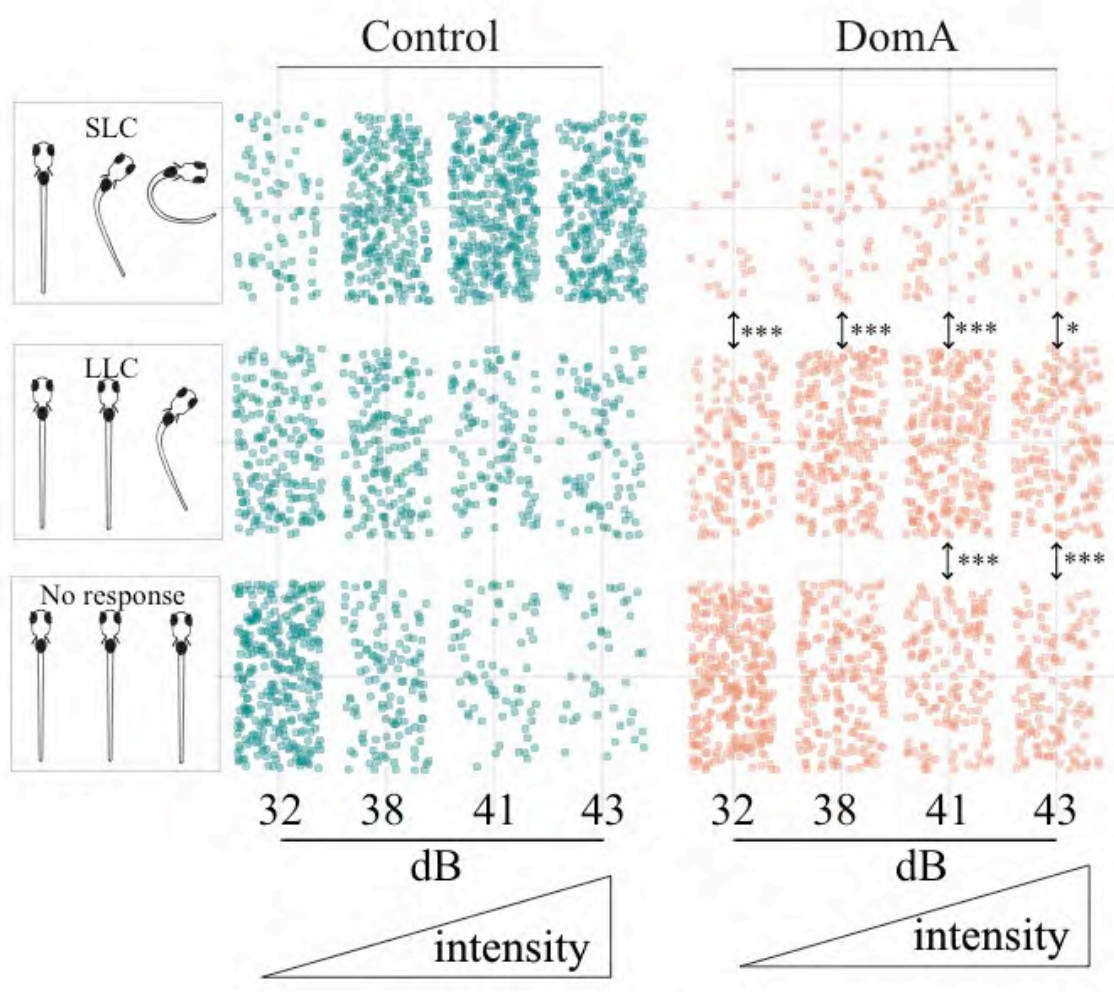

Figure 17: Behavioral choice for control and DomA-treated larvae with increasing stimulus intensities

Furthermore, when I look at the initial maximal bend angle, I find that DomA-exposed fish have reduced bend angles relative to control fish at all intensities tested (Fig. 18).
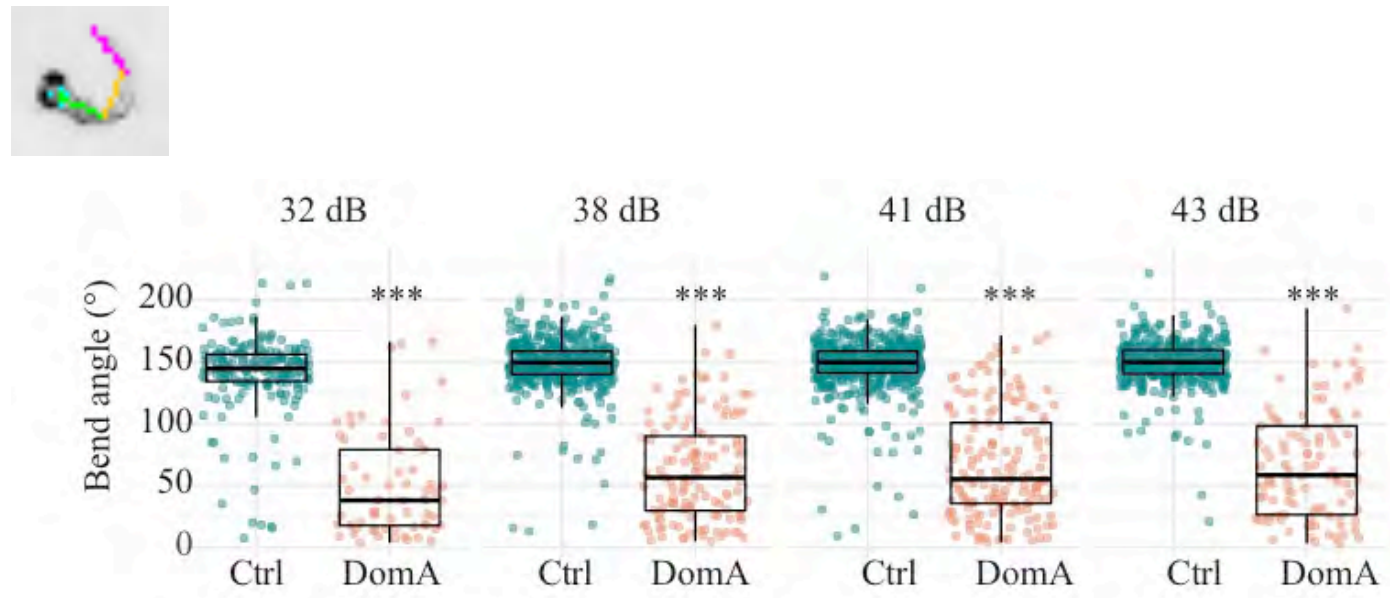

Figure 18: Maximal bend angles for control versus DomA exposed larvae 
We now know that these behavior deficits occur when the fish are exposed to auditory/ vibrational stimuli. I then wanted to see whether we could observe startle deficits when we take the sensory system out of the equation. Direct electrical stimulation does just that. When given a direct electric field, the sensory system is bypassed completely, and the Mauthner cell is directly activated (Fig. 19).

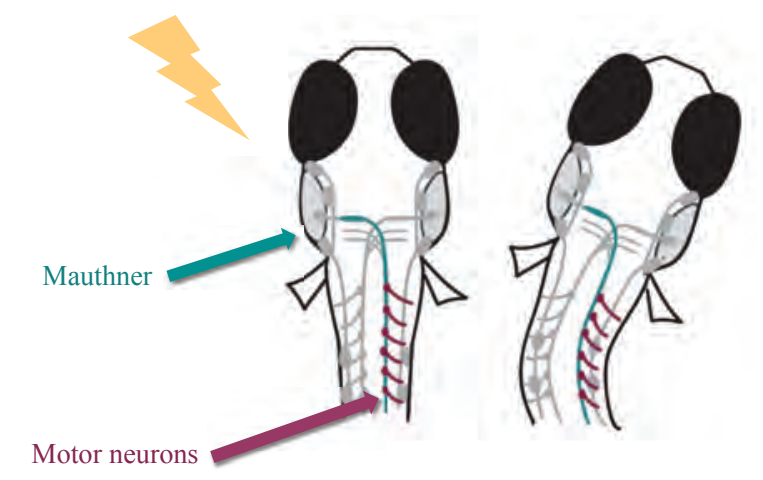

\section{Figure 19: Neural circuits that underlie startle from direct electric fields}

To assess startle responses from direct electric fields, larvae were head mounted in agarose and aligned to the electrodes. Their responses to a $4.4 \mathrm{~V} / \mathrm{cm}$ pulse were recorded using a high-speed camera. Each fish was given 7 replicate direct electric field pulses. From there, I calculated the percent of times an individual fish was responsive. 69 out of 74 control fish responded 100\% of the time. In contrast, 47/74 DomA-treated larvae never responded ( $0 \%$ responsiveness), and when they did, they had muted bend angles compared to controls (Fig. 20).

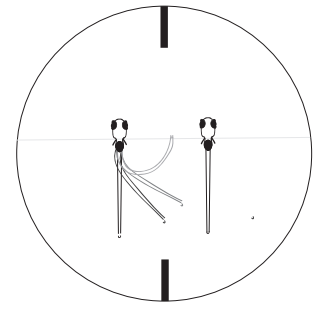

Points represent individual larvae

$\%$ of time they respond to 7 replicate stimuli

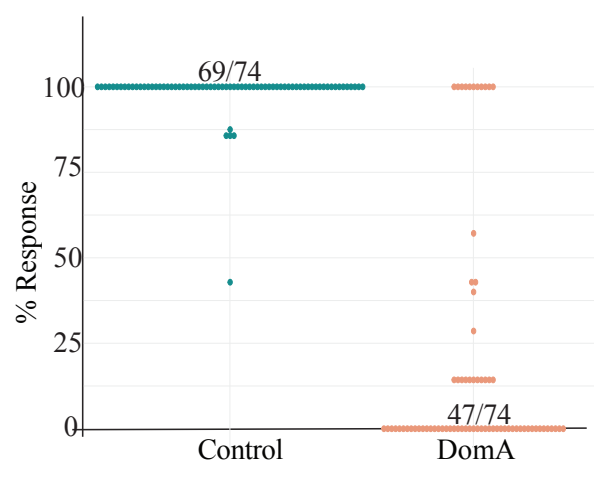

Figure 20: DomA-exposed larvae have reduced responsiveness to direct electric field stimulation. 


\section{Structural and cellular targets of DomA}

Behavioral results show that larvae exposed to DomA have aberrant startle responses, even when the sensory system is bypassed. Recall that the electric field stimulation directly activates the Mauthner cells and the downstream components like the motor neurons (Fig. 21). Furthermore, DomA-treated fish rarely undergo startle with this stimulus. We know that the SLC startle behavior (i.e. the short latency startle occurring after an auditory/vibrational stimulus) also requires the Mauthner cells and the downstream components like the motor neurons. Similar to what was observed after providing larvae with a direct-electric field, DomA-exposed animals were also less likely to perform SLCs. Since both the SLC-type startle after an auditory/vibrational stimulus and the startle in response to an electrical stimulus require the Mauthner cell and proper myelination, I investigated both of these features.

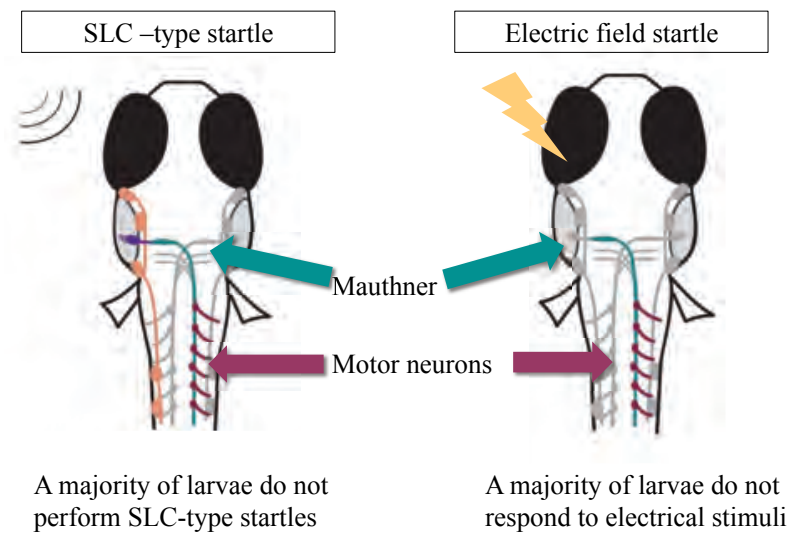

Figure 21: DomA-exposed larvae have aberrant startle behavior.

I first assessed myelination in the spinal cord. To accomplish this, I used a transgenic fish that has labeled myelin sheaths. In control fish, there are two myelinated regions with organized, elongated myelin sheaths. DomA-exposed fish not only have an overall reduction in myelin, but also have these really interesting circular features, a phenotype I will revisit later (Fig. 22). 


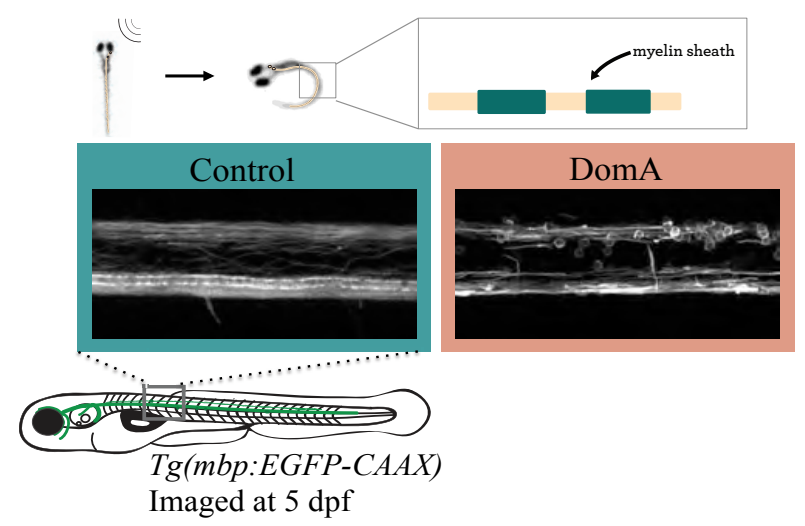

Figure 22: Myelin sheaths are disrupted in DomA-exposed fish.

I also wanted to look at the neurons that make up the circuit. In particular, I looked at the Mauthner cell. In the control fish, you can see both the Mauthner cells present (depicted in teal in the graphical representation, Fig. 23). In contrast, most of the DomA-treated fish (43/45) did not have Mauthner cells (Fig. 23)

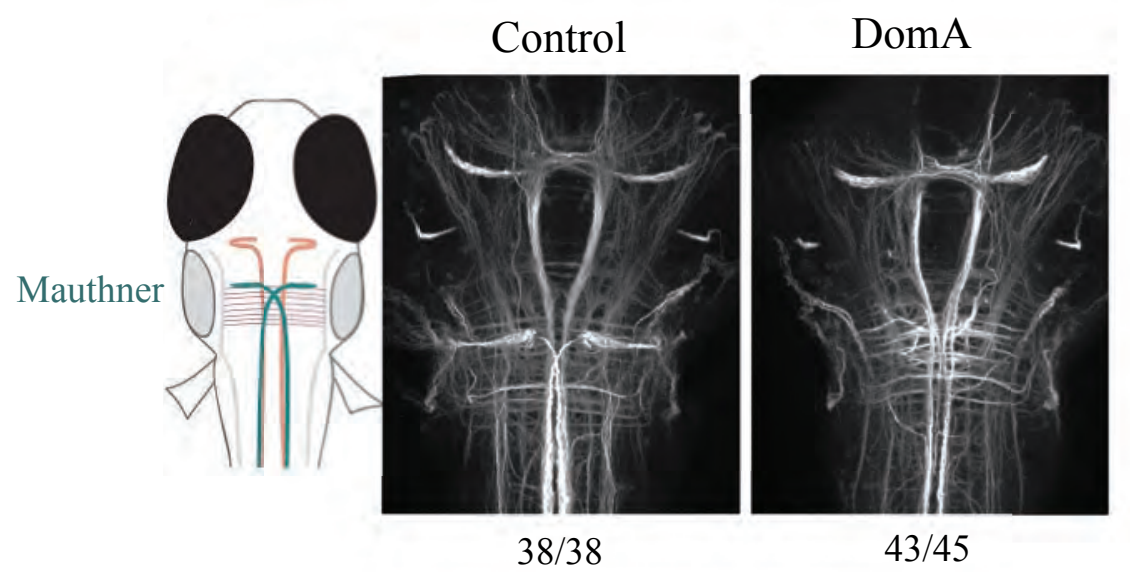

Figure 23: Mauthner cells are absent from DomA-exposed larvae.

Findings so far show that exposures at $2 \mathrm{dpf}$ led to measurable startle deficits, even when the sensory system is bypassed. In conjunction with these behavioral deficits, we also see the loss of the Mauthner cell and myelin defects during the larval period at $5 \mathrm{dpf}$ (Fig. 24). Note that all these endpoints were taken during the larval stages, well after exposures at $2 \mathrm{dpf}$ and after nascent myelination has occurred (orange region highlighted in Fig.24). 


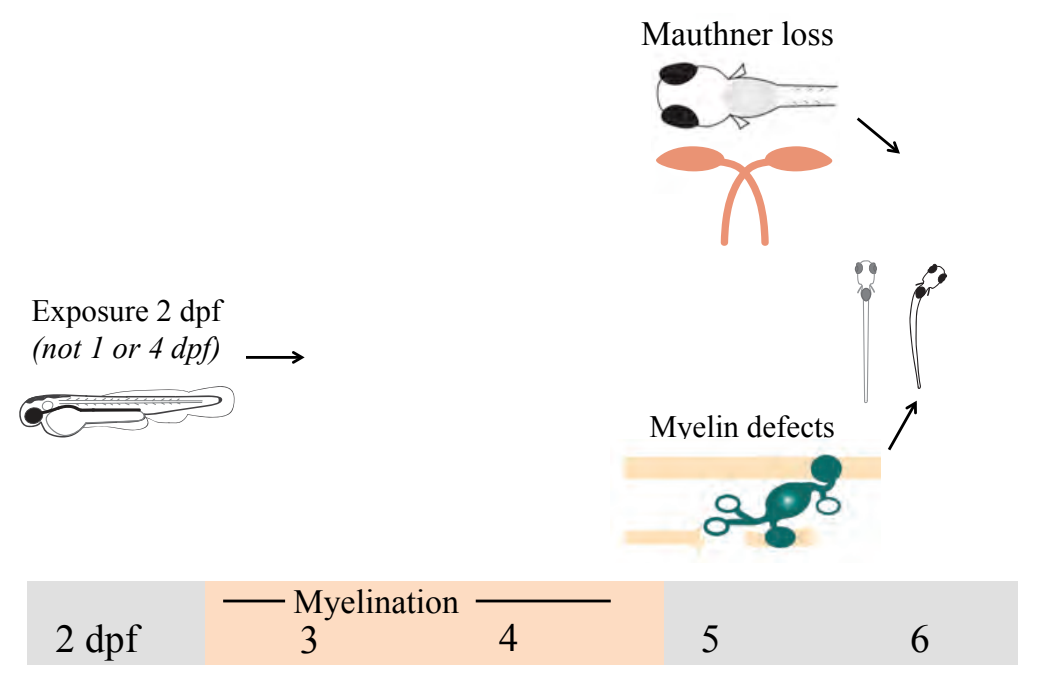

Figure 24: Building our proposed model: effects in later larval stages

\section{Initial cell target for DomA exposure}

Recall that at the larval stages I saw both the loss of the Mauthner cells and axons as well as myelin defects. We know that myelin cannot wrap around axons that do not exist, so if the axons are lost first, we expect to see myelin defects. We also know that myelin provides really important metabolic support for axons as the loss of myelin could also lead to the axonal defects (Fig. 25). The question then becomes, what happens first? Do we lose the myelin first, then the axons follow, or vice versa?

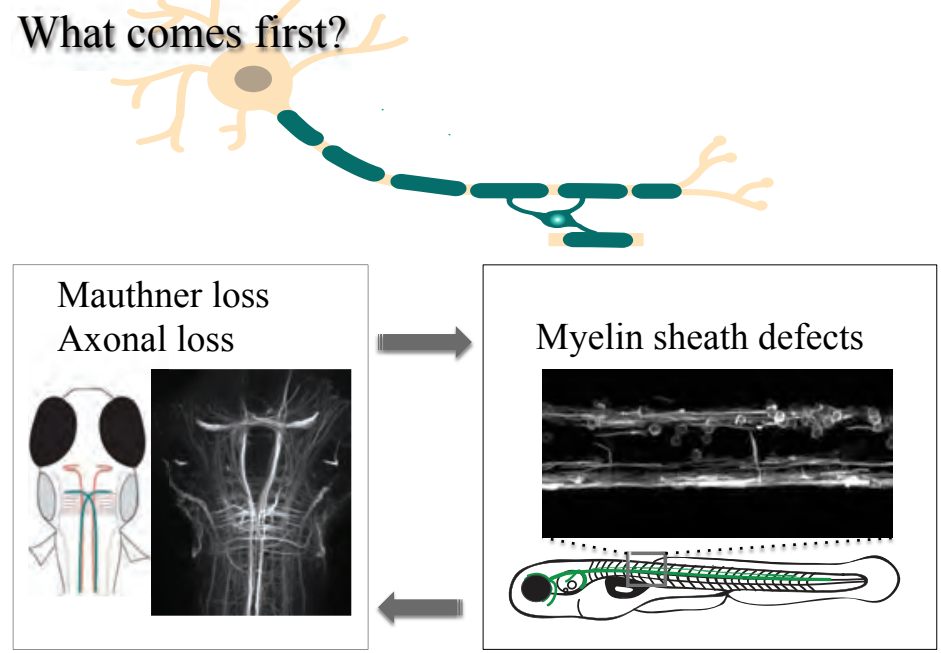

Figure 25: Identifying whether axonal loss or myelin sheath defects come first 
To determine whether DomA alters myelination, I turned to the lineage of cells that make the myelin. Specifically, I looked at how DomA perturbs oligodendrocyte development around the time myelination commences. To accomplish this, I quantified the number of oligodendrocyte precursor cells, which are cells that develop to eventually myelinate. I also quantified the number of oligodendrocytes later in development.

I first counted the number of oligodendrocyte precursor cells within the spinal cord. To do this, I have a fish line that has the oligodendrocyte cell bodies labeled in green and their membrane processes in red. I then counted the number of oligodendrocyte precursor cells within a single fish (as represented by individual points in Fig. 26). Following quantification, I found no differences between DomA-exposed fish and controls.
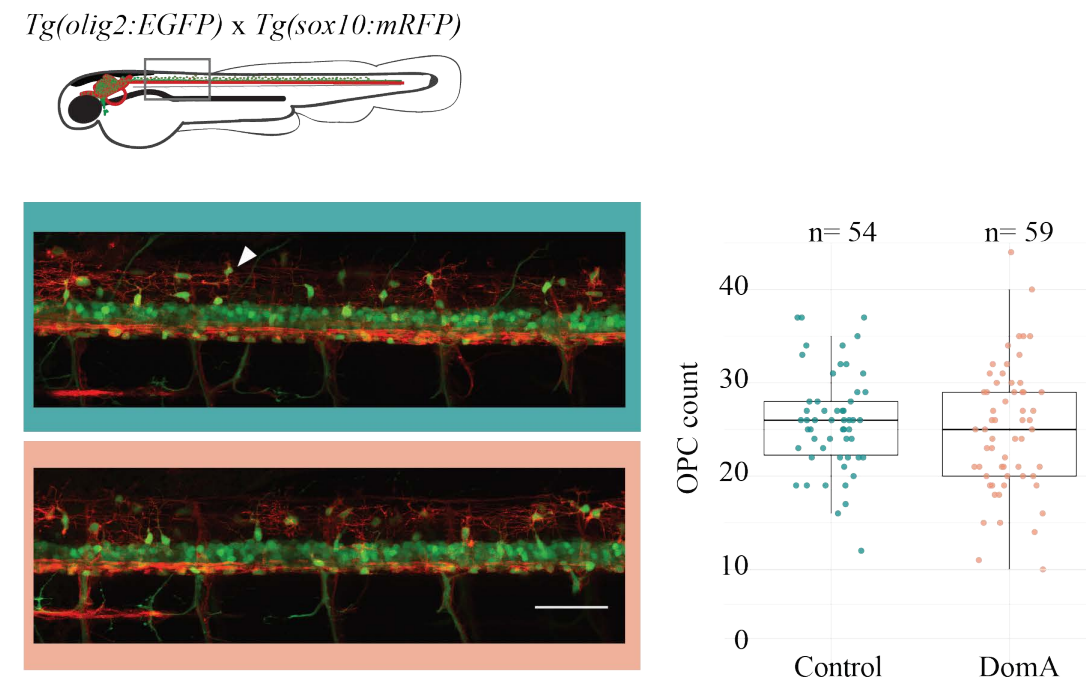

Figure 26: DomA exposure does not reduce the number of oligodendrocyte precursor cells prior to myelination.

Using another fish line, I quantified the number of myelinating oligodendrocytes a little later in development. Each point on the graph in Figure 27 represents the number of oligodendrocytes counted in an individual fish. DomA-exposed larvae had a significant reduction in the number of oligodendrocytes, especially when exposed to the higher dose of DomA. 

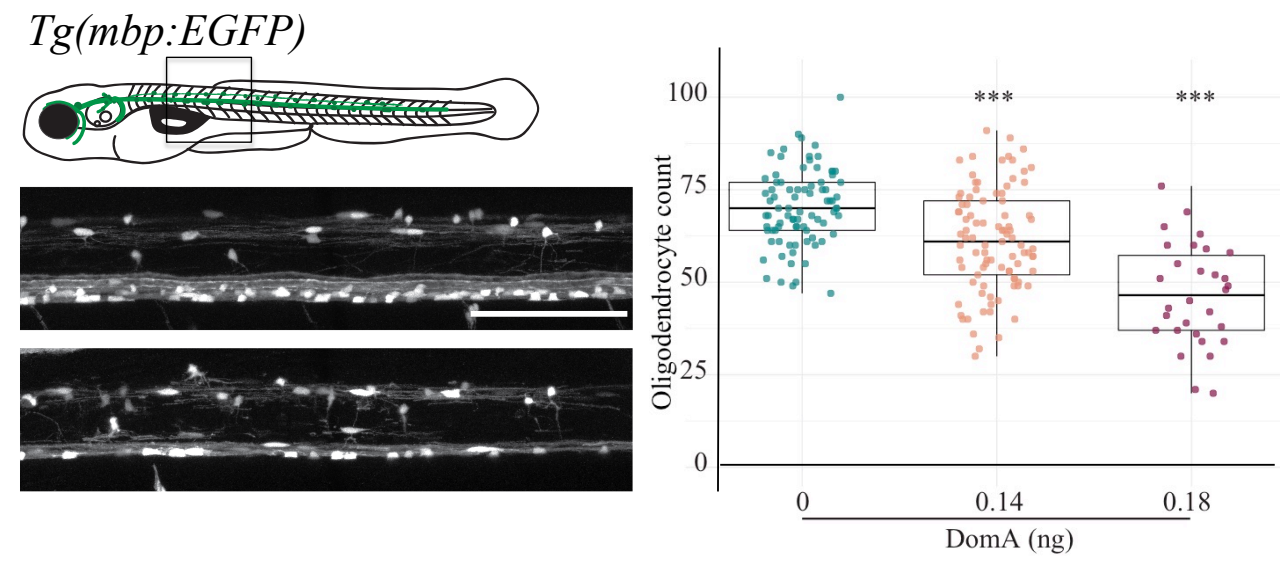

Figure 27: DomA exposure at 2 dpf reduces the number of mature oligodendrocytes by 4 dpf

Building on the proposed model, we know now that DomA does not reduce oligodendrocyte precursor cells (OPCs) prior to myelination, but it does lead to the loss of oligodendrocytes by 4 days post fertilization (dpf). The loss of oligodendrocytes could conceivably contribute to the myelin defects observed. With fewer myelinating oligodendrocytes, it may be possible that the reduced 'supply' of available oligodendrocytes to myelinate may contribute to less myelin being generated. The loss of the oligodendrocytes could be due to DomA directly affecting the oligodendrocyte cells, which have the receptors to which DomA binds (Fig. 28).

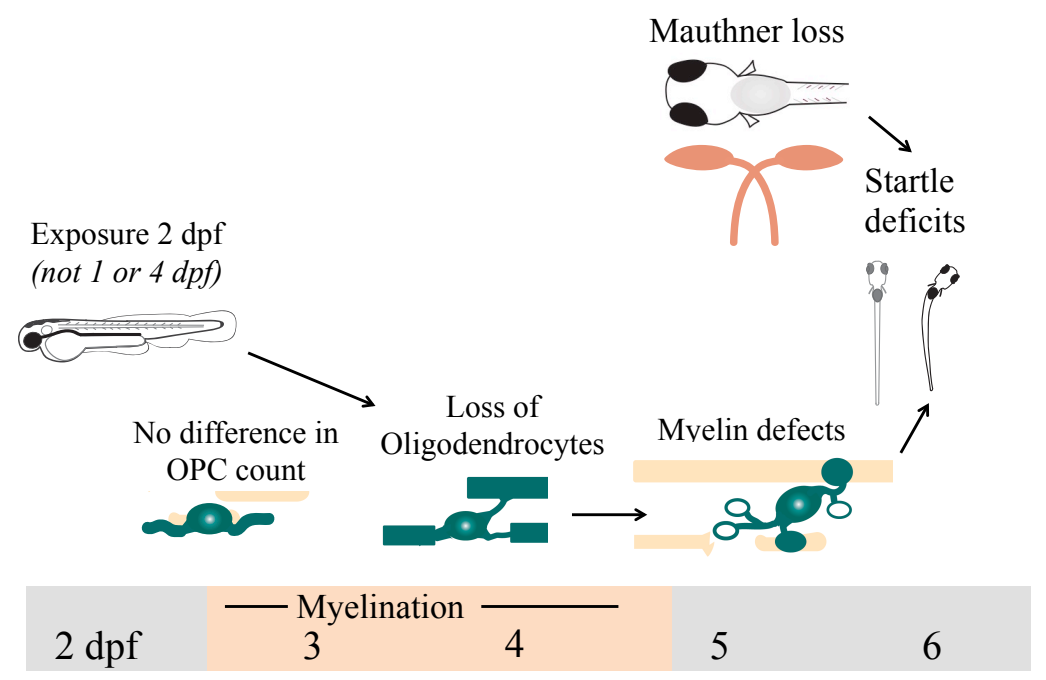

Figure 28: Building our proposed model: effects in oligodendrocyte lineage 
Now that we have looked at the oligodendrocytes, let's revisit the Mauthner cells. Recall that later in the larval stage, I found that the Mauthner cell that is required for startle responses was absent. I then wanted to determine whether the Mauthner cells were absent shortly after exposure, but prior to the initiation of myelination. Two teal arrows point to the two large Mauthner cells found in all control larvae (76/76) in Figure 29. In contrast, DomA-exposed larvae have a range of phenotypes. Some DomA-exposed larvae have 2 Mauthners. Some have 1 Mauthner, but the majority of DomA-exposed larvae have none.

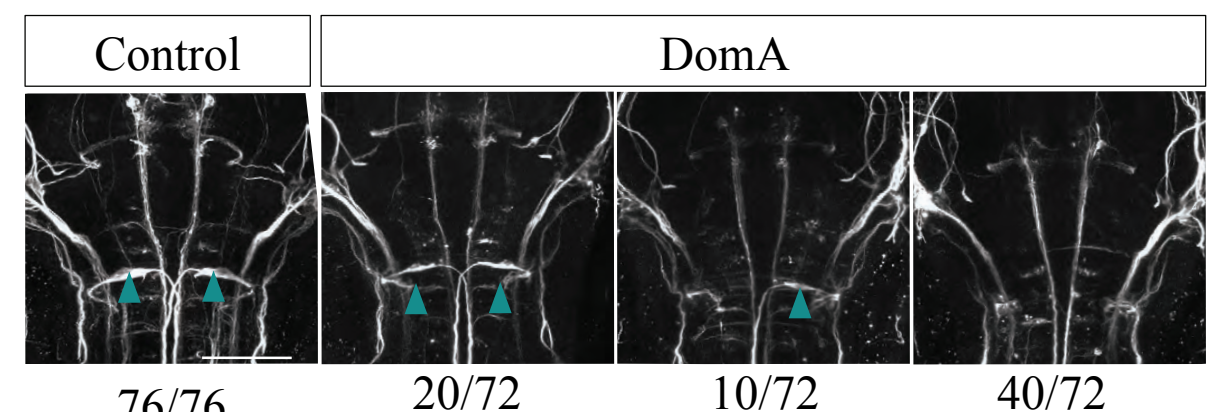

Figure 29: Mauthner cells are lost prior to myelination in DomA exposed fish.

We now know that the Mauthner is lost even earlier than when myelination occurs. This emphasizes that loss of the Mauthner cannot result from myelination defects. Thus, DomA may be targeting neurons like the Mauthner cell, leading to an altered cellular environment in the spinal cord prior to myelination. An aberrant cellular environment could then contribute to the observed effects we see in the oligodendrocyte lineage.

Speaking of this cellular environment, let us take a look at myelination in the spinal cord. In control animals, there is the correct balance between the number of oligodendrocytes that are ready to myelinate, and their targets, which are the spinal cord axons (Fig. 30).

Control

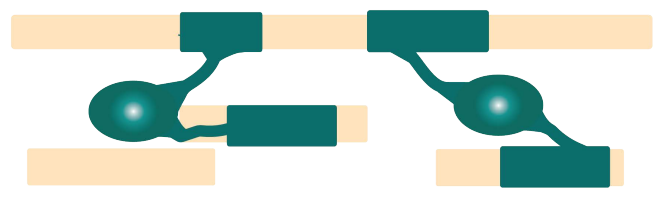

Figure 30: Proper myelination within the spinal cord is the result of the balance between oligodendrocytes and their targets (axons). 
Let's now take a situation where we have fewer axons in the spinal cord. Now that oligodendrocytes have fewer targets, what do they do? A study looked specifically at this conundrum by using a genetic mutant that has fewer spinal cord axons. ${ }^{22,23}$ They found that the reduction in axonal surface area also led to a reduction in the number of oligodendrocytes. This is potentially a feedback process, which attempts to correct the mismatch in oligodendrocytes to axons. They also saw the appearance of circular profiles and identified them as myelinated neuronal cell bodies. Normally, only axons are myelinated, so this is an aberrant phenotype. Researchers attributed these effects to the fact that there was a mismatch in axonal surface area relative to the number of oligodendrocytes. As there are fewer axons in the environment to myelinate, oligodendrocytes start to myelinate other features, including neuronal cell bodies (Fig. 31). It is possible that such a thing is occurring with DomA-exposed fish too. The loss of the Mauthner cell could contribute to the effects we are seeing with oligodendrocytes and myelin. Recall that DomA-exposed fish had similar characteristics to those seen in the genetic model with reduced axons. DomA-exposed larvae have reduced myelin and had these strange circular features as well.

Loss of axons $=$ loss of targets What do the oligodendrocytes do now?
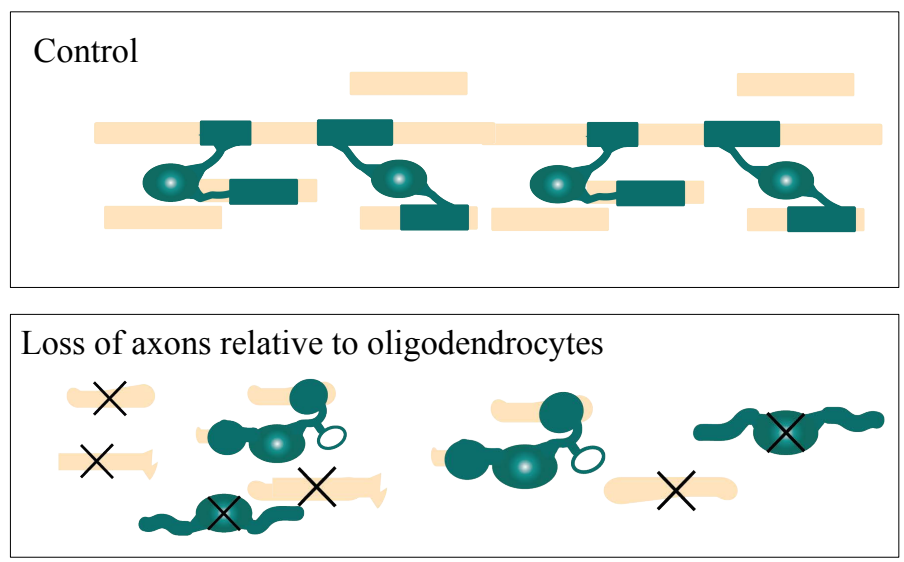

Figure 31: The loss of axonal targets leads to aberrant myelination patterns.

I used another transgenic fish line that labels the membranes of both oligodendrocytes and neurons. In red (Figure 32), you see these unusual circular profiles produced by the 
oligodendrocytes in DomA-exposed fish. In green, you see these almost honeycomb-like structures that represent densely packed neuronal cell bodies within the spinal cord.

In the merged image, I see that the circular oligodendrocyte membranes appear to be in the same location as neuronal cell bodies.
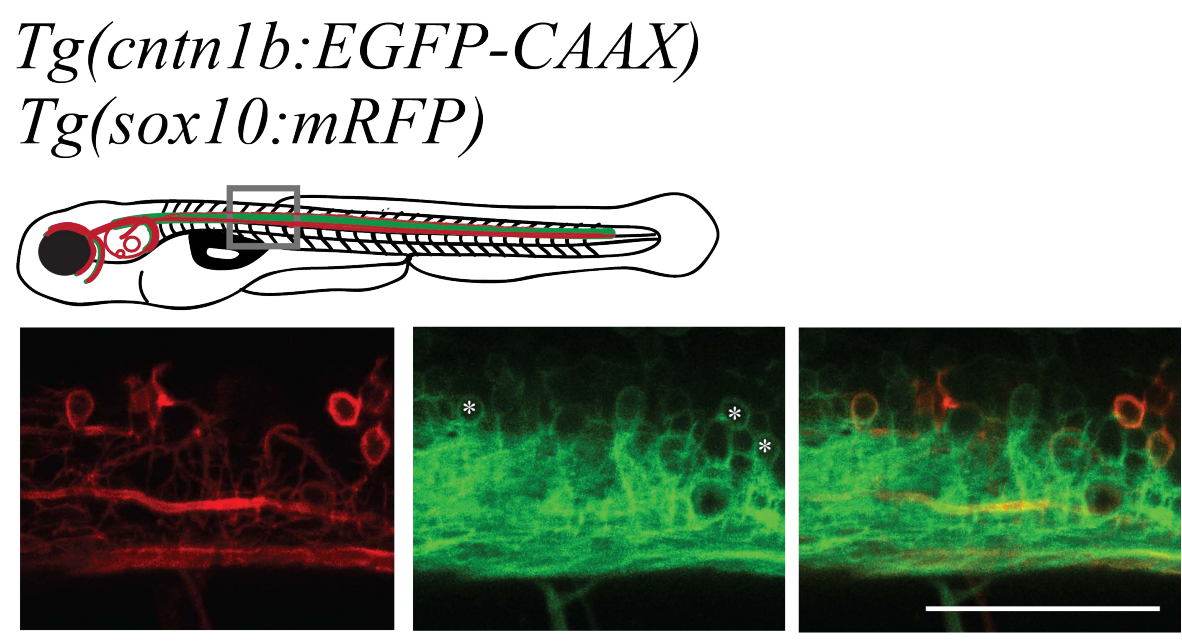

Figure 32: Light microscopy data suggests that these circular features may be ectopically myelinated neuronal cell bodies.

This suggests that the oligodendrocyte membrane is wrapping neuronal cell bodies (Fig. 33). To confirm this, I am doing electron microscopy, which gives me better resolution to identify these circular features. While still being processed, these data seem to confirm that these myelin circles are neuronal cell bodies wrapped in myelin.

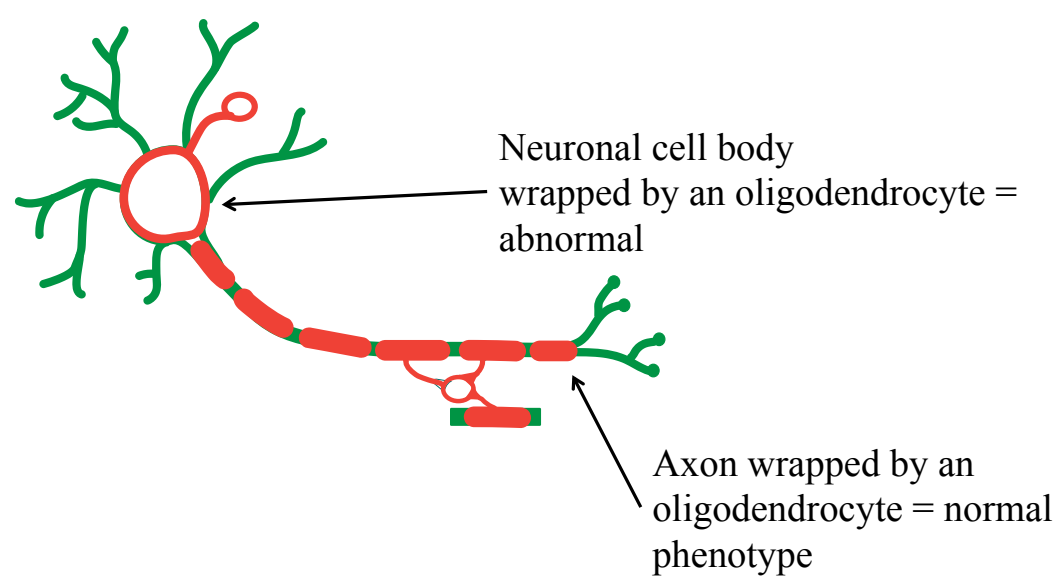

Figure 33: Schematic of a neuron wrapped by myelin 


\section{Proposed model for DomA toxicity}

With all this, I have a working model for how developmental exposures to DomA causes toxicity. There is a loss of Mauthner cells that precedes the disruptions in myelin. The loss of the Mauthner cell contributes to a reduced axonal surface area in the spinal cord (purple arrows in Figure 34). There is also the loss of oligodendrocytes. The loss of the oligodendrocytes may result from the reduced axonal surface area - a feedback process that attempts to correct the discrepancies between the axonal surface area and the oligodendrocytes present. Myelin defects may also result from the reduced axonal surface area, as oligodendrocytes start to myelinate neuronal cell bodies in the absence of enough axons. Both of these phenotypes have been found in a genetic model that lacks axons, so it is possible that the myelin defects and loss of oligodendrocytes are just secondary effects of the loss of axonal surface area (blue arrows).

I previously proposed (Figure 28) that DomA might directly bind to and target oligodendrocytes as well. This may still be possible as these cells have the receptors to respond to DomA (orange arrows in Fig. 34). All of these elements can then contribute to the aberrant startle behavior we observe (green arrows). There are many unknowns (question marks) in the proposed model. The glutamate receptors to which DomA binds are expressed in multiple cell types in the nervous system. DomA could conceivably be affecting multiple cell types and processes in concert.

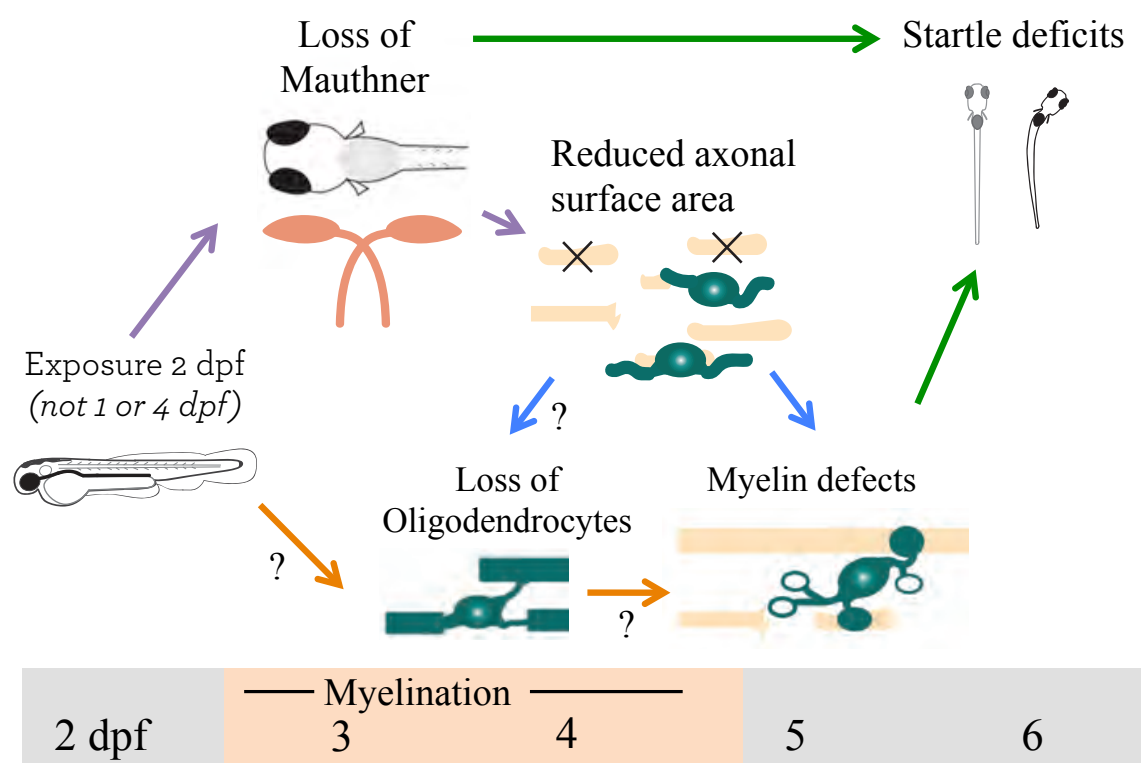

Figure 34: Proposed model for domoic acid toxicity 


\section{Implications for human health}

Stepping back from these details, we should ask - what could this mean for human health? My current research identifies a potential mechanism for domoic acid toxicity that occurs during a susceptible window of exposure in early development.

\section{$\underline{\text { Regional and cellular targets }}$}

I found pronounced changes in spinal cord structure following DomA treatment. This highlights how DomA targets not just the brain but also the spinal cord. I also found that selected cells were lost following DomA exposures. Humans do not have Mauthner cells, but have a group of functionally equivalent neurons. Thus, this research does not identify the specific neurons targeted in humans. However, it may provide us with clues as to which characteristics make some cell types more sensitive to DomA toxicity compared to other cell types. Perhaps neurons that have large axons, are excitable, and are located in the spinal cord are more sensitive to DomA exposure.

\section{Cellular mechanisms and timing of exposure}

I also found that the loss of axons right before a big wave of myelination leads to aberrant myelin phenotypes. We could of course speculate whether DomA exposures in humans prior to myelination could have important consequences. If myelination is truly a target during human exposure, this has important implications for human health. Unlike fish, myelination in humans is prolonged, and spans from late prenatal development all the way to early childhood or later. ${ }^{24}$ This would lead to a potentially prolonged window of susceptibility to DomA exposures.

\section{Overall implications: safe doses, important periods for exposure}

These findings would have to be tested in a variety of other model systems, but they do provide directions for future investigation. The identification of specific cellular and molecular targets has provided us with possible endpoints to assess in humans, along with clues as to which periods in human development are most vulnerable and thus most necessary to protect from exposure. 
Given what we already know about the effects from DomA for chronic consumers, along with these potential effects with developmental exposures, we may want to rethink what we consider is a 'safe' limit for DomA. Indeed, the Washington State Department of Health has released an official statement recommending that people not eat more than 15 razor clams per month, especially for women who are pregnant or nursing, or to young children. Such an advisory should be provided nationally, especially in places where algal blooms that contain DomA are known to occur.

\section{Future directions: adult phenotypes}

Finally, now that we have a better understanding of the underlying cellular and molecular mechanisms for developmental exposures to domoic acid, a next major step of this research is to understand the long-term effects. Harnessing the zebrafish model, we could assess different functional phenotypes in adults and connect them to initiating events in early development. This step will be critical to identifying whether there are long-term consequences, which will point the way towards more accurate hazard assessments and preventative measures. 


\section{REFERENCES}

1. Barker, D. J. P. The origins of the developmental origins theory. J. Intern. Med. 261, 4127 (2007).

2. Barker, D. J. et al. Fetal nutrition and cardiovascular disease in adult life. Lancet (London, England) 341, 938-41 (1993).

3. Haugen, A. C., Schug, T. T., Collman, G. \& Heindel, J. J. Evolution of DOHaD: the impact of environmental health sciences. J. Dev. Orig. Health Dis. 6, 55-64 (2015).

4. Tran, N. Q. V. \& Miyake, K. Neurodevelopmental Disorders and Environmental Toxicants: Epigenetics as an Underlying Mechanism. Int. J. Genomics 2017, 7526592 (2017).

5. Brulle, R. J. \& Pellow, D. N. Environmental Justice: Human Health and Environmental Inequalities. Annu. Rev. Public Health 27, 103-124 (2006).

6. Brooks, N. \& Sethi, R. The Distribution of Pollution: Community Characteristics and Exposure to Air Toxics. J. Environ. Econ. Manage. 32, 233-250 (1997).

7. Mikati, I., Benson, A. F., Luben, T. J., Sacks, J. D. \& Richmond-Bryant, J. Disparities in Distribution of Particulate Matter Emission Sources by Race and Poverty Status. Am. J. Public Health 108, 480-485 (2018).

8. Waalwijk, C. et al. Quantitative detection of Fusarium spp. and its correlation with fumonisin content in maize from South African subsistence farmers. World Mycotoxin J. 1, 39-47 (2008).

9. Shephard, G. S. et al. Multiple mycotoxin exposure determined by urinary biomarkers in rural subsistence farmers in the former Transkei, South Africa. Food Chem. Toxicol. 62, 217-225 (2013).

10. Hove, M. et al. Occurrence and risk assessment of mycotoxins in subsistence farmed maize from Zimbabwe. Food Control 69, 36-44 (2016).

11. Berdalet, E. et al. Marine harmful algal blooms, human health and wellbeing: challenges and opportunities in the 21st century. J. Mar. Biol. Assoc. United Kingdom 96, 61-91 (2016).

12. Anderson, D. M., Cembella, A. D. \& Hallegraeff, G. M. Progress in Understanding Harmful Algal Blooms: Paradigm Shifts and New Technologies for Research, Monitoring, and Management. Ann. Rev. Mar. Sci. 4, 143-176 (2012).

13. Dolah, F. M. Van, Roelke, D. \& Greene, R. M. Health and Ecological Impacts of Harmful Algal Blooms: Risk Assessment Needs. Hum. Ecol. Risk Assess. An Int. J. 7, 1329-1345 (2001).

14. Backer, L. C., McGillicuddy, D. J. \& Jr. Harmful Algal Blooms: At the Interface Between Coastal Oceanography and Human Health. Oceanography (Wash. D. C). 19, 94-106 (2006).

15. Grattan, L. M., Holobaugh, S. \& Morris, J. G. Harmful algal blooms and public health. Harmful Algae 57, 2-8 (2016).

16. Zhu, Z. et al. Understanding the blob bloom: Warming increases toxicity and abundance of the harmful bloom diatom Pseudo-nitzschia in California coastal waters. Harmful Algae 67, 36-43 (2017).

17. McKibben, S. M. et al. Climatic regulation of the neurotoxin domoic acid. Proc. Natl. Acad. Sci. U. S. A. 114, 239-244 (2017).

18. Washington Department of Fish and Wildlife. Razor Clams Latest Domoic acid levels. 
(2019). at <https://wdfw.wa.gov/fishing/shellfish/razorclams/domoic_levels.html $>$

19. Petroff, R. et al. Chronic, Low-Level Oral Exposure to Marine Toxin, Domoic Acid, Alters Whole Brain Morphometry in Nonhuman Primates. Neurotoxicology (2019). doi:10.1101/439109

20. Stiles, J. \& Jernigan, T. L. The basics of brain development. Neuropsychol. Rev. 20, $327-$ 48 (2010).

21. Ackerman, S. Discovering the Brain. (National Academies Press (US), 1992). doi:10.17226/1785

22. Almeida, R. \& Lyons, D. Oligodendrocyte Development in the Absence of Their Target Axons In Vivo. PLoS One 11, e0164432 (2016).

23. Almeida, R. G. et al. Myelination of Neuronal Cell Bodies when Myelin Supply Exceeds Axonal Demand. Curr. Biol. 28, 1296-1305.e5 (2018).

24. Jakovcevski, I., Filipovic, R., Mo, Z., Rakic, S. \& Zecevic, N. Oligodendrocyte development and the onset of myelination in the human fetal brain. Front. Neuroanat. 3, 5 (2009). 